\title{
Soil Characterization Database for the Area 5 Radioactive Waste Management Site, Nevada Test Site, Nye County, Nevada
}

by

\author{
Y. J. Lee, R. D. Van Remortel, and K. E. Snyder \\ Lockheed Martin Services Group \\ Las Vegas, Nevada
}

Prepared for:

Bechtel Nevada

Las Vegas, Nevada

and

U.S. Department of Energy

National Nuclear Security Administration

Nevada Site Office

Las Vegas, Nevada

January 2005 


\title{
DISCLAIMER STATEMENT
}

Reference herein to any specific commercial product, process, or service by trade name, trademark, manufacturer, or otherwise, does not necessarily constitute or imply its endorsement, recommendation, or favoring by the U.S. Government or any agency thereof or its contractors or subcontractors.

\section{AVAILABILITY STATEMENT}

Available to the public, in paper, from-

\author{
U.S. Department of Commerce \\ National Technical Information Service \\ 5285 Port Royal Road \\ Springfield, VA, 22161-0002 \\ Telephone: 800.553 .6847 \\ Fax: 703.605.6900 \\ E-mail: orders@ntis.gov \\ Online ordering: http://www.ntis.gov/ordering.htm
}

Available electronically at http://www.osti.gov/bridge.

Available for a processing fee to U.S. Department of Energy and its contractors, in paper, from-

U.S. Department of Energy

Office of Scientific and Technical Information

P.O. Box 62

Oak Ridge, TN 37831-0062

Telephone: 865.576 .8401

Fax: 865.576.5728

E-mail: reports@adonis.osti.gov 
This page intentionally left blank. 


\title{
Soil Characterization Database for the Area 5 Radioactive Waste Management Site, Nevada Test Site, Nye County, Nevada
}

DOE/NV/11718--1014

\begin{abstract}
Soils were characterized in an investigation at the Area 5 Radioactive Waste Management Site at the U.S. Department of Energy Nevada Test Site in Nye County, Nevada. Data from the investigation are presented in four parameter groups: sample and site characteristics, U.S. Department of Agriculture (USDA) particle size fractions, chemical parameters, and American Society for Testing MaterialsUnified Soil Classification System (ASTM-USCS) particle size fractions. Spread-sheet workbooks based on these parameter groups are presented to evaluate data quality, conduct database updates, and set data structures and formats for later extraction and analysis. This document does not include analysis or interpretation of presented data.
\end{abstract}


This page intentionally left blank. 


\section{Table of Contents}

Abstract ............................................ iii

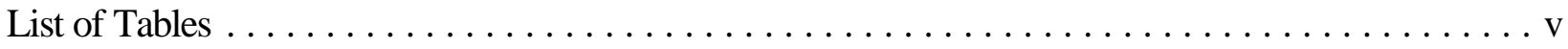

List of Acronyms and Abbreviations $\ldots \ldots \ldots \ldots \ldots \ldots \ldots \ldots \ldots \ldots \ldots \ldots \ldots \ldots \ldots \ldots \ldots$

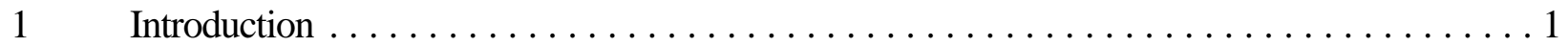

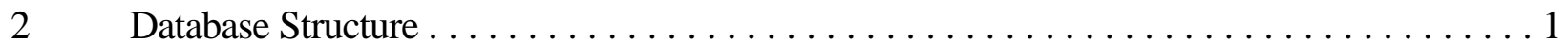

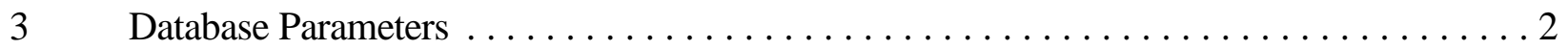

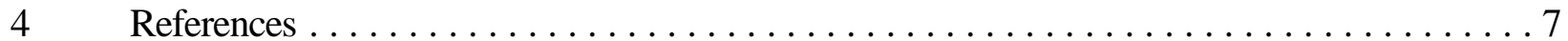

Appendix A Sample and Site Characteristics

Appendix B U.S. Department of Agriculture Particle Size Fractions

Appendix C Chemical Parameters

Appendix D American Society for Testing and Materials Unified Soil Classification System Particle Size Fractions

Distribution List

\section{List of Tables}

Number

Title

Page

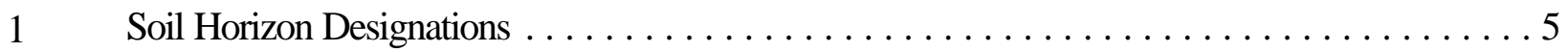

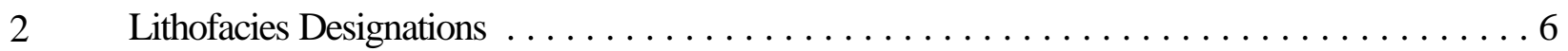

3 USDA Texture Class - Laboratory and Field $\ldots \ldots \ldots \ldots \ldots \ldots \ldots \ldots \ldots \ldots \ldots \ldots \ldots \ldots$

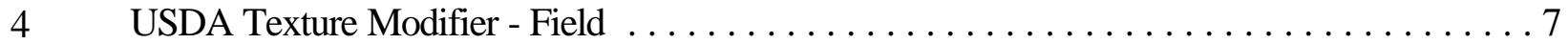

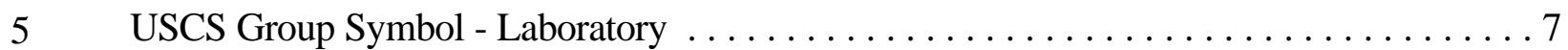




\section{List of Acronyms and Abbreviations}

ASTM

QA

RWSM

USCS

USDA
American Society for Testing and Materials

quality assurance

Radioactive Waste Management Site

Unified Soil Classification System

U.S. Department of Agriculture 


\section{INTRODUCTION}

This document presents the database of a soil characterization study conducted at the Area 5 Radioactive Waste Management Site (RWMS) on the U.S. Department of Energy Nevada Test Site in Nye County, Nevada. The database is maintained in a Microsoft ${ }^{\circledR}$ Excel Version 5.0 for Windows ${ }^{\mathrm{TM}}$ spreadsheet framework that is suitable for transfer into other spreadsheet or database formats. Column fields (parameters) include both descriptive field data and analytical laboratory data. Methods used to produce the analytical laboratory data are described in Snyder et al. (1996). Quality assurance (QA) criteria used to evaluate the quality of the data are described in Byers et al. (1996).

\section{DATABASE STRUCTURE}

The Excel Version 5.0 spreadsheet framework uses a workbook and worksheet data file structure. A workbook is made up of one or more worksheets, each containing data on a particular parameter, batch, or other characteristic.

The analytical laboratory constructed a batch workbook for each analytical batch of RWMS soil samples. The general naming convention for a batch workbook is "batch\#_ray.xls" (e.g., 401_ray.xls for Batch 401). An " $r$ " trailing the batch number (e.g., 401r_ray.xls) denotes a workbook that contains data for laboratory rerun of the batch for a specific parameter; the rerun workbook supersedes the initial batch data submission in the database. Within a batch workbook are several worksheets containing batch-wide data grouped by parameter (e.g., particle size analysis or pH). The batch workbooks were submitted by the laboratory to the QA staff for data evaluation and analysis.

The QA staff created evaluation workbooks (i.e., A5_work.xls) in which parameter data are linked to the batch workbooks using Excel formula linkages established between two or more workbooks, or between two or more worksheets within a workbook. Evaluation workbooks use a structure of parameter fields in sequential columns and specific sample records in sequential rows sorted by batch and sample numbers. These workbooks are used for evaluating data quality, conducting database updates, and setting data structures and formats for later extraction. Field and record cells are locked after editing to prevent errors from being entered into the database. Evaluation workbooks are not suitable for routine user data analysis because of their linkages.

Data from the appropriate evaluation workbooks or worksheets were extracted as non-linked (hard-data) values into a user workbook suitable for analysis purposes. The A5_data.xls workbook may include multiple worksheets, each containing selected hard-data values extracted from the evaluation workbooks. It is the repository for appropriate final parameter data and has undergone formatting, rounding, and thorough QA evaluation.

A primary label variable for the database and user workbooks is Field Sample Code. Its value is unique and illustrative to each record and sufficient to identify any sample in the database. Secondary label variables are Batch and Sample, which link an individual sample to the batch workbooks submitted by the laboratory. Field descriptive data, including horizon designation, sampling depths, and field texture, have been added to the evaluation and user workbooks for interpretive purposes. The 
remainder of the data represent the final reported values for the physical and chemical characteristics of the samples. Each physical and chemical parameter is reported in the units specified by the analytical method.

\section{DATABASE PARAMETERS}

The following column fields (parameters) represent the label variables and the physical and chemical soil characteristics in the Area 5 RWMS database. Appendix A contains data for the soil sample and site characteristics. Appendix B contains data for the U.S. Department of Agriculture (USDA) particle size fractions. Appendix C contains data for other physical and chemical parameters. Appendix D contains data for the American Society for Testing Materials (ASTM) Unified Soil Classification System (USCS) particle size fractions. Specific parameters, in the order of their appearance in the database workbook, are:

\section{Parameter \\ Batch \\ Sample \\ Field Sample Code}

Horizon

Upper Depth

Lower Depth

Location

Profile

Allostratigraphic Unit

Allostratigraphic Subunit

Lithofacies

Geomorphic Surface

Soil Texture - Lab
Description or Definition

Laboratory batch number

Laboratory sample number within a batch

Code assigned in the field identifying the sample. For routine samples, the alphanumeric code includes: (1) a 4- or 6-character location element; (2) a 3-character profile element; (3) a 2-character allostratigraphic unit element; and (4) a 1- or 3-character lithofacies element. For QA samples, the code includes a 2- or 3-letter alpha element followed by a 2- or 5-character alphanumeric element; the alpha elements are DBD for double-blind duplicate, RM for reference material, and CU for referee laboratory reference material.

Soil horizon designation of the sample (Table 1)

Upper depth (centimeters) of soil horizon below ground surface

Lower depth (centimeters) of soil horizon below ground surface

Type of sampling site: $\mathrm{ST}=$ soil trench; $\mathrm{SC}=$ streamcut; $\mathrm{RC}=$ roadcut; $\mathrm{WB}=\mathrm{RWMS}$ facility pit or trench.

Profile number (P\#\#) described and sampled

Allostratigraphic unit (A\#) of the sample

Allostratigraphic subunit of the sample

Lithofacies designation of the sample (Table 2)

Geomorphic surface at the sampling location

USDA texture class estimated using analytical laboratory data; free of carbonate and organic matter (Table 3) 


\section{Parameter}

Soil Texture - Field

Texture Modifier-Field

Air-Dry Sample Weight

Air-Dry Fine Earth Weight

Ground Elevation

Northing

Easting

Total Pebbles

Coarse Pebbles

Medium Pebbles

Fine Pebbles

Total Sand

Very Coarse Sand

Coarse Sand

Medium Sand

Fine Sand

Very Fine Sand

Total Silt

\section{Description or Definition}

USDA textural class estimated by field personnel (Table 3)

USDA rock fragment volume textural modifier estimated by field personnel (Table 4)

Total air-dry weight (grams) of sample before sieving

Air-dry weight (grams) of the <2-millimeter (mm) sample

Elevation (feet) at ground surface of sampling location

Nevada State Plane northing coordinate (feet) of sampling location

Nevada State Plane easting coordinate (feet) of sample location

USDA total pebbles (air-dry weight percent) ranging from $75 \mathrm{~mm}$ to 2 $\mathrm{mm}$ within the $<75$-mm (<3-inch) sample; large fragments (cobble size and larger) were left in the field

USDA coarse pebbles (air-dry weight percent) ranging from $75 \mathrm{~mm}$ to $20 \mathrm{~mm}$ within the $<75$-mm (<3-inch) sample

USDA medium pebbles (air-dry weight percent) ranging from $20 \mathrm{~mm}$ to $5 \mathrm{~mm}$ within the $<75-\mathrm{mm}(<3$-inch) sample

USDA fine pebbles (air-dry weight percent) ranging from $5 \mathrm{~mm}$ to $2 \mathrm{~mm}$ within the $<75$ - $\mathrm{mm}(<3$-inch) sample

USDA total sand content (oven-dry weight percent) ranging from $2.0 \mathrm{~mm}$ to $0.05 \mathrm{~mm}$ within the $<2-\mathrm{mm}$ sample

USDA very coarse sand (oven-dry weight percent) ranging from $2.0 \mathrm{~mm}$ to $1.0 \mathrm{~mm}$ within the $<2-\mathrm{mm}$ sample

USDA coarse sand (oven-dry weight percent) ranging from $1.0 \mathrm{~mm}$ to $0.50 \mathrm{~mm}$ within the $<2-\mathrm{mm}$ sample

USDA medium sand (oven-dry weight percent) ranging from $0.50 \mathrm{~mm}$ to $0.25 \mathrm{~mm}$ within the $<2-\mathrm{mm}$ sample

USDA fine sand (oven-dry weight percent) ranging from $0.25 \mathrm{~mm}$ to $0.10 \mathrm{~mm}$ within the $<2-\mathrm{mm}$ sample

USDA very fine sand (oven-dry weight percent) ranging from $0.10 \mathrm{~mm}$ to $0.05 \mathrm{~mm}$ within the $<2-\mathrm{mm}$ sample

USDA total silt content (oven-dry weight percent) ranging from $50 \mu \mathrm{m}$ to $2 \mu \mathrm{m}$ within the <2-mm sample 


\section{Parameter}

Coarse Silt

Fine Silt

Total Clay

Moist

$\mathrm{pH}$

CaCO3_Eq

CBD_Fe

CBD_Si

AOX_Fe

AOX_Si

USCS Group-Lab

Gravel_USCS

Sand_USCS

Silt+Clay_USCS

$\mathrm{P}<3$-inch

$\mathrm{P}<2$-inch

\section{Description or Definition}

USDA coarse silt (oven-dry weight percent) ranging from 50 micrometers ( $\mu \mathrm{m})$ to $20 \mu \mathrm{m}$ within the <2-mm sample

USDA fine silt (oven-dry weight percent) ranging from $20 \mu \mathrm{m}$ to $2 \mu \mathrm{m}$ within the $<2$-mm sample

USDA total clay (oven-dry weight percent) less than $2 \mu \mathrm{m}$ within the $<2$-mm sample

Air-to-oven-dry moisture content (oven-dry weight percent) of the $<2$-mm sample

$\mathrm{pH}$ of soil $(<2-\mathrm{mm}$ sample $)$ in water

Calcium carbonate $\left(\mathrm{CaCO}_{3}\right)$ equivalent (oven-dry weight percent) of the $<2$-mm sample

Iron extracted by citrate-bicarbonate-dithionite (oven-dry weight percent) from the $<2$-mm sample

Silicon extracted by citrate-bicarbonate-dithionite (oven-dry weight percent) from the $<2$-mm sample

Iron extracted by ammonium oxalate (oven-dry weight percent) from the $<2$-mm sample

Silicon extracted by ammonium oxalate (oven-dry weight percent) from the $<2$-mm sample

ASTM-USCS group symbol, using laboratory data for the $<3$-inch $(<75-\mathrm{mm})$ sample, including carbonate (Table 5); based on D2488 classification criteria.

ASTM-USCS gravel content (air-dry weight percent) ranging from 5 to $75 \mathrm{~mm}$ within the $<3$-inch $(<75-\mathrm{mm})$ sample; based on D2488 classification criteria

ASTM-USCS sand content (oven-dry weight percent) within the <3-inch (<75-mm) sample; based on D2488 classification criteria

ASTM-USCS total silt and clay content (oven-dry weight percent) within the $<3$-inch (<75-mm) sample, including carbonate; based on D2488 classification criteria

Calculated cumulative weight percentage of particles passing 3-inch sieve, reported on a $<3$-inch base.

Calculated cumulative weight percentage of particles passing 2-inch sieve, reported on a $<3$-inch base. 


Parameter
$P<1 \frac{1 / 2-i n c h}{}$
$P<1$-inch
$P<3 / 4$-inch
$P<d-$ inch
$P<\# 4$
$P<\# 10$
$P<\# 40$
$P<\# 200$

\section{Description or Definition}

Calculated cumulative weight percentage of particles passing $1 \frac{1}{2}-$-inch sieve, reported on a $<3$-inch base.

Calculated cumulative weight percentage of particles passing 1-inch sieve, reported on a $<3$-inch base.

Calculated cumulative weight percentage of particles passing 3/4-inch sieve, reported on a $<3$-inch base.

Calculated cumulative weight percentage of particles passing $d$-inch sieve, reported on a $<3$-inch base.

Calculated cumulative weight percentage of particles passing No. 4 $(4.75-\mathrm{mm})$ sieve, reported on a $<3$-inch base.

Calculated cumulative weight percentage of particles passing No. 10 (2.0-mm) sieve, reported on a $<3$-inch base.

Calculated cumulative weight percentage of particles passing No. 40 $(0.42-\mathrm{mm})$ sieve, reported on a $<3$-inch base.

Calculated cumulative weight percentage of particles passing No. 200 (0.074-mm) sieve, reported on a $<3$-inch base.

\section{Table 1. Soil Horizon Designations}

\begin{tabular}{ll}
\hline \hline Master Horizon or Suffix & Description \\
\hline A & A horizon \\
B & B horizon \\
C & C horizon \\
b & Buried horizon \\
k & Accumulation of secondary carbonate \\
m & Cemented in $>90 \%$ of horizon \\
q & Accumulation of secondary silica \\
t & Accumulation of clay \\
w & Color or textural B horizon \\
\hline \hline
\end{tabular}

Note: Leading numerals indicate a discontinuity from overlying materials. Trailing numerals indicate a sequence of similar horizons. Dual letters indicate transitional characteristics. A virgule (/) separating 2 horizons designations indicates sample contains distinct parts of each horizon. A prime is used when a horizon has the same master horizon and suffix designation as an overlying horizon. 
Table 2. Lithofacies Designations

\begin{tabular}{|c|c|}
\hline Lithofacies & Description \\
\hline a & Observable pedogenic development \\
\hline $\mathrm{b}$ & Fine-grained (pebbles and smaller); no bedding; moderately to poor sorting \\
\hline c & $\begin{array}{l}\text { Coarse-grained (medium pebbles and larger); no bedding; moderately to well } \\
\text { sorted }\end{array}$ \\
\hline$d$ & Fine-grained; bedded; moderately to poorly sorted \\
\hline e & Fine-grained; bedded; moderately to well sorted \\
\hline$f$ & $\begin{array}{l}\text { Coarse-grained with } 25 \text { to } 50 \% \text { clasts }>75-\mathrm{mm} \text {; little to no bedding; poorly } \\
\text { sorted }\end{array}$ \\
\hline g & $\begin{array}{l}\text { Coarse-grained with } 50 \text { to } 75 \% \text { clasts }>75 \text {-mm; little to no bedding; poorly } \\
\text { sorted }\end{array}$ \\
\hline $\mathrm{h}$ & Coarse-grained with $>75 \%$ clasts $>75-\mathrm{mm}$; little to no bedding; poorly sorted \\
\hline $\mathrm{i}$ & Pebbly; bedded; moderately sorted \\
\hline
\end{tabular}

Table 3. USDA Texture Class - Laboratory and Field

\begin{tabular}{cll}
\hline \hline Abbreviation & Definition \\
\hline VCOS & Sand \\
COS & Very coarse sand \\
FS & Coarse sand \\
VFS & Fine sand \\
LS & Very fine sand \\
LCOS & Loamy sand \\
LFS & Loamy coarse sand \\
LVFS & Loamy fine sand \\
SL & Loamy very fine sand \\
COSL & Sandy loam \\
FSL & Coarse sandy loam \\
VFSL & Fine sandy loam \\
L & Very fine sandy loam \\
SCL & Loam \\
CL & Sandy clay loam \\
\hline \hline
\end{tabular}


Table 4. USDA Texture Modifier - Field

\begin{tabular}{ll}
\hline \hline Abbreviation & Definition \\
\hline $\mathrm{GR}$ & Gravelly \\
$\mathrm{CB}$ & Cobbly \\
$\mathrm{CN}$ & Channery \\
$\mathrm{GRV}$ & Very gravelly \\
$\mathrm{CBV}$ & Very cobbly \\
$\mathrm{CNV}$ & Very channery \\
$\mathrm{GRX}$ & Extremely gravelly \\
$\mathrm{CBX}$ & Extremely cobbly \\
$\mathrm{CNX}$ & Extremely channery \\
\hline \hline
\end{tabular}

Table 5. USCS Group Symbol - Laboratory

\begin{tabular}{cl}
\hline \hline Group Symbol & Group Name \\
\hline GM & Sandy silt (with sand) \\
SM & Silty sand (with gravel) \\
SW & Well-graded sand (with gravel) \\
SW-SM & Well-graded sand with silt (and gravel) \\
\hline \hline
\end{tabular}

\section{REFERENCES}

Byers, G.E., R.D. Van Remortel, and K.E. Snyder, 1996. Quality Assurance Report for a Soil Characterization Study at the Area 5 Radioactive Waste Management Facility, Nevada Test Site, Nye County, Nevada. U.S. Department of Energy, Las Vegas, NV.

Snyder, K.E., G.E. Byers, and R.D. Van Remortel, 1996. Handbook of Laboratory Methods of Soil Analysis. U.S. Department of Energy, Las Vegas, NV. 
This page intentionally left blank. 


\section{APPENDIX A}

Sample and Site Characteristics 


\begin{tabular}{|c|c|c|c|c|c|c|c|c|c|c|c|c|c|c|c|c|c|c|c|c|c|}
\hline (H) 6u!nseg & $\begin{array}{l}\text { 焉 } \\
\text { 足 } \\
\text { p. }\end{array}$ & $\begin{array}{l} \\
0 \\
0 \\
5 \\
0 \\
0\end{array}$ & $\mathbb{C}$ & 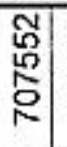 & 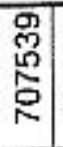 & $\begin{array}{l}\text { 呺 } \\
\text { 号 } \\
\text { 员 }\end{array}$ & $\begin{array}{l}\text { 品 } \\
\text { 员 } \\
\text { 总 }\end{array}$ & 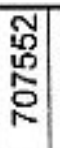 & $\begin{array}{l}0 \\
0 \\
0 \\
0 \\
0\end{array}$ & 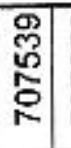 & $\begin{array}{l}\text { त్ } \\
\text { م్ } \\
\text { م }\end{array}$ & $\mathbb{Z}$ & 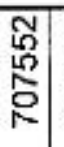 & 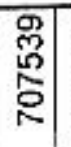 & 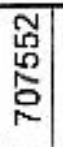 & 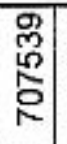 & 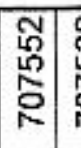 & 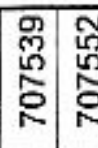 & 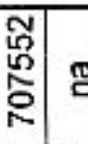 & पू & Tू \\
\hline (ม) ธน!บนัN & $\begin{array}{l}0 \\
0 \\
0 \\
0 \\
\mathbb{P} \\
\end{array}$ & 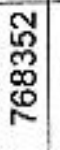 & $\Phi$ & 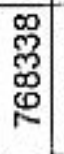 & $\begin{array}{l}N \\
N \\
\infty \\
0 \\
\mathbb{N}\end{array}$ & 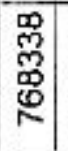 & 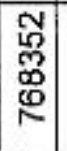 & 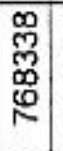 & 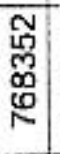 & 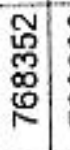 & 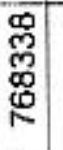 & $\stackrel{\square}{\square}$ & 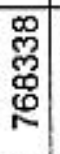 & $\begin{array}{l}N \\
\mathbb{N} \\
\infty \\
\mathbb{\infty} \\
\mathbb{N}\end{array}$ & 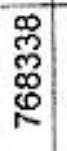 & $\begin{array}{l}\mathbb{N} \\
\mathbb{D} \\
\mathbb{D} \\
\mathbb{N}\end{array}$ & 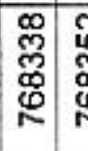 & 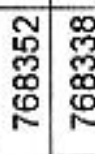 & & g & $\mathbb{T}$ \\
\hline 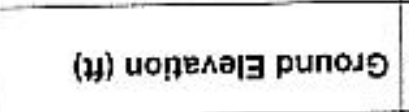 & $\frac{\vec{\sigma}}{m}$ & $\frac{\hat{\sigma}}{m}$ & g & के & $\frac{\grave{\sigma}}{m}$ & 兽 & $\frac{\mathrm{s}}{\mathrm{m}}$ & $\frac{5}{\circ}$ & 可 & $\frac{5}{5}$ & 点 & $\mathbb{T}$ & के & $\frac{5}{m}$ & 莺 & के & ⿳亠口冋口幺 & के & $\bar{m}$ & $\underline{\underline{g}}$ & $\mathbb{M}$ \\
\hline 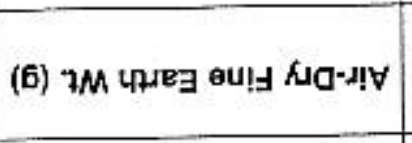 & 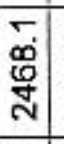 & 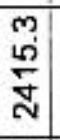 & 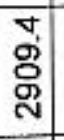 & $\begin{array}{l}0 \\
0 \\
\end{array}$ & ঙ্ষ & $\begin{array}{l}\infty \\
\mathfrak{N} \\
\stackrel{N}{\sim}\end{array}$ & 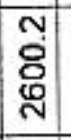 & $\begin{array}{l}0 \\
\infty \\
0 \\
0 \\
\\
\end{array}$ & 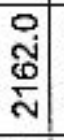 & $\begin{array}{l}\stackrel{\oplus}{0} \\
\sigma \\
\stackrel{2}{=}\end{array}$ & 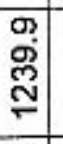 & 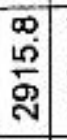 & 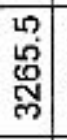 & 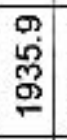 & 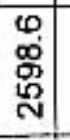 & $\begin{array}{l}\dot{v} \\
\dot{v} \\
\stackrel{5}{*}\end{array}$ & $\begin{array}{ll} \\
\text { J } \\
\text { 今 }\end{array}$ & 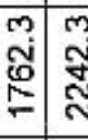 & 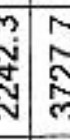 & & \begin{tabular}{l}
$\infty$ \\
\multirow{2}{*}{} \\
\\
\\
\end{tabular} \\
\hline 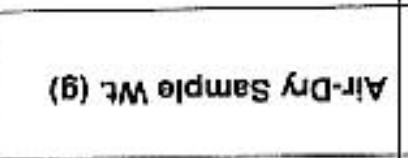 & 袋 & 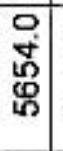 & 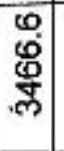 & 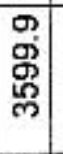 & 年 & 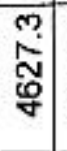 & 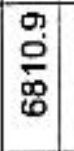 & $\begin{array}{c}\bar{g} \\
\stackrel{8}{\sigma}\end{array}$ & क् & $\begin{array}{l}m \\
0 \\
0 \\
0 \\
8\end{array}$ & 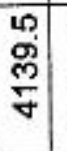 & 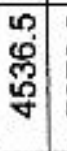 & $\begin{array}{l}0 \\
\stackrel{N}{N} \\
\stackrel{N}{*}\end{array}$ & 管 & $\begin{array}{l}0 \\
\dot{v} \\
0 \\
v\end{array}$ & $\begin{array}{l}\text { O } \\
\text { V } \\
\text { S }\end{array}$ & \begin{tabular}{l|l} 
N̦ \\
\multirow{2}{*}{}
\end{tabular} & 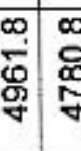 & 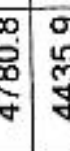 & & 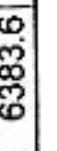 \\
\hline 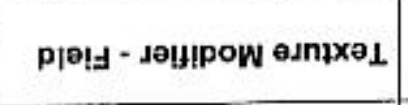 & $\frac{\alpha}{0}$ & $\begin{array}{l}\text { 采 } \\
\end{array}$ & 믇 & 赁 & 齐 & 采 & 紊 & $\begin{array}{l}\geq \\
⿱ 亠 䒑 \\
0\end{array}$ & $\begin{array}{l}\mathbb{1} \\
0\end{array}$ & 至 & $\underset{\substack{0 \\
0}}{\gtrless}$ & 읃 & 菏 & 采 & $\frac{\pi}{0}$ & 爻 & 临 & 惫 & $\frac{x}{5}$ & $=$ & 읻 \\
\hline 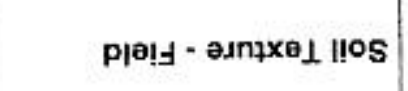 & $\dot{\omega}$ & 9 & 무 & 0 & 0 & $\vec{\omega}$ & 0 & $\underline{-1}$. & $\Omega$ & $\infty$ & es & ㄹ & 0 & 2 & $\vec{\omega}$ & $\infty$ & $\vec{\omega}$ & $\Omega$ क & ऊ) & 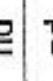 & 믈 \\
\hline 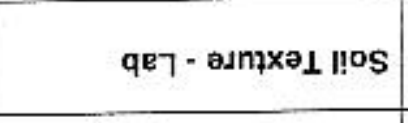 & $\begin{array}{l}\overrightarrow{1} \\
\\
\mathcal{U}\end{array}$ & \% & $\stackrel{心}{\rightleftarrows}$ & $\begin{array}{l}\infty \\
\mathrm{S}\end{array}$ & \begin{tabular}{|l|}
0 \\
\\
\\
\end{tabular} & $\begin{array}{l}\text { n } \\
0 \\
\text { S } \\
\end{array}$ & $\begin{array}{l}0 \\
0 \\
0\end{array}$ & 嵒 & $\begin{array}{l}\text { क } \\
\text { S } \\
\text { S }\end{array}$ & $\begin{array}{l}0 \\
0 \\
\end{array}$ & $\begin{array}{l}0 \\
0 \\
0\end{array}$ & $\underset{\square}{\mathbb{Z}}$ & $\begin{array}{l}50 \\
0 \\
\end{array}$ & क & $\infty$ & 8 & $\omega$ & $\begin{array}{ll}0 \\
\mathcal{S} \\
\end{array}$ & $\underset{4}{w}$ & $\frac{0}{5}$ & $\stackrel{0}{u}$ \\
\hline 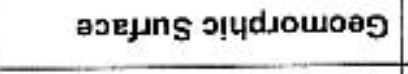 & $\mathscr{~}$ & 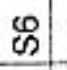 & $\mathbb{E}$ & 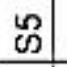 & $\mathscr{8}$ & 织 & $\mathscr{0}$ & 㤎 & $\begin{array}{l}\mathscr{0} \\
\vdots\end{array}$ & $\%$ & 员 & 刃्ञ & D & 号 & 峞 & $\begin{array}{c}\infty \\
0\end{array}$ & 峞 & 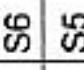 & 只 & 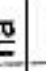 & 刃्) \\
\hline 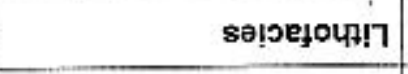 & $\infty$ & D. & $\mathbb{2}$ & 0 & $\infty$ & $\pi$ & ำ & (v) & 查 & 0 & $\frac{0}{\pi}$ & $\stackrel{\mathbb{C}}{\mathrm{C}}$ & $\pi$ & $\pi$ & $\pi$ & D & $\pi 2$ & 0 & $\pi$ & E & 픈 \\
\hline 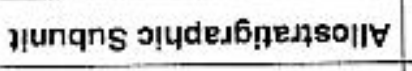 & $\Xi$ & () & $\mathbb{E}$ & $\widehat{\mathrm{d}}$ & $\Xi$ & $E$ & $\equiv$ & $\widehat{\mathrm{v}}$ & $E$ & 3 & $E$ & $\stackrel{\pi}{c}$ & $\Xi$ & $E$ & $\mathbb{\mathfrak { d }}$ & ल) & $E$ & 5 & $E$ & E) & 뜯 \\
\hline 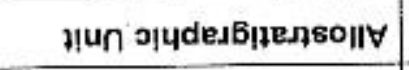 & $\stackrel{1}{\&}$ & 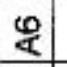 & $\underset{\mathcal{T}}{\mathrm{T}}$ & $\stackrel{\mathscr{Q}}{\varangle}$ & 足 & $\stackrel{0}{<}$ & 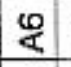 & 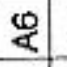 & $\stackrel{0}{<}$ & $\mathscr{8}$ & 足 & g & $\stackrel{40}{\&}$ & $\underset{8}{4}$ & $\mathscr{Q}$ & $\mathscr{8}$ & $\stackrel{\infty}{\&}$ & $\stackrel{0}{8} 4$ & 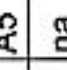 & ख̃ & 믇 \\
\hline elyodd & 용 & 욤 & $\mathbb{\Xi}$ & $\bar{\circ}$ & $\begin{array}{l}\text { 엉 } \\
\end{array}$ & Б & 今 & $\overline{8}$ & 용 & סे & ¿ & פ & 뭉 & ్ㅇㅁ & 횸. & О) & $\bar{\delta}$ & 응 & 5 & T2 & $\mathbb{E}$ \\
\hline uopมsoา & 它 & 它 & (2) & $\stackrel{5}{\circ}$ & 总 & 官 & 它 & 怘 & $\frac{5}{\infty}$ & $\frac{5}{5}$ & 5 & 은 & 它 & 它 & 家 & 它 & 它 & $\stackrel{5}{b}$ & 5 & 跑 & ⿷匚 \\
\hline (wכ) чวdeg دәмоา & 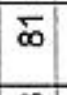 & 品 & T & $\bar{T}$ & $\stackrel{\circ}{\infty}$ & $\stackrel{N}{5}$ & 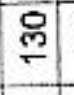 & 유 & 은 & 으 & फ़ & I & $\stackrel{\oplus}{\sim}$ & $\stackrel{5}{Ð}$ & $\begin{array}{l}\mathscr{O} \\
\end{array}$ & $\mathscr{m}$ & sे & $\stackrel{2}{2}$ & 吕 & $\mathbb{8}$ & 思 \\
\hline (wכ) ułdəa dəddn & $\begin{array}{l} \\
0 \\
2\end{array}$ & 焉 & (8) & g & $\begin{array}{ll}0 \\
0\end{array}$ & $\stackrel{40}{-2}$ & $\bar{\infty}$ & 0 & 욤 & 0 & 今े & $\mathbb{\Xi}$ & $\begin{array}{l}0 \\
\infty \\
\div\end{array}$ & 움 & 우 & ผ & $\bar{\Sigma} \$$ & 으 & ్ָ & व & 要 \\
\hline UOZ!̣JOH & 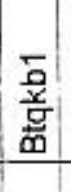 & 3 & 空 & 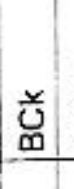 & 恙 & 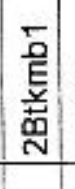 & 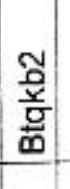 & $\leq$ & 总 & $\mathcal{Q}$ & $\begin{array}{l}\text { vo } \\
\frac{\mathbf{y}}{\bar{y}} \\
\overline{0}\end{array}$ & $\mathbb{E}$ & 丞 & $\begin{array}{c}\text { 号 } \\
\text { 恙 } \\
\text { N }\end{array}$ & 音 & $\mathcal{J}$ & 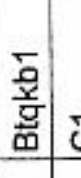 & 心 & 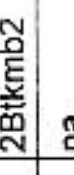 & 또 & 미다 \\
\hline apos әdues pjol! & 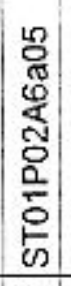 & 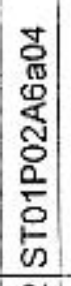 & $\begin{array}{l}\underbrace{}_{0} \\
\sum_{X}\end{array}$ & $\begin{array}{l}3 \\
0 \\
0 \\
0 \\
\frac{1}{2} \\
0 \\
0 \\
0 \\
0 \\
0\end{array}$ & 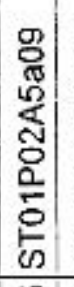 & 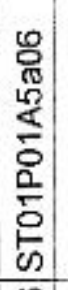 & 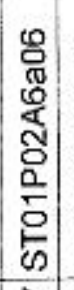 & & 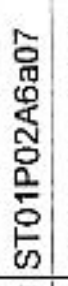 & 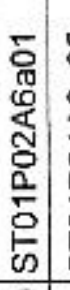 & 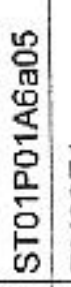 & 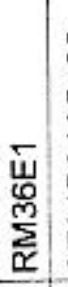 & 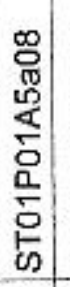 & 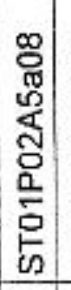 & 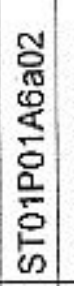 & $\begin{array}{l}3 \\
0 \\
0 \\
0 \\
0 \\
0 \\
0 \\
0 \\
0 \\
0 \\
0\end{array}$ & 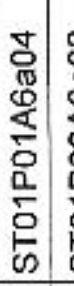 & 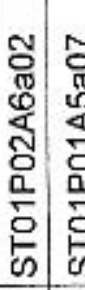 & 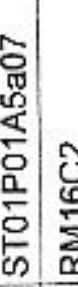 & 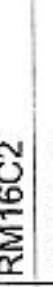 & 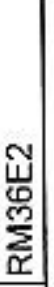 \\
\hline әdurs & - & $N$ & $m$ & $\nabla$ & $n$ & $\omega$ & $N$ & $\infty$ & $\sigma \mid$ & 유 & $\mp$ & 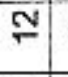 & $m$ & 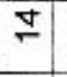 & $\stackrel{n}{-}$ & 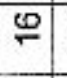 & $\approx$ & $\stackrel{\infty}{2} \div$ & 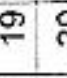 & ก) & $\bar{\sim}$ \\
\hline чэग्8 & 5 & 亏 & $\bar{g}$ & 5 & 守 & 守 & 5 & $\bar{g}$ & g & g & 与 & 윰 & 亏 & 함 & 웅 & 5 & 합 & $\overline{8}$ & 马 & 5 & 하 \\
\hline
\end{tabular}




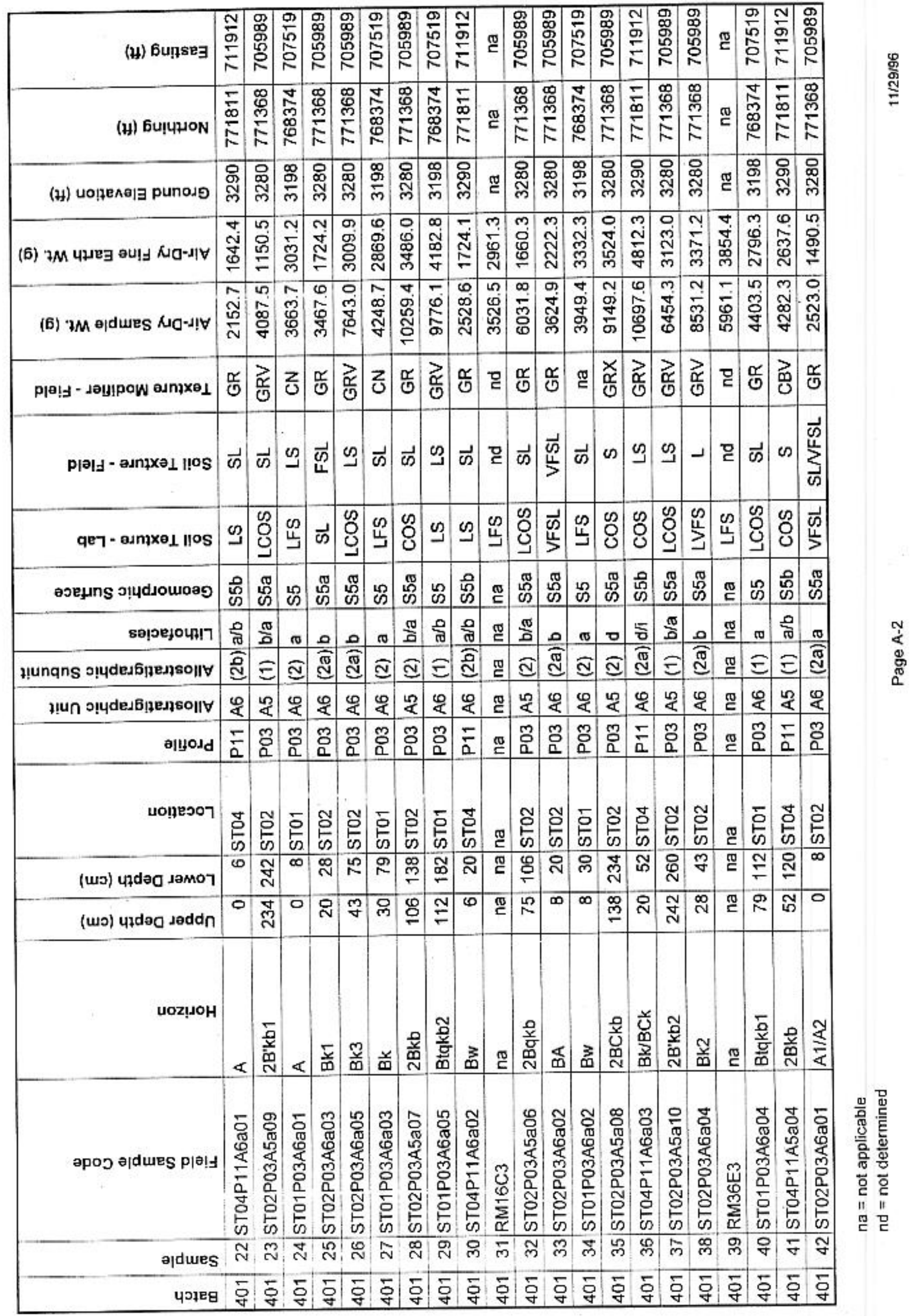




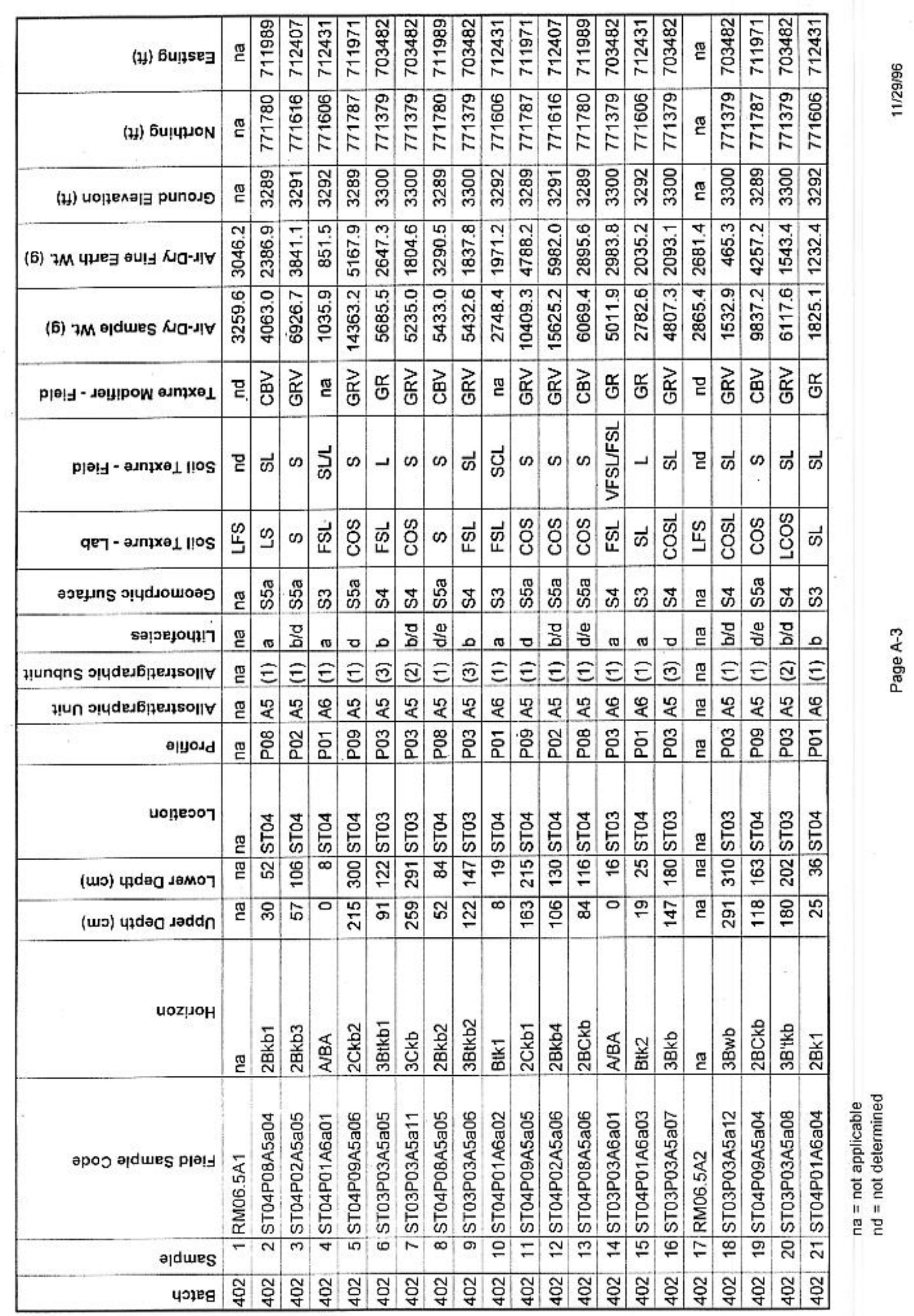




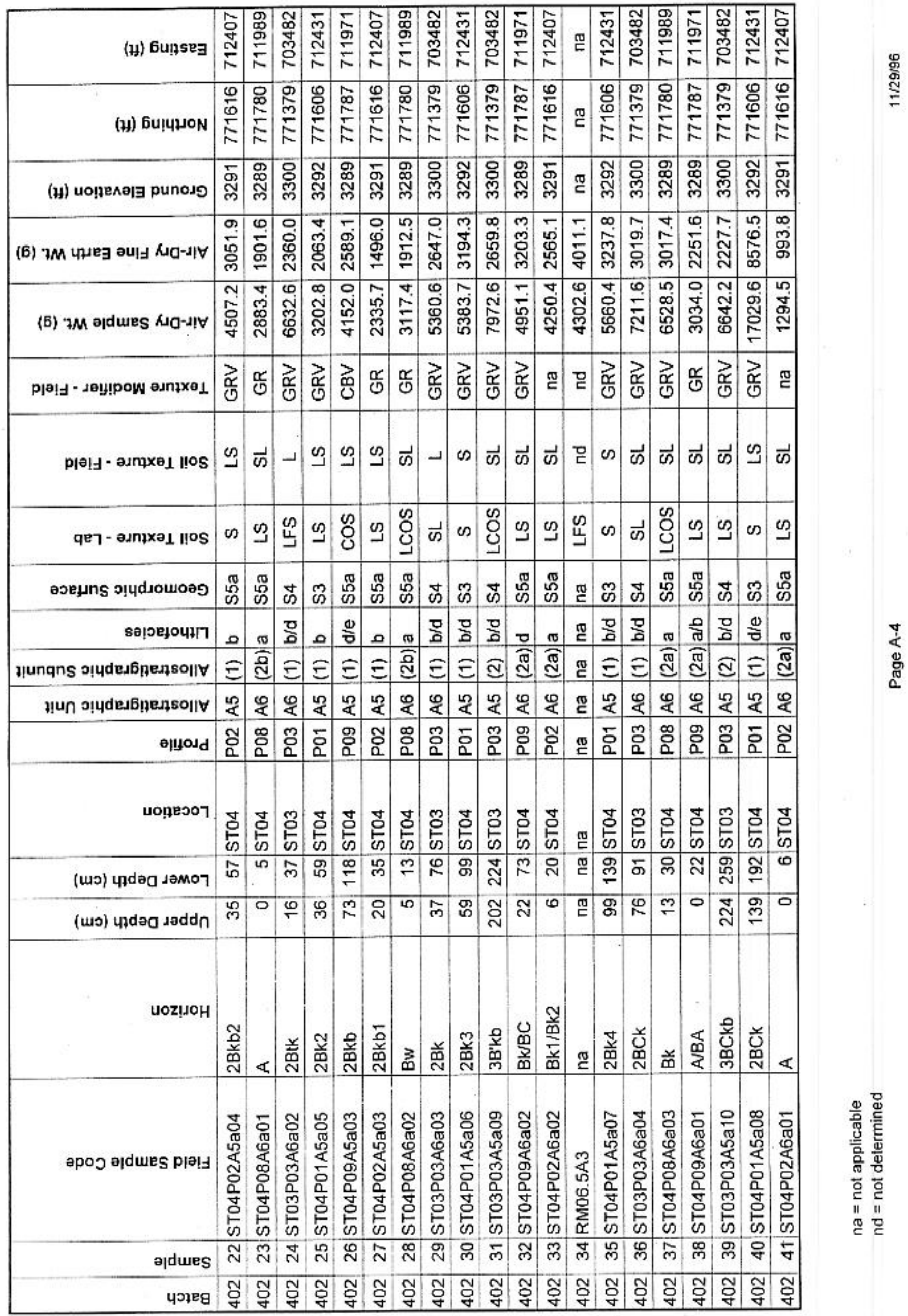




\begin{tabular}{|c|c|c|c|c|c|c|c|c|c|c|c|c|c|c|c|c|c|c|c|c|c|}
\hline (4) 6u!̣seg & 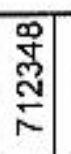 & $\stackrel{\mathbb{\Xi}}{\complement}$ & 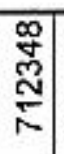 & \begin{tabular}{l|} 
\\
\\
O \\
O \\
\end{tabular} & $\stackrel{\mathbb{I}}{\mathbf{\Xi}}$ & $\begin{array}{l}\text { 呆 } \\
\text { 员 } \\
\text { P }\end{array}$ & $\begin{array}{c}\bar{y} \\
\bar{N} \\
\stackrel{2}{p}\end{array}$ & 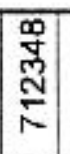 & $\begin{array}{l}\text { 导 } \\
\text { 号 } \\
\end{array}$ & 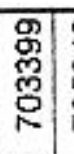 & 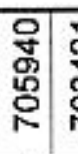 & 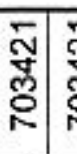 & ড় & 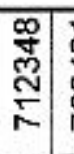 & 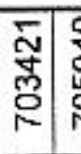 & 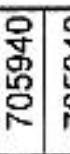 & $\begin{array}{l}\text { 导 } \\
\text { 号 } \\
\text { م }\end{array}$ & 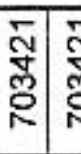 & 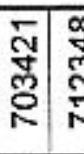 & 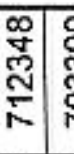 & 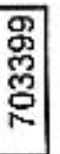 \\
\hline (अ) 6u!पนON & $\begin{array}{l}\infty \\
\mathbb{N} \\
\stackrel{N}{N}\end{array}$ & $\stackrel{\mathbb{I}}{\mathbf{I}}$ & 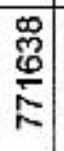 & $\begin{array}{l} \\
0 \\
5 \\
5 \\
\end{array}$ & (I) & 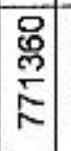 & $\begin{array}{l}\dot{W} \\
\text { ల్ } \\
E\end{array}$ & 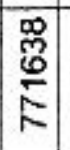 & 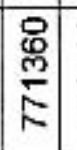 & 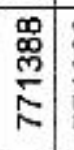 & $\begin{array}{l}\stackrel{8}{0} \\
\stackrel{N}{N}\end{array}$ & 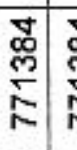 & 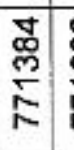 & $\begin{array}{l}\infty \\
\mathbb{N} \\
\stackrel{0}{0} \\
\Gamma\end{array}$ & 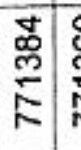 & 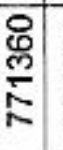 & 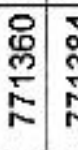 & $\begin{array}{l}\vec{J} \\
\stackrel{m}{m} \\
\stackrel{5}{N}\end{array}$ & 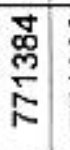 & $\begin{array}{l} \\
\tilde{0} \\
0 \\
\Sigma \\
\Sigma\end{array}$ & 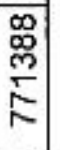 \\
\hline (H) uo!neselg punoנפ & $\begin{array}{l}\stackrel{N}{్} \\
\text { N }\end{array}$ & $\mathbb{2}$ & స్ల్లె & 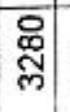 & $\mathbb{\widetilde { T }}$ & \begin{tabular}{l|} 
O్ \\
లె \\
\end{tabular} & 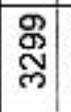 & $\begin{array}{l}N \\
\text { స్ల్ } \\
\end{array}$ & 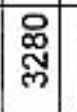 & 总 & $\begin{array}{l}\text { 品 } \\
\text { d }\end{array}$ & 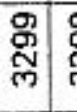 & 号 & $\begin{array}{l}\text { N } \\
\text { N }\end{array}$ & 㞼 & 品 & $\begin{array}{l}\text { : } \\
\text { స్ల }\end{array}$ & 眾 & 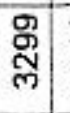 & 今్ & ర్ల్ల \\
\hline 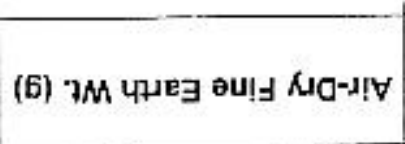 & $\begin{array}{l}0 \\
0 \\
\dot{8} \\
\mathbf{S}\end{array}$ & 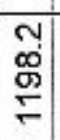 & 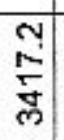 & $\begin{array}{l}\mathrm{N} \\
\mathbb{g} \\
\mathrm{g}\end{array}$ & $\begin{array}{l}\forall \\
\dot{y} \\
\tilde{y}\end{array}$ & 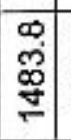 & $\begin{array}{l} \pm \\
E \\
E\end{array}$ & 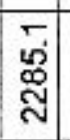 & 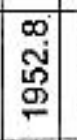 & $\begin{array}{l}\text { ¿ } \\
\text { ర్ల }\end{array}$ & 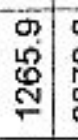 & 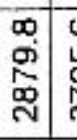 & $\begin{array}{l} \\
\omega \\
\rho \\
\tilde{m} \\
\end{array}$ & $\begin{array}{l}\text { ํ. } \\
\text { ํ. } \\
\text { ํ. }\end{array}$ & 立 & ज़ & 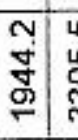 & $\begin{array}{l}\text { L } \\
\text { L } \\
\text { लि }\end{array}$ & $\begin{array}{l}n \\
\infty \\
\infty \\
\wp\end{array}$ & $\begin{array}{l}\stackrel{M}{\Sigma} \\
\Sigma \\
\Sigma\end{array}$ & $\begin{array}{l}0 \\
\stackrel{0}{0} \\
\stackrel{\circ}{\circ}\end{array}$ \\
\hline (6) ' $7 M$ әdures $M 0-\lambda \mid \forall$ & 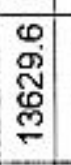 & 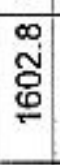 & 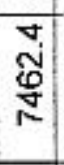 & 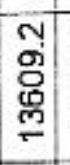 & $\begin{array}{l}\infty \\
\\
\dot{D} \\
\infty \\
\infty\end{array}$ & 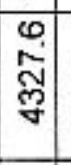 & 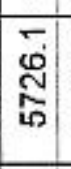 & 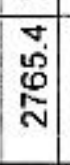 & ᄋ̊․ & $\begin{array}{l}\text { v } \\
\infty \\
\text { Oे } \\
\text { J }\end{array}$ & 柋 & $\begin{array}{l}0 \\
0 \\
0 \\
2 \\
\end{array}$ & $\begin{array}{l}0 \\
0 \\
0 \\
⿱ 亠 䒑 \\
\infty\end{array}$ & 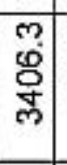 & 我 & $\begin{array}{l}m \\
\infty \\
\infty \\
\sigma\end{array}$ & 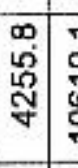 & $\frac{\check{\sigma}}{\bar{\phi}}$ & 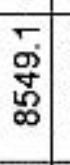 & N & \begin{tabular}{l}
0 \\
0 \\
0 \\
0 \\
\hdashline
\end{tabular} \\
\hline 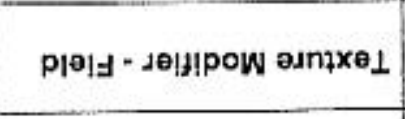 & $\frac{z}{0}$ & $\frac{1}{0}$ & 营 & 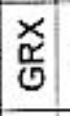 & D & 芺 & 怘 & 四 & 离 & $\underset{\substack{0 \\
0}}{\gtrless}$ & 齐 & 兴 & 永 & $\frac{\alpha}{0}$ & $\frac{\pi}{\mathbb{N}}$ & 品 & 永 & 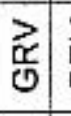 & 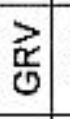 & 怘 & 片 \\
\hline pla!y - a dm & $\infty$ & $\vec{\omega}$ & $\omega$ & in & Q & - & $\vec{\omega}$ & $\frac{5}{\omega}$ & $\overrightarrow{0}$ & $\omega$ & $\vec{\omega}$ & $\vec{\omega}$ & $\infty$ & $\vec{W}$ & - & $\pi$ & -1 & $\infty$ & $\vec{\omega}$ & - & $\vec{\omega}$ \\
\hline 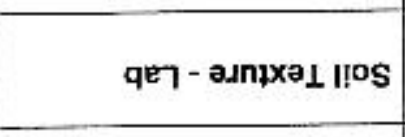 & \begin{tabular}{l|} 
\\
0 \\
0
\end{tabular} & $\begin{array}{l}\overrightarrow{0} \\
0 \\
0\end{array}$ & क & \begin{tabular}{|l|}
0 \\
0 \\
\end{tabular} & 出 & 0 & \begin{tabular}{|c|} 
कर \\
0 \\
\end{tabular} & 嵅 & $\begin{array}{l}\overrightarrow{0} \\
0 \\
0\end{array}$ & $\begin{array}{l} \\
0 \\
0\end{array}$ & $\begin{array}{l}0 \\
0 \\
0\end{array}$ & $\begin{array}{l}\text { Lo } \\
8 \\
\end{array}$ & $\begin{array}{l}0 \\
0 \\
0\end{array}$ & \begin{tabular}{|c|} 
\\
0
\end{tabular} & $\vec{\omega}$ & $\begin{array}{l}\text { en } \\
\text { O } \\
\end{array}$ & $\begin{array}{l}0 \\
0 \\
\end{array}$ & $\begin{array}{l}0 \\
0 \\
0\end{array}$ & $\begin{array}{l}\infty \\
8\end{array}$ & $\vec{\omega}$ & $\begin{array}{l}\vec{\omega} \\
\text { Оુ }\end{array}$ \\
\hline 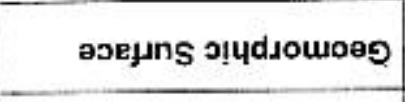 & $\mathscr{B}$ & $\mathbb{\mathbb { E }}$ & $\boldsymbol{\infty}$ & 员 & 刃 & $\begin{array}{l}\text { 怘 } \\
\text { 员 }\end{array}$ & 冓 & ஜ & $\begin{array}{l}0 \\
0 \\
0 \\
0\end{array}$ & む゙ & 员 & $\stackrel{10}{0}$ & 㞧 & $\tilde{\mathscr{D}}$ & 心 & $\begin{array}{l}0 \\
\text { 号 } \\
\end{array}$ & 号 & 岕 & 心 & E & फ \\
\hline รə!อำu!! & $\stackrel{\Perp}{0}$ & 뜯. & 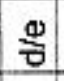 & D & 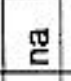 & $\approx$ & 뭉 & $\varpi$ & $\frac{\pi}{0}$ & 0 & (1) & 음 & (1) & $\omega$ & 읗 & $\frac{\pi}{0}$ & م. & 0 & 음 & $\pi$ & مـ \\
\hline 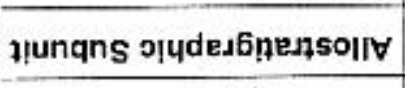 & $\Xi$ & 胥 & $E$ & $\bar{d}$ & 四 & 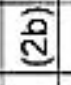 & (c) & (⿹ & $E$ & $E$ & $\begin{array}{l}\mathrm{a} \\
\mathrm{d}\end{array}$ & $\widehat{\mathrm{d}}$ & $\mathrm{d}$ & $\widetilde{\mathrm{d}}$ & (2) & $E$ & $\stackrel{\mathbb{d}}{\mathbb{d}}$ & $\equiv$ & $\widehat{\mathrm{d}}$ & $\widehat{\mathbb{V}}$ & $E$ \\
\hline 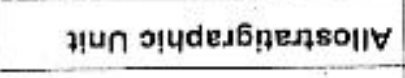 & $\&$ & 茎 & 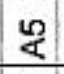 & 4 & 巴ृ. & $\stackrel{\mathscr{Q}}{\mathbb{\alpha}}$ & $\&$ & 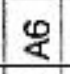 & 4 & 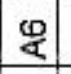 & $\underset{\leftarrow}{\propto}$ & $\stackrel{40}{\&}$ & $\mathscr{Q}$ & $\stackrel{0}{\alpha}$ & $\underset{0}{0}$ & $\stackrel{10}{2}$ & 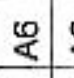 & 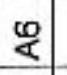 & $\stackrel{40}{<}$ & $\stackrel{\mathscr{c}}{<}$ & $\stackrel{\varphi}{8}$ \\
\hline शแ०גd & : & ฐ & 骂 & $\begin{array}{l} \pm \\
0\end{array}$ & ख & : & $\begin{array}{l}\text { g } \\
口\end{array}$ & $\begin{array}{l}3 \\
0 \\
0\end{array}$ & 豆 & 명 & $\begin{array}{l} \pm \\
0 \\
0\end{array}$ & $\begin{array}{l}8 \\
8 \\
\end{array}$ & $\begin{array}{l}8 \\
\end{array}$ & m & \begin{tabular}{|l|} 
\\
\\
\end{tabular} & $\begin{array}{l}\text { ¿े } \\
0\end{array}$ & $\begin{array}{l}\mathbf{D} \\
\mathbf{2}\end{array}$ & $\begin{array}{l}0 \\
8 \\
\end{array}$ & $\begin{array}{l}\mathscr{8} \\
\mathbf{2}\end{array}$ & $\begin{array}{l}\text { m } \\
0\end{array}$ & 응 \\
\hline uo!neวoา & 志 & $\stackrel{\mathbb{S}}{\mathbb{2}}$ & 志 & $\begin{array}{l} \\
\\
\\
\text { CS } \\
\end{array}$ & $\mathbb{\mathbb { Z }}$ & 锆 & 点 & 苍 & 员 & $\stackrel{m}{\circ}$ & $\underset{0}{0}$ & $\frac{8}{6}$ & $\underset{\infty}{8}$ & $\begin{array}{l} \\
0 \\
0\end{array}$ & 兽 & $\stackrel{8}{8}$ & 点 & $\underset{\infty}{\circ}$ & $\stackrel{8}{\circ}$ & $\frac{8}{\circ}$ & $\frac{B}{\infty}$ \\
\hline (uv) uldao samoา & 吕 & $\mathbb{\Xi}$ & 志 & \begin{tabular}{|l}
\multirow{N}{*}{} \\
\end{tabular} & 可 & $\stackrel{m}{?}$ & $\mathscr{\infty}$ & $\pi$ & ल & $\mathscr{8}$ & ลี & लొ & ก) & $\stackrel{\infty}{\infty}$ & 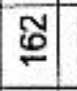 & 앙 & 5 & 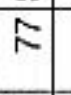 & $\stackrel{\infty}{\sim}$ & N & 6 \\
\hline (سэ) uldeg Jəddn & 吉 & $\stackrel{\mathscr{D}}{2}$ & 吕 & $\stackrel{\mathbb{N}}{\stackrel{N}{*}}$ & $\stackrel{\mathbb{D}}{C}$ & 0 & $\hat{N}$ & 0 & $\begin{array}{l}\mathscr{N} \\
\mathbb{N} \\
\end{array}$ & $\tilde{\omega}$ & $\stackrel{m}{\circ}$ & $\stackrel{\infty}{\sim}$ & $\infty$ & क & \begin{tabular}{|l|} 
g \\
\end{tabular} & స్ల్ల & $\stackrel{N}{N}$ & पे & 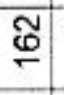 & $\stackrel{\infty}{\infty}$ & 웜 \\
\hline uoz!JoH & 空 & 塄 & 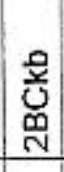 & 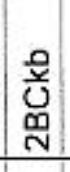 & జ & $\varangle$ & $\begin{array}{l}\overline{\mathbf{g}} \\
\text { 辛 } \\
\text { 惫 }\end{array}$ & $\begin{array}{l}\mathbb{4} \\
\mathbf{m}\end{array}$ & 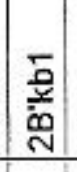 & 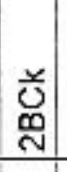 & 宫 & 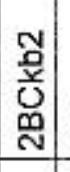 & కె口 & Е & 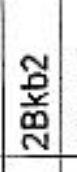 & 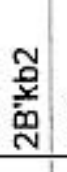 & 空 & 总 & $\begin{array}{l}\overline{0} \\
\overline{0} \\
\underline{\mathrm{N}} \\
\mathrm{N}\end{array}$ & 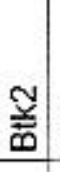 & 兑 \\
\hline әроว 리 & 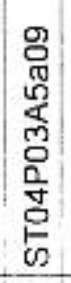 & 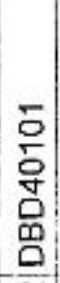 & 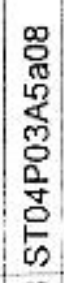 & 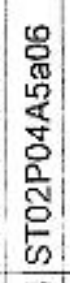 & $\mid$ & 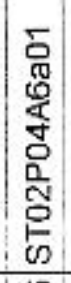 & 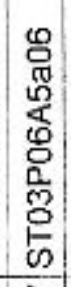 & $\begin{array}{c}0 \\
0 \\
0 \\
0 \\
0 \\
0 \\
0 \\
0 \\
0 \\
0 \\
0\end{array}$ & 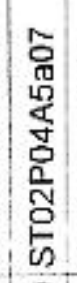 & $\begin{array}{l}\text { 岩 } \\
0 \\
0 \\
0 \\
5 \\
0 \\
0 \\
0 \\
0 \\
0\end{array}$ & $\begin{array}{l}0 \\
0 \\
0 \\
0 \\
0 \\
0 \\
0 \\
0 \\
0 \\
0 \\
0 \\
0\end{array}$ & $\begin{array}{l}\frac{5}{0} \\
0 \\
0 \\
0 \\
0 \\
0 \\
0 \\
0 \\
0 \\
0 \\
0\end{array}$ & $\begin{array}{l}0 \\
0 \\
0 \\
0 \\
8 \\
0 \\
0 \\
0 \\
0 \\
0 \\
0\end{array}$ & $\begin{array}{l}0 \\
0 \\
0 \\
0 \\
8 \\
0 \\
0 \\
0 \\
0 \\
0 \\
0 \\
0\end{array}$ & \begin{tabular}{|l|}
0 \\
0 \\
0 \\
0 \\
0 \\
0 \\
0 \\
0 \\
0 \\
0 \\
0 \\
\end{tabular} & 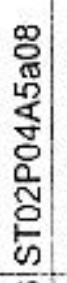 & 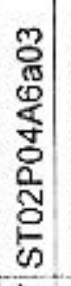 & 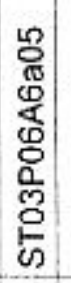 & $\begin{array}{l}0 \\
\frac{0}{\pi} \\
0 \\
0 \\
0 \\
0 \\
0 \\
0 \\
0 \\
0 \\
0\end{array}$ & 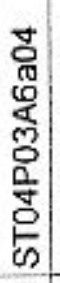 & $\begin{array}{l} \pm \\
\mathbb{8} \\
0 \\
0 \\
5 \\
0 \\
0 \\
0 \\
0 \\
0\end{array}$ \\
\hline apdues & $T$ & $N$ & $m$ & $\nabla$ & 10 & 10 & $N$ & $|\infty|$ & 0 & 으 & $=$ & $\mathscr{N}$ & 2 & \pm & $\stackrel{40}{2}$ & $\oplus$ & $F$ & $\stackrel{\infty}{-}$ & $\sigma$ & ㅇ & $\bar{v}$ \\
\hline & g & 要 & 韋 & 寻 & \% & 韋 & $\begin{array}{l}m \\
\end{array}$ & \begin{tabular}{|c|} 
\\
\end{tabular} & 总 & O & g & 旁 & g & 晜 & 兑 & gु & g & गे & \% & ? & gु \\
\hline
\end{tabular}




\begin{tabular}{|c|c|c|c|c|c|c|c|c|c|c|c|c|c|c|c|c|c|}
\hline (4) 6upseg & ্ָণু & ঙัু & 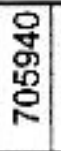 & $\begin{array}{l}\text { D } \\
\text { ल } \\
\text { ल }\end{array}$ & $\begin{array}{l}\text { क } \\
\text { ल } \\
\text { ल } \\
\text { 이 }\end{array}$ & $\begin{array}{l}\text { 용 } \\
\text { 。요 }\end{array}$ & 离 & $\mathbb{E}$ & $\begin{array}{l}\text { क् } \\
\text { ల్లై } \\
\text { م }\end{array}$ & 氙 & 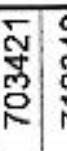 & 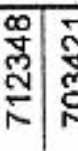 & 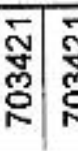 & 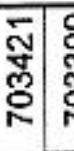 & 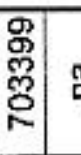 & $\stackrel{\mathbb{E}}{\mathrm{C}}$ & $\begin{array}{l}\text { 品 } \\
\text { స్ } \\
\stackrel{N}{N}\end{array}$ \\
\hline (H) BuIULION & 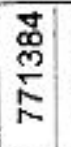 & 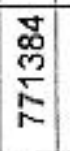 & 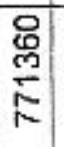 & $\begin{array}{l}\text { 品 } \\
\text { m } \\
⿱ ⺊\end{array}$ & 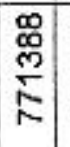 & 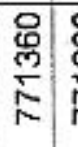 & 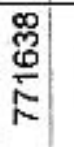 & $\mathbb{E}$ & 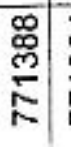 & 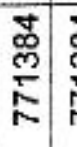 & 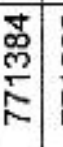 & 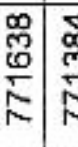 & 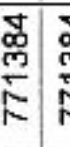 & 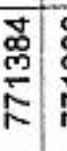 & 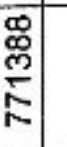 & $\mathbb{\widetilde { C }}$ & 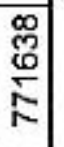 \\
\hline 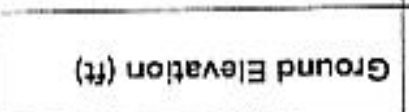 & $\begin{array}{l}\text { g } \\
\text { g్ల }\end{array}$ & 怘 & 品 & 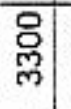 & 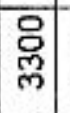 & 怘 & ণू & $\mathbb{C}$ & স্লু & 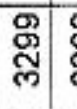 & ब্লু & ণ্লু & 尺्रे & : & 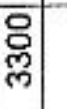 & d & స్ \\
\hline (6) 7м чมе & $\begin{array}{l}\infty \\
1 \\
\infty \\
\rho \\
\rho \\
\end{array}$ & $\begin{array}{c}0 \\
\mathbf{N} \\
\end{array}$ & $\begin{array}{l}\infty \\
o \\
\infty \\
\\
\end{array}$ & $\begin{array}{l}m \\
\infty \\
N \\
N\end{array}$ & 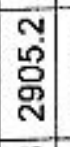 & 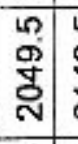 & $\begin{array}{l}0 \\
\frac{5}{\sigma} \\
\frac{N}{N}\end{array}$ & 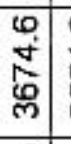 & $\begin{array}{l}\text { Nุ } \\
\stackrel{5}{n} \\
\text { N }\end{array}$ & 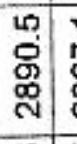 & 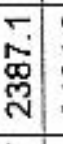 & 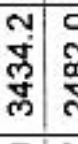 & 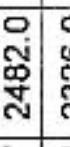 & 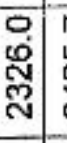 & 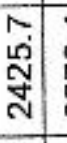 & $\begin{array}{l}\text { J } \\
\infty \\
\text { N } \\
\text { ले }\end{array}$ & 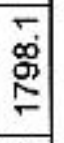 \\
\hline (6) $2 M$ o|dues $M d-J ! \forall$ & 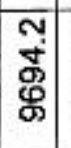 & 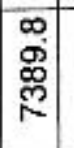 & 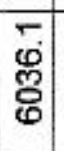 & 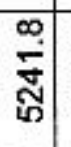 & 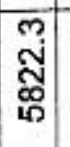 & $\begin{array}{l}m \\
\stackrel{9}{0} \\
\end{array}$ & $\begin{array}{l}n \\
\text { ñ } \\
\frac{n}{n}\end{array}$ & m & 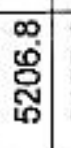 & $\begin{array}{l}0 \\
\stackrel{0}{0} \\
\bar{\varphi}\end{array}$ & $\begin{array}{l}\frac{1}{\sigma} \\
\frac{v}{6}\end{array}$ & 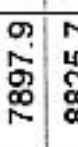 & 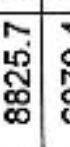 & 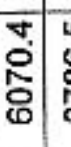 & 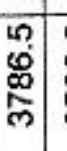 & 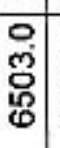 & $\begin{array}{l}\text { m } \\
\text { लू } \\
\text { 员 }\end{array}$ \\
\hline 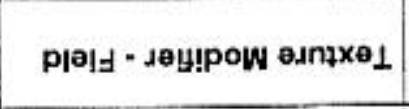 & $\underset{0}{\mathbb{\alpha}}$ & 齐 & 品 & 怘 & 兑 & 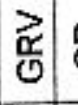 & 息 & 묻 & $\frac{\alpha}{0}$ & 怘 & 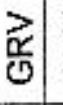 & $\underset{\mathbb{1}}{\mathbb{1}}$ & 罚 & 品 & $\frac{⿱ 夭 ⿰ 丿 ㇅}{0}$ & g & 品 \\
\hline 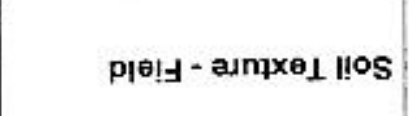 & 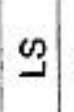 & $\vec{\omega}$ & $\vec{\omega}$ & $\vec{\omega}$ & - & $\vec{\omega}$ & $\vec{\omega}$ & 맏 & - & 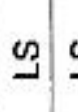 & 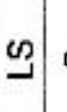 & $\infty$ & ऍ & 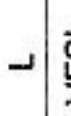 & $\begin{array}{l}\vec{\omega} \\
\frac{1}{>}\end{array}$ & 몯 & 足 \\
\hline qeר - $2 x n \times x_{1} \perp$ llos & \begin{tabular}{|c|} 
\\
0 \\
0 \\
\end{tabular} & 3 & $\Omega$ & $\overrightarrow{\mathscr{\omega}}$ & $\vec{\sigma}$ & s & $\Omega$ & $\begin{array}{l}0 \\
\amalg\end{array}$ & $\vec{\omega}$ & $\begin{array}{l}0 \\
0 \\
\end{array}$ & 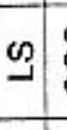 & ๑ & $\vec{\omega}$ & 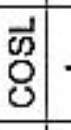 & ــ & 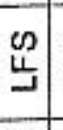 & ש \\
\hline 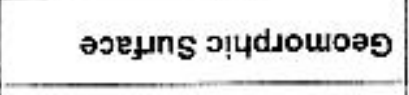 & 冓 & 足 & $\begin{array}{l}\stackrel{0}{0} \\
0\end{array}$ & ڤ & 怘 & $\begin{array}{l}0 \\
0 \\
0\end{array}$ & $\tilde{\Omega}$ & I & 芯 & ณ̊ & 只 & (i) & 心 & 8 & फ & ख & $\stackrel{m}{\infty}$ \\
\hline sөอрерочमา & 0 & ๘ & $\frac{\pi}{2}$ & 물 & مـ & $\frac{\pi}{3}$ & 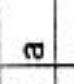 & $\underline{\mathbb{v}}$ & $\varpi$ & 0 & 음 & 07 & 뭉 & ـ & $w$ & 프. & $\pi$ \\
\hline 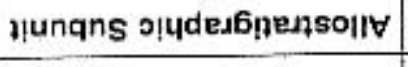 & ल्ర & त & $\vec{v}$ & $\widehat{3}$ & $\Xi$ & d & त) & 四 & $\Xi$ & $E:$ & त् & ลิ & क्? & $\Xi$ & $E$ & 胥 & $\Xi$ \\
\hline 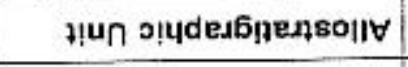 & $\&$ & $\mathbb{8}$ & 过 & 岁 & $\dddot{1}$ & $<$ & $\&$ & 胥 & 里 & $\&$ & 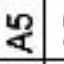 & $\stackrel{\infty}{4}$ & $\stackrel{4}{4}$ & $\stackrel{0}{4}$ & $\stackrel{2}{<}$ & 䠉 & 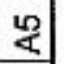 \\
\hline 리어d & $\begin{array}{l}9 \\
\\
\end{array}$ & 8 & $\begin{array}{l}\text { ¿ } \\
0\end{array}$ & $\begin{array}{l}1 \\
0 \\
0\end{array}$ & $\begin{array}{l}\text { 豆 } \\
\end{array}$ & $\begin{array}{l}\text { पे } \\
\text { 口. }\end{array}$ & \% & $\mathbb{E}$ & ¿ & 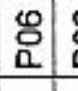 & 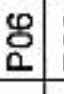 & 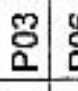 & $\mathscr{8}$ & $\mathscr{8}$ & $\begin{array}{l}1 \\
0 \\
0\end{array}$ & ฐ) & ஜ̊ㅁㅁ \\
\hline uo!̣Eอ0า & $\frac{8}{6}$ & $\frac{m}{0}$ & $\frac{N}{\infty}$ & $\begin{array}{l}8 \\
\\
0 \\
\end{array}$ & $\begin{array}{l}8 \\
\\
\end{array}$ & 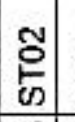 & $\frac{5}{6}$ & 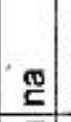 & 总 & 总 & ${ }_{\infty}^{3}$ & 志 & 点 & $\stackrel{9}{\circ}$ & 品 & $\underset{\mathrm{g}}{\mathrm{g}}$ & 吉 \\
\hline 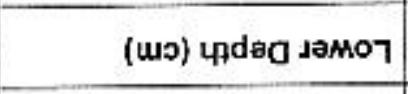 & क् & $\infty$ & P & 운 & 웜 & $\stackrel{m}{\sim}$ & ஜ & ख्य & $\approx$ & फ & $\begin{array}{l}\text { 足 } \\
\text { N }\end{array}$ & $\widehat{6}$ & $\stackrel{2}{\circ}$ & $\bar{\nabla}$ & $\omega$ & 茎 & 8 \\
\hline (wo) 47dəo sedd & \begin{tabular}{|l|}
$\mathscr{O}$ \\
$\mathscr{O}$
\end{tabular} & 0 & 员 & 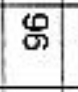 & $F$ & क & $\mathrm{N}$ & 要 & $\omega$ & $\overline{8}$ & ल् & 朋 & 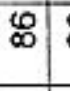 & $\stackrel{\mathfrak{m}}{N}$ & 0 & 믿 & $\overline{6}$ \\
\hline & $\begin{array}{l}\stackrel{5}{\mathbf{p}} \\
\text { 商 }\end{array}$ & $\alpha$ & $\begin{array}{c}\mathrm{e} \\
\stackrel{\mathrm{v}}{\mathrm{g}} \\
\stackrel{\mathrm{q}}{\mathrm{v}} \\
\end{array}$ & \begin{tabular}{|} 
恙 \\
⿳亠丷厂心 \\
\end{tabular} & 德 & $\begin{array}{l}\text { 盖 } \\
\text { 商 }\end{array}$ & 㩊 & $\mathbb{E}$ & $\begin{array}{l}\mathbb{6} \\
\mathbf{0}\end{array}$ & 盖 & 总 & 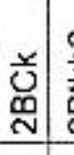 & 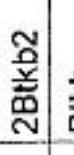 & 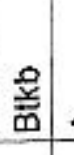 & $\varangle$ & $\mathbb{Z}$ & $\begin{array}{l}\text { 辛 } \\
\text { 总 }\end{array}$ \\
\hline әроэ 키dures p|!ㅣ & $\begin{array}{l}0 \\
0 \\
0 \\
0 \\
0 \\
0 \\
0 \\
0 \\
0 \\
0 \\
0 \\
0\end{array}$ & $\begin{array}{l}5 \\
0 \\
0 \\
0 \\
0 \\
0 \\
0 \\
0 \\
0 \\
0\end{array}$ & 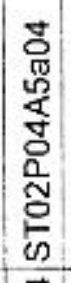 & $\begin{array}{l}0 \\
0 \\
0 \\
0 \\
4 \\
5 \\
0 \\
\frac{1}{9} \\
0 \\
0 \\
0\end{array}$ & $\begin{array}{l}8 \\
0 \\
0 \\
0 \\
0 \\
0 \\
0 \\
5 \\
0 \\
0 \\
0\end{array}$ & 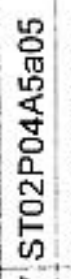 & 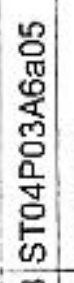 & 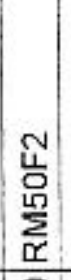 & 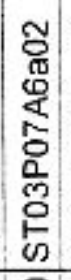 & $\begin{array}{l}5 \\
0 \\
\mathbb{0} \\
\$ \\
0 \\
0 \\
0 \\
0 \\
0 \\
0 \\
0\end{array}$ & 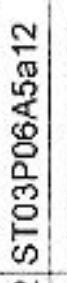 & 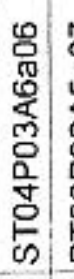 & 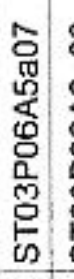 & 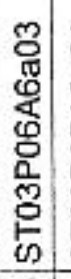 & $\begin{array}{l}\overline{0} \\
0 \\
0 \\
0 \\
0 \\
0 \\
\frac{0}{0} \\
0 \\
0 \\
0\end{array}$ & 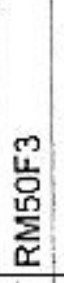 & 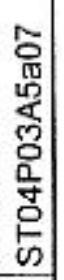 \\
\hline a|dues & $N$ & M & 吾 & $\stackrel{2}{2}$ & $\stackrel{\text { N }}{ }$ & 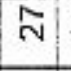 & $\stackrel{\infty}{\sim}$ & 모 & ले & $\bar{m}$ & న్ & m: & ले। & लू & 足 & $m$ & $\underset{m}{\infty}$ \\
\hline पगЕछ & \% & \% & 寽 & 筞 & \begin{tabular}{l|} 
\\
\\
$\sigma$
\end{tabular} & \begin{tabular}{|l|}
$g$ \\
\end{tabular} & \begin{tabular}{|l|}
$\begin{array}{c}3 \\
0\end{array}$ \\
\end{tabular} & 旁 & m & $\begin{array}{l}\text { m } \\
\text { go } \\
\end{array}$ & 奚 & $\begin{array}{l}3 \\
y \\
y\end{array}$ & 乌. & \% & 亭 & 䎌 & 酋 \\
\hline
\end{tabular}




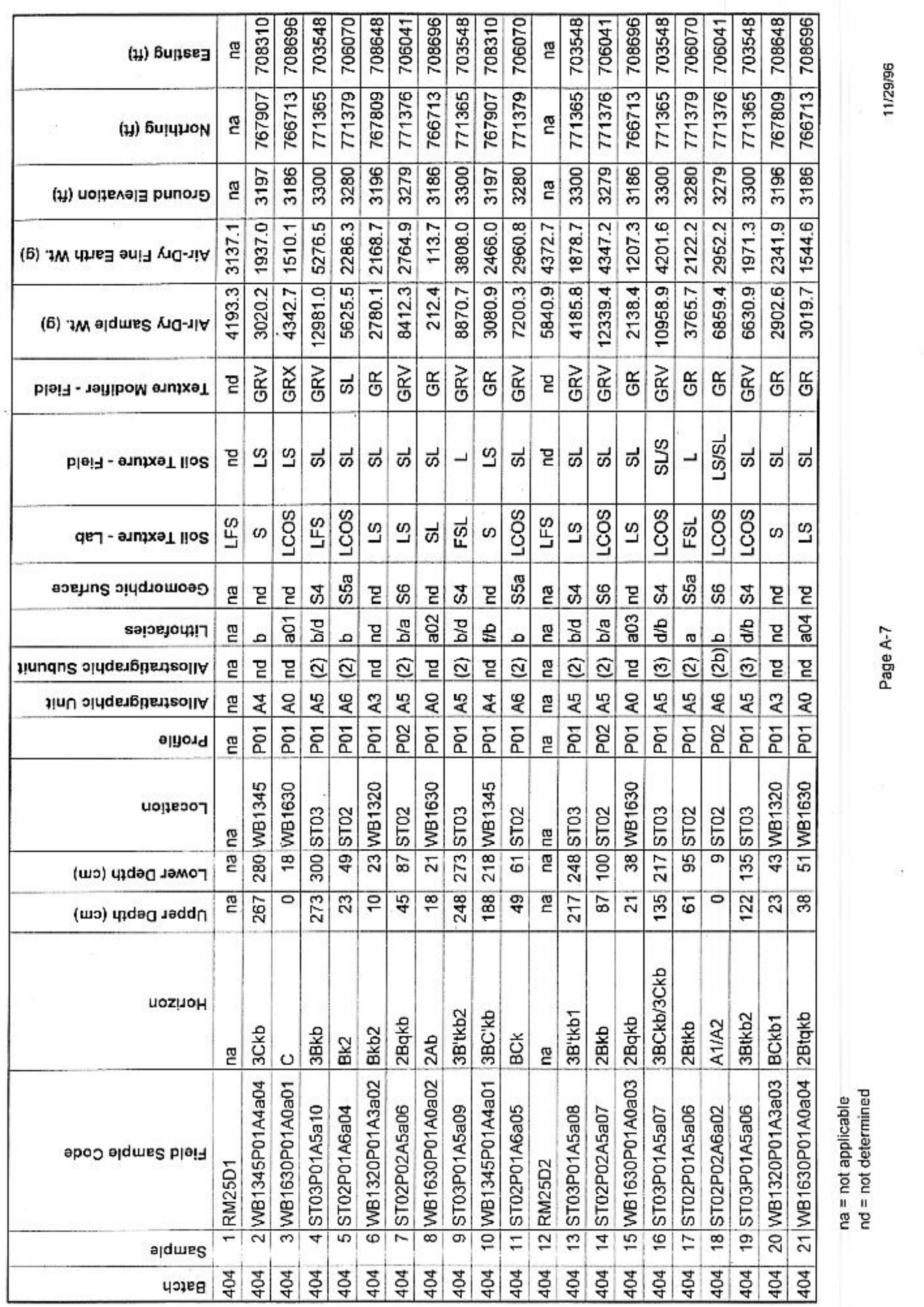




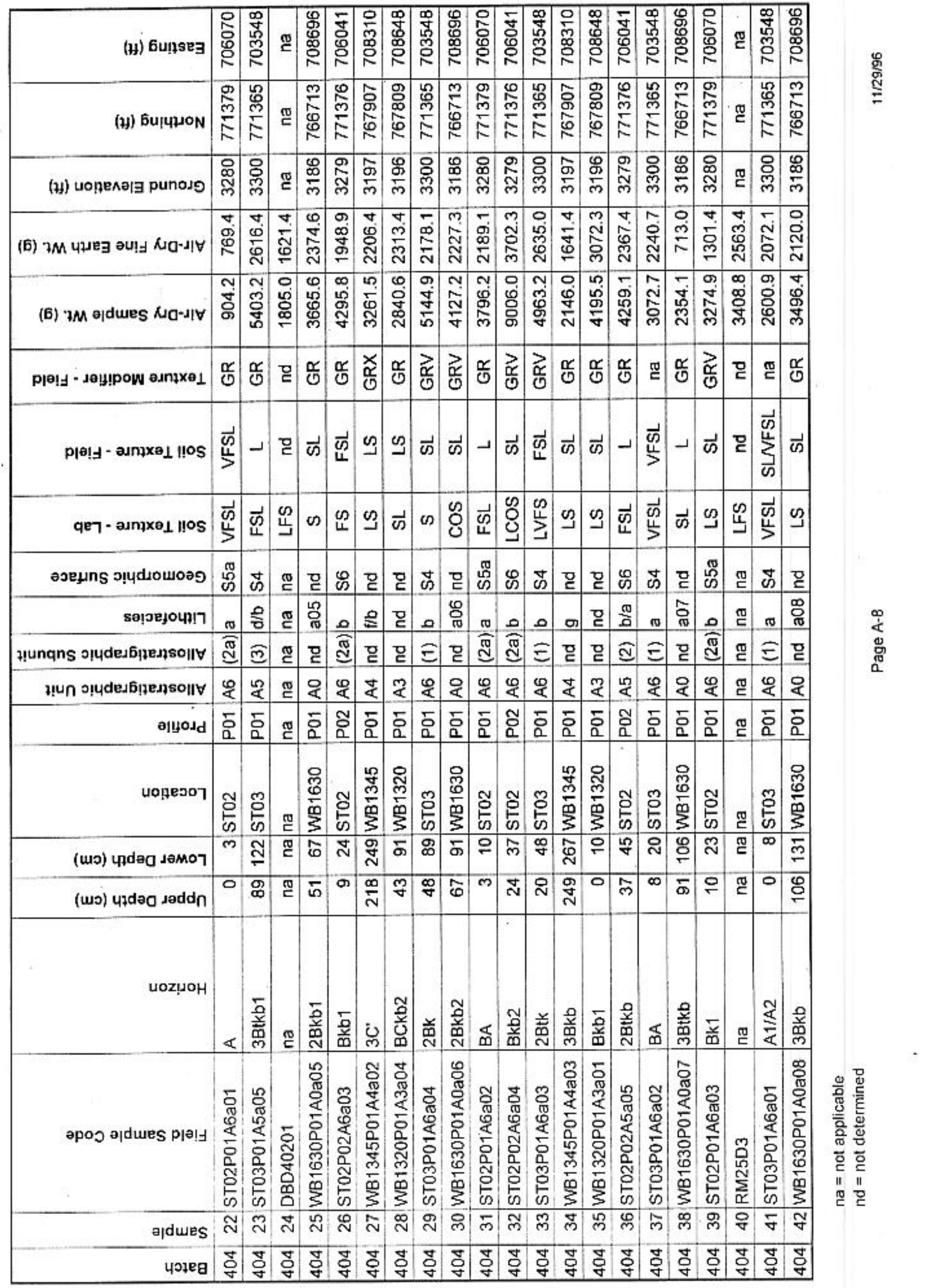




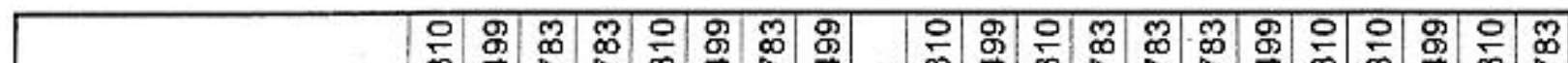

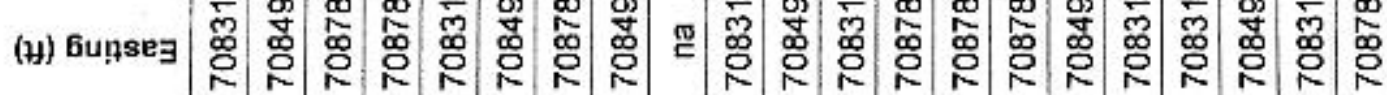
$\begin{array}{lll}0 & 0.000 \\ 0\end{array}$

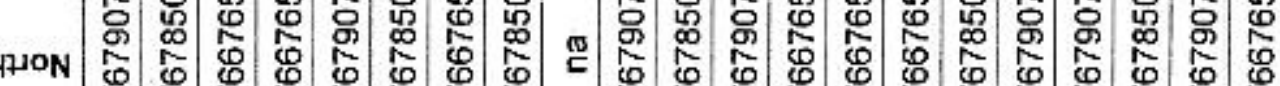
(н) Биl470N

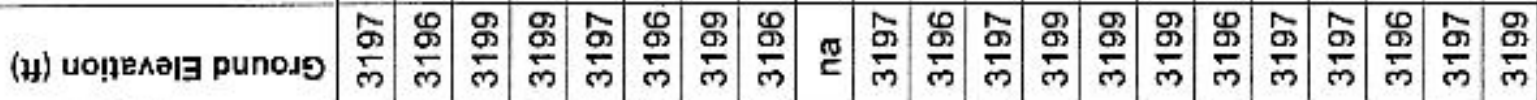

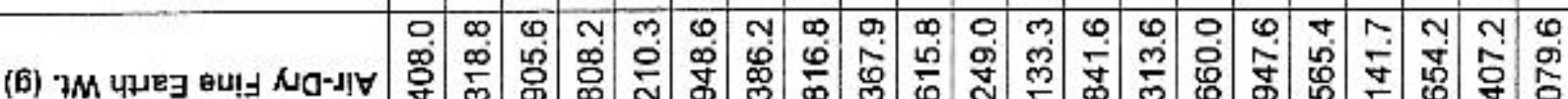

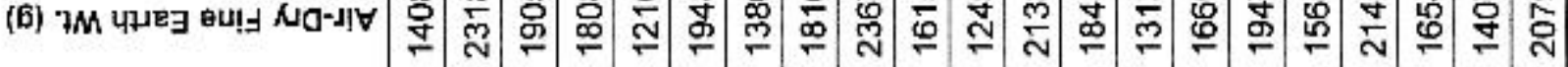

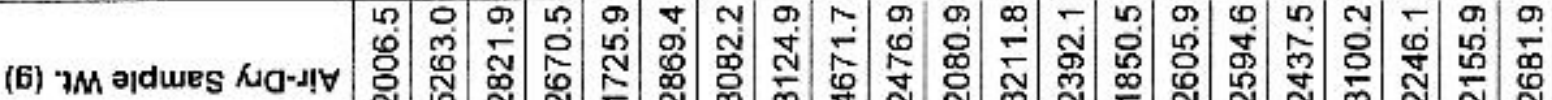

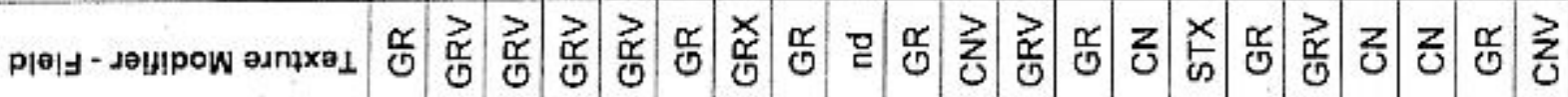

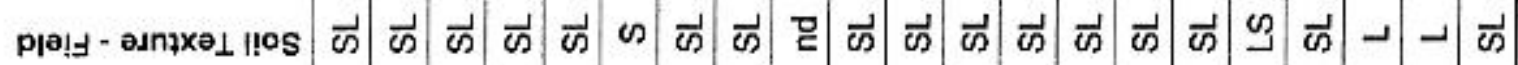

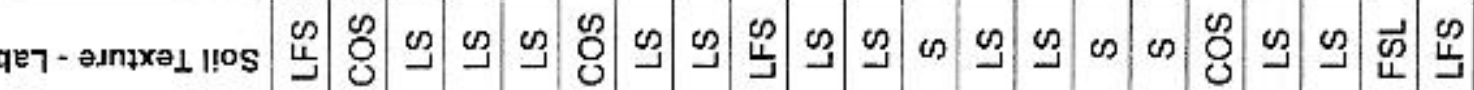

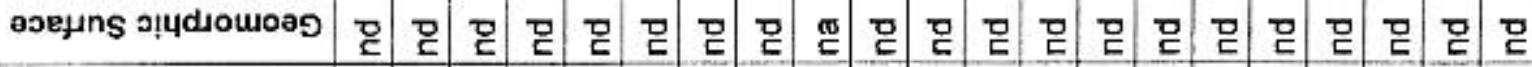

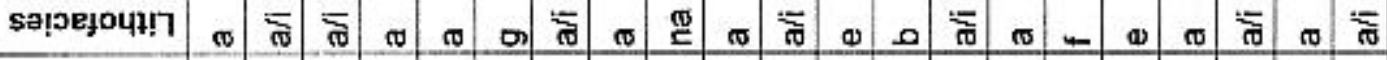

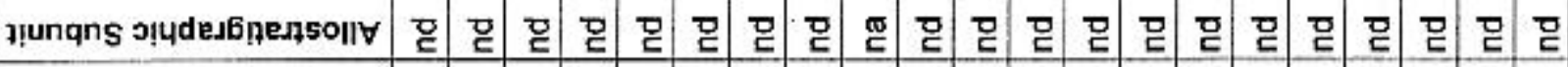

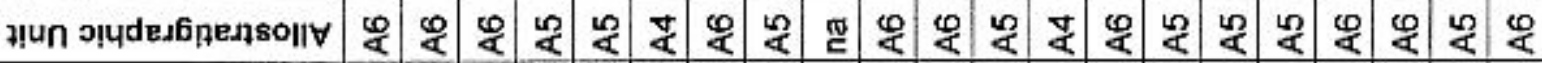

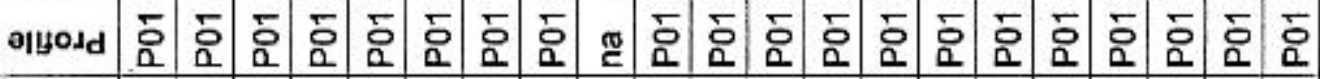

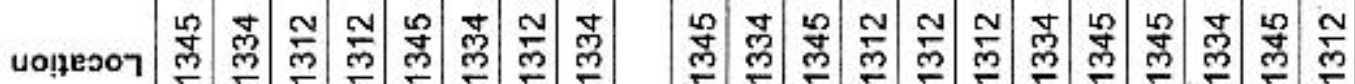

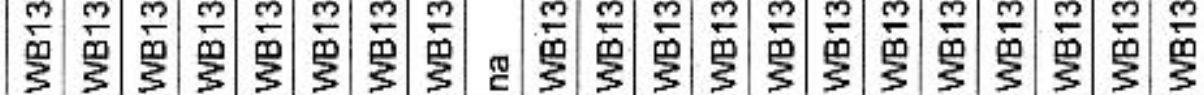

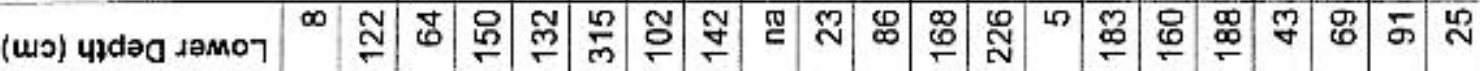
(шэ) uาden Jadd 0 品

UOZ!ıOH

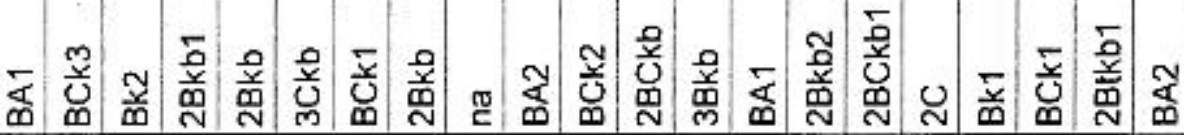
-

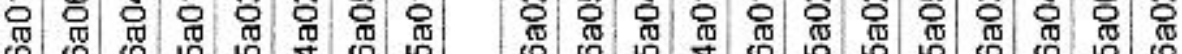

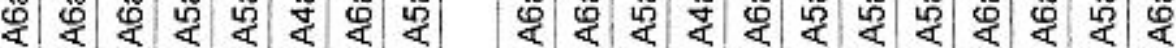
әроว ә땅 p|ㅣㅂ

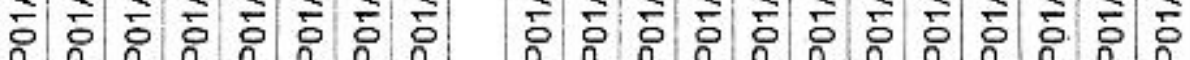

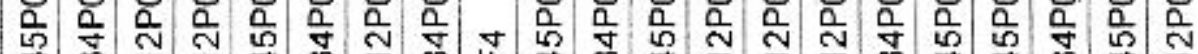

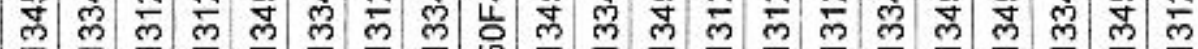

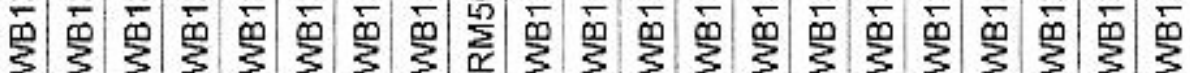
ㅂdues $-N$ ल

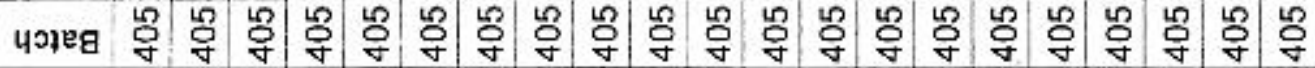




\begin{tabular}{|c|c|c|c|c|c|c|c|c|c|c|c|c|c|c|c|c|}
\hline (4) Bu!nse & 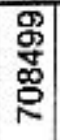 & 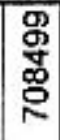 & 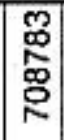 & \begin{tabular}{|l|} 
을 \\
営 \\
\end{tabular} & $\begin{array}{l}\text { क } \\
\text { 号 } \\
\text { 今 }\end{array}$ & \begin{tabular}{|l|}
0 \\
兽 \\
总
\end{tabular} & $\mathbb{\mathscr { C }}$ & 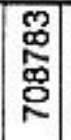 & \begin{tabular}{|l|}
0 \\
g \\
员 \\
\end{tabular} & $\begin{array}{l}\text { gू } \\
\text { 高 } \\
\text { 员 }\end{array}$ & 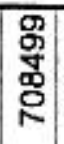 & 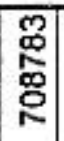 & \begin{tabular}{|l|} 
을 \\
员 \\
\end{tabular} & $\begin{array}{l}\text { क } \\
\text { 怘 } \\
\text { 号 } \\
\end{array}$ & $\begin{array}{l}\text { 옹 } \\
\text { 品 } \\
\end{array}$ & 里 \\
\hline (H) 6U!บนON & 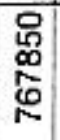 & $\begin{array}{l}0 \\
0 \\
0 \\
0 \\
6 \\
0\end{array}$ & 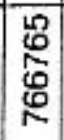 & 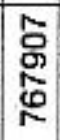 & 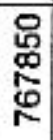 & 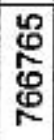 & $\mathbb{E}$ & 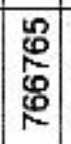 & $\begin{array}{l}\mathbf{5} \\
0 \\
0 \\
0 \\
0\end{array}$ & 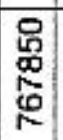 & 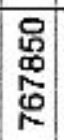 & 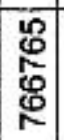 & $\begin{array}{l}5 \\
0 \\
0 \\
0 \\
0\end{array}$ & 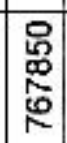 & 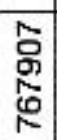 & $\mathbb{R}$ \\
\hline (H) uopenelヨ punosg & $\frac{\mathscr{\Phi}}{\mathrm{m}}$ & $\frac{\mathscr{S}}{m}$ & $\frac{\mathrm{g}}{\mathrm{m}}$ & $\frac{\hat{\sigma}}{\mathrm{m}}$ & $\frac{\mathscr{\sigma}}{m}$ & 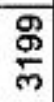 & $\mathbb{E}$ & $\frac{\mathrm{g}}{\mathrm{m}}$ & 高 & $\frac{\mathscr{9}}{\mathrm{m}}$ & $\frac{\mathscr{O}}{m}$ & $\frac{\mathrm{g}}{\mathrm{m}}$ & $\frac{5}{m}$ & $\frac{\mathscr{m}}{\mathrm{m}}$ & $\frac{\hat{m}}{m}$ & 똗 \\
\hline (6) 7 М чमе & 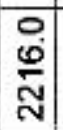 & 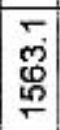 & 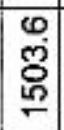 & 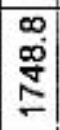 & 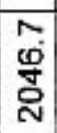 & 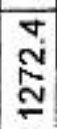 & 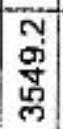 & 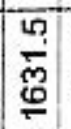 & 㐌 & 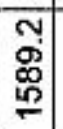 & 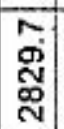 & $\begin{array}{l}0 \\
g \\
g \\
m\end{array}$ & \begin{tabular}{|l|}
\multirow{u}{*}{} \\
号 \\
\end{tabular} & $\begin{array}{l}5 \\
0 \\
0\end{array}$ & 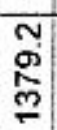 & 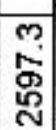 \\
\hline (6) $7 M$ ㅇdurs $\Lambda a \cdot \lambda \mid \forall$ & 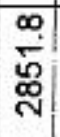 & 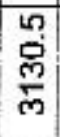 & \begin{tabular}{|l|} 
\\
dु \\
d्.
\end{tabular} & 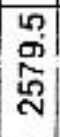 & $\underset{+}{\stackrel{\infty}{\sigma}}$ & 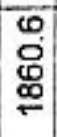 & $\stackrel{0}{\mathrm{~N}}$ & 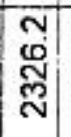 & $\underset{\varnothing}{\square}$ & 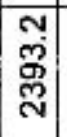 & \begin{tabular}{l|} 
\\
$\dot{g}$ \\
$\mathscr{g}$ \\
$\mathscr{g}$
\end{tabular} & $\begin{array}{l}\infty \\
\stackrel{N}{N} \\
\stackrel{N}{N}\end{array}$ & 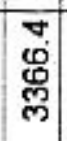 & 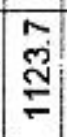 & 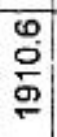 & 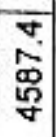 \\
\hline ple! & 采 & $\sum_{0}^{3}$ & 竞 & z & 离 & z & 믇 & 永 & $\frac{\pi}{0}$ & 乓 & 竝 & $\begin{array}{l}\times \\
\mathbb{0} \\
\mathbb{0}\end{array}$ & z & $z$ & $\frac{\Upsilon}{0}$ & 芯 \\
\hline 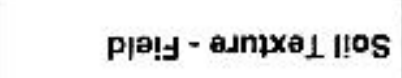 & $\vec{\omega}$ & $\vec{\omega}$ & 足 & $\vec{\omega}$ & $\infty$ & $\vec{\omega}$ & ? & $\vec{\omega}$ & - & - & $\omega$ & $\vec{\omega}$ & $\vec{\omega}$ & - & $\vec{\omega}$ & en \\
\hline qeJ - esmxel ilos & $\infty$ & 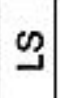 & D్ & 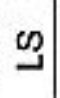 & D & $\Omega$ & $\begin{array}{l}\infty \\
\Psi\end{array}$ & $\infty$ & 3 & $\omega$ & \& & $\omega$ & $\begin{array}{l}\mathscr{0} \\
\mathrm{J}\end{array}$ & 足 & $\infty$ & 只 \\
\hline องejuns ग!4dגauras & 믇 & 묻 & 묻 & 묻 & 믇 & 묻 & $\mathbb{E}$ & g & 을 & 空 & 을 & $\mathbf{g}$ & 묻 & 믇 & 을 & Tू \\
\hline 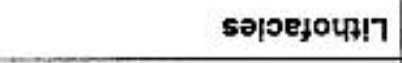 & $\leftarrow$ & 囦 & م & $\infty$ & ㅁ] & 莿 & T & - & $\pi$ & $\bar{\pi}$ & $\leftarrow$ & $\bar{\pi}$ & $\pi$ & $\bar{\pi}$ & $\approx$ & 끋 \\
\hline 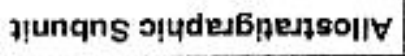 & 믄 & 일 & 므 & 마 & प्र & g & 空 & 묻 & 을 & 읃 & Q & 모 & gृ & 묻 & 을 & 要 \\
\hline 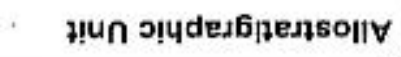 & 量 & $\stackrel{\varphi}{<}$ & \& & $\mathscr{\&}$ & 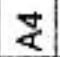 & $\stackrel{1}{\&}$ & 吼 & $<$ & 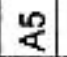 & $\mathscr{\&}$ & 48 & $\stackrel{1}{\&}$ & 旪 & \& & $<$ & 뜯 \\
\hline อщఋ०دd & $\overline{8}$ & $\overline{0}$ & 묭 & 용 & 욤 & 묨 & $\mathbb{\mathfrak { g }}$ & 횸 & 횽 & 힘 & 힘 & 宫 & 훙 & $\begin{array}{l}5 \\
0\end{array}$ & 5 & 몯 \\
\hline บo!̣ยอวา & $\begin{array}{l}\text { ल्ల } \\
\frac{\text { m}}{\xi}\end{array}$ & $\begin{array}{l}\stackrel{\mathbb{m}}{\mathrm{m}} \\
\stackrel{\text { go }}{\xi}\end{array}$ & $\stackrel{N}{\frac{N}{m}}$ & 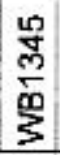 & 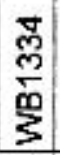 & $\frac{N}{\frac{N}{m}}$ & ֻே & $\frac{\mathrm{N}}{\mathrm{m}}$ & $\frac{n}{\frac{m}{m}}$ & $\begin{array}{l}\text { ॠ్ } \\
\frac{\mathrm{m}}{\xi} \\
\xi\end{array}$ & $\frac{\text { 亗 }}{\text { 㤩 }}$ & $\frac{N}{\frac{N}{m}}$ & $\frac{n}{\mathrm{~m}}$ & 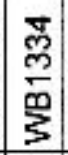 & 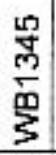 & 믈 \\
\hline (wо) чрdә & $\stackrel{\infty}{\circ}$ & 禺 & 芯 & $\mathscr{8}$ & $\begin{array}{l}\text { क } \\
\text { N }\end{array}$ & 守 & פ & 怘 & 8 & $\stackrel{2}{2}$ & ग्ले & $\stackrel{\sim}{\sim}$ & 5 & 60 & $\frac{9}{7}$ & 四 \\
\hline (uv) undeg seddn & $\stackrel{8}{\circ}$ & $\frac{2}{2}$ & $\stackrel{\mathscr{N}}{\mathrm{N}}$ & 9 & $\underset{\sim}{\stackrel{్}{~}}$ & $\stackrel{4}{N}$ & Q & $\begin{array}{l} \\
\\
\end{array}$ & $\bar{\sigma}$ & L) & 罳. & 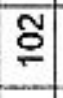 & 8 & 0 & g & TI \\
\hline uoz!NOH & \begin{tabular}{l} 
N \\
\multirow{2}{*}{} \\
D. \\
N
\end{tabular} & 弟 & U & 产 & 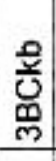 & 总 & $\stackrel{\mathbb{g}}{c}$ & U & 京 & జ̂ & U & 离 & 0 & $\underset{\mathrm{\Phi}}{\overline{\mathbf{c}}}$ & 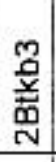 & $\stackrel{\mathbb{2}}{\check{C}}$ \\
\hline әроว ә|dues p|ㅣㅣ & 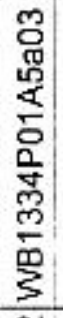 & 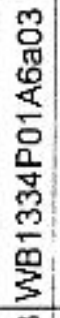 & 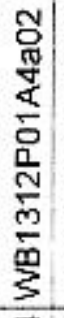 & 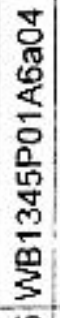 & 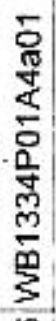 & 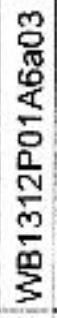 & 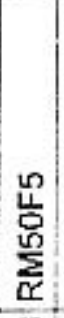 & 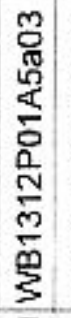 & 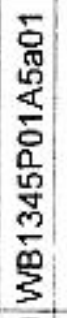 & 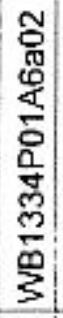 & 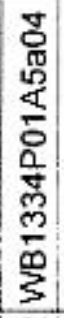 & 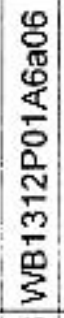 & 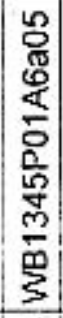 & 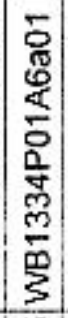 & 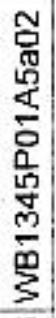 & 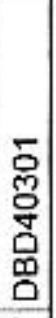 \\
\hline a|dues & $\widetilde{N}$ & ? & 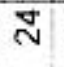 & $\stackrel{\infty}{N}$ & $\mathscr{N}$ & $\sqrt[N]{ }$ & $\stackrel{\infty}{N}$ & i & প। & $\bar{m}$ & लु & $m$ & जे & 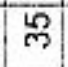 & 品 & nิ \\
\hline чэฺ्घ & $\stackrel{\text { 용 }}{\mathrm{g}}$ &. & $\begin{array}{l}\text { 岁 } \\
\text { q }\end{array}$ & 㟒 & 岁 & 总 & 岁 & \& & 岁 & 号 & 总 & 品 & 용 & 낭 & 总 & 남 \\
\hline
\end{tabular}




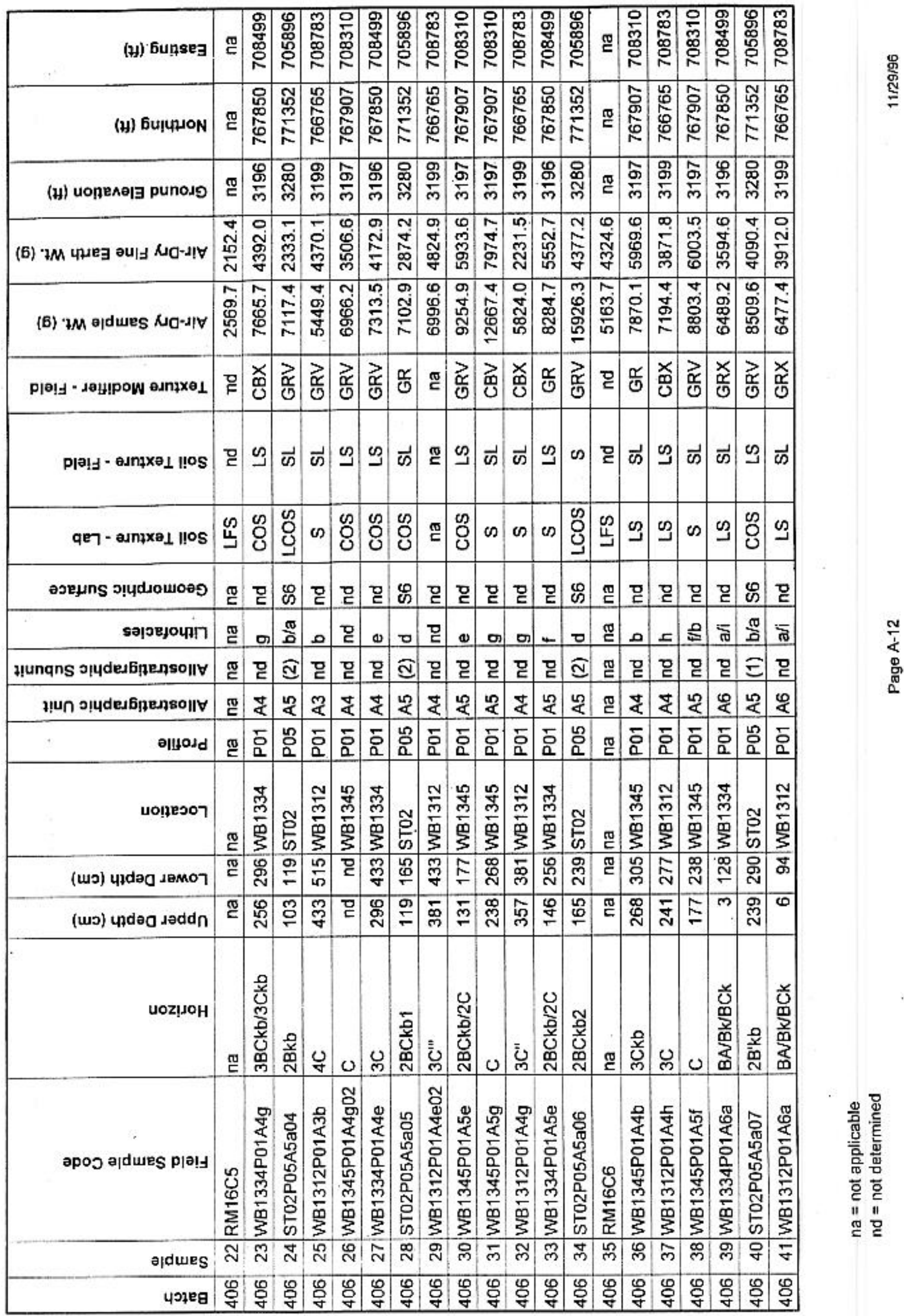




\begin{tabular}{|c|c|c|c|c|c|c|c|c|c|c|c|c|c|c|c|c|c|c|c|c|c|}
\hline (д) Gu!nsez & 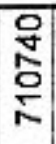 & 品 & 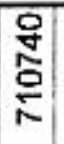 & $\begin{array}{l}\frac{9}{\mathrm{~V}} \\
\mathbf{9} \\
\frac{1}{N}\end{array}$ & 인 & $\begin{array}{l} \\
\\
\end{array}$ & \begin{tabular}{|l|}
$\frac{w}{m}$ \\
$\frac{m}{n}$ \\
\end{tabular} & 贻 & 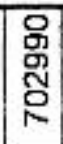 & 吕 & $\stackrel{0}{\stackrel{0}{N}}$ & $\stackrel{\mathbb{2}}{C}$ & $\begin{array}{l}\text { g. } \\
\text { 高 } \\
\text { g }\end{array}$ & $\begin{array}{l} \\
\\
\\
\\
\end{array}$ & $\frac{\stackrel{m}{m}}{\stackrel{m}{m}}$ & 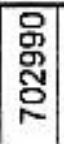 & 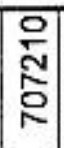 & \begin{tabular}{|l|} 
\\
\\
0 \\
0 \\
0
\end{tabular} & $\begin{array}{l} \\
\\
\\
\\
\end{array}$ & \begin{tabular}{|l|}
$\frac{w}{N}$ \\
$\stackrel{2}{5}$ \\
\end{tabular} & \begin{tabular}{|l|} 
号 \\
్ㅇㅇ
\end{tabular} \\
\hline (H) GulıนHON & 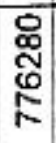 & $\begin{array}{l}\text { 导 } \\
\text { 号 } \\
\text { ผ } \\
\text { م }\end{array}$ & 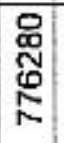 & 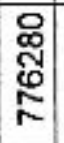 & $\begin{array}{l}\text { 昌 } \\
\text { F } \\
\text { F }\end{array}$ & $\begin{array}{l}9 \\
6 \\
0 \\
0 \\
0\end{array}$ & 蛋 & 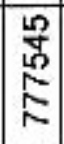 & 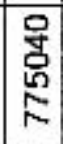 & 蛋 & $\begin{array}{l}\text { 怘 } \\
\text { N } \\
\text { 上 }\end{array}$ & $\mathbb{E}$ & 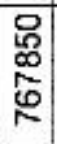 & 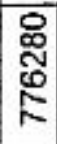 & $\frac{\substack{\mathrm{N} \\
\mathrm{N}}}{\mathrm{N}}$ & 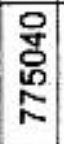 & 号 & 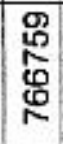 & 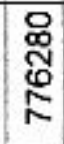 & 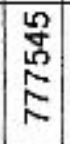 & $\begin{array}{l}\text { 号 } \\
\text { 员 } \\
\text { F }\end{array}$ \\
\hline 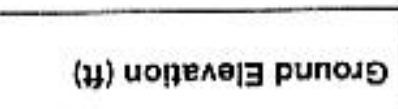 & 吕 & 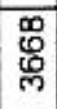 & $\begin{array}{l}9 \\
\stackrel{0}{0}\end{array}$ & 商 & $\begin{array}{l}\stackrel{w}{p} \\
5 \\
p\end{array}$ & $\frac{\infty}{\infty}$ & \begin{tabular}{|c|} 
量 \\
峞
\end{tabular} & $\begin{array}{ll}n \\
\text { 点 } \\
\end{array}$ & $\begin{array}{l}0 \\
0 \\
0 \\
0\end{array}$ & \begin{tabular}{|l|} 
品 \\
㐌
\end{tabular} & $\mid \begin{array}{l}n \\
5 \\
5 \\
m\end{array}$ & $\mathbb{E}$ & $\frac{\mathscr{D}}{\mathrm{m}}$ & छ & 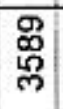 & $\begin{array}{l}\infty \\
0 \\
\mathbb{d}\end{array}$ & $\begin{array}{l}\frac{n}{2} \\
\text { 点 }\end{array}$ & $\frac{\infty}{\infty}$ & 怘 & $\begin{array}{l}5 \\
5 \\
5 \\
m\end{array}$ & $\begin{array}{l}\mathscr{D} \\
\mathbb{0} \\
\mathbb{W}\end{array}$ \\
\hline 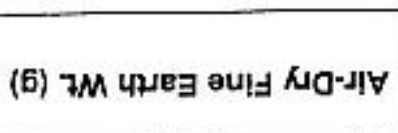 & 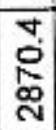 & 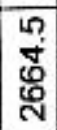 & $\underset{\substack{n \\
m}}{\stackrel{n}{n}}$ & 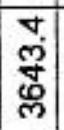 & 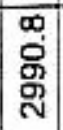 & $\begin{array}{l}\infty \\
\text { ป̀ } \\
\text { Na }\end{array}$ & \begin{tabular}{l|}
$\infty$ \\
0 \\
0 \\
4 \\
\end{tabular} & 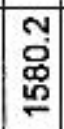 & 品 & 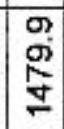 & \begin{tabular}{|l|}
0 \\
5 \\
5 \\
50
\end{tabular} & $\begin{array}{l}\infty \\
0 \\
0 \\
\text { ले } \\
\text { ले }\end{array}$ & $\begin{array}{l}\infty \\
5 \\
0\end{array}$ & 咅 & $\hat{\bar{\sigma}}$ & 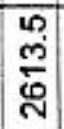 & 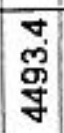 & $\begin{array}{l}0 \\
\infty \\
\infty \\
\\
\end{array}$ & 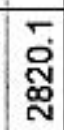 & \begin{tabular}{|l|} 
D \\
$\dot{\mathrm{g}}$ \\
$\mathrm{E}$ \\
\end{tabular} & 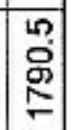 \\
\hline (6) $7 M$ 비ues $N$ a-d & $\frac{n}{0}$ & 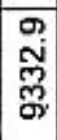 & 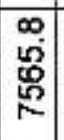 & \begin{tabular}{l|}
0 \\
$g$ \\
$\mathbb{e}$ \\
$\mathrm{p}$
\end{tabular} & 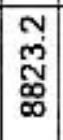 & 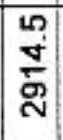 & 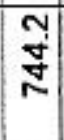 & 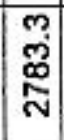 & \begin{tabular}{|l|}
0 \\
0 \\
0 \\
0 \\
0
\end{tabular} & \begin{tabular}{l|} 
N \\
लू \\
ले
\end{tabular} & 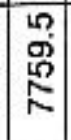 & 艿 & $\frac{\sigma}{\stackrel{\sigma}{\dot{Z}}}$ & $\begin{array}{l}0 \\
\dot{\mathrm{t}} \\
\mathrm{g}\end{array}$ & 离 & 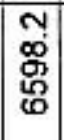 & 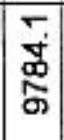 & $\begin{array}{l}0 \\
0 \\
0 \\
\end{array}$ & $\begin{array}{l}\infty \\
\text { 禹 } \\
\text { 色 } \\
\text { ले }\end{array}$ & \begin{tabular}{|c|}
$\infty$ \\
$M$ \\
g్ \\
\end{tabular} & $\begin{array}{l}\text { m } \\
\text { gू } \\
\text { g } \\
\text { p. }\end{array}$ \\
\hline plei!d - & $\underset{\frac{0}{0}}{3}$ & 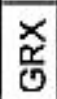 & 永 & 齐 & 竝 & 采 & 寽 & 荧 & $\begin{array}{l}\times \\
\mathbb{N} \\
\mathcal{O}\end{array}$ & 离 & 営 & ? & $\mathbb{E}$ & 罚 & 悤 & $\underset{\mathbb{2}}{\mathbb{2}}$ & 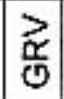 & 品 & 肴 & 营 & 喜 \\
\hline
\end{tabular}

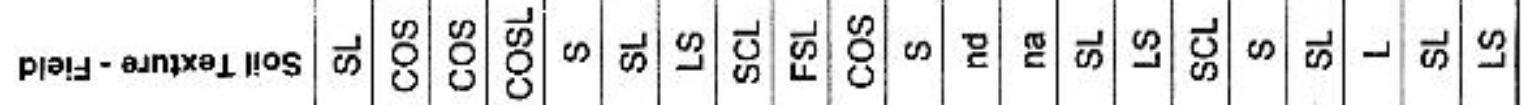

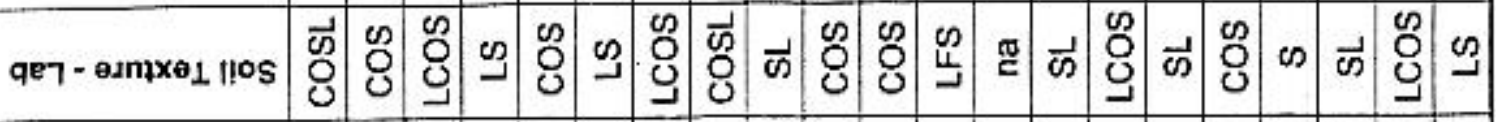

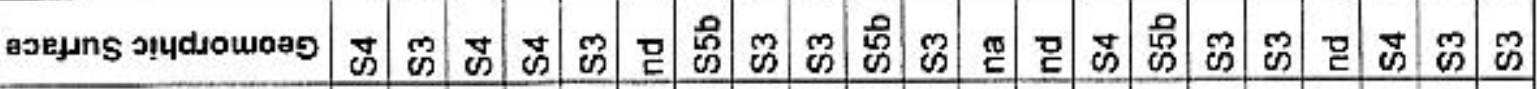

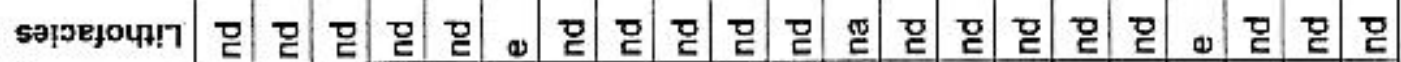

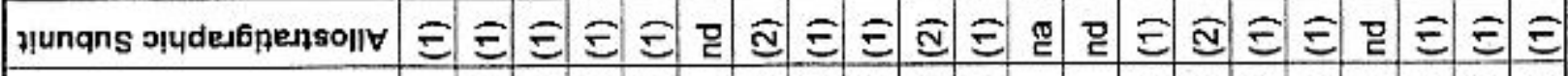

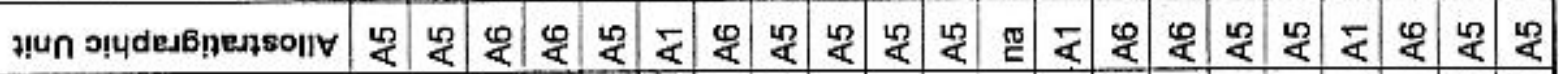

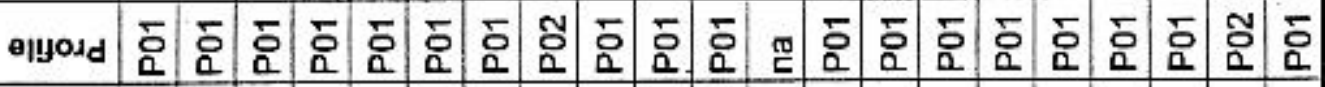
unper

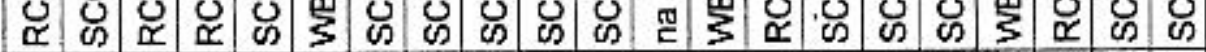

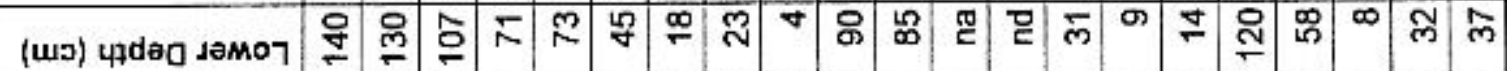

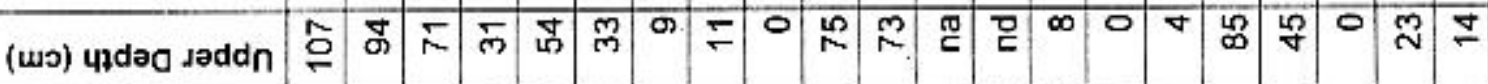
uoz!̣oH

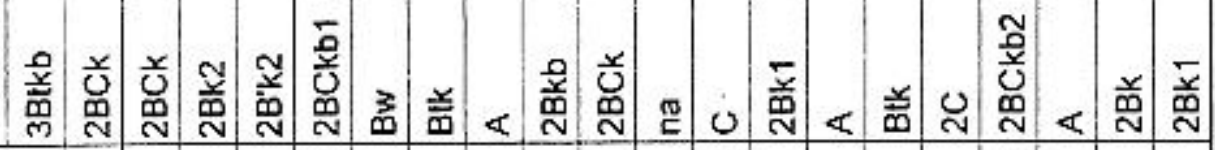

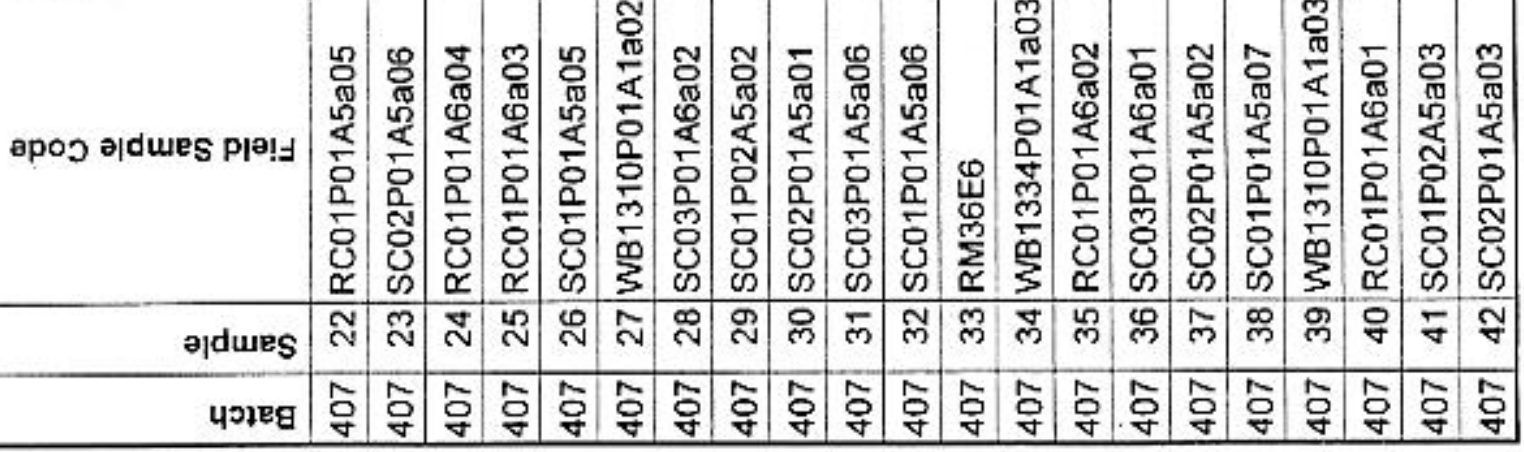




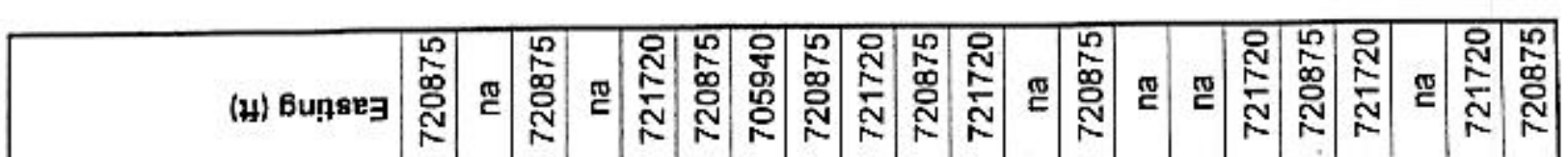

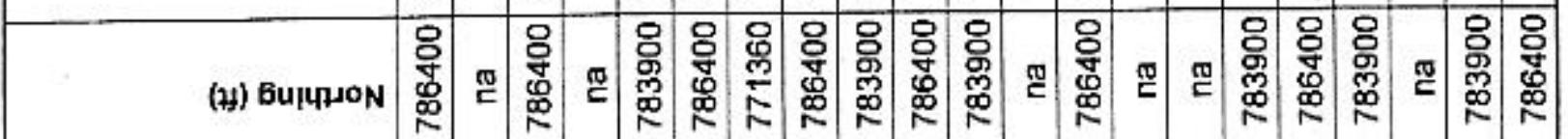

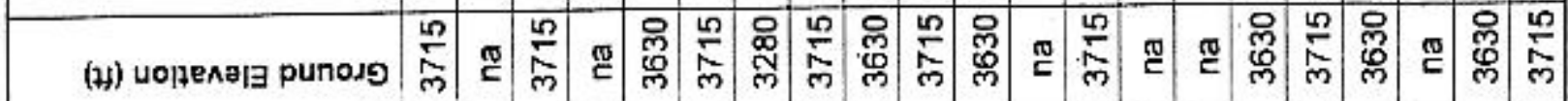

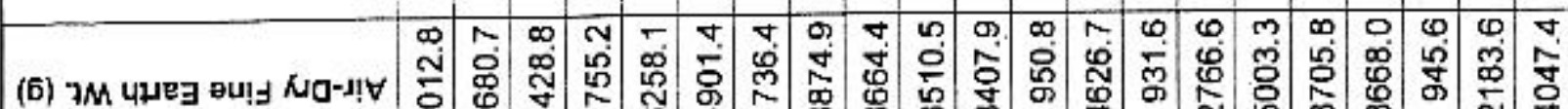

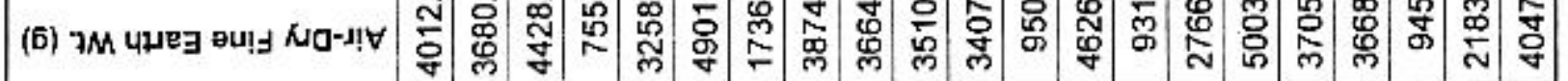

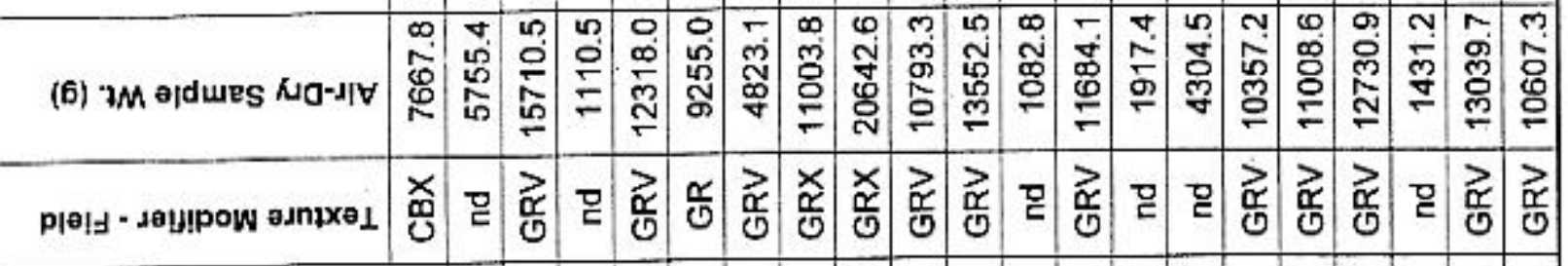

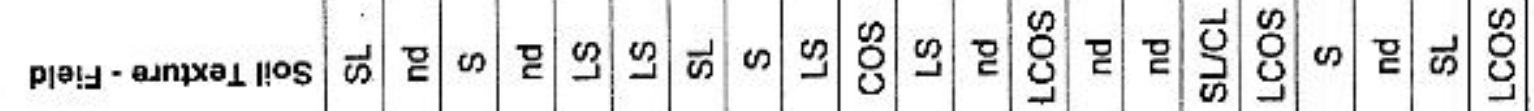

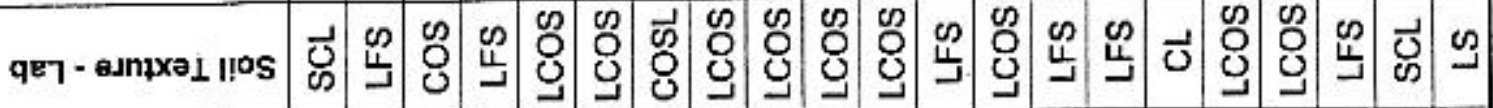

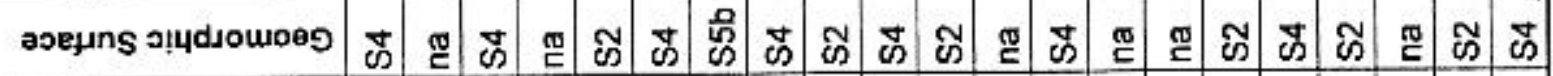

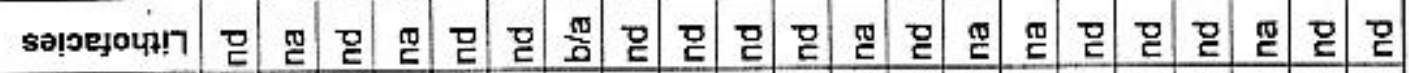

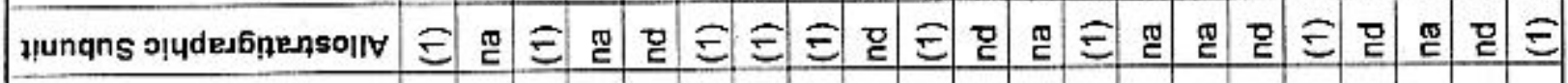

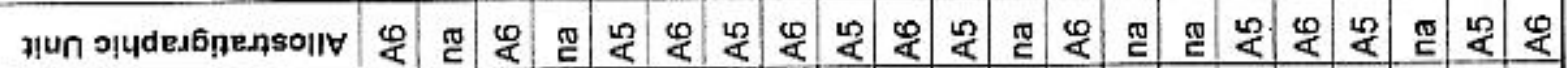

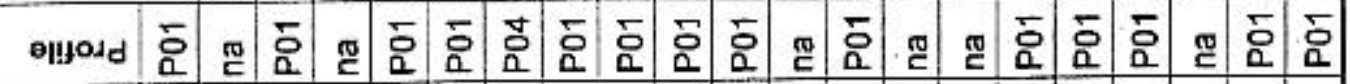

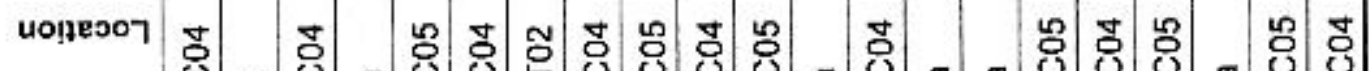

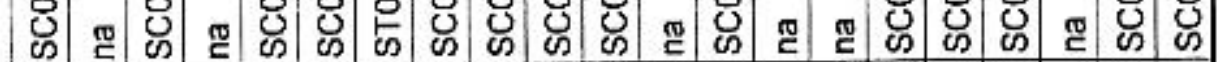

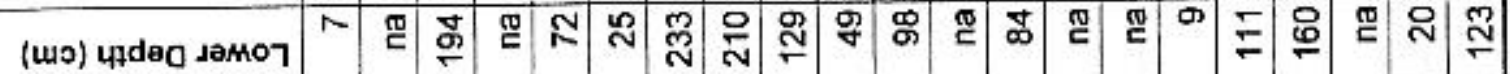

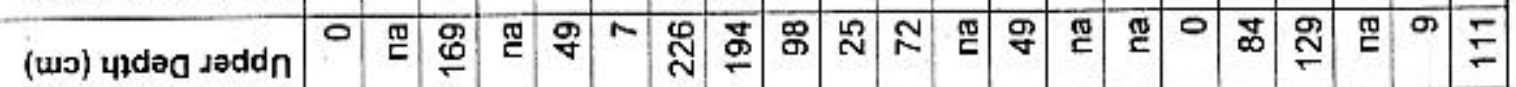

uoz!̣oH

E

×

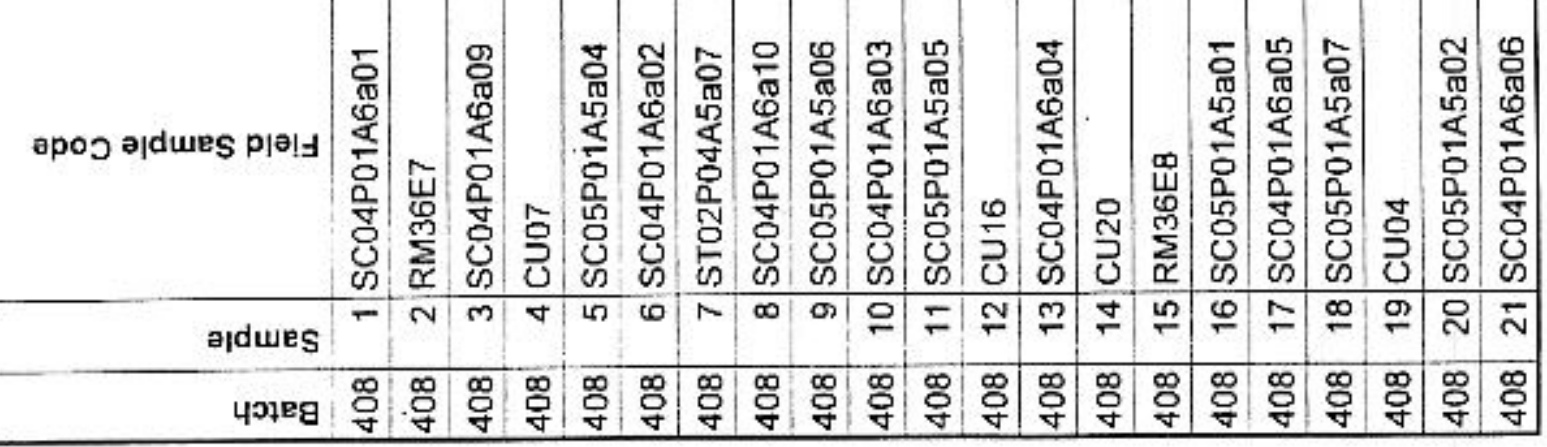

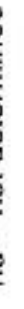




\begin{tabular}{|c|c|c|c|c|c|c|}
\hline (स) Gupseg & \begin{tabular}{l|}
$\mathbf{m}$ \\
$\mathbf{5}$ \\
$\stackrel{0}{\mathrm{~N}}$ \\
\end{tabular} & $\mathbb{E}$ & $\mathbb{\mathbb { Z }}$ & 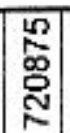 & 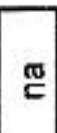 & 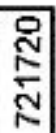 \\
\hline (н) Бแ!ฺน०N & $\begin{array}{l} \\
\\
\\
\mathscr{D} \\
0 \\
1\end{array}$ & $\stackrel{\mathbb{I}}{\mathrm{E}}$ & $\mathbb{E}$ & 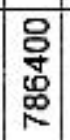 & $\stackrel{\pi}{C}$ & 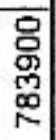 \\
\hline (ม) บoำ & $\frac{n}{n}$ & $\mathbb{E}$ & $\mathbb{8}$ & $\frac{10}{\stackrel{5}{m}}$ & $\stackrel{\pi}{5}$ & 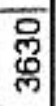 \\
\hline (6) 'ТM чมе & $\begin{array}{l}0 \\
\text { Oे } \\
\text { o }\end{array}$ & 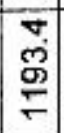 & \begin{tabular}{|l|}
$m$ \\
$\infty$ \\
$\hat{N}$ \\
$m$
\end{tabular} & \begin{tabular}{|c|} 
\\
$\sigma$ \\
$g$ \\
$g$ \\
\end{tabular} & $\frac{\bar{\alpha}}{\sigma}$ & 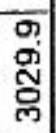 \\
\hline (6) $7 M$ ejdures Aug-d & $\begin{array}{l}0 \\
\stackrel{0}{0} \\
\stackrel{0}{0} \\
0 \\
0\end{array}$ & \begin{tabular}{|c|} 
\\
$\stackrel{9}{m}$ \\
$\stackrel{5}{b}$ \\
\end{tabular} & \begin{tabular}{|l|}
0 \\
00 \\
$\mathscr{0}$ \\
$\wp$ \\
\end{tabular} & \begin{tabular}{|l|}
$n$ \\
$\tilde{\infty}$ \\
$\mathscr{8}$ \\
\end{tabular} & 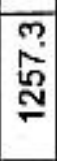 & $\frac{m}{\dot{m}}$ \\
\hline 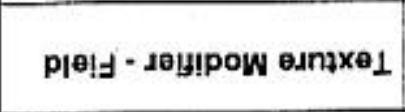 & 点 & $\underset{\substack{0 \\
\mathcal{O}}}{\gtrless}$ & 읃 & $\mid \begin{array}{l}\underset{\alpha}{0} \\
\stackrel{\sim}{0}\end{array}$ & 믈 & $\frac{\mathbb{0}}{\mathbb{0}}$ \\
\hline 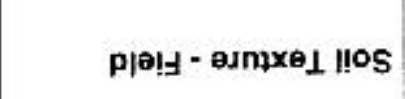 & $\vec{\omega}$ & $\stackrel{\vec{\omega}}{\breve{a}}$ & g & $\infty$ & 몯 & S \\
\hline qeך - edmxe & \begin{tabular}{|l|} 
\\
0 \\
0
\end{tabular} & $\frac{1}{\omega}$ & 号 & $\begin{array}{l}\omega \\
0 \\
0\end{array}$ & 邑 & S \\
\hline exeyns ग!ydsowoeg & 范 & 믈 & 胥 & है & 뜯 & ชี \\
\hline 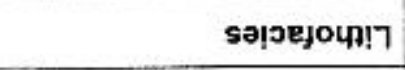 & 믇 & $\mathbb{E}$ & $\mathbb{E}$ & g & $\stackrel{\mathbb{Z}}{\mathrm{C}}$ & 묻 \\
\hline 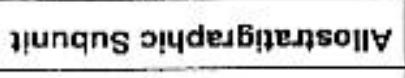 & $E$ & 胥 & 꼬. & $\Xi$ & 四 & 음 \\
\hline JuU ग!ุdes6!̨ensoll & $\stackrel{0}{\varangle}$ & $\mathbb{E}$ & $\mathbb{2}$ & $\stackrel{10}{\&}$ & 四 & 这 \\
\hline eltgosd & 뭉 & $\mathbb{E}$ & $\mathbb{\pi}$ & 뭉 & 모 & $\overline{0}$ \\
\hline บ०!ฏEכoา & $\begin{array}{l}\text { 芦 } \\
\text { 心 }\end{array}$ & $\mathbb{C}$ & $\stackrel{\mathbb{m}}{=}$ & $\begin{array}{l}\text { D } \\
\text { U్ } \\
\end{array}$ & w & $\begin{array}{l}\text { 号 } \\
\text { ஸ }\end{array}$ \\
\hline (wo) 4łdag دamoา & $\stackrel{9}{\circ}$ & $\stackrel{\pi}{5}$ & 要 & $\begin{array}{l}\mathscr{g} \\
\wp \\
\end{array}$ & TI & g \\
\hline (uxo) updea dəddn & $\stackrel{\mathscr{m}}{\mathrm{T}}$ & (2) & $\stackrel{\text { g }}{5}$ & $\stackrel{\text { M }}{\longrightarrow}$ & व & 오 \\
\hline uozuor & $\begin{array}{l}\mathscr{D} \\
\text { 茴 }\end{array}$ & 몸 & 또 & 帝 & $\stackrel{\mathbb{g}}{\mathrm{E}}$ & 盖 \\
\hline әро弓 비ures P|!ㅣ & 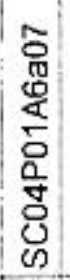 & $\begin{array}{l}5 \\
\text { 号 } \\
\text { 号 } \\
\text { 商 }\end{array}$ & 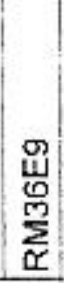 & $\begin{array}{l}0 \\
0 \\
0 \\
0 \\
0 \\
0 \\
0 \\
0 \\
0 \\
0 \\
0 \\
0\end{array}$ & $\frac{40}{5}$ & 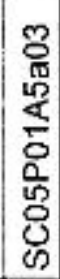 \\
\hline әdues & $N$ & M & N & $\stackrel{4}{2}$ & 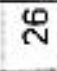 & $\stackrel{N}{N}$ \\
\hline 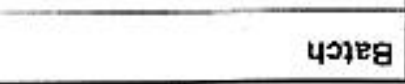 & $\begin{array}{l}\infty \\
\text { \& } \\
\end{array}$ & 寽 & \begin{tabular}{|l}
0 \\
\\
\end{tabular} & $\begin{array}{l}\text { o } \\
\text { g }\end{array}$ & 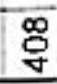 & 尊 \\
\hline
\end{tabular}




\begin{tabular}{|c|c|c|c|c|c|c|c|c|c|c|c|c|c|c|c|c|c|}
\hline (H) 6u!nseg & 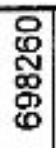 & 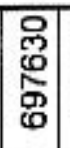 & 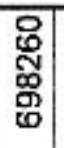 & 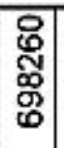 & 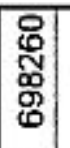 & 政 & 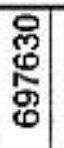 & 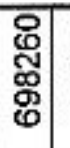 & 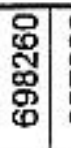 & 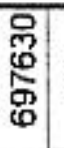 & $\mathbb{Z}$ & 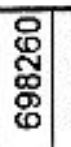 & 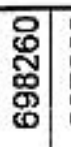 & $\begin{array}{l}\text { 유 } \\
\stackrel{5}{5} \\
8 \\
\end{array}$ & ㄸ & 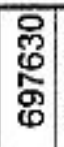 & 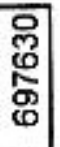 \\
\hline (H) 6u!บนบON & 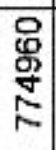 & 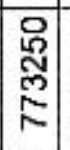 & 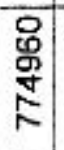 & 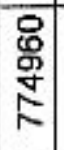 & 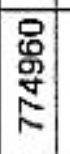 & 四 & $\begin{array}{l}\text { 怘 } \\
\text { N్ } \\
\stackrel{N}{N}\end{array}$ & 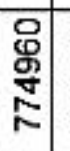 & 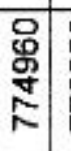 & $\begin{array}{l}\text { 怘 } \\
\text { N } \\
\text { F }\end{array}$ & 뜰 & 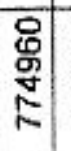 & 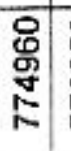 & $\begin{array}{l}\text { 怘 } \\
\stackrel{N}{0} \\
\stackrel{N}{N}\end{array}$ & g & 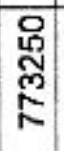 & $\begin{array}{l}\text { 온 } \\
\text { 옹 } \\
\text { N }\end{array}$ \\
\hline 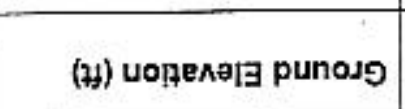 & 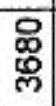 & $\frac{1}{6}$ & 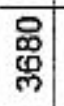 & \begin{tabular}{l|} 
吕 \\
心 \\
\end{tabular} & \begin{tabular}{|l|} 
요 \\
怘 \\
\end{tabular} & (ृ) & 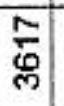 & $\begin{array}{l}\text { : } \\
\text { @્ల్ల }\end{array}$ & $\begin{array}{l}\text { 怘 } \\
\text { @્ల }\end{array}$ & $\stackrel{\bar{D}}{\bar{D}}$ & $\mathbb{\mathbb { E }}$ & \begin{tabular}{l|}
0 \\
0 \\
0 \\
0
\end{tabular} & $\begin{array}{l}0 \\
0 \\
0 \\
0\end{array}$ & 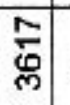 & $\mathbb{T}$ & $\frac{1}{6}$ & 点 \\
\hline 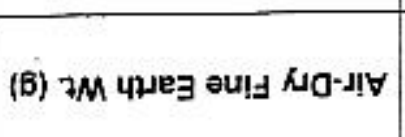 & 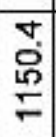 & 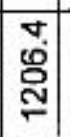 & $\stackrel{m}{-}$ & 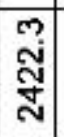 & \begin{tabular}{|c|} 
\\
$\dot{\sigma}$ \\
$\Phi$ \\
$\stackrel{\sigma}{\sigma}$ \\
\end{tabular} & \begin{tabular}{l|}
0 \\
0 \\
0 \\
0 \\
5 \\
\end{tabular} & 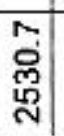 & 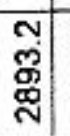 & $\begin{array}{l}m \\
\dot{m} \\
\text { W }\end{array}$ & \begin{tabular}{l} 
\pm \\
$\square$ \\
\hdashline
\end{tabular} & స్ & 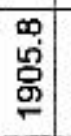 & 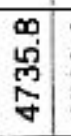 & 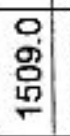 & $\frac{\square}{\frac{\dot{y}}{m}}$ & 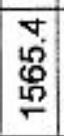 & 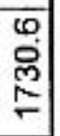 \\
\hline (B) $э M$ e|dures $\Lambda$ a-d & 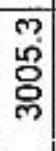 & $\begin{array}{l}\infty \\
\\
\\
\end{array}$ & 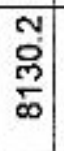 & 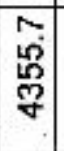 & $\frac{\infty}{\sim}$ & 原 & 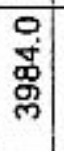 & $\underset{\mathrm{N}}{\mathrm{N}}$ & 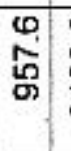 & 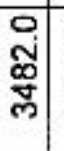 & $\begin{array}{l}\infty \\
\Phi \\
\mathbb{N}\end{array}$ & 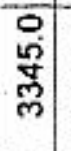 & 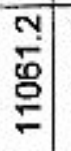 & $\begin{array}{l}m \\
\text { 今. } \\
\text { N }\end{array}$ & 产 & 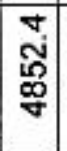 & 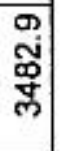 \\
\hline 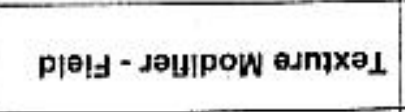 & 永 & $\left|\begin{array}{l}\mathbb{x} \\
0\end{array}\right|$ & 齐 & $\underset{\mathbb{0}}{\mathbb{0}}$ & 希 & $\stackrel{q}{E}$ & 总 & $\underset{\mathbb{N}}{\mathbb{0}}$ & 质 & 总 & 总 & 总 & $\underset{\mathbb{0}}{\mathbb{0}}$ & 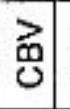 & 몯 & $\begin{array}{l}\underset{\alpha}{0} \\
\mathbb{0}\end{array}$ & $\frac{B}{\infty}$ \\
\hline 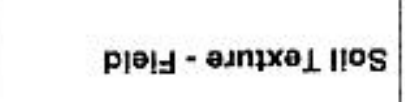 & $\vec{\omega}$ & - & ల్ల & - & $\vec{\omega}$ & 足 & $\vec{\omega}$ & $\vec{\omega}$ & $\vec{\omega}$ & $\vec{\omega}$ & 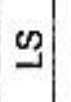 & - & $\vec{\omega}$ & - & 믇 & $\vec{\omega}$ & $\vec{\omega}$ \\
\hline 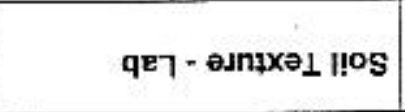 & $\vec{\omega}$ & $\vec{\omega}$ & $\vec{\omega}$ & $\vec{\omega}$ & 0 & 足 & $\vec{\omega}$ & $\vec{\omega}$ & $\vec{\omega}$ & $\vec{\omega}$ & $\infty$ & $\vec{\omega}$ & $\vec{\omega}$ & $\vec{\omega}$ & $\underset{\mathscr{u}}{\mathbb{u}}$ & 占 & $\vec{\omega}$ \\
\hline 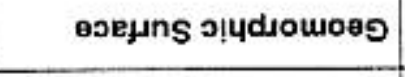 & 8 & ஜ & $\ddot{3}$ & $\mathscr{B}$ & B & 默 & के & 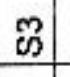 & ڤ్ & 心) & $\mathbb{2}$ & 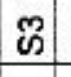 & 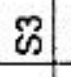 & षे & జ & ظे & फे \\
\hline se!̣อยุoun!า & 믈 & 물. & 믇 & 믇 & ? & 墕 & $\mathrm{g}$ & 을 & 믈 & ? & $\mathbb{E}$ & 믇 & 믿 & 믈 & $\mathbb{Z}$ & 믈 & 을 \\
\hline 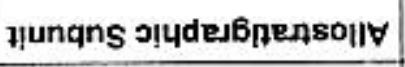 & $\bar{d}$ & $\Xi$ & $E$ & $\overleftarrow{\mathrm{d}}$ & ป & $\mathbb{E}$ & $E$ & $=$ & 으 & $E$ & $\mathbb{E}$ & d & $E$ & $E$ & $\mathbb{E}$ & $E$ & $=$ \\
\hline 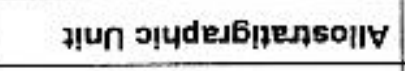 & 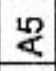 & $\stackrel{0}{4}$ & $<$ & 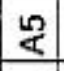 & 这 & 胥 & $\stackrel{8}{4}$ & $\&$ & $\&$ & $\stackrel{\infty}{\&}$ & $\mathbb{E}$ & $\underset{4}{4}$ & $\underset{1}{10}$ & $\mathscr{L}$ & $\underset{c}{c}$ & $\stackrel{\leftrightarrow}{\varangle}$ & $\stackrel{\mathscr{L}}{\mathbb{\alpha}}$ \\
\hline 이엄 & 효 & $\overline{8}$ & $\begin{array}{l}5 \\
0 \\
\end{array}$ & 宫 & 훙 & 政 & 혼 & 혼 & $\overline{0}$ & 흠 & $\stackrel{0}{\mathrm{C}}$ & 훙 & 훙 & Б & w & $\bar{\alpha}$ & $\overline{8}$ \\
\hline uัnูeอoา & 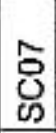 & $\begin{array}{l} \\
\\
\\
0\end{array}$ & 灾 & \begin{tabular}{l} 
\\
\\
0 \\
\hdashline
\end{tabular} & $\begin{array}{l}\hat{0} \\
0 \\
0\end{array}$ & (I) & 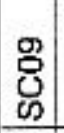 & $\begin{array}{l}0 \\
0 \\
0\end{array}$ & 엉 & $\begin{array}{l}\text { O } \\
\text { O্ } \\
\end{array}$ & () & $\begin{array}{l} \\
0 \\
0 \\
0\end{array}$ & 氖 & 总 & $\stackrel{\mathbb{g}}{\mathrm{C}}$ & $\begin{array}{l}\circ \\
\circ \\
0 \\
0\end{array}$ & $\begin{array}{l}\circ \\
\circ \\
\end{array}$ \\
\hline (шэ) ч)dag دәмоา ! & $\overline{6}$ & ని & $\mathscr{O}$ & 要 & กิ & 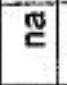 & $\mathbb{8}$ & $\stackrel{\sigma}{=}$ & $\mathrm{N}$ & $\mathscr{\varnothing}$ & एँ & $\stackrel{9}{=}$ & 吕 & $\infty$ & T) & 筞 & 읃 \\
\hline (uכ) uł dəg Jaddn & $\tilde{ก}$ & $\infty$ & $\overline{0}$ & $\simeq$ & g & एव & F & 密 & 0 & 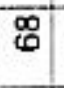 & 要 & $N$ & $\stackrel{9}{=}$ & 0 & 可 & I্ & $\mathscr{\square}$ \\
\hline & 嚍 & 音 & 总 & 离 & \begin{tabular}{|l|l} 
ָ̃ \\
בे
\end{tabular} & 밀 & 恙 & 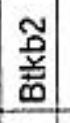 & $\bar{s}$ & 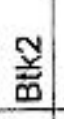 & Tा & 2 & 竞 & 4 & 胥 & $\stackrel{\mathcal{Y}}{\mathbf{m}}$ & U. \\
\hline әроว өөdurs ple!y & 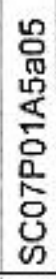 & 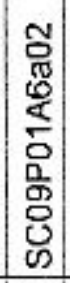 & 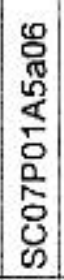 & 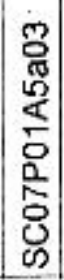 & 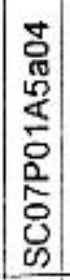 & 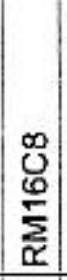 & 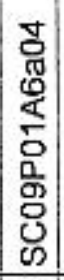 & $\begin{array}{l}0 \\
0 \\
0 \\
5 \\
5 \\
0 \\
0 \\
0 \\
0 \\
0 \\
0\end{array}$ & 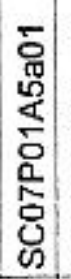 & 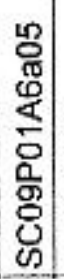 & 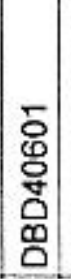 & 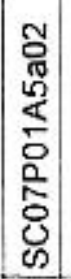 & $\begin{array}{l}0 \\
0 \\
0 \\
0 \\
0 \\
5 \\
0 \\
0 \\
0 \\
0 \\
0 \\
0\end{array}$ & $\mid \begin{array}{l}5 \\
0 \\
0 \\
\mathbb{\$} \\
5 \\
0 \\
0 \\
0 \\
0 \\
0 \\
0\end{array}$ & 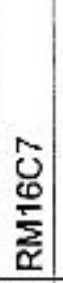 & 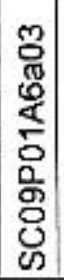 & \begin{tabular}{l}
0 \\
0 \\
0 \\
0 \\
0 \\
0 \\
0 \\
0 \\
8 \\
8 \\
0 \\
\hdashline
\end{tabular} \\
\hline e|dues & - & $N$ & $m$ & $\nabla$ & 10 & $\omega$ & $m$ & $\infty$ & 0 & 으 & $=$ & 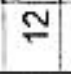 & $\stackrel{9}{\square}$ & प & 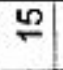 & 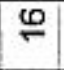 & 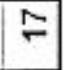 \\
\hline पग्ड & g & $\begin{array}{l}9 \\
9\end{array}$ & g & $\frac{9}{8}$ & 8 & \% & 宫 & $\begin{array}{l}8 \\
\\
\end{array}$ & 总 & g & g & $\begin{array}{l}\text { gi } \\
\text { V }\end{array}$ & g & $\begin{array}{l}\text { g } \\
\end{array}$ & g & 总 & 总 \\
\hline
\end{tabular}




\begin{tabular}{|c|c|c|c|c|c|c|c|c|c|c|c|c|c|c|c|c|c|c|c|c|c|c|c|}
\hline (y) 6upseg & 몬 & 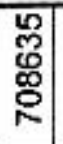 & 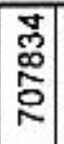 & 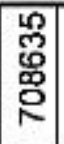 & 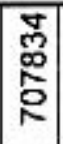 & 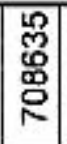 & 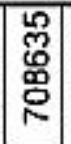 & 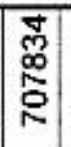 & \begin{tabular}{l|}
0 \\
0 \\
0 \\
0 \\
0
\end{tabular} & 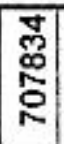 & 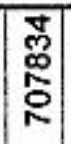 & क & 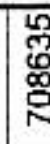 & 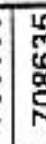 & & 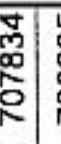 & 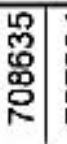 & 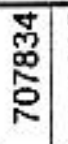 & \begin{tabular}{l|} 
\\
0 \\
0 \\
0 \\
0
\end{tabular} & 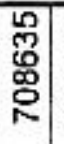 & 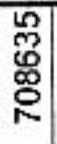 & 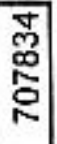 & \\
\hline (H) Gu!บนoN & 昰 & 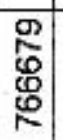 & 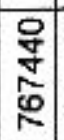 & 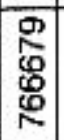 & 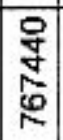 & 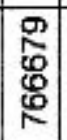 & 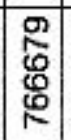 & 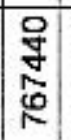 & $\begin{array}{l}\text { क } \\
0 \\
0 \\
0 \\
0\end{array}$ & 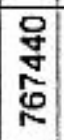 & $\mid$\begin{tabular}{|l|} 
\\
$\frac{V}{4}$ \\
$\frac{5}{1}$ \\
\end{tabular} & $\underset{c}{\pi}$ & $\begin{array}{l}9 \\
0 \\
6 \\
6 \\
1\end{array}$ & $\begin{array}{ll}0 \\
8\end{array}$ & 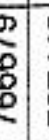 & 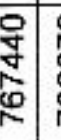 & 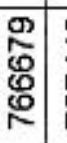 & 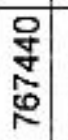 & 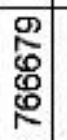 & $\begin{array}{l}9 \\
\stackrel{9}{0} \\
\mathbb{8} \\
\mathbb{R}\end{array}$ & 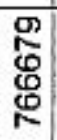 & 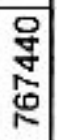 & \\
\hline (4) чоремәэ punosפ & $\stackrel{5}{2}$ & $\frac{N}{\infty}$ & 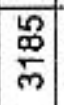 & $\frac{N}{m}$ & $\frac{1}{m}$ & $\frac{\infty}{m}$ & $\frac{\infty}{m}$ & 离 & $\frac{\Phi}{m}$ & $\frac{1}{\infty}$ & $\frac{10}{5}$ & $\mathbb{Z}$ & $\frac{\infty}{m}$ & $\frac{1}{2}$ & 攵 & $\frac{10}{m}$ & ळ & $\frac{\infty}{m}$ & జ్ & $\frac{\pi}{m}$ & $\frac{\infty}{m}$ & $\stackrel{\infty}{\infty}$ & \\
\hline 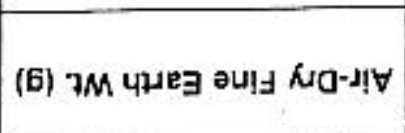 & 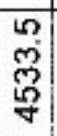 & 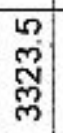 & 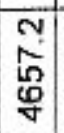 & 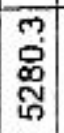 & $\begin{array}{l}\mathrm{N} \\
\hat{5} \\
\mathrm{~g} \\
\end{array}$ & $\begin{array}{l}m \\
m \\
\text { v } \\
\dot{\varphi}\end{array}$ & $\frac{v}{5}$ & $\begin{array}{l}0 \\
0 \\
0 \\
0 \\
6\end{array}$ & $\begin{array}{l}n \\
\text { 号 } \\
\text { \% }\end{array}$ & $\begin{array}{l}N \\
\frac{N}{n} \\
\frac{\omega}{n}\end{array}$ & \begin{tabular}{|c|}
\multirow{N}{*}{} \\
$\underset{\sim}{*}$
\end{tabular} & $\underset{m}{\stackrel{G}{*}}$ & ำ & 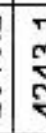 & $\begin{array}{l}5 \\
y \\
y\end{array}$ & 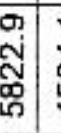 & 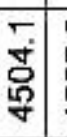 & $\begin{array}{l}m \\
\stackrel{m}{0} \\
\stackrel{9}{0}\end{array}$ & 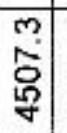 & 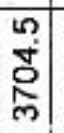 & 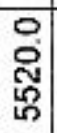 & $\frac{\nabla}{\sigma}$ & \\
\hline (6) $M M$ शdues $M a-\lrcorner \square$ & 今̊ & 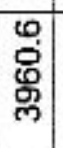 & 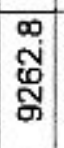 & 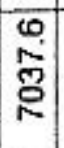 & \begin{tabular}{|c|}
$m$ \\
g \\
点
\end{tabular} & 品 & 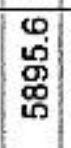 & $\begin{array}{l}\infty \\
0 \\
0 \\
\delta\end{array}$ & $\mid \begin{array}{l}0 \\
\text { M } \\
\text { ğ }\end{array}$ & \begin{tabular}{|c|}
0 \\
0 \\
0 \\
0 \\
\end{tabular} & 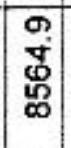 & $\frac{m}{\varphi}$ & है & 5 & 8 & 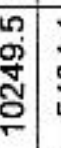 & $\begin{array}{l}\text { 苟 } \\
\text { 岕 }\end{array}$ & 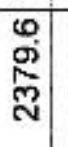 & 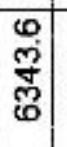 & 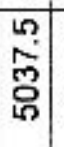 & 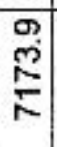 & '̄ & \\
\hline 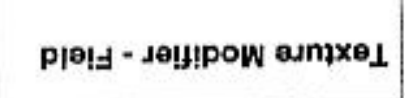 & 믇 & $\frac{\mathscr{c}}{0}$ & $\begin{array}{l}\underset{1}{0} \\
\text { 잉 }\end{array}$ & 怘 & $\sum_{0}^{3}$ & 恕 & $\sum_{0}^{3}$ & 荧 & 品 & 吝 & Z & Q & $\frac{r}{0}$ & c & $z$ & $\frac{\pi}{0}$ & $\frac{\sqrt{0}}{5}$ & 采 & 苑 & 采 & 离 & $\frac{\alpha}{0}$ & \\
\hline ple!y - ejnixe1 l!os & 믇 & 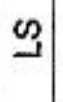 & 0 & 9 & $\vec{n}$ & 0 & 0 & $\vec{\omega}$ & 0 & $\infty$ & 0 & 읃 & a & & $\vec{n}$ & (s) & ळ. & $\vec{\omega}$ & $\vec{\omega}$ & 急 & $\infty$ & $\ddot{\omega}$ & \\
\hline qeר - aגmxa $\perp$ I!os & $\stackrel{心}{\square}$ & a & $\begin{array}{l}0 \\
9 \\
\end{array}$ & 0 & us & 0 & $\omega$ & 9 & $\begin{array}{l}n \\
0 \\
0\end{array}$ & $\infty$ & $\begin{array}{l}0 \\
0 \\
0 \\
\end{array}$ & 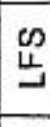 & $\infty$ & 0 & 3 & o & בת & 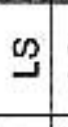 & 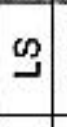 & Оి & 今) & 0 & \\
\hline әэejuns ग!प & $\underset{\square}{\square}$ & 믇 & 마 & 물 & 물 & ㅁ & g & T) & 묻 & 믇 & 묻 & $\stackrel{\text { 区 }}{C}$ & 믇 & $\tau$ & 묻 & 믈 & 므 & 을 & 至 & g & g & 몯 & \\
\hline 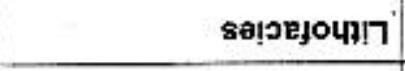 & $\stackrel{\mathbb{2}}{\mathrm{C}}$ & - & 0 & 0 & $\omega$ & $\pi$ & $\pi$ & 은 & مـ & $\leftarrow$ & $\pi$ & 똔 & . & & $\pi$ & 0 & $\omega$ & $\pi$ & 으 & 4 & - & 으 & \\
\hline મฺUnqn & 世 & 이 & 마 & ? & 믈 & 묻 & 믈 & 물 & 믄 & 읃 & 일 & $\stackrel{\mathbb{2}}{\mathrm{C}}$ & g & $\underline{z}$ & 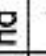 & Q & 몯 & 믇 & 므 & 믇 & 므 & 믇 & \\
\hline 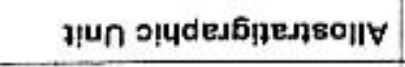 & T & 莚 & 安 & 2 & $\dddot{Q}$ & 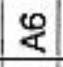 & $\frac{\infty}{4}$ & $\bar{\longleftarrow}$ & 岁 & $\frac{8}{2}$ & $\frac{1}{4}$ & 모 & 8 & $\underline{s}$ & 迹 & $\bar{\longleftarrow}$ & $\bar{x}$ & 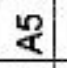 & 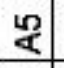 & 茫 & \&ุ & $\stackrel{m}{\&}$ & \\
\hline 이어d & 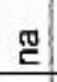 & 员 & 훔 & - & $\overline{0}$ & $\overline{0}$ & 흠 & $\overline{0}$ & 훙 & $\overline{0}$ & $\begin{array}{l}\overline{0} \\
0\end{array}$ & $\stackrel{8}{5}$ & 등 & C & 5 & $\begin{array}{c}5 \\
0 \\
\end{array}$ & ס & 훙 & - & ¿ & 훔 & 흠 & \\
\hline บоำองา & $\mathbb{E}$ & $\frac{\mathfrak{N}}{\stackrel{\sim}{\sim}}$ & 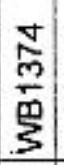 & 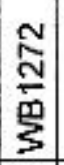 & $\frac{N}{\frac{N}{m}}$ & 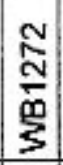 & $\frac{N}{\stackrel{N}{N}}$ & $\frac{\mathbb{N}}{\frac{m}{m}}$ & $\frac{N}{\mathbf{N}}$ & 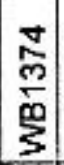 & $\frac{ \pm}{\frac{J}{m}}$ & 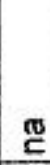 & $\frac{N}{2}$ & $\frac{2}{5}$ & $\frac{v}{\frac{v}{z}}$ & $\frac{⿱ 亠 乂}{m}$ & 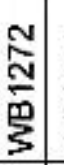 & $\underset{⿱ 亠 䒑}{\frac{m}{m}}$ & $\frac{\mathbb{N}}{\mathrm{N}}$ & 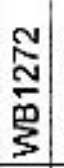 & 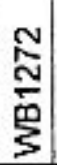 & 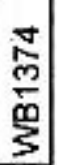 & \\
\hline (uv) yłdeg damoา & $\mathbb{T}$ & s & 哥 & $\stackrel{\oplus}{\sim}$ & क्व & 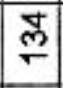 & 움 & \begin{tabular}{|l|} 
罥 \\
\end{tabular} & ลั & \% & 옹 & $\sqrt{5}$ & รู & 5 & $\bar{\nabla}$ & 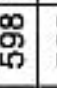 & ष्ठ & $\stackrel{\substack{\infty \\
\hdashline}}{-}$ & 蛋 & 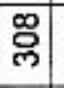 & พั้ & $\overline{\mathrm{N}}$ & \\
\hline (wo) पhdəa dəddn & $\mathbb{Z}$ & 福 & Y & $\begin{array}{ll}\stackrel{N}{W} \\
\stackrel{4}{\leftarrow}\end{array}$ & 5 & 9 & 竝 & $\overline{\mathrm{N}}$ & $\frac{\infty}{v}$ & 商 & $\stackrel{9}{5}$ & $\stackrel{\text { g }}{ᄃ}$ & $n$ & 8 & 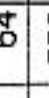 & 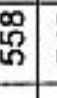 & 骨 & $\stackrel{0}{=}$ & $\stackrel{0}{\varnothing}$ & กี & $\underset{v}{5}$ & รั & \\
\hline uoz!noH & $\stackrel{\mathbb{Z}}{\check{C}}$ & $\begin{array}{l}\text { 总 } \\
\text { 品 }\end{array}$ & ¿ & 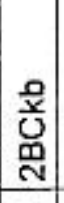 & 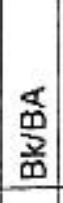 & 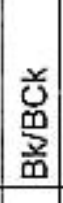 & \begin{tabular}{|l|} 
음 \\
\end{tabular} & 0 & \begin{tabular}{|c} 
\\
u \\
\end{tabular} & 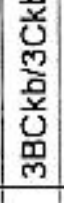 & 仓̌ & 또 & e & 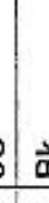 & 号 & u. & U. & 善 & 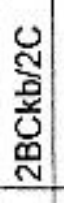 & $\begin{array}{l}\text { e्m } \\
\text { 总 } \\
\text { 品 }\end{array}$ & 包 & U & \\
\hline apoj ә|dues pla!j] & 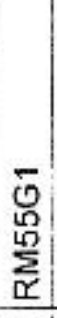 & 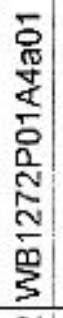 & 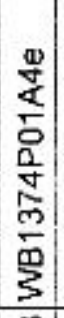 & 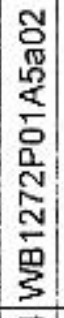 & 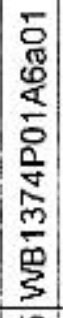 & 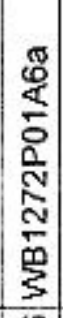 & 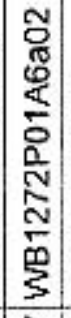 & 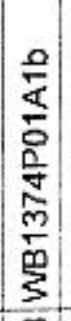 & 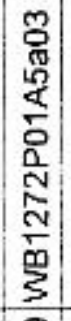 & 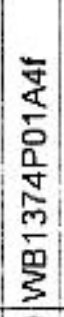 & 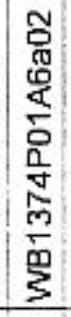 & 峁 & 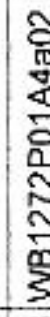 & 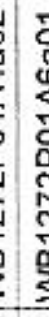 & 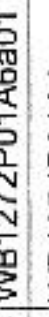 & 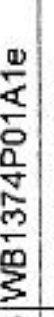 & 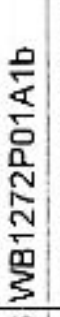 & 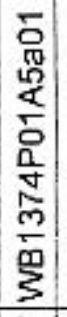 & 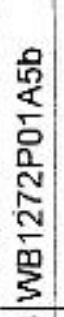 & 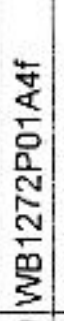 & 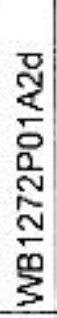 & 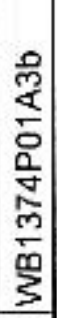 & 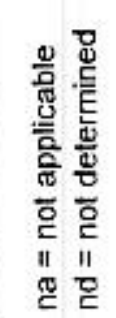 \\
\hline ejdures & -1 & $N$ & $m$ & 8 & 60 & 0 & $N$ & $\infty$ & $\sigma$ & 은 & $F$ & $\stackrel{2}{2}$ & $m$ & $?$ & 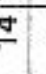 & $\frac{n}{2}$ & $\dddot{0}$ & 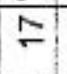 & $\stackrel{\infty}{\infty}$ & $\sigma$ & 이 & $\bar{N}$ & \\
\hline чээЕ & $\frac{0}{4}$ & $\frac{O}{8}$ & $\frac{0}{7}$ & $\frac{0}{8}$ & $\frac{0}{8}$ & $\frac{0}{4}$ & $\frac{0}{4}$ & $\frac{0}{4}$ & $\frac{9}{4}$ & 䱛 & 号 & $\frac{0}{8}$ & 号 & 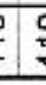 & $\frac{1}{4}$ & $\begin{array}{l} \\
\\
\end{array}$ & $\frac{9}{8}$ & $\frac{9}{8}$ & $\frac{9}{4}$ & $\frac{9}{\square}$ & $\frac{0}{4}$ & $\frac{0}{8}$ & \\
\hline
\end{tabular}




\begin{tabular}{|c|c|c|c|c|c|c|c|c|c|c|c|c|}
\hline (H) Gunsez & \begin{tabular}{|l|}
0 \\
0 \\
0 \\
0 \\
\end{tabular} & 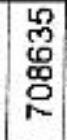 & 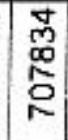 & 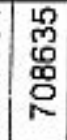 & $\stackrel{\mathbb{2}}{\mathrm{C}}$ & 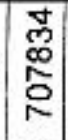 & 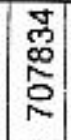 & 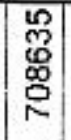 & 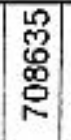 & $\stackrel{w}{c}$ & $\begin{array}{l} \pm \\
m \\
0 \\
5 \\
0 \\
N\end{array}$ & $\begin{array}{l}\text { J } \\
\text { D } \\
5 \\
5 \\
\end{array}$ \\
\hline (ఘ) Бน!นमоN & 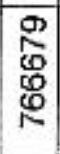 & 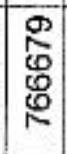 & $\begin{array}{l}P \\
\mathbb{Z} \\
\mathfrak{E} \\
\mathfrak{R}\end{array}$ & 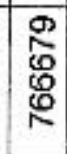 & $\stackrel{\mathbb{T}}{\mathscr{C}}$ & 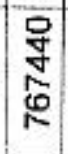 & $\begin{array}{l}q \\
\text { 足 } \\
\text { 员 } \\
\text { N }\end{array}$ & $\begin{array}{l}\text { D } \\
\mathbb{6} \\
\mathbb{\Phi} \\
\mathbb{N}\end{array}$ & \begin{tabular}{l|} 
\\
\\
0 \\
8 \\
$\mathbb{D}$ \\
\end{tabular} & $\stackrel{\mathbb{Z}}{\mathbb{C}}$ & $\begin{array}{l}9 \\
\text { g } \\
0 \\
0 \\
1\end{array}$ & 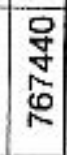 \\
\hline 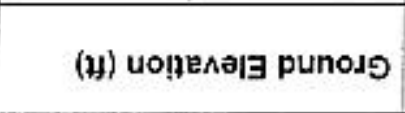 & $\frac{N}{\infty}$ & $\frac{N}{m}$ & $\frac{n}{\infty}$ & $\underset{m}{\infty}$ & 胥 & $\underset{m}{\frac{10}{m}}$ & $\frac{m}{m}$ & $\frac{\widetilde{\delta}}{\tilde{m}}$ & $\frac{N}{\infty}$ & $\stackrel{\pi}{C}$ & $\frac{n}{\infty}$ & $\begin{array}{l}\infty \\
\infty \\
\end{array}$ \\
\hline 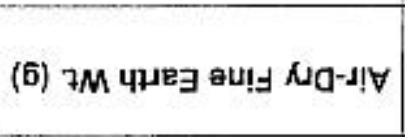 & \begin{tabular}{|l|}
0 \\
0 \\
0 \\
9 \\
\end{tabular} & $\begin{array}{l}m \\
m \\
\text { ले }\end{array}$ & \begin{tabular}{|c|} 
\\
\\
\\
\\
\end{tabular} & $\begin{array}{l}m \\
0 \\
0 \\
0 \\
\infty \\
m\end{array}$ & $\begin{array}{c}N \\
\stackrel{N}{S} \\
\stackrel{m}{m}\end{array}$ & $\left|\begin{array}{c}\infty \\
\text { g } \\
\text { g } \\
\text { f }\end{array}\right|$ & $\begin{array}{l}0 \\
\frac{\sigma}{\sigma} \\
\frac{\sigma}{\sigma}\end{array}$ & $\begin{array}{l}10 \\
0 \\
\stackrel{0}{0} \\
\text { D. }\end{array}$ & \begin{tabular}{|c|} 
\\
0 \\
0 \\
$\tilde{\sigma}$ \\
\end{tabular} & $\begin{array}{l}\text { P. } \\
\text { जे }\end{array}$ & \begin{tabular}{|c|} 
\\
ల్ \\
ஸे \\
\end{tabular} & \begin{tabular}{|c|} 
\\
$\infty$ \\
$\tilde{m}$ \\
$\tilde{m}$ \\
\end{tabular} \\
\hline (6) JM eqdures $S \Delta \sigma-\lambda \mid \forall$ & 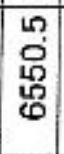 & $\begin{array}{l}N \\
\stackrel{0}{N} \\
\stackrel{D}{O}\end{array}$ & $\begin{array}{l}0 \\
\text { ci } \\
\text { o } \\
\text { 员 }\end{array}$ & $\frac{J}{\sigma}$ & m & $\begin{array}{l} \\
\text { 品 } \\
\text { g } \\
\end{array}$ & 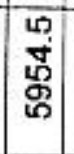 & 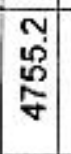 & 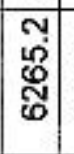 & $\begin{array}{l}\vec{Z} \\
\dot{J} \\
\dot{v}\end{array}$ & 官 & $\begin{array}{l}5 \\
\stackrel{6}{0} \\
0\end{array}$ \\
\hline 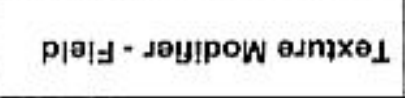 & $\frac{\alpha}{0}$ & $\begin{array}{l}\times \\
\text { 怘 } \\
\end{array}$ & 品 & 采 & Ф & $\frac{\alpha}{0}$ & 采 & 采 & 临 & 昰 & $\underset{\mathbb{x}}{\mathbb{0}}$ & 永 \\
\hline 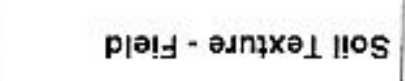 & $\bar{\omega}$ & $\vec{\omega}$ & 0 & $\Omega$ & 무 & $\vec{\omega}$ & s & $\vec{\omega}$ & $\vec{ळ}$ & ळ & क & $\omega$ \\
\hline qeר - ammxə] IIOS & 0 & $\infty$ & $\omega$ & $\begin{array}{l}0 \\
0 \\
0\end{array}$ & 足 & s & $\infty$ & $\infty$ & $\begin{array}{l}0 \\
0 \\
0\end{array}$ & $\dot{\omega}$ & $\infty$ & 号 \\
\hline อэe & 믇 & 묻 & 을 & 미 & $\stackrel{\varpi}{c}$ & 묻 & 물 & $\mathrm{g}$ & $\mathrm{g}$ & 焉 & 읻 & 묻 \\
\hline sә!ฺㄹㅇำ!7 & 0 & $\stackrel{0}{\check{c}}$ & مـ & $\frac{0}{0}$ & 茫 & - & ـ & of & ـ & $\mathbb{C}$ & ـ & 0 \\
\hline 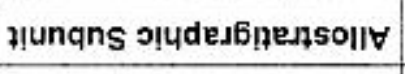 & 믇 & 믇 & 무 & 믇 & (ָ) & 믇 & 믇 & 믿 & tg & $\mathbb{Z}$ & 무 & g \\
\hline 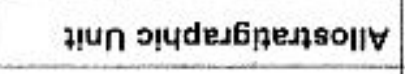 & $\&$ & \& & 48 & \& & 胥 & 8 & 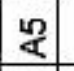 & $<$ & $\stackrel{m}{q}$ & 四 & \& & \& \\
\hline 이어d & $\begin{array}{l}\overline{0} \\
0\end{array}$ & 흠 & $\overline{0}$ & $\begin{array}{l}\overline{0} \\
\end{array}$ & $\stackrel{\mathbb{D}}{\mathrm{D}}$ & 官 & $\begin{array}{l} \\
\\
\end{array}$ & 둠 & 员 & 모 & $\begin{array}{l}5 \\
0 \\
\end{array}$ & 5 \\
\hline บำ & $\frac{N}{N}$ & $\frac{N}{\stackrel{N}{N}}$ & $\frac{\mathrm{s}}{\mathrm{m}}$ & $\frac{\mathrm{N}}{\mathrm{N}}$ & $\stackrel{\mathbb{2}}{\mathrm{C}}$ & $\begin{array}{l}\mathbf{N} \\
\stackrel{m}{\mathbf{m}} \\
\mathbf{3}\end{array}$ & 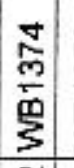 & $\begin{array}{l}\frac{N}{N} \\
\frac{1}{3} \\
3\end{array}$ & $\frac{\mathrm{N}}{\stackrel{\mathrm{N}}{\mathrm{m}}}$ & $\mathbb{\mathfrak { I }}$ & 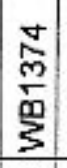 & 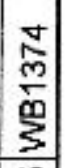 \\
\hline (سо) чldag sәмоา & 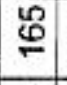 & $\begin{array}{l}0 \\
1 \\
\infty\end{array}$ & $\stackrel{\mathbb{N}}{N}$ & 峖 & 元 & 怘 & $\mathbb{N}$ & Nָ & 守 & 要 & $\begin{array}{l}I \\
N\end{array}$ & స్లి \\
\hline (us) uldəa dəddn & ले & \begin{tabular}{|l|} 
\\
0
\end{tabular} & $\begin{array}{l}\infty \\
\stackrel{\infty}{-}\end{array}$ & 守 & 묻 & $\underset{N}{N}$ & \begin{tabular}{|l|}
$\mathscr{N}$ \\
$\mathbb{N}$
\end{tabular} & ำ & $\begin{array}{l}\infty \\
⿱ ⺊ \\
m\end{array}$ & "ָ & $\stackrel{\infty}{\stackrel{\infty}{2}}$ & 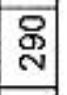 \\
\hline UOZ!ฺOH & $\begin{array}{l}\mathrm{g} \\
\text { 㩊 }\end{array}$ & ن & $\begin{array}{l}\stackrel{9}{8} \\
\text { 鱼 } \\
\text {. }\end{array}$ & u & 苋 & 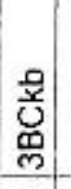 & U & $\begin{array}{l}\text { 号 } \\
\text { ⿳亠口冋. } \\
\text { N }\end{array}$ & U & 写 & 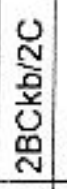 & 总 \\
\hline epos әjdues ple! & 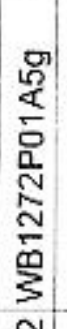 & 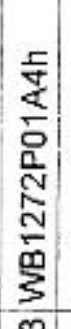 & 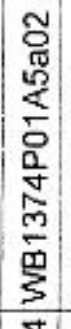 & 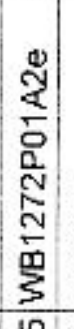 & 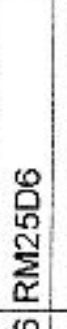 & 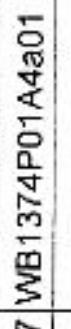 & $\begin{array}{l}0 \\
0 \\
0 \\
0 \\
0 \\
0 \\
0 \\
0 \\
0 \\
5\end{array}$ & 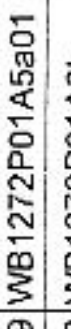 & 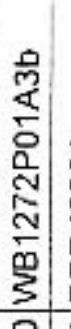 & 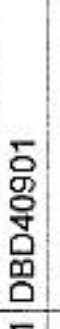 & $\begin{array}{l}\frac{0}{2} \\
\frac{0}{4} \\
\frac{0}{0} \\
\frac{5}{5} \\
\frac{m}{5} \\
\frac{m}{5}\end{array}$ & 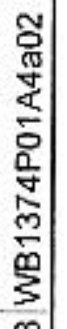 \\
\hline शdures & $\approx$ & ঙ & ন & $\stackrel{W}{N}$ & $\mathbb{N}$ & ง & $\stackrel{\sim}{\sim}$ & g: & 이 & $\bar{m}$ & $\mathbb{N}$ & $m$ \\
\hline yग1Eg & 임 & $\frac{0}{\dot{v}}$ & $\frac{0}{\dot{v}}$ & 음 & $\frac{0}{4}$ & $\frac{0}{4}$ & $\frac{9}{7}$ & $\frac{9}{4}$ & $\frac{9}{4}$ & \begin{tabular}{l|} 
\\
\\
\end{tabular} & $\frac{0}{\dot{y}}$ & $\frac{0}{\dot{y}}$ \\
\hline
\end{tabular}




\section{This Page Intentionally Left Blank}




\section{APPENDIX B}

U.S. Department of Agriculture Particle Size Fractions 


\begin{tabular}{|c|c|c|c|c|c|c|c|c|c|c|c|c|c|c|c|c|c|c|c|c|c|}
\hline (\%2M) KEIO IETOL & เึ & $\stackrel{n}{m}$ & $\begin{array}{l}10 \\
10\end{array}$ & 요 & $\begin{array}{ll}\sigma \\
6\end{array}$ & m. & $\begin{array}{l}50 \\
60\end{array}$ & $\stackrel{\mathscr{v}}{\dot{v}}$ & $\underset{\infty}{+}$ & $\stackrel{\infty}{m}$ & $\bar{\oplus}$ & 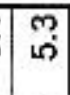 & $\begin{array}{l} \\
\end{array}$ & $\stackrel{\text { L? }}{=}$ & \begin{tabular}{l|} 
\\
\\
\end{tabular} & $\underset{v}{N}$ & $\bar{\sigma}$ & $\begin{array}{l}\mathscr{v} \\
\dot{v}\end{array}$ & $\begin{array}{l}\Phi \\
\stackrel{\sim}{*}\end{array}$ & $\begin{array}{l}\sigma \\
\dot{\varphi}\end{array}$ & $\begin{array}{l}\infty \\
\omega \\
\end{array}$ \\
\hline (\%łM) मIS eu!y & 음 & $\stackrel{\infty}{m}$ & $\begin{array}{l}M \\
\nabla\end{array}$ & $\bar{m}$ & $\underset{y}{y}$ & $\stackrel{4}{+}$ & V̛ & $\stackrel{\infty}{+}$ & $\stackrel{O}{N}$ & ल & is & F & \begin{tabular}{|l|}
0 \\
\\
\end{tabular} & $\stackrel{\infty}{\forall}$ & $\bar{N}$ & \begin{tabular}{|l|}
0 \\
\end{tabular} & 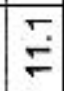 & $\stackrel{M}{m}$ & $\begin{array}{l}\infty \\
\omega^{\circ}\end{array}$ & in & ले \\
\hline$(\%$ (M) d!IS asJeos & की & $\stackrel{\infty}{\forall}$ & $\stackrel{\bullet}{\infty}$ & 足 & O & $\because$ & $\stackrel{m}{\mathrm{~N}}$ & $\stackrel{\infty}{\infty}$ & $\stackrel{N}{-}$ & $\begin{array}{l}0 \\
4\end{array}$ & $\stackrel{\square}{-}$ & 100 & \begin{tabular}{|l|} 
क \\
ल
\end{tabular} & 步 & ণे & \begin{tabular}{|l|} 
\\
0
\end{tabular} & $\check{\infty}^{-}$ & \begin{tabular}{|l|}
$m$ \\
Li
\end{tabular} & 苗 & $\underset{\text { N }}{N}$ & $\omega$ \\
\hline (\%みM) म!S IEOL & ָั & $\Gamma_{\infty}^{\prime}$ & 뭉 & $\hat{\infty}_{\infty}$ & $\begin{array}{c}m \\
\infty\end{array}$ & $\omega$ & $\begin{array}{l}0 \\
0\end{array}$ & $\stackrel{n}{+}$ & $\begin{array}{l}\infty \\
\infty\end{array}$ & $\underset{\infty}{N}$ & $\begin{array}{l}10 \\
6\end{array}$ & $\bar{\varphi}$ & $\begin{array}{l}\omega \\
\text { के }\end{array}$ & ल & $\begin{array}{l}\omega \\
\varphi\end{array}$ & $\begin{array}{l}40 \\
6\end{array}$ & ? & $\begin{array}{l}\sigma \\
\text { के }\end{array}$ & m & ஸั & $\begin{array}{l}\infty \\
0 \\
0\end{array}$ \\
\hline$(\% \geqslant M)$ pues euly Mon & $\stackrel{\infty}{\infty}$ & $\stackrel{+}{=}$ & $\begin{array}{l}0 \\
\text { ஸें }\end{array}$ & 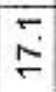 & $\underset{\infty}{\infty}$ & $\begin{array}{l}\infty \\
0 \\
0\end{array}$ & $\stackrel{\infty}{\infty}$ & लु & $\bar{\sigma}$ & $\stackrel{m}{\stackrel{m}{\rho}}$ & $\stackrel{N}{N}$ & J & ¿ & $\stackrel{m}{N}$ & $\stackrel{m}{\mathcal{N}}$ & $\begin{array}{l}0 \\
\stackrel{9}{-}\end{array}$ & $\bar{\sigma}$ & $\stackrel{0}{=}$ & ली & $\begin{array}{c}\checkmark \\
\stackrel{\sim}{\mathrm{N}}\end{array}$ & $\bar{d}$ \\
\hline (\%7M) pues auly & $\begin{array}{l}\infty \\
\sigma \\
\sigma\end{array}$ & $\stackrel{m}{\sim}$ & $\overrightarrow{\mathrm{j}}$ & $\underset{N}{N}$ & Nָ & $\stackrel{\vec{m}}{\stackrel{2}{\sigma}}$ & 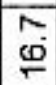 & $\begin{array}{l}\text { Ln } \\
\stackrel{\infty}{N}\end{array}$ & $\begin{array}{l}\infty \\
\stackrel{\infty}{\sigma}\end{array}$ & $\begin{array}{l}\infty \\
\text { N̦ }\end{array}$ & N & 它 & \begin{tabular}{|l|}
$\infty$ \\
$\dot{n}$
\end{tabular} & 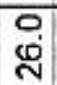 & $\begin{array}{l}m \\
\dot{\vec{d}}\end{array}$ & $\stackrel{0}{\circ}$ & $\begin{array}{l} \\
\sigma \\
\end{array}$ & $\begin{array}{l}\infty \\
\stackrel{\leftrightarrow}{~}\end{array}$ & 㲾 & ल) & ल) \\
\hline (\%ұM) pues wn!̣pew & m & $\begin{array}{l}\infty \\
\stackrel{\infty}{2}\end{array}$ & $\begin{array}{l}\mathscr{O} \\
\sigma^{\prime}\end{array}$ & $\stackrel{\Xi}{\circ}$ & $\begin{array}{l}m \\
\mathbb{T}\end{array}$ & ñ & ஜே & $\begin{array}{l}\infty \\
\infty\end{array}$ & $\check{\infty}$ & $\ddot{\sigma}$ & $\begin{array}{l}\stackrel{0}{\circ} \\
\infty\end{array}$ & \begin{tabular}{|l|}
$\infty$ \\
$\infty$ \\
$\sigma$
\end{tabular} & \begin{tabular}{|l|}
$\infty$ \\
$\stackrel{\infty}{*}$
\end{tabular} & $\begin{array}{l}\stackrel{\varphi}{ } \\
\stackrel{2}{2}\end{array}$ & $\begin{array}{l}\infty \\
0 \\
0\end{array}$ & $\stackrel{\infty}{\check{\infty}}$ & 응 & 党 & 음 & \% & $\bar{\sigma}$ \\
\hline (\%2M) pues asuroo & 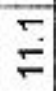 & के & $\begin{array}{l} \\
0 \\
\end{array}$ & $\begin{array}{l}\stackrel{m}{m} \\
\stackrel{\sim}{*}\end{array}$ & $\begin{array}{l}\varphi \\
\stackrel{5}{\circ}\end{array}$ & ल) & $\overline{\mathrm{N}}$ & $\overline{6}$ & $\begin{array}{l}\text { ¿ } \\
\text { Nิ }\end{array}$ & $\begin{array}{l}10 \\
6 \\
\end{array}$ & $\begin{array}{l}\omega \\
\sigma \\
\sigma\end{array}$ & $\bar{N}$ & 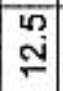 & $\stackrel{0}{\circ}$ & $\bar{\sigma}$ & $\begin{array}{l}0 \\
\infty \\
\infty\end{array}$ & $\stackrel{0}{=}$ & $\stackrel{\omega}{\square}$ & $\hat{M}$ & $\stackrel{N}{N}$ & $\bar{r}$ \\
\hline (\%1M) pUES esueoO KNA & $\stackrel{\sim}{\omega}$ & $\stackrel{\square}{\stackrel{\sim}{\sigma}}$ & $\begin{array}{l}0 \\
\infty\end{array}$ & $\begin{array}{l}\stackrel{\sim}{m} \\
\stackrel{m}{-}\end{array}$ & $\begin{array}{l}m \\
\dot{\square}\end{array}$ & m. & 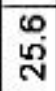 & o & $\begin{array}{l}\sigma \\
\sigma \\
\sigma\end{array}$ & $\stackrel{0}{=}$ & $\begin{array}{l}\infty \\
4 \\
2\end{array}$ & $\begin{array}{c}N \\
\sigma\end{array}$ & $\begin{array}{l}0 \\
\dot{ \pm} \\
\end{array}$ & $m$ & $\stackrel{n}{\mathrm{n}}$ & \begin{tabular}{l|}
$\infty$ \\
山⿱⺈ \\
\end{tabular} & $\stackrel{T}{=}$ & $\begin{array}{l}\infty \\
\dot{U}\end{array}$ & N & $\begin{array}{l}0 \\
\infty\end{array}$ & $M$ \\
\hline$(\%$ (M) pues I & $\bar{N}$ & $\stackrel{\infty}{\infty}$ & $\overline{\mathrm{J}}$ & $\begin{array}{l}3 \\
\dot{\infty}\end{array}$ & $\begin{array}{l}\infty \\
\dot{\Phi} \\
\dot{\Phi}\end{array}$ & $\check{\varpi}$ & 怘 & O̊ & $\underset{\infty}{\infty}$ & $\begin{array}{l}0 \\
\infty \\
\infty \\
\infty\end{array}$ & 峁 & $\begin{array}{l}\mathbf{2} \\
\mathbf{D} \\
\end{array}$ & \begin{tabular}{|l|}
0 \\
$\infty$ \\
$\infty$ \\
$\infty$
\end{tabular} & 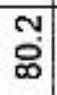 & $\begin{array}{l}0 \\
\infty \\
\infty\end{array}$ & 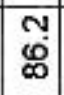 & $\frac{0}{i}$ & $\begin{array}{l}\mathbf{0} \\
\mathscr{\infty}\end{array}$ & $\bar{F}$ & 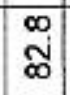 & ปู \\
\hline (\%ұM) sapqqed eu!y & 은 & $\stackrel{5}{n}$ & $\bar{\oplus}$ & $\begin{array}{l}\mathscr{\varphi} \\
\infty \\
\sigma\end{array}$ & $\begin{array}{l}0 \\
60 \\
\end{array}$ & 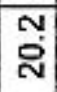 & $\stackrel{10}{\stackrel{10}{5}}$ & $\stackrel{\varphi}{\Gamma}$ & $\stackrel{m}{=}$ & $\stackrel{F}{\leftarrow}$ & $\begin{array}{l} \\
\infty \\
\infty\end{array}$ & \begin{tabular}{|c|} 
\\
\end{tabular} & $\frac{10}{=}$ & $\begin{array}{l}\infty \\
\wp \\
\wp\end{array}$ & $\begin{array}{l}\infty \\
\stackrel{N}{\leftarrow}\end{array}$ & 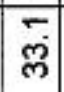 & m & $\stackrel{\infty}{\Sigma}$ & $\underline{Y}$ & ஸึ & $\stackrel{\emptyset}{=}$ \\
\hline 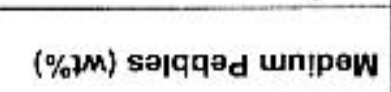 & m & $\begin{array}{l}\text { क } \\
m \\
m\end{array}$ & 우 & 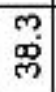 & 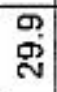 & $\frac{8}{8}$ & $\frac{N}{m}$ & $\stackrel{N}{\sigma}$ & $m$ & $\stackrel{M}{m}$ & $\begin{array}{l}m \\
\dot{q}\end{array}$ & \begin{tabular}{|l|}
$\mid \mathfrak{n}$ \\
$\tilde{N}$
\end{tabular} & 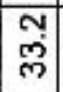 & 吕 & $\begin{array}{l}\omega \\
\stackrel{\omega}{\mathrm{N}}\end{array}$ & "్ల & $\begin{array}{c}\text { N̦ } \\
\text { ָे }\end{array}$ & m & 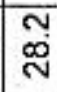 & के & $\frac{\infty}{\sim}$ \\
\hline (\%łM) selqqәd asueoo & $\begin{array}{l}\infty \\
\text { N }\end{array}$ & $\stackrel{5}{-}$ & 영 & $\begin{array}{l}\infty \\
\tilde{m}\end{array}$ & $\stackrel{5}{=}$ & \begin{tabular}{|l|}
0 \\
$\infty$
\end{tabular} & $\stackrel{\omega}{\mathrm{N}}$ & $\begin{array}{l}n \\
\text { ले }\end{array}$ & $\bar{\sigma}$ & \% & $\begin{array}{l}\text { mo } \\
\text { in }\end{array}$ & \begin{tabular}{l|l|} 
\\
$\sim$
\end{tabular} & $\begin{array}{l} \\
\\
\end{array}$ & \begin{tabular}{l|}
$\infty$ \\
$\omega$ \\
$\omega$
\end{tabular} & 80 & \begin{tabular}{|c|} 
\\
m.
\end{tabular} & 눙 & $N$ & 꽁 & 음 & 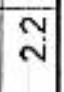 \\
\hline 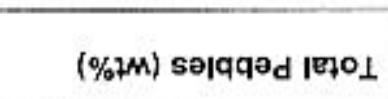 & $\begin{array}{l}\infty \\
\infty \\
\infty\end{array}$ & $\stackrel{m}{n}$ & 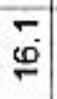 & $\tilde{\circ}$ & 0 & $\dot{O}$ & $\sum_{\infty}^{\infty}$ & क & एँ & $\begin{array}{l}\infty \\
10 \\
0\end{array}$ & 웅 & $\begin{array}{l}M \\
\text { Li }\end{array}$ & \begin{tabular}{|l|} 
号 \\
号
\end{tabular} & $\overrightarrow{\dot{H}}$ & $\begin{array}{l}\text { क } \\
\text { एक }\end{array}$ & $\bar{r}$ & $\begin{array}{l} \\
\dot{g} \\
\end{array}$ & $\begin{array}{l}\dot{U} \\
\dot{U}\end{array}$ & $\overline{\text { त्र }}$ & \begin{tabular}{|c|}
$\Phi$ \\
$\dot{m}$ \\
\end{tabular} & 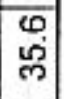 \\
\hline (шэ) чاdอם دөмоา & $\Phi$ & 品 & TI & $E$ & 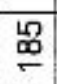 & 즘 & প్ల & 음 & 윰 & 은 & $\stackrel{\circ \mathrm{O}}{\mathrm{N}}$ & 要 & $\frac{\mathscr{N}}{\sim}$ & $\stackrel{\square}{\square}$ & $\mathscr{q}$ & $\mathscr{m}$ & Бे & 茨 & 吕 & 『 & T \\
\hline (uv) u’dag saddn & 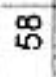 & $\mathscr{m}$ & $\stackrel{\pi}{c}$ & $\mathscr{q}$ & $\stackrel{\circ}{6}$ & $\stackrel{\text { m }}{\stackrel{2}{2}}$ & $\bar{\infty}$ & 0 & 음 & 0 & $\hat{\infty}$ & एव & 吕 & 品 & 우 & $\sqrt[W]{ }$ & $E$ & 은 & 苟 & (I) & 읻 \\
\hline & $\begin{array}{l}\frac{5}{0} \\
\text { 咅 } \\
\text { ஸे }\end{array}$ & 3 & 垔 & 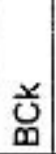 & $\begin{array}{l}\text { 羔 } \\
\text { 总 } \\
\text {. }\end{array}$ & 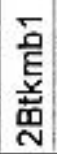 & $\frac{N}{\frac{N}{0}}$ & $<$ & 总 & U⿺ & 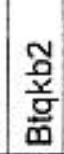 & 包 & 竝 & 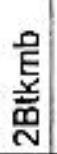 & ڤ & $\mathbb{S}$ & 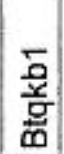 & 5 & $\begin{array}{l}\text { N } \\
\text { है } \\
\text { 总 } \\
\text { ․ }\end{array}$ & Tु & 몯 \\
\hline әpos 리wes plo!y & 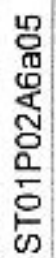 & 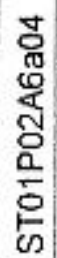 & $\frac{\grave{\Phi}}{\sum_{\alpha}}$ & 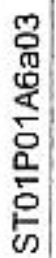 & 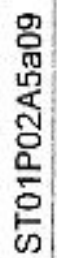 & 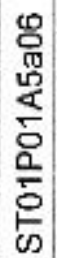 & 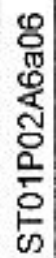 & $\begin{array}{l}5 \\
0 \\
0 \\
0 \\
0 \\
0 \\
0 \\
\frac{0}{0} \\
0 \\
0\end{array}$ & 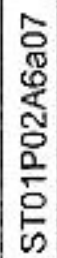 & 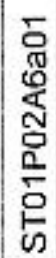 & 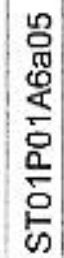 & $\mid$ & 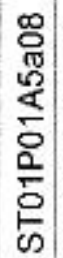 & 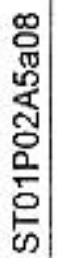 & 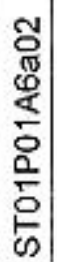 & 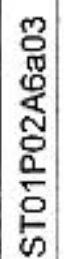 & 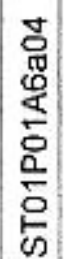 & 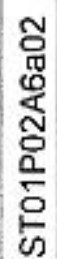 & 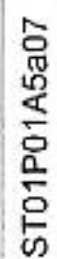 & 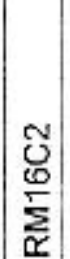 & 罣 \\
\hline a|dues & 5 & $N$ & $m$ & $\nabla$ & का & $\omega$ & $M$ & $\infty$ & $\sigma$ & 음 & $F$ & $\simeq$ & $\stackrel{m}{2}$ & $\nabla$ & $\dddot{2}$ & 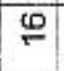 & $=$ & $\stackrel{\infty}{=}$ & $\stackrel{\circ}{-}$ & 8 & $\bar{N}$ \\
\hline पวक्ष & ర్ & 웜 & 守 & ¿্口 & ছ্ & 守 & 家 & S & 守 & 守 & 守 & 움 & 守 & 守 & 守 & 5 & 항 & 亏 & 5 & 守 & 함 \\
\hline
\end{tabular}




\begin{tabular}{|c|c|c|c|c|c|c|c|c|c|c|c|c|c|c|c|c|c|c|c|c|}
\hline 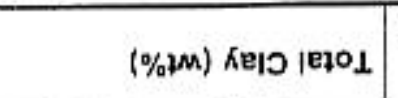 & $\begin{array}{l}9 \\
\dot{\nabla}\end{array}$ & \begin{tabular}{l|l}
$n$ \\
$N$
\end{tabular} & $\begin{array}{l}m \\
\dot{\nabla}\end{array}$ & 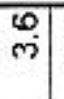 & 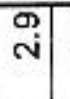 & $\stackrel{7}{\nabla}$ & $\bar{N}$ & $\begin{array}{l}m \\
\oplus\end{array}$ & \begin{tabular}{l|l}
$\begin{array}{l}8 \\
\end{array}$ & 1
\end{tabular} & $\overline{L i}$ & \begin{tabular}{l|l} 
& \\
4 &
\end{tabular} & \begin{tabular}{l|} 
क \\
\end{tabular} & \begin{tabular}{l|l} 
& 7 \\
& 2
\end{tabular} & \begin{tabular}{l|l} 
& $\infty$ \\
$m$ & $\infty$
\end{tabular} & \begin{tabular}{l|l}
$\infty$ & $\infty$ \\
$m$ & $m$
\end{tabular} & \begin{tabular}{l|l}
\multirow{2}{*}{} & L \\
\end{tabular} & \begin{tabular}{l|l} 
& 6 \\
& 0
\end{tabular} & $\begin{array}{l}\dot{0} \\
\end{array}$ & \begin{tabular}{l|}
$\infty$ \\
$\infty$ \\
$\omega^{\prime}$
\end{tabular} & $\stackrel{m}{\nabla}$ \\
\hline 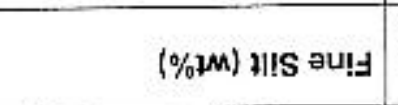 & $\begin{array}{l}\oplus \\
\oplus \\
\end{array}$ & 0 & ले & $\begin{array}{l}+ \\
+\end{array}$ & $\vec{m}$ & $\vec{\nabla}$ & $\begin{array}{l}N \\
m \\
m\end{array}$ & $\begin{array}{l}m \\
\dot{v}\end{array}$ & \begin{tabular}{l|l} 
& \\
$m$ &
\end{tabular} & \begin{tabular}{l|l}
$\infty$ \\
$\dot{v}$
\end{tabular} & $\begin{array}{ll}\text { m. } \\
\text { फे }\end{array}$ & प⿺ & $\begin{array}{c}\sigma \\
\text { ले }\end{array}$ & $\overline{\mathrm{N}}$ & \begin{tabular}{l|l}
$\underset{N}{N}$ & $\nabla$ \\
& $\nabla$
\end{tabular} & \begin{tabular}{l|l} 
& $\overline{\mathrm{d}}$ \\
\end{tabular} & $\begin{array}{l}\dot{\infty} \\
i\end{array}$ & 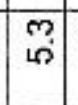 & $\stackrel{\oplus}{\sim}$ & $\begin{array}{l}\underset{1}{*} \\
\text { S. }\end{array}$ \\
\hline 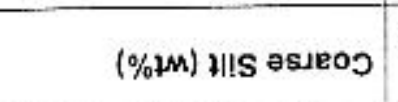 & बे & क & $\begin{array}{l}\infty \\
\infty\end{array}$ & $\stackrel{?}{=}$ & $\begin{array}{l}\omega \\
\infty\end{array}$ & $\begin{array}{l}100 \\
100\end{array}$ & \begin{tabular}{|l|}
0 \\
0 \\
0
\end{tabular} & \begin{tabular}{l|l}
$\infty$ \\
$\dot{\sigma}$ \\
\end{tabular} & $\begin{array}{l}6 \\
6\end{array}$ & $\begin{array}{c}N \\
\text { in }\end{array}$ & $\underset{\omega}{N}$ & $\begin{array}{l}\omega \\
\stackrel{\omega}{\sigma}\end{array}$ & $\begin{array}{l}m \\
m\end{array}$ & \begin{tabular}{l|l}
\multirow{2}{*}{} & ल
\end{tabular} & \begin{tabular}{c|c}
0 & 0 \\
$m$ & 0
\end{tabular} & \begin{tabular}{c|c}
$\infty$ \\
\hdashline
\end{tabular} & $\begin{array}{l}\nabla \\
\nabla\end{array}$ & $\underset{\nabla}{\sim}$ & $\stackrel{\infty}{-}$ & $\begin{array}{l}\underset{1}{N} \\
\infty\end{array}$ \\
\hline (\%みM) H!S IEOL & $\begin{array}{l}0 \\
\varphi \\
\varphi\end{array}$ & $\begin{array}{l}9 \\
\dot{J}\end{array}$ & $\bar{\sigma}$ & $\stackrel{n}{\stackrel{n}{\sigma}}$ & $\stackrel{\oplus}{=}$ & $\hat{\omega}$ & $\hat{\omega}$ & $\bar{\sigma}$ & mo & 응 & $\stackrel{\text { L }}{=}$ & $\begin{array}{l}\text { 임 } \\
\text { की }\end{array}$ & $\hat{\sigma}$ & $\begin{array}{l}\infty \\
\dot{v}\end{array}$ & $\stackrel{m}{\sim}$ & $\begin{array}{l}m \\
= \\
=\end{array}$ & \% & \begin{tabular}{|l|}
0 \\
$\sigma$
\end{tabular} & $\underset{v}{\nabla}$ & $\begin{array}{l}\text { ஸे } \\
\text { ஸे }\end{array}$ \\
\hline (\%2M) pues aU!- KaAN & $\stackrel{\mathrm{N}}{\mathrm{N}}$ & $\frac{5}{\mathrm{~N}}$ & $\vec{m}$ & $\begin{array}{l}\underset{n}{N} \\
\stackrel{\infty}{N}\end{array}$ & $\begin{array}{l}m \\
m \\
\text { जै }\end{array}$ & \begin{tabular}{|l|} 
\\
0 \\
$\mathrm{~N}$
\end{tabular} & \begin{tabular}{l|} 
엄 \\
\end{tabular} & $\stackrel{m}{\grave{N}}$ & N & $\begin{array}{l}\stackrel{\varphi}{ } \\
\stackrel{2}{N}\end{array}$ & 今ે & m & $\begin{array}{l}\forall \\
\text { g. }\end{array}$ & 응 & 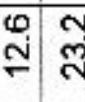 & \begin{tabular}{c|c} 
Ny \\
Nin
\end{tabular} & $\begin{array}{l}0 \\
\stackrel{0}{N} \\
\stackrel{0}{*}\end{array}$ & $\mid \begin{array}{l}\infty \\
\oplus \\
\oplus\end{array}$ & $\stackrel{+}{\rightleftarrows}$ & 옹 \\
\hline (\%2M) pues ou!- & $\overline{\mathfrak{N}}$ & $\stackrel{N}{N}$ & Mo & 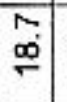 & $\begin{array}{l}0 \\
0 \\
0\end{array}$ & $\begin{array}{l}\mathrm{N} \\
\text { do } \\
\mathrm{m}\end{array}$ & $\begin{array}{l}m \\
\tilde{N}\end{array}$ & $\overline{\dot{j}}$ & 点 & \begin{tabular}{l|l}
$\infty$ & \\
लెల &
\end{tabular} & $\stackrel{\infty}{\bar{v}}$ & $\begin{array}{l}m \\
\infty \\
\infty\end{array}$ & $\frac{9}{8}$ & 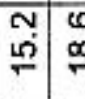 & \begin{tabular}{l|c}
$\infty$ & $m$ \\
$\infty$ & \multicolumn{2}{c|c}{}
\end{tabular} & \begin{tabular}{l|l}
$\mathrm{m}$ \\
$\overline{\mathrm{N}}$
\end{tabular} & 峞 & กิ & $\stackrel{10}{\mathrm{~N}}$ & 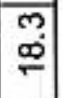 \\
\hline$(\%$ qM) pues un!pew & $\stackrel{0}{=}$ & ָָ' & 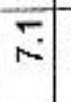 & $\underset{\infty}{+}$ & $\begin{array}{l} \\
\end{array}$ & $\overline{\sigma i}$ & $\begin{array}{l}\infty \\
\infty \\
\infty\end{array}$ & \begin{tabular}{l|l}
$\infty$ \\
$\stackrel{N}{N}$ \\
$\sim$
\end{tabular} & $\underset{m}{m}$ & ले & $\begin{array}{l} \\
\sigma \\
\sigma\end{array}$ & \begin{tabular}{l|}
0 \\
in
\end{tabular} & $\bar{\infty}$ & 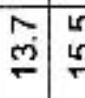 & 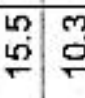 & \begin{tabular}{l|l}
$n$ & $\infty$ \\
$\dot{p}$ & $N$ \\
\end{tabular} & $\stackrel{\infty}{\infty}$ & $\begin{array}{l}0 \\
\\
\end{array}$ & 옹 & 이 \\
\hline$(\% 2 M)$ pues esueos & कृ & $\stackrel{0}{=}$ & $\vec{m}$ & $\stackrel{0}{=}$ & $\begin{array}{l}0 \\
\pm\end{array}$ & $\begin{array}{l}0 \\
0 \\
0\end{array}$ & \begin{tabular}{l|l}
0 \\
0 \\
0
\end{tabular} & $\stackrel{N}{\leftarrow}$ & $\stackrel{m}{\rightleftarrows}$ & M & $\stackrel{\infty}{=}$ & 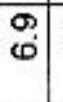 & 守 & 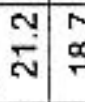 & $\begin{array}{l}m \\
\infty \\
\infty\end{array}$ & $=\begin{array}{l}\infty \\
\infty\end{array}$ & 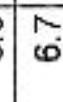 & $\begin{array}{l}\mathbf{v} \\
\dot{\Xi}\end{array}$ & $\begin{array}{l}\varphi \\
\stackrel{0}{ }\end{array}$ & $\begin{array}{l}9 \\
\dot{\sigma}\end{array}$ \\
\hline$(\%$ \%M) pues asueoo kuen & $\begin{array}{l} \\
\\
\end{array}$ & $\begin{array}{l}\underset{v}{v} \\
\dot{\tau}\end{array}$ & 0 & $\begin{array}{l}0 \\
\stackrel{m}{?}\end{array}$ & ปี & $\div$ & $\begin{array}{l} \\
\mathscr{\sigma}\end{array}$ & \begin{tabular}{l|l|l|l|l|l}
$N$ & \\
$\sigma$ &
\end{tabular} & \begin{tabular}{l|l}
$\infty$ & \\
$\infty$ &
\end{tabular} & $\begin{array}{c}m \\
\dot{\infty}\end{array}$ & $\begin{array}{l}N \\
\emptyset \\
\emptyset\end{array}$ & $\underset{\infty}{\infty}$ & $m$ & 产 & 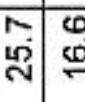 & \begin{tabular}{l|c}
$\tilde{\sigma}$ \\
$\stackrel{\sigma}{\sigma}$ & $\tilde{\sigma}$ \\
\end{tabular} & \begin{tabular}{|c}
$\mathscr{1}$ \\
\end{tabular} & $\stackrel{\pi}{\bar{N}}$ & $\stackrel{+}{\stackrel{0}{-}}$ & 0 \\
\hline (\%वM) pues feqOL & $\underset{N}{N}$ & $M$ & $\begin{array}{l}\mathbb{G} \\
\mathbb{S}\end{array}$ & $\begin{array}{l}0 \\
\\
\end{array}$ & $\begin{array}{l}n \\
\mathscr{\infty} \\
\infty \\
\infty\end{array}$ & \begin{tabular}{|l|}
$\mathscr{\infty}$ \\
$\stackrel{\infty}{\infty}$ \\
$\infty$
\end{tabular} & \begin{tabular}{|l|}
$\mathbf{S}$ \\
$\mathbf{\omega}$ \\
\end{tabular} & $\begin{array}{l}\infty \\
\dot{\Xi}\end{array}$ & $\begin{array}{l}m \\
m \\
\infty\end{array}$ & $\begin{array}{l}3 \\
\dot{\omega}\end{array}$ & స్j & : & in & $\frac{5}{5}$ & $\bar{\sigma}$ & \begin{tabular}{l|l}
$\sigma_{\infty}^{\prime}$ & $\frac{\infty}{\infty}$ \\
\end{tabular} & Е & $\begin{array}{l}3 \\
10 \\
\infty\end{array}$ & $\begin{array}{c}\infty \\
\infty \\
\infty\end{array}$ & $\stackrel{N}{N}$ \\
\hline (\%) salqqad au! s & 뭉 & $\stackrel{N}{\stackrel{N}{2}}$ & $\begin{array}{l}\omega \\
\omega \\
\omega\end{array}$ & $\begin{array}{l}m \\
\dot{m} \\
\dot{v}\end{array}$ & $\begin{array}{l}m \\
\stackrel{m}{-}\end{array}$ & $\begin{array}{c}\infty \\
\infty\end{array}$ & $\overline{\dot{m}}$ & 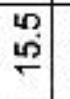 & $\underset{\mathrm{N}}{\mathrm{N}}$ & in & $\stackrel{\sim}{\approx}$ & $\stackrel{0}{\longrightarrow}$ & ल) & \begin{tabular}{l|l}
$m$ & \\
m &
\end{tabular} & 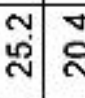 & $\begin{array}{ll} \\
\text { ते }\end{array}$ & $\stackrel{\mathbb{N}}{\stackrel{N}{N}}$ & $\begin{array}{l}0 \\
\emptyset \\
0\end{array}$ & $\stackrel{\varphi}{=}$ & ب0 \\
\hline 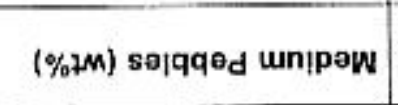 & $\stackrel{N}{?}$ & $\begin{array}{l}\omega \\
m \\
m\end{array}$ & \begin{tabular}{l|} 
\\
0 \\
0
\end{tabular} & $\begin{array}{l}\dot{\varphi} \\
\text { ஸें }\end{array}$ & m & $\begin{array}{l}\omega \\
= \\
=\end{array}$ & \begin{tabular}{|l|} 
\\
\\
$m$
\end{tabular} & $\begin{array}{l}\text { क } \\
\mathrm{N}\end{array}$ & $\begin{array}{l}\text { क } \\
\stackrel{2}{2}\end{array}$ & \begin{tabular}{l|l}
$\infty$ & 0 \\
0 & \\
\end{tabular} & 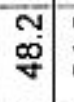 & $\begin{array}{l}0 \\
\dot{J}\end{array}$ & $\begin{array}{l}\infty \\
\infty\end{array}$ & 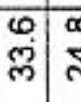 & 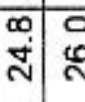 & 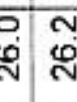 & 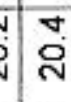 & $\begin{array}{l}0 \\
\text { o. }\end{array}$ & $\begin{array}{c}m \\
\sigma \\
\sigma\end{array}$ & 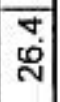 \\
\hline$(\%$ (\%) səlqqad esJeos & 음 & $\vec{\sigma}$ & \begin{tabular}{l|}
$\infty$ \\
0 \\
0
\end{tabular} & Nָ & $\begin{array}{ll}0 \\
\end{array}$ & $\begin{array}{ll}9 \\
6\end{array}$ & 함 & $\begin{array}{l}\infty \\
\infty\end{array}$ & $\cong$ & 잉 & $\stackrel{0}{\leftarrow}$ & $\overline{\mathrm{N}}$ & $\stackrel{40}{=}$ & \begin{tabular}{l|l}
$\stackrel{\circ}{N}$ & 5
\end{tabular} & \begin{tabular}{l|l}
0 & \\
6 & L
\end{tabular} & 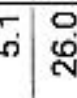 & $\stackrel{m}{n}$ & $\begin{array}{l}0 \\
0\end{array}$ & $\begin{array}{l}v \\
N\end{array}$ & $\begin{array}{l}\infty \\
\text { m }\end{array}$ \\
\hline$(\% \not M)$ selqq9d $\mid \mathrm{E} \% \perp$ & 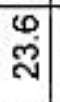 & $\stackrel{\infty}{\infty}$ & $\stackrel{m}{=}$ & $\bar{c}$ & \begin{tabular}{|l|} 
\\
0 \\
0 \\
0
\end{tabular} & 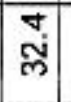 & \begin{tabular}{l|} 
\\
$\mathscr{8}$ \\
8
\end{tabular} & Nָ & $\stackrel{\infty}{\infty}$ & $\stackrel{0}{\oplus}$ & 疋 & $\stackrel{m}{\infty}$ & $\begin{array}{l}\varphi \\
\stackrel{\varphi}{0} \\
\square\end{array}$ & $\frac{50}{6}$ & 앵 in & \begin{tabular}{l|l}
$n$ & 15 \\
5 & 8 \\
5 & 8
\end{tabular} & $\begin{array}{l}m \\
m \\
m\end{array}$ & 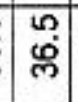 & 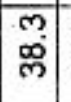 & $\begin{array}{l}\infty \\
\dot{g}\end{array}$ \\
\hline (шэ) ч)deg дәмоา & $\omega$ & ָั & $\infty$ & 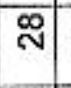 & $m$ & 9 & \begin{tabular}{|l|}
\multirow{2}{*}{} \\
\end{tabular} & $\underset{\text { న్ల }}{\leftarrow}$ & 으 & $\stackrel{\mathbb{E}}{\complement}$ & $\stackrel{9}{\circ}$ & 워 & 유 & స్ & 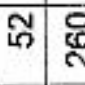 & \begin{tabular}{c|c}
$\stackrel{\mathfrak{N}}{\mathrm{N}}$ & ? \\
\end{tabular} & (I) & $\stackrel{N}{\mp}$ & 용 & $\infty$ \\
\hline (wэ) पวdə0 Jeddn & O & స్ & 0 & 요 & g & ले & 8 & $\underset{\mp}{\mp}$ & $\infty$ & $\pi$ & $m$ & $\infty$ & $\infty$ & 产 & 워 & \begin{tabular}{lll}
\multirow{N}{*}{} \\
N
\end{tabular} & (7) & क & กิ & 0 \\
\hline uoz!ld & $\leq$ & 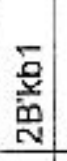 & $\alpha$ & 总 & 旁 & 酋 & $\begin{array}{l}\text { 号 } \\
\text { 畄 } \\
\end{array}$ & 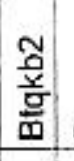 & 竞 & : & 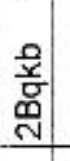 & $\underset{\infty}{\mathbb{c}}$ & 宫 & 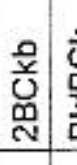 & 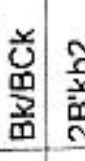 & 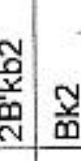 & gू & $\begin{array}{l}\frac{\overline{0}}{\mathrm{z}} \\
\frac{\mathrm{g}}{\bar{\omega}} \\
\end{array}$ & $\begin{array}{l}\text { 盖 } \\
\text { 僾 }\end{array}$ & $\frac{z}{2}$ \\
\hline әроว әdسes P|a!s & 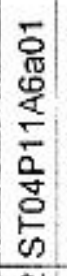 & 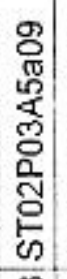 & $\begin{array}{l}5 \\
0 \\
0 \\
0 \\
8 \\
0 \\
0 \\
\frac{0}{0} \\
0 \\
0\end{array}$ & 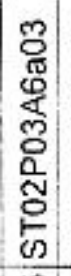 & $\begin{array}{l}0 \\
0 \\
0 \\
0 \\
0 \\
0 \\
0 \\
0 \\
0 \\
0 \\
0 \\
0\end{array}$ & \begin{tabular}{|l|}
0 \\
0 \\
0 \\
0 \\
8 \\
0 \\
0 \\
0 \\
5 \\
0 \\
0 \\
0
\end{tabular} & 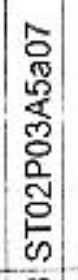 & $\begin{array}{l}\text { 号 } \\
\mathbb{8} \\
\mathbb{0} \\
\mathbb{8} \\
8 \\
\vdots \\
0 \\
0\end{array}$ & 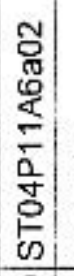 & 芯 & 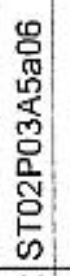 & 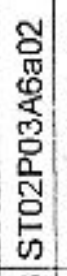 & 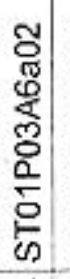 & 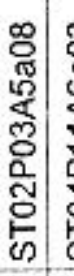 & 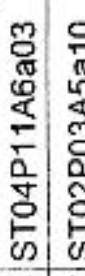 & 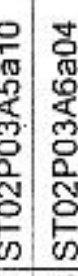 & 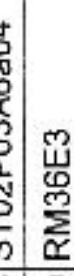 & 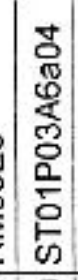 & 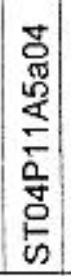 & 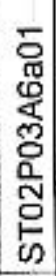 \\
\hline ajdues & $N$ & $\stackrel{3}{2}$ & $\stackrel{N}{4}$ & 岕 & $\stackrel{9}{9}$ & 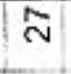 & $\stackrel{\infty}{\sim}$ & 2 & 유 & ल & กี & $m$ & ले & 学8 & $\stackrel{9}{D}$ & m & : & 肙 & 守 & T \\
\hline पगנeg & g & 守 & g & g & g & 守 & 5 & 5 & 5 & $\overline{8}$ & $\overline{0}$ & $\bar{g}$ & 号 & 守 & $\bar{g}$ & 8 & 5 & $\bar{g}$ & 守 & $\overline{8}$ \\
\hline
\end{tabular}




\begin{tabular}{|c|c|c|c|c|c|c|c|c|c|c|c|c|c|c|c|c|c|c|c|c|c|}
\hline (\%7M) Kelo IEjol & $\stackrel{\mathscr{O}}{\omega}$ & m & $\hat{\mathrm{N}}$ & $\stackrel{n}{=}$ & $\begin{array}{l}\mathrm{v} \\
\mathrm{v}\end{array}$ & \begin{tabular}{|l|}
0 \\
$\dot{0}$ \\
\end{tabular} & $\stackrel{\sim}{\sim}$ & N & $\begin{array}{l}N \\
\text { us }\end{array}$ & $\begin{array}{c}n \\
\infty\end{array}$ & $\begin{array}{l}0 \\
0\end{array}$ & \begin{tabular}{|l|} 
\\
$\dot{m}$
\end{tabular} & $\begin{array}{l}\oplus \\
\stackrel{v}{v}\end{array}$ & $\hat{0}$ & \begin{tabular}{|c|}
\multirow{2}{*}{} \\
in
\end{tabular} & $\begin{array}{l}0 \\
\end{array}$ & \begin{tabular}{|l|} 
\\
0 \\
\end{tabular} & \begin{tabular}{l|}
10 \\
$\infty$
\end{tabular} & $\stackrel{m}{\forall}$ & $\begin{array}{l}\infty \\
1 \\
1\end{array}$ & $\stackrel{\infty}{\leftarrow}$ \\
\hline$(\%)$ M ) मI!S au!t & $\begin{array}{l}\varphi \\
\dot{v}\end{array}$ & $\begin{array}{l}\mathscr{Q} \\
\dot{v}\end{array}$ & $\stackrel{D}{\sim}$ & $\tilde{\sigma}$ & $\mp$ & $\begin{array}{l}0 \\
6 \\
6\end{array}$ & 오 & N & 웅 & 욷 & 今. & m & $\underset{\sim}{N}$ & 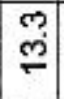 & $\begin{array}{l}m \\
\text { dे } \\
\end{array}$ & $N$ & $\stackrel{m}{\nabla}$ & $\stackrel{2}{\circ}$ & $\stackrel{N}{-}$ & $\begin{array}{c} \pm \\
6\end{array}$ & $\stackrel{\text { gr }}{\forall}$ \\
\hline (\%) मIIS esuro? & $\overline{10}$ & $\begin{array}{l}\nabla \\
\dot{\nabla}\end{array}$ & $\begin{array}{l}\infty \\
\dot{\sigma}\end{array}$ & 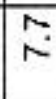 & $\stackrel{\infty}{-\infty}$ & \begin{tabular}{|l|} 
\\
\end{tabular} & $\begin{array}{l}\infty \\
\infty\end{array}$ & $\begin{array}{l}\nabla \\
N\end{array}$ & $\begin{array}{l}0 \\
\dot{\nabla}\end{array}$ & $\begin{array}{l}n \\
0\end{array}$ & $\begin{array}{l}\mathrm{d} \\
\mathrm{j}\end{array}$ & " & $\stackrel{\circ}{\circ}$ & $\bar{\oplus}$ & 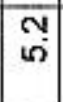 & \begin{tabular}{|l|}
$\mathscr{\omega}$ \\
\end{tabular} & $\begin{array}{l}N \\
\text { in }\end{array}$ & $\stackrel{\infty}{=}$ & $\vec{m}$ & $\stackrel{m}{r}$ & í \\
\hline (\% ҰM) มIS IEJOL & $\bar{\omega}$ & $\begin{array}{l}0 \\
\text { के }\end{array}$ & $\begin{array}{l}\nabla \\
\end{array}$ & 总 & \begin{tabular}{l|} 
ก \\
\end{tabular} & \begin{tabular}{|c|}
$\oplus$ \\
लि \\
\end{tabular} & $\begin{array}{l}\infty \\
\infty \\
\infty\end{array}$ & vi & $\begin{array}{l}v \\
\stackrel{2}{\sim}\end{array}$ & $\stackrel{N}{\sim}$ & 草 & $\begin{array}{ll} \\
\mathrm{N}\end{array}$ & ָั & $\begin{array}{c}\dot{\square} \\
\stackrel{a}{N}\end{array}$ & 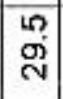 & \begin{tabular}{|l|}
$\infty$ \\
0 \\
0
\end{tabular} & \begin{tabular}{|l|}
$n$ \\
0 \\
\end{tabular} & $\begin{array}{l}n \\
\mathrm{~N}\end{array}$ & $\begin{array}{l}\text { क } \\
\end{array}$ & $\begin{array}{l}\infty \\
\stackrel{\infty}{\sim}\end{array}$ & 广ं \\
\hline (\%ఖM) pues әu!」 AJaA & $\begin{array}{l}\text { O } \\
\dot{N}\end{array}$ & 옹 & 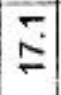 & $\begin{array}{l}0 \\
\mathbb{Z}\end{array}$ & $M$ & $\begin{array}{l}m \\
g \\
d\end{array}$ & $\begin{array}{l}\infty \\
\text { Ni }\end{array}$ & $\begin{array}{l}\infty \\
⿱ 亠 \\
+\end{array}$ & స్ & $\begin{array}{l}\mathscr{Q} \\
\stackrel{+}{ \pm}\end{array}$ & $\stackrel{\mathfrak{N}}{\mathfrak{N}}$ & $\hat{\mathrm{n}}$ & $\begin{array}{c}m \\
\infty\end{array}$ & 号 & $\stackrel{m}{\stackrel{m}{m}}$ & $\begin{array}{l}\infty \\
\stackrel{\infty}{=}\end{array}$ & $\begin{array}{l}0 \\
\dot{J} \\
\end{array}$ & \begin{tabular}{|l|}
\multirow{N}{*}{} \\
\end{tabular} & $\begin{array}{l}\text { N } \\
\text { I }\end{array}$ & Nָ & $\begin{array}{l}\infty \\
+ \\
+\end{array}$ \\
\hline (\%/M) pues auly & $\begin{array}{l}\text { क } \\
\text { ले }\end{array}$ & $\check{N}$ & $\begin{array}{l}0 \\
\stackrel{0}{d} \\
\stackrel{d}{*}\end{array}$ & 寈 & $\begin{array}{l}\mathbf{J} \\
\text { J }\end{array}$ & $\stackrel{M}{\mathscr{Q}}$ & $\begin{array}{l}3 \\
\text { N }\end{array}$ & $\begin{array}{l}N \\
\text { g }\end{array}$ & 总 & $\frac{\dot{v}}{\mathrm{~N}}$ & 穴 & 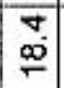 & $\begin{array}{l}m \\
\stackrel{m}{v} \\
\stackrel{0}{*}\end{array}$ & $\begin{array}{l}\infty \\
\oplus \\
\oplus\end{array}$ & \begin{tabular}{|l|}
0 \\
\\
\end{tabular} & $\begin{array}{l} \\
\end{array}$ & गे & $\begin{array}{l}\Phi \\
\omega \\
\square\end{array}$ & $\begin{array}{l}\omega \\
\text { N̦ }\end{array}$ & $\stackrel{\sigma}{\sigma}$ & $\bar{N}$ \\
\hline (\%1M) pues unjpaw & $\begin{array}{l}n \\
\stackrel{0}{\circ}\end{array}$ & $\stackrel{m}{\stackrel{m}{5}}$ & กิ & เே & $\mid \begin{array}{c}\infty \\
\text { d्̀ } \\
\text { | }\end{array}$ & के & 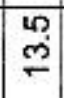 & $\begin{array}{l}\infty \\
\text { ָู่ }\end{array}$ & $\bar{N}$ & $\begin{array}{l}\infty \\
\dot{\square} \\
\dot{\square}\end{array}$ & $\frac{0}{\mathrm{~N}}$ & 勇 & $\stackrel{N}{N}$ & $\bar{\varphi}$ & \begin{tabular}{|l|}
\multirow{2}{*}{} \\
$\dot{0}$ \\
\end{tabular} & $\sigma^{-}$ & $\mathscr{\sigma}$ & \begin{tabular}{|c|} 
\\
$\oplus$
\end{tabular} & \begin{tabular}{l|} 
\\
$\infty$ \\
$c$
\end{tabular} & $\bar{\sigma}$ & $\begin{array}{l}\infty \\
\infty\end{array}$ \\
\hline (\%2M) pues esuroo & $\stackrel{\Delta}{N}$ & $\stackrel{\oplus}{\stackrel{m}{\sim}}$ & $\begin{array}{c}\infty \\
\stackrel{m}{m}\end{array}$ & $\bar{\sigma}$ & $\begin{array}{l}0 \\
\mathbb{N}\end{array}$ & \begin{tabular}{|l|}
$m$ \\
$n$
\end{tabular} & $\stackrel{m}{=}$ & 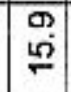 & $\stackrel{N}{N}$ & \begin{tabular}{|l|}
0 \\
0 \\
\end{tabular} & क & $\begin{array}{l}\dot{J} \\
\stackrel{d}{d}\end{array}$ & $\stackrel{m}{\infty}$ & \begin{tabular}{l|} 
\\
$\dot{\omega}$
\end{tabular} & \begin{tabular}{|l|}
0 \\
0 \\
0
\end{tabular} & $\begin{array}{l} \\
\\
\end{array}$ & $\stackrel{N}{N}$ & \begin{tabular}{|l|} 
\\
$\infty$ \\
$\infty$
\end{tabular} & $\begin{array}{c}\infty \\
\infty \\
\infty\end{array}$ & $\underset{\sim}{\mathbb{N}}$ & $\stackrel{\sim}{\stackrel{N}{m}}$ \\
\hline (\%дM) pues asieoo KiəA & 임 & $\underset{\infty}{\sim}$ & $M$ & क & $\frac{m}{5}$ & $\begin{array}{l}\sigma \\
\varphi \\
\end{array}$ & \begin{tabular}{|l|}
$\mathscr{6}$ \\
$\stackrel{\varphi}{0}$
\end{tabular} & $\begin{array}{l}\hat{\sigma} \\
\end{array}$ & $\stackrel{N}{N}$ & $\begin{array}{l}N \\
\dot{v}\end{array}$ & 옹 & \begin{tabular}{|l|} 
\\
$\Phi$ \\
\end{tabular} & $\underset{\sim}{\sim}$ & o & \begin{tabular}{|l|}
5 \\
15
\end{tabular} & $\frac{m}{\mathrm{~m}}$ & $\begin{array}{l}\omega \\
\infty\end{array}$ & $\stackrel{\square}{\oplus}$ & \begin{tabular}{|l|}
$\mathscr{L}$ \\
$\oplus$ \\
\end{tabular} & 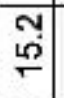 & $m$ \\
\hline (\%]M) pues 1 leqo1 & $\begin{array}{l}m \\
\dot{c}\end{array}$ & $\bar{\infty}$ & $\begin{array}{l}\mathbf{g} \\
g \\
\end{array}$ & $\frac{5}{6}$ & \begin{tabular}{l|}
$\infty$ \\
$\dot{\sigma}$
\end{tabular} & $\begin{array}{l} \\
8 \\
8\end{array}$ & \begin{tabular}{|l|}
0 \\
9 \\
8 \\
\end{tabular} & 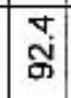 & $\begin{array}{l}4 \\
8\end{array}$ & $\begin{array}{l}0 \\
\dot{0} \\
\end{array}$ & \begin{tabular}{|l|}
0 \\
$\infty$ \\
\hdashline
\end{tabular} & $\begin{array}{l} \\
\text { में }\end{array}$ & 岗 & O' & $\begin{array}{l}0 \\
8 \\
8\end{array}$ & $\begin{array}{l}\mathbb{N} \\
6 \\
6\end{array}$ & 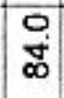 & \begin{tabular}{|l|}
0 \\
0 \\
8
\end{tabular} & $\begin{array}{l}\infty \\
0 \\
0\end{array}$ & $\begin{array}{l}\square \\
\stackrel{0}{*}\end{array}$ & $\stackrel{\infty}{\infty}$ \\
\hline (\%дM) selqqed au!d & $\underset{\sim}{\mathrm{N}}$ & $\begin{array}{l}\infty \\
\infty\end{array}$ & $\begin{array}{l}m \\
\infty\end{array}$ & के & के & \begin{tabular}{l|} 
\\
\\
\end{tabular} & M & $\begin{array}{l} \\
\infty \\
\end{array}$ & $\dddot{0}$ & $\stackrel{\square}{=}$ & mi & \begin{tabular}{|l|}
0 \\
$\stackrel{3}{\circ}$
\end{tabular} & 흠 & $\stackrel{\sigma}{\stackrel{m}{\circ}}$ & $\stackrel{m}{=}$ & \begin{tabular}{|l|}
$\infty$ \\
N่
\end{tabular} & 웅 & 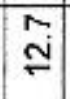 & $\check{\varphi}$ & $\begin{array}{l}\oplus \\
\circ \\
\end{array}$ & 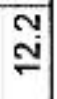 \\
\hline$(\% \not M)$ sejqqed un!pow & ल) & $\begin{array}{l}\dot{v} \\
\stackrel{0}{0}\end{array}$ & $\begin{array}{l}r \\
\varphi\end{array}$ & $\tilde{N}$ & $\stackrel{i}{\sim}$ & $\bar{E}$ & \begin{tabular}{|l|}
$\infty$ \\
9 \\
\end{tabular} & $\begin{array}{l}0 \\
\stackrel{6}{\rightleftharpoons}\end{array}$ & $\begin{array}{l}\mathrm{Ln} \\
\mathrm{N}\end{array}$ & 웅 & 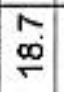 & 일 & 跑 & लू & $\begin{array}{l}m \\
\stackrel{m}{m}\end{array}$ & \begin{tabular}{|l|} 
吡 \\
\end{tabular} & \begin{tabular}{l|} 
\\
\end{tabular} & $\frac{5}{5}$ & $\bar{g}$ & 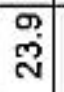 & $\begin{array}{l}\infty \\
\stackrel{0}{0}\end{array}$ \\
\hline (\%2M) selqqad asieo & $\begin{array}{l}\infty \\
0 \\
0\end{array}$ & $\begin{array}{l}\sigma \\
6 \\
6\end{array}$ & 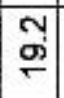 & ले & 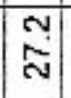 & 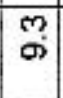 & $\begin{array}{l} \\
\\
\end{array}$ & $\begin{array}{l}0 \\
\dot{v}\end{array}$ & \begin{tabular}{|l|} 
का \\
ले
\end{tabular} & $\begin{array}{l}4 \\
6\end{array}$ & బ) & $\bar{\infty}$ & $\begin{array}{l}\infty \\
\oplus \\
\oplus\end{array}$ & $\stackrel{m}{-}$ & $\mathrm{N}$ & \begin{tabular}{|c|}
0 \\
ले
\end{tabular} & O্. & $\begin{array}{c}9 \\
01\end{array}$ & $\stackrel{40}{-}$ & $\begin{array}{l}\text { No } \\
\text { ô }\end{array}$ & m) \\
\hline 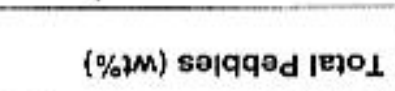 & $\stackrel{\emptyset}{\oplus}$ & $\frac{5}{\square}$ & $\begin{array}{l}0 \\
\dot{q}\end{array}$ & 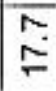 & $\begin{array}{l} \\
\text { 우 }\end{array}$ & \begin{tabular}{ll} 
\\
\hdashline \\
\end{tabular} & \begin{tabular}{|l|}
5 \\
56 \\
60
\end{tabular} & \begin{tabular}{|l|} 
in \\
op
\end{tabular} & $\overline{8}$ & 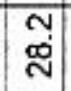 & 宫 & $\begin{array}{c}\varphi \\
\overline{0}\end{array}$ & त్ & $\begin{array}{l}0 \\
0 \\
\end{array}$ & 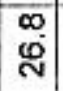 & \begin{tabular}{|l|}
$t$ \\
0 \\
8 \\
\end{tabular} & \begin{tabular}{|l|} 
\\
6 \\
\end{tabular} & $\begin{array}{l} \\
g \\
g\end{array}$ & $\begin{array}{l}M \\
00 \\
0\end{array}$ & 永 & గ్ల \\
\hline 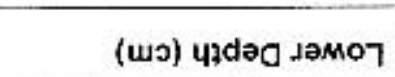 & $\mathbb{Z}$ & กิ & $\mathscr{8}$ & $\infty$ & 要 & ลี & 兽 & ळ & 守 & $\stackrel{\text { DI }}{\leftarrow}$ & $\frac{40}{2}$ & প্ল & $\stackrel{\square}{\doteqdot}$ & 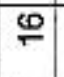 & ผ & 邑 & 茎 & 을 & $\mathscr{8}$ & 次 & 迎 \\
\hline (wכ) पpde0 dedd & $\stackrel{\overparen{D}}{=}$ & 品 & 5 & 0 & $\frac{L 8}{N}$ & $\bar{\sigma}$ & 吕 & ชู & ָָ & $\infty$ & $\bigoplus$ & 8 & 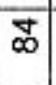 & 0 & क & $\underset{工}{ \pm}$ & 可 & 丞 & $\stackrel{\infty}{=}$ & $\stackrel{\square}{\dddot{\Phi}}$ & $\stackrel{\sim}{\sim}$ \\
\hline & T & 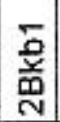 & $\begin{array}{l}\text { 总 } \\
\text { 产 } \\
\text { 空 }\end{array}$ & 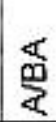 & 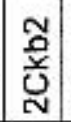 & 总 & 总 & $\begin{array}{l}\text { N } \\
\frac{\mathbf{x}}{\sim} \\
\text { N }\end{array}$ & 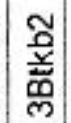 & $\overline{\mathbf{m}}$ & $\begin{array}{l}\overline{\mathbf{0}} \\
\mathrm{D} \\
\mathrm{N}\end{array}$ & 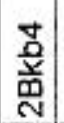 & $\begin{array}{l}\stackrel{ }{\mathrm{v}} \\
\mathrm{D} \\
\stackrel{\mathrm{N}}{\mathrm{N}}\end{array}$ & $\begin{array}{l}\mathbb{6} \\
\text { 㐭 }\end{array}$ & $\begin{array}{c}\text { 盖 } \\
\end{array}$ & $\begin{array}{l}\text { 盖 } \\
\text { 恶 }\end{array}$ & $\mathbb{Z}$ & $\mid \begin{array}{l}0 \\
\text { 点} \\
\text { 号 }\end{array}$ & 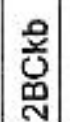 & $\begin{array}{l}\text { 章 } \\
\text { 㟧 }\end{array}$ & 总 \\
\hline әро弓 ә|dwes ple!y & 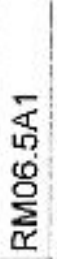 & 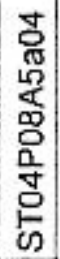 & 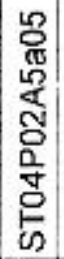 & 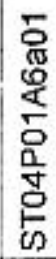 & 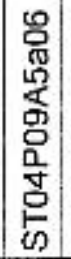 & 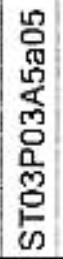 & 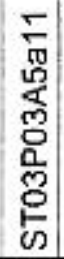 & 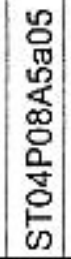 & 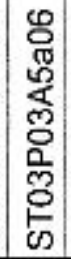 & 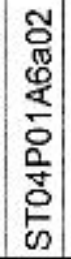 & 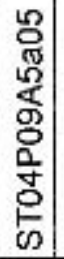 & 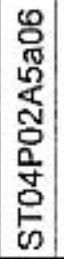 & 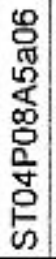 & 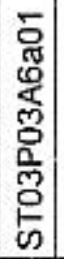 & 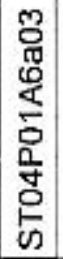 & 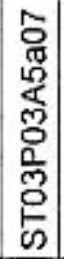 & 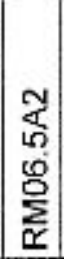 & 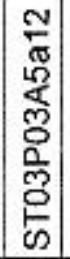 & 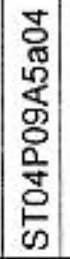 & 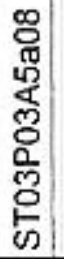 & 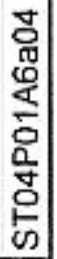 \\
\hline әjdures & $r$ & $N$ & $m$ & $\theta$ & in & $\omega$ & $N$ & $\infty$ & $\infty$ & 은 & $=$ & $\dddot{T}$ & $\stackrel{m}{=}$ & 士 & $\frac{20}{2}$ & $\because$ & $\approx$ & $\stackrel{\infty}{\infty}$ & क & 尺 & $\bar{N}$ \\
\hline प깔ㅇ & 맘 & 강 & ণ & O & ป & \% & ণ্ & 夺 & 尺̊. & フุ & ช్ & ชิ & 굼 & 号 & 总 & 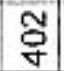 & 守 & ชิ & $\mathscr{8}$ & Õ & ชั \\
\hline
\end{tabular}




\begin{tabular}{|c|c|c|c|c|c|c|c|c|c|c|c|c|c|c|c|c|c|c|c|c|}
\hline (\%मM) KEIO |EZOL & \begin{tabular}{l|} 
\\
Li
\end{tabular} & \begin{tabular}{l|l}
\multirow{N}{*}{} & \\
\end{tabular} & \begin{tabular}{l|} 
\\
$\infty$ \\
$\infty$
\end{tabular} & \begin{tabular}{l|l|} 
& \\
10
\end{tabular} & \begin{tabular}{|l|}
0 \\
ले
\end{tabular} & $\bar{\infty}$ & \begin{tabular}{c|} 
\\
m.
\end{tabular} & \begin{tabular}{l|}
2 \\
$m$
\end{tabular} & \begin{tabular}{l|} 
का \\
ले
\end{tabular} & ले & \begin{tabular}{l|} 
\\
$\dot{v}$
\end{tabular} & \begin{tabular}{l|}
$N$ \\
$N$
\end{tabular} & \begin{tabular}{l|l} 
& \\
\end{tabular} & $\mathrm{N}$ & \begin{tabular}{|l|} 
\\
\end{tabular} & $\bar{\omega}^{\circ}$ & $\begin{array}{l}\infty \\
\infty \\
\infty\end{array}$ & 户 & $\overline{\mathrm{N}}$ & $\begin{array}{l}\varphi \\
\dot{\nabla}\end{array}$ \\
\hline$(\% 2 M)$ ㄱ!s au!d & \begin{tabular}{l|l}
\multirow{v}{*}{} & \\
\end{tabular} & $\bar{m}$ & $\dot{0}$ & $\begin{array}{l}m \\
\dot{v}\end{array}$ & \begin{tabular}{|l|} 
o \\
तi
\end{tabular} & $\vec{f}$ & 임 & 茴 & $\begin{array}{l}0 \\
\dot{m}\end{array}$ & $\begin{array}{l}\infty \\
\dot{m}\end{array}$ & 广্ं & $\begin{array}{l}\infty \\
\dot{v}\end{array}$ & \begin{tabular}{l|}
0 \\
$\dot{v}$
\end{tabular} & \begin{tabular}{l|}
$\infty$ \\
$N$
\end{tabular} & \begin{tabular}{|l|}
$n$ \\
$\infty$ \\
\end{tabular} & $\begin{array}{l}10 \\
8\end{array}$ & 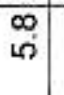 & \begin{tabular}{l|l|l|} 
& \\
$\forall$
\end{tabular} & $\stackrel{\Upsilon}{\sim}$ & $\widehat{\omega}^{\circ}$ \\
\hline (\%2M) มा!S asieoว & $\mathrm{i}$ & 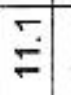 & $\begin{array}{l}m \\
\dot{y}\end{array}$ & $\mathrm{~m}$ & \begin{tabular}{|l|}
0 \\
\end{tabular} & m. & $\begin{array}{l}8 \\
i \circ\end{array}$ & 号 & $\begin{array}{l}0 \\
\\
m\end{array}$ & 虽 & $\begin{array}{l}\mathbf{V} \\
0\end{array}$ & $\begin{array}{l}0 \\
0 \\
0\end{array}$ & 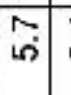 & $\dot{m}$ & \begin{tabular}{|l|}
$\oplus$ \\
\end{tabular} & $\stackrel{0}{N}$ & ח. & $\begin{array}{l}10 \\
\sigma\end{array}$ & $\stackrel{6}{-}$ & $\begin{array}{l}0 \\
\sigma\end{array}$ \\
\hline$(\% 2 M)$ AIS IEIOL & $\begin{array}{l}\infty \\
\infty\end{array}$ & $\begin{array}{l}m \\
\square\end{array}$ & $\stackrel{\sigma}{T}$ & $\bar{g}$ & 吕 & $\begin{array}{l}0 \\
0\end{array}$ & 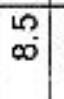 & $\begin{array}{l}0 \\
\text { N }\end{array}$ & $\begin{array}{l}\varphi \\
\bullet \\
0\end{array}$ & 禹 & $\begin{array}{l}n \\
0 \\
0\end{array}$ & $\stackrel{+}{=}$ & m. & $\begin{array}{l}N \\
\bullet\end{array}$ & $\overline{\mathrm{N}}$ & $\underset{\mathrm{i}}{\sigma}$ & $\begin{array}{l}0 \\
\stackrel{2}{2}\end{array}$ & $\begin{array}{l}\mathbb{Y} \\
\mathbb{V}\end{array}$ & \begin{tabular}{l|}
$\infty$ \\
$-i$
\end{tabular} & $\stackrel{m}{\mathscr{m}}$ \\
\hline (\%¥M) pues әu!d Kuen & $m$ & 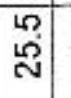 & क् & $\begin{array}{l} \pm \\
\stackrel{5}{-}\end{array}$ & $\frac{5}{\mathrm{~N}}$ & $\stackrel{9}{\circ}$ & $\check{\check{C}}$ & กี & $\begin{array}{l}\mathscr{\varphi} \\
\emptyset\end{array}$ & Mid & $\begin{array}{l}0 \\
\dot{v}\end{array}$ & ָ̇ & $\begin{array}{l}m \\
\dot{v}\end{array}$ & $\begin{array}{l}\infty \\
\dot{Z} \\
+\end{array}$ & \begin{tabular}{|l|}
0 \\
0 \\
N
\end{tabular} & $\stackrel{0}{\mp}$ & 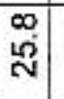 & 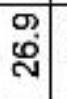 & N & $\begin{array}{l}\text { क } \\
\text { ले }\end{array}$ \\
\hline (\%+M) pues ou!- & $\begin{array}{l}\text { O } \\
\text { gi }\end{array}$ & ले & $\begin{array}{l}\infty \\
\text { กิ }\end{array}$ & 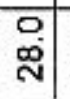 & \begin{tabular}{|l|}
50 \\
$\mathrm{n}$ \\
$\mathrm{N}$
\end{tabular} & $\begin{array}{c}\oplus \\
\dot{J}\end{array}$ & $\begin{array}{l}0 \\
\text { 吕 } \\
\text { N }\end{array}$ & $\stackrel{N}{\check{N}}$ & वें & $\begin{array}{l}0 \\
\mathbf{d}\end{array}$ & 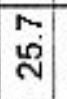 & N & हूల & m & $\stackrel{m}{\sigma}$ & $\begin{array}{l}m \\
\stackrel{m}{\sigma}\end{array}$ & $\begin{array}{l} \pm \\
\text { స }\end{array}$ & $\begin{array}{l}\infty \\
\stackrel{m}{\sim}\end{array}$ & $\frac{0}{2}$ & $\frac{\Phi}{\sim}$ \\
\hline (\%łM) pues un!̣paw & $\bar{i}$ & $\begin{array}{l}\sigma \\
\dot{\omega} \\
\sigma\end{array}$ & $\ddot{0}$ & $\begin{array}{l} \\
\Phi \\
\sigma\end{array}$ & \begin{tabular}{|l|}
$\oplus$ \\
$\stackrel{\odot}{ }$ \\
\end{tabular} & $\begin{array}{l}\mathscr{\infty} \\
\rightleftharpoons\end{array}$ & $\begin{array}{l}\infty \\
\stackrel{0}{0} \\
\wp\end{array}$ & $\bar{\infty}$ & î̀ & $\stackrel{0}{=}$ & $\begin{array}{l}\infty \\
\dot{m}\end{array}$ & $\begin{array}{l}\stackrel{\varphi}{\varphi} \\
\stackrel{\varphi}{\sigma}\end{array}$ & $\begin{array}{l}\text { no } \\
0 \\
0\end{array}$ & \begin{tabular}{|l|}
0 \\
足
\end{tabular} & \begin{tabular}{l|l} 
\\
0 \\
0
\end{tabular} & \begin{tabular}{l|}
$\infty$ \\
$\dot{\infty}$ \\
$\stackrel{0}{-}$
\end{tabular} & $\stackrel{N}{\simeq}$ & $\stackrel{r}{\circ}$ & $\begin{array}{l}\infty \\
\stackrel{\infty}{\mathrm{N}}\end{array}$ & $\begin{array}{l}0 \\
\dot{+}\end{array}$ \\
\hline (\%tM) pues asueoo & $\begin{array}{l}m \\
\dot{q}\end{array}$ & $\stackrel{F}{=}$ & مי & $\stackrel{\substack{N \\
m}}{m}$ & $\begin{array}{c}0 \\
\stackrel{0}{*} \\
\leftarrow\end{array}$ & $\begin{array}{l}\text { ले } \\
\text { के }\end{array}$ & 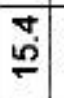 & $\begin{array}{l} \\
\sigma \\
\sigma\end{array}$ & $\stackrel{\sim}{ \pm}$ & 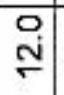 & $\underset{c}{\mathfrak{c}}$ & $\stackrel{m}{\stackrel{M}{\sim}}$ & $\tilde{N}$ & $\stackrel{N}{=}$ & 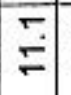 & $\begin{array}{l}\mathscr{Q} \\
\mathscr{0}\end{array}$ & $\bar{\sigma}$ & $\begin{array}{l}\sigma \\
\sigma \\
\sigma\end{array}$ & 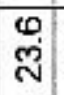 & $\begin{array}{l}\stackrel{N}{\sim} \\
\text { Na }\end{array}$ \\
\hline 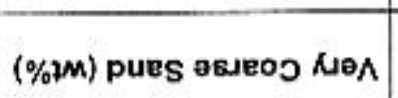 & \begin{tabular}{l|} 
\\
$\infty$
\end{tabular} & $\begin{array}{l}\omega \\
\infty\end{array}$ & $\stackrel{8}{*}$ & $\bar{\infty}$ & 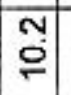 & $\bar{r}$ & $\begin{array}{l} \\
\dot{0} \\
\dot{t}\end{array}$ & $\begin{array}{l}\infty \\
\dot{+} \\
\dot{+}\end{array}$ & $\bar{\infty}$ & $\underset{\dot{m}}{\vec{m}}$ & $\begin{array}{l}N \\
\sigma\end{array}$ & $m$ & $m$ & \begin{tabular}{l|}
$\infty$ \\
$m$ \\
$\stackrel{p}{\Gamma}$
\end{tabular} & \begin{tabular}{l|}
$\infty$ \\
$\mathbb{N}$
\end{tabular} & $\begin{array}{l}0 \\
\div\end{array}$ & $\begin{array}{l} \\
0 \\
0\end{array}$ & $\stackrel{\square}{\leftarrow}$ & $\stackrel{r}{N}$ & $\overrightarrow{6}$ \\
\hline$(\% 2 M)$ pues |EqOL & 임 & $\overrightarrow{⿱ 亠 凶}$ & $\begin{array}{l}\infty \\
\dot{0} \\
\dot{0}\end{array}$ & $\vec{\Phi}$ & Б্ & $\stackrel{m}{\infty}$ & \begin{tabular}{l|l} 
\\
0 \\
1 \\
0 \\
0
\end{tabular} & $\begin{array}{l}n \\
\mathrm{~N}\end{array}$ & 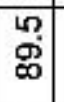 & 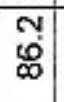 & \begin{tabular}{l|}
0 \\
$\dot{\Phi}$ \\
\end{tabular} & $\begin{array}{l}\infty \\
\infty \\
\infty\end{array}$ & $\begin{array}{l}\infty \\
\dot{\square} \\
\infty\end{array}$ & $\bar{\sigma}$ & $\begin{array}{l}m \\
\dot{m}\end{array}$ & $\underset{\infty}{\infty}$ & $\frac{\square}{\infty}$ & 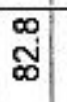 & $\begin{array}{l}\text { J } \\
\text { जे }\end{array}$ & $\bar{S}^{\circ}$ \\
\hline$(\%$ p $)$ selqqod әu! & 훙 & 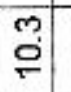 & $\begin{array}{l}\text { ?ִ } \\
\stackrel{0}{\circ}\end{array}$ & $\stackrel{0}{\leftarrow}$ & $\stackrel{0}{=}$ & ơ & $\underset{\infty}{\stackrel{\phi}{c}}$ & $\begin{array}{l}\infty \\
\emptyset \\
\emptyset\end{array}$ & $\stackrel{0}{=}$ & $\stackrel{\text { no }}{=}$ & $\overline{\mathrm{N}}$ & \% & $\stackrel{\varphi}{-}$ & $\stackrel{y}{=}$ & $\begin{array}{l}0 \\
50 \\
-2\end{array}$ & $\begin{array}{l}0 \\
\dot{U}\end{array}$ & \begin{tabular}{l|l}
$\sigma$ \\
$\infty$
\end{tabular} & $\stackrel{\infty}{=}$ & 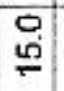 & $\stackrel{4}{\infty}$ \\
\hline$(\%$ ( $M)$ so|qqod wn!̣pow & $\stackrel{\oplus}{=}$ & $\stackrel{m}{m}$ & $\begin{array}{l}8 \\
\text { กิ }\end{array}$ & $\begin{array}{l}\Phi \\
\stackrel{\infty}{\sigma}\end{array}$ & 吕 & $\begin{array}{l}m \\
\stackrel{m}{\circ}\end{array}$ & $\begin{array}{l}m \\
\stackrel{m}{\sigma}\end{array}$ & $\begin{array}{l}N \\
\stackrel{p}{p}\end{array}$ & $\bar{\sim}$ & एँ & $\begin{array}{l}\infty \\
\infty \\
\sim\end{array}$ & $\begin{array}{l}\omega \\
\\
\end{array}$ & $\begin{array}{l}0 \\
\dot{v}\end{array}$ & $\begin{array}{l}\infty \\
0 \\
\end{array}$ & \begin{tabular}{l|} 
\\
$\tilde{m}$
\end{tabular} & $\begin{array}{l} \\
\dot{m}\end{array}$ & 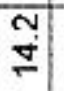 & $\begin{array}{l}0 \\
\dot{y}\end{array}$ & 어 & $\stackrel{\circ}{=}$ \\
\hline (\%qM) sə|qqәd asieo & $\stackrel{n}{0}$ & $\overline{50}$ & $\tilde{0}$ & \begin{tabular}{|c|} 
का \\
ம்
\end{tabular} & 잉 & $\stackrel{\infty}{m}$ & $\begin{array}{l}\infty \\
0 \\
0\end{array}$ & \begin{tabular}{l|}
0 \\
ले
\end{tabular} & $\begin{array}{l}0 \\
\infty \\
\infty\end{array}$ & $\begin{array}{l}0 \\
\text { D } \\
\text {. }\end{array}$ & vi & $\underset{\sim}{\sim}$ & 5 & $\stackrel{\infty}{=}$ & m & $\underset{v}{N}$ & 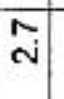 & $\begin{array}{l} \\
\\
\end{array}$ & $\stackrel{\sim}{\sim}$ & mे \\
\hline 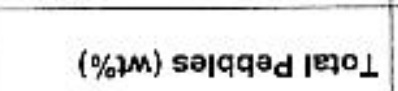 & న్ & एं & $\begin{array}{l}\dot{0} \\
\dot{0}\end{array}$ & 吕 & $\begin{array}{l}0 \\
\infty \\
\text { ले }\end{array}$ & $\begin{array}{l}\text { के } \\
\text { ले }\end{array}$ & \begin{tabular}{l|}
$\infty$ \\
$\infty$ \\
$\infty$ \\
$\infty$
\end{tabular} & 员 & $\tilde{q}$ & \begin{tabular}{l|}
0 \\
8 \\
8
\end{tabular} & $\begin{array}{l}m \\
\text { min } \\
\text { m. }\end{array}$ & \begin{tabular}{|l|}
$\infty$ \\
0 \\
\end{tabular} & \begin{tabular}{l|}
$\infty$ \\
$\infty$
\end{tabular} & \begin{tabular}{l|}
$\infty$ \\
$\stackrel{y}{*}$
\end{tabular} & $\begin{array}{c}\overline{8} \\
\infty\end{array}$ & M & $\begin{array}{l}\infty \\
w \\
w\end{array}$ & $\begin{array}{l}9 \\
\varnothing \\
\varnothing\end{array}$ & 峁 & ָับ \\
\hline (uכ) पदdәg ләмоך & 5 & $\omega$ & $m$ & का & $\stackrel{\infty}{=}$ & 点 & m & $\infty$ & \% & $\stackrel{\mathbb{N}}{\mathrm{N}}$ & $m$ & ำ & व्व & \begin{tabular}{l|} 
क \\
p
\end{tabular} & $\bar{\infty}$ & 品 & N & 嵒 & ๙ & $\omega$ \\
\hline (wo) uldeg dəddn & m & 0 & 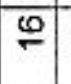 & 品 & $\mathbb{R}$ & 윳 & $\omega$ & $M$ & 9 & 유 & N & $\infty$ & TI & क & $\infty$ & $?$ & 0 & స్ & g & 0 \\
\hline & 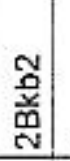 & 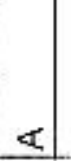 & $\begin{array}{l}\text { 恙 } \\
\text { c }\end{array}$ & 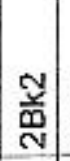 & $\begin{array}{l}\text { 总 } \\
\text { 产 }\end{array}$ & $\begin{array}{l}\text { 总 } \\
\text { 善 }\end{array}$ & 家 & 兽 & 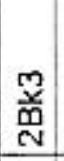 & 鉝 & 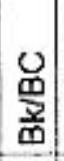 & 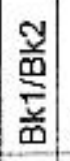 & $\mathbb{\mathbb { E }}$ & 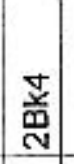 & 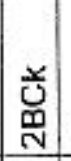 & 前 & $\begin{array}{l}\text { 茴 } \\
\text { \& }\end{array}$ & $\begin{array}{l}\stackrel{e}{\mathrm{~s}} \\
\text { 品 }\end{array}$ & $\begin{array}{l}\text { 总 } \\
\text { 总 }\end{array}$ & $<$ \\
\hline әро弓 히ues pla!d & 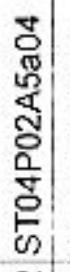 & 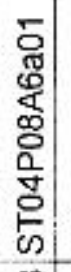 & 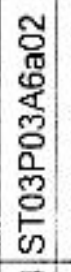 & $\begin{array}{l}5 \\
0 \\
0 \\
8 \\
8 \\
0 \\
0 \\
0 \\
0 \\
0 \\
0 \\
0\end{array}$ & $\begin{array}{c}0 \\
0 \\
0 \\
0 \\
0 \\
0 \\
0 \\
0 \\
0 \\
0 \\
0 \\
0 \\
0 \\
\end{array}$ & 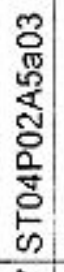 & $\begin{array}{l}0 \\
0 \\
\mathbb{N} \\
\mathbb{2} \\
\mathbb{2} \\
0 \\
0 \\
\overline{0} \\
0 \\
\omega\end{array}$ & 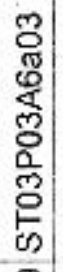 & 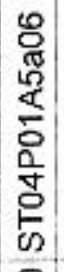 & 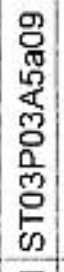 & 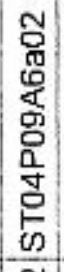 & 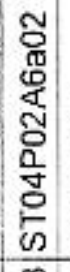 & 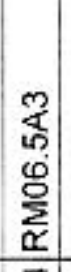 & \begin{tabular}{|l}
0 \\
0 \\
0 \\
0 \\
0 \\
$\vdots$ \\
0 \\
0 \\
0 \\
0 \\
0 \\
0 \\
0 \\
\end{tabular} & \begin{tabular}{|l|} 
\\
0 \\
0 \\
0 \\
0 \\
0 \\
0 \\
0 \\
0 \\
0 \\
0 \\
0 \\
\end{tabular} & $\begin{array}{c}0 \\
0 \\
0 \\
0 \\
0 \\
0 \\
0 \\
0 \\
0 \\
0 \\
0 \\
0 \\
0\end{array}$ & 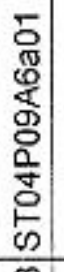 & 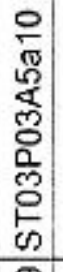 & 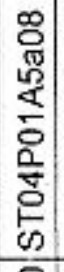 & 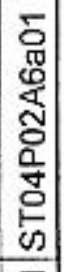 \\
\hline ㅋdues & $N$ & 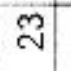 & 茨 & D & 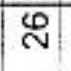 & 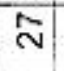 & $\stackrel{\infty}{\sim}$ & is & 이 & $\bar{m}$ & ल) & $m$ & ले & 歺 & 吕 & $m$ & क्ల & ल & 昌 & $\overline{8}$ \\
\hline 748g & $\stackrel{8}{8}$ & O & $\begin{array}{l}\mathcal{O} \\
8\end{array}$ & O & $\underset{\gamma}{\mathcal{Y}}$ & $\stackrel{8}{8}$ & $\stackrel{\mathcal{O}}{\mathrm{g}}$ & \& & \& & ช్ & $\begin{array}{l}\text { V } \\
\text { O }\end{array}$ & O & Ṽ & O & O & 定 & $\stackrel{\mathcal{O}}{\mathrm{g}}$ & $\underset{8}{O}$ & 守 & O \\
\hline
\end{tabular}




\begin{tabular}{|c|c|c|c|c|c|c|c|c|c|c|c|c|c|c|c|c|c|c|c|c|c|}
\hline 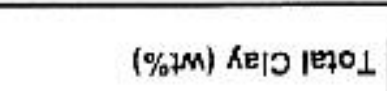 & $\vec{\sim}$ & \begin{tabular}{l|}
$\square$ \\
\end{tabular} & $\begin{array}{l} \\
\text { ले }\end{array}$ & $\begin{array}{l}\overrightarrow{0} \\
\text { ले }\end{array}$ & जi & $\bar{v}$ & \begin{tabular}{l|} 
का \\
की
\end{tabular} & $\begin{array}{l} \\
\mathrm{in}\end{array}$ & \begin{tabular}{c|c|}
$n$ \\
$\infty$
\end{tabular} & $\vec{m}$ & 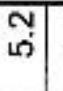 & $\bar{\omega}$ & $\begin{array}{l}m \\
\dot{v}\end{array}$ & $\begin{array}{l}n \\
\text { Na }\end{array}$ & $\hat{\sigma}$ & $\vec{m}$ & Nu & ल) & \begin{tabular}{l|}
$m$ \\
ले
\end{tabular} & क़ & $\begin{array}{l}9 \\
\text { Li⿱ }\end{array}$ \\
\hline (\%+M) ) & $\stackrel{\text { Oे }}{\leftarrow}$ & $\stackrel{0}{\circ}$ & N & $\underset{\forall}{\infty}$ & ำ & $\ddot{q}$ & $\begin{array}{l}\text { m. } \\
0\end{array}$ & $\begin{array}{l}\mathbf{L} \\
\infty \\
\infty\end{array}$ & के & $\begin{array}{l}\sigma \\
\mathrm{m}\end{array}$ & $\begin{array}{l}\infty \\
10\end{array}$ & m & $\begin{array}{l}9 \\
\dot{v}\end{array}$ & $\begin{array}{l}\checkmark \\
\sigma^{\prime}\end{array}$ & 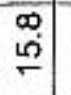 & m. & $\begin{array}{l}\sigma \\
6\end{array}$ & \begin{tabular}{l|l}
$\mathscr{0}$ & 9 \\
$\dot{\forall}$ & $c$
\end{tabular} & $\begin{array}{l}\sigma \\
m \\
m\end{array}$ & m & $\begin{array}{l}\text { Na } \\
\sigma\end{array}$ \\
\hline (\%) & $\stackrel{\infty}{\sim}$ & $\begin{array}{l}\infty \\
\sigma^{\prime}\end{array}$ & $\stackrel{\circ}{\mathrm{N}}$ & $\begin{array}{l}\infty \\
\infty\end{array}$ & 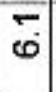 & $\overline{\mathrm{i}}$ & $\begin{array}{l}0 \\
\stackrel{\circ}{\circ}\end{array}$ & $\begin{array}{l}\forall \\
0\end{array}$ & $\begin{array}{l}0 \\
0 \\
10\end{array}$ & $\begin{array}{c}\text { N } \\
\text { in }\end{array}$ & $\begin{array}{l}m \\
\infty\end{array}$ & 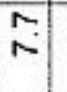 & $\bar{N}$ & $\begin{array}{l}50 \\
\nabla\end{array}$ & $\overline{0}$ & $\begin{array}{l}0 \\
\dot{\nabla}\end{array}$ & $\stackrel{\infty}{N}$ & $\overline{0}$ & $m$ & गे & $\stackrel{1}{N}$ \\
\hline 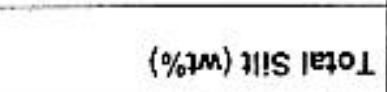 & ले & 穴 & $\stackrel{m}{v}$ & $\underset{\sim}{\stackrel{\sim}{c}}$ & $\stackrel{m}{=}$ & $\begin{array}{l}10 \\
\Phi \\
\emptyset\end{array}$ & $\underset{N}{\stackrel{N}{N}}$ & 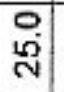 & Nָ & $\bar{\sigma}$ & $\begin{array}{l}0 \\
\dot{v} \\
\leftarrow\end{array}$ & $\stackrel{\text { क }}{=}$ & $\begin{array}{l}0 \\
\stackrel{0}{*}\end{array}$ & \begin{tabular}{l|} 
\\
ल) \\
\end{tabular} & $\begin{array}{l}0 \\
\stackrel{2}{N}\end{array}$ & 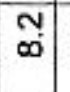 & $\begin{array}{l}0 \\
\dot{J}\end{array}$ & $\begin{array}{l}\infty \\
\stackrel{+}{+}\end{array}$ & $\begin{array}{l}0 \\
\stackrel{1}{*}\end{array}$ & $\hat{N}$ & $\stackrel{M}{\mathrm{~N}}$ \\
\hline (\%) pues au!d Kan & $\overline{6}$ & 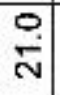 & ले & $\stackrel{\mathrm{m}}{\mathrm{N}}$ & 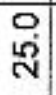 & 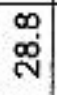 & $\begin{array}{l}\text { के } \\
\text { के }\end{array}$ & 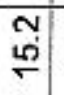 & $\stackrel{2}{=}$ & $\stackrel{\infty}{\leftarrow}$ & \begin{tabular}{l|l|}
$\infty$ \\
$\infty$ \\
-
\end{tabular} & $\begin{array}{l}\text { ผ } \\
\text { ผ }\end{array}$ & $\underset{\sim}{\mathbb{N}}$ & $\stackrel{\oplus}{=}$ & $\begin{array}{l}\infty \\
\dot{N} \\
\dot{N}\end{array}$ & 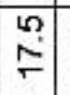 & $\frac{\dot{V}}{\mathrm{~N}}$ & $\begin{array}{l}\infty \\
\stackrel{\infty}{0}\end{array}$ & $\begin{array}{l}\dot{v} \\
\dot{v}\end{array}$ & 꼬 & $\stackrel{\text { क् }}{\stackrel{2}{*}}$ \\
\hline (\%वm) pues au!g & $\begin{array}{l}\sigma \\
\dot{\omega} \\
\underline{\sigma}\end{array}$ & 足 & 足 & $\begin{array}{l}\infty \\
\sigma \\
\sigma\end{array}$ & जे & Nָ & $\begin{array}{l}\dot{v} \\
\varphi\end{array}$ & $\begin{array}{l}0 \\
\text { N }\end{array}$ & $\stackrel{\leftrightarrow}{\sim}$ & $\begin{array}{l}0 \\
\infty \\
-\end{array}$ & $\begin{array}{l}\mathscr{0} \\
\Phi \\
\sigma\end{array}$ & $\overline{\mathrm{N}}$ & $\begin{array}{l}m \\
\text { D. }\end{array}$ & $\begin{array}{l}\circ \\
\text { क् }\end{array}$ & $\begin{array}{l}\infty \\
\infty \\
\infty\end{array}$ & $\frac{\infty}{\sim}$ & $\begin{array}{c}\forall \\
\text { i }\end{array}$ & $\begin{array}{l}40 \\
=\end{array}$ & $\begin{array}{l}\infty \\
\text { ma } \\
\text { v }\end{array}$ & ले & $\begin{array}{l}\varphi \\
\Phi\end{array}$ \\
\hline (\%力m) pues un!paw & $\begin{array}{l}0 \\
\text { गें } \\
\text {. }\end{array}$ & लू & $\begin{array}{l}\text { ก } \\
\text { Nู }\end{array}$ & 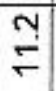 & \begin{tabular}{l|}
$\infty$ \\
$\sigma^{\prime}$
\end{tabular} & $\begin{array}{l}m \\
\infty \\
\infty\end{array}$ & \begin{tabular}{l|}
\multicolumn{1}{|c|}{} \\
$\boldsymbol{\infty}$
\end{tabular} & $\begin{array}{l}0 \\
\square \\
\end{array}$ & $\underset{N}{N}$ & $\begin{array}{l}\square \\
\stackrel{2}{\circ}\end{array}$ & $\overline{0}$ & $\begin{array}{l}m \\
\end{array}$ & गे & $\begin{array}{l}v \\
6\end{array}$ & $\begin{array}{l}\infty \\
6 \\
0\end{array}$ & 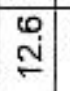 & $\begin{array}{ll}\text { का } \\
\text { के }\end{array}$ & $\begin{array}{ll}0 & 5 \\
5 & 5\end{array}$ & $\begin{array}{l}0 \\
0 \\
\circ\end{array}$ & $\begin{array}{l}\mathscr{N} \\
\mathbb{N}\end{array}$ & m. \\
\hline (\%дM) pues osjeoo & $\begin{array}{l}r \\
\stackrel{N}{N}\end{array}$ & $\begin{array}{l}\text { क) } \\
\text { 응 }\end{array}$ & $\stackrel{0}{N}$ & $\begin{array}{l}\text { के } \\
\text { m. }\end{array}$ & 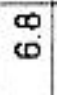 & $\begin{array}{l}\infty \\
\infty \\
\infty\end{array}$ & $\stackrel{\vec{p}}{\square}$ & के & $\begin{array}{l}\infty \\
m \\
\sim\end{array}$ & $\begin{array}{l}\mathscr{0} \\
\stackrel{\infty}{\sigma}\end{array}$ & $\begin{array}{l}\mathbb{N} \\
\stackrel{ \pm}{ \pm}\end{array}$ & \begin{tabular}{l|}
$\infty$ \\
0 \\
0
\end{tabular} & $\underset{\sigma}{\circ}$ & $\overline{0}$ & $\bar{r}$ & 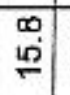 & 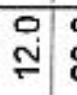 & $\begin{array}{l}0 \\
\stackrel{0}{0} \\
\end{array}$ & $\stackrel{+}{5}$ & $\underset{\oplus}{\check{C}}$ & $\stackrel{\infty}{\stackrel{\infty}{\sim}}$ \\
\hline 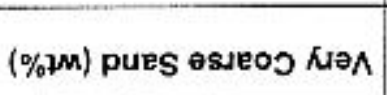 & 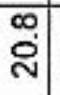 & 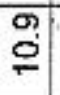 & $\begin{array}{l}0 \\
=\end{array}$ & $=$ & 웅 & $\stackrel{m}{=}$ & $\underset{+}{+}$ & is & $\stackrel{10}{ \pm}$ & $\begin{array}{l}0 \\
\stackrel{0}{\circ} \\
\text { | }\end{array}$ & $\underset{-}{-}$ & $\underset{\dot{U}}{\mathrm{U}}$ & $\begin{array}{l}\dot{0} \\
\stackrel{6}{*}\end{array}$ & $\begin{array}{l}n \\
0\end{array}$ & $\begin{array}{l}0 \\
0 \\
\end{array}$ & 守 & $\stackrel{m}{\dot{m}}$ & $\begin{array}{l}\infty \\
\stackrel{\infty}{\sim} \\
\sim\end{array}$ & $\stackrel{N}{=}$ & $\begin{array}{c}3 \\
\infty\end{array}$ & $\stackrel{\overbrace{}}{\mathscr{C}}$ \\
\hline (\%?M) pues feqol & \begin{tabular}{l|}
$\mathscr{m}$ \\
రై
\end{tabular} & $\begin{array}{l}\infty \\
m\end{array}$ & $\begin{array}{c}\text { m } \\
\text { ๙ू }\end{array}$ & $\begin{array}{l}\infty \\
\infty \\
\infty\end{array}$ & $m_{\infty}^{m}$ & $\begin{array}{l}+ \\
\text { o. }\end{array}$ & $\begin{array}{l}0 \\
\stackrel{2}{*} \\
\mathrm{~N}\end{array}$ & 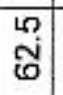 & $\begin{array}{l}\omega \\
0 \\
5\end{array}$ & 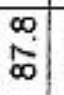 & m. & 옹 & $m$ & 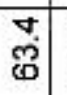 & $\begin{array}{l}m \\
\tilde{g}\end{array}$ & $\begin{array}{l}\bar{\infty} \\
\infty\end{array}$ & 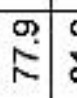 & $\stackrel{\infty}{\infty}$ & \begin{tabular}{l|l}
5 & 2 \\
$\infty$ \\
$\infty$
\end{tabular} & $\begin{array}{l}0 \\
0 \\
8 \\
8\end{array}$ & $\begin{array}{l}\nabla \\
m\end{array}$ \\
\hline (\%?M) selqqod auly & $\begin{array}{l}\text { L2) } \\
\stackrel{2}{2}\end{array}$ & $\begin{array}{l} \pm \\
0\end{array}$ & $\stackrel{m}{\stackrel{m}{2}}$ & \pm & $\begin{array}{l}\infty \\
m \\
m\end{array}$ & $\begin{array}{l}M \\
\pm\end{array}$ & $\begin{array}{l}0 \\
6 \\
6\end{array}$ & $\begin{array}{c}n \\
m\end{array}$ & $\underset{\sigma}{\infty}$ & 品 & $\begin{array}{l}m \\
0 \\
2\end{array}$ & $\stackrel{\vec{m}}{\rightleftharpoons}$ & $\stackrel{0}{=}$ & 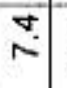 & $\vec{m}$ & $\stackrel{N}{\approx}$ & $\begin{array}{lll}0 & ? \\
\stackrel{0}{6}\end{array}$ & $\stackrel{\square}{ \pm}$ & 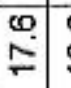 & $\begin{array}{l}\stackrel{0}{\circ} \\
\stackrel{0}{*}\end{array}$ & $\bar{\sigma}$ \\
\hline$(\% \mathrm{~m})$ selqqed u & m & $\begin{array}{l} \\
\stackrel{\infty}{N} \\
\end{array}$ & $\underset{\sim}{\stackrel{N}{\sim}}$ & $\dot{m}$ & 일 & $\begin{array}{l}\text { क } \\
\text { v }\end{array}$ & $\begin{array}{l}0 \\
\text { di } \\
\end{array}$ & $\begin{array}{l}\text { Na } \\
\text { ó }\end{array}$ & $\begin{array}{l}\dot{\varphi} \\
\dot{\mathrm{H}}\end{array}$ & $\frac{\square}{\square}$ & $\begin{array}{l}8 \\
\text { v }\end{array}$ & 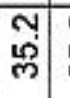 & 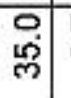 & $\hat{\sigma}$ & 嗾 & $\begin{array}{l}\text { m. } \\
\dot{g} \\
\dot{v}\end{array}$ & 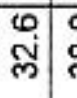 & 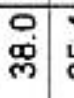 & $\begin{array}{l}\Delta \\
\stackrel{\sim}{\sim}\end{array}$ & $\begin{array}{l}\text { กี } \\
\text { ปิ }\end{array}$ & $\begin{array}{l}\infty \\
\stackrel{m}{\infty}\end{array}$ \\
\hline (\%४M) salqqad as.eo & $\begin{array}{l}5 \\
10\end{array}$ & $\begin{array}{l}9 \\
6 \\
10\end{array}$ & $\stackrel{r}{\dot{Z}}$ & $\begin{array}{l} \\
\text { के }\end{array}$ & $\begin{array}{l}\mathbf{\infty} \\
\stackrel{N}{v}\end{array}$ & ले & \begin{tabular}{l}
0 \\
0 \\
\hdashline
\end{tabular} & 召 & 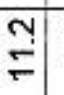 & บֶ. & $\begin{array}{l}0 \\
\dot{v}\end{array}$ & $\begin{array}{l}\stackrel{\leftrightarrow}{\mathrm{N}} \\
\stackrel{\mathrm{N}}{*}\end{array}$ & $\begin{array}{l}\infty \\
\mathrm{N}\end{array}$ & \begin{tabular}{l|}
$\infty$ \\
$\infty$
\end{tabular} & ז' & $\begin{array}{l}m \\
\dot{v}\end{array}$ & $\begin{array}{l}\infty \\
\infty \\
\infty\end{array}$ & $\stackrel{\check{c}}{\mathscr{c}}$ & $\overline{\mathrm{v}}$ & $\begin{array}{c}+ \\
\text { cे. }\end{array}$ & $\overline{\mathrm{i}}$ \\
\hline$(\% \not M)$ so|qqण्d IE) & กี่ & $\overline{\mathrm{N}}$ & $\begin{array}{l}N \\
\dot{W}\end{array}$ & ஜ) & $\begin{array}{l}\forall \\
\text { g }\end{array}$ & $\begin{array}{l}\omega \\
6 \\
0\end{array}$ & $\begin{array}{l}0 \\
\infty \\
0 \\
0\end{array}$ & m & $\begin{array}{l}0 \\
\tilde{D} \\
\tilde{D}\end{array}$ & $\overline{\mathscr{B}}$ & $\overline{0}$ & $\begin{array}{l}0 \\
8 \\
0\end{array}$ & $\begin{array}{l}8 \\
\text { L }\end{array}$ & $\begin{array}{l}0 \\
0 \\
\text { N }\end{array}$ & $\begin{array}{l}m \\
\text { mi } \\
\text { v }\end{array}$ & $\begin{array}{l}\infty \\
\dot{0}\end{array}$ & 它 & $\begin{array}{l}\infty \\
\infty \\
\infty\end{array}$ & ig & 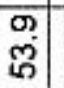 & 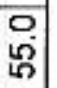 \\
\hline (uv) чlfdea demo & 운 & 田 & $\underset{\square}{\square}$ & $\stackrel{\mathbb{N}}{\mathbb{N}}$ & 哭 & $\stackrel{2}{2}$ & $\mathscr{M}$ & का & ल्ల్v & 8 & ֶָ & 营 & N & $\stackrel{\infty}{\leftarrow}$ & ֻ & 足 & 员 & $E$ & $\frac{\infty}{N}$ & $\tilde{N}$ & $\tilde{6}$ \\
\hline (w৩) updəg Jəddn & $\underset{\square}{\check{I}}$ & 만. & 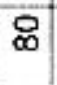 & $\stackrel{M}{v}$ & פ & 0 & $\hat{n}$ & o & $\begin{array}{l}\mathscr{N} \\
\mathrm{N}\end{array}$ & $\tilde{\bullet}$ & $\stackrel{m}{=}$ & $\frac{\infty}{\sim}$ & $\infty$ & का & $\stackrel{g}{\mathrm{~m}}$ & $\begin{array}{c}\text { ల్ } \\
\text { N }\end{array}$ & mis & Lे & 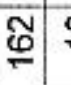 & $\stackrel{\infty}{\leftarrow}$ & 운 \\
\hline & 总 & ฐ & $\begin{array}{l}\text { 总 } \\
\text { 岕 } \\
\end{array}$ & $\begin{array}{l}\text { 竎 } \\
\text { 㓢 } \\
\end{array}$ & $\mathbb{E}$ & $<$ & $\begin{array}{l}\text { 总 } \\
\text { 商 }\end{array}$ & 悤 & $\begin{array}{l}\frac{5}{\mathbf{0}} \\
\frac{\mathbf{u}}{\mathrm{N}}\end{array}$ & $\begin{array}{l}\text { 总 } \\
\text { 足 }\end{array}$ & 芯 & $\begin{array}{l}\frac{N}{0} \\
\text { D. } \\
\text { N }\end{array}$ & 离 & $\overline{\underline{z}}$ & $\begin{array}{l}\text { N } \\
\text { 商 } \\
\text { 㟧 }\end{array}$ & 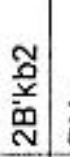 & 盖 & c & 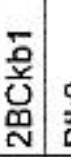 & 节 & 崽 \\
\hline әpoว ә|dwes pla! & 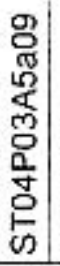 & $\begin{array}{l}\overline{0} \\
\text { ○ } \\
\text { 巳 } \\
\text { 品 }\end{array}$ & 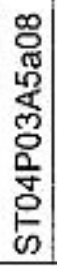 & 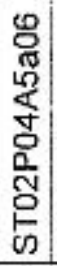 & 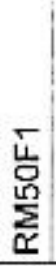 & 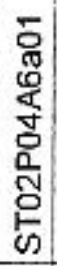 & 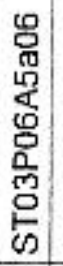 & 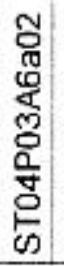 & 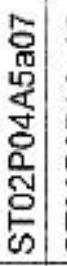 & 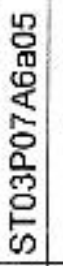 & 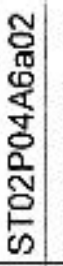 & $\begin{array}{l}\bar{c} \\
0 \\
\& \\
\& \\
0 \\
0 \\
0 \\
0 \\
0 \\
0\end{array}$ & 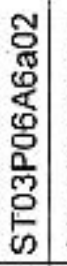 & 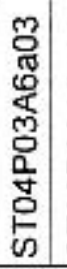 & 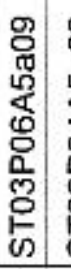 & 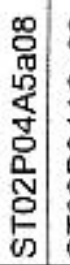 & 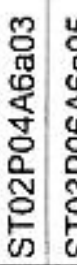 & 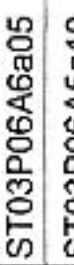 & 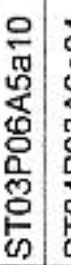 & 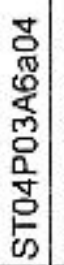 & 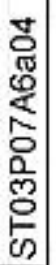 \\
\hline opdues & - & $N$ & $m$ & $\nabla$ & $n$ & $\omega$ & $N$ & $\infty$ & का & 우 & $\mp$ & 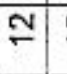 & $m$ & 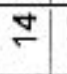 & 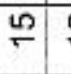 & $\oplus$ & $\approx:$ & $\stackrel{\infty}{\infty}$ & $\%$ & i & $\bar{v}$ \\
\hline & 豸్ & gु & 马ำ & 学 & 8 & \& & 8 & $\stackrel{8}{8}$ & ஜֶ) & g্ & ্ֻ & ஓ्? & Oे. & M̊ & $\stackrel{m}{O}$ & ğ & g & g & 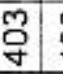 & 旁 & gु \\
\hline
\end{tabular}




\begin{tabular}{|c|c|c|c|c|c|c|c|c|c|c|c|c|c|c|c|c|c|}
\hline 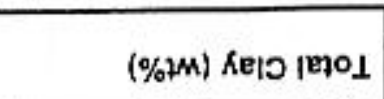 & $m$ & 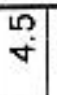 & \begin{tabular}{l|}
5 \\
$50^{\circ}$
\end{tabular} & $M$ & \begin{tabular}{l|l}
$\omega$ \\
\end{tabular} & \begin{tabular}{l|} 
\\
$\dot{m}$
\end{tabular} & \begin{tabular}{l|l} 
\\
0
\end{tabular} & \begin{tabular}{l|l}
$\infty$ \\
$\omega$
\end{tabular} & $\begin{array}{l} \\
\sigma\end{array}$ & \begin{tabular}{|l|l|}
$\infty$ & \\
$\dot{m}$ &
\end{tabular} & 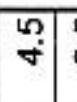 & $\begin{array}{l}0 \\
\text { ñ }\end{array}$ & \begin{tabular}{l|l}
$\infty$ & 1 \\
+ &
\end{tabular} & 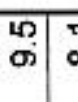 & \begin{tabular}{l|l}
$\bar{\infty}$ & $\overline{5}$ \\
\end{tabular} & \begin{tabular}{l|l} 
& 1 \\
0 & 1
\end{tabular} & 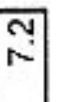 \\
\hline (\%2M) 게!S au!- & 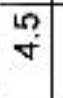 & $\begin{array}{l}\infty \\
\dot{\sigma} \\
\end{array}$ & $\begin{array}{l}6 \\
6\end{array}$ & लें & $\bar{\sigma}$ & $\begin{array}{l}m \\
\dot{v}\end{array}$ & Niv & $\begin{array}{l} \\
\dot{\sigma} \\
\dot{v}\end{array}$ & $\begin{array}{l}\infty \\
\omega^{\prime}\end{array}$ & $\stackrel{n}{\nabla}$ & $\begin{array}{l}0 \\
6\end{array}$ & $\stackrel{n}{\circ}$ & \begin{tabular}{l|l}
$\infty$ & \\
&
\end{tabular} & $\stackrel{0}{\circ}$ & \begin{tabular}{l}
$\infty$ \\
\multirow{N}{N}{}
\end{tabular} & $\begin{array}{c}m \\
\text { L }\end{array}$ & $\underset{\nabla}{\nabla}$ \\
\hline 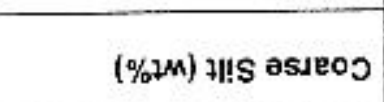 & 임 & $\begin{array}{l}0 \\
0 \\
0\end{array}$ & $\begin{array}{l}\infty \\
\infty\end{array}$ & $\begin{array}{l}0 \\
\text { m. }\end{array}$ & $\begin{array}{l}0 \\
\infty \\
\infty\end{array}$ & ז & \begin{tabular}{l|l|} 
& \\
$\infty$ &
\end{tabular} & กิ & $\begin{array}{l}0 \\
\dot{\square}\end{array}$ & $\bar{\omega}$ & $\underset{1}{\mp}$ & $\stackrel{m}{-}$ & $\begin{array}{l}m \\
\stackrel{m}{\circ}\end{array}$ & $\begin{array}{l}\infty \\
\infty \\
\infty\end{array}$ & $\stackrel{\text { ma }}{\infty}$ & \begin{tabular}{l|}
$\infty$ \\
$\infty$ \\
$\omega$
\end{tabular} & O. \\
\hline$(\% \not M)$ ZIIS |EDOL & 茴 & $\begin{array}{l}0 \\
6 \\
2\end{array}$ & $\underset{+0}{\stackrel{5}{\circ}}$ & $\begin{array}{l}\text { ले } \\
\text { లై }\end{array}$ & $\bar{\sim}$ & $\begin{array}{l}\square \\
=\end{array}$ & $\begin{array}{l}\infty \\
0 \\
0\end{array}$ & $\stackrel{M}{\circ}$ & d. & $\begin{array}{l}\varphi \\
0\end{array}$ & $=$ & a) & $\overrightarrow{\mathrm{n}}$ & $\begin{array}{l}\infty \\
\stackrel{5}{=}\end{array}$ & 守 & $F$ & ন \\
\hline (\%) pues әu! & $\begin{array}{l}\sigma \\
\oplus \\
\oplus\end{array}$ & $\begin{array}{l}\infty \\
\stackrel{\sim}{\sim}\end{array}$ & i্ & $\bar{d}$ & 잉 & 워 & $\stackrel{\oplus}{\circ}$ & $\bar{d}$ & 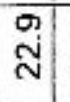 & $\stackrel{\mathrm{O}}{\mathrm{C}}$ & \begin{tabular}{l|} 
\\
\\
s.
\end{tabular} & $\begin{array}{l}\mathscr{0} \\
\mathscr{\omega}\end{array}$ & \begin{tabular}{l|l}
0 \\
$\stackrel{n}{*}$
\end{tabular} & \begin{tabular}{ll}
\multirow{2}{*}{} & \\
6 &
\end{tabular} & $\begin{array}{ll}\infty & \\
\sim & \sim\end{array}$ & एே. & $\begin{array}{l}\dot{J} \\
\dot{I}\end{array}$ \\
\hline (\%+M) pues au!y & సิ & 沗 & ลิ & $\begin{array}{l}\omega \\
\infty \\
\sigma\end{array}$ & $\begin{array}{l} \\
\infty \\
\leftarrow\end{array}$ & స̊. & 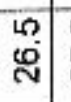 & $\begin{array}{l}\omega \\
\omega \\
j\end{array}$ & 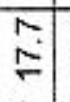 & $\underset{i s}{\leftarrow}$ & ล่ & 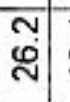 & $\begin{array}{l}\square \\
\sigma\end{array}$ & $\stackrel{m}{\oplus}$ & \begin{tabular}{l|l}
$\omega$ \\
$\stackrel{4}{N}$
\end{tabular} & $\begin{array}{l}\varphi \\
\text { mi }\end{array}$ & ঠे \\
\hline$(\% 1 M)$ pues unjpaw & $\vec{m}$ & $\stackrel{\text { 웅 }}{=}$ & $\stackrel{m}{\sigma}$ & $M$ & $\begin{array}{l}\infty \\
\omega \\
\infty\end{array}$ & $\hat{\circ}$ & 离 & m & $\begin{array}{l}\infty \\
m \\
m\end{array}$ & \begin{tabular}{|c|}
0 \\
10 \\
\end{tabular} & \begin{tabular}{l|l|}
0 \\
0 \\
0
\end{tabular} & 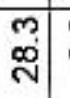 & \begin{tabular}{l|l}
$\sigma$ \\
$\infty$
\end{tabular} & $\stackrel{m}{=}$ & $\bar{v}$ & क. & $\begin{array}{l}0 \\
0 \\
-\end{array}$ \\
\hline (\%ҰM) pues asueoo & $\begin{array}{l}\text { का } \\
\stackrel{2}{\circ}\end{array}$ & 둥 & $\stackrel{\text { m }}{=}$ & \begin{tabular}{l|l}
$\infty$ \\
$\infty$
\end{tabular} & $\stackrel{0}{\leftarrow}$ & $\overline{0}$ & $\begin{array}{l}\check{2} \\
\stackrel{2}{\sigma}\end{array}$ & N & $\begin{array}{l}\varphi \\
\infty\end{array}$ & $\overline{\mathrm{N}}$ & \begin{tabular}{l|}
$\infty$ \\
$\stackrel{\infty}{0}$ \\
$\stackrel{0}{*}$
\end{tabular} & 品 & के & : & 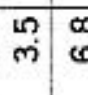 & | & مُ \\
\hline (\%M) purs osueoJ MeA & $\begin{array}{l}m \\
\infty \\
=\end{array}$ & $\begin{array}{l}0 \\
\stackrel{0}{\circ}\end{array}$ & ㅁ. & \begin{tabular}{l|}
$\infty$ \\
$\infty$ \\
$\infty$
\end{tabular} & 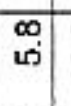 & $\begin{array}{l}\text { No } \\
\text { 으 }\end{array}$ & $\begin{array}{l}6 \\
\dot{m}\end{array}$ & $\begin{array}{l}\nabla \\
\oplus\end{array}$ & $\begin{array}{l}N \\
\sigma\end{array}$ & \begin{tabular}{|l|}
$\infty$ \\
$\stackrel{n}{n}$
\end{tabular} & $\overline{\mathrm{d}}$ & $\begin{array}{l}\dot{0} \\
\stackrel{\rho}{\circ}\end{array}$ & 요 & \begin{tabular}{l|l} 
& \\
0 & 0
\end{tabular} & \begin{tabular}{l|l}
0 & 4 \\
$ن$ & 4
\end{tabular} & 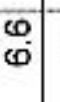 & స్ \\
\hline 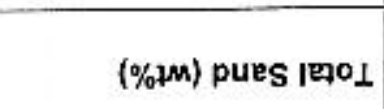 & 芦 & 岂 & 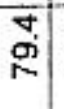 & $\begin{array}{l}0 \\
g \\
g\end{array}$ & $\begin{array}{l}m \\
m \\
0\end{array}$ & ए广 & \begin{tabular}{l|} 
\\
$\grave{\infty}$ \\
$\infty$
\end{tabular} & 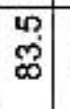 & $\bar{\Phi}$ & \begin{tabular}{|l|} 
\\
$\mathscr{O}$ \\
$\mathscr{\infty}$
\end{tabular} & $\begin{array}{c} \\
\infty \\
0\end{array}$ & $\stackrel{M}{\mathrm{~S}}$ & $\bar{c}$ & $\stackrel{N}{N}$ & 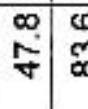 & \begin{tabular}{l|}
$\mathscr{D}$ \\
$\mathscr{\infty}$ \\
$\infty$
\end{tabular} & $\begin{array}{l}\bar{\Xi} \\
\infty \\
\infty\end{array}$ \\
\hline (\%2M) səlqqәd өu! & $\stackrel{亡}{\leftarrow}$ & $\begin{array}{l}n \\
\stackrel{n}{*}\end{array}$ & $\begin{array}{l}\infty \\
\dot{v} \\
\dot{ \pm}\end{array}$ & $\begin{array}{l}\sigma \\
\emptyset\end{array}$ & $\stackrel{+}{=}$ & $\begin{array}{l}\infty \\
\sim \\
\sim\end{array}$ & 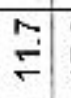 & $\stackrel{M}{\stackrel{M}{=}}$ & $\mathrm{m}$ & \begin{tabular}{|l|}
0 \\
$\infty$ \\
$\leftarrow$
\end{tabular} & $\begin{array}{l}0 \\
\stackrel{0}{=}\end{array}$ & $\stackrel{m}{m}$ & N & $\begin{array}{ll}0 \\
+\end{array}$ & $\hat{\omega}$ & 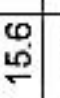 & $\tilde{\sigma}$ \\
\hline (\%łM) selqqed un!pew & $\begin{array}{l}\bar{y} \\
\text { vis }\end{array}$ & $\begin{array}{l}\mathbf{D} \\
\text { 号 }\end{array}$ & \begin{tabular}{l}
\multirow{2}{*}{} \\
$\stackrel{\text { m. }}{ }$
\end{tabular} & $\begin{array}{l} \\
\infty \\
\infty \\
\infty\end{array}$ & $\bar{p}$ & $\begin{array}{l}0 \\
\dot{N}\end{array}$ & $\underset{\sim}{\stackrel{*}{*}}$ & 吕 & लें & 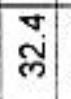 & \begin{tabular}{l|} 
\\
$g$ \\
$m$
\end{tabular} & $\frac{\mathrm{n}}{\mathrm{m}}$ & : & $\begin{array}{l}\text { क } \\
\text { से }\end{array}$ & $\stackrel{\mathrm{N}}{\mathrm{N}}$ & Бें & $\vec{N}$ \\
\hline (\%дM) selqqәd asseo? & 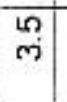 & $\begin{array}{l}0 \\
\stackrel{0}{ }\end{array}$ & $\stackrel{F}{E}$ & $\begin{array}{l}\infty \\
m\end{array}$ & $\stackrel{\circ}{-}$ & $\begin{array}{c}\dot{y} \\
\dot{m}\end{array}$ & $\vec{m}$ & $\ddot{\sim}$ & $\stackrel{\infty}{\sim}$ & \begin{tabular}{l|} 
in \\
ñ
\end{tabular} & $\tilde{m}$ & $\begin{array}{l}\circ \\
\stackrel{0}{-1}\end{array}$ & $\begin{array}{l}0 \\
\text { 㟧 }\end{array}$ & $\stackrel{\text { N̦ }}{\sim}$ & 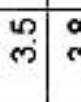 & \begin{tabular}{l|}
$\infty$ \\
$\infty$ \\
$\dot{\omega}$
\end{tabular} & $\begin{array}{l}N \\
\text { ले }\end{array}$ \\
\hline$(\% \not M)$ sa|qqed IEOL & $\begin{array}{c}m \\
\text { ले }\end{array}$ & $\begin{array}{l}10 \\
60 \\
6 \\
6\end{array}$ & ల్ & $\begin{array}{l}\nabla \\
0 \\
0 \\
0\end{array}$ & 只 & $\frac{m}{10}$ & गू & $\begin{array}{l}0 \\
\dot{q}\end{array}$ & 号 & $\begin{array}{l} \\
\text { กิ }\end{array}$ & 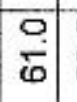 & $\begin{array}{l}40 \\
0 \\
0 \\
03\end{array}$ & $\stackrel{\infty}{i}$ & $\overline{6}$ & 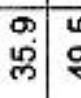 & 吕 & $\begin{array}{l}\varphi \\
\stackrel{\varphi}{\varphi}\end{array}$ \\
\hline (шэ) чұdag sәмот & कृ & $\infty$ & a & 음 & 守 & $\stackrel{\mathbb{m}}{\stackrel{N}{*}}$ & Ln & $\mathbb{T}$ & 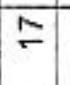 & 落 & 只 & $\widehat{\omega}$ & 号 & $\overline{8}$ & $\infty$ & I & 요 \\
\hline (uv) पłdeg doddn & 号 & 0 & 4 & $\mathscr{S}$ & $=$ & क & vi & $\stackrel{\pi}{C}$ & 6 & $\overline{7}$ & న్ & लొ & 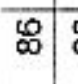 & m & 0 & 可 & $\mathscr{\varphi}$ \\
\hline uoz!®ö & 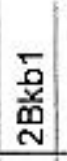 & $\leq$ & 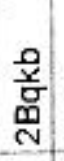 & $\begin{array}{l}\text { 童 } \\
\text { 萹 }\end{array}$ & 恙 & $\begin{array}{l}\text { 总 } \\
\text { 兴 } \\
\end{array}$ & 兽 & 뜸 & 芯 & 善 & 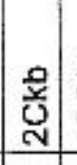 & 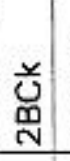 & 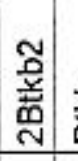 & $\begin{array}{l}\text { 辛 } \\
\text { 离 }\end{array}$ & $<$ & $\underset{\mathrm{E}}{\mathrm{E}}$ & $\begin{array}{l}\text { 总 } \\
\text { 总 }\end{array}$ \\
\hline әроว 리ues p|ə!」] & 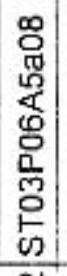 & $\begin{array}{l}5 \\
0 \\
0 \\
\mathbb{0} \\
0 \\
0 \\
\frac{0}{m} \\
0 \\
\omega \\
\end{array}$ & 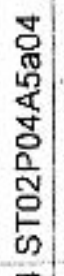 & 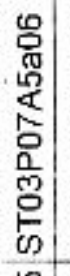 & $\begin{array}{l}0 \\
0 \\
0 \\
0 \\
5 \\
0 \\
0 \\
0 \\
0 \\
0 \\
0 \\
0\end{array}$ & 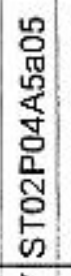 & $\begin{array}{l}0 \\
0 \\
0 \\
\mathbb{1} \\
0 \\
0 \\
0 \\
0 \\
0 \\
0 \\
0\end{array}$ & 员 & 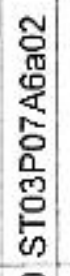 & $\begin{array}{l}0 \\
0 \\
0 \\
0 \\
0 \\
0 \\
0 \\
0 \\
0 \\
0 \\
0 \\
0\end{array}$ & $\mid \begin{array}{l}N \\
\mathbb{N} \\
0 \\
8 \\
0 \\
0 \\
0 \\
0 \\
0 \\
0 \\
0\end{array}$ & $\begin{array}{l}0 \\
0 \\
0 \\
0 \\
0 \\
0 \\
0 \\
0 \\
0 \\
0 \\
0\end{array}$ & 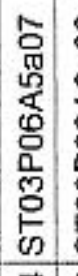 & 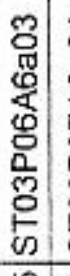 & 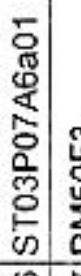 & 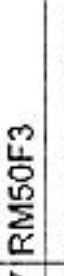 & 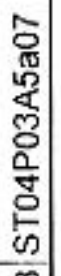 \\
\hline e्pdurs & $\mathbb{N}$ & $\stackrel{M}{N}$ & $\stackrel{\sim}{\sim}$ & $\stackrel{2}{\sim}$ & 9 & s & $\infty$ & I & 品 & 5 & $\sqrt{2}$ & m & फ & $\stackrel{m}{m}$ & 包! & m. & $\infty$ \\
\hline чำा & O্ & m & ल) & g & g & g & g & g & g & m & g & g & \begin{tabular}{|l|l|}
$g$ \\
\end{tabular} & 荬 & 多 & gु & \% \\
\hline
\end{tabular}

0
$\dot{\omega}$
0
0
0
0 


\begin{tabular}{|c|c|c|c|c|c|c|c|c|c|c|c|c|c|c|c|c|c|c|c|c|c|}
\hline 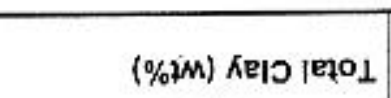 & $\begin{array}{l}\mathscr{0} \\
0\end{array}$ & \begin{tabular}{l|} 
\\
ले
\end{tabular} & $\begin{array}{l} \\
\text { ले }\end{array}$ & m & $\overrightarrow{\mathrm{N}}$ & m & ले & \begin{tabular}{l|}
$\infty$ \\
6 \\
6
\end{tabular} & $\begin{array}{l}m \\
\infty\end{array}$ & \begin{tabular}{l|}
$\infty$ \\
$\dot{\nabla}$
\end{tabular} & $\begin{array}{l}N \\
m\end{array}$ & ' & \begin{tabular}{|l|}
$\infty$ \\
0 \\
\end{tabular} & ले & \begin{tabular}{|l|l|}
50 \\
0
\end{tabular} & \begin{tabular}{|l|}
$n$ \\
$m$
\end{tabular} & $\bar{\infty}$ & $\underset{\forall}{\sim}$ & $\begin{array}{l} \\
+\end{array}$ & $\stackrel{N}{v}$ & $\begin{array}{l} \\
0 \\
\end{array}$ \\
\hline$(\% 2 \mathrm{M})$ zI!S au!t & $\overline{10}$ & m. & $\begin{array}{l}\sigma \\
\dot{m}\end{array}$ & ז & $\begin{array}{l}n \\
\text { m }\end{array}$ & $\vec{v}$ & ले & ب़ & $\underset{-}{\square}$ & $\begin{array}{c}N \\
m\end{array}$ & $\underset{v}{Y}$ & 5 & $\begin{array}{l}10 \\
60\end{array}$ & $\begin{array}{c}N \\
\forall\end{array}$ & \begin{tabular}{|l|}
$n$ \\
0
\end{tabular} & \begin{tabular}{|l|}
$\begin{array}{l}n \\
\text { on }\end{array}$ \\
\end{tabular} & $\underset{\sim}{\stackrel{N}{N}}$ & $\begin{array}{l}N \\
\forall\end{array}$ & $\bar{\theta}$ & \begin{tabular}{|l|} 
\\
$\mathrm{N}$
\end{tabular} & O \\
\hline (\%2M ) मा5 esueoo & $\ddot{m}$ & $\stackrel{m}{\sim}$ & $\bar{n}$ & ָָ & $\begin{array}{l}\nabla \\
\infty\end{array}$ & $\bar{v}$ & $\stackrel{N}{N}$ & $\bar{i}$ & $\begin{array}{l}0 \\
\text { o. }\end{array}$ & $\bar{\sim}$ & $\begin{array}{l}M \\
\omega\end{array}$ & $\begin{array}{l}\infty \\
\dot{v}\end{array}$ & $\begin{array}{l}0 \\
m\end{array}$ & $\stackrel{0}{\circ}$ & \begin{tabular}{|l|}
0 \\
\end{tabular} & \begin{tabular}{|l|} 
\\
\\
$\sigma$
\end{tabular} & $\mp$ & $\bar{\infty}$ & \begin{tabular}{l|l} 
\\
$\infty$ \\
$\infty$
\end{tabular} & $\overline{\vec{m}}$ & $\begin{array}{l}\infty \\
\dot{v}\end{array}$ \\
\hline 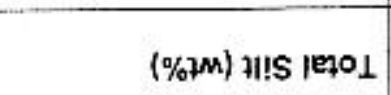 & $\underset{\square}{\tau}$ & \begin{tabular}{l|}
0 \\
$\dot{\omega}$
\end{tabular} & \begin{tabular}{|l|}
0 \\
$\sigma$ \\
\end{tabular} & 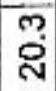 & $\stackrel{0}{=}$ & $\bar{N}$ & $\begin{array}{l}\mathscr{O} \\
\stackrel{\circ}{\circ}\end{array}$ & 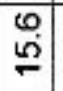 & 옹 & $\begin{array}{c}m \\
0\end{array}$ & $\begin{array}{l}\text { का } \\
\text { के }\end{array}$ & $\begin{array}{l} \\
\end{array}$ & \begin{tabular}{|l|}
$\vec{m}$ \\
$\stackrel{2}{r}$
\end{tabular} & 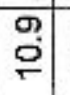 & \begin{tabular}{l|}
3 \\
0 \\
0
\end{tabular} & $\begin{array}{l}40 \\
f\end{array}$ & $\begin{array}{c}\mathrm{m} \\
\mathrm{N}\end{array}$ & $\begin{array}{c}\mathfrak{N} \\
\mathfrak{v}\end{array}$ & $\underset{\square}{ \pm}$ & $\bar{\omega}$ & $\hat{\sigma}$ \\
\hline 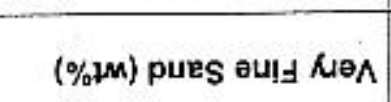 & $\begin{array}{l}\infty \\
\text { నె่ }\end{array}$ & $\begin{array}{l}\dot{J} \\
\dot{v}\end{array}$ & $\stackrel{N}{\sim}$ & N & 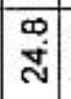 & $\stackrel{5}{2}$ & गิ & 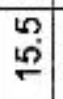 & $\begin{array}{l}\text { ম } \\
\text { J্ণ }\end{array}$ & $\begin{array}{l}\text { 요 } \\
\stackrel{2}{\circ}\end{array}$ & $\stackrel{\infty}{\stackrel{\infty}{\perp}}$ & \begin{tabular}{l|} 
\\
0 \\
0
\end{tabular} & 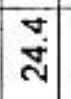 & $\begin{array}{l}0 \\
\dot{N}\end{array}$ & $\stackrel{2}{\stackrel{2}{*}}$ & \begin{tabular}{l|} 
\\
$\infty$ \\
$\infty$
\end{tabular} & $\begin{array}{l}0 \\
\dot{N}\end{array}$ & $\frac{N}{N}$ & $\begin{array}{l}0 \\
\infty \\
\infty\end{array}$ & $\begin{array}{l}0 \\
\stackrel{0}{\circ}\end{array}$ & $\begin{array}{l}\sigma \\
\text { g }\end{array}$ \\
\hline (\%) $\%$ pues au!s & $\begin{array}{l}N \\
0 \\
0\end{array}$ & 을 & $\begin{array}{l}0 \\
\omega \\
\sim\end{array}$ & N & $\underset{\infty}{\rightleftharpoons}$ & $\frac{\vec{m}}{\mathrm{~m}}$ & \begin{tabular}{l|l} 
\\
ले
\end{tabular} & $\begin{array}{l}\infty \\
\text { d } \\
\end{array}$ & స̇ & ब. & $\bar{N}$ & 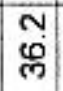 & $\frac{m}{\stackrel{5}{N}}$ & $\hat{\mathrm{N}}$ & \begin{tabular}{l|}
$n$ \\
\multirow{2}{*}{}
\end{tabular} & \begin{tabular}{l|}
0 \\
\\
$\sim$
\end{tabular} & $\begin{array}{l}\infty \\
\stackrel{\infty}{\sim}\end{array}$ & $\stackrel{0}{\sim}$ & $\begin{array}{l}\text { 웅 } \\
\text { की }\end{array}$ & 品 & ल \\
\hline (\%џM) pues un!pew & $\begin{array}{l}\sigma \\
\sigma^{\prime}\end{array}$ & $\begin{array}{l}0 \\
\text { ஸे }\end{array}$ & \begin{tabular}{|l|}
0 \\
40 \\
20
\end{tabular} & 号 & 훙 & $\begin{array}{l}\stackrel{N}{N} \\
\stackrel{N}{n}\end{array}$ & \begin{tabular}{l|l|} 
\\
$\infty$
\end{tabular} & $\stackrel{2}{\sim}$ & \begin{tabular}{l|}
0 \\
$\infty$
\end{tabular} & 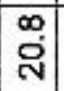 & $\stackrel{y}{\dot{y}}$ & बे & ष & No & 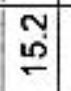 & T: & $\underset{N}{N}$ & $\stackrel{+}{=}$ & ตे & $\begin{array}{l}0 \\
\stackrel{0}{\mathbf{N}}\end{array}$ & $\underset{\square}{\Phi}$ \\
\hline [\%2M] pues esuroo & $\begin{array}{l}40 \\
0\end{array}$ & $\begin{array}{l}\text { के } \\
\stackrel{m}{\sigma}\end{array}$ & $\stackrel{m}{m}$ & $\stackrel{N}{N}$ & $\stackrel{v}{\square}$ & $\begin{array}{l}\mathrm{N} \\
\mathfrak{T}\end{array}$ & $\begin{array}{l}\infty \\
\infty \\
\sigma\end{array}$ & 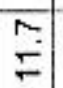 & $\begin{array}{c}y \\
0\end{array}$ & $\begin{array}{l}50 \\
0\end{array}$ & 只 & 岁 & $=$ & $\begin{array}{l} \\
0 \\
0\end{array}$ & $\begin{array}{l}\mathrm{M} \\
\mathrm{N}\end{array}$ & $\begin{array}{l}m \\
m\end{array}$ & n & $\begin{array}{l}m \\
\square \\
\square\end{array}$ & $\begin{array}{l}\dot{d} \\
\dot{T}\end{array}$ & $\stackrel{r}{\sim}$ & $\begin{array}{l}\circ \\
\text { ó }\end{array}$ \\
\hline h) pues asueoj NəA & n) & $m$ & $\begin{array}{l}0 \\
\stackrel{0}{\circ} \\
\end{array}$ & 9 & $\underset{7}{2}$ & ஸे & $\begin{array}{l}\stackrel{0}{\rho} \\
\stackrel{m}{\longrightarrow}\end{array}$ & $\overline{\mathrm{i}}$ & $\begin{array}{l}\text { Oे } \\
\end{array}$ & $\begin{array}{l}50 \\
10 \\
10\end{array}$ & \begin{tabular}{|l|}
$\infty$ \\
$\dot{j}$
\end{tabular} & $\begin{array}{l}3 \\
6 \\
0\end{array}$ & \begin{tabular}{l|}
$m$ \\
$m$ \\
$m$
\end{tabular} & \begin{tabular}{|c|} 
\\
$\varphi$ \\
$\varphi$
\end{tabular} & $\stackrel{+}{\tau}$ & \begin{tabular}{|c|} 
\\
$\infty$ \\
\end{tabular} & $\begin{array}{l}\omega \\
\infty\end{array}$ & $\begin{array}{c}\underset{\sim}{n} \\
\stackrel{4}{\sim}\end{array}$ & $\frac{+}{N}$ & \begin{tabular}{l|} 
\\
6 \\
\end{tabular} & $\check{\sigma}$ \\
\hline$(\%$;M) pues & 옳 & $\begin{array}{l}0 \\
8 \\
8\end{array}$ & 怘 & vo & \begin{tabular}{c|}
8 \\
$i$ \\
$\infty$
\end{tabular} & $\begin{array}{l}0 \\
\wp \\
\infty\end{array}$ & 今 & $\begin{array}{l}0 \\
\tilde{E}\end{array}$ & $\frac{M}{R}$ & $\begin{array}{l}\text { 옹 } \\
\text { 8. }\end{array}$ & \begin{tabular}{l|} 
\\
$\dot{\infty}$ \\
$\dot{\infty}$
\end{tabular} & $\begin{array}{l} \\
\text { में }\end{array}$ & $\begin{array}{l}m \\
\sigma \\
\end{array}$ & \begin{tabular}{l|}
$\infty$ \\
ஸ் \\
\end{tabular} & \begin{tabular}{l|}
$N$ \\
$\tilde{D}$ \\
\end{tabular} & \begin{tabular}{|c|} 
ஸू. \\
\end{tabular} & $\begin{array}{l}\infty \\
\infty \\
\infty\end{array}$ & $\begin{array}{l}\mathscr{D} \\
\mathscr{\infty}\end{array}$ & $\underset{\infty}{\mathbf{N}}$ & $\stackrel{m}{9}$ & $\stackrel{\infty}{\infty}$ \\
\hline$(\% 2 M)$ se|qq日d ou!y & $\begin{array}{l}9 \\
\oplus\end{array}$ & $\begin{array}{l}\infty \\
\infty \\
\omega\end{array}$ & i & एक & $\begin{array}{l}\infty \\
\stackrel{m}{2}\end{array}$ & $\sigma_{\infty}$ & $\stackrel{m}{+}$ & \begin{tabular}{l} 
Na \\
\multirow{2}{*}{}
\end{tabular} & m. & $\bar{\omega}$ & \begin{tabular}{|l|} 
\\
$\stackrel{2}{5}$ \\
\end{tabular} & $\begin{array}{l}0 \\
\infty\end{array}$ & 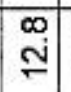 & $\stackrel{m}{q}$ & $\begin{array}{l}0 \\
\stackrel{0}{2} \\
\end{array}$ & $\underset{\infty}{\infty}$ & $\vec{m}$ & ¿্ণ & $\begin{array}{l}\infty \\
\infty \\
\infty\end{array}$ & O. & $\stackrel{+}{\stackrel{5}{2}}$ \\
\hline (\%2M) se|qqश्d & $\begin{array}{l}\infty \\
\stackrel{\infty}{\circ} \\
\sim\end{array}$ & $\stackrel{2}{\approx}$ & $\overline{\mathrm{g}}$ & $\begin{array}{l}\text { m } \\
\text { ô }\end{array}$ & 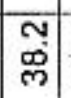 & $\stackrel{\square}{\mp}$ & $\begin{array}{l}\nabla \\
\text { in }\end{array}$ & $\begin{array}{l}\infty \\
\text { o } \\
\stackrel{N}{0}\end{array}$ & $\begin{array}{l}\infty \\
\infty \\
\infty\end{array}$ & $\begin{array}{l}\infty \\
0 \\
0\end{array}$ & $\begin{array}{c}N \\
\infty \\
\infty \\
m\end{array}$ & $\begin{array}{l}m \\
4 \\
L\end{array}$ & \begin{tabular}{l|} 
No \\
ஸ̃
\end{tabular} & $\bar{\sim}$ & \begin{tabular}{|c|} 
\\
$\tilde{N}$ \\
$\tilde{J}$
\end{tabular} & \begin{tabular}{|c|} 
\\
\end{tabular} & 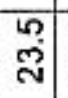 & $\begin{array}{l}6 \\
\text { స్ల } \\
\end{array}$ & $\begin{array}{l}0 \\
\\
\end{array}$ & $\begin{array}{l}m \\
\stackrel{m}{0} \\
\end{array}$ & $\begin{array}{l}\stackrel{ }{\mathrm{N}} \\
\stackrel{-}{2}\end{array}$ \\
\hline (\%1m) selqqәd asueo & $\begin{array}{l}\infty \\
\stackrel{\infty}{v}\end{array}$ & $\stackrel{m}{=}$ & 范 & กี & No & N & 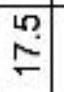 & 잉 & 品 & i & m & $\stackrel{\infty}{-}$ & $\begin{array}{l}0 \\
\\
\end{array}$ & 茎 & \begin{tabular}{|l|} 
\\
\\
\end{tabular} & \begin{tabular}{l|}
$\infty$ \\
$\sim$ \\
$\sim$
\end{tabular} & \begin{tabular}{l|} 
\\
0 \\
0
\end{tabular} & $\begin{array}{l}\text { ल) } \\
\text { m. }\end{array}$ & $\tilde{\sigma}$ & O잉 & ฟ่ \\
\hline 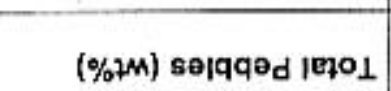 & N & $\begin{array}{l}\infty \\
\text { బల }\end{array}$ & $\begin{array}{l}N \\
y \\
0\end{array}$ & m & $\begin{array}{l}m \\
\tilde{g}\end{array}$ & 올 & 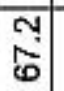 & $\begin{array}{l} \\
\\
\mathscr{v} \\
\dot{v}\end{array}$ & $\overline{5}$ & \begin{tabular}{l|} 
क \\
के
\end{tabular} & $\begin{array}{l}\infty \\
\infty \\
\infty \\
0\end{array}$ & $\overline{\mathrm{N}}$ & \begin{tabular}{l|}
0 \\
0 \\
$n$ \\
6
\end{tabular} & 岀 & \begin{tabular}{|l|}
0 \\
$m$ \\
8
\end{tabular} & $\frac{\omega}{\sigma}$ & $\begin{array}{l}n \\
\tilde{S}\end{array}$ & $\begin{array}{l}0 \\
0 \\
0 \\
0\end{array}$ & ָ̃ & $\begin{array}{l}m \\
\dot{m} \\
\stackrel{\sigma}{\sigma}\end{array}$ & $\begin{array}{l}\infty \\
\dot{q}\end{array}$ \\
\hline (แ⿰) чpdag Jamoา & 苋 & 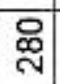 & $\stackrel{\infty}{\sim}$ & 足 & g & กิ & 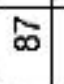 & $\bar{\sim}$ & 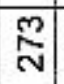 & $\frac{\infty}{\mathrm{v}}$ & $\bar{\varphi}$ & प्ञ & 文 & 8 & m & $\stackrel{N}{\bar{N}}$ & ळ & $\sigma$ & m్ & ? & 5 \\
\hline (wo) yłdea Jeddn & 器 & $\begin{array}{l}\stackrel{N}{\mathrm{~N}} \\
\stackrel{\mathrm{N}}{ }\end{array}$ & 0 & $\stackrel{m}{N}$ & ָี & 은 & 年 & $\stackrel{\infty}{\sim}$ & \begin{tabular}{l|} 
\\
$\stackrel{\infty}{N}$ \\
|
\end{tabular} & \begin{tabular}{|l|}
\multirow{2}{\infty}{} \\
$\stackrel{2}{\circ}$ \\
\end{tabular} & 要 & व & $\frac{m}{n}$ & 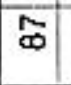 & $\bar{v}$ & $\begin{array}{l}\mathscr{2} \\
\stackrel{2}{2}\end{array}$ & $\bar{\varphi}$ & 0 & $\underset{N}{ }$ & M & $\stackrel{\infty}{\infty}$ \\
\hline & (⿻) & 总 & 0 & $\begin{array}{l}\text { 总 } \\
\text { ले }\end{array}$ & $\frac{\Upsilon}{\mathbf{m}}$ & $\begin{array}{l}\text { 잉 } \\
\text { 商 }\end{array}$ & $\begin{array}{l}\text { 辛 } \\
\text { 产 } \\
\text { N }\end{array}$ & $\underset{\mathbb{N}}{\stackrel{0}{0}}$ & 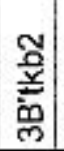 & 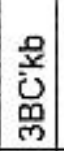 & 임 & 要 & 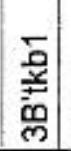 & 总 & 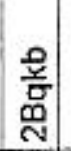 & 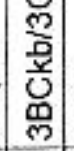 & 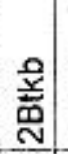 & $\frac{\pi}{2}$ & 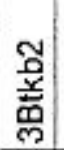 & $\begin{array}{l}\overline{\mathbf{s}} \\
\text { 仓 }\end{array}$ & $\begin{array}{l}\frac{0}{y} \\
\text { 产 } \\
\text { N }\end{array}$ \\
\hline apoว 미dues p|ㅣㅂ & 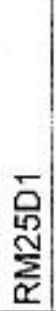 & 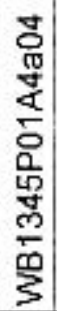 & 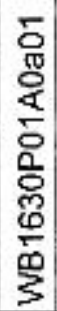 & 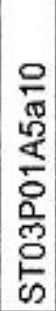 & $\begin{array}{l} \pm \\
0 \\
\mathbb{8} \\
\mathbb{8} \\
0 \\
0 \\
0 \\
0 \\
0 \\
0\end{array}$ & 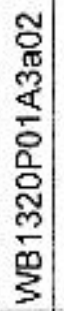 & 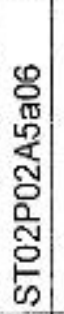 & 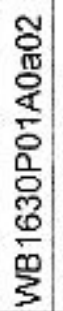 & 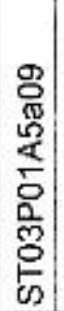 & 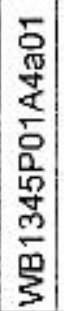 & 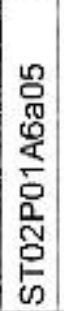 & 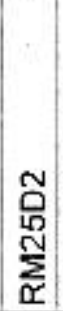 & $\begin{array}{l}0 \\
0 \\
0 \\
0 \\
5 \\
5 \\
0 \\
0 \\
0 \\
0 \\
0 \\
0\end{array}$ & 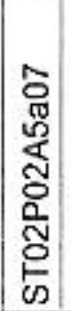 & $\begin{array}{l}0 \\
0 \\
0 \\
0 \\
0 \\
0 \\
0 \\
0 \\
0 \\
0 \\
0 \\
0 \\
3\end{array}$ & $\begin{array}{l} \\
5 \\
0 \\
0 \\
0 \\
\$ \\
0 \\
0 \\
0 \\
0 \\
0 \\
0 \\
0\end{array}$ & 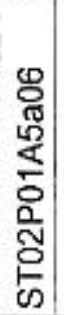 & 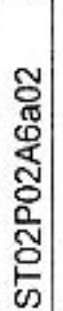 & 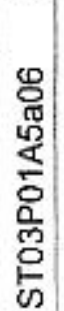 & 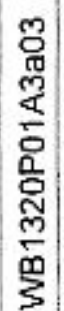 & 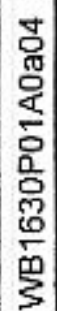 \\
\hline P|dwes & - & $N$ & $m$ & $\nabla$ & का & $\infty$ & $N$ & $\infty$ & क) & 은 & $F$ & $\stackrel{2}{2}$ & 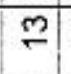 & $\square$ & $\because$ & 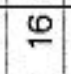 & $\approx$ & $\stackrel{\infty}{\sim}$ & 임 & 오 & $\bar{N}$ \\
\hline บээеg & 号 & 马े & gु & 京 & 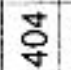 & $\stackrel{\nabla}{\nabla}$ & 守! & $\begin{array}{l}\vec{D} \\
\text { V }\end{array}$ & 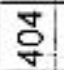 & $\begin{array}{l}\text { 巳 } \\
\text { g }\end{array}$ & gे & $\begin{array}{l}\text { J } \\
\text { f }\end{array}$ & g & 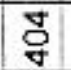 & ষ & 守 & 马 & 导 & 苛 & 导 & पे \\
\hline
\end{tabular}




\begin{tabular}{|c|c|c|c|c|c|c|c|c|c|c|c|c|c|c|c|c|c|c|c|c|c|}
\hline 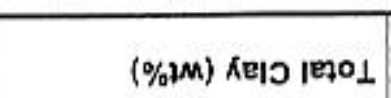 & $\vec{m}$ & \begin{tabular}{|l|} 
\\
\\
us
\end{tabular} & $\begin{array}{l}m \\
\text { is }\end{array}$ & $\begin{array}{l}\text { O } \\
\dot{v}\end{array}$ & $\begin{array}{l}\Phi \\
\end{array}$ & ल) & $\begin{array}{l} \\
\end{array}$ & \begin{tabular}{l|}
$\vec{m}$ \\
ले
\end{tabular} & ஸ્ & $\begin{array}{l}\dot{0} \\
\dot{v}\end{array}$ & $\begin{array}{l} \\
\text { Na }\end{array}$ & 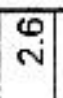 & $\stackrel{N}{M}$ & \begin{tabular}{l|}
0 \\
0 \\
0
\end{tabular} & \begin{tabular}{|l|} 
\\
\end{tabular} & \begin{tabular}{l|} 
\\
$\infty$
\end{tabular} & $\bar{i}$ & ले & (1) & \begin{tabular}{|l|} 
\\
\\
10
\end{tabular} & क \\
\hline (\%ұм) ग!S बu! & $\stackrel{8}{*}$ & \begin{tabular}{|l|} 
\\
$\stackrel{5}{2}$ \\
\end{tabular} & $\dot{v}$ & $\stackrel{m}{q}$ & $\begin{array}{l}0 \\
0\end{array}$ & m & ง & \begin{tabular}{l|l}
$\infty$ \\
$\infty$ \\
$\infty$
\end{tabular} & $\bar{m}$ & $\begin{array}{l}5 \\
6 \\
0\end{array}$ & $\vec{\nabla}$ & $\stackrel{m}{r}$ & $\stackrel{N}{N}$ & \begin{tabular}{|l|}
0 \\
10 \\
\end{tabular} & \begin{tabular}{l|} 
\\
\end{tabular} & \begin{tabular}{l|} 
\\
$\sigma^{\prime}$
\end{tabular} & $\begin{array}{l}m \\
\infty \\
\infty\end{array}$ & $\begin{array}{ll}\text { 이 } \\
\end{array}$ & $\begin{array}{l}\infty \\
\stackrel{\infty}{+}\end{array}$ & \begin{tabular}{|c|}
$m$ \\
$\infty$ \\
$\infty$
\end{tabular} & $\hat{m}$ \\
\hline (\%2M) มIIS Esuroว & $\begin{array}{l}\text { N̦ } \\
\text { o. }\end{array}$ & $\begin{array}{l}M \\
\infty\end{array}$ & $\bar{\sigma}^{\circ}$ & $\stackrel{m}{-}$ & $\stackrel{?}{=}$ & $\stackrel{\Delta}{N}$ & $\underset{N}{N}$ & $\begin{array}{l}\infty \\
\dot{\nabla}\end{array}$ & $\begin{array}{l}0 \\
\mathrm{i}\end{array}$ & $\begin{array}{l}0 \\
\dot{D} \\
\dot{Z}\end{array}$ & $\stackrel{\infty}{\infty}$ & $\begin{array}{l}\dot{0} \\
\oplus\end{array}$ & \begin{tabular}{l|} 
\\
\end{tabular} & $\begin{array}{l}N \\
m\end{array}$ & 웅 & \begin{tabular}{l|}
50 \\
60 \\
5
\end{tabular} & $\stackrel{\leftrightarrow}{\dot{v}}$ & $\overline{\mathrm{i}}$ & m & $\begin{array}{l}40 \\
20 \\
م\end{array}$ & in \\
\hline 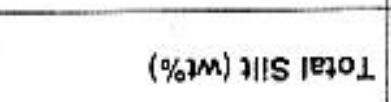 & $\begin{array}{l}\stackrel{\infty}{0} \\
\stackrel{0}{\sim}\end{array}$ & $\begin{array}{l}\text { N } \\
\text { o్ } \\
\text { o }\end{array}$ & $\begin{array}{l}0 \\
\dot{m} \\
\end{array}$ & $\begin{array}{l}\mathscr{6} \\
\omega 0\end{array}$ & $\stackrel{3}{5}$ & $\begin{array}{l}\infty \\
\dot{v}\end{array}$ & $\overline{5}$ & 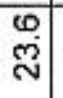 & 茫 & $\frac{\dot{N}}{\mathrm{~N}}$ & $\stackrel{9}{=}$ & गे & $\overline{0}$ & $\begin{array}{l}0 \\
\infty\end{array}$ & 옹 & $\begin{array}{c}0 \\
\text { d. } \\
\text { N }\end{array}$ & 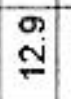 & $\stackrel{m}{=}$ & 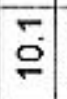 & \begin{tabular}{l|}
$\infty$ \\
্ָ
\end{tabular} & $\stackrel{\infty}{\infty}$ \\
\hline (\%वM) pues au!d Kü $\mathrm{A}$ & m. & $\begin{array}{l}\infty \\
\text { ○े }\end{array}$ & $\begin{array}{l}0 \\
0 \\
\infty \\
\leftarrow\end{array}$ & $\overline{\mathrm{Q}}$ & \begin{tabular}{l|}
$\infty$ \\
\\
\end{tabular} & $\stackrel{\mathscr{C}}{=}$ & 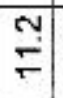 & $\stackrel{\oplus}{\grave{N}}$ & $\stackrel{m}{m}$ & $\vec{m}$ & $\begin{array}{l}\infty \\
\text { స్ }\end{array}$ & $\frac{m}{m}$ & $\begin{array}{c}m \\
\oplus \\
\oplus\end{array}$ & \begin{tabular}{l|}
$\mathbb{O}$ \\
$\stackrel{N}{=}$
\end{tabular} & \begin{tabular}{|l|} 
\\
0 \\
$\mathbb{N}$
\end{tabular} & \begin{tabular}{|c|}
0 \\
m.
\end{tabular} & $\stackrel{N}{N}$ & $\begin{array}{l}\sigma \\
g \\
g \\
\end{array}$ & \begin{tabular}{l|}
0 \\
이 \\
$\mathrm{N}$
\end{tabular} & \begin{tabular}{|l|}
$n$ \\
0 \\
0 \\
\end{tabular} & क \\
\hline$(\% 2 M)$ pues oult & $\begin{array}{l}\text { Na } \\
\text { के }\end{array}$ & $\underset{+}{+}$ & M & $\begin{array}{l}\infty \\
\dot{m} \\
\end{array}$ & $\stackrel{\varphi}{\sim}$ & $\begin{array}{l}m \\
m\end{array}$ & $\begin{array}{l}\infty \\
\text { ले }\end{array}$ & 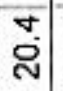 & $\begin{array}{l}0 \\
\text { 吕 } \\
\text { | }\end{array}$ & $\stackrel{m}{\sim}$ & m & $\tilde{N}$ & 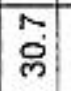 & $\begin{array}{l}\infty \\
\dot{p}\end{array}$ & 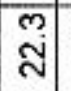 & $\begin{array}{l}40 \\
0 \\
0\end{array}$ & $\begin{array}{c}N \\
\text { og } \\
\text { N }\end{array}$ & $\frac{0}{\mathrm{~N}}$ & $\begin{array}{l}0 \\
\text { 吕 } \\
\text { | }\end{array}$ & 㐫 & $\stackrel{\infty}{\text { क }}$ \\
\hline (\%?M) pues un!pow & $\begin{array}{l}\infty \\
\dot{v}\end{array}$ & $\begin{array}{l}\sigma \\
\text { की }\end{array}$ & \begin{tabular}{l|} 
\\
\\
$\dot{v}$
\end{tabular} & \begin{tabular}{|l|}
$\infty$ \\
0 \\
0
\end{tabular} & $\begin{array}{l}0 \\
\infty \\
0\end{array}$ & $\overrightarrow{\mathrm{n}}$ & $\begin{array}{c}n \\
\stackrel{N}{*}\end{array}$ & $\stackrel{\oplus}{\sigma}$ & $\begin{array}{l}\infty \\
\infty \\
\infty\end{array}$ & $\bar{\varphi}$ & $\stackrel{\omega}{\stackrel{\omega}{N}}$ & $\begin{array}{l}m \\
\omega \\
\end{array}$ & 苍 & 옹 & \begin{tabular}{l|} 
\\
\\
\end{tabular} & \begin{tabular}{|c|}
$m$ \\
$\forall$
\end{tabular} & ĩ & $\stackrel{N}{r}$ & $\begin{array}{l}\infty \\
\sigma \\
\sigma\end{array}$ & $\vec{v}$ & $\stackrel{\mathbb{N}}{\stackrel{N}{N}}$ \\
\hline (\%2M) pues esueoJ & $\begin{array}{l} \\
\\
\end{array}$ & $\overline{6}$ & \begin{tabular}{c|}
5 \\
$\infty$
\end{tabular} & \begin{tabular}{l|}
$\infty$ \\
0
\end{tabular} & \begin{tabular}{l|}
$\infty$ \\
$\infty$ \\
$\infty$
\end{tabular} & $\underset{\sim}{\sim}$ & $\begin{array}{l}\text { Na } \\
\stackrel{N}{\sim}\end{array}$ & \begin{tabular}{l|}
$\sigma$ \\
$\sigma$ \\
$\sigma$
\end{tabular} & $\begin{array}{l}0 \\
\dot{q} \\
\mathbb{j}\end{array}$ & $\bar{\omega}$ & $\begin{array}{l}\stackrel{0}{\mathrm{~N}} \\
\mathrm{~N}\end{array}$ & si & $\stackrel{m}{=}$ & 웅 & \begin{tabular}{l|l|} 
\\
$\infty$ \\
$\infty$
\end{tabular} & \begin{tabular}{|c|} 
क \\
\end{tabular} & $\begin{array}{l}\infty \\
\infty\end{array}$ & $\begin{array}{l} \\
\infty\end{array}$ & \begin{tabular}{|l|} 
\\
0 \\
0
\end{tabular} & $\begin{array}{l}m \\
\forall\end{array}$ & $\stackrel{\oplus}{\sigma}$ \\
\hline$(\%$ MM) pues asaros $\mathrm{N} \theta \mathrm{A}$ & $\begin{array}{l}\text { क } \\
\text { ले }\end{array}$ & $\begin{array}{l}N \\
\infty\end{array}$ & $N$ & $\bar{N}$ & \begin{tabular}{l|}
0 \\
$\infty$ \\
\end{tabular} & $\bar{\sigma}$ & \begin{tabular}{l|}
9 \\
मn
\end{tabular} & $\stackrel{0}{=}$ & $\begin{array}{l}0 \\
\dot{0} \\
\mathbb{5}\end{array}$ & 只 & ָู & $\infty$ & \begin{tabular}{|l|}
\multicolumn{1}{|c|}{} \\
\end{tabular} & \begin{tabular}{l|}
0 \\
6
\end{tabular} & $\begin{array}{l}\infty \\
N\end{array}$ & \begin{tabular}{|c|}
$m$ \\
\end{tabular} & $\begin{array}{l}5 \\
6\end{array}$ & $\stackrel{5}{E}$ & \begin{tabular}{|l|}
0 \\
\end{tabular} & $\underset{\forall}{+}$ & $\begin{array}{l}\text { Ln } \\
0\end{array}$ \\
\hline (\%)M pues leqOL & 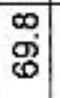 & $\begin{array}{l}4 \\
0 \\
6\end{array}$ & $\frac{5}{\infty}$ & \begin{tabular}{|l|} 
\\
\\
\\
$\infty$
\end{tabular} & $\begin{array}{l}N \\
S \\
S\end{array}$ & $\frac{\infty}{\sigma}$ & 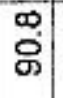 & $\begin{array}{l}0 \\
M \\
N\end{array}$ & $\frac{5}{\circ}$ & $\underset{N}{\mathbb{N}}$ & 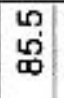 & $M$ & \begin{tabular}{|c|} 
\\
$\mathrm{d}$ \\
$\mathrm{\Phi}$
\end{tabular} & \begin{tabular}{|l|}
$\infty$ \\
\\
0 \\
\end{tabular} & $\mathrm{~N}$ & $\begin{array}{c}- \\
6 \\
\end{array}$ & $\begin{array}{l}0 \\
\text { N }\end{array}$ & $\begin{array}{l}40 \\
00 \\
1\end{array}$ & $\underset{\infty}{⿰}$ & 훙 & $\begin{array}{l}\mathscr{1} \\
\stackrel{\infty}{\infty}\end{array}$ \\
\hline 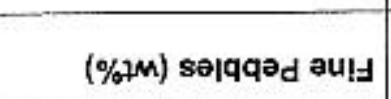 & $\check{N}$ & $\begin{array}{l}\infty \\
\dot{0} \\
\dot{v}\end{array}$ & 户े & $\begin{array}{l} \\
\end{array}$ & $\begin{array}{l}\mathcal{N} \\
\text { O }\end{array}$ & क. & $\begin{array}{l}0 \\
\infty\end{array}$ & $\stackrel{\infty}{=}$ & 今. & $\begin{array}{l}m \\
\infty \\
\infty\end{array}$ & 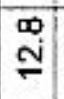 & $\stackrel{m}{\circ}$ & $\stackrel{9}{\sim}$ & 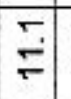 & \begin{tabular}{|l|l|}
$\sigma$ \\
$m$ \\
\end{tabular} & $N$ & $\begin{array}{l}3 \\
0\end{array}$ & ?ִ & \begin{tabular}{l|} 
\\
$\infty$ \\
$\infty$
\end{tabular} & N̦ & $\stackrel{\infty}{\stackrel{m}{2}}$ \\
\hline (\%łM) sejqqod wn!pew & $\stackrel{\infty}{N}$ & $\overrightarrow{\mathrm{m}}$ & \begin{tabular}{|l|}
$\infty$ \\
0 \\
\end{tabular} & $\begin{array}{l}N \\
\stackrel{N}{\longrightarrow}\end{array}$ & $\begin{array}{l}0 \\
\text { ले }\end{array}$ & $\begin{array}{l}\stackrel{N}{\oplus} \\
\stackrel{2}{*}\end{array}$ & ơ & में & $\begin{array}{l}\text { की } \\
\text { సิ }\end{array}$ & $\begin{array}{l}0 \\
\text { d }\end{array}$ & $\frac{7}{\dot{y}}$ & $\bar{m}$ & $\stackrel{m}{=}$ & $\vec{m}$ & 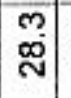 & $\begin{array}{l}0 \\
\dot{J}\end{array}$ & $\begin{array}{l}0 \\
\omega \\
N\end{array}$ & $\begin{array}{l}N \\
y\end{array}$ & $\stackrel{0}{\dot{v}}$ & $\begin{array}{ll}0 \\
0 \\
\end{array}$ & $\stackrel{\infty}{\infty}$ \\
\hline 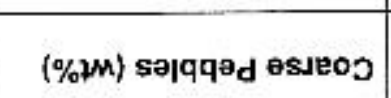 & 잉 & \begin{tabular}{|l|} 
\\
\\
\end{tabular} & \begin{tabular}{|l|} 
\\
\end{tabular} & 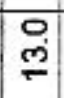 & $\begin{array}{l}\omega \\
\infty \\
\end{array}$ & $\bar{N}$ & $\stackrel{m}{-}$ & 궁 & $\stackrel{\sim}{\sim}$ & $\begin{array}{l} \\
\sigma \\
\sigma\end{array}$ & $\stackrel{m}{*}$ & क & \begin{tabular}{|l|}
0 \\
\end{tabular} & \begin{tabular}{l|}
$\mathrm{O}$ \\
$\mathrm{i}$
\end{tabular} & N & ช่ & $\begin{array}{l}\infty \\
m \\
m\end{array}$ & 今) & $\stackrel{?}{-}$ & $\stackrel{\omega}{\sim}$ & ○ \\
\hline 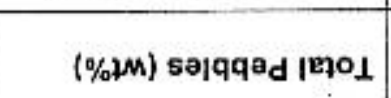 & $\begin{array}{l}0 \\
+ \\
+\end{array}$ & \begin{tabular}{|l|}
0 \\
5 \\
\end{tabular} & 용 & $\begin{array}{l}N \\
\text { ind }\end{array}$ & $\begin{array}{l}0 \\
\text { फ }\end{array}$ & लू & $\begin{array}{l}\infty \\
\infty \\
\infty\end{array}$ & $\begin{array}{l}0 \\
\stackrel{0}{0} \\
\end{array}$ & $\begin{array}{l}0 \\
0 \\
\dot{g}\end{array}$ & $\begin{array}{l}\dot{J} \\
\text { ฟ }\end{array}$ & 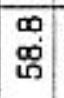 & $\begin{array}{l} \\
6 \\
\end{array}$ & \begin{tabular}{|l|}
\multirow{2}{*}{} \\
$\tilde{N}$
\end{tabular} & \begin{tabular}{|l|}
$\infty$ \\
0 \\
\end{tabular} & $\begin{array}{l}\dot{y} \\
\dot{y}\end{array}$ & ลे & m & $\begin{array}{l}N \\
0 \\
\end{array}$ & \begin{tabular}{l|}
$\infty$ \\
$\dot{J}$
\end{tabular} & @ì & ल) \\
\hline (uv) 47dag Jamo & m & $\underset{\mathfrak{N}}{\mathbb{N}}$ & ख्व & $\widehat{\omega}$ & N & 亮 & $\bar{\sigma}$ & 8 & $\bar{\sigma}$ & 은 & $\hat{m}$ & $\stackrel{\infty}{q}$ & $\widehat{\mathscr{N}}$ & 으 & $\frac{5}{4}$ & 요 & 号 & N & 覀 & $\infty$ & $\bar{m}$ \\
\hline (uv) updog seddn & 0 & ஐ & 政. & 5 & $\infty$ & $\stackrel{\infty}{\sim}$ & m & 文 & $\tilde{\omega}$ & m & $\bar{n}$ & ก & 胥 & 0 & m & $\infty$ & 5 & 운 & 要 & 0 & $\mathscr{8}$ \\
\hline & 4 & 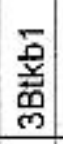 & 垔 & 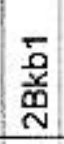 & 总 & ¿ & $\begin{array}{l}\text { O } \\
\text { 总 } \\
\end{array}$ & 兽 & 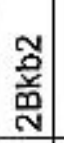 & 向 & $\begin{array}{l}\text { N } \\
\text { 盖 }\end{array}$ & 恙 & $\begin{array}{l}0 \\
\text { 盖 } \\
\end{array}$ & $\begin{array}{l}\overline{2} \\
\text { 盖 }\end{array}$ & $\begin{array}{l}\text { 羔 } \\
\text { 产 } \\
\end{array}$ & ळ & $\begin{array}{l}\text { 羙 } \\
\text { 商 }\end{array}$ & 兽 & 믇 & $\frac{\mathbb{Z}}{\mathbb{K}}$ & $\begin{array}{l}\text { 盖 } \\
\text { 惫 }\end{array}$ \\
\hline apoj ә әdwes pla!t & 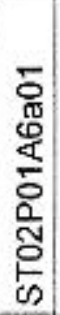 & 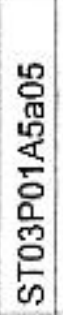 & 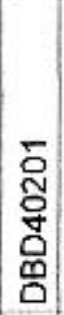 & 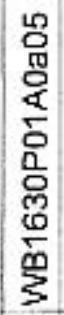 & 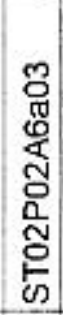 & 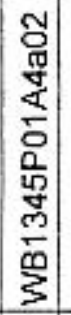 & 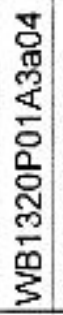 & 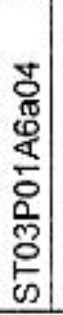 & 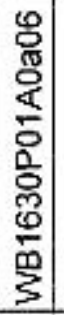 & 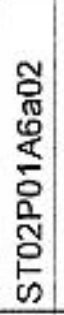 & 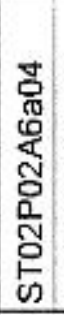 & 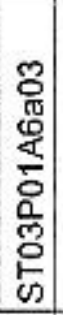 & 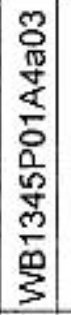 & 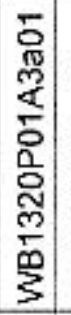 & 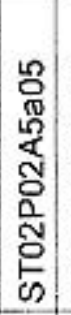 & $\begin{array}{l} \\
8 \\
0 \\
0 \\
8 \\
5 \\
0 \\
0 \\
8 \\
0 \\
0 \\
0\end{array}$ & $\begin{array}{l}0 \\
0 \\
0 \\
\vdots \\
5 \\
0 \\
0 \\
0 \\
0 \\
0 \\
\vdots \\
\vdots\end{array}$ & 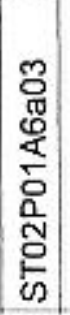 & m & 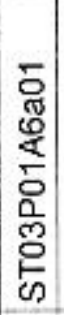 & 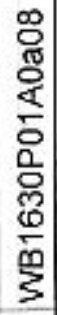 \\
\hline apdues & N & $\stackrel{N}{N}$ & N & $\stackrel{n}{2}$ & $\ddot{d}$ & กิ & $\stackrel{\infty}{\sim}$ & g & প্ & $\bar{m}$ & స్ల & m & ले & 奌 & $\ddot{~}$ & $m$ & $\infty$ & $\mathrm{m}$ & 육 & $\overline{7}$ & T \\
\hline чगีЕ & ț & ষ্ & S & $\underset{V}{O}$ & ঠ্ & 守 & ষ̀ & 守 & 守 & 守 & 导 & 寸 & 声 & 导 & 吾 & g & 巳 & 巳 & 巳 & ষ & ষ \\
\hline
\end{tabular}




\begin{tabular}{|c|c|c|c|c|c|c|c|c|c|c|c|c|c|c|c|c|c|c|c|c|c|}
\hline (\%〉M) KEㅣㅣ |E‡OL & $\begin{array}{l}\mathscr{6} \\
\qquad\end{array}$ & $\begin{array}{l}\mathscr{Q} \\
\dot{\nabla}\end{array}$ & $\begin{array}{l}\text { का } \\
\text { फ่ }\end{array}$ & $\begin{array}{l} \\
0\end{array}$ & $\begin{array}{l}\infty \\
\mathbf{r}\end{array}$ & लె & $\overline{\omega^{\circ}}$ & \begin{tabular}{l|l|} 
\\
1
\end{tabular} & \begin{tabular}{|l|}
$\infty$ \\
ஸे
\end{tabular} & $\begin{array}{l}8 \\
\end{array}$ & $\begin{array}{l}m \\
\dot{y}\end{array}$ & \begin{tabular}{|c|} 
\\
\end{tabular} & $\begin{array}{l}3 \\
6\end{array}$ & Nֵ & i & $\begin{array}{c}\mathrm{N} \\
\text { ल }\end{array}$ & \begin{tabular}{|l|l|} 
ज \\
\end{tabular} & $\underset{y}{N}$ & $\begin{array}{l}0 \\
10\end{array}$ & $\bar{\sigma}$ & \begin{tabular}{|l|} 
\\
0 \\
\end{tabular} \\
\hline (\%) & $\underset{v}{V}$ & $\stackrel{M}{N}$ & $\begin{array}{l}\nabla \\
\dot{v}\end{array}$ & $\begin{array}{l}\text { O } \\
\dot{v}\end{array}$ & $\begin{array}{l}\varphi \\
\omega\end{array}$ & $\stackrel{\leftrightarrow}{\circ}$ & $\begin{array}{l}4 \\
m\end{array}$ & $\begin{array}{l}\infty \\
m \\
\end{array}$ & $\ddot{\nabla}$ & $\vec{m}$ & M & ㅁ. & 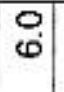 & $\begin{array}{l}0 \\
0 \\
0\end{array}$ & \begin{tabular}{l|} 
\\
\end{tabular} & $\begin{array}{l}\infty \\
\sim\end{array}$ & $\stackrel{0}{-}$ & $\begin{array}{l}\sigma \\
\text { q }\end{array}$ & $\bar{c}$ & $\stackrel{ }{\circ}$ & $\underset{\nabla}{\sim}$ \\
\hline$(\% 2 M)$ aIIS әsueOO & $\underset{\infty}{ }$ & $\stackrel{\varphi}{\oplus}$ & $\begin{array}{l}m \\
0\end{array}$ & $\mathrm{i}$ & 잉 & 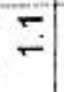 & $\begin{array}{l}9 \\
\text { ले }\end{array}$ & $\bar{i}$ & \begin{tabular}{|l|}
$\mathbb{N}$ \\
Li
\end{tabular} & \begin{tabular}{|l|} 
\\
Li⿱
\end{tabular} & $\begin{array}{l}7 \\
\text { Lí }\end{array}$ & \begin{tabular}{|l|} 
\\
0 \\
$N$
\end{tabular} & $\stackrel{m}{\mathrm{~N}}$ & $\begin{array}{l}\varphi \\
\dot{8}\end{array}$ & $\begin{array}{c}\sim \\
\mathrm{v}\end{array}$ & $\begin{array}{c}\mathscr{N} \\
\mathrm{N}\end{array}$ & $\stackrel{m}{-}$ & 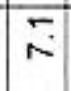 & $\begin{array}{l}\omega \\
\omega\end{array}$ & $\stackrel{m}{+}$ & No \\
\hline 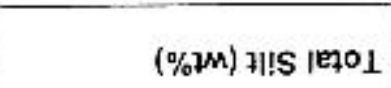 & $\begin{array}{l}\infty \\
\stackrel{0}{0}\end{array}$ & $\ddot{\varphi}$ & $\hat{\circ}$ & $\hat{\omega}$ & $\begin{array}{l}\omega^{\prime} \\
\end{array}$ & 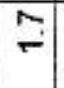 & $\begin{array}{l} \\
N\end{array}$ & \begin{tabular}{|c|}
$\begin{array}{c}0 \\
\text { no }\end{array}$ \\
\end{tabular} & \begin{tabular}{|l|}
$\Phi$ \\
6 \\
\end{tabular} & $\begin{array}{l}\infty \\
\infty\end{array}$ & $\begin{array}{l}\mathrm{N} \\
\mathrm{N}\end{array}$ & $\begin{array}{l}0 \\
\end{array}$ & $\begin{array}{l}3 \\
\infty\end{array}$ & $\begin{array}{l}\mathscr{O} \\
\Phi\end{array}$ & 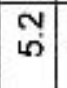 & $\begin{array}{l} \\
5 \\
50\end{array}$ & $\begin{array}{l}N \\
\text { m }\end{array}$ & $\begin{array}{l}\text { 어 } \\
\text { ㄱ. }\end{array}$ & $\stackrel{\oplus}{\stackrel{N}{\sim}}$ & $\stackrel{n}{I}$ & बे \\
\hline (\%ఖM) pues au!y אUA & $\overline{\mathrm{N}}$ & $\stackrel{m}{\stackrel{2}{2}}$ & $\stackrel{\circ}{\circ}$ & $\stackrel{N}{\sim}$ & $\begin{array}{l}\infty \\
\infty \\
\leftarrow\end{array}$ & $\stackrel{2}{0}$ & $\begin{array}{l}0 \\
\oplus \\
\varrho\end{array}$ & \begin{tabular}{|l|} 
\\
$\dot{\sigma}$ \\
\end{tabular} & \begin{tabular}{|l|}
$\mathrm{v}$ \\
$\mathrm{N}$ \\
$\mathrm{N}$
\end{tabular} & 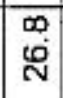 & $\begin{array}{l}\infty \\
\infty \\
\infty\end{array}$ & $\begin{array}{c}0 \\
\sigma \\
\end{array}$ & $\begin{array}{l}0 \\
\dot{\square}\end{array}$ & $\begin{array}{l}\varphi \\
\omega \\
\omega\end{array}$ & $\begin{array}{l}\oplus \\
\bullet \\
\bullet\end{array}$ & 高 & $\underset{\sim}{\sim}$ & $\begin{array}{c} \\
\stackrel{0}{ } \\
\sim\end{array}$ & $\begin{array}{l}\dot{v} \\
\dot{N}\end{array}$ & $\stackrel{\infty}{\sim}$ & $\begin{array}{l}\mathscr{\omega} \\
\stackrel{\mathscr{N}}{ }\end{array}$ \\
\hline (\%1M) pues au! & స్లె & $\begin{array}{l}\infty \\
\text { g్ } \\
\text { d. }\end{array}$ & ल) & के & $\begin{array}{l}m \\
\text { m }\end{array}$ & $\begin{array}{l}\mathscr{\omega} \\
\stackrel{N}{N}\end{array}$ & \begin{tabular}{|l|}
$\infty$ \\
N \\
\end{tabular} & 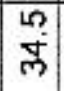 & $\begin{array}{ll}m \\
m \\
m\end{array}$ & $\begin{array}{l}\infty \\
\text { Nj } \\
\text { na }\end{array}$ & \begin{tabular}{l|} 
m \\
o্ं
\end{tabular} & $\begin{array}{l}N \\
m \\
m\end{array}$ & \begin{tabular}{l|} 
क्ष \\
న్స
\end{tabular} & $\begin{array}{l}\mathrm{N} \\
\mathrm{N}\end{array}$ & \begin{tabular}{l|}
$\infty$ \\
我 \\
$m$
\end{tabular} & $\begin{array}{l}\text { च. } \\
\text { m. }\end{array}$ & $\begin{array}{c}0 \\
\mathscr{N} \\
\stackrel{N}{ }\end{array}$ & \begin{tabular}{|l|}
\multirow{2}{*}{} \\
हिं
\end{tabular} & 灾 & ले & $\begin{array}{l}\mathscr{\varphi} \\
\stackrel{0}{0}\end{array}$ \\
\hline (\%qM) pues wn!pew & $\begin{array}{l}\forall \\
\circ\end{array}$ & $\stackrel{m}{m}$ & $\begin{array}{l}0 \\
\dot{r} \\
\end{array}$ & \begin{tabular}{l|} 
\\
0 \\
0
\end{tabular} & $\begin{array}{l}\text { क } \\
\dot{+}\end{array}$ & స్. & $\begin{array}{l}\forall \\
6 \\
6\end{array}$ & $\begin{array}{l}\forall \\
6\end{array}$ & 웅 & $\stackrel{N}{N}$ & $\begin{array}{l}N \\
\stackrel{N}{q}\end{array}$ & $=$ & $\stackrel{0}{-}$ & $\begin{array}{l}\text { क् } \\
\text { Oे }\end{array}$ & m.ं & $\begin{array}{l} \pm \\
F\end{array}$ & \begin{tabular}{|l|}
$\infty$ \\
లై \\
\end{tabular} & 뭉 & $\underset{\leftarrow}{\stackrel{\infty}{\leftarrow}}$ & $\stackrel{\vec{m}}{\sim}$ & $\stackrel{m}{\circ}$ \\
\hline (\%)M pues esueos & $\bar{\infty}$ & $\stackrel{\vec{m}}{\stackrel{m}{\sim}}$ & 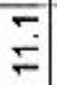 & के & $\stackrel{m}{\infty}$ & $\begin{array}{l}\text { N̦ } \\
\text { స్ }\end{array}$ & \begin{tabular}{l}
$\infty$ \\
$\stackrel{\infty}{\sim}$ \\
\multirow{2}{*}{}
\end{tabular} & \begin{tabular}{|l|}
$\infty$ \\
$\sigma^{\prime}$
\end{tabular} & \begin{tabular}{|l|} 
\\
$\omega$ \\
0
\end{tabular} & $\begin{array}{l}\nabla \\
\infty\end{array}$ & $\stackrel{\circ}{\circ}$ & $\mathrm{O}$ & $\stackrel{\mathbb{v}}{\mathrm{N}}$ & స్ & $\stackrel{\omega}{=}$ & \begin{tabular}{|l|} 
\\
$\mathscr{\sigma}$ \\
\end{tabular} & $\check{\emptyset}$ & $\begin{array}{c}0 \\
\infty\end{array}$ & के & $\stackrel{N}{\infty}$ & $\stackrel{\bullet}{\oplus}$ \\
\hline (\% \%M) pues asueoJ NüA & $\begin{array}{l}\text { की } \\
40^{\circ}\end{array}$ & $\stackrel{\sim}{\text { m }}$ & $\begin{array}{l}\nabla \\
\infty\end{array}$ & $\bar{N}$ & $\begin{array}{l}\circ \\
0\end{array}$ & $\begin{array}{l}0 \\
0 \\
0\end{array}$ & $\underset{\infty}{\infty}$ & \begin{tabular}{|l|}
0 \\
0
\end{tabular} & $\begin{array}{l}\sigma \\
6\end{array}$ & $N_{\infty}^{\circ}$ & 믐 & 둥 & $\bar{m}$ & $\begin{array}{l}0 \\
\\
\end{array}$ & $\bar{\infty}$ & \begin{tabular}{|l|}
0 \\
0 \\
\end{tabular} & \begin{tabular}{|c|}
$m$ \\
$\stackrel{m}{c}$
\end{tabular} & $\begin{array}{l}0 \\
\infty\end{array}$ & \begin{tabular}{|c|} 
\\
$\infty$
\end{tabular} & $\underset{\forall}{\forall}$ & $\begin{array}{l}0 \\
+\end{array}$ \\
\hline 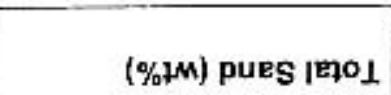 & $\begin{array}{l}40 \\
\infty \\
\infty\end{array}$ & $\overline{\sigma_{0}}$ & $\begin{array}{l} \pm \\
\infty\end{array}$ & \begin{tabular}{l|}
$\Phi$ \\
$\mathscr{\infty}$ \\
$\Phi$
\end{tabular} & $\begin{array}{l}\varphi \\
\stackrel{\aleph}{\infty}\end{array}$ & Гூ⿱㇒㠯 & \begin{tabular}{|l|}
0 \\
$\infty$ \\
$\infty$
\end{tabular} & \begin{tabular}{|l|} 
\\
$\dot{\infty}$ \\
$\dot{\infty}$
\end{tabular} & 莕 & $\begin{array}{l}0 \\
0 \\
\infty\end{array}$ & $\begin{array}{l}0 \\
M \\
M\end{array}$ & \begin{tabular}{|l|}
\multicolumn{2}{|c|}{} \\
$\mathrm{S}$
\end{tabular} & 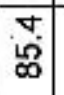 & 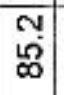 & ภु & $\frac{\square}{\sigma}$ & \begin{tabular}{|l|}
$\sigma$ \\
$\tilde{\beta}$ \\
\end{tabular} & \begin{tabular}{|c|}
$\infty$ \\
$\prod^{\infty}$ \\
$\infty$
\end{tabular} & 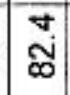 & $\stackrel{9}{6}$ & 只 \\
\hline$(\%$ (M) so|qqә & $\begin{array}{l}0 \\
\sigma^{\circ}\end{array}$ & $\begin{array}{l}\dot{J} \\
\underset{+}{*}\end{array}$ & $\begin{array}{l}0 \\
m\end{array}$ & $\begin{array}{l}\mathrm{m} \\
0 \\
0\end{array}$ & $\begin{array}{l}\nabla \\
\stackrel{m}{*}\end{array}$ & $\stackrel{\nabla}{=}$ & $\underset{\square}{\square}$ & \begin{tabular}{|c|}
$v$ \\
ind \\
2
\end{tabular} & $=$ & $\begin{array}{l}10 \\
0 \\
\end{array}$ & $\begin{array}{l}N \\
\mathscr{Q}\end{array}$ & 品 & $\begin{array}{l}M \\
\infty\end{array}$ & $=$ & $\begin{array}{l}0 \\
0 \\
0\end{array}$ & $\begin{array}{l}n \\
\infty\end{array}$ & $\overline{\check{\mathrm{i}}}$ & $\begin{array}{l}\mathrm{m} \\
\stackrel{N}{2}\end{array}$ & $\stackrel{m}{=}$ & $\begin{array}{l}\sigma \\
\sigma \\
\end{array}$ & $\mathbb{N}$ \\
\hline (\%१M) sə|qqәd un|pəw & $\begin{array}{l}\infty \\
i \\
\sim\end{array}$ & $\begin{array}{c}N \\
\text { o }\end{array}$ & $\stackrel{m}{\infty}$ & $\begin{array}{l}\mathscr{0} \\
\square \\
\square\end{array}$ & $\begin{array}{l}0 \\
\pm \\
\square\end{array}$ & \begin{tabular}{l|}
$\infty$ \\
$\infty$ \\
$\infty$
\end{tabular} & \begin{tabular}{|l|}
$\infty$ \\
融
\end{tabular} & \begin{tabular}{|l|}
$\infty$ \\
c่
\end{tabular} & $\begin{array}{l} \\
\text { g্j } \\
\text { N }\end{array}$ & $\overline{\mathrm{d}}$ & $\begin{array}{l}\mathbf{N} \\
\text { N }\end{array}$ & $\begin{array}{l}0 \\
\ddot{\omega} \\
\sim\end{array}$ & 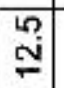 & $\begin{array}{l}\omega \\
\stackrel{0}{=}\end{array}$ & $\underset{\infty}{\infty}$ & $\begin{array}{ll} \\
= \\
\end{array}$ & \begin{tabular}{|l|}
$m$ \\
$\stackrel{m}{\sigma}$ \\
$\stackrel{0}{*}$
\end{tabular} & $\begin{array}{l}0 \\
5 \\
5\end{array}$ & $\begin{array}{l}\dot{0} \\
\dot{T}\end{array}$ & I & $\hat{\mathrm{i}}$ \\
\hline 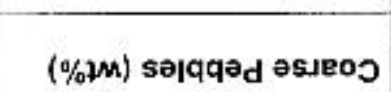 & $\stackrel{N}{N}$ & $\stackrel{\infty}{=}$ & 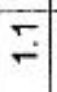 & गे & $\stackrel{0}{-}$ & $\begin{array}{l}\text { के } \\
\text { m. }\end{array}$ & $\stackrel{\nabla}{-}$ & 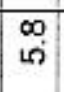 & NN & $\check{\sigma}$ & $\stackrel{4}{-}$ & $N$ & $\stackrel{\infty}{-}$ & ले & $\stackrel{M}{\stackrel{M}{*}}$ & \begin{tabular}{|l|} 
\\
\\
\end{tabular} & $\stackrel{m}{+}$ & $\stackrel{\varphi}{-}$ & 今. & Dे & $\stackrel{\leftrightarrow}{\longrightarrow}$ \\
\hline 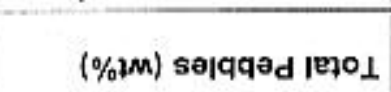 & $\begin{array}{l}\text { क) } \\
\text { g్ }\end{array}$ & $\begin{array}{l}\infty \\
\text { ก }\end{array}$ & $\begin{array}{l}\text { v } \\
\text { di }\end{array}$ & लू & $\begin{array}{l}0 \\
\text { g. } \\
\text { d. }\end{array}$ & $\overline{\mathrm{N}}$ & \begin{tabular}{|l|}
$m$ \\
$m$ \\
min
\end{tabular} & $\begin{array}{l}\infty \\
\\
\end{array}$ & $\begin{array}{l}N \\
g \\
g\end{array}$ & ले & \begin{tabular}{|c|} 
क \\
क्ల
\end{tabular} & \begin{tabular}{|l|}
$n$ \\
$m$ \\
$m$
\end{tabular} & 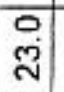 & $\begin{array}{l}\text { D } \\
\text { Dू }\end{array}$ & $\begin{array}{l}m \\
0 \\
0\end{array}$ & $\begin{array}{l}\text { क } \\
\dot{N}\end{array}$ & $\overrightarrow{\mathrm{m}}$ & के & $\begin{array}{l}\nabla \\
\stackrel{\mathbb{N}}{ }\end{array}$ & $\vec{m}$ & $\stackrel{\infty}{\mathrm{N}}$ \\
\hline (uiv) पldәg sемоך & $\infty$ & $\underset{\sim}{\mathbb{N}}$ & छे & 员 & लू & $\frac{50}{\infty}$ & 뭄 & F & 塄 & $\ddot{N}$ & $\mathscr{1}$ & 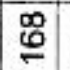 & ָ̃ & n) & $\stackrel{\mathscr{\infty}}{\boldsymbol{\infty}}$ & 인 & $\underset{\infty}{\infty}$ & ? & 8 & क & กี \\
\hline 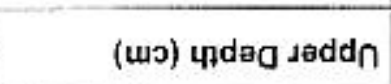 & 0 & $\$$ & $\varphi$ & $\stackrel{\mathbf{N}}{\mathrm{N}}$ & $\stackrel{\sigma}{=}$ & 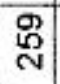 & फ & $\underset{N}{N}$ & 骂 & $\infty$ & 迥 & న్ & : & 0 & 웅 & $\stackrel{\mathcal{Y}}{\mathrm{V}}$ & $\stackrel{\infty}{\oplus}$ & $\stackrel{?}{N}$ & 9 & $\bar{\infty}$ & कo \\
\hline & 㐫 & 总 & 商 & $\begin{array}{l}\text { 总 } \\
\text { 总 }\end{array}$ & $\begin{array}{l}\text { 总 } \\
\text { 恖 }\end{array}$ & 总 & $\begin{array}{l}\bar{y} \\
\end{array}$ & $\begin{array}{l}\text { 音 } \\
\text { ㄱ }\end{array}$ & पू & 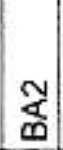 & $\mid \begin{array}{l}\mathscr{Y} \\
\text { J্ল } \\
\end{array}$ & $\begin{array}{l}\text { 辛 } \\
\text { 只 } \\
\text { | }\end{array}$ & 善 & 灾 & $\frac{\text { กิ }}{\frac{0}{\mathbf{~}}}$ & $\begin{array}{l}\frac{9}{\mathbf{n}} \\
\text { 商 }\end{array}$ & U & 咅 & $\overline{\breve{~}}$ & 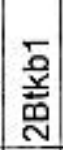 & ષิ \\
\hline 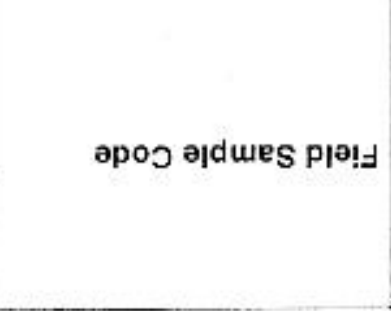 & 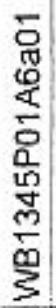 & 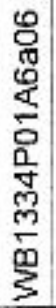 & 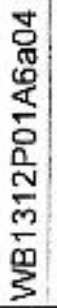 & 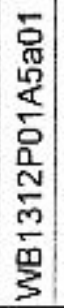 & 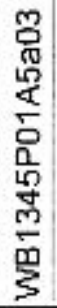 & 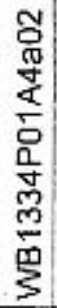 & 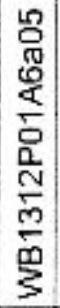 & 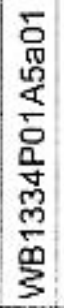 & 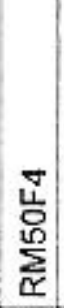 & 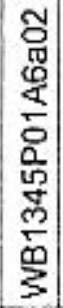 & 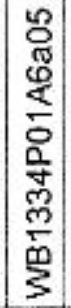 & 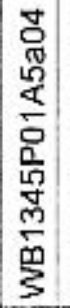 & $\begin{array}{l}\frac{5}{0} \\
\frac{0}{0} \\
\vdots \\
\dot{0} \\
\frac{0}{N} \\
\frac{m}{m} \\
\frac{m}{3}\end{array}$ & $\begin{array}{l}\frac{5}{0} \\
\frac{0}{0} \\
\frac{0}{0} \\
\frac{0}{n} \\
\frac{\operatorname{m}}{3} \\
\frac{1}{3}\end{array}$ & 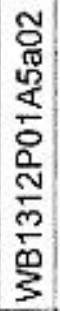 & 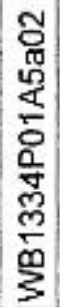 & 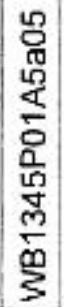 & 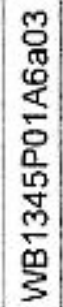 & 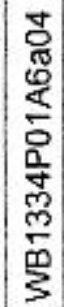 & 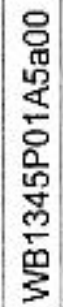 & 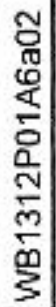 \\
\hline әdшеS & $r$ & $\mathrm{~N}$ & $m$ & $\theta$ & $\omega$ & $\omega$ & 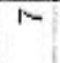 & $\infty$ & क & 으 & $=$ & $\cong$ & m & $\underset{Z}{Z}$ & $\stackrel{42}{\longrightarrow}$ & 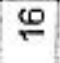 & $\approx$ & $\stackrel{\infty}{\leftarrow}$ & क & 모 & $\bar{v}$ \\
\hline чээеg & 号 & 号 & 号 & 웅 & 骂 & 岁 & 号 & 용 & 놈 & \& & 号 & 骂 & 号 & 号 & 号 & 용 & 용 & 总 & 总 & 욤 & 용 \\
\hline
\end{tabular}




\begin{tabular}{|c|c|c|c|c|c|c|c|c|c|c|c|c|c|c|c|c|}
\hline$(\% \not M)$ REIO & r & $\begin{array}{l}0 \\
\end{array}$ & $\begin{array}{ll}\infty \\
\dot{m}\end{array}$ & $\begin{array}{l}N \\
\dot{v}\end{array}$ & \begin{tabular}{l|}
$m$ \\
$\infty$ \\
$\infty$
\end{tabular} & \begin{tabular}{|l|}
0 \\
i⿱
\end{tabular} & \begin{tabular}{|l|}
$m$ \\
$n^{\circ}$
\end{tabular} & लू & 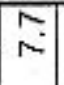 & \begin{tabular}{|l|}
8 \\
40
\end{tabular} & \begin{tabular}{l|}
$\infty$ \\
$i v$ \\
\end{tabular} & $\begin{array}{c}N \\
0\end{array}$ & \begin{tabular}{l|}
0 \\
$\dot{v}$
\end{tabular} & $\dot{\varphi}$ & \begin{tabular}{|l|}
0 \\
40
\end{tabular} & $\begin{array}{l}m \\
m\end{array}$ \\
\hline (\%1M) M!S әu! & लें & $\underset{\forall}{\tilde{y}}$ & $\stackrel{+}{-}$ & $\begin{array}{l}m \\
\varphi\end{array}$ & $\begin{array}{l}0 \\
\dot{\nabla}\end{array}$ & \begin{tabular}{|l|}
0 \\
$\dot{\forall}$
\end{tabular} & \begin{tabular}{|l|}
$g$ \\
$\dot{q}$
\end{tabular} & $\begin{array}{l}\text { Ni } \\
\text { I }\end{array}$ & $\begin{array}{ll}m \\
m\end{array}$ & $\underset{\forall}{\forall}$ & $\stackrel{\circ}{-}$ & \begin{tabular}{l|} 
\\
m
\end{tabular} & \begin{tabular}{|l|}
0 \\
$\infty$ \\
0
\end{tabular} & $\stackrel{N}{N}$ & $\stackrel{D}{\mathrm{~N}}$ & $\stackrel{20}{-}$ \\
\hline$(\%$ \% ) J!S EsJeOJ & $\stackrel{\nabla}{\mathrm{N}}$ & 6 & क्र & $\begin{array}{l}\nabla \\
\infty\end{array}$ & $\begin{array}{ll} \\
\end{array}$ & 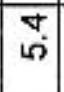 & \begin{tabular}{|l|}
0 \\
6
\end{tabular} & 임 & $m$ & \begin{tabular}{|l|}
$N$ \\
$\omega$
\end{tabular} & $\begin{array}{l}\varphi \\
0\end{array}$ & 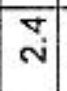 & \begin{tabular}{|l|}
$\oplus$ \\
$\mathbf{N}$
\end{tabular} & $\begin{array}{l}\omega \\
\infty\end{array}$ & ñ & i \\
\hline (\%)M ) MIS IEOL & $\begin{array}{l}\infty \\
\infty\end{array}$ & $\hat{\sigma}$ & $\hat{\mathrm{N}}$ & 方 & \begin{tabular}{|l|}
0 \\
\end{tabular} & \begin{tabular}{|l|}
3 \\
$\sigma$
\end{tabular} & \begin{tabular}{|l|} 
\\
0 \\
$j$
\end{tabular} & $\dot{\nabla}$ & $\stackrel{i}{i}$ & 吕 & $\stackrel{\oplus}{\circ}$ & $\begin{array}{l}\nabla \\
0\end{array}$ & $\begin{array}{l}0 \\
0\end{array}$ & $\begin{array}{ll}\infty \\
\substack{\infty\\
}\end{array}$ & กิ & कि \\
\hline (\%ఖM) pues әu!g KeA & $\stackrel{r}{\oplus}$ & $\stackrel{3}{\sim}$ & $\bar{N}$ & $\frac{m}{\mathrm{~N}}$ & $\stackrel{m}{N}$ & $\begin{array}{l}\infty \\
\mathbb{N} \\
\mathbb{N}\end{array}$ & $\stackrel{\mathrm{N}}{\mathrm{N}}$ & $\underset{\mathrm{N}}{\mathrm{N}}$ & $\begin{array}{l}\sigma \\
\dot{\varphi}\end{array}$ & 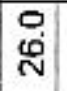 & ¿ & \begin{tabular}{l|}
$\varphi$ \\
$\mathscr{\sigma}$ \\
\end{tabular} & \begin{tabular}{|l|l|}
0 \\
\end{tabular} & $\begin{array}{l}M \\
\infty \\
\infty \\
N\end{array}$ & 号 & ले \\
\hline$(\% 2 M)$ pues ault & $\bar{g}$ & $\begin{array}{l}N \\
\text { on } \\
\text { N }\end{array}$ & 孞 & $\begin{array}{l}m \\
\text { m } \\
\text { (n) }\end{array}$ & \begin{tabular}{l|}
$\mathrm{N}$ \\
$\mathrm{N}$ \\
$\mathrm{N}$
\end{tabular} & 总 & $\begin{array}{l}\text { फ़ } \\
\text { m. }\end{array}$ & 商 & ஸ్ల & $\begin{array}{c}N \\
\text { D } \\
\text { లn }\end{array}$ & $\mid \begin{array}{c}1 \\
\text { ñ } \\
\text { | }\end{array}$ & $\begin{array}{l}m \\
\dot{m}\end{array}$ & i & 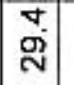 & 空 & $\tilde{\infty}$ \\
\hline (\%ұM) pues un!pew & 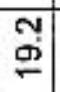 & 근 & 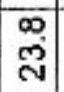 & $\overline{\mathrm{i}}$ & $\begin{array}{l}\mathrm{Ln} \\
\mathrm{N} \\
\mathrm{N}\end{array}$ & $\begin{array}{l}\infty \\
\stackrel{\infty}{\sim}\end{array}$ & 응 & N̦ & $\begin{array}{l}0 \\
\dot{v}\end{array}$ & $\begin{array}{l}0 \\
0 \\
0\end{array}$ & $\left|\begin{array}{l}0 \\
\infty \\
\sim\end{array}\right|$ & $\hat{N}$ & $\stackrel{\leftarrow}{\leftarrow}$ & $\begin{array}{l}m \\
\infty \\
\infty\end{array}$ & 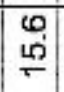 & ָั \\
\hline (\%2M) pues esjeop & $\stackrel{\varphi}{\circ}$ & $\stackrel{0}{\sim}$ & 蒙 & $=$ & $\frac{N}{N}$ & $\stackrel{N}{N}$ & $M$ & $\begin{array}{l}5 \\
\frac{10}{2}\end{array}$ & $\begin{array}{l}\text { के } \\
\text { के }\end{array}$ & के & $\begin{array}{l}\text { प } \\
\text { D }\end{array}$ & $\stackrel{5}{=}$ & \begin{tabular}{|l|}
0 \\
0 \\
\end{tabular} & \begin{tabular}{l|}
$\nabla$ \\
0
\end{tabular} & 웅 & $\begin{array}{l}\text { : } \\
\text { cे }\end{array}$ \\
\hline (\%1M) pues asueo J KaA & 웅 & $\stackrel{\mathrm{i}}{\mathrm{V}}$ & $\begin{array}{l}\sigma \\
\dot{\omega}\end{array}$ & $\stackrel{N}{=}$ & w & $\begin{array}{l}n \\
\infty\end{array}$ & 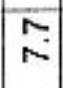 & $\underset{\sigma}{N}$ & $\underset{\oplus}{*}$ & ○ & $\underset{\sim i}{\sigma}$ & $\dot{m}$ & $\bar{N}$ & $\begin{array}{ll}m \\
\end{array}$ & $\begin{array}{ll}\infty \\
n \\
n\end{array}$ & $\stackrel{0}{2}$ \\
\hline (\%) pues ImoL & \begin{tabular}{l|}
0 \\
9 \\
9 \\
\end{tabular} & \begin{tabular}{l|}
$\infty$ \\
$\infty$ \\
$\infty$
\end{tabular} & \begin{tabular}{|l|}
0 \\
8 \\
$\alpha$
\end{tabular} & $\frac{5}{\infty}$ & \% & \begin{tabular}{|l|} 
\\
m \\
$\infty$ \\
$\infty$
\end{tabular} & \begin{tabular}{l|}
$\infty$ \\
$\dot{\infty}$ \\
\end{tabular} & \begin{tabular}{l}
\multirow{d}{*}{} \\
๙ે
\end{tabular} & $\begin{array}{l}0 \\
0 \\
2 \\
2\end{array}$ & $\bar{\infty}$ & \begin{tabular}{|c|}
$\infty$ \\
$\sigma$ \\
$\sigma$
\end{tabular} & 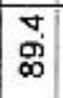 & 옹 & $\begin{array}{c}\bar{\infty} \\
\infty \\
p\end{array}$ & $\begin{array}{l}\infty \\
\\
\infty\end{array}$ & ๙ָ \\
\hline (\% \% & $\begin{array}{l}v_{\infty} \\
\end{array}$ & $\begin{array}{l}\infty \\
\stackrel{\infty}{\sim}\end{array}$ & $\begin{array}{l}\mathrm{U} \\
\stackrel{\mathrm{N}}{\mathrm{V}}\end{array}$ & 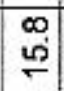 & $\begin{array}{l}\dot{0} \\
\dot{q}\end{array}$ & $\begin{array}{l}0 \\
\oplus\end{array}$ & $\begin{array}{l}\text { D) } \\
\text { m }\end{array}$ & $\bar{\infty}$ & $\begin{array}{l}\infty \\
\infty \\
\infty\end{array}$ & $\stackrel{0}{=}$ & \begin{tabular}{|c|} 
\\
\\
\end{tabular} & $\begin{array}{l}\mathrm{O} \\
\stackrel{N}{N}\end{array}$ & $\stackrel{N}{N}$ & \begin{tabular}{|l|}
0 \\
$\infty$
\end{tabular} & \begin{tabular}{|l|}
$\sigma$ \\
$\sigma$ \\
\end{tabular} & 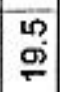 \\
\hline (\%ұM) sə|qqәd unjpow & $\stackrel{\sim}{\rightleftarrows}$ & $\begin{array}{l}0 \\
\text { w } \\
\text { N. }\end{array}$ & $\begin{array}{l}0 \\
\infty \\
\infty \\
\infty\end{array}$ & $\begin{array}{l} \\
\stackrel{\sigma}{\sigma}\end{array}$ & \begin{tabular}{l|}
$\mathrm{N}$ \\
$\mathrm{d}$ \\
$\mathrm{N}$
\end{tabular} & \begin{tabular}{|l|}
0 \\
\\
\end{tabular} & $\frac{0}{m}$ & 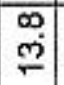 & $\begin{array}{l}m \\
\dot{m} \\
\underline{0}\end{array}$ & $\begin{array}{l}\text { N̦ } \\
\text { के }\end{array}$ & 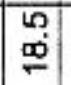 & 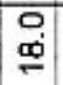 & $\begin{array}{l}\text { N̦ } \\
\text { m }\end{array}$ & $\begin{array}{l}0 \\
\end{array}$ & 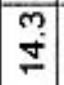 & ষ্ঠ \\
\hline$(\%$ (M) selqqәd asuroo & 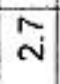 & 뭉 & $\begin{array}{l}m \\
\Gamma\end{array}$ & O & 웅 & O & \begin{tabular}{l|}
$\infty$ \\
m
\end{tabular} & 吕 & $m$ & $\begin{array}{l} \\
\text { Ni }\end{array}$ & \begin{tabular}{|c|}
$\infty$ \\
0 \\
\end{tabular} & \begin{tabular}{l|}
$\infty$ \\
$\omega$ \\
1
\end{tabular} & $\stackrel{\infty}{\sim}$ & \begin{tabular}{|l|}
0 \\
0 \\
\end{tabular} & \begin{tabular}{|l|}
0 \\
\end{tabular} & W \\
\hline (\%2M) selqqod lez०1 & $\ddot{N}$ & $\overline{8}$ & $\begin{array}{c}\tau \\
\dot{\infty} \\
\dot{q}\end{array}$ & N & $\begin{array}{l}\text { N } \\
0 \\
0\end{array}$ & $\stackrel{\infty}{m}$ & $\begin{array}{l}m \\
\text { g } \\
\text { g }\end{array}$ & $\begin{array}{l}\infty \\
\text { gi } \\
\text { D. }\end{array}$ & $\begin{array}{l}\vec{j} \\
\text { న్ల }\end{array}$ & 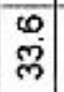 & \begin{tabular}{|c|} 
\\
क्व \\
\end{tabular} & $\begin{array}{l}M \\
9 \\
9\end{array}$ & $\vec{m}$ & 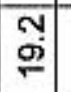 & \begin{tabular}{l}
0 \\
\multirow{n}{*}{} \\
\end{tabular} & $\begin{array}{l}\nabla \\
\dot{v}\end{array}$ \\
\hline (wo) updag נамоา & $\stackrel{\infty}{\sigma}$ & 点 & 葋 & 0 & 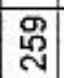 & $\mathscr{q}$ & 爱 & 总 & gi & $\frac{50}{7}$ & 总 & $\stackrel{N}{N}$ & $\bar{\infty}$ & $\ln$ & $\stackrel{\sigma}{=}$ & 垔 \\
\hline (wo) ułdeg seddn & $\stackrel{9}{\oplus}$ & $\stackrel{40}{2}$ & $\stackrel{\mathbb{N}}{\mathbb{N}}$ & m & 总 & 蜔 & $\stackrel{\mathbb{2}}{2}$ & \$ి & $\bar{\sigma}$ & $n$ & \begin{tabular}{|l|} 
罟 \\
\end{tabular} & $\underset{ }{O}$ & 6 & 0 & क & 要 \\
\hline uoz!joH & 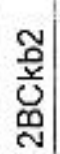 & 弟 & ن & $\frac{\Upsilon}{\mathbb{T}}$ & $\begin{array}{l}\text { o } \\
\text { 总 } \\
\text { ल }\end{array}$ & 盖 & $\stackrel{\mathbb{C}}{=}$ & U్ & 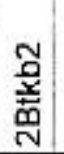 & 爻 & U్N & $\frac{\mathscr{Y}}{\mathrm{D}}$ & 0 & 芯 & $\mid \begin{array}{l}\tilde{3} \\
\text { 辛 } \\
\text { 商 }\end{array}$ & Iㄷ \\
\hline әpoว ә|dures p|ㅣㅣ & 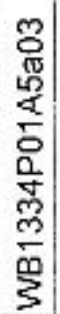 & 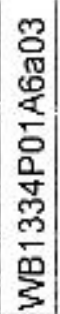 & 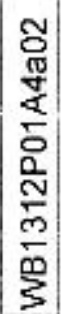 & 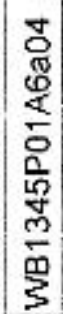 & 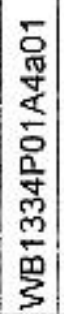 & 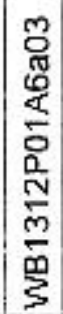 & 点 & 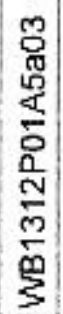 & 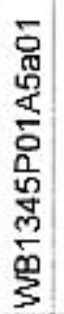 & 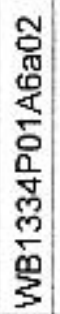 & 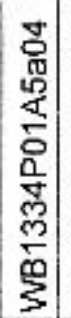 & 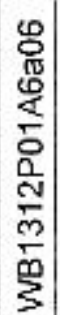 & 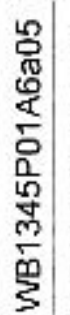 & 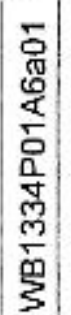 & 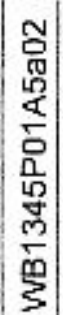 & 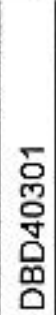 \\
\hline 키us & ป & m & 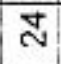 & $\stackrel{2}{2}$ & 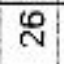 & N & $\infty$ & g & 品 & $\bar{m}$ & กี & M & से & 点 & 点 & ल \\
\hline ч०्यद्ध & 号 & 饮 & 韋 & 总 & 号 & $\stackrel{\text { L }}{\circ}$ & 岁 & 음 & $\stackrel{\mathscr{q}}{\mathrm{g}}$ & 总 & $\frac{\mathscr{Q}}{\mathrm{O}}$ & 8 & 号 & 告 & 号 & ๖̊ \\
\hline
\end{tabular}




\begin{tabular}{|c|c|c|c|c|c|c|c|c|c|c|c|c|c|c|c|c|c|c|c|c|c|}
\hline 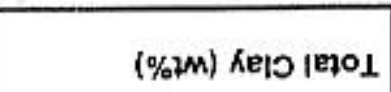 & $\begin{array}{l}0 \\
\end{array}$ & $\begin{array}{l}\mathbb{N} \\
\forall\end{array}$ & \begin{tabular}{|l|}
5 \\
60
\end{tabular} & $\begin{array}{ll}\infty \\
10\end{array}$ & 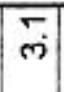 & \begin{tabular}{|l|} 
\\
uे
\end{tabular} & $\begin{array}{ll}n \\
\mathrm{~N}\end{array}$ & \begin{tabular}{l|}
$\infty$ \\
\\
$\dot{\infty}$
\end{tabular} & $\overline{\text { is }}$ & \begin{tabular}{|l|} 
\\
\end{tabular} & \begin{tabular}{|l|}
0 \\
0 \\
\end{tabular} & \begin{tabular}{|l|}
\multicolumn{1}{|c|}{} \\
$\omega^{\prime}$
\end{tabular} & \begin{tabular}{|l|} 
\\
\\
\end{tabular} & $\begin{array}{l}\dot{8} \\
\dot{\nabla}\end{array}$ & $\begin{array}{ll}\mathrm{N} \\
\mathrm{C}\end{array}$ & $\begin{array}{l} \\
\dot{\nabla}\end{array}$ & $\begin{array}{c}N \\
10\end{array}$ & v & \begin{tabular}{|c|} 
\\
$\dot{\nabla}$
\end{tabular} & $\begin{array}{l}\infty \\
\infty\end{array}$ & $\begin{array}{l}\infty \\
\dot{\nabla}\end{array}$ \\
\hline (\%)M) भा!s әu! & $\stackrel{\sim}{\sim}$ & $\begin{array}{l}\nabla \\
\text { ले }\end{array}$ & $\bar{m}$ & 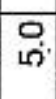 & 5 & Nָ & $\stackrel{0}{-}$ & $\stackrel{N}{\longrightarrow}$ & \begin{tabular}{|l|}
$\infty$ \\
$\infty$ \\
\end{tabular} & $\begin{array}{c}\text { na } \\
\text { Ni }\end{array}$ & $\begin{array}{l}\mathrm{N} \\
\mathrm{v}\end{array}$ & \begin{tabular}{|l|}
$\infty$ \\
m. \\
\end{tabular} & $\bar{i}$ & $\stackrel{\nabla}{\sim}$ & 임 & $\cong$ & $\begin{array}{c}N \\
L\end{array}$ & $\begin{array}{l}\infty \\
\text { N }\end{array}$ & $\begin{array}{l} \\
\text { ले }\end{array}$ & $\stackrel{?}{-}$ & $\stackrel{\infty}{\sim}$ \\
\hline (\%+M) H!S asueo & $\begin{array}{l} \\
\\
0\end{array}$ & $\begin{array}{l}+ \\
\dot{\omega}\end{array}$ & $\stackrel{\infty}{-}$ & $\begin{array}{l}\infty \\
\dot{v}\end{array}$ & \begin{tabular}{|l|}
40 \\
0
\end{tabular} & \begin{tabular}{|c|} 
\\
\\
0
\end{tabular} & $M$ & पे & \begin{tabular}{|l|} 
\\
0
\end{tabular} & $\tilde{\sim}$ & \begin{tabular}{l|}
0 \\
10
\end{tabular} & 吕 & Nִ & $\stackrel{0}{\circ}$ & $\begin{array}{l}\infty \\
0\end{array}$ & $\begin{array}{l}0 \\
0\end{array}$ & $\begin{array}{l}\nabla \\
0\end{array}$ & $\stackrel{M}{=}$ & $\overline{\mathrm{N}}$ & O & $\stackrel{4}{\longrightarrow}$ \\
\hline (\%+M) H!S IE) & $\overline{\mathrm{N}}$ & \begin{tabular}{l|}
$\infty$ \\
$\sigma$ \\
$\sigma$
\end{tabular} & O & $\begin{array}{l}\infty \\
\text { ஸे }\end{array}$ & $\stackrel{T}{F}$ & $\hat{\mathrm{N}}$ & 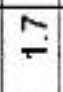 & $\stackrel{0}{-}$ & \begin{tabular}{|l|}
\multirow{2}{*}{} \\
$\mathrm{N}$
\end{tabular} & \begin{tabular}{|c|} 
여 \\
in
\end{tabular} & $\hat{\sigma}$ & \begin{tabular}{|c|} 
\\
in
\end{tabular} & $\begin{array}{c}m \\
m\end{array}$ & $\begin{array}{l}\text { मे } \\
\text { ले }\end{array}$ & $\stackrel{\infty}{-}$ & N & $\underset{-}{\square}$ & $\stackrel{0}{\forall}$ & 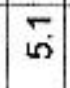 & $\stackrel{m}{\sim}$ & $\stackrel{m}{\nabla}$ \\
\hline 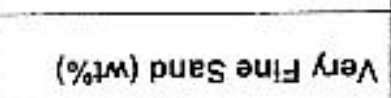 & $\begin{array}{l}\infty \\
0 \\
0\end{array}$ & 灾 & $\stackrel{m}{m}$ & 尚 & is & $\begin{array}{l}\Delta \\
0\end{array}$ & $\begin{array}{ll}0 \\
60\end{array}$ & 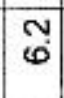 & $\stackrel{5}{=}$ & $\begin{array}{l}5 \\
+\end{array}$ & \begin{tabular}{|l|}
$\infty$ \\
$\dot{N}$ \\
\end{tabular} & $\stackrel{\mathrm{m}}{\mathrm{N}}$ & 六 & \begin{tabular}{|l|}
$\sigma$ \\
$\sigma$
\end{tabular} & $\begin{array}{l}\infty \\
\dot{v}\end{array}$ & $\begin{array}{l}O \\
\infty\end{array}$ & $\underset{\sigma}{v}$ & \begin{tabular}{l}
0 \\
0 \\
\hdashline
\end{tabular} & $\begin{array}{l}N \\
N\end{array}$ & 吕 & $\begin{array}{l}\sigma \\
\text { के }\end{array}$ \\
\hline (\% \% pul pus ouly & $\begin{array}{l}0 \\
0 \\
0 \\
0\end{array}$ & ळ) & $\begin{array}{l}N \\
\text { ma }\end{array}$ & ए్ & $\begin{array}{l}\dot{\nabla} \\
\infty \\
\stackrel{0}{0}\end{array}$ & \begin{tabular}{|l|} 
\\
g্ \\
\end{tabular} & 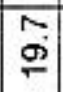 & $\begin{array}{l}\nabla \\
\text { d্ }\end{array}$ & m & $\begin{array}{l}m \\
\mathrm{~N} \\
\mathrm{~N}\end{array}$ & m & Бे & $\begin{array}{l}+ \\
0 \\
\text { d }\end{array}$ & 足 & $\begin{array}{l}m \\
\varphi \\
\varphi\end{array}$ & $\frac{\text { L }}{m}$ & $\begin{array}{l}n \\
=\end{array}$ & $\frac{m}{m}$ & $\begin{array}{l}\text { N } \\
\text { Ni }\end{array}$ & $\begin{array}{l}\text { m } \\
\text { D }\end{array}$ & ल्户 \\
\hline (\%џM) pues un!̣paw & @্ & $\stackrel{T}{=}$ & क़ & $\stackrel{\oplus}{\sigma}$ & $\begin{array}{l}\mathbf{U} \\
\stackrel{9}{\circ}\end{array}$ & స & $\frac{n}{m}$ & 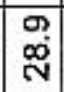 & 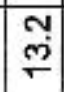 & 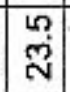 & \begin{tabular}{|l|} 
\\
\\
\end{tabular} & 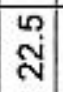 & 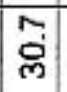 & $\underset{⿱ 亠 凶}{\mathbb{V}}$ & $\begin{array}{l}\forall \\
\text { ه }\end{array}$ & $\begin{array}{l}\infty \\
\stackrel{N}{N}\end{array}$ & $\begin{array}{l}\infty \\
\stackrel{\sim}{\sim}\end{array}$ & 定 & $\begin{array}{l}\infty \\
\stackrel{\infty}{\circ} \\
\sim\end{array}$ & लु & İ \\
\hline$(\%$ \% $)$ pues asueos & $\stackrel{\mathscr{N}}{\stackrel{0}{\sim}}$ & $\begin{array}{l}\text { 웅 } \\
\stackrel{0}{\circ}\end{array}$ & $\stackrel{\circ}{=}$ & 용 & $\begin{array}{l}\infty \\
\infty \\
\text { ஸे }\end{array}$ & $\underset{\infty}{-\infty}$ & $\overline{\mathrm{N}}$ & 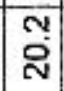 & 20 & $\bar{\infty}$ & 只 & \begin{tabular}{|l|} 
\\
$i$ \\
20
\end{tabular} & $\begin{array}{l}m \\
\mathrm{D}\end{array}$ & $\begin{array}{c}0 \\
0 \\
\end{array}$ & $\frac{m}{m}$ & م) & $\begin{array}{l}0 \\
\infty \\
\infty\end{array}$ & $\underset{⿱}{ \pm}$ & $\begin{array}{l}0 \\
\mathrm{~N} \\
\mathrm{~N}\end{array}$ & $\stackrel{\check{N}}{\grave{N}}$ & $\stackrel{\infty}{+}$ \\
\hline (\%ఖM) puES asueō NOA & i) & $\stackrel{0}{\mathrm{I}}$ & $\overrightarrow{0}$ & $\dot{v}$ & $\begin{array}{l}\mathscr{L} \\
\stackrel{\varphi}{\sigma}\end{array}$ & \begin{tabular}{l|} 
\\
\\
0
\end{tabular} & \begin{tabular}{|l|}
$\stackrel{\circ}{\mathrm{i}}$ \\
\end{tabular} & \begin{tabular}{|l|}
$\infty$ \\
0 \\
0
\end{tabular} & \begin{tabular}{|l|}
\multirow{2}{*}{} \\
Li \\
\end{tabular} & $\begin{array}{l}\text { 움 } \\
\end{array}$ & \begin{tabular}{|c|} 
\\
$\infty$
\end{tabular} & के & $\begin{array}{c}- \\
\infty\end{array}$ & \begin{tabular}{|l|}
0 \\
0 \\
\end{tabular} & \begin{tabular}{|l|}
$\infty$ \\
$\varphi$ \\
$\varphi$
\end{tabular} & $\begin{array}{c}\text { No } \\
\text { O }\end{array}$ & $\begin{array}{l}\nabla \\
\infty\end{array}$ & 음 & $\stackrel{m}{m}$ & 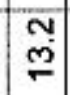 & $\underset{\infty}{\sim}$ \\
\hline (\%\%M) pues $\mid E O_{\perp}$ & $\begin{array}{l}m \\
\dot{\sigma}\end{array}$ & \begin{tabular}{l|}
0 \\
0 \\
$\infty$
\end{tabular} & $\begin{array}{l}1 \\
8 \\
\infty\end{array}$ & $\begin{array}{l}\dot{J} \\
\dot{\infty}\end{array}$ & 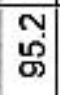 & क़े & \begin{tabular}{|l|}
$\infty$ \\
$\delta$ \\
$\sigma$ \\
$\sigma$
\end{tabular} & \begin{tabular}{l|} 
\\
$\mathrm{g}$ \\
$\mathrm{g}$
\end{tabular} & \begin{tabular}{|l|}
$\mathbf{J}$ \\
స్
\end{tabular} & $\frac{9}{5}$ & \begin{tabular}{|l|} 
\\
$\dot{m}$ \\
$\dot{m}$
\end{tabular} & $\begin{array}{l}\mathbf{Q} \\
\stackrel{8}{\infty} \\
\mathbf{g}\end{array}$ & 'ू & ুั & $\begin{array}{l}0 \\
20 \\
50\end{array}$ & $\begin{array}{l} \\
\text { ğ }\end{array}$ & $\begin{array}{l}N \\
\mathcal{\infty}\end{array}$ & 穴 & \begin{tabular}{|l|} 
\\
\\
\end{tabular} & \begin{tabular}{|l|} 
\\
$\dot{j}$ \\
\end{tabular} & \% \\
\hline (\%2M) sə|qqed au!y & $\begin{array}{l}0 \\
\mathbf{N}\end{array}$ & $\begin{array}{l}m \\
\omega \\
\omega\end{array}$ & $\omega$ & is & $\begin{array}{l}3 \\
0 \\
\varphi\end{array}$ & $\stackrel{m}{=}$ & $\begin{array}{l}\infty \\
\infty \\
\infty\end{array}$ & 웅 & 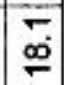 & 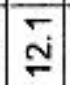 & \begin{tabular}{|l|} 
\\
$\infty$
\end{tabular} & $\begin{array}{l}M \\
\infty\end{array}$ & $\begin{array}{l} \\
\end{array}$ & ஸू & $\underset{⿱ 亠}{+}$ & \begin{tabular}{|c|} 
\\
$\sigma$
\end{tabular} & I & $\stackrel{\infty}{\infty}$ & $\stackrel{\nabla}{\sigma}$ & $\begin{array}{l}0 \\
\dot{\nabla} \\
\end{array}$ & 茴 \\
\hline (\%дM) selqqәd un!̣pow & $\begin{array}{l}\text { क } \\
4 \\
\square\end{array}$ & 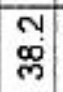 & $\stackrel{N}{N}$ & 웅 & 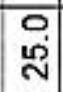 & \begin{tabular}{|l|}
$\infty$ \\
$\infty$ \\
\end{tabular} & \begin{tabular}{|l|}
$\underline{\omega}$ \\
$\Phi$ \\
\end{tabular} & $\overline{\dot{d}}$ & \begin{tabular}{l|}
0 \\
$\dot{g}$ \\
\end{tabular} & $\begin{array}{l}0 \\
\text { N } \\
N\end{array}$ & స్ & $F$ & $\begin{array}{l}0 \\
\\
\end{array}$ & $\frac{0}{\mathrm{~N}}$ & \begin{tabular}{|l|}
0 \\
0 \\
\end{tabular} & $\begin{array}{l}\mathbf{v} \\
\text { N }\end{array}$ & $\begin{array}{c}N \\
\mathfrak{y} \\
\mathrm{v}\end{array}$ & $\check{\square}$ & \begin{tabular}{c}
\multirow{2}{*}{} \\
$\sigma$ \\
$\sigma$
\end{tabular} & $\underset{\oplus}{\mathscr{\sigma}}$ & $\stackrel{\check{c}}{\leftarrow}$ \\
\hline (\%¥M) sa|qqәd asieoว & $\begin{array}{l}\infty \\
\stackrel{0}{\circ}\end{array}$ & $\stackrel{\text { क }}{-}$ & $\underset{\forall}{N}$ & $\overrightarrow{0}$ & $\begin{array}{l}\varphi \\
\Gamma\end{array}$ & $\begin{array}{l}0 \\
0\end{array}$ & \begin{tabular}{|c|}
0 \\
0 \\
0
\end{tabular} & \begin{tabular}{|l|} 
\\
6
\end{tabular} & $\begin{array}{l} \\
\text { i }\end{array}$ & $\begin{array}{l}\forall \\
0\end{array}$ & 品 & $\stackrel{T}{\square}$ & \begin{tabular}{|c|}
$\infty$ \\
0 \\
0
\end{tabular} & $\begin{array}{l}9 \\
\circ\end{array}$ & $\tilde{m}$ & $\stackrel{\infty}{\stackrel{\infty}{\sim}}$ & $\stackrel{\nabla}{\sim}$ & F & $\frac{n}{\mathrm{~N}}$ & $\overline{\mathrm{N}}$ & $\stackrel{L}{-}$ \\
\hline$(\% \not M)$ solqq. & gे & 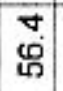 & $\overline{\mathrm{N}}$ & $\begin{array}{l}\stackrel{9}{0} \\
\stackrel{0}{\circ}\end{array}$ & \begin{tabular}{l|} 
\\
0 \\
0 \\
\end{tabular} & N & \begin{tabular}{|l|}
0 \\
\\
\end{tabular} & 吕 & $\frac{\bar{\sigma}}{\overline{6}}$ & \begin{tabular}{|l|}
0 \\
0 \\
f \\
\end{tabular} & \begin{tabular}{l|} 
\\
ले
\end{tabular} & से & $\overline{\mathrm{N}}$ & \begin{tabular}{l|}
$\infty$ \\
0 \\
q
\end{tabular} & $\mid \begin{array}{c}\infty \\
m \\
m\end{array}$ & $\begin{array}{l}\infty \\
\dot{J}\end{array}$ & $\begin{array}{l}m \\
\mathcal{C}\end{array}$ & $\stackrel{\infty}{\mathbb{N}}$ & ปั่ & लूं & $\overline{\mathfrak{N}}$ \\
\hline (ws) undag semoา & 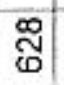 & ㄱ & 8 & 28 & $\hat{\mathrm{m}}$ & $\overline{8}$ & 铝 & $\begin{array}{l}0 \\
0 \\
0 \\
6\end{array}$ & ชี & 묻 & 要 & ন্ড & \begin{tabular}{|l|}
$\mathscr{D}$ \\
ñ \\
\end{tabular} & $\widetilde{0}$ & 몯 & $\frac{m}{60}$ & \% & $\frac{N}{4}$ & 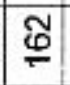 & 勇 & 产 \\
\hline (wo) uldəa seddn & 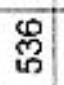 & 0 & $\begin{array}{l}\infty \\
\mathbb{8}\end{array}$ & 要 & $\mathrm{N}$ & \begin{tabular}{|l|}
0 \\
$\mathbb{N}$ \\
$\mathbb{0}$
\end{tabular} & 号 & $\overline{\mathscr{0}}$ & in & 몯 & पू & $\cong$ & $\frac{N}{i n}$ & 足 & g & 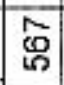 & 刃 & $\stackrel{M}{\mathscr{Y}}$ & চ & $\frac{\infty}{6}$ & 过 \\
\hline & 迆 & 呆 & 0 & 包 & ऐ్ల & ن & U్ల & U & 啚 & 0 & 딷 & 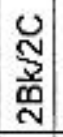 & 0 & ऐ્ळ & 0 & U. & $\begin{array}{l}\frac{0}{y} \\
\text { 号 } \\
\text { ( }\end{array}$ & O & $\begin{array}{l}\text { 盖 } \\
\text {. }\end{array}$ & 0 & U \\
\hline әpos 리uㅏ ple!y & 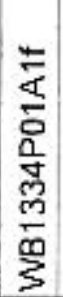 & 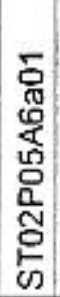 & 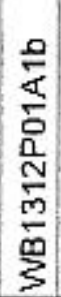 & 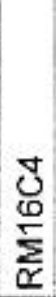 & 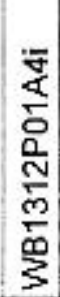 & 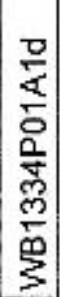 & 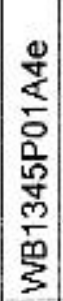 & 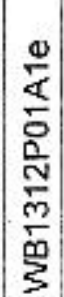 & 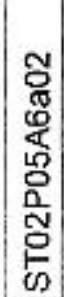 & 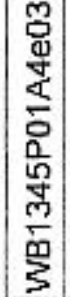 & $\begin{array}{l} \\
\text { 宫 } \\
\text { 号 } \\
\text { 絗 }\end{array}$ & $\begin{array}{l}\frac{0}{0} \\
\frac{\Phi}{0} \\
\frac{0}{0} \\
\frac{\mathrm{m}}{\mathrm{m}} \\
\frac{\mathrm{m}}{3}\end{array}$ & 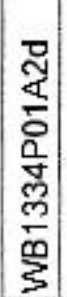 & 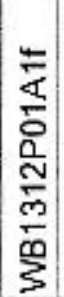 & 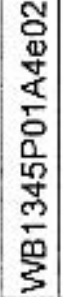 & 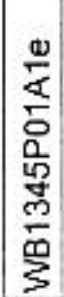 & 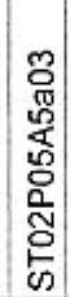 & 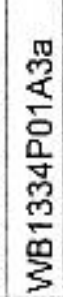 & 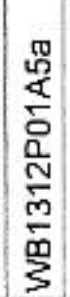 & 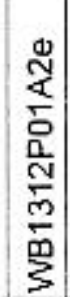 & 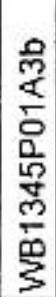 \\
\hline a|dwes & - & $N$ & $m$ & $\nabla$ & $\omega 0$ & $\infty$ & $r$ & $\infty$ & o & 음 & $F$ & $\stackrel{N}{T}$ & $m$ & $\stackrel{\square}{\square}$ & 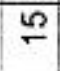 & 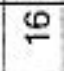 & 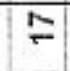 & $\stackrel{\infty}{\sim}$ & क & 요 & $\bar{N}$ \\
\hline чэग7еg & $\stackrel{8}{8}$ & $\stackrel{\mathscr{g}}{\mathrm{g}}$ & $\mathscr{8}$ & $\stackrel{\mathscr{P}}{\stackrel{2}{*}}$ & $\stackrel{8}{8}$ & 8 & 8 & 导 & 号 & 号 & 总 & 号 & 尊 & 寽 & O & 夏 & 号 & $\mathscr{8}$ & $\begin{array}{l}0 \\
8\end{array}$ & $\begin{array}{l}\mathscr{O} \\
\end{array}$ & $\stackrel{8}{8}$ \\
\hline
\end{tabular}




\begin{tabular}{|c|c|c|c|c|c|c|c|c|c|c|c|c|c|c|c|c|c|c|c|c|}
\hline 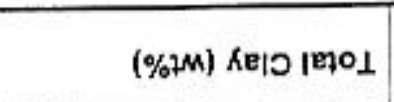 & $\begin{array}{l}\infty \\
\omega \\
\omega\end{array}$ & $\stackrel{m}{\mathrm{v}}$ & $\overrightarrow{L i}$ & 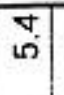 & $\stackrel{N}{m}$ & ले & م) & \begin{tabular}{|c|}
$n$ \\
$\infty$
\end{tabular} & लि & 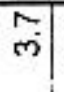 & $\begin{array}{l}0 \\
\text { m. }\end{array}$ & \begin{tabular}{|l|} 
\\
$\dot{m}$
\end{tabular} & m) & $\begin{array}{l}m \\
\omega \sigma^{\prime}\end{array}$ & $\begin{array}{l}50 \\
6\end{array}$ & \begin{tabular}{l|l|}
5 \\
6
\end{tabular} & $\begin{array}{l}9 \\
\mathrm{~m}\end{array}$ & 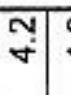 & $\begin{array}{l}\infty \\
\dot{v}\end{array}$ & $\begin{array}{l}m \\
\oplus\end{array}$ \\
\hline (\%,M) मा!S au!s & $\begin{array}{l}\dot{\nabla} \\
\dot{\nabla}\end{array}$ & $\stackrel{\nabla}{-}$ & $\begin{array}{l}10 \\
6\end{array}$ & 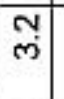 & $\begin{array}{l}\text { Oे } \\
\text { Oे }\end{array}$ & $\stackrel{\infty}{-\infty}$ & $\vec{m}$ & $\stackrel{+}{-}$ & Nָ & $\begin{array}{l}n \\
\text { in }\end{array}$ & $\cong$ & in & $\stackrel{10}{8}$ & $\dot{v}$ & \begin{tabular}{l|} 
\\
\multicolumn{1}{|c|}{}
\end{tabular} & \begin{tabular}{l|l} 
के & \\
ले
\end{tabular} & $\stackrel{\oplus}{\mathrm{N}}$ & m) & ن̀ & $\stackrel{\varphi}{\dot{v}}$ \\
\hline$(\% 2 M)$ म!!s asueos & $\begin{array}{l}n \\
\infty \\
\infty\end{array}$ & $\overline{0}$ & $\overline{0}$ & $\stackrel{\text { ㅇํ }}{-}$ & $\check{0}$ & 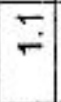 & \begin{tabular}{l|}
$\infty$ \\
$\infty$ \\
$\omega^{\prime}$
\end{tabular} & $\begin{array}{l}0 \\
\mathrm{~N}\end{array}$ & $\Rightarrow$ & $\mathrm{N}$ & $\begin{array}{l}\mathscr{\omega} \\
\mathrm{N}\end{array}$ & $\tilde{n}$ & $\begin{array}{l}n \\
\sigma\end{array}$ & \begin{tabular}{|l|}
10 \\
10 \\
0
\end{tabular} & $\begin{array}{l}\infty \\
\mathbf{N}\end{array}$ & $\begin{array}{ll}m \\
\text { ले }\end{array}$ & $\overline{c i}$ & $\begin{array}{l}8 \\
\text { in }\end{array}$ & $\stackrel{\Delta}{\mathrm{N}}$ & $\stackrel{m}{\forall}$ \\
\hline (\%?M) AIS IE & $\begin{array}{l}\text { के } \\
\text { के }\end{array}$ & $\overline{i i}$ & $\begin{array}{l}\mathscr{Q} \\
\varrho\end{array}$ & $\tilde{v}$ & $\stackrel{\circ}{-}$ & $\begin{array}{l}0 \\
\mathrm{i}\end{array}$ & $m$ & \begin{tabular}{|l|} 
\\
ले
\end{tabular} & \begin{tabular}{l|} 
क \\
ले
\end{tabular} & No & $\begin{array}{l}m \\
\dot{m}\end{array}$ & $\begin{array}{l}\infty \\
\dot{v}\end{array}$ & $\stackrel{\vec{m}}{\mathrm{~m}}$ & 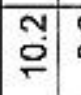 & $\begin{array}{l}m \\
\infty\end{array}$ & บุ & $m$ & 숭 & $\begin{array}{l}\infty \\
\dot{\nabla}\end{array}$ & $\begin{array}{l}\sigma \\
\infty\end{array}$ \\
\hline (\%) pues әul] NieA & $\begin{array}{l}\infty \\
\text { लें }\end{array}$ & $\stackrel{\infty}{\infty}$ & $\overline{\mathrm{N}}$ & $\stackrel{m}{=}$ & $\ddot{q}$ & 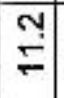 & \begin{tabular}{|l|}
$\mathrm{N}$ \\
$\mathrm{D}$ \\
\end{tabular} & $\frac{0}{F}$ & 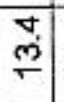 & $\doteqdot$ & $\begin{array}{l}\text { O } \\
\text { m }\end{array}$ & $\stackrel{\check{L}}{\square}$ & $\overline{\mathrm{N}}$ & \begin{tabular}{|l|}
$\infty$ \\
$\dot{\sim}$ \\
\end{tabular} & $\begin{array}{l}\infty \\
\omega \\
\omega\end{array}$ & ڤi & $\underset{\sim}{\infty}$ & $\frac{N}{N}$ & \begin{tabular}{l|}
$\infty$ \\
$\infty$ \\
$\omega^{\prime}$
\end{tabular} & $\overline{\mathfrak{N}}$ \\
\hline (\%\M) pues ouly & पी & $\begin{array}{l}\text { Nu } \\
\text { 山े }\end{array}$ & $\begin{array}{l}\mathbf{N} \\
\stackrel{5}{\sigma}\end{array}$ & స్ల & $\begin{array}{l}m \\
0 \\
0\end{array}$ & ìd & \begin{tabular}{|l|} 
\\
\multirow{J}{*}{}
\end{tabular} & \begin{tabular}{|c|} 
\\
0 \\
$\stackrel{D}{0}$
\end{tabular} & $\frac{\infty}{m}$ & $\begin{array}{l}\text { क्र } \\
\text { N }\end{array}$ & 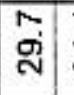 & घे & $\bar{N}$ & $\begin{array}{l}N \\
0 \\
0\end{array}$ & 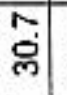 & $\frac{\mathrm{N}}{\mathrm{m}}$ & $\begin{array}{l}L \\
0 \\
0\end{array}$ & 눙 & $\begin{array}{l}0 \\
10\end{array}$ & $\begin{array}{l}\text { I } \\
\text { ס्ల }\end{array}$ \\
\hline (\%1M) pues untpew & $\stackrel{N}{N}$ & $\begin{array}{l}\text { के } \\
\text { O্ं }\end{array}$ & के & 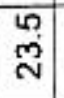 & 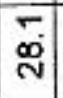 & 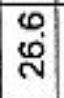 & $\begin{array}{l} \\
\stackrel{G}{v} \\
\sim\end{array}$ & \begin{tabular}{|c|c|} 
\\
$\stackrel{D}{*}$
\end{tabular} & $\overline{\mathrm{N}}$ & 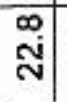 & \begin{tabular}{|c|}
0 \\
\multirow{N}{*}{} \\
\end{tabular} & ঠ. & 弪 & \begin{tabular}{|c|}
$\sigma^{\prime}$ \\
\end{tabular} & $\begin{array}{l}\text { O } \\
\text { S. }\end{array}$ & ஸे & $\begin{array}{l}0 \\
0 \\
0\end{array}$ & $\stackrel{m}{m}$ & $\begin{array}{l}\mathscr{D} \\
\mathbb{I}\end{array}$ & $\bar{c}$ \\
\hline (\%גM) pues әsteo & N & $\begin{array}{l}7 \\
\stackrel{2}{2}\end{array}$ & 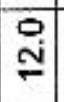 & 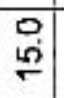 & 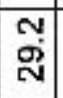 & \begin{tabular}{l|}
0 \\
0 \\
$\infty$
\end{tabular} & 触 & 定 & $\begin{array}{l}m \\
\dot{q} \\
+\end{array}$ & $\stackrel{5}{\longrightarrow}$ & \begin{tabular}{|l|}
$?$ \\
$\mathscr{O}$ \\
\end{tabular} & $\stackrel{\square}{=}$ & $\begin{array}{l}\square \\
= \\
=\end{array}$ & \begin{tabular}{|l|l|}
0 & \\
0 & \\
\end{tabular} & $\stackrel{0}{\circ}$ & $\begin{array}{l}m \\
\stackrel{m}{m}\end{array}$ & ब) & $\begin{array}{l} \\
\\
\end{array}$ & 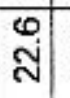 & O \\
\hline 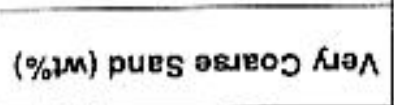 & $\stackrel{\infty}{m}$ & 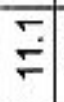 & 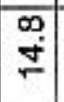 & 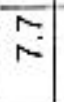 & $\begin{array}{l}0 \\
= \\
=\end{array}$ & $\stackrel{N}{=}$ & $\begin{array}{l}n \\
\phi \\
\sigma\end{array}$ & \begin{tabular}{l|}
0 \\
0 \\
\end{tabular} & $\stackrel{\infty}{=}$ & N & $\begin{array}{l}0 \\
\infty\end{array}$ & $\begin{array}{l}\text { mo } \\
\dot{0}\end{array}$ & $\begin{array}{l}\square \\
\rightleftharpoons\end{array}$ & O: & $\underset{\infty}{N}$ & $\begin{array}{l}0 \\
0\end{array}$ & $\overline{0}$ & สุ & $\begin{array}{l}\infty \\
\stackrel{0}{0} \\
\sim\end{array}$ & 10 \\
\hline (\%2M) pues IeqOL & $\begin{array}{l}m \\
\dot{m}\end{array}$ & 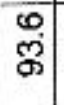 & $\hat{N}$ & \begin{tabular}{l|l|} 
' \\
ळ. \\
\end{tabular} & \begin{tabular}{l|} 
\\
$\dot{\sigma}$ \\
$\dot{\sigma}$
\end{tabular} & \begin{tabular}{|c|} 
\\
$\delta$ \\
\end{tabular} & \begin{tabular}{l|}
$\mathbf{N}$ \\
$\infty$ \\
$\infty$ \\
$\infty$
\end{tabular} & ळু & $\bar{\sigma}$ & \begin{tabular}{|c|} 
\\
\\
\end{tabular} & $\frac{\infty}{\infty}$ & $\begin{array}{l}0 \\
\sigma\end{array}$ & \begin{tabular}{|l|} 
\\
$\dot{D}$ \\
$\infty$
\end{tabular} & \begin{tabular}{|l|}
0 \\
$\dot{5}$ \\
\end{tabular} & $\vec{\infty}$ & $\begin{array}{l}0 \\
\mathscr{D}\end{array}$ & $\begin{array}{l}\infty \\
\stackrel{\circ}{\circ}\end{array}$ & $\bar{\infty}$ & 8. & $\begin{array}{l}\infty \\
\dot{D}\end{array}$ \\
\hline$(\% \nmid \mathrm{m})$ səlqqә्d au!s & \begin{tabular}{l|}
$\infty$ \\
$\omega$ \\
$\omega$
\end{tabular} & $\begin{array}{l}\infty \\
0 \\
0\end{array}$ & $\begin{array}{l}N \\
\text { w } \\
\leftarrow\end{array}$ & $\begin{array}{l}\mathbf{w} \\
\infty\end{array}$ & 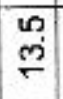 & \begin{tabular}{l|}
$m$ \\
$\sigma$ \\
\end{tabular} & \begin{tabular}{|l|} 
\\
\\
\end{tabular} & $\stackrel{?}{\tilde{q}}$ & $\frac{9}{=}$ & $\begin{array}{l}0 \\
N\end{array}$ & 今o & $\begin{array}{l}\infty \\
\infty\end{array}$ & $\frac{n}{5}$ & \begin{tabular}{l|l}
3 \\
6
\end{tabular} & $\bar{\infty}$ & 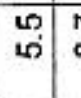 & $\stackrel{\infty}{\infty}$ & $\begin{array}{l}0 \\
\dot{v} \\
\forall\end{array}$ & $\begin{array}{l}\text { Na } \\
\text { న }\end{array}$ & 웅 \\
\hline (\%qM) selqqed un!pew & 후 & $\underset{\infty}{\mathbb{N}}$ & \begin{tabular}{|c|} 
\\
$\dot{g}$
\end{tabular} & N) & $\begin{array}{l}m \\
\stackrel{m}{ }\end{array}$ & \begin{tabular}{l|}
0 \\
0 \\
0
\end{tabular} & \begin{tabular}{|l|} 
\\
में
\end{tabular} & \begin{tabular}{|l|} 
\\
$\dot{\sigma}$ \\
\end{tabular} & $\begin{array}{c}\bar{\infty} \\
+\end{array}$ & $\begin{array}{c}\infty \\
m \\
\stackrel{2}{2}\end{array}$ & 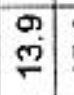 & \begin{tabular}{l|}
$m$ \\
mi \\
Lे
\end{tabular} & \begin{tabular}{|l|}
$O$ \\
\\
\end{tabular} & \begin{tabular}{|l|l|}
0 & \\
$\infty$ & \\
\end{tabular} & $\stackrel{40}{=}$ & N) & $\begin{array}{ll}0 \\
\stackrel{0}{\rightleftharpoons}\end{array}$ & 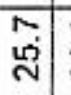 & $\begin{array}{l}\mathrm{N} \\
\mathrm{N}\end{array}$ & $\underset{-}{-}$ \\
\hline (\%मM) selqqed asjeoJ & m. & $\stackrel{m}{\sim}$ & $\stackrel{\nabla}{\square}$ & i & \begin{tabular}{|c|}
$\sigma$ \\
$\sigma \mathrm{o}$ \\
\end{tabular} & $\stackrel{\sigma}{\stackrel{m}{\sigma}}$ & \begin{tabular}{|l|}
$n$ \\
\end{tabular} & \begin{tabular}{|l|}
$\infty$ \\
$\sim$ \\
$\sim$
\end{tabular} & \begin{tabular}{|l|}
\multicolumn{1}{|c|}{} \\
$\leftrightarrow$ \\
\end{tabular} & $\begin{array}{l}2 \\
6\end{array}$ & $\begin{array}{c}\bar{v} \\
\text { V. }\end{array}$ & $\sigma^{\prime}$ & \begin{tabular}{|l|} 
\\
$\infty$ \\
$\infty$ \\
$\stackrel{0}{*}$
\end{tabular} & $\stackrel{m}{-}$ & 岁 & $\begin{array}{ll}\omega \\
\text { लై }\end{array}$ & N̦: & $\begin{array}{l}0 \\
\dot{v}\end{array}$ & $\begin{array}{l}10 \\
0 \\
0\end{array}$ & $\stackrel{0}{=}$ \\
\hline 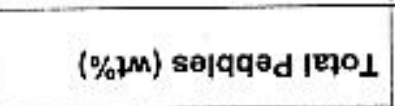 & $\begin{array}{l}\underset{N}{N} \\
\stackrel{\circ}{*}\end{array}$ & $\tilde{\mathcal{G}}$ & 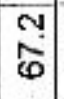 & $\begin{array}{l}\tilde{\sigma} \\
\stackrel{2}{\circ}\end{array}$ & $\begin{array}{l}M \\
g \\
g\end{array}$ & 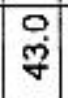 & \begin{tabular}{|c|}
0 \\
8
\end{tabular} & 穴 & $\begin{array}{l}\text { क } \\
\text { फ़ }\end{array}$ & ले & $\frac{1}{\infty}$ & \begin{tabular}{l|} 
\\
$m$ \\
$m$
\end{tabular} & $\begin{array}{l}10 \\
\mathrm{~N} \\
\mathrm{~N}\end{array}$ & $\begin{array}{l}\mathscr{N} \\
\mathscr{6}\end{array}$ & ন্ড & $\begin{array}{l}\mathbb{N} \\
\mathscr{q}\end{array}$ & क & $\begin{array}{l}0 \\
y\end{array}$ & $\frac{0}{5}$ & $\stackrel{\infty}{g}$ \\
\hline (wo) чұdag Jәmoา & 읃 & స్. & $\stackrel{\text { g }}{\rightleftharpoons}$ & $\begin{array}{ll}50 \\
5 \\
5\end{array}$ & 믄 & $\begin{array}{l}\text { m } \\
\tilde{v} \\
\end{array}$ & 邑 & \begin{tabular}{|c|} 
M \\
T.
\end{tabular} & $E$ & 怘 & $\underset{\infty}{\bar{\infty}}$ & \begin{tabular}{|l|}
$\mathscr{0}$ \\
\multirow{N}{*}{}
\end{tabular} & 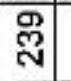 & (I) & 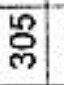 & E & 心్ల్ల & $\stackrel{\text { : }}{\sim}$ & 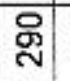 & कें \\
\hline (wo) 47dog Joddn & 믇 & 怘 & g & 多 & 믿 & $\begin{array}{l}\mathscr{S}_{1} \\
\end{array}$ & $\frac{\sigma}{\square}$ & $\bar{m}$ & $\bar{m}$ & 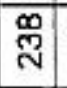 & 点 & 思 & $\stackrel{\square}{Ð}$ & $\stackrel{\mathbb{2}}{\mathrm{C}}$ & \begin{tabular}{l|l}
\multirow{8}{*}{} \\
$\stackrel{8}{*}$
\end{tabular} & $\underset{\sim}{\mathbb{N}}$ & $\approx$ & $m$ & ग & $\omega$ \\
\hline uozpoH & $\stackrel{0}{\mathrm{C}}$ & 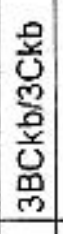 & \begin{tabular}{|l} 
o \\
㩊 \\
\end{tabular} & U & 0 & 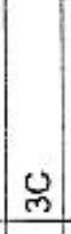 & $\begin{array}{l}\overline{\mathbf{D}} \\
\mathrm{i} \\
\mathrm{M} \\
\mathrm{N}\end{array}$ & 它 & $\mid \begin{array}{l}\mathrm{U} \\
\mathrm{N} \\
\mathrm{D} \\
\mathrm{v} \\
\mathrm{d} \\
\mathrm{N}\end{array}$ & 0 & 足 & $\begin{array}{l}\mathrm{U} \\
\mathrm{N} \\
\mathrm{o} \\
\mathrm{v} \\
\mathrm{d} \\
\mathrm{N}\end{array}$ & $\begin{array}{l}\mathrm{N} \\
\mathrm{d} \\
\mathrm{D} \\
\mathrm{d} \\
\mathrm{N}\end{array}$ & $\stackrel{\pi}{\complement}$ & 勇 & U & 0 & 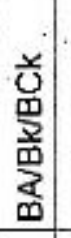 & 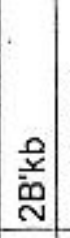 & 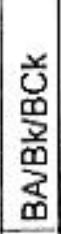 \\
\hline әроэ 히யues P|비 & 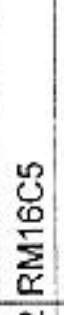 & 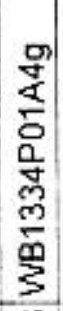 & 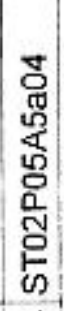 & 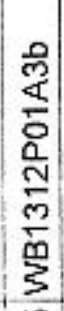 & 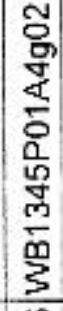 & 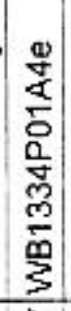 & $\begin{array}{l}0 \\
0 \\
0 \\
0 \\
0 \\
0 \\
0 \\
0 \\
0 \\
0 \\
0 \\
0 \\
0\end{array}$ & 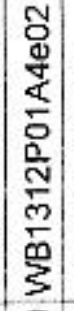 & $\begin{array}{l}0 \\
0 \\
5 \\
5 \\
0 \\
0 \\
5 \\
5 \\
5 \\
5 \\
5\end{array}$ & 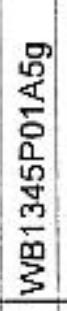 & 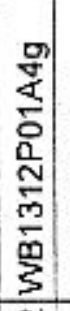 & 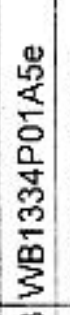 & 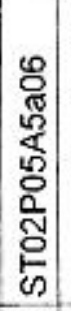 & 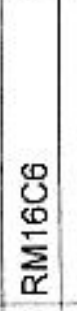 & $\begin{array}{l}0 \\
0 \\
\vdots \\
0 \\
0 \\
0 \\
m \\
0 \\
\vdots \\
\vdots\end{array}$ & $\begin{array}{l}\frac{c}{y} \\
\frac{1}{0} \\
0 \\
\frac{1}{N} \\
\frac{1}{m} \\
\frac{m}{3}\end{array}$ & 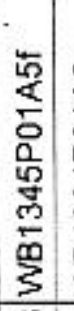 & 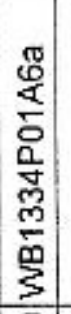 & 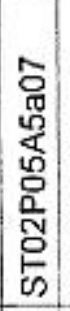 & 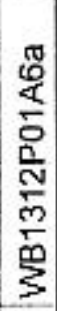 \\
\hline श्रdweS & กิ & $\stackrel{\sim}{N}$ & $\vec{N}$ & $\stackrel{4}{\mathrm{~N}}$ & 9 & $\hat{N}$ & $\stackrel{\infty}{\sim}$ & g) & ৪) & $\bar{m}$ & กู & m & ले & $m$ & 吕 & $\hat{m}$ & $\begin{array}{ll}\infty \\
m\end{array}$ & ल & 웅 & 寸 \\
\hline чэฺ्घ & ğ & 号 & $\stackrel{8}{8}$ & $\begin{array}{l}8 \\
8 \\
\end{array}$ & 8 & $\stackrel{9}{8}$ & $\stackrel{0}{g}$ & $\stackrel{8}{8}$ & 8 & $\begin{array}{l} \\
\end{array}$ & 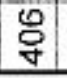 & 号 & $\stackrel{\mathscr{O}}{\square}$ & $\begin{array}{l}0 \\
\\
\end{array}$ & $\stackrel{9}{8}$ & $\begin{array}{l}9 \\
\end{array}$ & 号 & 号 & 号 & $\stackrel{\circ}{g}$ \\
\hline
\end{tabular}

$\frac{N}{\dot{\alpha}}$ 


\begin{tabular}{|c|c|c|c|c|c|c|c|c|c|c|c|c|c|c|c|c|c|c|c|c|c|}
\hline 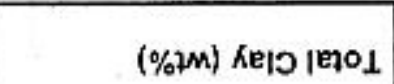 & $\begin{array}{l}\mathscr{W} \\
\mathscr{\varphi}\end{array}$ & $\begin{array}{l}\bar{\nabla} \\
\end{array}$ & \begin{tabular}{|l|}
0 \\
$\mathrm{~N}$
\end{tabular} & $\overrightarrow{\dot{m}}$ & $\bar{N}$ & $\dot{8}$ & No & 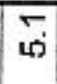 & $\begin{array}{l}\mathbf{0} \\
0\end{array}$ & \begin{tabular}{l|}
$\infty$ \\
m.
\end{tabular} & ले & $\begin{array}{l}m \\
0\end{array}$ & \begin{tabular}{l|} 
\\
\end{tabular} & \begin{tabular}{|l|}
$\mathbf{m}$ \\
\end{tabular} & \begin{tabular}{c|} 
\\
\end{tabular} & \begin{tabular}{|l|}
$\mathrm{m}$ \\
$\mathrm{N}$
\end{tabular} & \begin{tabular}{l|}
$\infty$ \\
$m$ \\
$m$
\end{tabular} & $\begin{array}{l} \\
10\end{array}$ & $m$ & $\stackrel{d}{\mathrm{j}}$ & $\stackrel{6}{8}$ \\
\hline (\%جM) $2 ! ! 5$ au! & $\mathscr{\sigma}$ & $\begin{array}{l}\infty \\
\dot{\nabla}\end{array}$ & $\begin{array}{l}m \\
m \\
m\end{array}$ & ले & $\begin{array}{l}0 \\
\text { N }\end{array}$ & $\mathrm{N}$ & $\begin{array}{l}\infty \\
\dot{v}\end{array}$ & กै & $\frac{8}{5}$ & $\bar{\omega}$ & \begin{tabular}{|l|}
0 \\
0
\end{tabular} & $\begin{array}{l}\forall \\
\forall\end{array}$ & $\begin{array}{l}m \\
m\end{array}$ & \begin{tabular}{|c|}
$m$ \\
mo \\
\end{tabular} & \begin{tabular}{|l|}
$n$ \\
0
\end{tabular} & \begin{tabular}{|l|}
0 \\
\end{tabular} & $\stackrel{\infty}{\forall}$ & $\sigma^{\circ}$ & $\begin{array}{l}0 \\
\dot{v}\end{array}$ & \begin{tabular}{l|}
$\infty$ \\
ले
\end{tabular} & $\stackrel{\oplus}{-}$ \\
\hline (\%みM) H!S esueoว & $\stackrel{m}{=}$ & $M$ & $\begin{array}{l}\dot{y} \\
\dot{m}\end{array}$ & $\stackrel{M}{M}$ & $\stackrel{\oplus}{\leftarrow}$ & $\stackrel{+}{\sim}$ & $\begin{array}{l}10 \\
10 \\
0\end{array}$ & $\underset{6}{N}$ & $\bar{\sigma}$ & 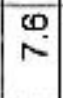 & $\begin{array}{c}N \\
0\end{array}$ & \begin{tabular}{l|}
$\infty$ \\
$\omega$ \\
$\omega$
\end{tabular} & $\begin{array}{c}5 \\
\sigma^{\prime}\end{array}$ & $\overline{L i}$ & $\begin{array}{l}40 \\
\square \\
\end{array}$ & \begin{tabular}{|l|}
\multicolumn{2}{c}{} \\
$\stackrel{2}{\circ}$
\end{tabular} & $\begin{array}{l}m \\
c\end{array}$ & $\begin{array}{l}\nabla \\
0\end{array}$ & $\begin{array}{l}m \\
m\end{array}$ & in & $\stackrel{10}{-}$ \\
\hline 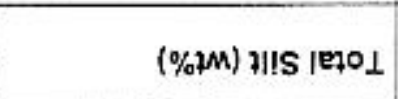 & $\begin{array}{l}\sigma \\
\text { 어 }\end{array}$ & $\begin{array}{l}10 \\
0 \\
0\end{array}$ & \begin{tabular}{|l|}
0 \\
$\Phi$ \\
\end{tabular} & $\stackrel{N}{\circ}$ & $\begin{array}{l}10 \\
+8\end{array}$ & $\underset{v}{N}$ & $\begin{array}{l}m \\
0 \\
0\end{array}$ & $\stackrel{\oplus}{=}$ & 品 & $\underline{\underline{N}}$ & $\begin{array}{l}0 \\
0 \\
\end{array}$ & $\begin{array}{l}\text { No } \\
\underline{g}\end{array}$ & $\begin{array}{l}\sigma \\
\infty\end{array}$ & $\begin{array}{l}\dot{v} \\
\dot{D}\end{array}$ & $\begin{array}{l}0 \\
\dot{\rho}\end{array}$ & $\frac{n}{m}$ & $\stackrel{\omega}{\leftarrow}$ & 吕 & m & $\begin{array}{l} \\
\sigma\end{array}$ & N \\
\hline$(\% 2 M)$ pues au! Kua & $\begin{array}{l}\infty \\
\infty \\
\sim\end{array}$ & 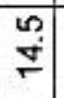 & $\begin{array}{l}\text { N } \\
\text { N }\end{array}$ & $\overline{\mathrm{N}}$ & $\begin{array}{l}\dot{*} \\
\dot{0}\end{array}$ & $\stackrel{F}{F}$ & $\begin{array}{l}0 \\
\text { ले } \\
\stackrel{4}{ }\end{array}$ & $\begin{array}{l}0 \\
+\end{array}$ & $\stackrel{5}{\mathrm{~N}}$ & $\begin{array}{l}v_{0} \\
\infty \\
\sim\end{array}$ & \begin{tabular}{|l|}
$\infty$ \\
N
\end{tabular} & $\begin{array}{l}\mathbf{N} \\
\mathrm{N}\end{array}$ & ले & $\begin{array}{l}0 \\
5 \\
2\end{array}$ & $\frac{\infty}{\square}$ & \begin{tabular}{|c|} 
\\
0 \\
0
\end{tabular} & $\begin{array}{l}m \\
\text { m }\end{array}$ & $\begin{array}{l}m \\
\stackrel{N}{ }\end{array}$ & $\begin{array}{l}\stackrel{0}{2} \\
\dot{T}\end{array}$ & \begin{tabular}{l|}
$\dot{L n}$ \\
$m$ \\
$m$
\end{tabular} & 등 \\
\hline (\%2M) pues oul] & $\begin{array}{l}\infty \\
\dot{\Xi}\end{array}$ & $\begin{array}{l}\mathbf{N} \\
\text { N }\end{array}$ & స్ & $\frac{\nabla}{\sim}$ & $\begin{array}{l}40 \\
\text { Jे }\end{array}$ & $\begin{array}{l}0 \\
\text { O }\end{array}$ & ले & $\begin{array}{l}\sigma \\
\dot{v}\end{array}$ & $\stackrel{\infty}{\sim}$ & $\begin{array}{l}0 \\
\mathbf{d}\end{array}$ & $\left|\begin{array}{c}\infty \\
\dot{\omega} \\
\end{array}\right|$ & के & $\stackrel{5}{=}$ & $\begin{array}{l}\infty \\
\infty \\
\infty\end{array}$ & $\underset{\sigma}{\sigma}$ & \begin{tabular}{l|}
$\infty$ \\
$\stackrel{\infty}{2}$
\end{tabular} & $\stackrel{\varphi}{\sim}$ & $\bar{m}$ & $\begin{array}{c}\text { N } \\
\infty \\
\infty\end{array}$ & $\stackrel{F}{E}$ & $\stackrel{M}{m}$ \\
\hline (\%ұM) pues un!pew & वi & $\vec{\phi}$ & 물 & $\bar{g}$ & $\begin{array}{l}T \\
\stackrel{m}{N}\end{array}$ & $\begin{array}{l}n \\
\mathrm{~d} \\
\mathrm{~d}\end{array}$ & $\begin{array}{l}\sigma \\
\sigma\end{array}$ & 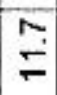 & 웅 & 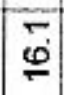 & $\begin{array}{l} \\
\dot{\theta} \\
\end{array}$ & $\dot{\sigma}$ & $\begin{array}{l}\infty \\
\dot{I} \\
\square\end{array}$ & $\begin{array}{l}0 \\
\stackrel{6}{\circ}\end{array}$ & $\begin{array}{l} \\
0 \\
\end{array}$ & $\begin{array}{l}\hat{\omega} \\
\hat{\theta}\end{array}$ & $\begin{array}{l} \pm \\
5\end{array}$ & $\overline{0}$ & $\begin{array}{l}\text { N. } \\
\text { D }\end{array}$ & $\begin{array}{l}N \\
\check{F}\end{array}$ & $\stackrel{n}{\mathrm{U}}$ \\
\hline (\%7M) pues asueoว & $\begin{array}{l}0 \\
\text { 움 }\end{array}$ & $\stackrel{⿱}{\stackrel{5}{\sim}}$ & $\begin{array}{l}0 \\
0 \\
0\end{array}$ & $\begin{array}{l}\infty \\
\dot{m}\end{array}$ & $\stackrel{m}{\stackrel{M}{N}}$ & $\begin{array}{l}\varphi \\
\end{array}$ & $\stackrel{\nabla}{\sim}$ & $\stackrel{\infty}{\sim}$ & $\bar{\sigma}$ & \begin{tabular}{|l|}
$m$ \\
$m$ \\
$\stackrel{m}{r}$
\end{tabular} & \begin{tabular}{|c|}
$\nabla$ \\
$\infty$ \\
$\square$
\end{tabular} & \begin{tabular}{|l|}
9 \\
0 \\
\end{tabular} & $\begin{array}{l}\dot{v} \\
\text { d }\end{array}$ & $\underset{\infty}{\infty}$ & 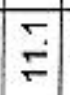 & \begin{tabular}{|l|}
$m$ \\
$\infty$
\end{tabular} & $\begin{array}{l}\square \\
0\end{array}$ & O & $\begin{array}{l}0 \\
\infty\end{array}$ & $\frac{0}{\bar{n}}$ & 함 \\
\hline (\%みM) pues asieoJ KUEA & $\begin{array}{l}\omega \\
\infty\end{array}$ & 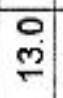 & $\underset{\sim}{\stackrel{N}{\tau}}$ & $\underset{\infty}{N}$ & No & $\stackrel{v}{*}$ & $\begin{array}{l}\omega \\
\infty \\
\infty\end{array}$ & $\underset{N}{\mathbb{N}}$ & $\begin{array}{l}\infty \\
\infty\end{array}$ & $\stackrel{1}{=}$ & $\begin{array}{l}\infty \\
\Gamma\end{array}$ & $\hat{n}$ & $\frac{a}{\grave{N}}$ & $\begin{array}{l}n \\
0 \\
\sigma\end{array}$ & $\begin{array}{l}n \\
0\end{array}$ & $\begin{array}{l}\infty \\
\dot{\omega}\end{array}$ & $\underset{\dot{T}}{T}$ & $\begin{array}{l}N \\
\omega\end{array}$ & $\underset{\nabla}{+}$ & 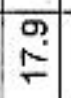 & $\widehat{0}$ \\
\hline$(\%$ M $)$ pues 1 eqo & $\begin{array}{l}\mathbb{D} \\
\mathbb{\infty}\end{array}$ & 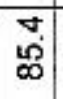 & \begin{tabular}{|l|}
$\infty$ \\
\\
\end{tabular} & $\mathscr{\wp}$ & $\begin{array}{l}10 \\
\infty \\
\infty\end{array}$ & $\frac{\square}{0}$ & $\begin{array}{l}10 \\
\infty \\
\infty\end{array}$ & m & $\bar{m}$ & \begin{tabular}{l|}
0 \\
$\infty$ \\
$\infty$
\end{tabular} & \begin{tabular}{|l|}
0 \\
6 \\
$\sigma$
\end{tabular} & $\begin{array}{l}0 \\
\mathscr{\infty}\end{array}$ & \begin{tabular}{|c|}
0 \\
$\infty$ \\
$\infty$
\end{tabular} & \begin{tabular}{l|}
$\sigma$ \\
4 \\
$\infty$
\end{tabular} & $\begin{array}{l}0 \\
\text { m }\end{array}$ & $\begin{array}{l}0 \\
0 \\
6 \\
6\end{array}$ & $\vec{\Phi}$ & $\begin{array}{l}\mathscr{O} \\
\grave{\infty}\end{array}$ & $\begin{array}{l}\dot{y} \\
\infty\end{array}$ & $\begin{array}{c}\text { y } \\
\infty \\
\infty\end{array}$ & $\begin{array}{l}\text { तू } \\
\text { W }\end{array}$ \\
\hline (\%дM) selqqәd әu!d & ले & 苞 & 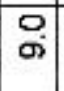 & $\begin{array}{l}\infty \\
\dot{\Xi} \\
\mathbb{Z}\end{array}$ & $\stackrel{\oplus}{=}$ & $\begin{array}{l}5 ? \\
\omega\end{array}$ & $\stackrel{m}{=}$ & $\stackrel{10}{5}$ & 뭉 & $\begin{array}{l}\infty \\
\stackrel{m}{\sim}\end{array}$ & $\begin{array}{l}m \\
\square\end{array}$ & $\begin{array}{l}0 \\
\stackrel{0}{\circ}\end{array}$ & $\begin{array}{l} \\
\\
\end{array}$ & $\infty$ & $\stackrel{m}{\dot{m}}$ & $\stackrel{\square}{\leftarrow}$ & $\begin{array}{l}0 \\
\\
\end{array}$ & $\begin{array}{l}0 \\
\mathbb{N}\end{array}$ & $\bar{N}$ & $\begin{array}{c}M \\
0\end{array}$ & $\stackrel{O}{N}$ \\
\hline (\%qM) sәqqәd unjpəw & $\begin{array}{l} \\
\text { 에 }\end{array}$ & $\begin{array}{l}\text { बें } \\
\text { ( }\end{array}$ & $\begin{array}{l}0 \\
\vdots \\
\text { N }\end{array}$ & $\begin{array}{l}0 \\
\dot{g} \\
y\end{array}$ & $\begin{array}{l}\text { क } \\
\text { gं }\end{array}$ & \begin{tabular}{|l|}
0 \\
0 \\
0
\end{tabular} & مें & $\begin{array}{l}\infty \\
\text { m }\end{array}$ & ָุ & $\begin{array}{l}\infty \\
\stackrel{0}{0}\end{array}$ & $\begin{array}{l}\omega \\
\text { gे }\end{array}$ & $\begin{array}{l}\text { क } \\
\text { N̦ }\end{array}$ & $\frac{7}{8}$ & $\begin{array}{l}0 \\
\dot{\mathrm{v}}\end{array}$ & $\begin{array}{c}\text { N } \\
\text { N }\end{array}$ & $\begin{array}{l}\dot{\sigma} \\
\ddot{\sigma}\end{array}$ & $\begin{array}{l}r \\
\stackrel{\sigma}{\sigma}\end{array}$ & $\begin{array}{l}0 \\
\mathscr{0} \\
\sigma\end{array}$ & $\underset{N}{N}$ & $\stackrel{m}{-}$ & $\underset{\infty}{\infty}$ \\
\hline (\%џM) salqqed asseop & $\begin{array}{l}0 \\
\stackrel{N}{N}\end{array}$ & $\begin{array}{l}\sigma \\
\varphi\end{array}$ & $\begin{array}{l}\infty \\
\omega \\
\end{array}$ & $\begin{array}{l}0 \\
\stackrel{0}{\circ} \\
\end{array}$ & 号 & $\stackrel{\sigma}{\circ}$ & $\bar{m}$ & $\begin{array}{l}\sigma \\
\infty \\
\infty\end{array}$ & $\begin{array}{l}\omega \\
\stackrel{10}{0}\end{array}$ & $\stackrel{\circ}{\circ}$ & $\bar{m}$ & 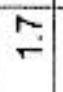 & \begin{tabular}{|l|}
0 \\
\end{tabular} & \begin{tabular}{l|} 
\\
\\
p.
\end{tabular} & $\begin{array}{l}\infty \\
\end{array}$ & 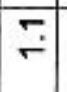 & \begin{tabular}{|l|}
+0 \\
$\square$
\end{tabular} & $m$ & $\begin{array}{l}0 \\
\end{array}$ & $\underset{\omega}{N}$ & 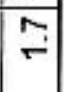 \\
\hline 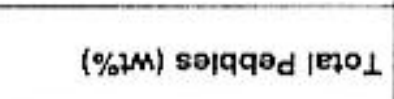 & $\begin{array}{l}\infty \\
1 \\
1\end{array}$ & 菏 & \begin{tabular}{|l|}
0 \\
ชิ
\end{tabular} & $\stackrel{m}{n}$ & \begin{tabular}{l}
$\omega$ \\
\multirow{N}{*}{}
\end{tabular} & $\begin{array}{l}\infty \\
\infty \\
\infty\end{array}$ & $\begin{array}{c}\text { d } \\
\text { m }\end{array}$ & 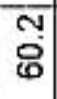 & 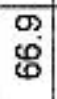 & \begin{tabular}{|l|} 
\\
$\dot{g}$ \\
\end{tabular} & \begin{tabular}{|l|l|}
0 \\
$\dot{g}$ \\
\end{tabular} & $\begin{array}{l}5 \\
5 \\
5\end{array}$ & \begin{tabular}{|l|} 
\\
$\dot{g}$
\end{tabular} & \begin{tabular}{l|}
$\sigma$ \\
$\rho$ \\
$\Gamma$
\end{tabular} & $\overline{8}$ & 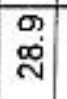 & $\bar{r}$ & $\begin{array}{l}m \\
m \\
m\end{array}$ & $\begin{array}{l}0 \\
\stackrel{N}{\leftarrow}\end{array}$ & $\stackrel{0}{0}$ & $\begin{array}{l}\mathbb{N} \\
\infty \\
\sim\end{array}$ \\
\hline (wэ) чұdeव Jәмоา & $F$ & $\frac{2}{2}$ & $\stackrel{\sim}{\circ}$ & षे & 믇 & 总 & 要 & $\stackrel{\sim}{\sim}$ & $\bar{\sigma}$ & g & ম & (2) & मे & 암 & $\stackrel{M}{L}$ & $\mp$ & g) & 茎 & $m$ & ถึ & D \\
\hline (wว) uldeg səddn & 0 & क & Б্ & n) & 몯 & 9 & $\mathbb{C}$ & $\mp$ & $\stackrel{m}{F}$ & D & 0 & 四 & ถ⿻ & $\stackrel{\sim}{\sim}$ & 导 & 0 & $\stackrel{\infty}{-}$ & $\stackrel{\mathbb{D}}{\mathrm{C}}$ & W & g & $\overline{ }$ \\
\hline & 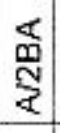 & 胥 & $\begin{array}{l}\text { m } \\
\text { 离 } \\
\text { 惫 }\end{array}$ & $\begin{array}{l}\stackrel{N}{\mathrm{~N}} \\
\text { 寍 }\end{array}$ & 0. & U & 可 & 兽 & 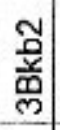 & 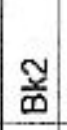 & 0 & 刃 & 畄 & 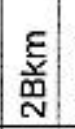 & $\left|\begin{array}{l}\mathbf{0} \\
\mathbf{0} \\
\text { 心 }\end{array}\right|$ & 孚 & 音 & జู & $\begin{array}{l}\text { 号 } \\
\text { 畄 }\end{array}$ & 眔 & 0 \\
\hline 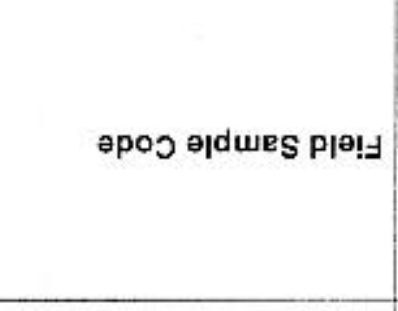 & 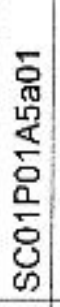 & 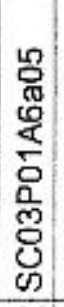 & 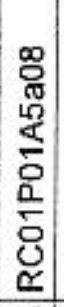 & 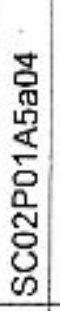 & 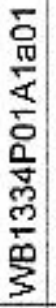 & 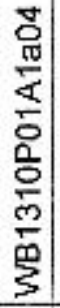 & 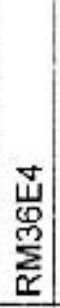 & 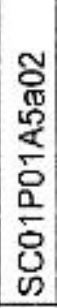 & 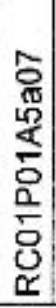 & $\begin{array}{l}\mathbf{Z} \\
0 \\
0 \\
0 \\
0 \\
0 \\
0 \\
0 \\
0 \\
0 \\
0 \\
0\end{array}$ & 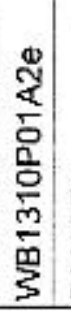 & 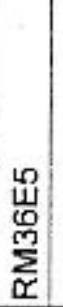 & 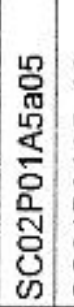 & 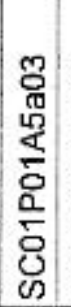 & 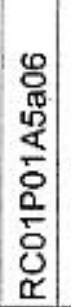 & 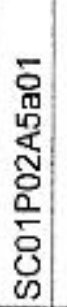 & 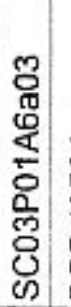 & 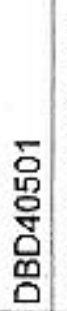 & $\begin{array}{l}\frac{\delta}{0} \\
\frac{0}{5} \\
\overline{0} \\
\frac{0}{0} \\
\frac{0}{0} \\
\frac{m}{5}\end{array}$ & 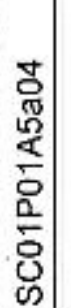 & 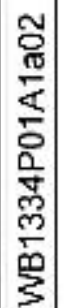 \\
\hline ә|dues & - & $N$ & ला & 8 & का & $\omega$ & $N$ & $\infty$ & क & 음 & $\mp$ & $\stackrel{1}{4}$ & $\stackrel{m}{-}$ & $\stackrel{+}{\square}$ & $\because$ & $\dddot{\varphi}$ & $\approx$ & $\stackrel{\infty}{\infty}$ & क & ㅇ & $\bar{v}$ \\
\hline Чગ્Е & 亏 & $\stackrel{8}{8}$ & 亏 & 今 & $\tilde{g}$ & 号 & D & 点 & 守 & 点 & 宫 & F & So & to & 今 & $\widehat{O}$ & $\frac{\pi}{8}$ & I & 亏 & 守 & 守 \\
\hline
\end{tabular}




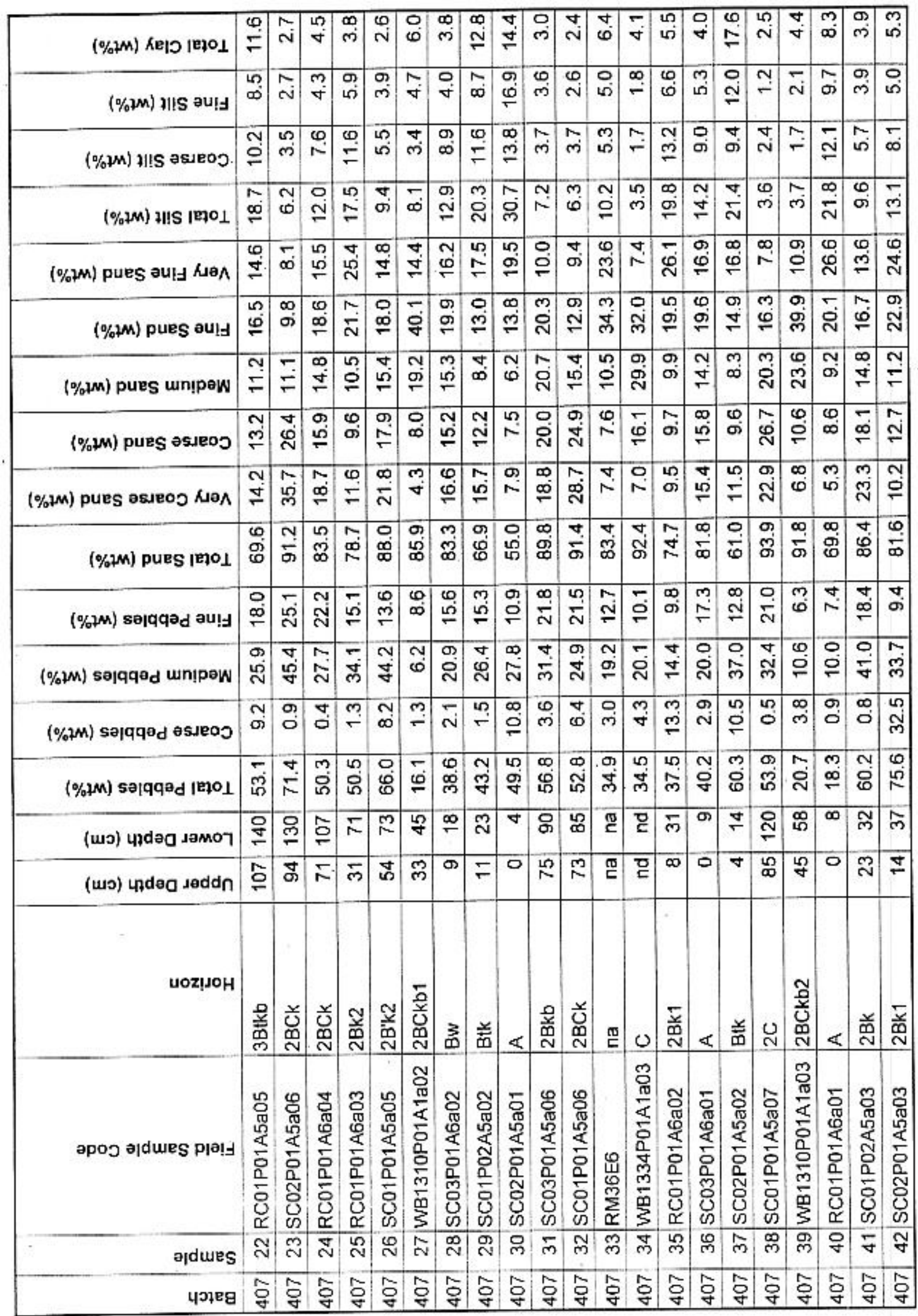




\begin{tabular}{|c|c|c|c|c|c|c|c|c|c|c|c|c|c|c|c|c|c|c|c|c|c|}
\hline (\%मM) КE।O IEZOL & 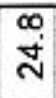 & $\hat{\omega}$ & is & \begin{tabular}{|c|}
$\infty$ \\
$\omega$
\end{tabular} & $\stackrel{\omega}{\omega}$ & $\begin{array}{l}\infty \\
\infty\end{array}$ & m. & की & \begin{tabular}{|l|}
$\infty$ \\
ले \\
\end{tabular} & $\begin{array}{l}\oplus \\
\infty\end{array}$ & $\nabla_{\infty}$ & $\vec{\omega}$ & $\begin{array}{l} \\
0\end{array}$ & $\begin{array}{l}1 \\
\omega\end{array}$ & \begin{tabular}{|l|}
$\infty$ \\
$\oplus$ \\
\end{tabular} & 面 & $\begin{array}{l}\text { का } \\
\text { के }\end{array}$ & กู & $\dot{0}$ & \begin{tabular}{|l|}
$\mathfrak{W}$ \\
$\stackrel{m}{N}$ \\
$\stackrel{2}{*}$
\end{tabular} & $\begin{array}{l}\infty \\
\infty \\
\infty\end{array}$ \\
\hline (\%ұM) H!S ou!d & $\stackrel{m}{v}$ & is & $\begin{array}{l}\sigma \\
\text { N }\end{array}$ & is & 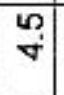 & \begin{tabular}{l|}
$\infty$ \\
0 \\
\end{tabular} & $\begin{array}{l}0 \\
\omega^{\circ}\end{array}$ & ले & \begin{tabular}{|l|}
\multirow{v}{*}{} \\
$\omega$
\end{tabular} & \begin{tabular}{|l|} 
\\
$\omega$ \\
0
\end{tabular} & $\bar{i}$ & \begin{tabular}{l|}
$\infty$ \\
$\dot{\sigma}$
\end{tabular} & 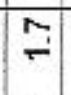 & \begin{tabular}{l|}
5 \\
40
\end{tabular} & $\begin{array}{l} \\
\text { in }\end{array}$ & $\stackrel{\vec{m}}{\sim}$ & $\begin{array}{l}\mathscr{\varphi} \\
\stackrel{m}{*}\end{array}$ & $\begin{array}{l}\text { 임 } \\
\forall\end{array}$ & \begin{tabular}{l|}
$\infty$ \\
$\dot{\sigma}$ \\
$\dot{\sigma}$
\end{tabular} & $\begin{array}{l}\forall \\
0 \\
\end{array}$ & $\stackrel{\oplus}{\omega}$ \\
\hline (\%+M) गI!S osueos & $\stackrel{\varphi}{M}$ & m & $\stackrel{m}{N}$ & $\begin{array}{l}\infty \\
\dot{v}\end{array}$ & ì & $\begin{array}{l}\dot{O} \\
\dot{v}\end{array}$ & $\stackrel{N}{\mathbb{N}}$ & $\underset{\nabla}{\tau}$ & \begin{tabular}{l|}
$m$ \\
$\dot{v}$
\end{tabular} & \begin{tabular}{|l|} 
\\
ले
\end{tabular} & $\begin{array}{l}m \\
m\end{array}$ & $\begin{array}{l}\infty \\
\text { wi }\end{array}$ & $\underset{1}{N}$ & is & $\begin{array}{c}4 \\
\text { in }\end{array}$ & 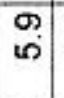 & m. & 悹 & $\begin{array}{l}m \\
\text { is }\end{array}$ & $\stackrel{0}{\sim}$ & m \\
\hline (\%みM) IIS IEłOL & 엄 & 巳ั & ஸ் & \begin{tabular}{|l|}
$\sigma$ \\
$\sigma$
\end{tabular} & $\begin{array}{ll}0 \\
0\end{array}$ & 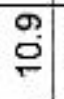 & $\underset{\sim}{\sim}$ & n? & \begin{tabular}{|l|}
$n$ \\
0 \\
0 \\
0
\end{tabular} & \begin{tabular}{|l|}
\multirow{2}{*}{} \\
$\underline{0}$
\end{tabular} & 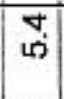 & 兑 & $\begin{array}{l}\text { o } \\
\text { s. }\end{array}$ & \begin{tabular}{|l|}
0 \\
\\
\end{tabular} & $\begin{array}{l}\square \\
0\end{array}$ & $\begin{array}{l}\mathscr{0} \\
\stackrel{\sigma}{\sigma}\end{array}$ & 웅 & $\stackrel{\sigma}{\sigma}$ & No & $\underset{\infty}{N}$ & 옷 \\
\hline 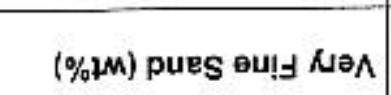 & $\stackrel{\square}{\square}$ & $\begin{array}{l}\infty \\
\text { D. } \\
\text { N }\end{array}$ & $\stackrel{0}{=}$ & 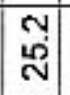 & $\stackrel{\text { no }}{=}$ & 品 & సู & $\begin{array}{l}\text { o) } \\
\dot{\nabla}\end{array}$ & ָָ & $\stackrel{\Xi}{=}$ & \begin{tabular}{l|}
0 \\
$\infty$ \\
\end{tabular} & \begin{tabular}{|l|}
$\stackrel{\omega}{0}$ \\
$\stackrel{N}{N}$
\end{tabular} & $\sigma^{\circ}$ & $\begin{array}{l}0 \\
\text { ปें }\end{array}$ & \begin{tabular}{l|}
$\infty$ \\
$\dot{\sim}$ \\
\end{tabular} & $\begin{array}{l}\infty \\
0 \\
\end{array}$ & $\begin{array}{l}\text { षे } \\
\text { के }\end{array}$ & 훙 & $\begin{array}{l}\infty \\
\grave{3} \\
\end{array}$ & $\vec{\omega}$ & $\bar{m}$ \\
\hline$(\%+M)$ pues ault & $\begin{array}{l}\nabla \\
\oplus\end{array}$ & $\begin{array}{l}0 \\
0 \\
\end{array}$ & $\overline{\mathrm{N}}$ & \begin{tabular}{|l|} 
\\
$\emptyset$ \\
\end{tabular} & $\begin{array}{l}N \\
\stackrel{N}{N}\end{array}$ & $\stackrel{\infty}{\stackrel{\infty}{\sim}}$ & $\begin{array}{l}\mathbb{d} \\
\stackrel{d}{ }\end{array}$ & $\begin{array}{l}\mathbb{N} \\
\stackrel{\sim}{*}\end{array}$ & \begin{tabular}{l|} 
\\
ฟิ \\
\end{tabular} & స్ & \begin{tabular}{|l|} 
ํ. \\
กิ
\end{tabular} & 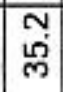 & $\begin{array}{l}m \\
\text { लें }\end{array}$ & \begin{tabular}{c|}
$m$ \\
me
\end{tabular} & ले & $\begin{array}{l}\sigma \\
\dot{0}\end{array}$ & $\overline{\mathrm{D}}$ & $\bar{d}$ & $\begin{array}{l}\infty \\
\dot{m} \\
\end{array}$ & $\begin{array}{l}\dot{v} \\
\text { N }\end{array}$ & g্ \\
\hline (\%2M) pues un!paw & $\stackrel{\square}{\circ}$ & षे & ָั & \begin{tabular}{|c|}
$\infty$ \\
$\sigma^{\prime}$ \\
\end{tabular} & $\overline{\stackrel{一}{\mathrm{~S}}}$ & $\begin{array}{l}\mathscr{0} \\
\bullet \\
\bullet\end{array}$ & $\begin{array}{l}\sigma \\
\sigma \\
\end{array}$ & $\begin{array}{l}\oplus \\
\oplus \\
\oplus\end{array}$ & $\begin{array}{l} \\
\text { di } \\
\end{array}$ & $\begin{array}{ll}m \\
\mathscr{6}\end{array}$ & $\overline{\mathrm{d}}$ & \begin{tabular}{|l|}
$\stackrel{\leftrightarrow}{\circ}$ \\
\end{tabular} & \begin{tabular}{l|} 
\\
M \\
ले
\end{tabular} & $\begin{array}{c}m \\
\omega\end{array}$ & \begin{tabular}{|l|}
$\omega$ \\
$\sigma$ \\
\end{tabular} & ळ. & 엄 & 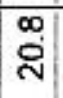 & 훙 & \begin{tabular}{l}
$N$ \\
\hdashline \\
\hdashline
\end{tabular} & Г్ \\
\hline$(\% 2 M)$ pues esuro & क & $\begin{array}{l}40 \\
6\end{array}$ & $\stackrel{m}{\mathscr{e}}$ & $\begin{array}{c}\infty \\
0 \\
0\end{array}$ & $\begin{array}{l}* \\
\oplus\end{array}$ & $\stackrel{\dddot{0}}{\mathscr{0}}$ & 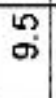 & $\stackrel{N}{\stackrel{N}{*}}$ & $\begin{array}{ll}N \\
\stackrel{N}{2}\end{array}$ & $\stackrel{m}{=}$ & \begin{tabular}{|l|}
$\infty$ \\
$\infty$ \\
0 \\
\end{tabular} & $\overline{6}$ & \begin{tabular}{|l|}
$\infty$ \\
$\infty$ \\
\end{tabular} & $\begin{array}{l}0 \\
0\end{array}$ & $\begin{array}{l}40 \\
0\end{array}$ & $\begin{array}{l}m \\
m\end{array}$ & a & 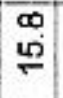 & m) & $\begin{array}{l}N \\
0 \\
O\end{array}$ & \\
\hline (\%2M) pues asieoj KaA & ปे & $\hat{n o s}^{\circ}$ & क & $\begin{array}{l}0 \\
6\end{array}$ & $\begin{array}{l}40 \\
\infty \\
\infty\end{array}$ & $\stackrel{N}{=}$ & $\begin{array}{l}m \\
\dot{q}\end{array}$ & $\stackrel{\check{\mathrm{i}}}{\mathrm{i}}$ & ma & \begin{tabular}{|l|} 
\\
$\dot{6}$ \\
\end{tabular} & $\stackrel{5}{=}$ & $\begin{array}{l} \\
0\end{array}$ & \begin{tabular}{|l|}
\multirow{2}{*}{} \\
$\stackrel{2}{ }$
\end{tabular} & N & $m$ & $\stackrel{\infty}{-}$ & $\begin{array}{l}\text { 어 } \\
\stackrel{\circ}{\circ}\end{array}$ & $\underset{I}{T}$ & $\begin{array}{c}N \\
\infty\end{array}$ & $\begin{array}{l}0 \\
1 \\
\end{array}$ & $\bar{m}$ \\
\hline$(\%+\mathrm{M})$ pue & लై & $\begin{array}{l}0 \\
\text { ल } \\
\infty\end{array}$ & $\bar{\infty}$ & \begin{tabular}{|l|}
$m$ \\
$\tilde{D}$
\end{tabular} & $\begin{array}{l}0 \\
\infty \\
\infty \\
\infty\end{array}$ & \% & $\begin{array}{l}\infty \\
\infty \\
\infty\end{array}$ & 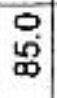 & $\begin{array}{l}M \\
\dot{\infty} \\
\infty\end{array}$ & $\frac{N}{\infty}$ & $\begin{array}{l}y \\
0 \\
0 \\
0\end{array}$ & ल) & $\begin{array}{l}\mathbf{1} \\
\mathbf{c}\end{array}$ & $\begin{array}{l}\mathrm{N} \\
\mathrm{N}\end{array}$ & \begin{tabular}{|c|}
$\infty$ \\
$\tilde{N}$ \\
\end{tabular} & $\begin{array}{l}\text { N̦ } \\
\text { 寸 }\end{array}$ & ָे & \begin{tabular}{l|} 
क \\
$\dot{\text { Dे }}$
\end{tabular} & \begin{tabular}{|l|}
$n$ \\
$\infty$ \\
$\infty$
\end{tabular} & $\begin{array}{ll} \\
0 \\
8 \\
0\end{array}$ & $\underset{\infty}{+}$ \\
\hline (\%дM) sepqqed ou!y & $\stackrel{\nabla}{\grave{N}}$ & $\stackrel{v}{i}$ & $\begin{array}{l}\infty \\
m \\
m\end{array}$ & $\stackrel{\text { ㅁ }}{\sim}$ & m. & $\stackrel{\oplus}{=}$ & $m$ & m. & \begin{tabular}{|l|}
5 \\
\\
\end{tabular} & \begin{tabular}{l|}
$\infty$ \\
$\stackrel{\infty}{ }$
\end{tabular} & 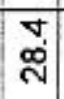 & $\stackrel{?}{?}$ & $\begin{array}{l}m \\
\therefore\end{array}$ & ले & $\tilde{\mathrm{N}}$ & $\bar{d}$ & $\begin{array}{l}\infty \\
\infty \\
\infty\end{array}$ & $\stackrel{40}{=}$ & $\stackrel{\Delta}{\sim}$ & $\mid \begin{array}{l}40 \\
\varphi \\
q\end{array}$ & $\begin{array}{l}\forall \\
0\end{array}$ \\
\hline$(\%$ భM) se|qqәd w & $\begin{array}{l}0 \\
9 \\
\varrho\end{array}$ & 守 & \begin{tabular}{|l|} 
\\
$\stackrel{g}{\mathrm{~N}}$
\end{tabular} & $\stackrel{\Sigma}{\Sigma}$ & $\begin{array}{c}\sim \\
\text { ले }\end{array}$ & $\begin{array}{l}10 \\
\text { ปี }\end{array}$ & $\frac{\infty}{\dot{\sigma}}$ & 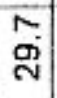 & क & $\begin{array}{l}9 \\
\text { gi }\end{array}$ & 일 & $n$ & 吕 & 占 & 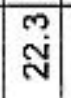 & $\overline{\mathrm{N}}$ & 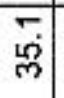 & $\begin{array}{l}\infty \\
\text { v } \\
\end{array}$ & \begin{tabular}{|l|}
$\sigma$ \\
$\dot{\sigma}$ \\
\\
\end{tabular} & 商 & $\frac{5}{8}$ \\
\hline (\%qm) selqqәd әsjeos & $\underset{\infty}{N}$ & 穴 & क & $\stackrel{m}{M}$ & م. & 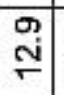 & $\stackrel{\square}{\mathscr{0}}$ & $\stackrel{\infty}{\leftarrow}$ & กิ่ & $\stackrel{\mathrm{m}}{\mathrm{m}}$ & $\stackrel{m}{=}$ & $\begin{array}{l} \\
m \\
\end{array}$ & $\stackrel{\varphi}{=}$ & $\begin{array}{l}40 \\
5\end{array}$ & $\begin{array}{l}0 \\
0 \\
\end{array}$ & $\begin{array}{l}\square \\
\bullet\end{array}$ & 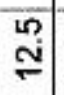 & $\begin{array}{l}\mathbf{O} \\
6 \\
6\end{array}$ & $\begin{array}{l}\stackrel{0}{0} \\
\stackrel{2}{*}\end{array}$ & \begin{tabular}{|l|} 
\\
$\dot{\omega}$
\end{tabular} & $\stackrel{\text { mे }}{\stackrel{2}{r}}$ \\
\hline$(\%)$ selqqed ImoL & \begin{tabular}{l}
$\varphi$ \\
\multirow{v}{*}{}
\end{tabular} & ¿্ল & $\frac{M}{F}$ & \begin{tabular}{l|} 
लूं \\
ల్
\end{tabular} & $\begin{array}{l}m \\
\stackrel{n}{2}\end{array}$ & $\frac{0}{9}$ & $\begin{array}{l}0 \\
\dot{0}\end{array}$ & $\begin{array}{l}m \\
\dot{0} \\
0\end{array}$ & त్ & $\begin{array}{l}7 \\
6 \\
6\end{array}$ & $\hat{\mathbb{N}}$ & $\stackrel{\bar{v}}{-}$ & \begin{tabular}{|l|} 
\\
\\
\end{tabular} & \begin{tabular}{l|}
$m$ \\
$\bar{n}$ \\
\end{tabular} & \begin{tabular}{|c|}
$\underline{\varphi}$ \\
ల్
\end{tabular} & 品 & $\begin{array}{l}\widetilde{N} \\
\mathscr{8}\end{array}$ & $\bar{\Sigma}$ & \begin{tabular}{|l|}
$\sigma$ \\
pep
\end{tabular} & $\overline{\check{\infty}}$ & $\stackrel{\infty}{\infty}_{0}$ \\
\hline 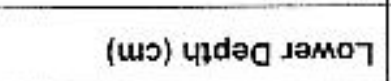 & $N$ & 受 & क्ष & 梠 & 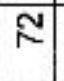 & 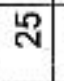 & ঙ্লে & $\frac{0}{2}$ & 尽 & g & $\stackrel{\infty}{\circ}$ & 몯 & ఐ & 可 & פ & $\sigma$ & $\bar{F}$ & 음 & 焉 & 이 & $\stackrel{2}{\sim}$ \\
\hline (wo) uldea səddn & 0 & $\stackrel{\mathbb{V}}{C}$ & 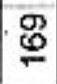 & 政 & g & $N$ & $\stackrel{\mathbb{N}}{\mathbb{N}}$ & $\underset{\sigma}{\sigma}$ & का & nू & 2 & 吅 & g & $\stackrel{\mathbb{D}}{C}$ & 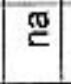 & 0 & \$ & $\stackrel{\text { న్ }}{2}$ & $\stackrel{\mathbb{N}}{C}$ & क & 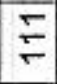 \\
\hline $\mathrm{NOH}$ & $\leq$ & 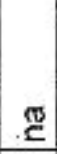 & $\begin{array}{l}\boldsymbol{\infty} \\
\text { 离 }\end{array}$ & T) & 咅 & 盖 & 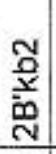 & 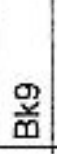 & \begin{tabular}{|l|} 
\\
\\
\end{tabular} & 商 & 总 & 뽇 & $\frac{m}{\tilde{D}}$ & ฐ & $\stackrel{\mathbb{Z}}{\Sigma}$ & $\begin{array}{l}\bar{E} \\
\text { 盇 } \\
\text { 急 }\end{array}$ & $\begin{array}{l}\text { 音 } \\
\text {. }\end{array}$ & $\breve{ठ ~}$ & $\mathbb{2}$ & $\begin{array}{l}\tilde{N} \\
\text { 盖 } \\
\end{array}$ & 岦 \\
\hline әpos 미dues pja! 더 & 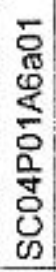 & 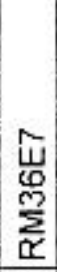 & 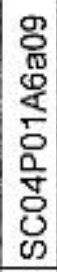 & 号 & 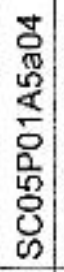 & 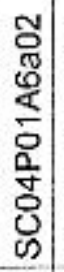 & 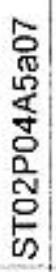 & 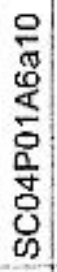 & 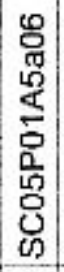 & 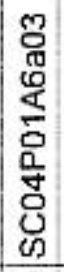 & 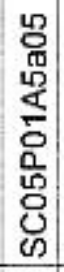 & $\frac{\infty}{5}$ & \begin{tabular}{|c|} 
\\
0 \\
0 \\
0 \\
$\$$ \\
0 \\
0 \\
0 \\
8 \\
0 \\
0 \\
0
\end{tabular} & $\begin{array}{l} \\
.8 \\
\\
0 \\
\end{array}$ & 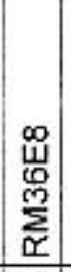 & 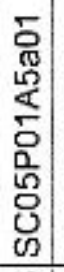 & 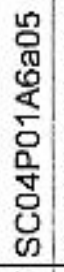 & 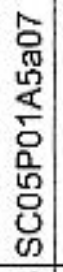 & ఫે & 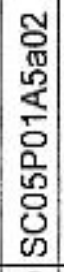 & 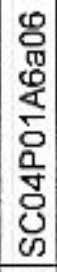 \\
\hline әdues & - & $N$ & $m$ & $\nabla$ & 10 & 0 & $N$ & $\infty$ & $\infty$ & 은 & $\mp$ & 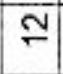 & $\stackrel{m}{\div}$ & 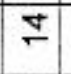 & $\stackrel{2}{\sim}$ & $\mathscr{\varphi}$ & 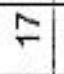 & $\stackrel{\infty}{\sim}$ & क & 이 & $\bar{N}$ \\
\hline ग7еg & $\stackrel{\infty}{\circ}$ & 号 & 号 & $\mid \begin{array}{l}\text { O } \\
\text { f }\end{array}$ & 罗 & 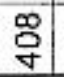 & $\begin{array}{l}\infty \\
8 \\
\dot{\gamma}\end{array}$ & $\stackrel{\infty}{\circ}$ & 号 & 总 & $\stackrel{\circ}{\%}$ & $\begin{array}{l}9 \\
9\end{array}$ & $\begin{array}{l}\text { o } \\
\text { O }\end{array}$ & 号 & $\begin{array}{l} \\
\\
\end{array}$ & $\stackrel{\circ}{\circ}$ & 号 & 号 & \begin{tabular}{|l|} 
o \\
\end{tabular} & 韋 & 号 \\
\hline
\end{tabular}




\begin{tabular}{|c|c|c|c|c|c|c|}
\hline 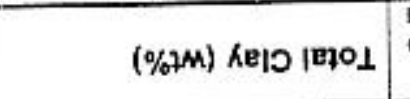 & $\infty$ & $\begin{array}{l}\omega \\
\varphi\end{array}$ & $\begin{array}{l}0 \\
0\end{array}$ & $m$ & $\stackrel{\infty}{\infty}$ & $\begin{array}{l}\mathscr{0} \\
\dot{0}\end{array}$ \\
\hline 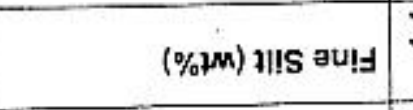 & 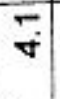 & $\begin{array}{l}\infty \\
0 \\
0\end{array}$ & 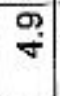 & $\stackrel{20}{-1}$ & $m$ & Ni \\
\hline (\%1M) HI!S әsJeO & $\stackrel{\infty}{-\infty}$ & $\stackrel{+}{\sim}$ & Lึ & Nָ & $\begin{array}{l}\infty \\
\text { เก }\end{array}$ & ก) \\
\hline$(\% \not M)$ 7IIS $\mid \mathrm{E}+\perp$ & की & $\stackrel{N}{\mathrm{~N}}$ & ㅁ. & $\hat{\mathrm{N}}$ & $\bar{\sigma}$ & $\stackrel{\varphi}{0}$ \\
\hline (\%1M) pues әu! אנал & $\stackrel{N}{\circ}$ & $\underset{m}{i}$ & $\begin{array}{l}\infty \\
\tilde{V}^{\prime}\end{array}$ & $m$ & m & की \\
\hline (\%2M) pues ou! & a & $\stackrel{5}{\leftarrow}$ & min & $\frac{\dot{v}}{\mathrm{~N}}$ & mín & స̃ \\
\hline$(\%) M)$ pues unjpow & 穴 & $\infty$ & क & N & 웅 & iv \\
\hline (\%) pues asjeop & $\begin{array}{l}0 \\
\text { กุ } \\
-\end{array}$ & $\stackrel{\infty}{\circ}$ & $\tilde{\omega}$ & $\begin{array}{l}\text { vi } \\
\text { बे }\end{array}$ & M & ? \\
\hline (\%2M) pues osueos NHOA & $\stackrel{m}{r}$ & 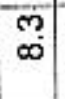 & 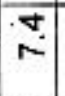 & $\stackrel{\sim}{\rightleftarrows}$ & $m$ & $\stackrel{\infty}{0}$ \\
\hline (\%) pues lejo $\perp$ & $\begin{array}{l}\text { पे } \\
\infty \\
\infty\end{array}$ & 吕 & ๓ं & $\begin{array}{l}\varphi \\
\grave{\infty} \\
\infty\end{array}$ & ले & लूं \\
\hline (\%zm) sajqqad әu!d & $\begin{array}{r}\text { U. } \\
\text { v }\end{array}$ & $\stackrel{\oplus}{=}$ & $\begin{array}{l}0 \\
m\end{array}$ & 品 & $\stackrel{0}{\circ}$ & क \\
\hline$(\%$ yM) sejqqod un!pow & Uั: & $\begin{array}{c}N \\
\infty\end{array}$ & $\frac{m}{N}$ & $\check{\sim}$ & 옴 & $\begin{array}{l}\infty \\
\tilde{N}\end{array}$ \\
\hline (\%) salqqәd asueoj. & $\stackrel{\oplus}{\oplus}$ & ले & m & $\begin{array}{l}\infty \\
\stackrel{\infty}{\leftarrow}\end{array}$ & vi & 눙 \\
\hline (\%?M) səJqqad IejoL & นุ' & $\frac{O}{c}$ & $\begin{array}{l}0 \\
\text { लें }\end{array}$ & 吕 & vi & $\begin{array}{l}N \\
N\end{array}$ \\
\hline (wo) पมdag samoา & क & 뜯 & 昰 & $\underset{\mathbb{0}}{\mathbb{9}}$ & 띧 & 守 \\
\hline (wo) updeg soddn & $\stackrel{2}{2}$ & 品 & $\underset{\mathrm{T}}{\mathrm{T}}$ & 梁 & 塄 & 요 \\
\hline 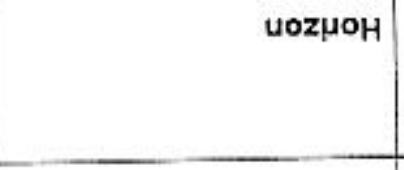 & 里 & 距 & $\underset{\mathbb{C}}{ }$ & 盖 & 昰 & Е \\
\hline әpoэ ㅎdues p|!ㅓ & 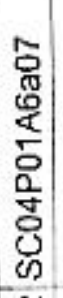 & $\begin{array}{l}5 \\
0 \\
0 \\
\text { 足 } \\
0 \\
0 \\
\end{array}$ & 焉 & $\begin{array}{l}\infty \\
0 \\
0 \\
\vdots \\
\vdots \\
0 \\
0 \\
\vdots \\
0 \\
0 \\
0\end{array}$ & $\frac{0}{5}$ & 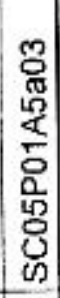 \\
\hline 리ues & N & M & ম & $\stackrel{4}{\mathrm{~N}}$ & 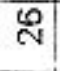 & $\pi$ \\
\hline 4ว7eg & o & 舫 & $\stackrel{\infty}{0}$ & $\stackrel{\infty}{0}$ & $\begin{array}{l}\infty \\
\theta\end{array}$ & $\stackrel{\infty}{\circ}$ \\
\hline
\end{tabular}




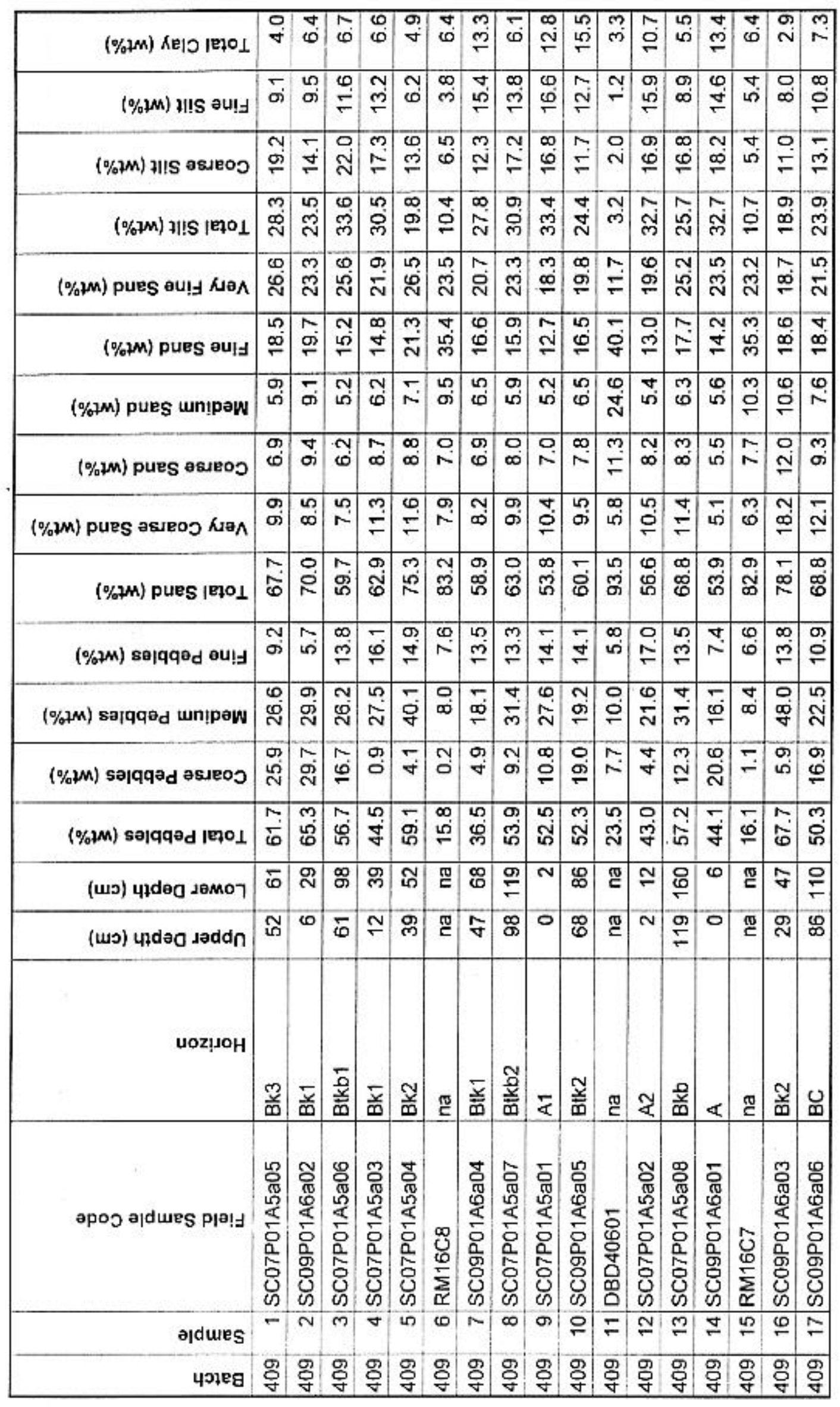

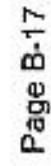

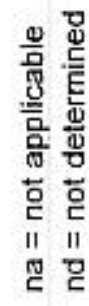




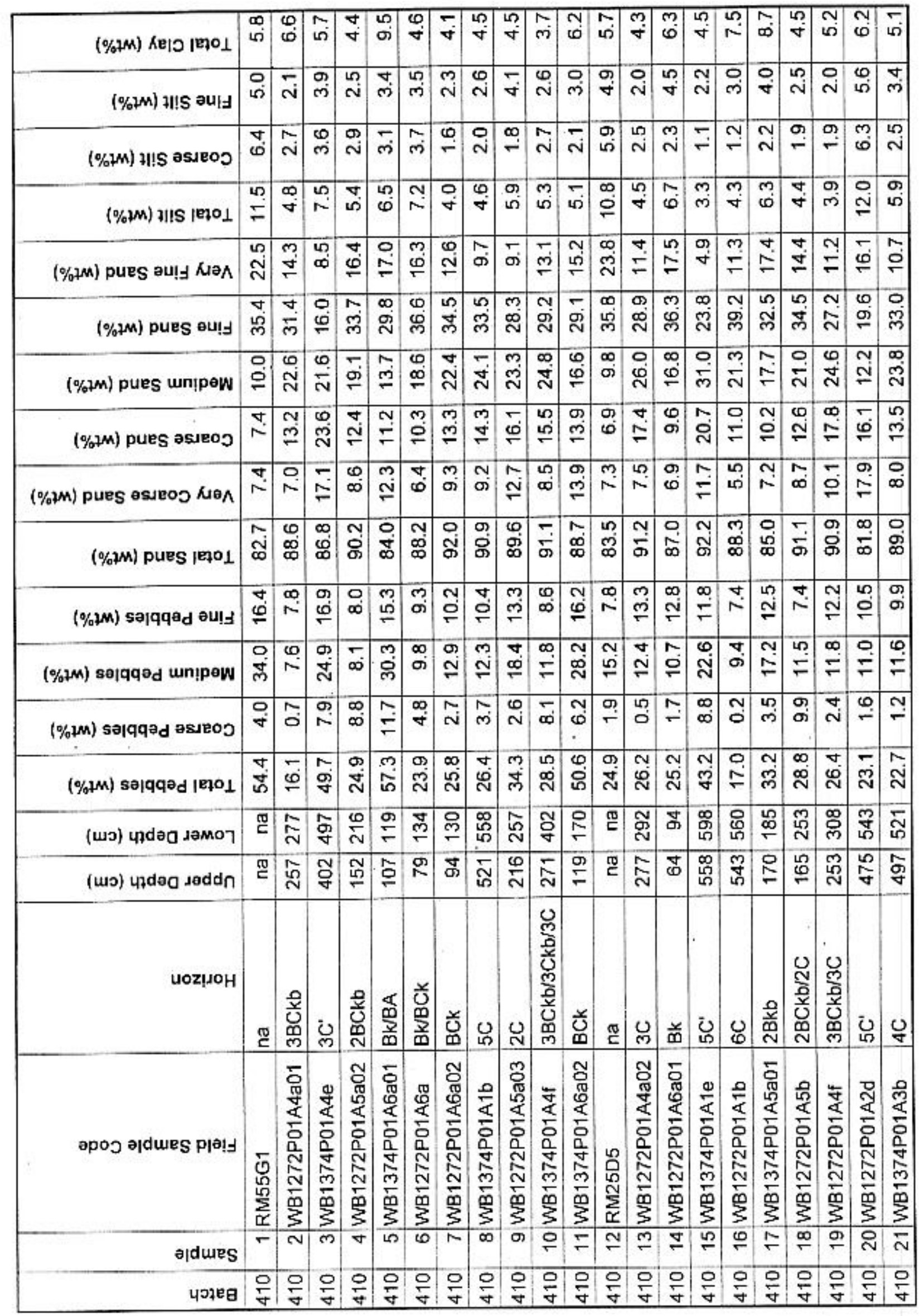

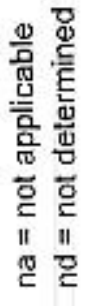




\begin{tabular}{|c|c|c|c|c|c|c|c|c|c|c|c|c|}
\hline 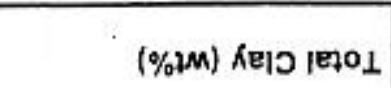 & 吕 & $\bar{j}$ & गु & \begin{tabular}{l|}
$\omega$ \\
$m$
\end{tabular} & \begin{tabular}{|l|}
0 \\
$\dot{\omega}$
\end{tabular} & $\omega$ & \begin{tabular}{|l|}
$\infty$ \\
$m$ \\
$m$
\end{tabular} & \begin{tabular}{l|}
$m$ \\
$\varphi$
\end{tabular} & $\begin{array}{l}8 \\
15\end{array}$ & $\underset{\nabla}{+}$ & \begin{tabular}{|l|} 
\\
$\dot{\nabla}$
\end{tabular} & ষ \\
\hline (\%\%M) म!!S घu!y & $\stackrel{w}{N}$ & $\stackrel{\circ}{\circ}$ & $\stackrel{\mathscr{N}}{\mathrm{N}}$ & $\stackrel{M}{=}$ & $\underset{\nabla}{\nabla}$ & ले & 雚 & $\begin{array}{l}N \\
\text { m }\end{array}$ & $\stackrel{N}{m}$ & $\begin{array}{l}\text { 足 } \\
0 \\
0\end{array}$ & $\bar{m}$ & $\stackrel{m}{\leftarrow}$ \\
\hline (\%?M) मI!S Osueoว & $\stackrel{m}{-}$ & ले & \begin{tabular}{|l|}
$\infty$ \\
$m$
\end{tabular} & $\stackrel{-1}{-}$ & \begin{tabular}{l|} 
\\
$c$
\end{tabular} & $\stackrel{m}{+}$ & \begin{tabular}{|l|}
0 \\
$\mathrm{~N}$
\end{tabular} & \begin{tabular}{l|} 
\\
m
\end{tabular} & $\stackrel{m}{\nabla}$ & $\begin{array}{l}\phi \\
\infty\end{array}$ & $\bar{i}$ & क \\
\hline$(\% 2 M)$ ZIIS |E¥OL & \begin{tabular}{l|}
$\infty$ \\
$\infty$ \\
$m$
\end{tabular} & $\begin{array}{l}+ \\
\dot{v}\end{array}$ & $\bar{c}$ & ल) & $\stackrel{2}{\circ}$ & $\nabla$ & $\begin{array}{l}0 \\
\dot{v}\end{array}$ & $\begin{array}{l} \pm \\
0\end{array}$ & n? & ন্ & Ni & $\underset{N}{N}$ \\
\hline (\%+M) pues au!d Kuan & $\begin{array}{l}5 \\
0 \\
0\end{array}$ & $\stackrel{\sigma}{\leftarrow}$ & 운 & in & $\begin{array}{l}\text { ๙ } \\
\text { ฟ }\end{array}$ & $\begin{array}{l}0 \\
2 \\
2\end{array}$ & \begin{tabular}{|l|}
0 \\
0 \\
\end{tabular} & \begin{tabular}{|l|}
0 \\
$\varphi$ \\
\end{tabular} & ?ํํㅇ & $\overline{\mathrm{N}}$ & $\begin{array}{l}m \\
\dot{v}\end{array}$ & O \\
\hline (\%2M) pues euly & 品 & $\begin{array}{l}\text { N్ } \\
\text { ల్m }\end{array}$ & $\overline{\dot{g}}$ & $\begin{array}{l}\infty \\
= \\
=\end{array}$ & $\begin{array}{l}\mathrm{M} \\
\mathrm{m}\end{array}$ & \begin{tabular}{l|}
$m$ \\
gi \\
\end{tabular} & $\begin{array}{c}N \\
\text { g } \\
\text { | }\end{array}$ & \begin{tabular}{|c|} 
\\
0 \\
0
\end{tabular} & $\begin{array}{l}\text { की } \\
\text { ஸी }\end{array}$ & $\stackrel{\infty}{\sim}$ & $\begin{array}{l}\text { क } \\
\dot{m}\end{array}$ & $\begin{array}{l}\mathbf{Z} \\
\text { N }\end{array}$ \\
\hline (\%ұM) pues un!paw & $\begin{array}{l}\mathbb{L}_{0} \\
\infty \\
\leftarrow\end{array}$ & 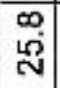 & $\stackrel{N}{\infty}$ & $\overline{\mathrm{m}}$ & 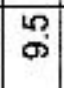 & กี & İ & $\begin{array}{l} \\
\Phi \\
\emptyset\end{array}$ & जे & No & $\begin{array}{l}\infty \\
\text { ○े }\end{array}$ & 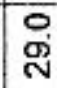 \\
\hline (\%ҰM) pues asueoJ & $\stackrel{\mathbb{Z}}{=}$ & $\underset{\square}{\square}$ & ? & 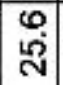 & \begin{tabular}{|l|}
$\infty$ \\
0 \\
0
\end{tabular} & $\stackrel{\circ}{\stackrel{0}{\sim}}$ & 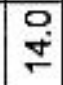 & $\bar{\sigma}$ & $\stackrel{5}{5}$ & 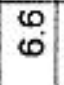 & $\stackrel{\circ}{\sim}$ & $\stackrel{\infty}{\check{N}}$ \\
\hline (\%1M) pues EsueoJ KäA & $\begin{array}{l}0 \\
\infty\end{array}$ & 50 & ro & \begin{tabular}{|l|}
\multirow{2}{*}{} \\
$\mathrm{m}$ \\
$\mathrm{n}$
\end{tabular} & \begin{tabular}{|l|}
$\infty$ \\
\\
\end{tabular} & \begin{tabular}{|l|}
$\infty$ \\
$\infty$
\end{tabular} & $\infty$ & $m$ & $\aleph_{\infty}$ & के & $\check{\infty}^{\prime}$ & $\begin{array}{l}N \\
\text { w }\end{array}$ \\
\hline 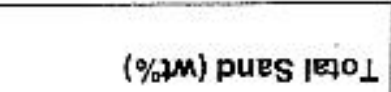 & $\frac{N}{\square}$ & $\begin{array}{l}\text { ஸे } \\
\text { ऊु }\end{array}$ & $\stackrel{m}{\mathscr{\infty}}$ & $\begin{array}{l}m \\
m \\
\delta \\
\delta\end{array}$ & ָָ & $\bar{\infty}$ & ชู & $\begin{array}{c}N \\
\stackrel{\infty}{\infty} \\
\end{array}$ & $\bar{\infty}$ & $\begin{array}{l}40 \\
6 \\
6\end{array}$ & $\begin{array}{l}\text { ํ. } \\
\text { ه̆ }\end{array}$ & एా \\
\hline (\% \% & \% & $\stackrel{m}{\forall}$ & $\stackrel{\infty}{N}$ & $\begin{array}{l}\underset{N}{N} \\
\mathfrak{m}\end{array}$ & $\bar{\infty}$ & $\begin{array}{l}0 \\
0\end{array}$ & os & $\begin{array}{l}0 \\
6\end{array}$ & $\stackrel{F}{=}$ & $\stackrel{\infty}{=}$ & $\begin{array}{l}m \\
\infty\end{array}$ & $\stackrel{m}{p}$ \\
\hline (\%)M) solqqed un!pew & $\stackrel{m}{=}$ & ํ. & $\begin{array}{c}5 \\
\sigma i\end{array}$ & $\begin{array}{l}0 \\
\stackrel{6}{\circ}\end{array}$ & $\begin{array}{l}m \\
m \\
m\end{array}$ & $\begin{array}{l}\sigma \\
\sigma\end{array}$ & 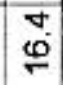 & $\underset{m}{\stackrel{2}{2}}$ & $\stackrel{\infty}{\leftarrow}$ & $\stackrel{\infty}{\infty}$ & 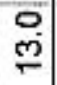 & J \\
\hline (\%1M) selqqad asueo? & $\stackrel{m}{\sigma}$ & $\bar{g}$ & is & \begin{tabular}{|l|} 
\\
ले
\end{tabular} & ì & $\begin{array}{ll} \\
\stackrel{N}{*}\end{array}$ & $\begin{array}{l}\infty \\
\varphi\end{array}$ & क़ & 虫 & 志 & $\bar{\infty}$ & ஸั \\
\hline 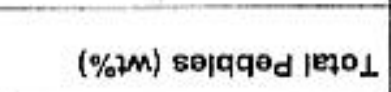 & 宛 & $\begin{array}{l}\infty \\
1 \\
10\end{array}$ & m & m. & $\begin{array}{l}\dot{N} \\
\dot{N}\end{array}$ & $\frac{\dot{2}}{\grave{N}}$ & ষ্ঠ & $\frac{\varphi}{\dot{m}}$ & $\begin{array}{l}N \\
\stackrel{N}{~}\end{array}$ & $\begin{array}{l}\infty \\
\tilde{N}\end{array}$ & $\begin{array}{l}\nabla \\
\text { I্. }\end{array}$ & 守 \\
\hline 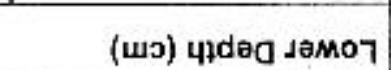 & $\stackrel{8}{\circ}$ & $\begin{array}{l}\infty \\
⿱ \\
m \\
m\end{array}$ & 足 & 等 & 界 & 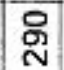 & $\stackrel{\mathbb{N}}{\mathbb{N}}$ & กิ & 守 & $\stackrel{\mathbb{8}}{\mathrm{C}}$ & $\begin{array}{c}E \\
N\end{array}$ & 峁 \\
\hline (wo) ułdəa Jəddn & ले & 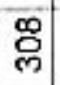 & $\stackrel{\text { 㟔 }}{=}$ & Y & 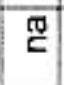 & $\stackrel{N}{N}$ & $\stackrel{\mathbb{N}}{\text { N }}$ & 임 & $\begin{array}{l}\infty \\
\text { ले }\end{array}$ & $\stackrel{\pi}{C}$ & 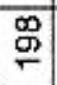 & 品 \\
\hline 4оz!」OH & 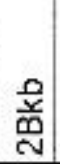 & ن्ల & $\begin{array}{l}\text { 总 } \\
\text { 怘 }\end{array}$ & U & 肯 & $\begin{array}{l}\text { 妾 } \\
\text { 品 }\end{array}$ & U & $\begin{array}{l}\text { 畣 } \\
\text { 总 }\end{array}$ & Y & $\stackrel{\mathbb{2}}{\check{2}}$ & 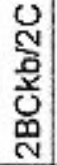 & ي \\
\hline apoj ㅋ|dures pla! & 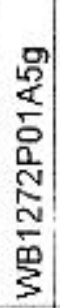 & 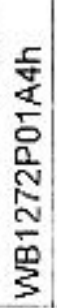 & 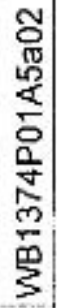 & 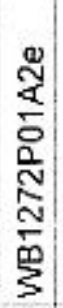 & $\begin{array}{l}\infty \\
0 \\
\sum_{\alpha}^{2}\end{array}$ & 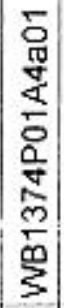 & 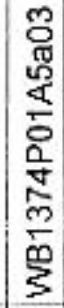 & 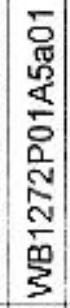 & 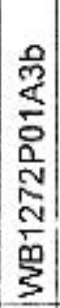 & 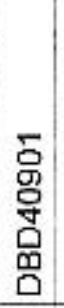 & 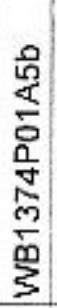 & 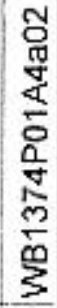 \\
\hline ә|durs & $\mathbb{N}$ & $\ddot{N}$ & 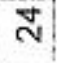 & $\stackrel{2}{\sim}$ & $\stackrel{\sim}{N}$ & 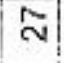 & $\stackrel{\infty}{N}$ & g & 유: & $\bar{m}$ & న్ & m \\
\hline чग्ञ & $\frac{0}{4}$ & $\frac{0}{8}$ & $\frac{0}{8}$ & $\frac{9}{4}$ & $\frac{0}{8}$ & $\frac{0}{8}$ & $\frac{\circ}{4}$ & $\frac{9}{8}$ & $\frac{9}{4}$ & $\frac{9}{8}$ & 임 & $\frac{0}{8}$ \\
\hline
\end{tabular}


This Page Intentionally Left Blank 


\section{APPENDIX C}

Chemical Parameters 


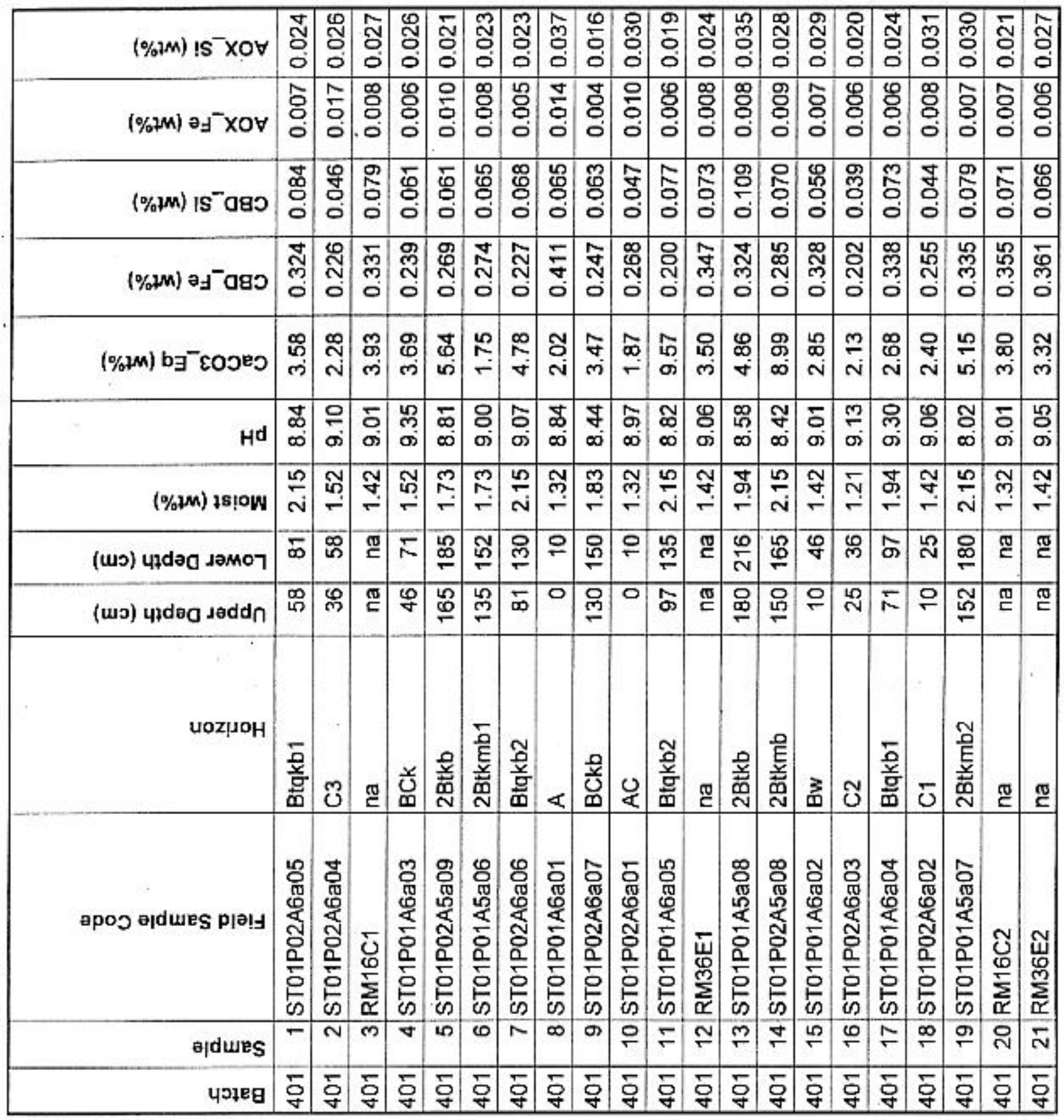




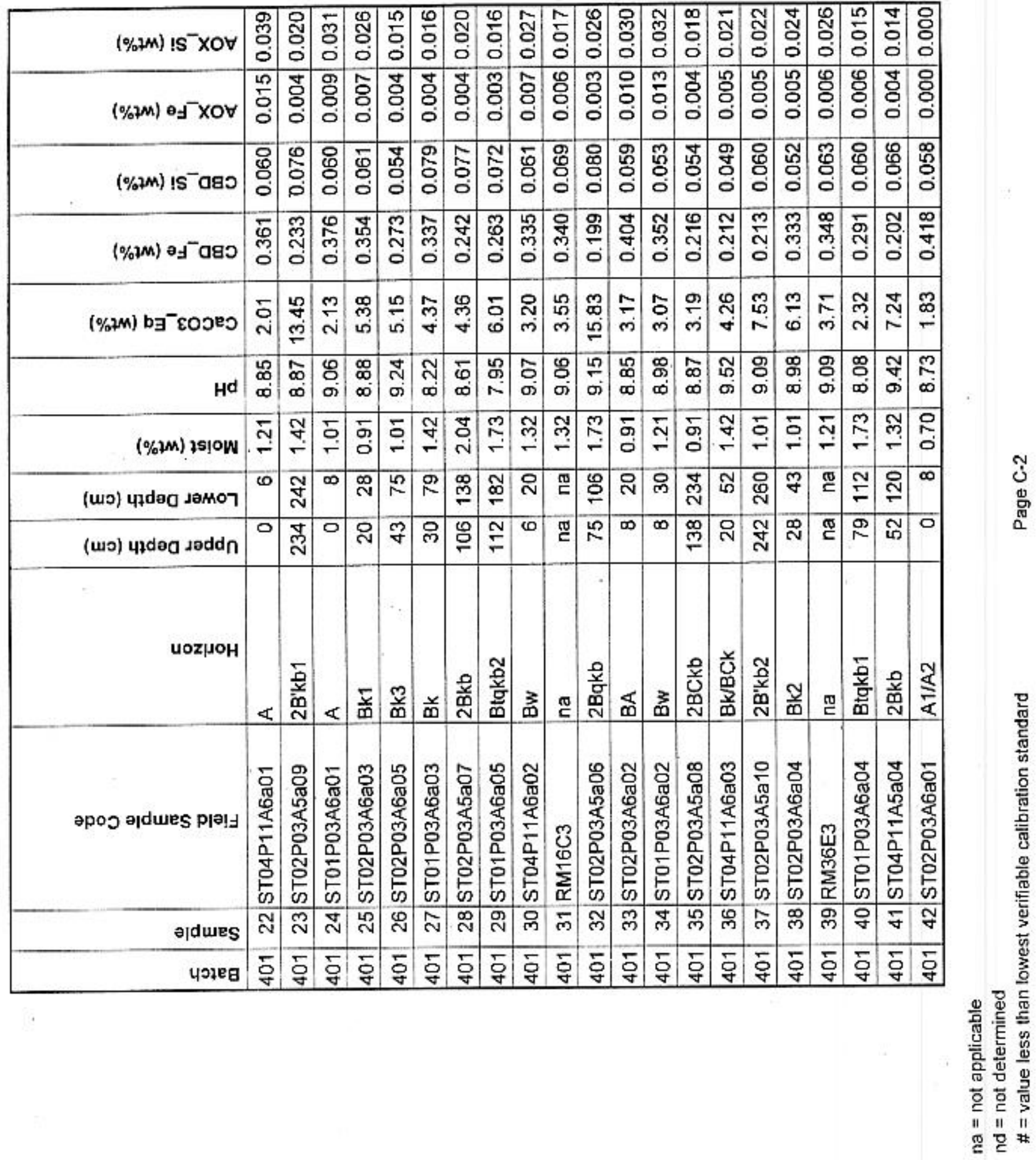




\begin{tabular}{|c|c|c|c|c|c|c|c|c|c|c|c|c|c|c|c|c|c|c|c|c|c|}
\hline$(\%$ qM) is $\mathrm{XOH}$ & $\stackrel{N}{\mathrm{~N}}$ & $\begin{array}{l}\infty \\
\vdots \\
0 \\
0\end{array}$ & 용 & 㸵 & $\begin{array}{l}\frac{42}{5} \\
0 \\
0\end{array}$ & $\begin{array}{l}\infty \\
\text { ర్ } \\
0 \\
0\end{array}$ & 공 & 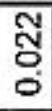 & $\begin{array}{l}\text { m } \\
\text { O }\end{array}$ & 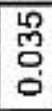 & 今̀ & $\begin{array}{l}\text { N } \\
\text { O }\end{array}$ & ָั & 喈 & $\begin{array}{l}\mathscr{D} \\
\text { ర్ } \\
0\end{array}$ & $\begin{array}{l}\text { S } \\
\text { O } \\
0\end{array}$ & 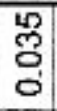 & $\begin{array}{l}\text { m } \\
\stackrel{8}{0} \\
0\end{array}$ & $\begin{array}{l}\mathscr{m} \\
\\
\end{array}$ & $\begin{array}{l}\text { 巳 } \\
0 \\
0\end{array}$ & 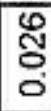 \\
\hline$(\% \not M) \partial^{-}$XOV & $\begin{array}{l}\infty \\
8 \\
0 \\
0\end{array}$ & 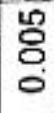 & 8 & $\stackrel{\circ}{\circ}$ & 농 & $\begin{array}{l}0 \\
\end{array}$ & 웅 & ֻூ & 욤 & 둥 & 닝 & 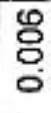 & 용 & $\begin{array}{l}\frac{N}{5} \\
0 \\
0\end{array}$ & $\begin{array}{l}\text { 영 } \\
\text { 웅 }\end{array}$ & 용 & $\begin{array}{l}\mathscr{O} \\
\\
0 \\
0\end{array}$ & 음 & $\begin{array}{l}\text { 옹 } \\
\text { ' }\end{array}$ & $\begin{array}{l}\text { ঠ } \\
\text { O } \\
\text { ○ }\end{array}$ & 응 \\
\hline (\%1M) is-ago & $\begin{array}{l}\stackrel{M}{8} \\
\stackrel{g}{0}\end{array}$ & $\begin{array}{l}\text { O } \\
\text { J } \\
\stackrel{0}{0}\end{array}$ & प్ & $\begin{array}{l}0 \\
\text { 웅 } \\
0\end{array}$ & $\begin{array}{l}\text { लू } \\
\text { ర్ }\end{array}$ & $\begin{array}{l}\text { 芦 } \\
\text { 웅 }\end{array}$ & 옹 & $\begin{array}{l}\text { V } \\
\text { O }\end{array}$ & 동 & 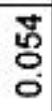 & 胥 & ల్ & $\begin{array}{l}\infty \\
\text { \& } \\
0 \\
0\end{array}$ & 氶 & 志 & $\begin{array}{l}0 \\
0 \\
0\end{array}$ & 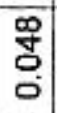 & 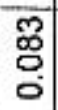 & ষ্ & $\begin{array}{l}9 \\
0 \\
0\end{array}$ & 훙 \\
\hline (\%) $\%$ 甲 & 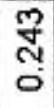 & $\frac{ \pm}{\sigma}$ & $\frac{\stackrel{n}{2}}{0}$ & సे & $\underset{0}{=}$ & $\begin{array}{l}\text { స్ } \\
\text { న్ }\end{array}$ & $\frac{5}{0}$ & $\stackrel{\sim}{\stackrel{N}{0}}$ & $\begin{array}{l}\stackrel{\mathscr{N}}{N} \\
\text { }\end{array}$ & $\begin{array}{l}\text { 呓 } \\
\underset{0}{0}\end{array}$ & 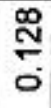 & $\frac{g}{\frac{g}{+}}$ & $\frac{10}{8}$ & 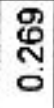 & 占 & $\begin{array}{l}\text { 옹 } \\
\text { ํ. }\end{array}$ & $\begin{array}{l}\mathscr{S} \\
\text { N } \\
0\end{array}$ & $\frac{m}{m}$ & 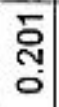 & $\begin{array}{l}\text { గ్ర } \\
\text { న్ } \\
0\end{array}$ & $\begin{array}{l}\text { J } \\
\text { ป̀ }\end{array}$ \\
\hline (\%? & $\frac{\sigma}{m}$ & $\begin{array}{l}\varnothing \\
\oplus 0 \\
\stackrel{0}{\circ}\end{array}$ & $\stackrel{0}{0}$ & $\frac{\varphi}{\text { v }}$ & $\stackrel{n}{m}$ & ले & $\begin{array}{l}\text { co } \\
\text { civ }\end{array}$ & $\underset{8}{\infty}$ & 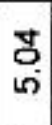 & in & $\stackrel{\text { న్ }}{\leftarrow}$ & $\underset{⿱}{\mathscr{O}}$ & $\stackrel{\mathscr{N}}{N}$ & $\begin{array}{l}\text { g. } \\
\text { ले }\end{array}$ & $\begin{array}{l}\stackrel{\sim}{N} \\
\text { m }\end{array}$ & $\underset{\omega}{\bar{\omega}}$ & $\underset{\text { ָे }}{\stackrel{N}{*}}$ & 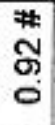 & 号 & m & ष \\
\hline Hd & $\begin{array}{l}\infty \\
\infty \\
\infty\end{array}$ & $\frac{\omega}{\sigma}$ & ले & $\begin{array}{l}3 \\
\text { Oे }\end{array}$ & $\underset{\infty}{\infty}$ & $\begin{array}{l}\stackrel{\mathscr{D}}{ } \\
\text { ळi }\end{array}$ & $\underset{\sigma}{\stackrel{\Xi}{~}}$ & $\begin{array}{l}\stackrel{\infty}{+} \\
\text { के }\end{array}$ & $\underset{\infty}{\stackrel{N}{\sim}}$ & 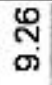 & $\begin{array}{l}\mathscr{O} \\
\infty \\
\infty\end{array}$ & $\begin{array}{l}\stackrel{0}{0} \\
\text { ఉ. }\end{array}$ & $\stackrel{N}{\infty}$ & $\begin{array}{l}\varnothing \\
\infty \\
\infty\end{array}$ & लై & $\begin{array}{l}\text { W } \\
\infty\end{array}$ & $\begin{array}{l}\text { J } \\
\text { क }\end{array}$ & $\underset{\infty}{\mathbb{N}}$ & $\begin{array}{l}\mathbb{N} \\
\text { in }\end{array}$ & $\underset{\infty}{\bar{\phi}}$ & $\frac{\mathrm{N}}{\mathrm{T}}$ \\
\hline$(\%+M)$ zs!ow & $\underset{\sim}{\stackrel{Y}{+}}$ & $\stackrel{m}{=}$ & ભำ & ָำ & $\underset{\leftarrow}{\leftarrow}$ & 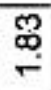 & $\begin{array}{l}0 \\
0 \\
0\end{array}$ & $\underset{7}{\digamma}$ & $\stackrel{m}{\infty}$ & $\stackrel{m}{\infty}$ & $\underset{\sim}{\mathrm{q}}$ & $\underset{\infty}{\infty}$ & $\begin{array}{l}\bar{\infty} \\
0\end{array}$ & 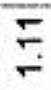 & 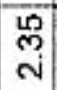 & $\underset{\leftarrow}{ָ}$ & 梁 & $\stackrel{n}{\mathrm{~N}}$ & $\begin{array}{c}\mathrm{N} \\
\mathrm{r}\end{array}$ & ल & $\stackrel{?}{?}$ \\
\hline (wo) uдdao Jamo & 党 & ก & $\stackrel{\circ}{\circ}$ & $\infty$ & 요 & $\underset{N}{N}$ & $\underset{\bar{N}}{\bar{\sigma}}$ & 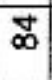 & $\stackrel{\Xi}{ \pm}$ & $\stackrel{\sigma}{\leftarrow}$ & $\frac{10}{i v}$ & 요 & $\stackrel{\varphi}{\div}$ & $\stackrel{\omega}{\circ}$ & เป & $\underset{0}{\mathscr{6}}$ & (돋 & 음 & $\mathscr{Q}$ & ్ㅗㅇ & 只 \\
\hline (wo) чมdә dəddn & 믇 & 유 & $n$ & 0 & $\frac{40}{\sqrt{N}}$ & 5 & 品 & ก & $\underset{N}{N}$ & $\infty$ & $\dddot{8}$ & $\mathscr{O}$ & 岕 & 0 & $\stackrel{\sigma}{-}$ & 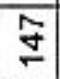 & $\stackrel{\pi}{\complement}$ & চ্ণ & $\stackrel{\infty}{\mp}$ & $\stackrel{\circ}{\infty}$ & n \\
\hline & 苋 & 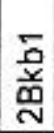 & 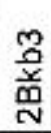 & 迎 & 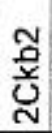 & $\begin{array}{l}\text { 总 } \\
\text { ले }\end{array}$ & 总. & $\frac{N}{\frac{N}{\Delta}}$ & $\begin{array}{l}\text { D } \\
\text { 总 } \\
\text { 号 }\end{array}$ & 总 & 总 & $\begin{array}{l}\text { 虽 } \\
\text { 兽 }\end{array}$ & $\begin{array}{l}\text { 号 } \\
\text { 睪 }\end{array}$ & @ & 咅 & $\begin{array}{l}\text { 总 } \\
\text { 品 }\end{array}$ & $\stackrel{\mathbb{Q}}{\mathrm{C}}$ & 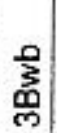 & 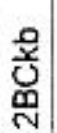 & 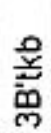 & $\frac{\bar{x}}{\text { m }}$ \\
\hline apos ㅎdues pן비 & 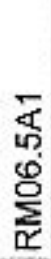 & 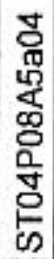 & 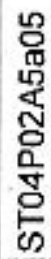 & 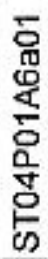 & 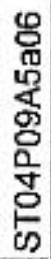 & 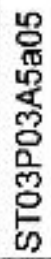 & 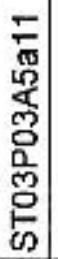 & 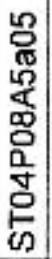 & 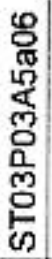 & 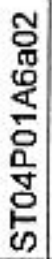 & 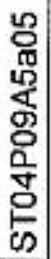 & 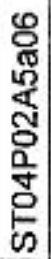 & 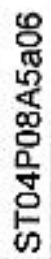 & 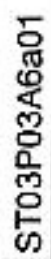 & $\begin{array}{l}\text { m } \\
\text { 员 } \\
\mathbb{1} \\
5 \\
0 \\
0 \\
0 \\
0 \\
0\end{array}$ & 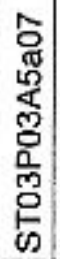 & 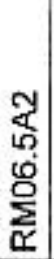 & $\begin{array}{l}\frac{N}{\pi} \\
\frac{1}{2} \\
\frac{1}{6} \\
\frac{0}{m} \\
0 \\
\frac{6}{6}\end{array}$ & 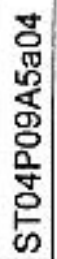 & 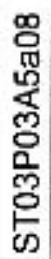 & 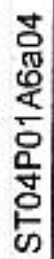 \\
\hline ajdues & - & $\sim$ & $m$ & ष & 10 & $\omega$ & $N$ & $\infty$ & o) & 은 & $F$ & $\stackrel{2}{2}$ & $\stackrel{m}{-}$ & 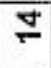 & $\frac{42}{5}$ & $\oplus$ & $\approx$ & $\stackrel{\infty}{\infty}$ & $\stackrel{D}{-}$ & 오 & $\bar{N}$ \\
\hline 4शำ & 尺 & 今 & 尺 & P & ญे & 尺 & 尺े & 욤 & S & Y & $\stackrel{\mathcal{O}}{\mathcal{O}}$ & O & $\stackrel{\mathrm{O}}{\mathrm{O}}$ & $\underset{\mathrm{V}}{\mathrm{V}}$ & $\underset{\forall}{\mathscr{V}}$ & $\stackrel{\mathcal{O}}{\mathrm{V}}$ & O & J & $\stackrel{\mathcal{O}}{\mathrm{O}}$ & $\stackrel{8}{8}$ & 용 \\
\hline
\end{tabular}




\begin{tabular}{|c|c|c|c|c|c|c|c|c|c|c|c|c|c|c|c|c|c|c|c|c|}
\hline$(\% 7 M) ! S^{-} X O \forall$ & $\begin{array}{l}\overline{0} \\
0 \\
0\end{array}$ & $\begin{array}{l}\text { m } \\
\text { ปे } \\
0\end{array}$ & $\begin{array}{l}\text { m్ } \\
\text { '. } \\
\text {. }\end{array}$ & 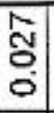 & $\begin{array}{l}\infty \\
\stackrel{0}{0} \\
0 \\
0\end{array}$ & 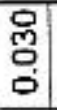 & 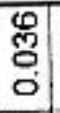 & $\begin{array}{l}\text { N } \\
\text { D. } \\
0\end{array}$ & 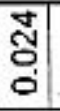 & $\begin{array}{l}0 \\
0 \\
0 \\
\end{array}$ & $\begin{array}{l}\text { N } \\
\text { O } \\
\dot{0}\end{array}$ & $\begin{array}{l}\text { क } \\
\text { o } \\
0\end{array}$ & $\begin{array}{l}\tilde{N} \\
0 \\
0 \\
0\end{array}$ & $\begin{array}{l}0 \\
\mathbb{O} \\
0 \\
0\end{array}$ & $\begin{array}{l}\text { 이 } \\
\text { 今. } \\
\text { d. }\end{array}$ & $\begin{array}{l}\text { M } \\
\text { g. } \\
0\end{array}$ & 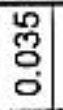 & 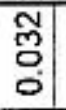 & 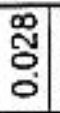 & $\begin{array}{l}\text { N } \\
\text { O } \\
\text { O }\end{array}$ \\
\hline$(\% 2 M)$ o ${ }^{-} \mathrm{XOV}$ & $\begin{array}{l}\text { 寸 } \\
\text { 口 } \\
0\end{array}$ & $\stackrel{m}{\circ}$ & $\begin{array}{l}4 \\
\text { 영 } \\
0\end{array}$ & $\begin{array}{l} \\
\\
0 \\
0\end{array}$ & $\begin{array}{l}0 \\
8 \\
8 \\
0\end{array}$ & $\begin{array}{l} \\
\\
0 \\
0\end{array}$ & $\begin{array}{l}\text { 영 } \\
\text { 영 }\end{array}$ & 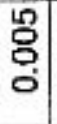 & $\begin{array}{l}\text { M } \\
\text { 잉 } \\
\end{array}$ & $\begin{array}{l}\text { 영 } \\
\text { 잉 }\end{array}$ & $\begin{array}{l}\infty \\
0 \\
\\
\\
0\end{array}$ & $\begin{array}{l}\overline{5} \\
0 \\
0\end{array}$ & $\begin{array}{l}9 \\
8 \\
0\end{array}$ & $\begin{array}{l}8 \\
8 \\
8 \\
0\end{array}$ & 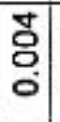 & $\begin{array}{l}\text { g. } \\
\text { 용 } \\
0\end{array}$ & $\underset{0}{\simeq}$ & $\begin{array}{l}\text { ठ } \\
\text { : } \\
\end{array}$ & $\begin{array}{l}\infty \\
\\
0\end{array}$ & $\begin{array}{l}0 \\
0 \\
0\end{array}$ \\
\hline$(\%+M)$ is & $\begin{array}{l}\text { g } \\
\text { 。 } \\
0\end{array}$ & $\begin{array}{l}\text { 足 } \\
\text { 。 } \\
0\end{array}$ & 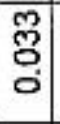 & $\begin{array}{l}\Omega \\
0 \\
0 \\
0\end{array}$ & $\begin{array}{l}\infty \\
\dot{U} \\
0 \\
0\end{array}$ & $\begin{array}{l}\text { N } \\
\text { 以 } \\
0 \\
0\end{array}$ & 동 & $\begin{array}{l}\mathscr{M} \\
0 \\
0 \\
0\end{array}$ & $\begin{array}{l} \\
\text { 잉 } \\
0 \\
0\end{array}$ & $\begin{array}{l}0 \\
0 \\
0 \\
0\end{array}$ & 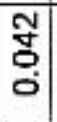 & $\begin{array}{l}\text { g } \\
\\
\\
\end{array}$ & 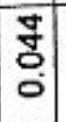 & $\begin{array}{l} \\
\text { fo } \\
0 \\
0\end{array}$ & $\begin{array}{l}\text { षे } \\
\text { Oे }\end{array}$ & $\begin{array}{l}\text { ญे } \\
\text { ¿ }\end{array}$ & 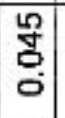 & $\begin{array}{l}\text { ח } \\
\text { \% } \\
0 \\
0\end{array}$ & $\begin{array}{l}\text { D్ } \\
\text { O } \\
0 \\
0\end{array}$ & $\begin{array}{l}\text { 응 } \\
\text { 웅 }\end{array}$ \\
\hline$(\%+M) \theta^{-}$- & $\frac{\widetilde{N}}{0}$ & $\begin{array}{l}r \\
N \\
\stackrel{N}{*}\end{array}$ & $\begin{array}{l}\infty \\
\infty \\
N \\
0 \\
0\end{array}$ & $\begin{array}{l}\mathscr{m} \\
\stackrel{\infty}{\circ} \\
\end{array}$ & $\begin{array}{l}\text { 운 } \\
\text { บै } \\
0\end{array}$ & 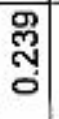 & 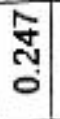 & $\begin{array}{l}10 \\
0 \\
\text { N. } \\
0\end{array}$ & $\begin{array}{l}0 \\
\frac{10}{0} \\
0\end{array}$ & $\frac{9}{\circ}$ & $\begin{array}{l}0 \\
\stackrel{\omega}{N} \\
0 \\
0\end{array}$ & ș & $\begin{array}{l}\mathscr{\mathscr { g }} \\
\text { ஸै } \\
0\end{array}$ & 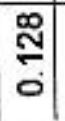 & Nָ & $\begin{array}{l}\mathscr{g} \\
\mathrm{y} \\
0 \\
0\end{array}$ & $\begin{array}{l}\text { N } \\
\text { న } \\
0\end{array}$ & $\frac{O}{0}$ & $\frac{N}{0}$ & $\begin{array}{l}\underset{\mathrm{N}}{\mathrm{N}} \\
0 \\
0\end{array}$ \\
\hline$\left(\%\right.$ (\%) b ${ }^{-}$cooes & $\begin{array}{l}n \\
\infty\end{array}$ & $\begin{array}{c}\text { mె } \\
\text { Ni }\end{array}$ & ড্য & $\begin{array}{l}0 \\
\text { N }\end{array}$ & $\begin{array}{l}\mathscr{D} \\
m\end{array}$ & $\begin{array}{l}\sigma \\
\underset{\sim}{W} \\
\infty\end{array}$ & $\begin{array}{l}\mathrm{O} \\
\mathrm{N}\end{array}$ & $\begin{array}{l}\mathscr{g} \\
\text { m. }\end{array}$ & $\begin{array}{l}\mathscr{6} \\
\text { के }\end{array}$ & E & $\begin{array}{l}0 \\
0\end{array}$ & $\begin{array}{l}\hat{v} \\
\dot{v}\end{array}$ & $\begin{array}{l}\text { 怘 } \\
\text { m }\end{array}$ & $\underset{\mathbb{N}}{\mathbb{N}}$ & $\underset{v}{\Delta}$ & $\begin{array}{l}\text { v } \\
\dot{v}\end{array}$ & $\begin{array}{c}m \\
\text { m. }\end{array}$ & $\begin{array}{l}5 \\
0\end{array}$ & $\begin{array}{l}\text { m. } \\
\text { ڤ. }\end{array}$ & $\underset{+}{\stackrel{N}{*}}$ \\
\hline $\mathrm{H}^{\mathrm{d}}$ & $\begin{array}{l}\text { g } \\
\infty\end{array}$ & $\begin{array}{l}\mathscr{0} \\
\boldsymbol{\infty}\end{array}$ & $\begin{array}{c}0 \\
\infty \\
\infty\end{array}$ & $\begin{array}{l}\text { mె } \\
\text { के }\end{array}$ & $\begin{array}{l}\bar{m} \\
\dot{\sigma}\end{array}$ & $\begin{array}{l}\checkmark \\
\text { के }\end{array}$ & $\begin{array}{l}\mathscr{S} \\
\sigma\end{array}$ & 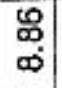 & $\frac{m}{\infty}$ & $\begin{array}{l}4 \\
\text { 员 } \\
\infty\end{array}$ & $\begin{array}{l}\text { पे } \\
\text { ம }\end{array}$ & $\begin{array}{l}\check{0} \\
\dot{\sigma}\end{array}$ & $\begin{array}{l}5 \\
\infty\end{array}$ & $\begin{array}{l}\mathbb{W} \\
\text { m. }\end{array}$ & $\begin{array}{l} \pm \\
\Phi\end{array}$ & $\underset{\sigma}{\sigma}$ & $\begin{array}{c}5 \\
\omega\end{array}$ & $\begin{array}{l}\mathscr{2} \\
\boldsymbol{\infty} \\
\dot{\infty}\end{array}$ & $\begin{array}{l}\text { ले } \\
\infty\end{array}$ & $\begin{array}{l}\mathscr{6} \\
\infty\end{array}$ \\
\hline (\%)M ) 18tow & ్ำ & $\bar{v}$ & $\stackrel{\check{ָ}}{\leftarrow}$ & ڤ్ & $\stackrel{v}{\checkmark}$ & ণ & గె & זั & 炾 & @ & ్ㅡㅁ & $\div$ & ח & 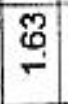 & $\stackrel{5}{\circ}$ & 岕 & $\underset{\leftarrow}{\check{T}}$ & $\stackrel{\bar{T}}{\longrightarrow}$ & $\mp$ & $\mp$ \\
\hline 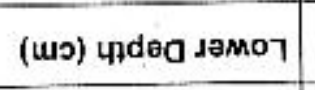 & 5 & LO & ल & 9 & $\stackrel{\infty}{亡}$ & ली & $m$ & $\infty$ & g) & ฟั. & $m$ & 유 & 蛋 & $\stackrel{g}{\circ}$ & 5 & 욤 & ฟิ & $\begin{array}{l}\text { 吕 } \\
\stackrel{N}{N}\end{array}$ & $\stackrel{N}{\sim}$ & $\omega$ \\
\hline (wo) updog seddn & 峁 & 0 & $\stackrel{\omega}{\longleftarrow}$ & $\stackrel{m}{m}$ & 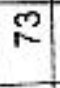 & 요 & 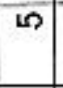 & $m$ & (9) & 옹 & N & $\omega$ & 焉 & $\mathscr{\sigma}$ & $Q$ & $\stackrel{m}{\sim}$ & 0 & N & $\stackrel{\text { m }}{-}$ & 0 \\
\hline uozщo & $\begin{array}{l}\text { ㅁ } \\
\text { ⿳亠丷厂巾 } \\
\text { N }\end{array}$ & $<$ & $\begin{array}{l}\text { 恙 } \\
\text { 㓢 }\end{array}$ & $\begin{array}{l}\mathbb{N} \\
\text { ⿳亠丷⿴囗十心 } \\
\mathbb{N}\end{array}$ & $\begin{array}{l}\frac{a}{\mathbf{y}} \\
\text { 心 } \\
\text { c }\end{array}$ & $\begin{array}{c}\frac{5}{2} \\
\text { 产 }\end{array}$ & 宅 & 愛 & $\begin{array}{l}\text { 育 } \\
\text { 产 }\end{array}$ & $\begin{array}{l}\text { 豆 } \\
\text { 商 } \\
\end{array}$ & 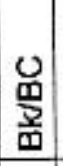 & 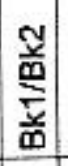 & 똗 & $\begin{array}{l}\dot{v} \\
\text { 诚 }\end{array}$ & 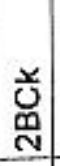 & 皿 & $\begin{array}{l}\text { ca } \\
\text { व }\end{array}$ & 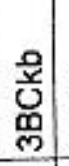 & $\begin{array}{l}\text { 仓 } \\
\text { 心 } \\
\text { N }\end{array}$ & 4 \\
\hline әроэ ㅎdues p|ㅣㅢ & 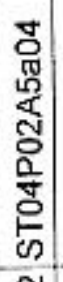 & 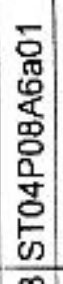 & 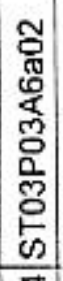 & $\begin{array}{l}\text { 음 } \\
\text { 员 } \\
\frac{4}{4} \\
0 \\
0 \\
\frac{0}{5} \\
0 \\
0\end{array}$ & 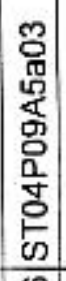 & 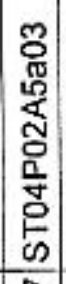 & 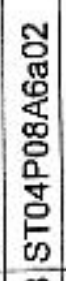 & 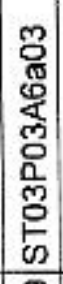 & 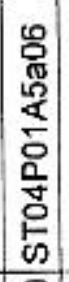 & 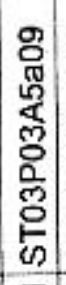 & 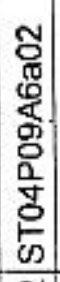 & 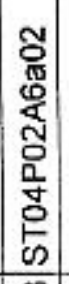 & 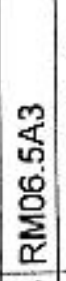 & 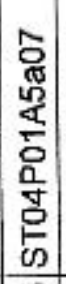 & 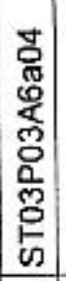 & $\begin{array}{l}3 \\
0 \\
0 \\
0 \\
0 \\
0 \\
0 \\
\frac{0}{8} \\
0 \\
0\end{array}$ & 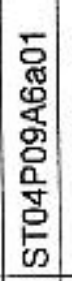 & 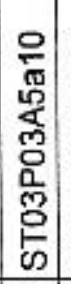 & 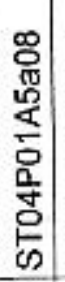 & 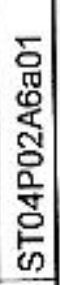 \\
\hline 키ues & N & $\stackrel{m}{N}$ & प্ & 虫 & $\stackrel{\sim}{\sim}$ & 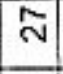 & $\stackrel{\sim}{N}$ & g & 요 & $\bar{m}$ & N్ & $m$ & ले & ஜึ & $\dot{m}$ & ल & $\begin{array}{ll}\infty \\
\infty\end{array}$ & ल & 용 & $\bar{\nabla}$ \\
\hline yगرe日 & 号 & 굥 & V & 记 & 용 & O & 尺̊ & O & 令 & ชิ & $\begin{array}{l}\text { V } \\
\text { g }\end{array}$ & 임 & 요 & 只 & 尺ั & $\stackrel{N}{8}$ & 次 & 용 & 尺ै & 尺ี \\
\hline
\end{tabular}




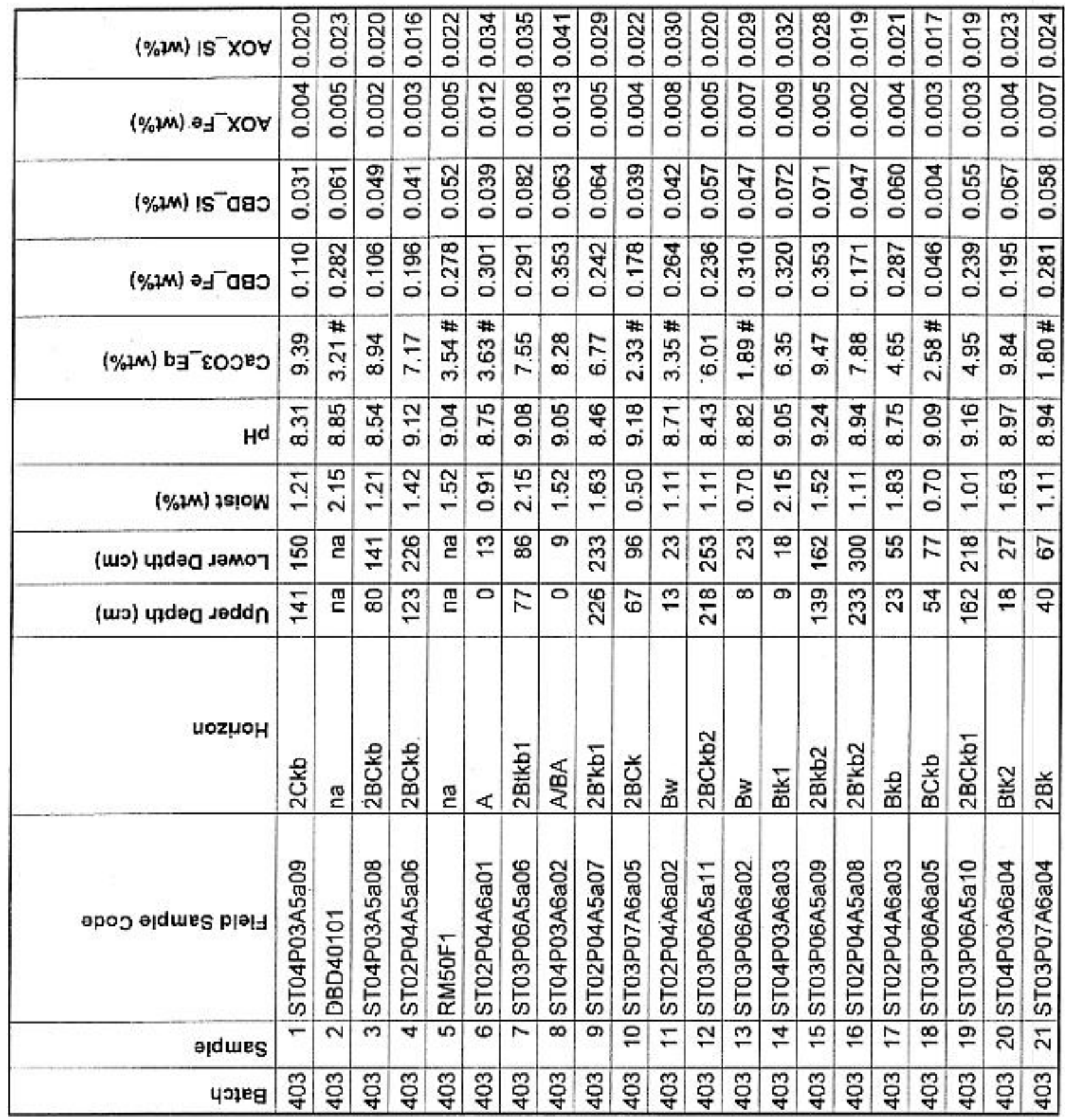




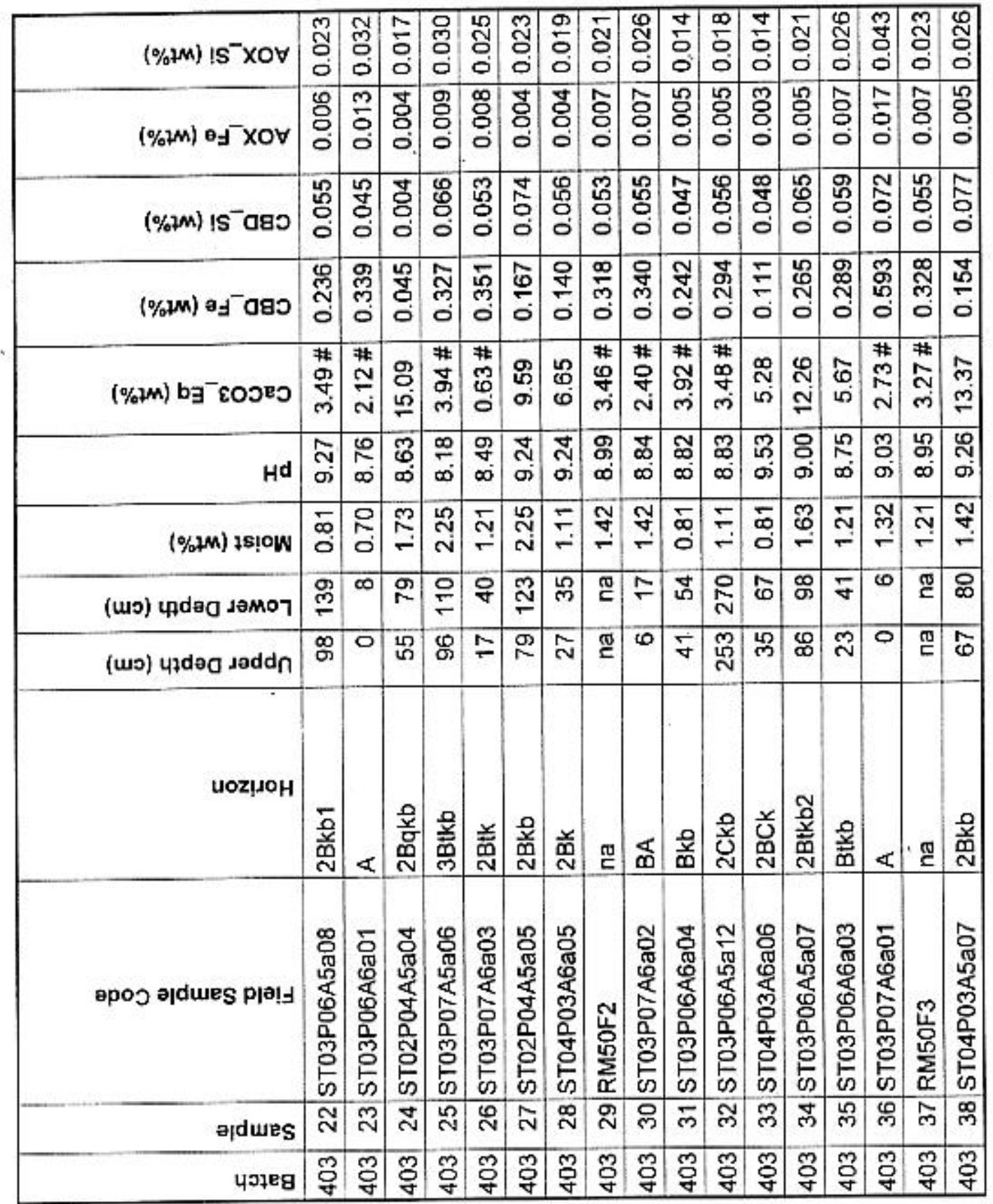




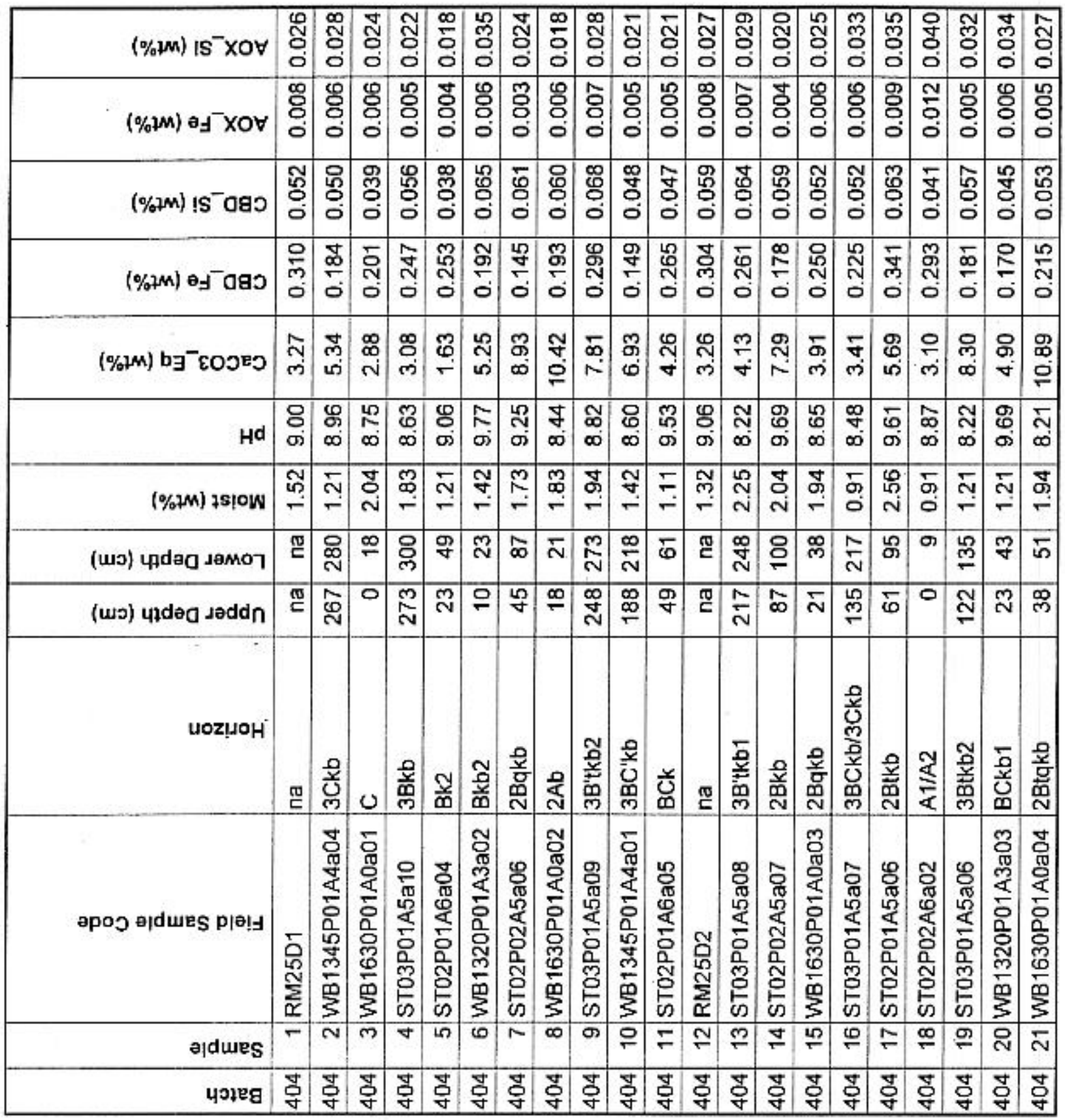




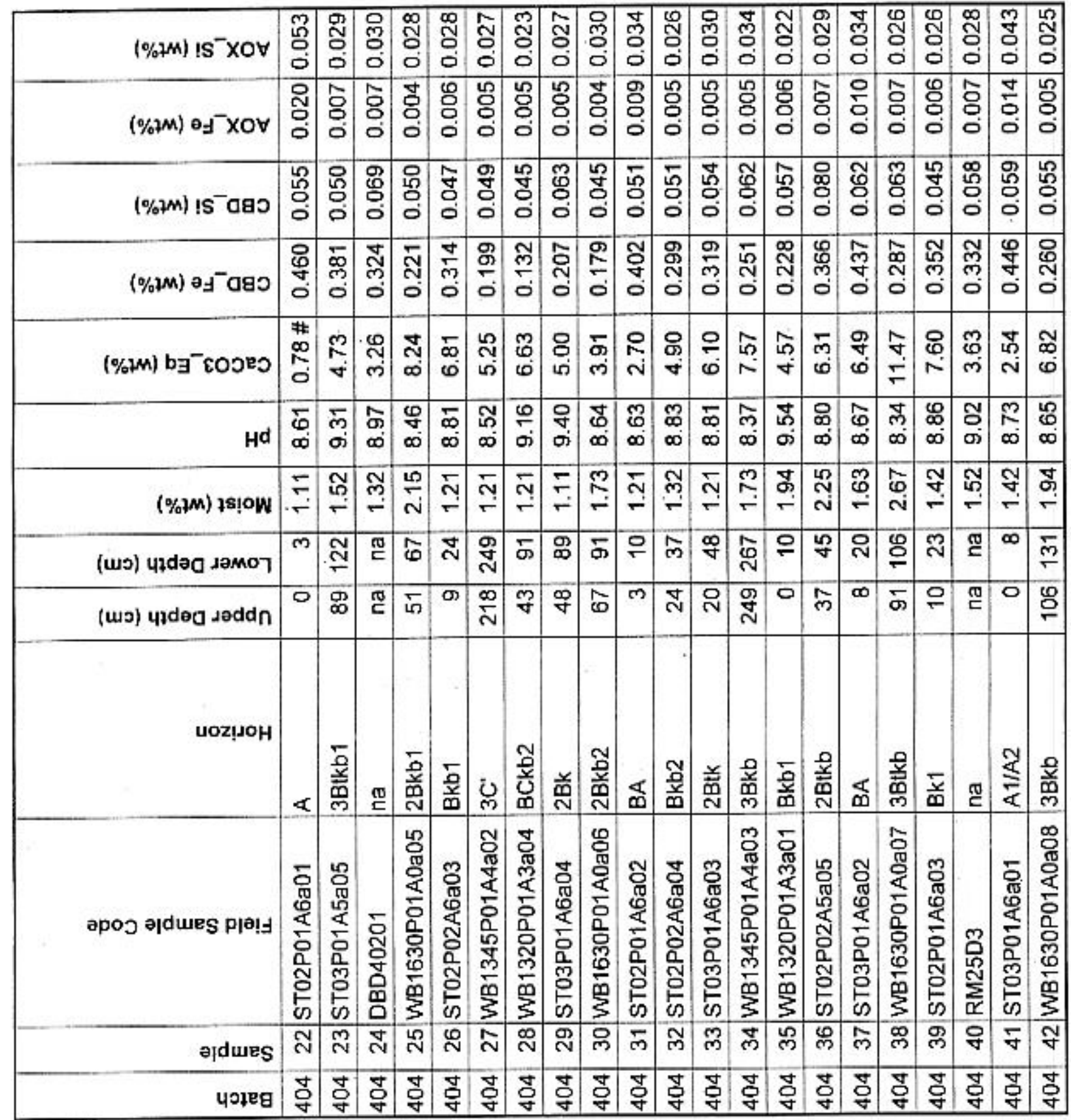




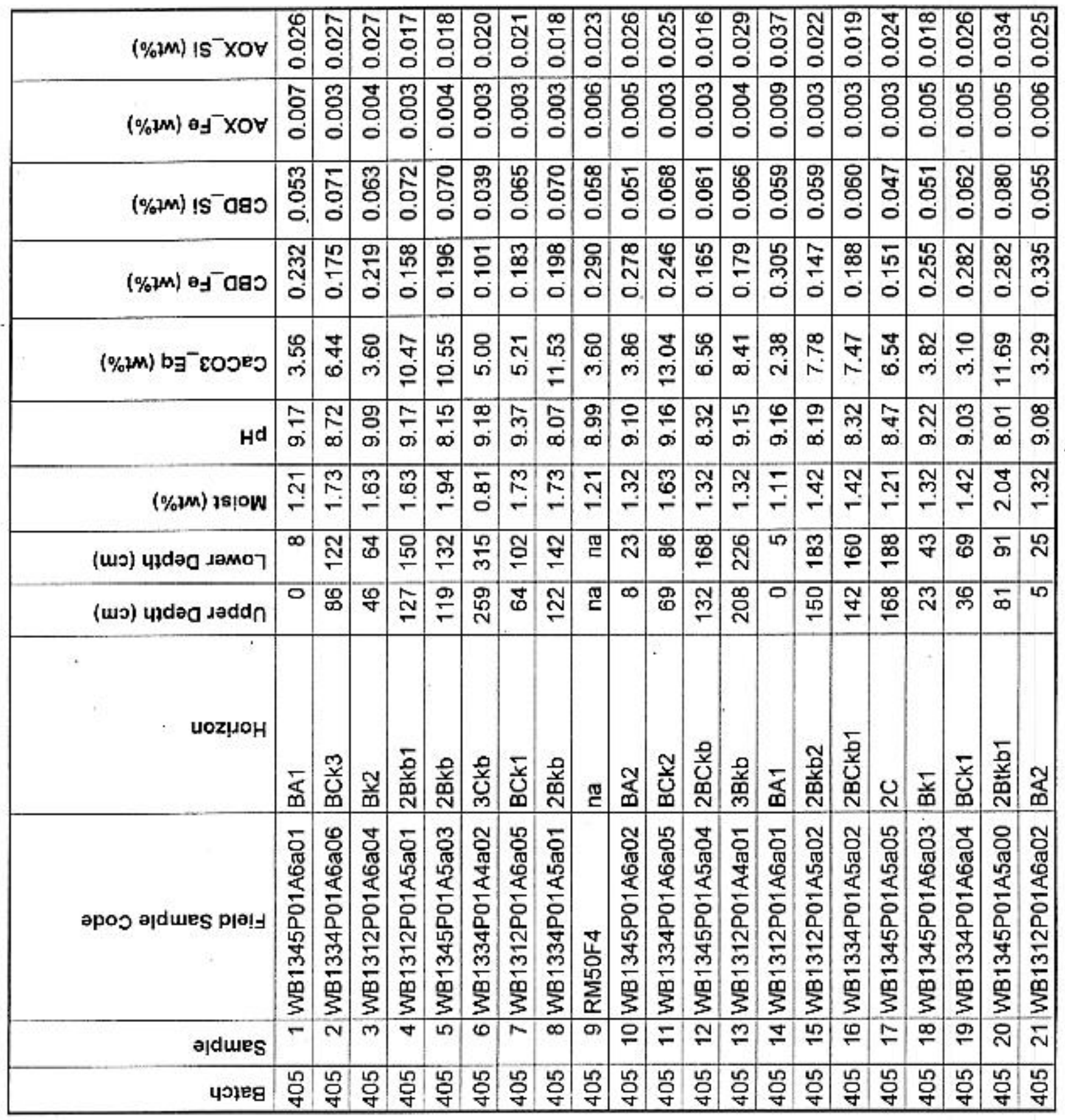




\begin{tabular}{|c|c|c|c|c|c|c|c|c|c|c|c|c|c|c|c|c|}
\hline$(\%+M)$ IS & 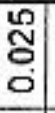 & $\begin{array}{l}9 \\
\stackrel{9}{0} \\
0\end{array}$ & $\begin{array}{l}5 \\
0 \\
0\end{array}$ & $\begin{array}{l}\mathbb{\infty} \\
\mathbb{O} \\
0\end{array}$ & 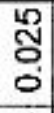 & 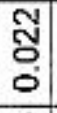 & 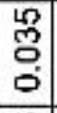 & $\begin{array}{l}1 \\
\text { No } \\
0\end{array}$ & 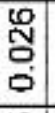 & $\begin{array}{l}\text { D } \\
\text { c } \\
\dot{0}\end{array}$ & $\begin{array}{l}\overline{0} \\
0 \\
0\end{array}$ & $\begin{array}{l}\infty \\
\vdots \\
0 \\
0\end{array}$ & $\begin{array}{l}\mathbf{5} \\
\text { ㅇ. } \\
0\end{array}$ & $\begin{array}{l}\text { 융 } \\
\text { ○ }\end{array}$ & 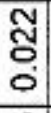 & $\stackrel{\wp}{\circ}$ \\
\hline$(\%) M)$ o $\mathrm{XOV}$ & $\begin{array}{l}3 \\
8 \\
\\
\end{array}$ & 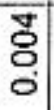 & 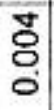 & $\begin{array}{l}\text { 농 } \\
\text { 웅 }\end{array}$ & $\begin{array}{l}\text { 巳 } \\
\text { ㅇ․ }\end{array}$ & $\begin{array}{l}\text { 농 } \\
\text { 웅 }\end{array}$ & $\begin{array}{l}\mathscr{8} \\
0 \\
0\end{array}$ & $\begin{array}{l}\text { 吕 } \\
\text { 口 }\end{array}$ & $\begin{array}{l}\text { 옹 } \\
\text { 웅 }\end{array}$ & $\begin{array}{l}\infty \\
\text { 号 } \\
0\end{array}$ & $\begin{array}{l} \pm \\
\\
\\
0\end{array}$ & $\begin{array}{l}\text { 号 } \\
\text { 马 }\end{array}$ & $\begin{array}{l}\text { ¿ } \\
\\
\\
\end{array}$ & 둥 & $\begin{array}{l}\text { 용 } \\
\text { 영 }\end{array}$ & $\begin{array}{l}\text { đ. } \\
\text { O. } \\
\text { ' }\end{array}$ \\
\hline (\%2M) Is ${ }^{-}$a日o & $\begin{array}{l}\square \\
\mathscr{O} \\
\dot{a}\end{array}$ & $\begin{array}{l}\text { 员 } \\
\stackrel{\circ}{\circ} \\
0 \\
0\end{array}$ & $\begin{array}{l}\text { 㖼 } \\
0 \\
0\end{array}$ & $\begin{array}{l}0 \\
\text { U⿱口口 } \\
0 \\
0\end{array}$ & $\begin{array}{l}\text { 웅 } \\
0 \\
0\end{array}$ & 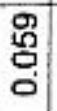 & 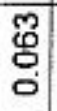 & $\begin{array}{l}\text { No } \\
0 \\
0\end{array}$ & $\begin{array}{l}1 \\
\\
0\end{array}$ & $\begin{array}{l}\square \\
5 \\
0\end{array}$ & $\begin{array}{l}\tau \\
\dot{g} \\
0\end{array}$ & $\begin{array}{l}\text { 운 } \\
5 \\
0\end{array}$ & $\begin{array}{l}\text { No } \\
0 \\
0\end{array}$ & $\begin{array}{l}0 \\
0 \\
0 \\
0 \\
0\end{array}$ & $\begin{array}{l}0 \\
\mathbb{8} \\
0 \\
0\end{array}$ & $\begin{array}{l}\text { 足 } \\
\text { \& } \\
0\end{array}$ \\
\hline (\%भม ) ว & 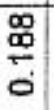 & $\begin{array}{l}\mathbb{N} \\
\stackrel{6}{0} \\
0\end{array}$ & $\frac{0}{0}$ & 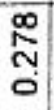 & $\frac{\mathscr{Q}}{\stackrel{\oplus}{0}}$ & $\begin{array}{l}\text { g } \\
\text { 心్d } \\
0\end{array}$ & $\begin{array}{l}\text { ల్ల } \\
\text { ले } \\
\text { o. }\end{array}$ & $\begin{array}{l}\text { 잉 } \\
\text { N. }\end{array}$ & 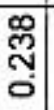 & $\begin{array}{l}\text { ले } \\
\text { ले }\end{array}$ & $\frac{0}{0}$ & $\frac{\bar{v}}{\mathrm{v}}$ & 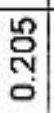 & $\begin{array}{l}\text { ల్ } \\
\text { ల్ } \\
0\end{array}$ & 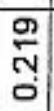 & 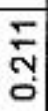 \\
\hline (\%?M) bə & $\underset{\sim}{5}$ & g & $\underset{N}{N}$ & $\begin{array}{l}\mathscr{m} \\
\text { N } \\
\text { N }\end{array}$ & $\underset{\infty}{\stackrel{ }{\nabla}}$ & $\begin{array}{l}\mathbb{W} \\
\text { ల' }\end{array}$ & $\begin{array}{l}\frac{ \pm}{2} \\
\text { ले }\end{array}$ & 吕 & $\begin{array}{l}\infty \\
\stackrel{p}{i} \\
\stackrel{2}{*}\end{array}$ & $\begin{array}{c}\mathcal{O} \\
\text { mi }\end{array}$ & $\begin{array}{l}\forall \\
\dot{y}\end{array}$ & $\begin{array}{c}\stackrel{N}{D} \\
\infty\end{array}$ & $\begin{array}{l}8 \\
8 \\
\end{array}$ & 热 & $\begin{array}{l}\text { on } \\
\text { 心 }\end{array}$ & $\underset{M}{\sigma}$ \\
\hline Hd & $\begin{array}{l}\stackrel{p}{p} \\
\infty\end{array}$ & $\frac{N}{\sigma}$ & $\begin{array}{l}\text { L } \\
0 \\
0\end{array}$ & $\begin{array}{l}\text { ల్ } \\
\text { சे }\end{array}$ & $\begin{array}{l}\mathscr{g} \\
\infty\end{array}$ & $\begin{array}{l}0 \\
0 \\
0\end{array}$ & $\begin{array}{l}\mathbf{0} \\
0 \\
0\end{array}$ & $\begin{array}{l}\infty \\
\dot{y} \\
\infty\end{array}$ & ळ) & $\begin{array}{l}\Delta \\
\text { Di } \\
\text { Di }\end{array}$ & $\begin{array}{l}0 \\
\varnothing \\
\infty\end{array}$ & $\begin{array}{l}\text { L } \\
0 \\
0\end{array}$ & $\underset{\infty}{\sigma}$ & $\stackrel{m}{\sigma}$ & ஸ্ & $\begin{array}{l}\infty \\
\infty\end{array}$ \\
\hline$(\%+m)$ 1s!̣ow & ণ & ָָ & б. & 约 & ले & $\underset{f}{\mathcal{Y}}$ & Nָ & $F$ & 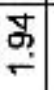 & Nָ & זั & @ִ & 次 & $\underset{F}{F}$ & $\stackrel{9}{\mathscr{C}}$ & $\begin{array}{l}\overleftarrow{\infty} \\
0\end{array}$ \\
\hline (us) updad Jәmoך & $\stackrel{9}{\circ}$ & 吕 & $\begin{array}{l}\text { ڤ్ } \\
\text { }\end{array}$ & $\mathscr{\complement}$ & $\begin{array}{l}\text { 怘 } \\
\text { 总 }\end{array}$ & 电 & 贾 & $\begin{array}{l}\infty \\
\text { 号 }\end{array}$ & क & $\stackrel{\text { Ln }}{\leftarrow}$ & 品 & $\stackrel{N}{N}$ & $\Sigma$ & $\infty$ & $\frac{\sigma}{\square}$ & 胥 \\
\hline (us) ypdea saddn & $\stackrel{8}{0}$ & 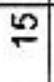 & $\begin{array}{l}\stackrel{\mathbb{N}}{\mathrm{N}} \\
\end{array}$ & m & 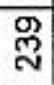 & w & Tू & 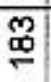 & $\bar{\sigma}$ & 40 & 番 & 응 & 吕 & 0 & g) & 穵 \\
\hline บozщоㅐ & $\begin{array}{l}\text { No } \\
\text { 总 } \\
\text { ․․ } \\
\end{array}$ & 皿 & ల్ల & $\frac{\mathcal{Y}}{0}$ & $\begin{array}{l}\text { 章 } \\
\text { 舀 }\end{array}$ & $\frac{\bar{x}}{\boldsymbol{D}}$ & 모 & ૫్ర & 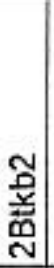 & $\begin{array}{l}\mathbb{\&} \\
\oplus\end{array}$ & ્ֻ. & 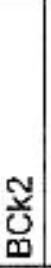 & 0 & 画 & 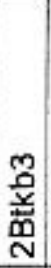 & $\stackrel{\mathbb{O}}{\mathrm{C}}$ \\
\hline 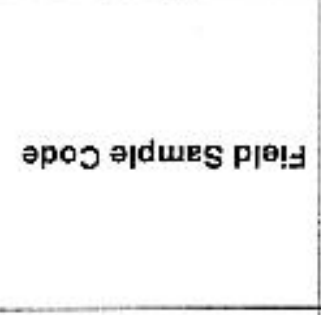 & 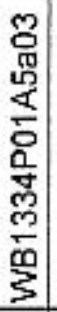 & 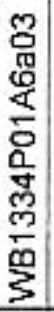 & 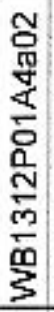 & 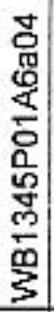 & 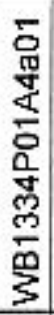 & 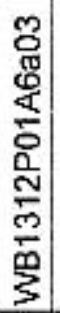 & 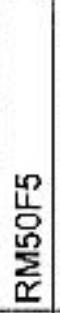 & $\begin{array}{l}\frac{m}{0} \\
\frac{1}{0} \\
\frac{4}{5} \\
\frac{0}{0} \\
\frac{0}{n} \\
\frac{n}{5} \\
\frac{1}{5}\end{array}$ & 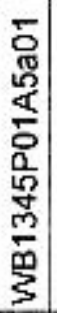 & 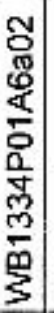 & 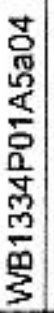 & 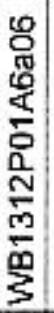 & 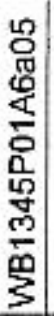 & 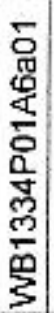 & 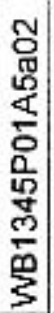 & 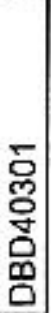 \\
\hline ydues & N & ma & पे & ก) & $\stackrel{\mathscr{v}}{\sim}$ & $\hat{N}$ & $\stackrel{\infty}{N}$ & D & m & $\bar{m}$ & N & $m$ & ले & लn & 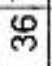 & $\bar{n}$ \\
\hline प0789 & 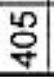 & $\begin{array}{l}5 \\
0 \\
\end{array}$ & 눙 & $\begin{array}{l}40 \\
8 \\
4\end{array}$ & $\begin{array}{l}50 \\
8 \\
4\end{array}$ & 웅 & $\begin{array}{l}50 \\
8\end{array}$ & $\begin{array}{l}40 \\
g\end{array}$ & $\begin{array}{l}40 \\
g\end{array}$ & $\begin{array}{l}\text { L } \\
\text { g }\end{array}$ & g) & 号 & $\begin{array}{l}40 \\
0 \\
\end{array}$ & $\begin{array}{l}40 \\
8\end{array}$ & Lo & $\begin{array}{l}10 \\
0 \\
\end{array}$ \\
\hline
\end{tabular}




\begin{tabular}{|c|c|c|c|c|c|c|c|c|c|c|c|c|c|c|c|c|c|c|c|c|c|}
\hline$(\% 2 M) 1 S^{-} X O \forall$ & 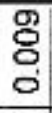 & 点 & 둥 & 잉 & 둥 & 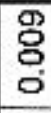 & $\begin{array}{l}9 \\
8 \\
8\end{array}$ & 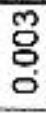 & 뭉 & $\begin{array}{l}\text { 吕 } \\
\text { 员 } \\
\end{array}$ & $\begin{array}{l}\frac{\sigma}{\tau} \\
0 \\
0 \\
0\end{array}$ & $\begin{array}{l}m \\
\\
\end{array}$ & $\begin{array}{l}5 \\
8 \\
0\end{array}$ & $\begin{array}{l}\text { m } \\
0 \\
0\end{array}$ & $\begin{array}{l}\text { Ln } \\
0 \\
0\end{array}$ & 年 & $\begin{array}{l}\overline{0} \\
0\end{array}$ & $\frac{1}{0}$ & 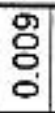 & 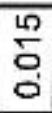 & Б \\
\hline (\%7m) of XOV & 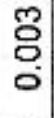 & $\begin{array}{l}\text { O̊口 } \\
\text { ㅇ } \\
0\end{array}$ & 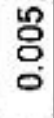 & 용 & 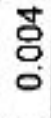 & 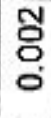 & 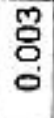 & 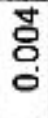 & $\begin{array}{l}9 \\
8 \\
\\
\end{array}$ & $\begin{array}{l}\text { 寸 } \\
\text { ㅇ } \\
0\end{array}$ & $\begin{array}{l}\text { पे } \\
\text { 영 }\end{array}$ & $\begin{array}{l}\text { 용 } \\
8 \\
0\end{array}$ & 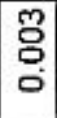 & $\begin{array}{l}\stackrel{ }{0} \\
\text { 뭉 }\end{array}$ & $\begin{array}{l}\text { 옹 } \\
\text { 잉 }\end{array}$ & 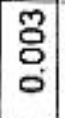 & $\begin{array}{l}\text { प } \\
0 \\
0\end{array}$ & $\begin{array}{l}\text { O̊ } \\
0 \\
0\end{array}$ & $\begin{array}{l}\text { M } \\
\\
\\
0\end{array}$ & $\begin{array}{l}\text { m } \\
\text { 웅 }\end{array}$ & 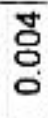 \\
\hline (\%+m) IS- & $\begin{array}{l}\text { 今. } \\
\text { ○ } \\
\text { ' }\end{array}$ & 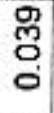 & $\begin{array}{l}\text { 융 } \\
\text { 웅 }\end{array}$ & 동 & ঙ্ণ & 号 & $\begin{array}{l}\bar{g} \\
\\
\end{array}$ & 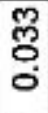 & $\begin{array}{l}\text { 号 } \\
\stackrel{0}{0} \\
0\end{array}$ & 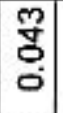 & $\begin{array}{l}5 \\
0 \\
0\end{array}$ & $\begin{array}{l}\text { m } \\
\text { i⿱口口 } \\
0 \\
0\end{array}$ & $\begin{array}{l}\text { ग } \\
\text { O } \\
\text { ○ }\end{array}$ & $\begin{array}{l}\text { 웜 } \\
\text { 잉 }\end{array}$ & $\begin{array}{l}\text { 겅 } \\
\text { O }\end{array}$ & 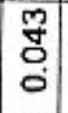 & 势 & $\begin{array}{l} \pm \\
\\
0\end{array}$ & $\begin{array}{l}\text { 웅 } \\
0 \\
0\end{array}$ & $\begin{array}{l}\text { लू } \\
\text { Oे }\end{array}$ & $\begin{array}{l}\text { ? } \\
\text { g. } \\
0\end{array}$ \\
\hline 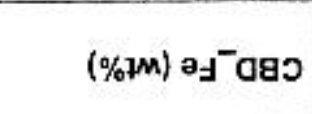 & $\frac{\mathscr{p}}{\circ}$ & $\begin{array}{l}\mathscr{0} \\
\text { ల్ } \\
0\end{array}$ & $\frac{\text { P }}{\dot{0}}$ & $\begin{array}{l}\underset{N}{N} \\
\text { O }\end{array}$ & $\frac{\substack{m \\
0}}{0}$ & $\frac{\bar{N}}{\check{O}}$ & $\frac{9}{\frac{9}{4}}$ & $\frac{g}{\frac{9}{8}}$ & 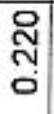 & 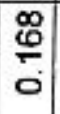 & $\begin{array}{l}\mathcal{N} \\
\underset{N}{\alpha} \\
0\end{array}$ & $\frac{\text { g }}{0}$ & $\frac{\sqrt{m}}{0}$ & $\frac{\mathscr{m}}{\circ}$ & 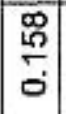 & $\begin{array}{l}\frac{2}{0} \\
\frac{0}{0}\end{array}$ & $\frac{\bar{\sigma}}{\sigma}$ & $\frac{\text { mे }}{0}$ & $\frac{5}{0}$ & $\frac{N}{\sigma}$ & $\frac{\text { Ð }}{\circ}$ \\
\hline (\%) bə & 实 & $\begin{array}{l}\text { g } \\
\text { i }\end{array}$ & 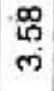 & $\begin{array}{l}\text { पि } \\
\text { ल }\end{array}$ & $\underset{\infty}{\infty}$ & $\begin{array}{l}\text { d } \\
6\end{array}$ & $\begin{array}{l}\text { Pै } \\
\text { w }\end{array}$ & $\begin{array}{l}\infty \\
\infty \\
N\end{array}$ & $\underset{v}{\frac{\sigma}{7}}$ & $\begin{array}{l}\underset{W}{ } \\
\text { in }\end{array}$ & $\underset{m}{\stackrel{m}{N}}$ & $\begin{array}{l}N \\
\dot{N}\end{array}$ & $\underset{\omega}{N}$ & $\begin{array}{c}0 \\
0 \\
\text { us }\end{array}$ & $\begin{array}{l}\infty \\
0 \\
0\end{array}$ & $\underset{0}{\stackrel{0}{0}}$ & $\begin{array}{l}\mathbb{N} \\
\dot{m}\end{array}$ & $\begin{array}{l}\square \\
0\end{array}$ & $\begin{array}{l} \pm \\
0\end{array}$ & $\begin{array}{l}\underset{v}{\square} \\
0\end{array}$ & $\underset{0}{\circ}$ \\
\hline $\mathrm{Hd}^{\mathrm{d}}$ & $\begin{array}{l}\mathbb{N} \\
\infty\end{array}$ & $\begin{array}{l}5 \\
\infty \\
\infty\end{array}$ & $\frac{0}{\sigma}$ & $\frac{\mathbb{t}}{\phi}$ & m & $\begin{array}{l}\text { क } \\
\text { कi }\end{array}$ & $\begin{array}{l}\Phi \\
\emptyset \\
\sigma\end{array}$ & $\begin{array}{l}\omega \\
0 \\
\infty\end{array}$ & $\begin{array}{l}\mathscr{Q} \\
\text { के }\end{array}$ & ๗ా & $\begin{array}{l}\text { オ } \\
\text { 'ं }\end{array}$ & $\underset{\infty}{\bar{q}}$ & $\begin{array}{l}\text { षे } \\
\infty\end{array}$ & ల్ & $\tilde{\sigma}$ & $\begin{array}{l}\mathbb{\Xi} \\
\sigma\end{array}$ & $\begin{array}{l}9 \\
0 \\
0\end{array}$ & $\begin{array}{l}\mathbb{N} \\
\infty \\
\infty\end{array}$ & $\begin{array}{l}\mathrm{O} \\
\sigma \\
\sigma\end{array}$ & $\begin{array}{l}\text { 员 } \\
\text { के }\end{array}$ & ஜ \\
\hline$(\% 7 M) 7810 w$ & $\begin{array}{l}\sigma \\
\sigma \\
0\end{array}$ & 只 & $\begin{array}{l}\widetilde{\sigma} \\
0\end{array}$ & $\underset{\sim}{\sim}$ & ๑ & 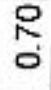 & $\begin{array}{l}0 \\
0\end{array}$ & $\stackrel{P}{0}$ & $\begin{array}{l}\bar{\sigma} \\
0\end{array}$ & $\underset{0}{\overleftarrow{0}}$ & Nָ & लू & $\begin{array}{l}0 \\
0\end{array}$ & $\begin{array}{c}5 \\
0\end{array}$ & $\begin{array}{l}0 \\
0 \\
0\end{array}$ & $\begin{array}{l}0 \\
0 \\
0\end{array}$ & $\underset{\square}{\square}$ & $\underset{-}{\check{-}}$ & U̦ & 只 & $\underset{\infty}{\infty}$ \\
\hline (wo) yวdeg damoา & 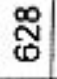 & 워 & 욤 & 贾 & N & 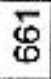 & पे & $\begin{array}{c}\infty \\
\Phi \\
\Phi\end{array}$ & $\mathbb{N}$ & 믇 & E & ¿্d & 忿 & 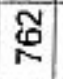 & 믇 & $\frac{m}{\infty}$ & 뭄 & $\frac{N}{60}$ & $\stackrel{\mathbb{N}}{\longleftarrow}$ & $\underset{W}{N}$ & 芯 \\
\hline (u৩) yłdəa səddn & 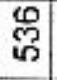 & 0 & $\begin{array}{l}\infty \\
\mathbb{6} \\
\mathbb{0}\end{array}$ & 要 & $\mathrm{N}$ & $\underset{\mathcal{N}}{\mathscr{N}}$ & 냉 & 煌 & O & 믇 & 苑 & $\stackrel{\mathbb{N}}{=}$ & $\frac{N}{i n}$ & 용 & D & 㤐 & 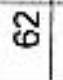 & m & J & $\frac{50}{50}$ & 离 \\
\hline UOZ!ツ애 & 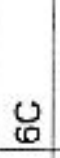 & $\frac{1}{3}$ & $\begin{array}{l}\overline{0} \\
\emptyset\end{array}$ & 苋 & ర్ల & ¿ & ల్ & 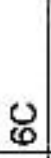 & 盖 & 0 & 空 & $\begin{array}{l}\text { U } \\
\text { s. } \\
\text { 心 } \\
\text { N }\end{array}$ & 员 & 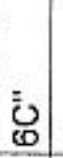 & 0 & 0 & 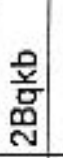 & U & $\begin{array}{l}\text { 号 } \\
\text { ⿳亠丷⿵冂丶 }\end{array}$ & 0 & $\mathcal{Y}$ \\
\hline әроэ ㅎdues p|키 & 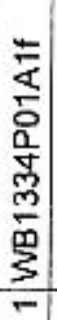 & 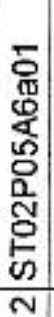 & $\begin{array}{l}\frac{0}{0} \\
\frac{a}{0} \\
0 \\
\frac{0}{n} \\
\frac{m}{\sigma} \\
\frac{m}{5} \\
m\end{array}$ & 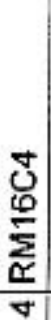 & 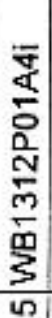 & 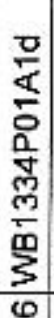 & 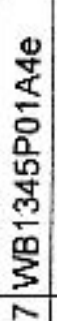 & 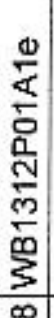 & 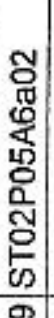 & 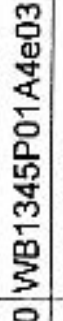 & 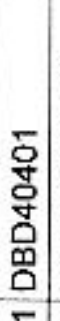 & $\begin{array}{l}\frac{0}{5} \\
\frac{1}{5} \\
\frac{5}{0} \\
\frac{1}{v} \\
\frac{\mathrm{m}}{m} \\
\frac{m}{5}\end{array}$ & 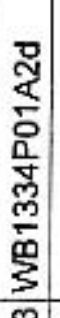 & 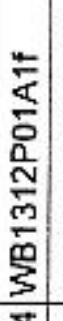 & 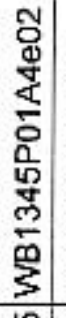 & $\begin{array}{l}\frac{0}{4} \\
\frac{5}{0} \\
\frac{1}{0} \\
\frac{5}{5} \\
\frac{1}{5} \\
\frac{0}{5}\end{array}$ & 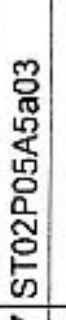 & 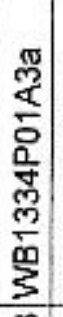 & 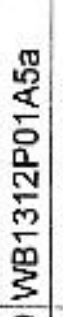 & 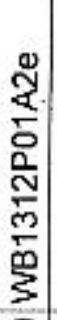 & 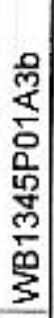 \\
\hline əodues & - & $N$ & $m$ & $\nabla$ & $n$ & $\varphi$ & $N$ & $\infty$ & (5) & 은 & $F$ & 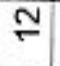 & $\dddot{m}$ & У & 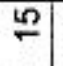 & 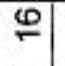 & $\approx$ & $\stackrel{\infty}{\square}$ & $\stackrel{\sigma}{-}$ & 오 & $\overline{\mathrm{N}}$ \\
\hline पээеg & $\begin{array}{l}\mathscr{Q} \\
\stackrel{8}{y}\end{array}$ & $\begin{array}{l}\bullet \\
\stackrel{9}{y}\end{array}$ & $\stackrel{\leftrightarrow}{\circ}$ & $\stackrel{8}{8}$ & $\stackrel{8}{8}$ & $\stackrel{0}{8}$ & 号 & $\mathscr{8}$ & 号 & 号 & $\stackrel{\mathscr{P}}{\nabla}$ & $\stackrel{\mathscr{P}}{\mathrm{P}}$ & $\begin{array}{l}\mathscr{8} \\
8\end{array}$ & $\mathscr{8}$ & $\mathscr{8}$ & $\stackrel{8}{8}$ & $\stackrel{\mathscr{P}}{8}$ & $\begin{array}{l}\mathscr{9} \\
\\
\end{array}$ & $\mathscr{g}$ & $\mathscr{g}$ & 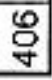 \\
\hline
\end{tabular}




\begin{tabular}{|c|c|c|c|c|c|c|c|c|c|c|c|c|c|c|c|c|c|c|c|c|}
\hline (\%+M) !s ${ }^{-} \times O \forall$ & $\begin{array}{l}\frac{10}{0} \\
0 \\
0\end{array}$ & $\begin{array}{l}\frac{m}{0} \\
0 \\
0\end{array}$ & 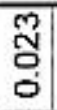 & $\begin{array}{l}\frac{\sigma}{5} \\
\text { 웅 }\end{array}$ & $\begin{array}{l}\overleftarrow{y} \\
\text { o. } \\
0\end{array}$ & $\begin{array}{l}5 \\
0 \\
0\end{array}$ & 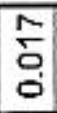 & 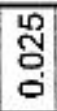 & $\begin{array}{l}\text { 동 } \\
\text { O } \\
\end{array}$ & $\frac{n}{\circ}$ & $\begin{array}{l}\overline{0} \\
0 \\
0\end{array}$ & $\begin{array}{l}n \\
0 \\
0\end{array}$ & $\begin{array}{l}\overleftarrow{N} \\
\text { ণ } \\
\end{array}$ & $\begin{array}{l}\sigma \\
\\
0 \\
0\end{array}$ & $\begin{array}{l}\overline{\mathrm{N}} \\
\mathrm{O}\end{array}$ & $\begin{array}{l}\text { क } \\
\\
0 \\
0\end{array}$ & 동 & 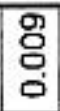 & 둥 & $\begin{array}{l}\overline{0} \\
\text { O }\end{array}$ \\
\hline$(\% 2 M)$ o & 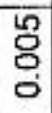 & 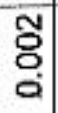 & $\begin{array}{l}m \\
\text { m. } \\
0\end{array}$ & $\begin{array}{l}\text { ¿ } \\
\text { 뭉 }\end{array}$ & $\begin{array}{l}\text { 吕 } \\
\text { 号 }\end{array}$ & $\begin{array}{l}\text { 욤 } \\
0 \\
0\end{array}$ & $\begin{array}{l}\text { 잉 } \\
\text { 웅 }\end{array}$ & 농 & 용 & 응 & $\begin{array}{l}\infty \\
0 \\
0 \\
0\end{array}$ & $\begin{array}{l}\text { 응 } \\
\text { O. }\end{array}$ & $\begin{array}{l}\text { 긍 } \\
\text { 엉 }\end{array}$ & 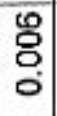 & $\begin{array}{l}\text { 농 } \\
\text { 웅 }\end{array}$ & 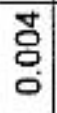 & 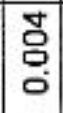 & 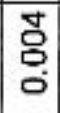 & $\begin{array}{l}\text { 농 } \\
\\
\end{array}$ & $\begin{array}{l}\text { L } \\
\text { 웅 } \\
0\end{array}$ \\
\hline$(\%+m)$ is ago & $\begin{array}{l}\overline{0} \\
0 \\
0\end{array}$ & $\begin{array}{l}7 \\
8 \\
0\end{array}$ & $\begin{array}{l}\text { 윰 } \\
0\end{array}$ & 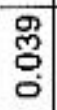 & $\begin{array}{l}\text { N } \\
\text { d. } \\
0\end{array}$ & $\begin{array}{l}\text { g } \\
\text { 。 } \\
\text { 。 }\end{array}$ & $\begin{array}{l}\qquad 8 \\
8 \\
0\end{array}$ & 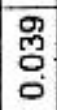 & 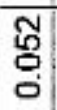 & 守 & $\begin{array}{l}\text { g } \\
\text { '. } \\
0\end{array}$ & $\begin{array}{l}\text { N } \\
\text { 员 } \\
0 \\
0\end{array}$ & 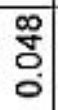 & $\begin{array}{l}\text { 点 } \\
0 \\
0 \\
0\end{array}$ & $\begin{array}{l}\text { 점 } \\
0 \\
0\end{array}$ & $\begin{array}{l}\text { प्र } \\
\text { ర్ } \\
0\end{array}$ & $\begin{array}{l}10 \\
0 \\
0 \\
0\end{array}$ & $\begin{array}{l}5 \\
0 \\
0 \\
0\end{array}$ & $\begin{array}{l}\text { J } \\
\text { O } \\
\text { O }\end{array}$ & 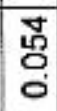 \\
\hline$(\% 7 m)$ ey & \begin{tabular}{l}
0 \\
\multirow{N}{N}{} \\
0
\end{tabular} & $\frac{\infty}{0}$ & $\frac{\infty}{0}$ & $\frac{8}{8}$ & $\frac{n}{\sigma}$ & $\frac{\widetilde{N}}{\stackrel{5}{0}}$ & $\frac{\mathscr{\varphi}}{\stackrel{\oplus}{\circ}}$ & $\frac{\text { L }}{\frac{\pi}{0}}$ & $\frac{\text { חొ }}{\circ}$ & $\underset{0}{\stackrel{\circ}{\infty}}$ & $\underset{\infty}{\check{\infty}}$ & $\frac{g}{\square}$ & $\frac{\pi}{\sigma}$ & $\begin{array}{l}\mathbb{N} \\
\text { Na } \\
0\end{array}$ & 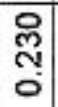 & 恋 & $\frac{⿱}{5}$ & $\begin{array}{l}\stackrel{m}{\sigma} \\
\\
\end{array}$ & $\begin{array}{l}\mathscr{D} \\
\stackrel{\infty}{\circ} \\
0\end{array}$ & $\begin{array}{l}\text { N } \\
\text { N } \\
0\end{array}$ \\
\hline 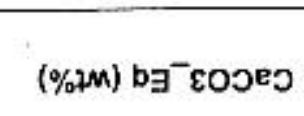 & $\begin{array}{l}\text { षे } \\
\text { ले }\end{array}$ & 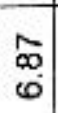 & ○ & 弪 & $\begin{array}{l}m \\
\infty \\
10\end{array}$ & $\begin{array}{c}\infty \\
\\
\text { เก́ }\end{array}$ & $\frac{\infty}{i n}$ & $m$ & ले & $\underset{v}{q}$ & ڤ & $\stackrel{m}{m}$ & ले & ले & $\begin{array}{l}\stackrel{J}{4} \\
\text { in }\end{array}$ & $\stackrel{n}{\sim}$ & $\begin{array}{l}\infty \\
N \\
10\end{array}$ & $\begin{array}{l}\mathscr{Q} \\
\oplus \\
\oplus\end{array}$ & 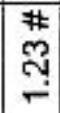 & ले \\
\hline $\mathrm{Hd}$ & $\underset{\sigma}{\sigma}$ & $\begin{array}{l}8 \\
\infty \\
\infty\end{array}$ & 농 & ڤั & $\begin{array}{l}\dddot{\wp} \\
\text { の }\end{array}$ & 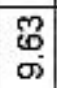 & $\begin{array}{l}\bar{\sim} \\
\text { oi }\end{array}$ & $\bar{\sigma}$ & $\begin{array}{l}\infty \\
\infty \\
\infty\end{array}$ & 号 & 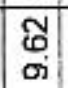 & $\underset{\infty}{\infty}$ & $\begin{array}{l}\mathscr{Q} \\
\infty\end{array}$ & $\frac{\text { 임 }}{2}$ & $\begin{array}{l}\stackrel{m}{*} \\
\infty\end{array}$ & 守 & $\frac{\sigma}{\infty}$ & क & $\begin{array}{l}\infty \\
\infty \\
\infty\end{array}$ & $\stackrel{m}{a}$ \\
\hline$(\% 2 M)$ Js! & $\underset{\sim}{\sim}$ & $\begin{array}{l}\bar{\infty} \\
0\end{array}$ & $\mp$ & 문 & 足 & Б্் & న్ & D & $\underset{-}{\sigma}$ & 운 & $\begin{array}{l}0 \\
\hat{n} \\
0\end{array}$ & $\underset{\Gamma}{\check{N}}$ & $\underset{F}{F}$ & ָָ & זָ & ले & $\stackrel{N}{\mathrm{~N}}$ & V & O & F \\
\hline (w) чł & 哭 & 吕 & $\stackrel{\text { क }}{\leftarrow}$ & $\frac{10}{6}$ & 믇 & m & $\stackrel{?}{\rightleftarrows}$ & m & 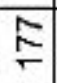 & 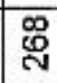 & 荋 & 品 & ్ㅐ & Q & 요 & N & $\begin{array}{l}\infty \\
\text { लु }\end{array}$ & $\stackrel{\infty}{\sim}$ & 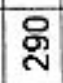 & 孛 \\
\hline (wo) updog saddn & 喿 & $\begin{array}{l}\text { 吕 } \\
\text { 茨 }\end{array}$ & 음 & m & T & 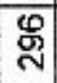 & $\stackrel{\sigma}{\leftarrow}$ & 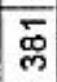 & $\bar{m}$ & ஸ̊ & 点 & $\stackrel{\infty}{ \pm}$ & $\stackrel{\omega}{6}$ & 嵒 & $\begin{array}{l}\infty \\
\Phi \\
\stackrel{N}{N}\end{array}$ & $\bar{J}$ & $\approx$ & $m$ & 品 & $\omega$ \\
\hline uoz!JoH & $\stackrel{\mathbb{U}}{\mathrm{C}}$ & 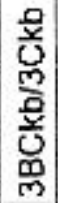 & 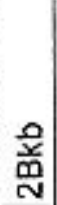 & U & 0 & U & 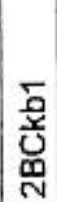 & 㐫 & 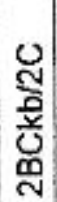 & 0 & 仓્ల & 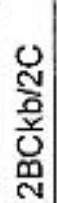 & $\frac{N}{\frac{N}{0}}$ & $\stackrel{\mathbb{C}}{=}$ & 总 & : & 0 & 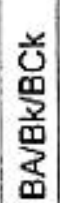 & 总 & 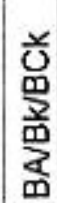 \\
\hline apoว 바dues pjə]ృ & $\frac{10}{\sum_{0}^{\infty}}$ & 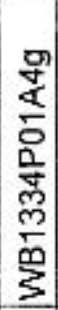 & 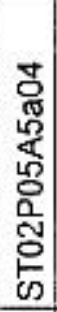 & 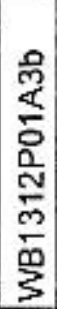 & 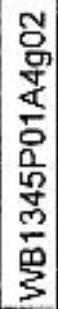 & 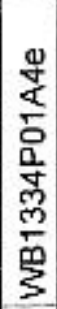 & 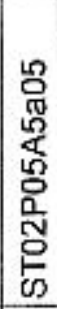 & 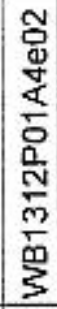 & $\begin{array}{l}\frac{0}{5} \\
\frac{1}{0} \\
\frac{0}{0} \\
\frac{0}{5} \\
\frac{5}{5}\end{array}$ & 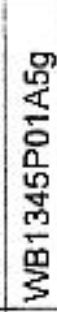 & 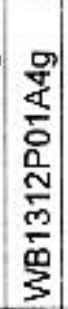 & 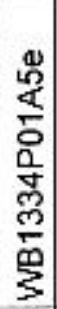 & 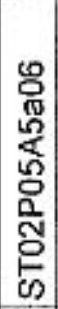 & 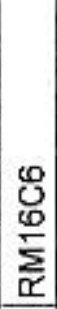 & $\begin{array}{l}\frac{9}{8} \\
\frac{5}{6} \\
0 \\
\frac{1}{5} \\
\frac{5}{2} \\
\frac{9}{3}\end{array}$ & 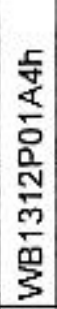 & 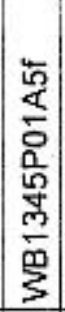 & 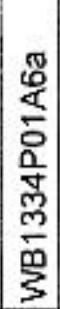 & 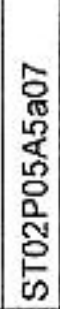 & 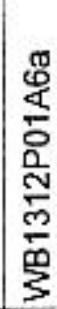 \\
\hline ә|dues & N & लु & पू & 常 & 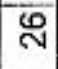 & $\tilde{N}$ & $\stackrel{\infty}{\sim}$ & (9 & 음 & $\bar{m}$ & N & $m$ & ले & 歺 & ஜூ & ले & m & g & 윰 & $\overline{8}$ \\
\hline บэุอg & ஜ & $\mathscr{8}$ & g & $\stackrel{\mathscr{Q}}{\stackrel{9}{q}}$ & 号 & $\mathscr{8}$ & $\stackrel{8}{8}$ & O & 号 & 号 & \begin{tabular}{|l|} 
\\
\\
\end{tabular} & 号 & $\mathscr{8}$ & $\mathscr{8}$ & ᄋ & O & $\begin{array}{l}8 \\
\end{array}$ & $\begin{array}{l}0 \\
\\
\end{array}$ & $\stackrel{9}{8}$ & $\mathscr{8}$ \\
\hline
\end{tabular}

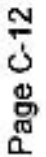




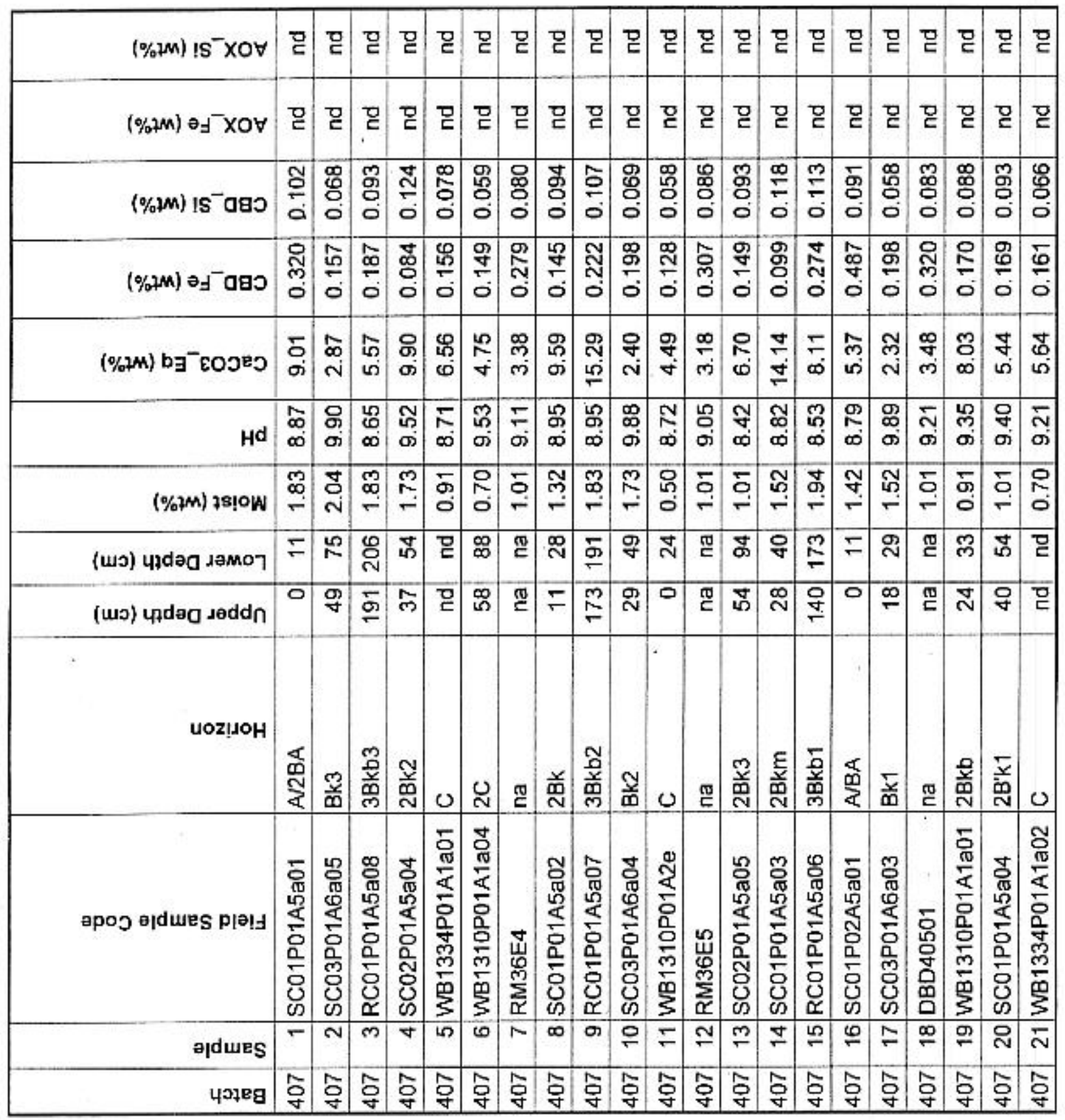




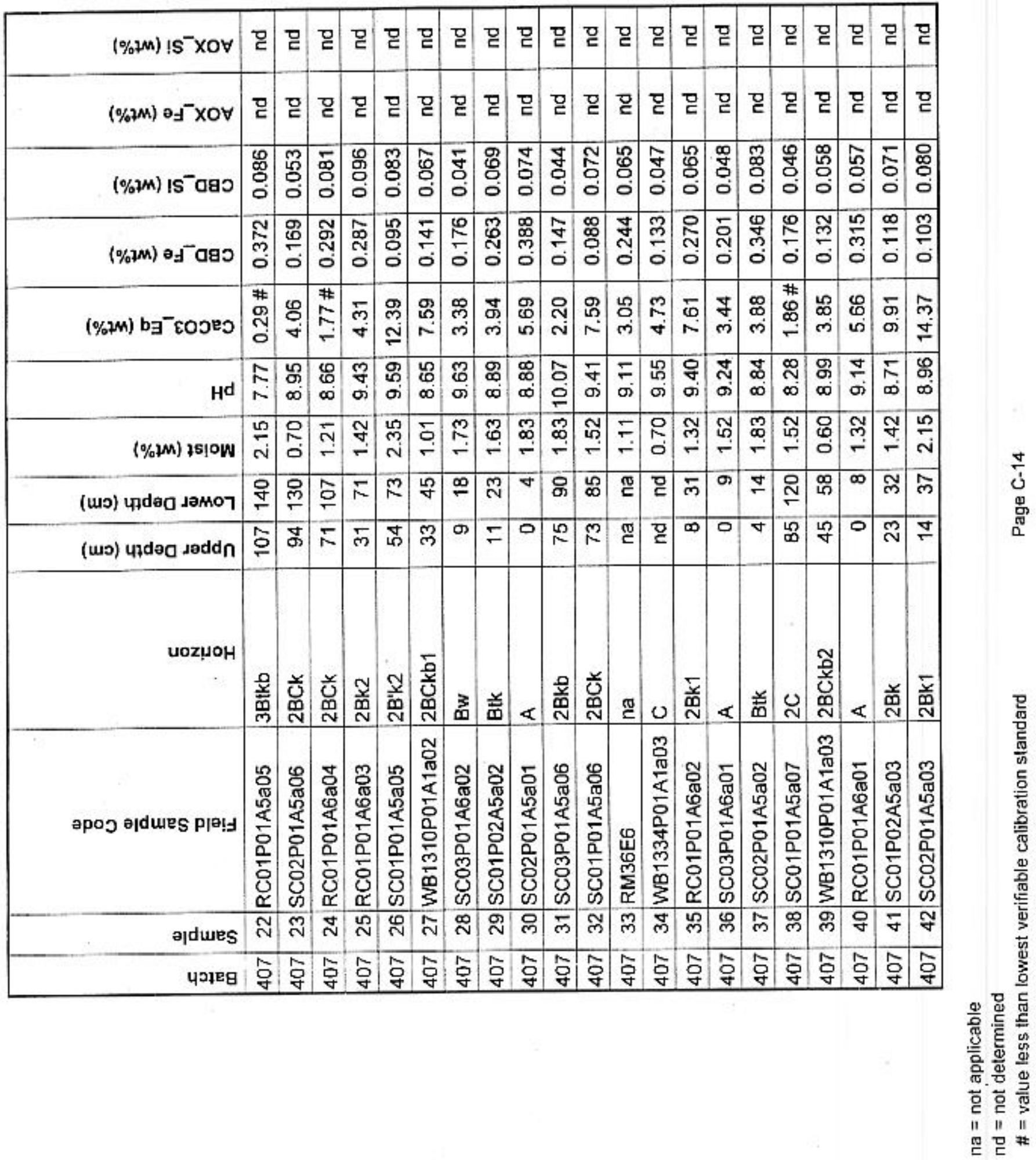




\begin{tabular}{|c|c|c|c|c|c|c|c|c|c|c|c|c|c|c|c|c|c|c|c|c|c|}
\hline$(\%+M) ! s^{-} X O \forall$ & 믄 & प् & 일 & ? & 믇 & ב & D & g & g & 맏 & T. & 产 & 읻 & 믇 & 믇 & 믇 & 읃 & 믿 & 음 & 읃 & 믿 \\
\hline$(\% \not M) \partial^{-} X O \forall$ & 읻 & 몯 & 믿 & 므 & प्र & 巳 & D & 묻 & 맏 & 믇 & 믇 & 믿 & ㅁ. & $\mathbf{q}$ & $\mathrm{z}$ & 묻 & 몯 & 묻 & 몯 & 몯 & 믿 \\
\hline$(\%+M)$ ! $5^{-}$- & $\bar{\sigma}$. & $\begin{array}{l} \\
\stackrel{D}{0} \\
0\end{array}$ & $\begin{array}{l}\text { 总 } \\
\text { O } \\
0\end{array}$ & 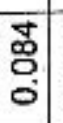 & $\begin{array}{l}\text { g } \\
\text { g. } \\
0 \\
0\end{array}$ & $\frac{\mathrm{O}}{\mathrm{O}}$ & $\begin{array}{l}\text { g } \\
\text { 号 } \\
\text { : }\end{array}$ & \begin{tabular}{l|} 
员 \\
员 \\
\end{tabular} & $\frac{\pi}{\sigma}$ & $\begin{array}{l}\mathscr{O} \\
\circ \\
0 \\
0\end{array}$ & 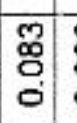 & \begin{tabular}{l|} 
\\
0 \\
0 \\
\\
\end{tabular} & $\begin{array}{l}9 \\
8 \\
0 \\
0 \\
0\end{array}$ & $\begin{array}{l}\text { प्र } \\
0 \\
0 \\
0\end{array}$ & $\begin{array}{l}\bar{\infty} \\
0 \\
0 \\
0\end{array}$ & $\begin{array}{l}\stackrel{\mathscr{N}}{\mathbb{N}} \\
\dot{0}\end{array}$ & $\begin{array}{l}\mathscr{8} \\
8 \\
0\end{array}$ & \begin{tabular}{l|}
0 \\
0 \\
0 \\
0 \\
0
\end{tabular} & \begin{tabular}{c|}
\multirow{2}{*}{} \\
0 \\
0
\end{tabular} & $\begin{array}{l}8 \\
\text { \% } \\
0\end{array}$ & 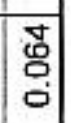 \\
\hline$(\%$ & $\begin{array}{l}\text { mे } \\
\text { m. }\end{array}$ & $\begin{array}{l}\text { D } \\
\text { N̦ } \\
0\end{array}$ & $\begin{array}{l}\stackrel{\square}{\Phi} \\
\stackrel{\circ}{0}\end{array}$ & 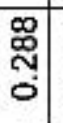 & $\frac{g}{\dot{\sigma}}$ & $\begin{array}{l}\tilde{m} \\
\stackrel{m}{0} \\
0\end{array}$ & $\begin{array}{l}\mathbb{J} \\
\stackrel{J}{0} \\
0\end{array}$ & $\frac{5}{0}$ & $\begin{array}{l}\text { 잉 } \\
0 \\
0\end{array}$ & $\begin{array}{l}\infty \\
\dddot{O} \\
0\end{array}$ & \begin{tabular}{|c}
$\mathbf{N}$ \\
$\frac{\infty}{0}$ \\
\end{tabular} & $\begin{array}{l}\infty \\
\\
0 \\
0\end{array}$ & $\frac{\bar{n}}{\overline{0}}$ & స్లె & $\begin{array}{l} \\
\text { o्ल } \\
0\end{array}$ & $\begin{array}{l}\text { מ } \\
\text { ల } \\
0\end{array}$ & $\begin{array}{l}0 \\
\stackrel{0}{\Omega} \\
0 \\
0\end{array}$ & $\begin{array}{l} \\
\\
\end{array}$ & \begin{tabular}{l|} 
\\
$\stackrel{2}{N}$ \\
0 \\
0
\end{tabular} & 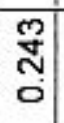 & $\frac{\text { Oด }}{\circ}$ \\
\hline (\% \% & 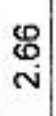 & 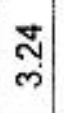 & సู & $\begin{array}{c}\mathscr{N} \\
\text { లె }\end{array}$ & 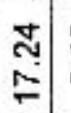 & $\underset{\infty}{\infty}$ & $\stackrel{+}{\stackrel{\Phi}{=}}$ & $\begin{array}{l}\stackrel{g}{N} \\
\text { vi }\end{array}$ & $\begin{array}{l}\stackrel{\omega}{\circ} \\
\stackrel{6}{\sigma}\end{array}$ & $\begin{array}{l}\bar{g} \\
\dot{v}\end{array}$ & $\begin{array}{c}\mathscr{g} \\
\stackrel{0}{\infty}\end{array}$ & $\begin{array}{l}\mathscr{m} \\
\text { ले }\end{array}$ & $\begin{array}{l}\infty \\
0 \\
0 \\
0\end{array}$ & $\begin{array}{l}\infty \\
\stackrel{\infty}{m} \\
m\end{array}$ & $\begin{array}{l}\text { g } \\
\text { ल }\end{array}$ & 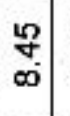 & $\hat{\varphi}$ & 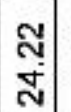 & $\begin{array}{l}\text { 员 } \\
\text { ल }\end{array}$ & 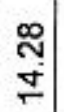 & $\left|\begin{array}{l}\mathbb{B} \\
0 \\
\mathrm{~N}\end{array}\right|$ \\
\hline $\mathrm{Hd}^{\mathrm{d}}$ & $\frac{\infty}{\sigma}$ & $\begin{array}{l}\text { S. } \\
\text { Oे } \\
\end{array}$ & $\begin{array}{l} \\
\delta \\
\infty\end{array}$ & 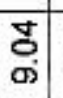 & $\begin{array}{l}\mathscr{S} \\
\infty\end{array}$ & $\begin{array}{c} \\
\sigma \\
\sigma\end{array}$ & $\begin{array}{l} \\
\stackrel{8}{0} \\
\infty\end{array}$ & $\frac{0}{\sigma i}$ & $\begin{array}{c}\mathscr{Q} \\
\infty\end{array}$ & $\begin{array}{l}m \\
\dot{m} \\
\infty\end{array}$ & $\begin{array}{l}\mathscr{Q} \\
\sigma\end{array}$ & $\begin{array}{l}\sigma \\
\infty \\
\infty\end{array}$ & $\begin{array}{l}\stackrel{p}{p} \\
\infty\end{array}$ & 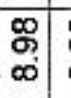 & $\begin{array}{l}\text { \% } \\
\text { के }\end{array}$ & 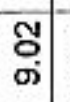 & $\begin{array}{l}50 \\
\infty \\
\infty\end{array}$ & $\begin{array}{l}\mathscr{S} \\
\infty \\
\infty\end{array}$ & \begin{tabular}{|c|}
$\mathscr{S}$ \\
$\infty$ \\
$\infty$
\end{tabular} & 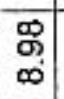 & \begin{tabular}{|l|}
9 \\
$\Phi$ \\
\end{tabular} \\
\hline 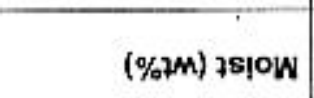 & 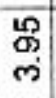 & $\stackrel{\text { ले }}{\circ}$ & $\begin{array}{l} \\
\mathrm{O} \\
\mathrm{N}\end{array}$ & $\mp$ & $\begin{array}{l}\varphi \\
\stackrel{\varphi}{v} \\
\sim\end{array}$ & $\begin{array}{l} \\
\dot{\sigma}\end{array}$ & $\underset{\sim}{\mathbb{Y}}$ & $\begin{array}{l}\mathrm{g} \\
\mathrm{g} \\
\mathrm{i}\end{array}$ & 荇 & \begin{tabular}{l}
8 \\
\hdashline \\
+
\end{tabular} & 焉 & $\stackrel{-}{\circ}$ & $\underset{\text { v }}{\leftarrow}$ & 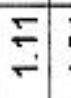 & $\stackrel{\bar{\sim}}{-}$ & $\begin{array}{l} \\
\dot{0}\end{array}$ & $\begin{array}{l} \\
\text { W } \\
\text { i }\end{array}$ & लू & $\begin{array}{l}\bar{\sigma} \\
\dot{\sigma}\end{array}$ & $\begin{array}{l}\infty \\
\infty \\
\sim \\
\sim\end{array}$ & \begin{tabular}{c}
$\mathscr{P}$ \\
\hdashline \\
\hdashline
\end{tabular} \\
\hline (uо) чpdeg temoך & $n$ & प्व & 志 & व & $\mathbb{N}$ & స & లెల్ & 은 & 昰 & g & \begin{tabular}{|l|}
$\mathscr{O}$ \\
\end{tabular} & 莫 & ळ & 믿 & (I) & क & $\mp$ & 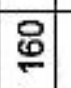 & 맏 & 이 & $\stackrel{\stackrel{N}{\sim}}{ }$ \\
\hline (யכ) unda Jaddก & 0 & 空 & $\stackrel{g}{\emptyset}$ & (ָ) & g & $N$ & స్ & g & \begin{tabular}{|l|} 
\% \\
\end{tabular} & ก & N & व & g & पू & 몯 & 0 & Џ & $\stackrel{\mathbb{N}}{\stackrel{9}{\sim}}$ & ख्व & का & $F$ \\
\hline чozuoㅐ & 4 & 四 & $\begin{array}{l}\infty \\
\text { 面 }\end{array}$ & జ̃ & 空 & 音 & 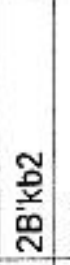 & $\begin{array}{l}\text { के } \\
\text { ⿳亠丷厂 }\end{array}$ & 总 & $\begin{array}{l}\text { 产 } \\
\end{array}$ & $\begin{array}{l}\frac{j}{2} \\
\text { 亩 }\end{array}$ & $\mathbb{Z}$ & 辛 & 四 & ஐே & 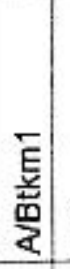 & 总 & 花 & 뜨 & $\begin{array}{l}\text { है } \\
\text { 章 }\end{array}$ & 恙 \\
\hline әpoว a|dues pןẹ̣ & 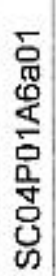 & 岕 & 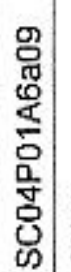 & 骂 & 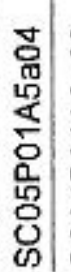 & 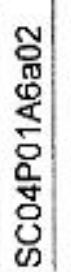 & 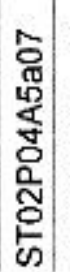 & 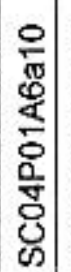 & 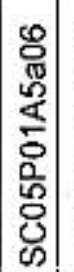 & 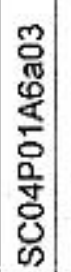 & 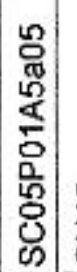 & $\frac{0}{3}$ & 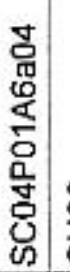 & 옹 & 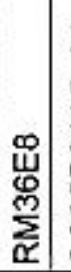 & 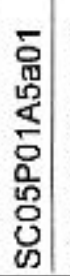 & 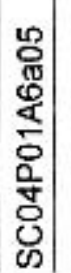 & 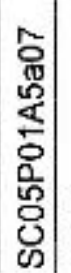 & प్ & 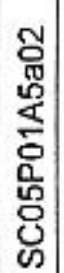 & 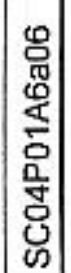 \\
\hline apdues & - & $N$ & $m$ & 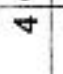 & in & $\omega$ & $m$ & $\infty$ & का & 으 & $=$ & 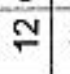 & $\stackrel{m}{m}$ & \pm & $\stackrel{2}{2}$ & $\stackrel{\varphi}{\circ}$ & $=$ & $\stackrel{\infty}{\infty}$ & $\stackrel{9}{-}$ & ก & $\bar{\sim}$ \\
\hline पग्सE & 品 & $\begin{array}{l} \\
\end{array}$ & \begin{tabular}{l|}
0 \\
\end{tabular} & $\begin{array}{l}\text { Oू } \\
\text { Oे }\end{array}$ & $\begin{array}{l}g \\
g\end{array}$ & \begin{tabular}{l|}
$\mathscr{g}$ \\
g
\end{tabular} & $\begin{array}{l}\infty \\
\text { o }\end{array}$ & 啴 & \begin{tabular}{|l|} 
号 \\
o
\end{tabular} & \begin{tabular}{l|} 
\\
o \\
v
\end{tabular} & \begin{tabular}{|l|}
$\circ$ \\
\end{tabular} & $\begin{array}{l} \\
\\
\end{array}$ & \begin{tabular}{l}
0 \\
\multirow{q}{*}{}
\end{tabular} & \begin{tabular}{l|l|}
\multirow{O}{*}{} & \\
\end{tabular} & $\begin{array}{ll}0 \\
\end{array}$ & 弟 & $\begin{array}{l}\infty \\
\text { g } \\
\end{array}$ & 品 & $\begin{array}{l}0 \\
0 \\
y\end{array}$ & 总 & \begin{tabular}{|c|}
0 \\
0 \\
\end{tabular} \\
\hline
\end{tabular}




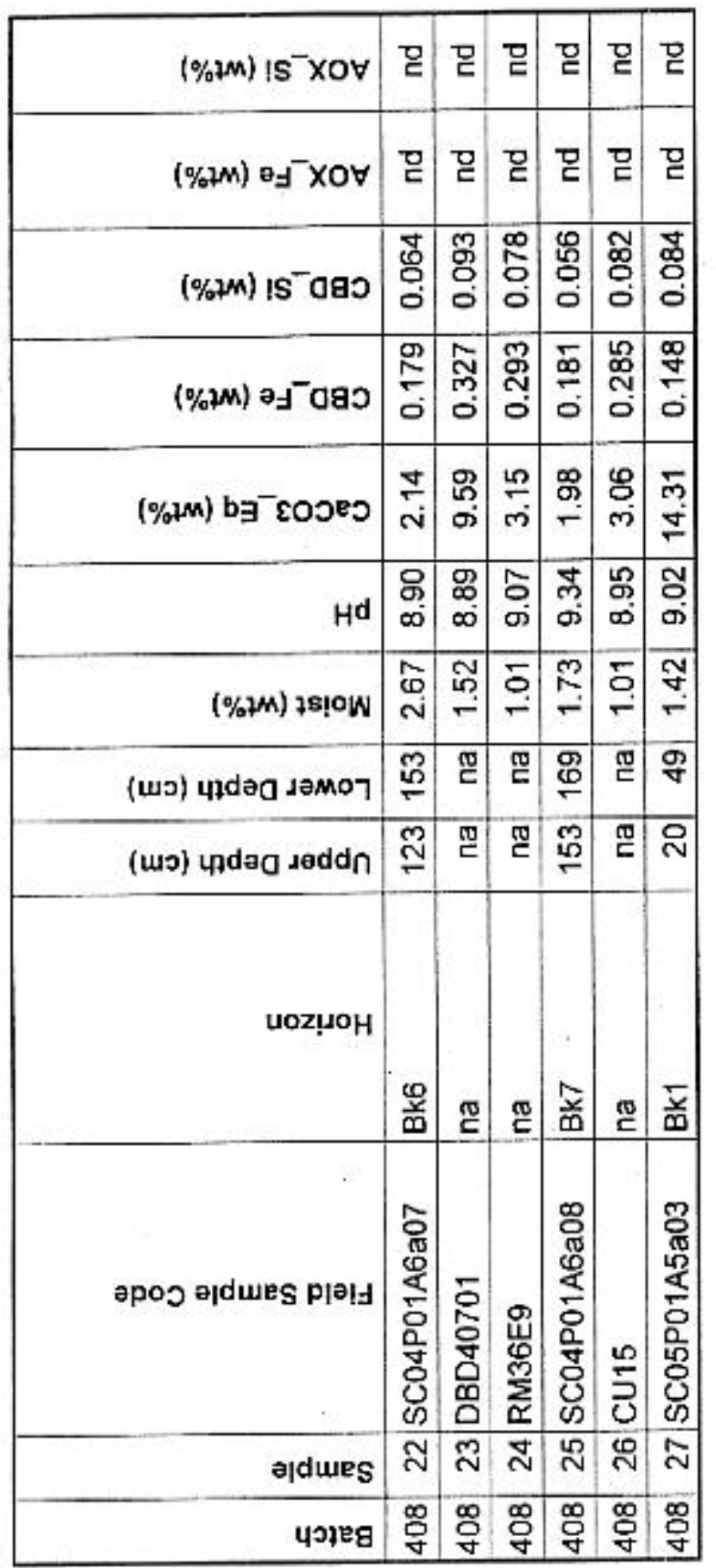




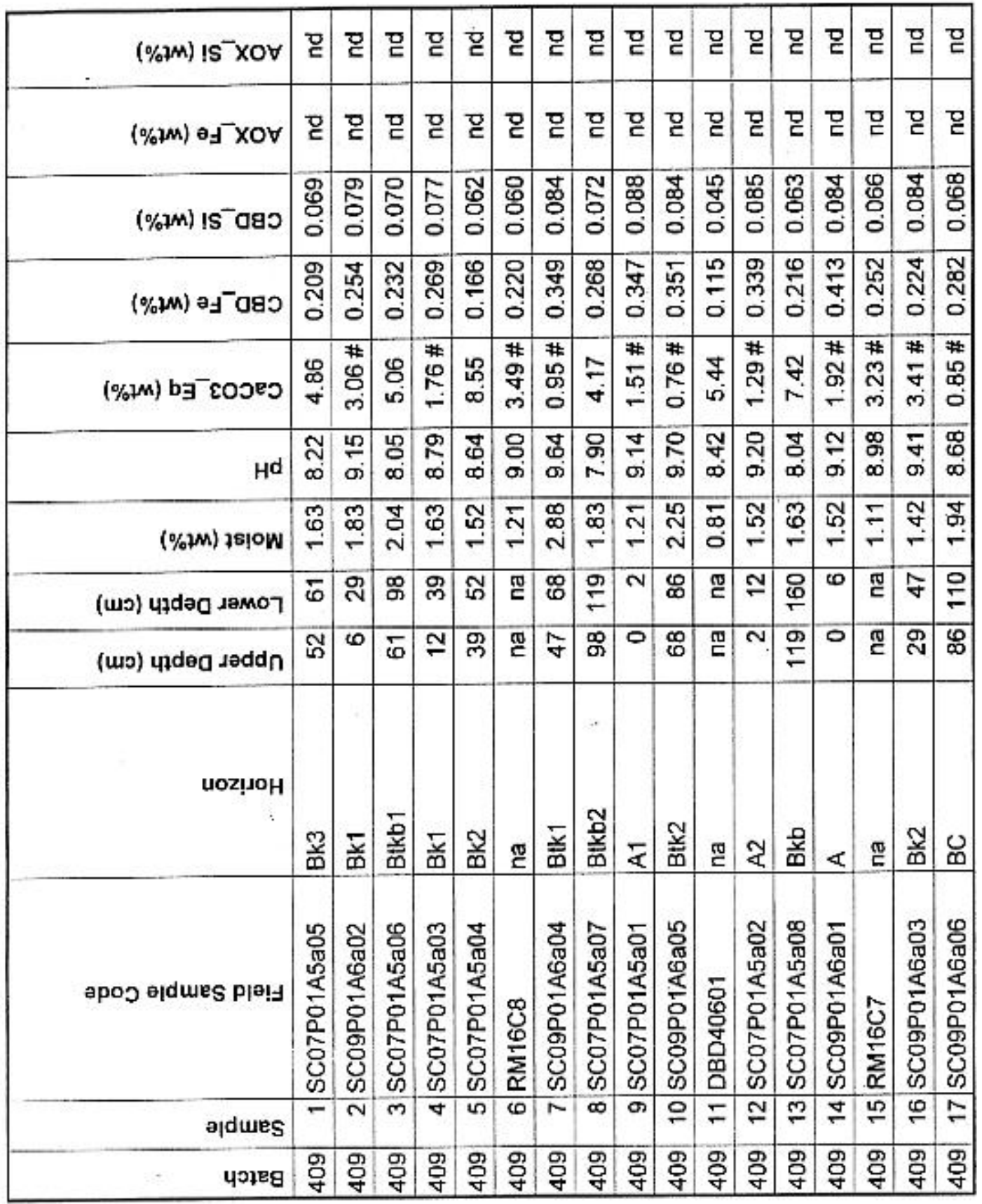




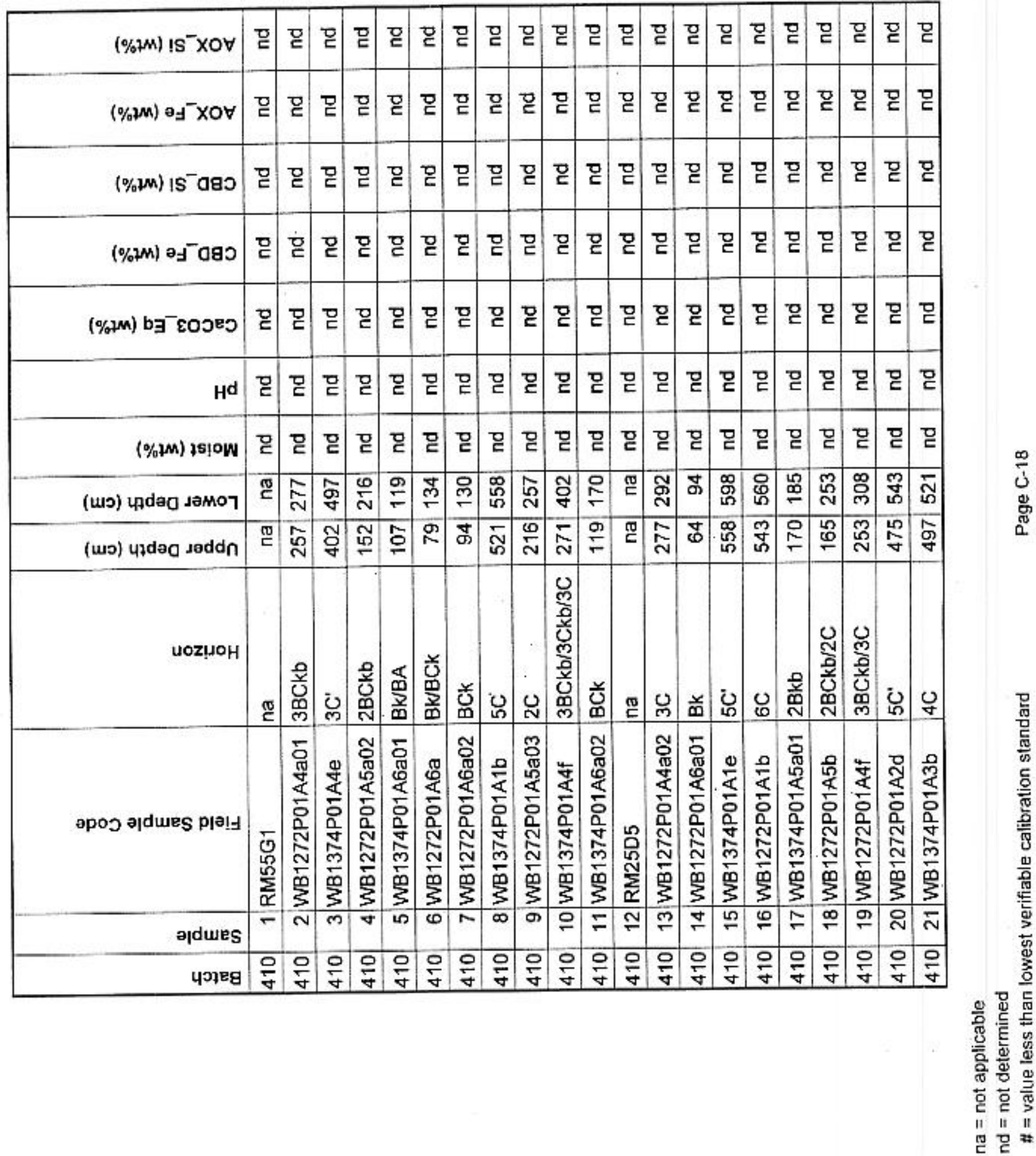




\begin{tabular}{|c|c|c|c|c|c|c|c|c|c|c|c|c|}
\hline$(\%+M)$ is $x O \forall$ & g & g & 무 & 마 & 맏 & g & g & $\mathrm{g}$ & $\mathrm{g}$ & g & है & g \\
\hline$(\% 2 M) \theta_{-}^{-} \mathrm{XOV}$ & है & T. & 닫 & 마 & $\mathrm{g}$ & g. & g & 말 & 묻 & 묻 & 하 & 므 \\
\hline$(\%+M) ! s^{-} a g o$ & ㅁ & g & g & 마 & 믿 & 마 & 믇 & 믿 & g & g & $\mathrm{g}$ & g \\
\hline (\% \% & g & है & 맏 & 맘 & 믿 & 맏 & g & g & g & g & $\mathrm{g}$ & g \\
\hline$(\% m)$ b $\exists^{-}$cooes & 묻 & 믿 & 마 & 묻 & g & g & 믿 & 묻 & 믿 & 믇 & 맏 & 믇 \\
\hline$H^{d}$ & g & ? & ह & $\bar{c}$ & 욷 & 믇 & 믇 & 믿 & 믇 & 묻 & g & ? \\
\hline$(\%$ (\%) נE!OW & ? & g & g & g & g & प्र & 마 & 믿 & ? & 핟 & g & Q \\
\hline (шכ) чұdeg دамоา & 峞 & $\begin{array}{l}\infty \\
\infty \\
\infty\end{array}$ & ลี & \begin{tabular}{ll}
\multirow{2}{*}{} \\
$\sigma$
\end{tabular} & $\mathbb{E}$ & ০্ণী & $\underset{N}{N}$ & ㄴ. & 告 & 要 & $\overline{\mathrm{N}}$ & న్లి \\
\hline 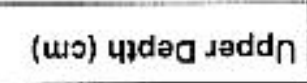 & $\stackrel{m}{\stackrel{m}{c}}$ & : & $\begin{array}{ll}\infty \\
\infty \\
\infty\end{array}$ & \begin{tabular}{|l|l}
$y$ \\
\end{tabular} & $\stackrel{m}{\circ}$ & กิ & N & 离 & 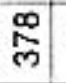 & $\approx$ & $\stackrel{\$}{\%}$ & 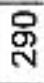 \\
\hline บOz!uOH & $\begin{array}{l}\frac{0}{2} \\
\text { 产 }\end{array}$ & ن्ల & $\begin{array}{l}0 \\
\text { 总 } \\
\text { N }\end{array}$ & 怘 & $\mathbb{2}$ & $\begin{array}{l}\text { 总 } \\
\text { 峁 }\end{array}$ & U & $\begin{array}{l}\text { 商 } \\
\text { N }\end{array}$ & U & $\mathbb{2}$ & 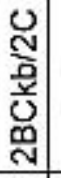 & 总 \\
\hline घpoว ә|dures p|a!y & 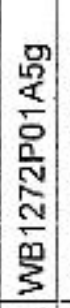 & 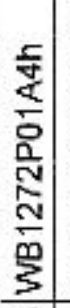 & 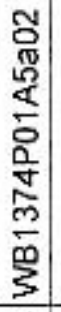 & 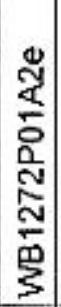 & 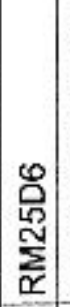 & 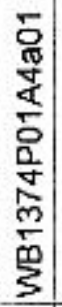 & 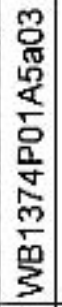 & 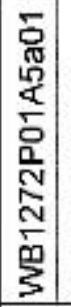 & 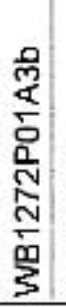 & 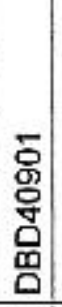 & 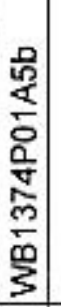 & 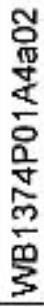 \\
\hline श्dures & $\mathbb{N}$ & N) & $\overline{4}$ & $\stackrel{2}{2}$ & $\mathscr{N}$ & $\hat{\sim}$ & : & 2ิ & क्ल & $\bar{m}$ & N & $m$ \\
\hline पวगอด & 임 & $\frac{0}{9}$ & $\frac{0}{4}$ & 임 & $\frac{9}{4}$ & $\frac{9}{y}$ & $\frac{0}{4}$ & $\frac{1}{y}$ & $\frac{0}{y}$ & $\frac{0}{4}$ & $\frac{9}{8}$ & $\frac{0}{8}$ \\
\hline
\end{tabular}


This Page Intentionally Left Blank 


\section{APPENDIX D}

\section{American Society for Materials and Testing - Unified Soil Classification System}

Particle Size Fractions 


\begin{tabular}{|c|c|c|c|c|c|c|c|c|c|c|c|c|c|c|c|c|c|c|c|c|c|}
\hline$z \#>d$ & ก & $\infty$ & 岗 & का & $F$ & 6 & $\pi$ & Q & 6 & & $\omega$ & क & m & एक & $\stackrel{\infty}{\leftarrow}$ & (5) & $\overline{n^{2}}$ & & & N. & ని \\
\hline Ot $\#>d$ & 寸 & $\stackrel{n}{N}$ & 8 & $\hat{N}$ & $\stackrel{\infty}{N}$ & $\stackrel{\theta}{\square}$ & 이 & $\overline{0}$ & क & ส & $\stackrel{\infty}{\sim}$ & N్ & N & 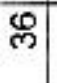 & $\overline{7}$ & $\frac{15}{2}$ & य? & ম & ल) & 8 & m \\
\hline $\mathrm{OL} \#>\mathrm{d}$ & $\overline{6}$ & \% & ळ & क्ला & ? & 官 & 邑 & $?$ & 要 & $\mathscr{D}$ & हे & 8 & का & g & फे & i & 8 & $\Phi$ & f & 5 & $\overline{0}$ \\
\hline$\forall \#>d$ & $A$ & $\Xi$ & 8 & $\stackrel{\infty}{\leftrightarrow}$ & 学 & 웅 & 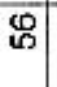 & 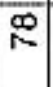 & 5 & ח & 票 & N & 5 & 8 & $\widehat{s}$ & $\overline{0}$ & $\kappa$ & 怘 & ฮू & 5 & $\stackrel{0}{2}$ \\
\hline$u !-8 / \varepsilon>d$ & $\widehat{\infty}$ & $\bar{\infty}$ & \% & 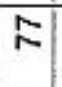 & N & $\bar{N}$ & $\Sigma$ & 足 & N & N & ก & 岕 & $\frac{1}{2}$ & $\stackrel{\infty}{N}$ & న్ల & 尺 & $\infty$ & 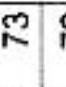 & $\frac{10}{10}$ & $5:$ & $\infty$ \\
\hline$u !-t / \varepsilon>d$ & 's & ळ & 8 & ळ & ळ & \% & - & के & б. & $\bar{\sigma}$ & \& & के & ৪ & के & $\mathscr{\sigma}$ & के & क & § & 8 & B: & \% \\
\hline$u !-\iota>d$ & 曲 & ळ & 웅 & 今े & $\bar{\sigma}$ & 8) & ৪) & s. & 8 & \%. & \& & $\stackrel{\infty}{\infty}$ & สิ & ๘ & \& & 5 & \% & के & ๙ & ৪: & \%ू \\
\hline $\mathrm{u} ! z / h \downarrow l>\mathrm{d}$ & 8 & \begin{tabular}{l|l} 
क \\
\end{tabular} & 8 & \% & ठे & $\%$ & के & $\stackrel{\infty}{\circ}$. & \% & $\mathscr{\%}$ & के & \% & ๘ & के & \% & 怘 & g & $\%$ & 4 & 8 & 8 \\
\hline$u !-z>d$ & \& & \% & ㅇ & \& & ज. & \% & 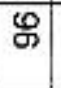 & \& & à & 尔 & ஜ & g. & के & 号 & g & \% & 8 & 必 & sे & 8 & 요 \\
\hline u! $-\varepsilon>d$ & 웅 & ㅇ & 음 & ㅇ & 은 & 8 & 임 & 은 & 8 & 음 & 웅 & 웅 & 움 & 음 & 웅 & 이 & § & छ & 요 & 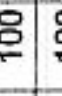 & 우 \\
\hline 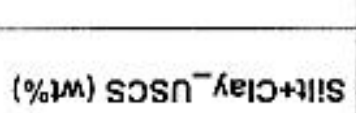 & 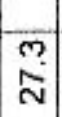 & 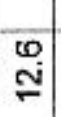 & $\begin{array}{l}0 \\
0 \\
i \\
\sim\end{array}$ & $\begin{array}{l}0 \\
0 \\
0\end{array}$ & $\stackrel{\vec{b}}{\stackrel{0}{*}}$ & $\begin{array}{l}\stackrel{N}{\mathrm{~N}} \\
\text { | }\end{array}$ & $\begin{array}{l}\text { के } \\
\stackrel{p}{6}\end{array}$ & $\begin{array}{l}m \\
\text { वे. } \\
\text { and }\end{array}$ & $\overline{\mathrm{i}}$ & 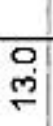 & $\begin{array}{l}m \\
m \\
g\end{array}$ & ্ָ & $\widehat{o}$ & $\begin{array}{l}0 \\
\dot{d}\end{array}$ & $\begin{array}{l}\infty \\
\stackrel{\infty}{\infty}\end{array}$ & $\begin{array}{l} \\
\dot{m} \\
\underline{m}\end{array}$ & 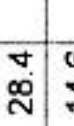 & $\begin{array}{l}\oplus \\
\square\end{array}$ & ָָ & & $\stackrel{m}{m}$ \\
\hline$(\%+M)$ sosn'pues & 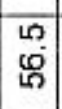 & $\begin{array}{l}0 \\
\dot{0} \\
\dot{0}\end{array}$ & $\begin{array}{l}\infty \\
\dot{\omega} \\
\dot{0}\end{array}$ & 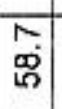 & $\bar{\phi}$ & $\begin{array}{c}\infty \\
0 \\
0 \\
0\end{array}$ & $\vec{\sigma}$ & $\begin{array}{l}\infty \\
\dot{\omega} \\
\dot{\omega}\end{array}$ & लि & $\begin{array}{c}\infty \\
\infty \\
\infty \\
\infty\end{array}$ & 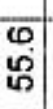 & क. & 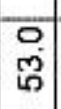 & \begin{tabular}{l}
0 \\
\multirow{\sim}{*}{}
\end{tabular} & $\begin{array}{l}\sigma \\
\tilde{0} \\
0\end{array}$ & $\begin{array}{l}\infty \\
\dot{0} \\
\end{array}$ & $\begin{array}{l}+ \\
\text { 咜 }\end{array}$ & & $\begin{array}{l}0 \\
0 \\
6 \\
6\end{array}$ & & 영 \\
\hline 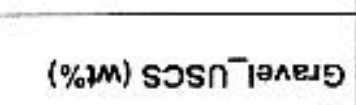 & 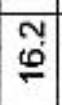 & הু & $\begin{array}{c}m \\
\infty \\
\infty\end{array}$ & $\begin{array}{l}0 \\
\stackrel{\leftrightarrow}{\mathrm{N}}\end{array}$ & 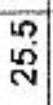 & $\begin{array}{c}0 \\
\stackrel{O}{N}\end{array}$ & $\begin{array}{l}0 \\
\stackrel{\mathrm{N}}{\mathrm{N}}\end{array}$ & $\begin{array}{l}\infty \\
\stackrel{\infty}{6} \\
\stackrel{6}{*}\end{array}$ & N & Nָ & $\begin{array}{l}\mathrm{N} \\
\mathrm{N} \\
\mathrm{N}\end{array}$ & 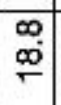 & $\begin{array}{l}\mathrm{N} \\
\mathbf{N} \\
\end{array}$ & ग़े & N & స̃ & $\stackrel{3}{=}$ & & m. & & $\stackrel{m}{=}$ \\
\hline qE7-dnoנפ SOSח & $\sum_{\infty}$ & 章 & $\sum_{\infty}$ & $\sum_{\omega}$ & $\sum_{\infty}$ & 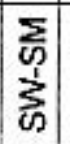 & $\begin{array}{l}\sum_{W} \\
\sum_{\omega \infty}^{\infty}\end{array}$ & $\sum$ & $\begin{array}{l}\sum_{0} \\
\sum_{\omega}\end{array}$ & 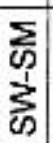 & ह & $\sum$ & $\Sigma$ & $\Sigma$ & $\sum_{\infty}$ & $\begin{array}{l}\sum_{\infty} \\
\vdots \\
\sum_{\infty}\end{array}$ & $\sum$ & $\begin{array}{c}\sum_{0} \\
\vdots \\
\omega \\
\omega\end{array}$ & के & ऊ & $\sum_{\text {ऍ }}$ \\
\hline 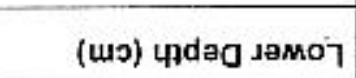 & $\bar{\infty}$ & 品 & g & $\Sigma$ & 蚛 & 뚣 & 总 & 우 & : & 우 & $\stackrel{2}{\stackrel{2}{2}}$ & $\cong$ & $\stackrel{0}{\sim}$ & 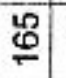 & $\mathscr{q}$ & $\mathscr{m}$ & के' & సू & \$ & $\mathbb{g}$ & 『 \\
\hline (wכ) पұdog Jədd ח & क & 而 & $\pi$ & \% & $\stackrel{\square}{\varrho}$ & $\stackrel{m}{m}$ & $\bar{\infty}$ & 0 & 윰 & 0 & के & व & ळ & 운 & 운 & ผ & $\bar{\kappa}$ & 우 & ָָ & ॠ? & ए \\
\hline iH & 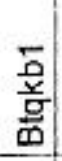 & 3 & $\stackrel{0}{c}$ & 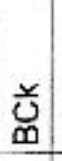 & 蒿 & $\begin{array}{l}\overline{0} \\
\text { है } \\
\text { 离 } \\
\text { ते }\end{array}$ & & $\varangle$ & $\begin{array}{l}\text { 章 } \\
\text { | }\end{array}$ & \& & 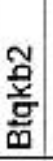 & $\mathbb{2}$ & 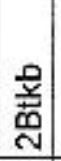 & & & $\mathcal{\mathcal { J }}$ & & & 梁 & ๕) & I \\
\hline әроэ घ घ|duEs p|ㅣㅂ & 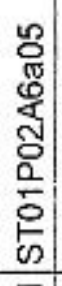 & 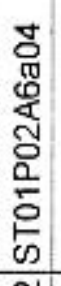 & 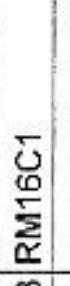 & $\begin{array}{l}8 \\
0 \\
0 \\
8 \\
5 \\
8 \\
\overline{0} \\
0 \\
5\end{array}$ & 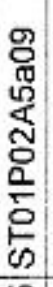 & $\begin{array}{l}0 \\
0 \\
0 \\
0 \\
5 \\
0 \\
0 \\
5 \\
0 \\
0\end{array}$ & 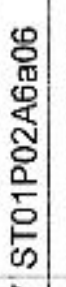 & 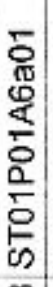 & $\begin{array}{l}5 \\
0 \\
\mathbb{N} \\
\mathbb{8} \\
\tilde{0} \\
0 \\
\overline{0} \\
0\end{array}$ & 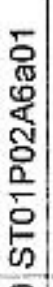 & 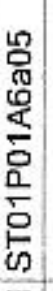 & 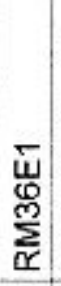 & 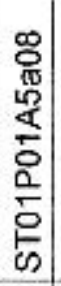 & 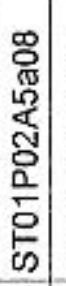 & 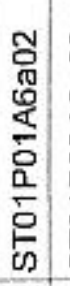 & 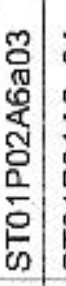 & $\begin{array}{l}\mathbf{5} \\
0 \\
0 \\
\frac{0}{0} \\
5 \\
0 \\
\vdots \\
0 \\
0\end{array}$ & 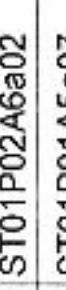 & 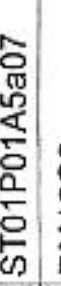 & & 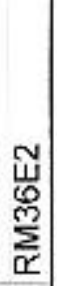 \\
\hline 리us & - & N & m & $\nabla$ & का & - & N & $\infty$ & का & 유 & $=$ & N & $\%$ & 古 & $\stackrel{\circ 1}{\square}$ & 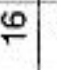 & $\mp:$ & $\stackrel{\infty}{\sim} \leqslant$ & 9 & i. & $\bar{N}$ \\
\hline эาе & 0 & g. & 宫 & 安 & ᄒ & ర్ & б & ᄒ⿱宀女子 & $\bar{g}$ & $\overline{8}$ & $\bar{g}$ & $\bar{g}$ & б & 宇 & $\bar{g}$ & $\overline{8}$ & 5 & $\bar{g}$ & S & $\bar{\sigma}$ & $\overline{8}$ \\
\hline
\end{tabular}




\begin{tabular}{|c|c|c|c|c|c|c|c|c|c|c|c|c|c|c|c|c|c|c|c|}
\hline $00 z \#>d$ & Nి & 으 & ה & @ & $=$ & ণ্ & & T/ & & $\overline{v^{\alpha}}$ & in & $\mathscr{N}$ & 6 & $N$ & & $6 \%$ & 5 & $\mp$ & I \\
\hline Ot\#>d & $\bar{\omega}$ & ㄱ & Q & D্ & ก & $\stackrel{\infty}{\circ}$ & ন & ma & & D: & in & $\stackrel{\infty}{n}$ & $=$ & $\stackrel{2}{2}$ & ले & 강 & क & $\overline{7}$ & $\bar{s}$ \\
\hline $\mathrm{Ol} \#>\mathrm{d}$ & 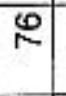 & : & ळ & (8) & q & 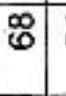 & से & ? & $8:$ & t. & is & $\Phi_{\infty}^{\not}$ & क्ल & 导 & $g$ & 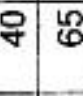 & \$ & ช & 욤 \\
\hline$t \#>d$ & $\stackrel{\infty}{\infty}$ & 学 & ळ & I & మ & $\approx$ & F & 㴓 & 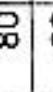 & 5 & M & \% & छ & ? & 8 & 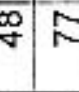 & 8 & $m$ & $?$ \\
\hline$u \div-8 / \varepsilon>d$ & \% & ธ & के & Q & 9 & \begin{tabular}{l|l}
$\infty$ & \\
$\infty$ &
\end{tabular} & 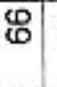 & 2 & ( & 8 & $\stackrel{\check{c}}{\infty}$ & 5 & 8 & $\infty$ & న & $\bar{\omega} \mid \infty$ & 8 & $\infty$ & œ \\
\hline$u !-t / \varepsilon>d$ & 음 & $\bar{\infty}$ & S & $\begin{array}{l}\infty \\
\infty\end{array}$ & \%) & के & 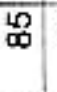 & $\bar{\infty}$ & 8 & $\xi^{\infty}$ & : & 9 & 5 & 48 & \% & 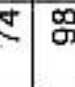 & g & \% & 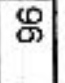 \\
\hline$u !-t>d$ & 음 & 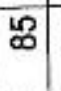 & ब & 8. & बे & ஃ & ఖ & 4 & 药 & 5 & : & 8 & $\mathscr{\circ}$ & 9 & 8 & \begin{tabular}{l|l}
0 & $\infty$
\end{tabular} & 8 & హ) & స \\
\hline แ!- $Z / l \iota>d$ & \% & क् & ㅇ․ & जे & 8 & के & \% & 5 & 8 & 5 & : & 8 & 8 & : & के & 8 & 8 & 边 & $\stackrel{\infty}{\infty}$ \\
\hline$u !-z>d$ & 우 & से & 음 & $\Phi$ & 品 & 今 & $\mathscr{8}$ & बू & 8 & 8 & : & : & क & 8 & \% & '大 & \% & : & g \\
\hline ull $-\varepsilon>d$ & $\stackrel{0}{\circ}$ & 웅 & ○े & 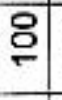 & 운 & 잉 & 인 & 8 & 8 & 9 & 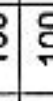 & 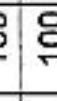 & 우 & : & 우 & 5 & 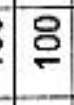 & 인 & 음 \\
\hline$(\%$ & $\begin{array}{l}\text { Na } \\
\text { Ni }\end{array}$ & $\begin{array}{l}\text { : } \\
\text { 虫 }\end{array}$ & m. & $\begin{array}{l}\text { 它 } \\
\text { ș }\end{array}$ & $\begin{array}{l}0 \\
\infty \\
\infty\end{array}$ & స్ల & 孞 & $\begin{array}{l}\odot \\
\stackrel{\circ}{\circ}\end{array}$ & הి & : & . & is & $\begin{array}{l}n \\
0 \\
0\end{array}$ & $\begin{array}{l}\mathrm{N} \\
\stackrel{\mathrm{N}}{ }\end{array}$ & $\stackrel{\nabla}{\triangle}$ & : & $\begin{array}{c}m \\
\infty\end{array}$ & \begin{tabular}{c|} 
\\
$\dot{\varphi}$
\end{tabular} & लि \\
\hline$(\%$ \% sosn pues & ल) & $\begin{array}{l} \\
\dot{y}\end{array}$ & $\begin{array}{l}0 \\
\tilde{D} \\
\tilde{0}\end{array}$ & $\begin{array}{l}\text { के } \\
\dot{n}\end{array}$ & 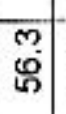 & mi & 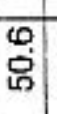 & $\begin{array}{l}0 \\
\dot{w} \\
\dot{w}\end{array}$ & דิ) & $\underset{\Phi}{\mathbb{c}}$ & & ic & $\mid \begin{array}{l}0 \\
\tilde{\omega}\end{array}$ & $\begin{array}{l}\text { ㅇ. } \\
\dot{0}\end{array}$ & \begin{tabular}{l|l} 
& i \\
\end{tabular} & $\begin{array}{l}5 \\
7 \\
\end{array}$ & $\mid \begin{array}{c}0 \\
\vdots \\
0\end{array}$ & 혛 & है \\
\hline (\%'M) SOSN & \begin{tabular}{l} 
Lొ \\
\hdashline \\
\hdashline
\end{tabular} & 苟 & क. & 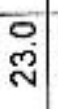 & o & $\stackrel{N}{\simeq}$ & $\overline{\dot{m}}$ & $\begin{array}{l}0 \\
\omega \\
N\end{array}$ & to & ơ & & $\begin{array}{lll}0 & \alpha \\
0 & 1\end{array}$ & $\begin{array}{l}0 \\
\text { ม̇ }\end{array}$ & $\begin{array}{c}T \\
\infty\end{array}$ & \begin{tabular}{lll}
\multirow{\sigma}{\sigma}{} \\
$\stackrel{\sigma}{\sigma}$
\end{tabular} & 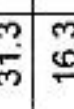 & $\stackrel{\square}{ \pm}$ & $\begin{array}{c}m \\
\infty \\
\infty\end{array}$ & $\frac{N}{N}$ \\
\hline qEר-dnos פ sosn & $\sum_{\omega}$ & $\sum_{\omega}$ & के & के & $\sum$ & 仿 & के & ह & 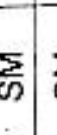 & $\sum_{\omega \infty}$ & & 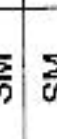 & 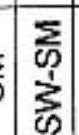 & $\begin{array}{c}\sum \\
\text { क } \\
\sum_{\infty}\end{array}$ & 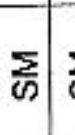 & $\sum_{n}$ & ई & ऊ) & $\sum_{\infty}$ \\
\hline (uv) पłdad Jamo 7 & 6 & ָั & $\infty$ & 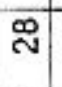 & $\frac{10}{2}$ & 2 & 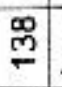 & 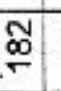 & స్ & 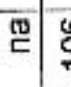 & 2. & ल & 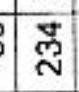 & กิ & \&্ণী & gु & $\stackrel{=}{\simeq}$ & กิ & $\infty$ \\
\hline (wo) 4łded Jaddn & 0 & స్ & 0 & i & \% & क्ल & $\stackrel{0}{0}$ & $\stackrel{N}{\mp}$ & $\omega$ & क्ष & 20 & $\begin{array}{l}0 \\
\end{array}$ & 要 & ลิ & ปี & 空 & 2 & م & 0 \\
\hline uoz!山OH & $<$ & $\begin{array}{l}\overline{\mathbf{a}} \\
\stackrel{\mathbf{u}}{\mathrm{N}}\end{array}$ & $\leq$ & $\overline{\mathbf{m}}$ & $\frac{2}{0}$ & 竞 & 爰 & $\begin{array}{l}\hat{v} \\
\text { 音 } \\
0 \\
0\end{array}$ & 岁 & T: & & $\underline{a}$ & 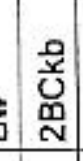 & 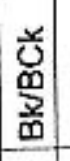 & 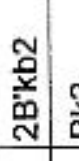 & ב⿱ ב⿱山 & 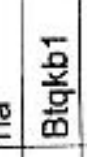 & $\begin{array}{l}\frac{0}{\mathbf{s}} \\
\text { 空 }\end{array}$ & $\frac{\pi}{2}$ \\
\hline apoj 키ues plal] & 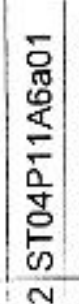 & 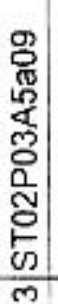 & $\begin{array}{l}5 \\
0 \\
0 \\
0 \\
0 \\
0 \\
0 \\
0 \\
0 \\
0 \\
0\end{array}$ & $\begin{array}{l}0 \\
0 \\
0 \\
\mathbb{2} \\
0 \\
0 \\
0 \\
0\end{array}$ & \begin{tabular}{|l|}
20 \\
80 \\
0 \\
8 \\
00 \\
0 \\
0 \\
0 \\
0 \\
0
\end{tabular} & 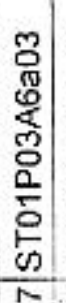 & 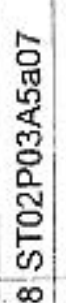 & $\begin{array}{l}2 \\
0 \\
0 \\
0 \\
0 \\
0 \\
0 \\
0 \\
5 \\
0 \\
0 \\
0\end{array}$ & 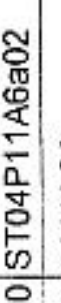 & 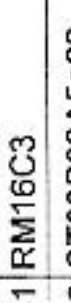 & 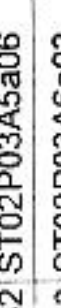 & 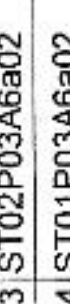 & 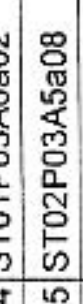 & 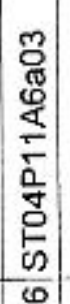 & 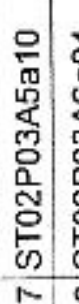 & 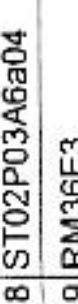 & 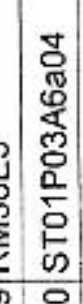 & $\begin{array}{l}0 \\
0 \\
0 \\
0 \\
0 \\
0 \\
0 \\
0 \\
0\end{array}$ & 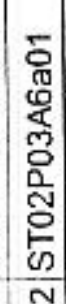 \\
\hline ㅁdues & ส & ल & 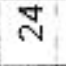 & & $\stackrel{\mathscr{N}}{\mathrm{N}}$ & $\stackrel{N}{N}$ & $\stackrel{\infty}{\sim}$ & N: & ले & $\bar{m}$ & , & $m$ & 点 & 邑 & m & $\infty$ & f & テ & ป \\
\hline чग्ष & $\bar{g}$ & $\bar{g}$ & $\bar{\sigma}$ & $\bar{g}$ & 5 & $\bar{q}$ & $\bar{g}$ & $\bar{q}$ & $\bar{q}$ & 5 & 5 & 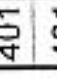 & $\bar{q}$ & $\bar{g}$ & $\bar{y}$ & $\bar{g}$ & $\bar{q}$ & $\bar{g}$ & $\overline{5}$ \\
\hline
\end{tabular}




\begin{tabular}{|c|c|c|c|c|c|c|c|c|c|c|c|c|c|c|c|c|c|c|c|c|}
\hline $00 Z \#>d$ & $\hat{N}$ & $\div$ & $\mp$ & $\begin{array}{lll}\infty & 0 & 0 \\
\end{array}$ & $m$ & 产 & $\infty$ & 은 & $\%$ & $\bar{m}$ & का & mा & का & N: & ले? & $\frac{2}{5}$ & ㄴ. & & का & $\bar{N}$ \\
\hline ot $\#>d$ & $\mathrm{~N}$ & 寸 & g & 8 & $\bar{N}$ & 广 & $\stackrel{ \pm}{N}$ & บั & $\stackrel{\infty}{\infty}$ & เด & 일 & $\stackrel{\infty}{\sim}$ & क्ल: & 8 & ஜ: & n & $=9$ & $\begin{array}{c}3 \\
3\end{array}$ & $\stackrel{\infty}{\sim}$ & E \\
\hline OLA $>d$ & 8 & 吕 & 吕 & $\infty$ & 賗 & F & ए户 & $\bar{\omega}$ & ले & $\frac{N}{N}$ & I & 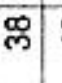 & g & 8 & ल/5 & 85 & $\Phi \bar{~}$ & $\begin{array}{l}3 \\
5\end{array}$ & $\mathbb{N}$ & ஜ \\
\hline$t \#>d$ & $\%$ & \begin{tabular}{l|l|}
0 \\
6
\end{tabular} & छ & $\infty$ & 守 & $\overline{0}$ & 8 & 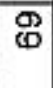 & 守 & $\begin{array}{l}\text { D } \\
\end{array}$ & 怘 & W & 吊 & 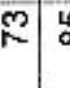 & 4 & \pm 4 & 89 & f & 由 & 8 \\
\hline $4 !-8 / \varepsilon>d$ & 5 & 贵 & n & สั & 8 & 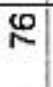 & ○ & N & $\mathscr{\circ}$ & 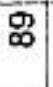 & 是 & $\widehat{6}$ & R: & $\infty: \bar{c}$ & Б) & 8 & 8 & 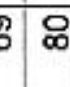 & \& & 遇 \\
\hline$u_{1}-\nabla / \varepsilon>d$ & g & 㽞 & $\bar{\infty}$ & ลे & $m$ & Б) & 8 & $\mathscr{\infty}$ & 6 & ठँ & N & $\mathbb{\infty}$ & 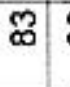 & \&: & ळ & \%: & 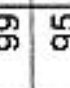 & 8 & 怘 & के \\
\hline$u !-b>d$ & g) & $\widehat{\infty}$ & 邑 & ธิ & 瞏 & \% & 우 & $\infty$ & $\frac{5}{4}$ & Һ & D & ఐ & ळ & 8 & \& & 5 & 84 & 品 & $\mathbb{8}$ & s̀ \\
\hline แ!- $-z / L l>d$ & 움 & ส & 8) & 曲 & 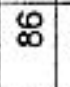 & 怘 & 웅 & の & एँ & ธิ & 分 & 5. & ส & g & 8 & $\stackrel{\infty}{\circ} 5$ & $8:$ & 8 & 8 & \% \\
\hline$u !-z>d$ & 임 & \% & 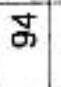 & \%) & \% & के & 응 & $\mathscr{g}$ & 8 & $\mathscr{\circ}$ & 8) & 迢 & 㟧 & 8 & S: & g & 8 & 要 & \begin{tabular}{|l|}
$\infty$ \\
$\infty$
\end{tabular} & \% \\
\hline$u !-\varepsilon>d$ & ㅇ & 은 & 음 & 움 & $\stackrel{9}{\circ}$ & ㅇ & 으 & 으 & 이 & 은 & 우 & 위 & 号 & 8 & 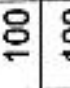 & $\stackrel{\circ}{\circ}$ & 8 & 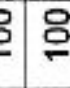 & 으 & 음 \\
\hline$(\% 2 M) \operatorname{sosn}^{-}$KE|O+11!S & $\begin{array}{l}N \\
\text { di }\end{array}$ & $\begin{array}{l}\vec{N} \\
\dot{N}\end{array}$ & $\underline{\underline{6}}$ & $\frac{7}{4}$ & $\begin{array}{l}40 \\
\infty\end{array}$ & 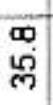 & $\begin{array}{l}\sigma \\
\mathrm{p} \\
\mathrm{m}\end{array}$ & $\vec{\sigma}$ & \begin{tabular}{|c|} 
\\
$\infty$ \\
\multirow{\infty}{*}{}
\end{tabular} & 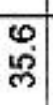 & $\begin{array}{l} \\
\stackrel{0}{\leftarrow}\end{array}$ & $\begin{array}{l}0 \\
\sigma \\
\sigma\end{array}$ & $\begin{array}{l}N \\
O \\
O\end{array}$ & $\begin{array}{l}\dot{m} \\
\vec{m}\end{array}$ & 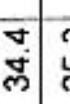 & స) & & 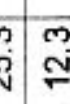 & $\overline{\mathrm{N}}$ & $\vec{d}$ \\
\hline (\%?M) sosn'pues & $\begin{array}{l}0 \\
\tilde{\omega} \\
\tilde{0}\end{array}$ & $\begin{array}{l}0 \\
\text { กิ่ }\end{array}$ & 葴 & 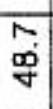 & $\begin{array}{l}\infty \\
\infty \\
\infty \\
6\end{array}$ & 宛 & $\begin{array}{l}\text { ปे. } \\
\text { U. }\end{array}$ & $\bar{G}$ & \begin{tabular}{|c|} 
\\
$\infty$ \\
$\infty$ \\
$ల$
\end{tabular} & $\frac{0}{i n}$ & 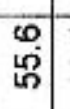 & $\overline{\mathcal{~}}$ & गु & 3 & 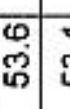 & 总 & & ơ & \begin{tabular}{|c|}
0 \\
$\bar{\sigma}$
\end{tabular} & $\begin{array}{l}\tilde{O} \\
\dot{0}\end{array}$ \\
\hline (\%) SOS & $\underset{F}{N}$ & $\begin{array}{l}\text { Lొ } \\
\text { वें }\end{array}$ & ָे & ָָ & $\frac{7}{m}$ & $\stackrel{\vec{L}}{\vec{L}}$ & ָু & $\frac{N}{N}$ & गें & $\begin{array}{l}\mathrm{J} \\
\mathrm{N}\end{array}$ & $\begin{array}{l}0 \\
0 \\
\stackrel{\infty}{0}\end{array}$ & 安 & 灾 & $\begin{array}{c}\infty \\
\infty \\
\infty\end{array}$ & & & & $\vec{n}$ & m & $\begin{array}{l}\infty \\
\dot{I}\end{array}$ \\
\hline qEר-dnodo sosn & ळ & इ & ऽ & $\sum$ & 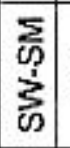 & § & 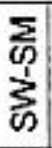 & $\begin{array}{l}\sum \\
\text { के } \\
\text { ड़ }\end{array}$ & $\sum_{\omega}$ & $\Sigma_{\omega}$ & क & $\begin{array}{l}\sum_{0} \\
\sum_{\omega}^{1}\end{array}$ & $\begin{array}{l}\sum_{0} \\
\sum_{\infty}^{1}\end{array}$ & क & ह & $\sum_{n}$ & $\sum_{n}$ & 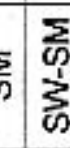 & $\sum_{\infty}$ & ๘ \\
\hline (шэ) पұdag Јәмоา & $\mathbb{g}$ & กิ & 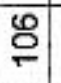 & $\infty$ & 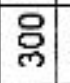 & $\underset{\mathbb{N}}{\mathbb{N}}$ & $\overline{\mathrm{s}}$ & $\bar{F}$ & $\bar{v}$ & $\stackrel{\sigma}{=}$ & $\frac{n}{N}$ & p & $\stackrel{\oplus}{\doteqdot}$ & 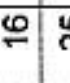 & $\stackrel{\sim}{\sim}$ & 㐫 & $\bar{m}$ & $\stackrel{\mathscr{8}}{0}$ & సิ & $\mathscr{ల}$ \\
\hline ( (U૭) updøg Jפddn & $\stackrel{0}{5}$ & প্ & 하 & 0 & $\frac{L}{N}$ & б. & 品 & N & $\stackrel{\mathbb{N}}{\sim}$ & $\infty$ & ळ) & \% & 志 & 0 & ㅇ & t & $\mathbb{2}$ & 司 & $\underset{\infty}{\circ}$ & 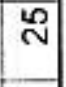 \\
\hline & 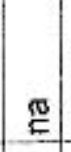 & 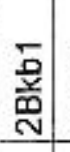 & $\begin{array}{l}\text { ? } \\
\text { 㩊 }\end{array}$ & 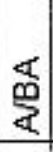 & $\begin{array}{l}\text { N̂} \\
\text { Dे } \\
\end{array}$ & 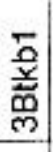 & $\begin{array}{l}\text { 离 } \\
\end{array}$ & $\begin{array}{l}\text { No } \\
\text { 离 } \\
\text { N }\end{array}$ & 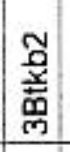 & $\overline{\mathbf{m}}$ & $\begin{array}{l}- \\
\text { 离 } \\
\end{array}$ & $\begin{array}{l}\text { 怘 } \\
\text { 商 }\end{array}$ & 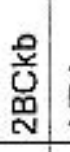 & $\frac{\mathbb{1}}{2}$ & & 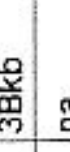 & & : & 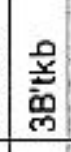 & 商 \\
\hline 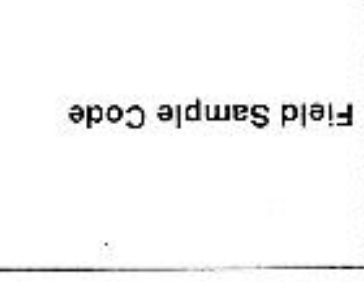 & $\begin{array}{l}\bar{x} \\
0 \\
\oplus \\
0 \\
\sum_{\underline{\Sigma}}\end{array}$ & 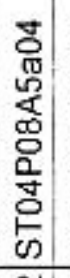 & 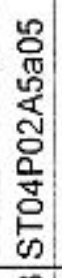 & $\begin{array}{l}5 \\
0 \\
0 \\
\frac{1}{0} \\
0 \\
0 \\
\frac{0}{5} \\
0 \\
0\end{array}$ & $\begin{array}{l}8 \\
0 \\
0 \\
8 \\
0 \\
0 \\
0 \\
0 \\
0\end{array}$ & $\begin{array}{l}2 \\
0 \\
0 \\
0 \\
0 \\
0 \\
0 \\
0 \\
0 \\
0 \\
0 \\
0\end{array}$ & 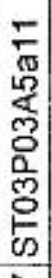 & 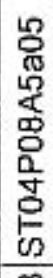 & 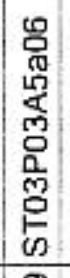 & 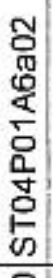 & $\begin{array}{l}0 \\
0 \\
0 \\
0 \\
0 \\
0 \\
0 \\
\frac{0}{5} \\
0 \\
0 \\
0\end{array}$ & 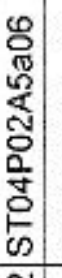 & 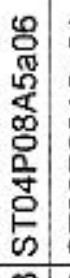 & 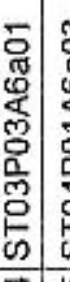 & 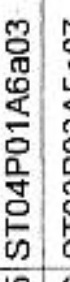 & 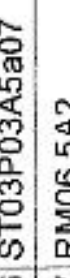 & 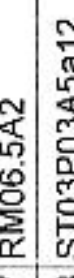 & 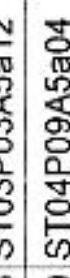 & 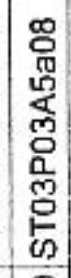 & $\begin{array}{l}0 \\
0 \\
0 \\
0 \\
0 \\
0 \\
0 \\
0 \\
0 \\
0 \\
0\end{array}$ \\
\hline a|dmes & 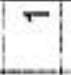 & $\sim$ & m & $\nabla$ & $\infty$ & c) & & $\infty$ & a & 으 & $\mp$ & $\frac{1}{4}$ & $m$ & I & 55 & (). & $F \div$ & $=9$ & ㄱ & $\bar{N}$ \\
\hline 298 & ণ্ণ & ণิ & $\underset{\gamma}{\mathscr{y}}$ & 额 & 跑 & $\frac{\mathrm{N}}{\mathrm{V}}$ & ণ్ర & O & ఫ్ & ్ָ & ปิ & ্ָড & テे| & 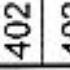 & $\begin{array}{c}\tilde{y} \\
y\end{array}$ & $\begin{array}{c}\text { So } \\
\text { S }\end{array}$ & ชิ & š & Õ & 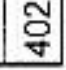 \\
\hline
\end{tabular}




\begin{tabular}{|c|c|c|c|c|c|c|c|c|c|c|c|c|c|c|c|c|c|c|c|}
\hline $00 z \#>d$ & $\nabla$ & $\bar{\sim}$ & 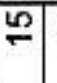 & 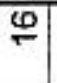 & 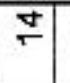 & $\begin{array}{l}\infty \\
\stackrel{\infty}{\circ}\end{array}$ & $\stackrel{m}{\square}$ & సิ & N & का: & का: & 27 & & $9 \div$ & $F$ & !n & $1=$ & 6 & 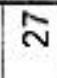 \\
\hline 0 t\# $>d$ & g) & (8) & 이 & $\stackrel{\infty}{q}$ & 寸 & 罗 & q & m & צ & $\stackrel{4}{2}$ & क & F & $=$ & 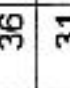 & กั & $\mathscr{E}$ & $\mathscr{N}$ & ని & $\notin$ \\
\hline $0 l \#>d$ & 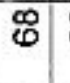 & 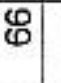 & 足 & 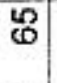 & 8 & है। & $\overline{6}$ & ใิ & 跑 & m: & 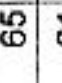 & $\bar{\omega}$ & 8 & กิ? & 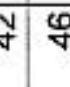 & $\frac{1}{2}$ & ले & $\overline{5 n}$ & N \\
\hline$t \#>d$ & $\stackrel{\infty}{\sim}$ & 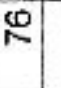 & o & 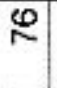 & 足 & 2 & 品 & 8 & ? & 昌: & N & 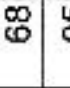 & Љ) & $\begin{array}{c}\infty \\
\infty\end{array}$ & 56 & $\infty$ & $\stackrel{9}{q}$ & 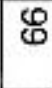 & \\
\hline $\mathrm{u}-8 / \mathcal{E}>d$ & $\infty$ & ळ & 2 & 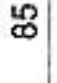 & $\mathscr{\infty}$ & 2 & $\mathbb{\infty}$ & ఎ & $\infty$ & ช & 金 & $\begin{array}{l}\infty \\
\wedge\end{array}$ & के & $\infty$ & $\pm \frac{9}{1}$ & ชั & 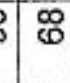 & R & $\bar{\sigma}$ \\
\hline$u !-t / \varepsilon>d$ & 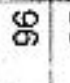 & ๘ & ஃ & के & $\%$ & ஜ & ब & के & Б) & $\begin{array}{l}9 \\
1\end{array}$ & 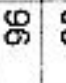 & ळ & का & $\infty \begin{array}{l}\infty \\
\infty\end{array}$ & 8 & a & 8 & $\stackrel{\infty}{\infty}$ & \\
\hline$u \mid-t>d$ & $\mathscr{8}$ & 8 & \% & ๕ & \& & ळ & का & ธิ & \%) & 声 & के & ब. & g) & के & '大 & 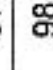 & \%ั & 8 & a \\
\hline$u !-z / L\llcorner>d$ & $\stackrel{\infty}{\infty}$ & సै. & 우 & s. & क & \%) & ! & 吕 & \% & 잉 & $\$$ & ने|ई & ? & जे & $\begin{array}{l}\infty \\
\S\end{array}$ & g & 4 & ङ & a \\
\hline$u-z>d$ & ' & \% & 이 인 & 思 & g) & $\mathscr{~}$ & 은 & g & 思 & के & g & $\mathscr{E} \mid \leqq$ & 吾 & के & 5 & g & 各 & $\mathscr{8}$ & g \\
\hline$u \leftarrow \varepsilon>d$ & $\stackrel{\circ}{\circ}$ & \% & @ & ㅇ & 위 & 잉 & 잉 & 인 & $\stackrel{8}{\circ}$ & 의 & 음 & ㅇ? & 8 & : & $\%$ & 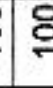 & $\%$ & 요 & 요 \\
\hline$(\% 2 M) \operatorname{sos}^{-}$(KE|כ+2!!s & mìn & స్ & 品 & $\stackrel{4}{\frac{1}{N}}$ & 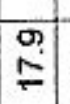 & 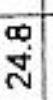 & $\begin{array}{l}\vec{t} \\
\dot{6}\end{array}$ & $\begin{array}{l}\mathrm{N} \\
\mathrm{N}\end{array}$ & $\bar{\sigma}$ & $\begin{array}{l}\infty \\
\infty \\
\infty \\
\tilde{c}\end{array}$ & $\begin{array}{l}\infty \\
\text { Na }\end{array}$ & $\overline{\mathbf{N}}$ & 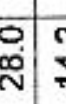 & & $\stackrel{9}{\circ}$ & & $\overline{\text { ন্ }}$ & $\stackrel{\infty}{\infty}^{\infty}$ & I \\
\hline (\%M) SOSn Sues & $\overline{\dot{G}}$ & 品 & $\frac{\dot{y}}{\dot{\sigma}}$ & $\frac{m}{5}$ & $\begin{array}{l} \\
8 \\
8\end{array}$ & 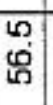 & \begin{tabular}{l|l} 
Na \\
of
\end{tabular} & 吕 & $\frac{N}{6}$ & $\begin{array}{l}m \\
\text { gु }\end{array}$ & $\begin{array}{l}50 \\
0 \\
0\end{array}$ & 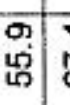 & 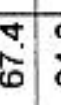 & & 5 & & $\begin{array}{l}0 \\
\vdots \\
\end{array}$ & $\check{\infty}$ & 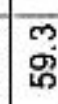 \\
\hline (\%) & 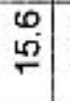 & 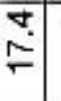 & $\frac{n}{\bar{m}}$ & $\bar{\Xi}$ & \begin{tabular}{|l|} 
\\
$\infty$ \\
$-\infty$
\end{tabular} & $\underset{\infty}{\infty}$ & $\begin{array}{l}\stackrel{0}{ \pm} \\
\dot{\square}\end{array}$ & & న్ & ले & $\begin{array}{l}\hat{T} \\
\hat{\varphi}\end{array}$ & స: & $\vec{\sigma}$ & & $\begin{array}{c}n \\
\stackrel{n}{*}\end{array}$ & & i & ฟู & 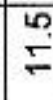 \\
\hline qE7-dnodo sosn & $\sum$ & क & क & $\Sigma_{\omega}$ & के & $\sum_{\infty}$ & $\sum_{\infty}$ & $\sum_{\infty}$ & क & ह & क & ल & $\sum_{\omega}$ & & क. & ब. & ह & 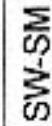 & 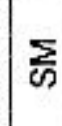 \\
\hline 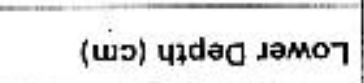 & 5 & n) & ले & : & $\stackrel{\infty}{\mp}$ & 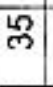 & $\stackrel{m}{\square}$ & 2 & \% & స్ & 2 & ลे & 可 & $9:$ & 5 & ก & 㶽 & \% & 0 \\
\hline (uэ) uzdøa Jedd n & $\stackrel{m}{m}$ & o & 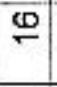 & 足 & $m$ & กิ & क & m & 용 & กิ้ & N & $\omega$ & 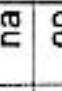 & 思 & e & $c$ & 就 & 总 & 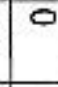 \\
\hline UOZ|АOH & 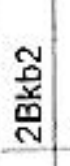 & $\varangle$ & 善 & 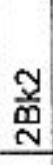 & 咅 & 商 & 忐 & 羔 & 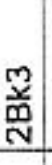 & 恙 & $\begin{array}{l}0 \\
\mathbf{s} \\
\mathbf{s} \\
\mathbf{m}\end{array}$ & $\frac{\frac{9}{9}}{\frac{9}{a}}$ & I & $\begin{array}{l}\mathbf{*} \\
\overrightarrow{\mathrm{N}}\end{array}$ & ت. & & 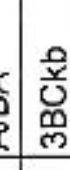 & $\begin{array}{l}\text { Y } \\
\text { N } \\
\end{array}$ & \\
\hline әроว 리dues plə!! & 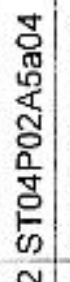 & 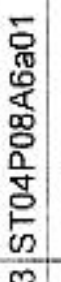 & 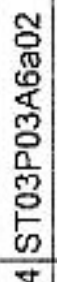 & 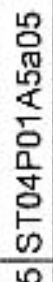 & $\begin{array}{l}0 \\
0 \\
0 \\
\frac{0}{0} \\
0 \\
0 \\
\frac{0}{0} \\
0 \\
0 \\
0\end{array}$ & 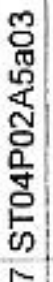 & 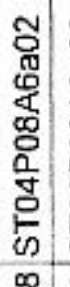 & $\begin{array}{l}8 \\
0 \\
0 \\
0 \\
0 \\
0 \\
0 \\
0 \\
0 \\
0 \\
0 \\
\\
\end{array}$ & 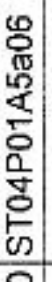 & 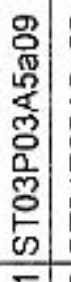 & 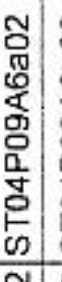 & 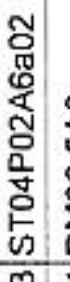 & 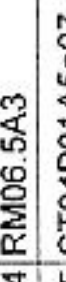 & 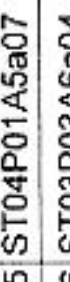 & 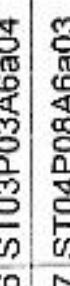 & 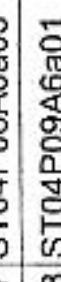 & $\begin{array}{ll}0 \\
0 \\
0\end{array}$ & 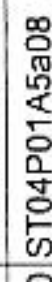 & $\begin{array}{l}5 \\
0 \\
0 \\
8 \\
0 \\
5 \\
0 \\
0 \\
0\end{array}$ \\
\hline aldures & ก & $\mathbb{N}$ & $\mathrm{n}$ & ลั & $\stackrel{\infty}{\circ}$ & N & $\stackrel{\infty}{N}$ & กิ & ద్లి| - & ले & m & $m$ & 茞 & m: & 照 & م) & : & 8 & $\overline{7}$ \\
\hline peg & O & & $\approx$ & & ूु & סु & 等 & స్ర & Sy & 岁 & סु & ซै & 尊 & రृ & \% & 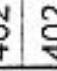 & $\mathscr{H}^{2}$ & ซु & 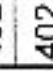 \\
\hline
\end{tabular}




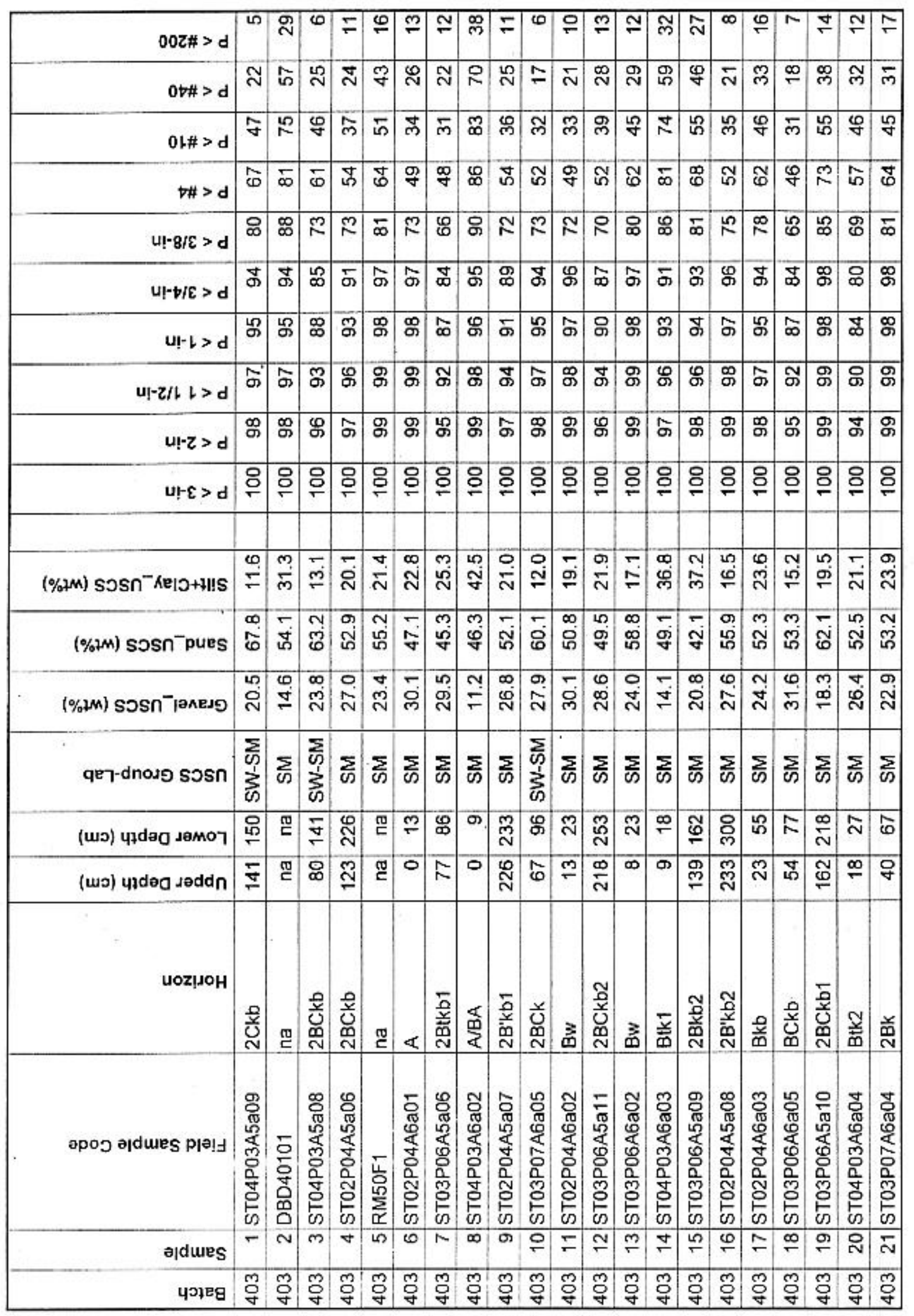




\begin{tabular}{|c|c|c|c|c|c|c|c|c|c|c|c|c|c|c|c|c|}
\hline $00 z \#>d$ & का & $\stackrel{42}{5}$ & $\stackrel{m}{\sim}$ & क & m & $\infty$ & 2 & $\frac{20}{2}$ & M & का & 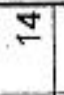 & 寸 & $\frac{917}{2}$ & Iभ & $\begin{array}{l}5 \\
\end{array}$ & $\pi$ \\
\hline $0 b \#>d$ & స & षे & 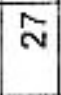 & 8 & 昌 & N & 多 & ชั & ल & 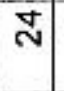 & g & $\stackrel{\leftrightarrow}{N}$ & $\stackrel{m}{N}$ & $\stackrel{\circ}{\circ}$ & 9 & $\stackrel{m}{N}$ \\
\hline $0 \mathrm{l} \#>d$ & ले & 8 & 帛 & Y & 8 & $\stackrel{\infty}{\sim}$ & 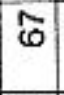 & 은 & g & F & g & 8 & $\stackrel{\sim}{\sim}$ & 足 & is & กี \\
\hline$t \#>d$ & 思 & 吕 & 5 & 思 & 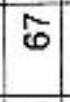 & 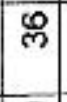 & 9 & \begin{tabular}{|l|}
8 \\
60
\end{tabular} & 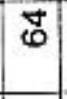 & 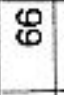 & 点 & 5 & 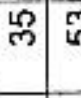 & S) & 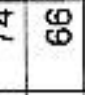 & $\mathscr{Y}$ \\
\hline$u !-8 / \varepsilon>d$ & $\infty$ & $\frac{m}{N}$ & ㅇ & $\infty$ & $m_{\infty}$ & g & $\infty$ & $\mathscr{\infty}$ & $\bar{\infty}$ & న్ & 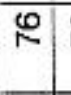 & 2 & 애 & $\bar{\sigma}=\frac{\mathscr{L}}{\alpha}$ & $\bar{\infty}$ & फ \\
\hline$u !-t / \varepsilon>d$ & ळ & 志 & \begin{tabular}{|l|}
$\infty$ \\
$\infty$
\end{tabular} & M & $\begin{array}{ll} \\
0\end{array}$ & 5 & 9 & \begin{tabular}{|l|}
$\mathscr{0}$ \\
\end{tabular} & \$ & $\$$ & $\mathscr{\%}$ & ळ & 8 & \begin{tabular}{l|l}
$\infty$ \\
$\infty$
\end{tabular} & 58 & $\mathscr{\varphi}$ \\
\hline$u !-l>d$ & $\tilde{\omega}$ & $\mathscr{8}$ & $\bar{\sigma}$ & 5 & 吕 & g & 5) & 怘 & g & 怘 & Бे & $\bar{\sigma}$ & m & $8: 1$ & 今 & $\mathfrak{i}$ \\
\hline$u !-Z / L \downarrow l>d$ & $\mathscr{\infty}$ & $\bar{\sigma}$ & ॠ & 怘 & \% & 吕 & \begin{tabular}{|l|} 
品 \\
\end{tabular} & g & 号 & 8 & 怘 & ङे & \begin{tabular}{l|l}
$\infty$ & 5 \\
$\infty$ & 5
\end{tabular} & \begin{tabular}{l|l} 
बे & \multicolumn{2}{c}{}
\end{tabular} & 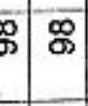 & $\infty$ \\
\hline$u !-z>d$ & 8 & $\stackrel{\infty}{\infty}$ & s. & g & g & (פ) & g & ' & g & g & g & $\stackrel{s}{s}$ & 怘 & $\mathscr{E}:$ & ুু & 号 \\
\hline$u \mathrm{u}-\varepsilon>d$ & 8 & 웅 & 量 & 8 & 용 & 웅 & 윰 & 웅 & 용 & 8 & 8 & 8 & ㅇํㄷ & 8 & 8 & 응 \\
\hline (\%2m) $\operatorname{sosn}^{-}$ke|ว+1!!s & $\begin{array}{l}m \\
\infty \\
\infty\end{array}$ & $\frac{\pi}{\mathrm{N}}$ & m & \begin{tabular}{l|}
$\sigma$ \\
$\infty$ \\
$\infty$ \\
$N$
\end{tabular} & Бें & $\frac{0}{-1}$ & 㔛 & 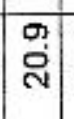 & $\frac{5}{m}$ & $\stackrel{5}{\square}$ & Nิ & \begin{tabular}{l|l}
$\infty$ & 0 \\
$\infty$ & 0 \\
$\infty$ & 0
\end{tabular} & 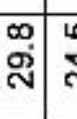 & 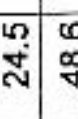 & 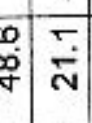 & $\begin{array}{l}\vec{\nabla} \\
\text { के }\end{array}$ \\
\hline$\left(\%\right.$ (\%) sosn ${ }^{-}$pues & $\begin{array}{l}0 \\
0 \\
6\end{array}$ & $\begin{array}{l}\infty \\
0 \\
0\end{array}$ & $\begin{array}{l} \\
\text { 多 }\end{array}$ & \begin{tabular}{|l|}
9 \\
\\
\end{tabular} & 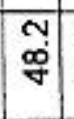 & $\begin{array}{l}m \\
m \\
q\end{array}$ & $\begin{array}{l}\infty \\
\dot{0} \\
\end{array}$ & 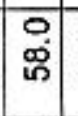 & 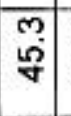 & $\overline{8}$ & $\frac{6}{50}$ & $\begin{array}{l}M \\
\dot{0}\end{array}$ & लि" & \begin{tabular}{l|l} 
ले \\
v
\end{tabular} & \begin{tabular}{l|l|}
$\tilde{N}$ & 0 \\
0 & 0 \\
$\leftrightarrow$
\end{tabular} & $\begin{array}{l}\infty \\
\infty \\
q\end{array}$ \\
\hline 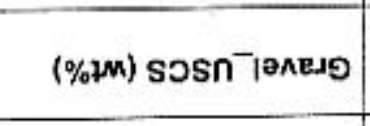 & N & $\stackrel{\sim}{\check{n}}$ & $\begin{array}{l}\Delta \\
\stackrel{n}{N}\end{array}$ & 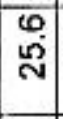 & $\stackrel{\omega}{\grave{N}}$ & $\begin{array}{l}m \\
m \\
m\end{array}$ & $\stackrel{\overrightarrow{s i j}}{\leftarrow}$ & $\overline{\mathrm{N}}$ & $\stackrel{\sim}{M}$ & స్ & 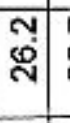 & ज़ & : & 察 & 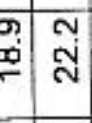 & $\begin{array}{l}0 \\
\text { N }\end{array}$ \\
\hline qeר-dno.s sosn & $\sum_{0}$ & $\overline{5}$ & $\overline{5}$ & $\sum_{\infty}$ & 市 & 心 & ह & $\sum_{\text {ڤ }}$ & फ & $\begin{array}{c}\sum_{0} \\
\sum_{0} \\
0\end{array}$ & $\bar{s}$ & 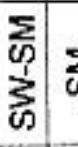 & $\frac{\sum}{5}$ & ळ & $\sum_{\infty}$ & क \\
\hline (uv) uldeg sәмо一 & 总 & $\infty$ & 9 & 음 & g & 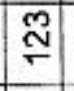 & फ & $\stackrel{\pi}{C}$ & $=$ & 藏 & 음 & $\sqrt{6}$ & 怘 & $\bar{z}$ & 맏 & 8 \\
\hline (uiv) पıdea deddn & $\infty$ & 0 & 贵 & $\mathscr{\%}$ & $=$ & क & N & 要 & $\omega$ & ए & న్ & 邑 & 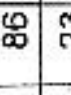 & M & "ृ & $\tilde{\omega}$ \\
\hline uoz!o애 & $\begin{array}{l}\text { 疍 } \\
\text { 产 }\end{array}$ & 4 & 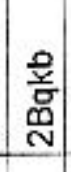 & 策 & $\begin{array}{c}\text { 恙 } \\
\text { 总 } \\
\end{array}$ & 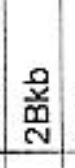 & 兽 & 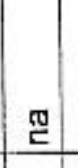 & 芯 & 点 & 恿 & $\begin{array}{l}\text { 总 } \\
\text { 岂 }\end{array}$ & 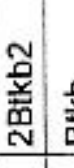 & 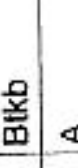 & ֻृ & 㩊 \\
\hline әpoว ә|dues plelı & 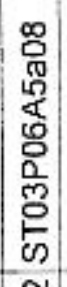 & 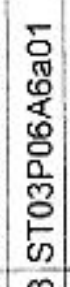 & 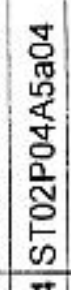 & $\begin{array}{l}0 \\
0 \\
0 \\
0 \\
0 \\
0 \\
5 \\
0 \\
0 \\
0 \\
0 \\
0 \\
0 \\
0\end{array}$ & $\begin{array}{l}0 \\
0 \\
0 \\
\mathbb{Q} \\
\mathbb{5} \\
0 \\
0 \\
\tilde{n} \\
0 \\
0 \\
0\end{array}$ & 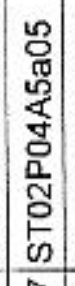 & \begin{tabular}{|l|}
0 \\
0 \\
8 \\
0 \\
$\mathbb{1}$ \\
8 \\
0 \\
0 \\
8 \\
8 \\
0 \\
0 \\
0
\end{tabular} & 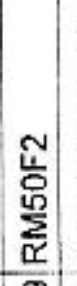 & $\begin{array}{l}0 \\
0 \\
0 \\
0 \\
0 \\
0 \\
0 \\
0 \\
0 \\
0 \\
0\end{array}$ & 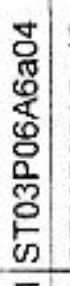 & 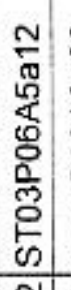 & $\begin{array}{c}0 \\
0 \\
\overline{0} \\
0 \\
\mathbb{1} \\
8 \\
0 \\
0 \\
0 \\
0 \\
0 \\
0\end{array}$ & 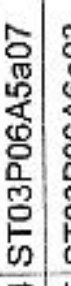 & 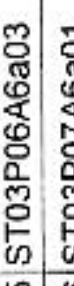 & 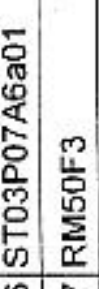 & $\begin{array}{l}5 \\
0 \\
0 \\
0 \\
0 \\
0 \\
0 \\
0 \\
\frac{1}{0} \\
0 \\
0 \\
\omega\end{array}$ \\
\hline श्रdues & N & ָे & D̃ & $\stackrel{2}{N}$ & 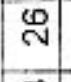 & $\approx$ & $\stackrel{\infty}{2}$ & (2) & 只 & $\bar{m}$. & लू & m: & ले & 요 & घ्ले ले & $\overbrace{ల}^{\infty}$ \\
\hline чэев & g & g & g & ğ & g & O & 8 & ? & 乌్ & 旁 & gु & g & g) & g) & \begin{tabular}{l|l}
3 & 3 \\
& 8 \\
\end{tabular} & g \\
\hline
\end{tabular}




\begin{tabular}{|c|c|c|c|c|c|c|c|c|c|c|c|c|c|c|c|c|c|c|c|}
\hline $00 Z \#>d$ & ম & $\mp$ & $\infty$ & $\approx$ & $\stackrel{2}{=}$ & $=1$ 응 & $2 F$ & 9 & 25 & 으 & लू & 12 & 의 & $\stackrel{4}{7} \div$ & $\stackrel{\mathscr{N}}{ }$ & 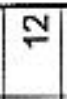 & का & 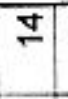 & \pm \\
\hline $0 t \#>d$ & छे & 等 & $\stackrel{\sim}{N}$ & जे & $\begin{array}{lll}\mathrm{N} & 0 \\
\end{array}$ & 呙 & क & \begin{tabular}{|l|}
$\infty$ \\
$\infty$
\end{tabular} & 芯 & $\stackrel{g}{\mathrm{~N}}$ & ष & $m$ & $\stackrel{4}{\mathrm{~N}}$ & $\underset{\forall}{\sim}$ & $\mathscr{F}$ & i & $\stackrel{\sigma}{\leftarrow}$ & 8 & 广 \\
\hline $0 \mathrm{L \#}>d$ & 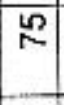 & \& & 匂 & $F$ & $\begin{array}{c}\tau \\
8\end{array}$ & m & 芯 & g & 8 & $\bar{v}$ & 20 & 18 & ल 1 & ஸ & 5 & g & 只 & 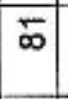 & $\overline{10}$ \\
\hline$t H>d$ & $\bar{\infty}$ & $\bar{r}$ & 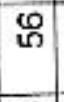 & 员 & \begin{tabular}{l|l} 
\\
$\sim$
\end{tabular} & \begin{tabular}{l|l}
$\mathscr{8}$ \\
\end{tabular} & $\bar{m}$ & N & 分 & 욤 & $\begin{array}{l}m \\
\infty\end{array}$ & $\mid$\begin{tabular}{l}
$\infty$ \\
\hdashline \\
$n$
\end{tabular} & $\stackrel{\infty}{q}$ & 25 & 웅 & 8 & 电 & के & $\mathscr{8}$ \\
\hline$u !-8 / \varepsilon>d$ & 怘 & ○ & $\mathscr{N}$ & 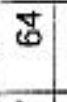 & 共 & 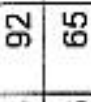 & $\mathscr{\infty}$ & ช్ & 8 & 9 & 용 & $\mathbb{N}$ & \begin{tabular}{l|l}
$\mathbb{0}$ & ळ
\end{tabular} & \$N & $\bar{\infty}$ & 8 & 9 & 48 & $\bar{r}$ \\
\hline $\mathrm{ul}-\not / \varepsilon>d$ & 5 & D & $\mathscr{\infty}$ & $E$ & $8 \%$ & \begin{tabular}{l|l}
$\%$ & $\infty$ \\
\end{tabular} & 8 & $\bar{N}$ & \begin{tabular}{|l|} 
o \\
\end{tabular} & 5 & : & 感 & \begin{tabular}{l|l} 
呙 & \%
\end{tabular} & 今. & \% & $\mathscr{O}$ & 욤 & 8 & $\hat{N}$ \\
\hline$u !-b>d$ & \% & $\bar{\sigma}$ & $\mathscr{8}$ & $\bar{\infty}$ & 芯票 & \begin{tabular}{l|l} 
& $\mathscr{O}$ \\
\end{tabular} & 응 & N & 兽 & 今. & 品 & \$ & \begin{tabular}{l|l} 
요 & $\mathscr{G}$
\end{tabular} & 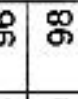 & 5 & के & ชิ & 용 & $\bar{\infty}$ \\
\hline u!-ZI $\iota>d$ & g & के & के & 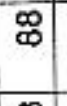 & \begin{tabular}{l|l}
8 \\
\end{tabular} & \begin{tabular}{l|l} 
& $\bar{\sigma}$
\end{tabular} & 웅 & $\mathscr{\infty}$ & I & 怘 & த) & మू & \begin{tabular}{l|l}
$\mathscr{\infty}$ & $\mathscr{\%}$
\end{tabular} & \begin{tabular}{l|l} 
& 8
\end{tabular} & के & 号 & เ & 음 & 丳 \\
\hline$u t z>d$ & g & 5 & \% & 8 & \% & \begin{tabular}{l|l} 
\\
\end{tabular} & 웅 & รั & g & g & g & $\mathscr{\sigma}$ & \%) & \begin{tabular}{l|l}
5 & 9
\end{tabular} & 号 & 8 & ڤ́ & 응 & \% \\
\hline u!l- $\varepsilon>d$ & 응 & 8 & 8 & 응 & 융유 & $8 \%$ & 음 & 인 & 은 & 인 & 은 & 인 & 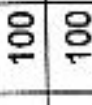 & 응 & 응 & 웅 & 8 & 용 & 웅 \\
\hline$(\% 2 m) \operatorname{sos}^{-}$Кe|0+1!!s & 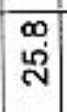 & 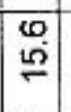 & $\begin{array}{ll}0 \\
\dot{T} \\
\end{array}$ & \begin{tabular}{|l|l|}
0 & 0 \\
$\dot{d}$ & 7
\end{tabular} & $\stackrel{\infty}{=}$ & 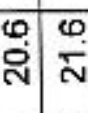 & 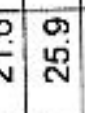 & \begin{tabular}{|l|} 
\\
0 \\
0
\end{tabular} & 总 & 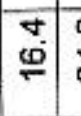 & \begin{tabular}{l|}
$\infty$ \\
$\dot{J}$ \\
\end{tabular} & 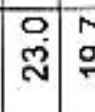 & $\stackrel{m}{\circ} \stackrel{m}{\circ}$ & 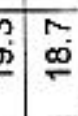 & $\bar{m}$ & $\begin{array}{l}\infty \\
\infty \\
\infty\end{array}$ & Nִ & $\begin{array}{l}\infty \\
\Phi \\
\oplus\end{array}$ & ले \\
\hline$(\% \not M)$ sosn $^{-}$pues & 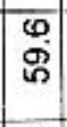 & $\begin{array}{l}\sigma \\
\ddot{0} \\
\ddot{0}\end{array}$ & 움 & \begin{tabular}{l|l}
$m$ & 0 \\
m & 0
\end{tabular} & 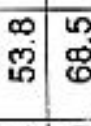 & $\begin{array}{lll}0 \\
0 \\
0 \\
0\end{array}$ & 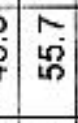 & \begin{tabular}{l|l}
\multirow{2}{*}{} \\
g
\end{tabular} & 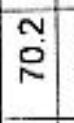 & \begin{tabular}{c|c}
$N$ \\
$\infty$ \\
$\infty$
\end{tabular} & क्ष & 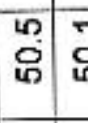 & है & 它 & 岁 & $\begin{array}{l}10 \\
0 \\
0 \\
60\end{array}$ & \begin{tabular}{|l|}
$\infty$ \\
0 \\
0 \\
\end{tabular} & $\begin{array}{l}\mathrm{r} \\
\end{array}$ & $\begin{array}{l}0 \\
\dot{1} \\
\dot{\omega}\end{array}$ \\
\hline (\%2M) sosn & $\begin{array}{l}n \\
\end{array}$ & 号 & फ़ & N: & 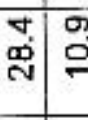 & 象 & 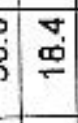 & O & 定 & 焗 & 芦 & 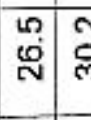 & 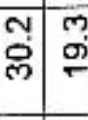 & 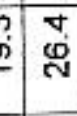 & 호 & $\begin{array}{l}\infty \\
\mathbb{N} \\
\mathbb{N}\end{array}$ & \begin{tabular}{l|} 
\\
के \\
cे
\end{tabular} & $\begin{array}{c}9 \\
\infty\end{array}$ & กี \\
\hline qeר-dnodg sosn & ऊ & 空 & $\begin{array}{l}\sum \\
\text { D } \\
\frac{1}{5} \\
\text { b }\end{array}$ & $\frac{5}{\omega}$ & $\sum_{\omega} \sum_{6}$ & $\sum_{\infty}$ & $\sum_{5}$ & 点 & 立 & $\sum$ & $\sum$ & $\sum_{\omega} \sum_{0}$ & $\sum_{\omega} \sum_{\omega}$ & $\sum_{W}$ & ज & 心 & $\sum_{\infty}$ & $\sum$ & ¿ \\
\hline (wo) чłdad גәмо一 & 要 & $\begin{array}{l}\text { 怘 } \\
\text { N }\end{array}$ & $\stackrel{\infty}{\circ}$ & 足 & g) & \begin{tabular}{c|c}
5 & 5 \\
\end{tabular} & $\bar{n}$ & ? & $\sum_{\infty}^{\infty}$ & 5 & \begin{tabular}{l|l} 
व & 9 \\
\end{tabular} & 品 & ్ㅣ & है & \%ั & $\infty$ & 惫 & ? & $\overline{5}$ \\
\hline (uv) upclea daddn & $\stackrel{\mathbb{Z}}{\mathrm{C}}$ & 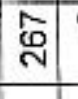 & $\circ$ & $\stackrel{m}{N}$ & ल) & $\frac{10}{8}$ & $\stackrel{\infty}{-}$ & స్ & $\stackrel{\infty}{\infty}$ & $\frac{9}{8}$ & $\stackrel{\mathbb{D}}{\mathrm{C}}$ & $\begin{array}{l}N \\
\sim\end{array}$ & $\bar{i}$ & 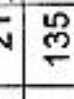 & $\bar{\infty}$ & 0 & $\underset{N}{\sim}$ & $\stackrel{\sim}{N}$ & m \\
\hline uoz!」OH & खี & 点 & 0 & 总 & 盖盖 & 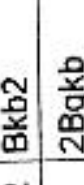 & 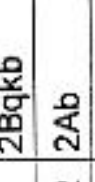 & $\mid \begin{array}{l}\text { N } \\
\text { 离 } \\
\text { ⿳亠丷厂心 }\end{array}$ & 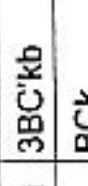 & 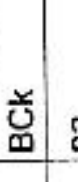 & ¿ & 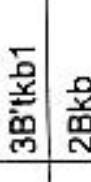 & $\begin{array}{l}\text { 产 } \\
\text { 愛 } \\
\end{array}$ & 总 & 总 & $\frac{\mathfrak{z}}{2}$ & 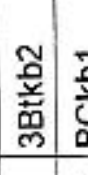 & 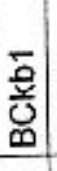 & 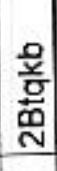 \\
\hline әроว ә|dwes p|ə!⿻ & 官 & 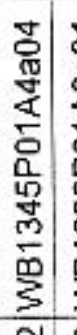 & 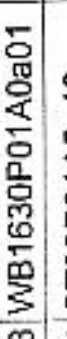 & 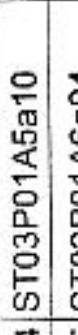 & & 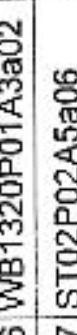 & 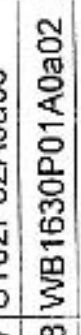 & 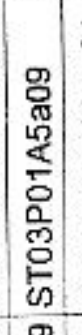 & 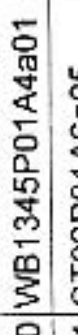 & 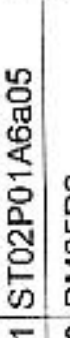 & 옹 & 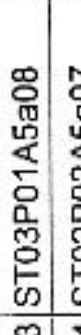 & 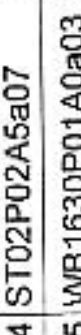 & 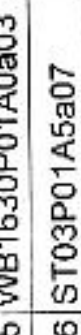 & 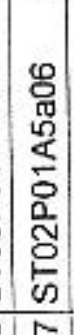 & 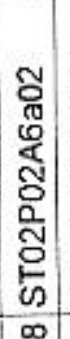 & 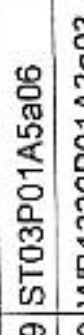 & 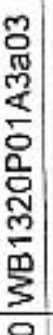 & 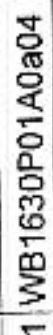 \\
\hline 리ues & -1 & $N$ & $m$ & +1 & n) & 01 & $\infty$ & $\infty$ & 이 & $=$ & $\cong$ & $m$ & $\frac{\pi}{2}$ & $\stackrel{\oplus}{\circ}$ & $=$ & $\stackrel{\infty}{\leftarrow}$ & $\stackrel{\sigma}{\div}$ & N & $\bar{N}$ \\
\hline पә7еg & 8 & gु & g & 导 & $\begin{array}{l}\text { g } \\
\text { g }\end{array}$ & $\begin{array}{lll} \\
\end{array}$ & 苛: & $\frac{5}{8}$ & Zु & 要 & $\begin{array}{l} \\
\text { वे }\end{array}$ & $\begin{array}{l}\text { J } \\
\text { व }\end{array}$ & 守 & \& & 广ั & J & g & 总 & 喜 \\
\hline
\end{tabular}

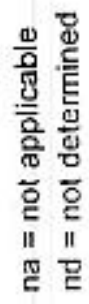




\begin{tabular}{|c|c|c|c|c|c|c|c|c|c|c|c|c|c|c|c|c|c|c|c|c|c|}
\hline $00 Z \#>d$ & Z & స & g) & 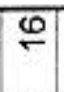 & $=$ & 응 & m & 9 & का & 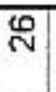 & $\mp$ & N) & হ্ & क & $\stackrel{\sim}{\sim}$ & का & 인 & 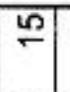 & ก & 암 & 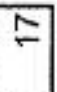 \\
\hline Ot\# $>d$ & $N$ & $\bar{v}$ & 2 & พั & $\mathscr{M}$ & 罗 & ণิ & N & m & 品 & 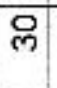 & $\frac{4}{8}$ & $\overline{0}$ & 5 & $\mathscr{q}$ & $\begin{array}{c}n \\
6\end{array}$ & $\vec{v}$ & $\bar{m}$ & 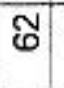 & $N$ & F \\
\hline Ol\# $>d$ & $\mathscr{\infty}$ & g & 용 & 号 & $\frac{5}{8}$ & \begin{tabular}{|l|}
$\infty$ \\
$\infty$
\end{tabular} & $\widetilde{\infty}$ & F & 8 & $\stackrel{\infty}{\circ}$ & ₹ & 8 & $\hat{N}$ & $m$ & $\begin{array}{l} \\
\end{array}$ & $m$ & ल्ल & 암 & $\mathfrak{R}$ & 吕 & 5 \\
\hline$t \#>d$ & กี & 8 & g & D & 号 & $N$ & 8 & 芯 & $\stackrel{L}{R}$ & $\mathscr{0}$ & ถั & 9 & Фా & $\Phi$ & P & 吕 & $\bar{\nabla}$ & 员 & 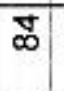 & $\stackrel{m}{M}$ & $\frac{10}{N}$ \\
\hline $\mathbf{u} \mid-8 / \varepsilon>d$ & $\mathscr{8}$ & 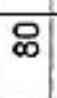 & के & 吕 & F & $\mathscr{\infty}$ & के & $\mathbb{N}$ & 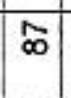 & $\infty$ & R & R & 8 & $\bar{\sigma}$ & ఫా & 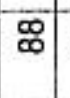 & m & $\mathbb{N}$ & \% & 8 & ळ \\
\hline $\mathbf{u} \cdot \bullet / \varepsilon>d$ & 8 & $\mathscr{\sigma}$ & 인 & $\infty$ & $\bar{\sigma}$ & 8 & $g$ & \% & 兽 & 号 & $\mathscr{G}$ & 5 & $\mathscr{G}$ & $\stackrel{\infty}{\circ}$ & \% & 5 & \begin{tabular}{|l|} 
\\
\end{tabular} & 志 & g & $\mathscr{\infty}$ & मू \\
\hline$u !-l>d$ & 웅 & ๙ & 응 & 요 & \% & ॄ & g & 용 & \% & รั & ๙ & $\mathscr{8}$ & $\mathscr{8}$ & $\mathscr{\infty}$ & 足 & $\mathscr{G}$ & $m$ & 4 & g & : & 용 \\
\hline ul-ZIL $L>d$ & 음 & $\stackrel{\circ}{\circ}$ & 응 & मे & 2 & $\mathscr{8}$ & \% & 59 & $g$ & ำ & $\stackrel{\infty}{\sigma}$ & 今 & \$ & ' & g & के & \begin{tabular}{|l|}
3 \\
$\infty$
\end{tabular} & के & g & 8 & 5 \\
\hline$u !-z>d$ & $\varnothing$ & g & 음 & $\mathscr{8}$ & 's & \% & 8 & 5) & gi & Бे & g & g & g & ' & g. & 号 & ○ & 品 & 음 & g & 号 \\
\hline ur $\varepsilon>d$ & 응 & 응 & 8 & 8 & 웅 & 웅 & 8 & 음 & 8 & 음 & 8 & 은 & 8 & 8 & 응 & 웅 & 웅 & 잉 & 용 & 음 & 옹 \\
\hline$(\% 2 M) \operatorname{SOSn}^{-}$KEIO+1I!S & गु & $\mathrm{m}$ & 茨 & $\overline{\mathrm{N}}$ & si & $\overline{\dot{I}}$ & $\overline{=}$ & $\begin{array}{l}m \\
\dot{N} \\
\stackrel{N}{*}\end{array}$ & $\underset{\mathrm{m}}{\mathrm{F}}$ & $\frac{m}{m}$ & $\stackrel{\sigma}{\circ}$ & $\frac{\mathrm{m}}{\mathrm{m}}$ & 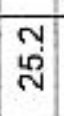 & 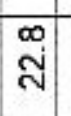 & $\frac{5}{m}$ & \begin{tabular}{|c|}
$m$ \\
$\dot{v}$
\end{tabular} & 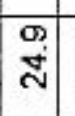 & $\begin{array}{l}N \\
g \\
d\end{array}$ & 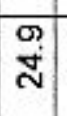 & 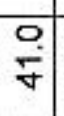 & $\overline{\mathrm{N}}$ \\
\hline$(\%+M)$ sosn'pues & ถั & $\begin{array}{l}\infty \\
\infty \\
\infty\end{array}$ & $\begin{array}{l}40 \\
60 \\
6\end{array}$ & $\begin{array}{l}\infty \\
\text { gi } \\
\end{array}$ & $\begin{array}{l}\infty \\
\text { ug } \\
\text { un }\end{array}$ & \begin{tabular}{l|} 
\\
0 \\
0 \\
0
\end{tabular} & \begin{tabular}{|l|} 
\\
\\
$m$
\end{tabular} & \begin{tabular}{c|}
0 \\
60 \\
\end{tabular} & 官 & $\begin{array}{c}N \\
5 \\
5\end{array}$ & \begin{tabular}{l}
$\infty$ \\
\multirow{N}{*}{}
\end{tabular} & $\begin{array}{l}10 \\
y\end{array}$ & $\begin{array}{l}10 \\
\text { กี่ }\end{array}$ & $\begin{array}{l}N \\
\text { U⿺ }\end{array}$ & $\begin{array}{l}0 \\
0 \\
\sigma\end{array}$ & $\frac{m}{\sigma}$ & $\begin{array}{l}m \\
\stackrel{m}{*}\end{array}$ & $\begin{array}{l}0 \\
\text { j }\end{array}$ & $\begin{array}{l}\infty \\
\tilde{\delta}\end{array}$ & $\begin{array}{l}m \\
m \\
q \\
q\end{array}$ & $\begin{array}{l}0 \\
0 \\
0\end{array}$ \\
\hline (\%ұM) sosn & $\widehat{\omega}$ & 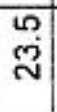 & \begin{tabular}{|l|}
0 \\
0 \\
\end{tabular} & 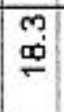 & $\begin{array}{l}n \\
\vdots \\
N\end{array}$ & $\begin{array}{l}\sigma \\
\Phi \\
\varphi\end{array}$ & $\underset{\infty}{\nabla}$ & $\begin{array}{c}N \\
\infty \\
\sim \\
\sim\end{array}$ & \begin{tabular}{l|} 
\\
$\Phi$ \\
$\mathscr{\sigma}$
\end{tabular} & $\begin{array}{l}\mathbb{L}^{\infty} \\
\stackrel{m}{\sim} \\
\sim\end{array}$ & 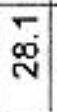 & का & 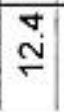 & $\begin{array}{l}\mathrm{O} \\
\stackrel{\mathrm{N}}{-}\end{array}$ & $\begin{array}{l}N \\
\text { N }\end{array}$ & \begin{tabular}{l|} 
\\
$\dot{T}$ \\
\end{tabular} & \begin{tabular}{l|}
$\infty$ \\
Nij
\end{tabular} & $\begin{array}{l}\infty \\
\stackrel{\infty}{ }\end{array}$ & $\begin{array}{l}\mathrm{d} \\
\mathrm{N}\end{array}$ & $\stackrel{2}{0}$ & $\stackrel{10}{=}$ \\
\hline qeר-dnoso sosn & $\sum_{\omega}$ & ఏ & $\sum_{\text {W }}$ & 心 & 施 & $\begin{array}{l}\sum \\
0 \\
3 \\
3 \\
\omega\end{array}$ & क & $\overline{5}$ & $\begin{array}{l}\sum_{0} \\
\vdots \\
\vdots \\
\infty\end{array}$ & $\sum_{N}$ & $\sum_{\omega}$ & ڤ) & $\sum_{\omega}$ & 心 & $\sum$ & 5 & ๘ & 5 & $\sum$ & $\sum_{\omega}$ & $\sum_{\text {ऊ }}$ \\
\hline 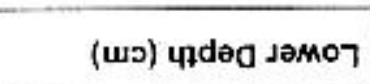 & $m$ & 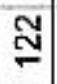 & $\stackrel{\mathbb{g}}{\mathrm{g}}$ & $\widehat{\varphi}$ & a & 守 & 5 & \begin{tabular}{|l|}
$\mathscr{D}$ \\
$\infty$
\end{tabular} & $\bar{\sigma}$ & 웅 & $\bar{\infty}$ & $\stackrel{\infty}{q}$ & 怘 & 운 & 㝵 & 이 & $\stackrel{\circ}{\circ}$ & 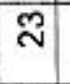 & 哭 & $\infty$ & $\stackrel{m}{\square}$ \\
\hline (wo) undeg dəddn & 0 & \$ & (D) & $\overline{15}$ & क & $\stackrel{\infty}{\sim}$ & พ & 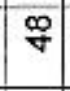 & $\ddot{\varphi}$ & $m$ & 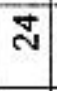 & 只 & ป & 0 & $m$ & $\infty$ & $\bar{\sigma}$ & 음 & $\mathbb{\pi}$ & 0 & 응 \\
\hline UOZ!JOH & $x$ & $\begin{array}{l}\overline{\mathbf{0}} \\
\text { 恙 } \\
\text { ल }\end{array}$ & $\stackrel{\mathbb{E}}{c}$ & $\begin{array}{l}\overline{\mathbf{o}} \\
\text { 畄 } \\
\end{array}$ & \begin{tabular}{|l|}
$\bar{\sigma}$ \\
离 \\
\end{tabular} & ن & 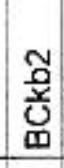 & 商 & 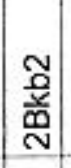 & $\underset{\mathbf{⿴ 囗 十}}{\mathbf{s}}$ & $\begin{array}{l}\text { N } \\
\text { 盖 }\end{array}$ & 恙 & $\begin{array}{l}\text { 盖 } \\
\text { ले }\end{array}$ & 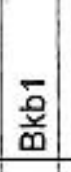 & $\begin{array}{l}\text { 辛 } \\
\text { 岕 } \\
\end{array}$ & 吕 & 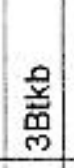 & 咅 & $\stackrel{\mathbb{E}}{\Sigma}$ & $\frac{\pi}{2}$ & 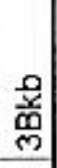 \\
\hline әpos 리யسes p|ㅣㅣ & 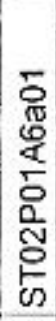 & 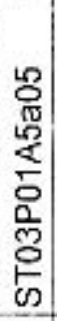 & 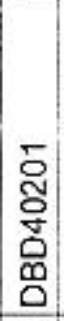 & 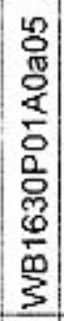 & 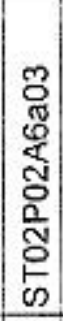 & 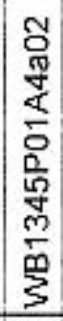 & 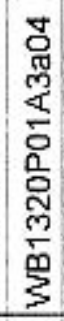 & 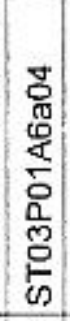 & $\begin{array}{l}\varphi \\
0 \\
0 \\
0 \\
0 \\
0 \\
0 \\
0 \\
0 \\
0 \\
5\end{array}$ & 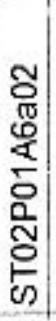 & 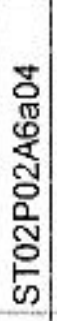 & 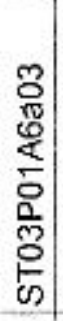 & 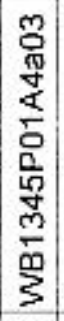 & 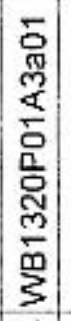 & 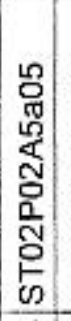 & 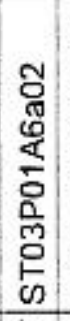 & 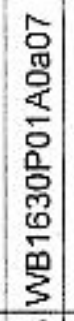 & $\begin{array}{l}m \\
0 \\
0 \\
0 \\
\mathbb{0} \\
\\
0 \\
0 \\
0 \\
0 \\
0 \\
0 \\
\end{array}$ & 总 & 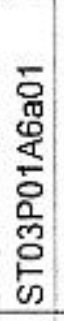 & 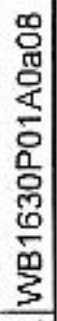 \\
\hline 리ues & 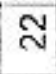 & $\vec{v}$ & N & 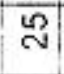 & 9 & $\bar{N}$ & $\stackrel{\infty}{N}$ & D & 8 & $\bar{m}$ & ल & M & ले & ल & ल & ले & 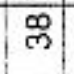 & ल & 움 & $\overline{7}$ & V \\
\hline בשבוכנ & $\bar{\nabla}$ & $\underset{8}{8}$ & $\underset{q}{\mathbb{O}}$ & $\underset{v}{\nabla}$ & 导 & 导 & 导 & $\begin{array}{l}f \\
g\end{array}$ & g & $\begin{array}{l}\forall \\
\dot{g}\end{array}$ & g & $\begin{array}{l}\nabla \\
\end{array}$ & $\begin{array}{l}J \\
\end{array}$ & $\begin{array}{l} \\
0 \\
\end{array}$ & $\begin{array}{l}J \\
O \\
q\end{array}$ & $\begin{array}{l}\nabla \\
\end{array}$ & $\begin{array}{l}\nabla \\
\end{array}$ & $\begin{array}{l} \\
\end{array}$ & 导 & 导 & J \\
\hline
\end{tabular}




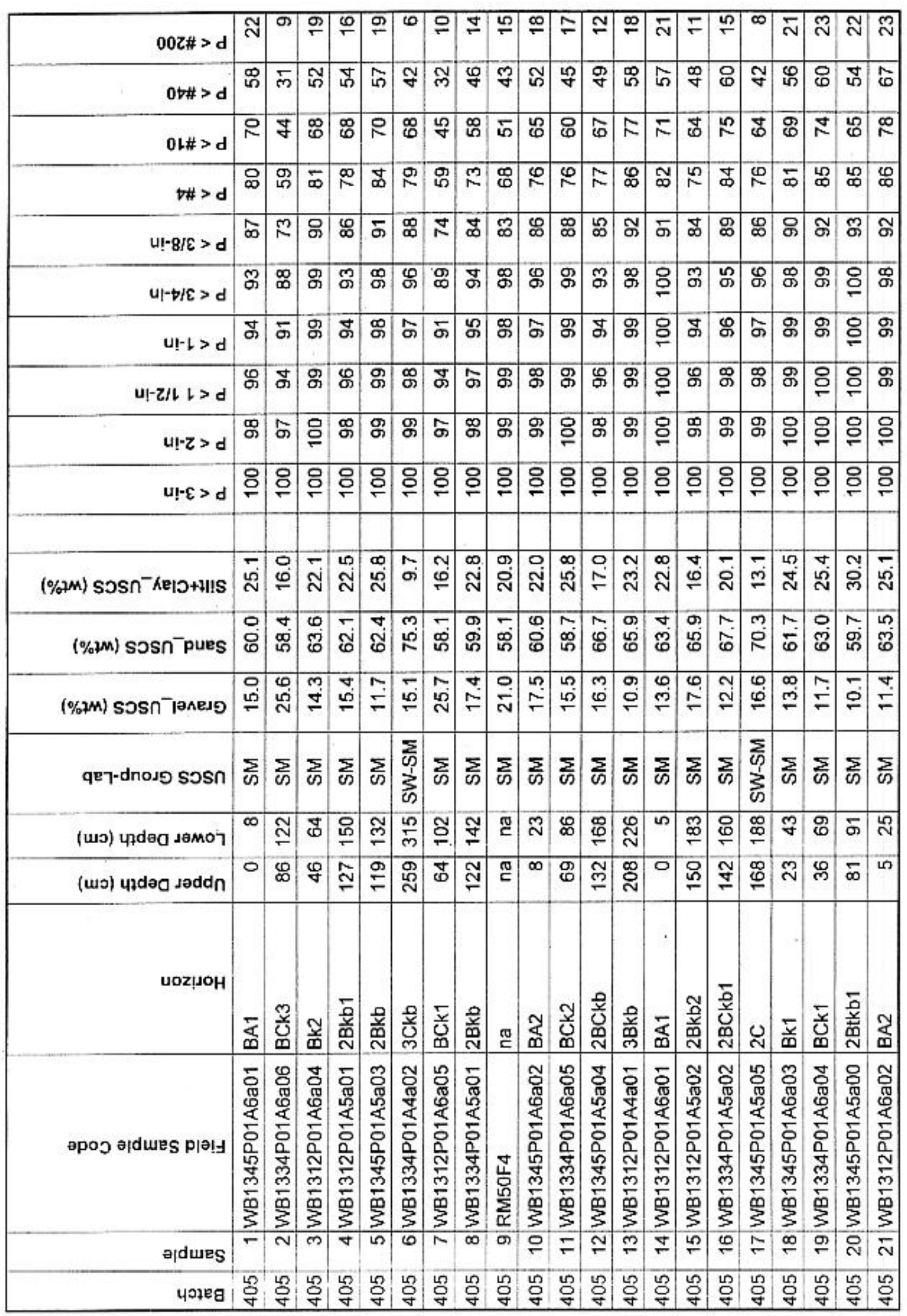




\begin{tabular}{|c|c|c|c|c|c|c|c|c|c|c|c|c|c|c|c|c|}
\hline $00 Z \#>d$ & 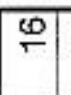 & $m$ & 6 & $\overline{\mathrm{N}}$ & $\omega$ & का & $\stackrel{\text { LI }}{\leftarrow}$ & 음 & ㅇ & হ & LI) & m. & $\omega$ & $\bar{m}$ & 56 & 6 \\
\hline OtH $>d$ & $\bar{\varphi}$ & 焉 & $\bar{N}$ & $\overline{5}$ & g & חొ & 可 & g & 只 & 8 & फू & F & N & $R$ & 穴 & I \\
\hline OlH $>d$ & $\infty$ & 인 & กิ & 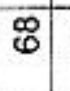 & 요 & \$ & 5 & R & 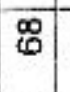 & 8 & $\bar{\varphi} \mid \overline{8}$ & (ֶ) & 8 & 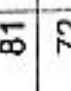 & $N$ & n \\
\hline$b \#>d$ & $\mathscr{\infty}$ & ष! & 志 & $\bar{\infty}$ & छี & चे & \begin{tabular}{|l|}
8 \\
0
\end{tabular} & 果 & 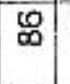 & 唄 & $\frac{10}{N}$ & $\varphi$ & 8 & (\$) & 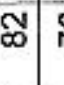 & $\stackrel{0}{1}$ \\
\hline$u !-8 / \varepsilon>d$ & 5 & $E$ & $m$ & \% & $R$ & ศั & 品 & \begin{tabular}{|l|l|}
50 \\
0
\end{tabular} & ชั & 5 & ळ & $\infty$ & $\bar{\infty}$ & का & 8 & $\infty$ \\
\hline$u !-t / \varepsilon>d$ & 5 & 8 & $\underset{\infty}{N}$ & 응 & 8 & 은 & $\mathscr{\circ}$ & ภु & $\bar{\infty}$ & 5 & 8 & छั & 思 & क & 8 & 5 \\
\hline$u !-b>d$ & \% & శ్ & $\mathscr{\infty}$ & 음 & สู & 음 & 今 & मे & के & 禺 & பூ & 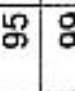 & क & 옹 & s. & ๙ิ \\
\hline u!-z/ $\iota$ l>d & g) & 58 & $\bar{\sigma}$ & 용 & \begin{tabular}{|l|l|}
\multicolumn{1}{|c|}{} \\
\end{tabular} & 욤 & 罗 & 9 & \% & क & के & 5 & क & 8 & 票 & $\mathscr{8}$ \\
\hline$u !-z>d$ & g & 5 & "ூ & 응 & $\hat{\sigma}$ & 은 & \% & 思 & का & g & \% & \% & g & 8 & $\begin{array}{l}\operatorname{s} \\
\end{array}$ & g \\
\hline$u \vdash \varepsilon>d$ & 8 & 용 & 8 & 음 & 용 & 음 & 욤 & 8 & 8 & 웅 & 웅 & 8 & 8 & 8 & 8 & 음 \\
\hline (\%2M) $\operatorname{sos}^{-}$Kelo+21Is & mo̊ & $\begin{array}{l}\infty \\
0 \\
\infty\end{array}$ & $\stackrel{\infty}{=}$ & $\begin{array}{l}\infty \\
\text { N }\end{array}$ & $\begin{array}{l}0 \\
\stackrel{N}{\leftarrow}\end{array}$ & $\overline{\mathrm{N}}$ & '̄ & $\begin{array}{l}\mathcal{N} \\
\underset{\square}{ \pm}\end{array}$ & \begin{tabular}{|l|}
$\mathrm{N}$ \\
$\mathrm{g}$ \\
$\mathrm{v}$
\end{tabular} & गें & $\begin{array}{l}m \\
\infty\end{array}$ & $\begin{array}{l}\varphi \\
\infty \\
\leftarrow \\
\leftarrow\end{array}$ & \begin{tabular}{l|l}
$\infty$ \\
$=$ \\
$=$ & \\
\end{tabular} & $\underset{m}{N}$ & $\begin{array}{l}m \\
\stackrel{2}{2}\end{array}$ & 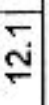 \\
\hline (\%qM) sosn ${ }^{-}$pues & Б্口: & $\begin{array}{l}0 \\
5 \\
5\end{array}$ & $\begin{array}{l}0 \\
\dot{0} \\
0\end{array}$ & $\begin{array}{l} \\
\dot{8} \\
0\end{array}$ & $\begin{array}{l}m \\
\dot{0}\end{array}$ & $\begin{array}{l}m \\
8\end{array}$ & \begin{tabular}{l|} 
\\
8 \\
8
\end{tabular} & \begin{tabular}{|l|} 
\\
0 \\
0
\end{tabular} & \begin{tabular}{|l|}
0 \\
\\
\end{tabular} & $\begin{array}{l}40 \\
8\end{array}$ & $\vec{N}$ & $\begin{array}{l}\infty \\
\dot{0}\end{array}$ & \begin{tabular}{l|l}
0 & \\
0 & \\
0 &
\end{tabular} & ת. & 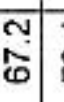 & $\bar{N}$ \\
\hline (\%7M) sosn & $\stackrel{\sim}{\circ}$ & $\begin{array}{l}\stackrel{40}{\infty} \\
\stackrel{N}{N}\end{array}$ & $\begin{array}{l}0 \\
\mathrm{~N}\end{array}$ & $\begin{array}{l}\mathrm{N} \\
\mathrm{N}\end{array}$ & \begin{tabular}{|l|} 
\\
$\mathfrak{N}$ \\
\end{tabular} & $\stackrel{0}{=}$ & N & $\stackrel{-}{\stackrel{\Phi}{\rightleftharpoons}}$ & \begin{tabular}{|l|}
0 \\
\end{tabular} & $\stackrel{\square}{\oplus}$ & $\stackrel{1}{2}$ & $\begin{array}{l}\dot{y} \\
\varphi \\
\varphi\end{array}$ & 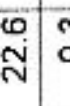 & $\stackrel{m}{m}$ & $\begin{array}{l} \\
\stackrel{2}{*}\end{array}$ & $\begin{array}{l}\infty \\
\stackrel{\infty}{\sim} \\
\stackrel{1}{*}\end{array}$ \\
\hline qeך-dnoג sosn & ह) & ज & $\begin{array}{l}\sum_{\infty} \\
\sum_{\infty}\end{array}$ & ङ & 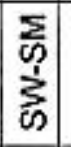 & 空 & ふ & $\begin{array}{l}\sum_{\infty} \\
\sum_{W} \\
\end{array}$ & क & ऊ & $\begin{array}{l}\sum_{0} \\
\vdots \\
\vdots \\
\vdots\end{array}$ & $\begin{array}{l}5 \\
0 \\
0 \\
0\end{array}$ & $\begin{array}{l}\sum_{b} \\
\frac{1}{5} \\
i s\end{array}$ & $\sum$ & $\sum$ & $\begin{array}{l}\sum_{W} \\
\frac{1}{\omega} \\
\sum_{W}\end{array}$ \\
\hline (uv) чpdag samoา & $\stackrel{\mathscr{\%}}{\$}$ & 帛 & 心్ & 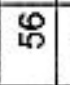 & \begin{tabular}{|l|}
\multicolumn{9}{|c}{} \\
$\stackrel{\mathrm{W}}{\mathrm{N}}$ \\
\end{tabular} & 9 & $\stackrel{\mathbb{Z}}{\mathrm{T}}$ & 思 & \% & 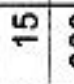 & $\begin{array}{l}\text { ग्ञ } \\
\text { సै }\end{array}$ & 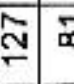 & $\bar{\infty}$ & L) & $\frac{\sigma}{\square}$ & 맏 \\
\hline (uv) undea Jeddn & 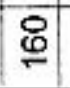 & $\cong$ & $\stackrel{N}{N}$ & 9 & लि & 虫 & $\stackrel{\mathbb{2}}{\square}$ & 兽 & $\bar{\sigma}$ & 10 & क्ष & ญิ & $\mathscr{\circ}$ & $0: 5$ & का & T \\
\hline uoz!山애 & 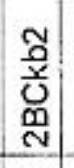 & 酋 & 4 & $\frac{\mathrm{T}}{\mathrm{m}}$ & $\begin{array}{l}\text { 总 } \\
\text { 品 }\end{array}$ & 嚍 & ख & O & $\begin{array}{c}\text { N } \\
\text { 害 } \\
\text { N } \\
\end{array}$ & 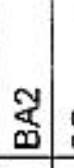 & U్స & c & 0 & $\underset{\mathbf{m}}{\mathbf{\alpha}}$ & 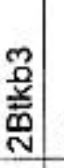 & 趈 \\
\hline әроэ 미uㅗ pla! & 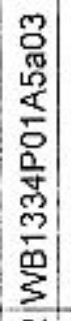 & 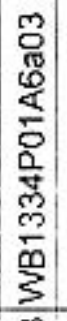 & 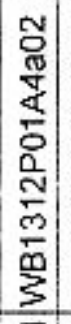 & 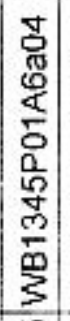 & 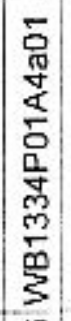 & 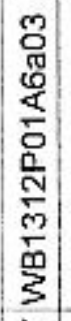 & 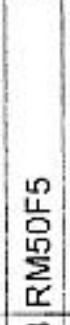 & 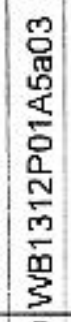 & 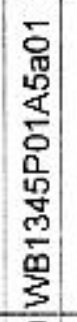 & 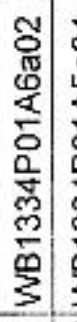 & 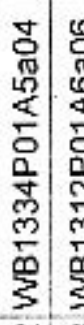 & 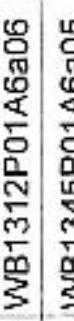 & 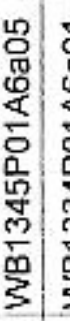 & 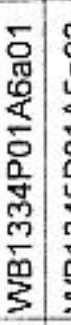 & 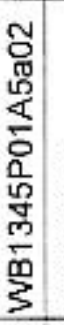 & $\begin{array}{l}\bar{\delta} \\
\text { סे } \\
\text { ปे } \\
\text { 品 }\end{array}$ \\
\hline әрdwes & ก & $\stackrel{m}{N}$ & $\overline{2}$ & $\infty$ & $\mathbb{N}$ & N & 韋 & g & 9 & $\bar{m}$ & ल ล & m ন & স & ผึ & 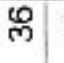 & $\hat{n}$ \\
\hline чэฺеg & 㱚 & 남 & O & 8 & $\stackrel{8}{7}$ & 号 & 总 & $\begin{array}{l}50 \\
\end{array}$ & 号 & 号 & 원 & $\stackrel{5}{8}$ & 8 & \& & 尔 & 号 \\
\hline
\end{tabular}




\begin{tabular}{|c|c|c|c|c|c|c|c|c|c|c|c|c|c|c|c|c|c|c|c|c|}
\hline $00 z \#>d$ & & $=$ & $\bar{\square}$ & 虾 & $\theta$ & 은 & F & 0 & $\mp$ & बा & i & $\mp$ & का & $\infty$ & $\overline{8} 0^{\circ}$ & ? & $=9$ & का & $\bar{\nabla}$ & 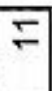 \\
\hline 0 b $\#>d$ & $\begin{array}{l}2 \\
\end{array}$ & 유 & 8 & $?$ & กั & 애 & $\bar{m}$ & म & 㟧 & চ & है & $\%$ & 8 & 俤 & a: & s. & $\begin{array}{lll}N & M \\
B O\end{array}$ & ले & 亗 & 岗 \\
\hline $\mathrm{Ol} \#>\mathrm{d}$ & 8 & 7 & $F$ & $\varpi$ & 5 & 这 & 迥 & 8 & 䍐 & 员 & 8 & 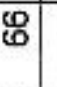 & Ri & की & 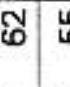 & 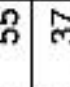 & 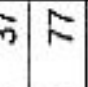 & | & t) & \\
\hline$t \#>d$ & $\begin{array}{c}\infty \\
\vdots\end{array}$ & 8 & $\infty$ & 足 & $\hat{0}$ & Б) & 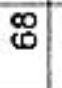 & 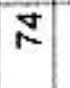 & 5 & 6 & R & 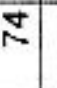 & 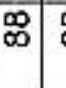 & 禺 & $\vec{s}$ & 色 & \begin{tabular}{|l|l|}
5 & $\infty$ \\
\end{tabular} & 9 & ल & 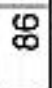 \\
\hline ul! $-8 / \varepsilon>d$ & $\stackrel{2}{\sim}$ & 2 & 8 & के & 足 & $\mathscr{G}$ & $\stackrel{\infty}{1}$ & ळ & $\infty$ & $\bar{\infty}$ & 遇 & क & का & का & 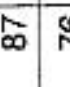 & e & $=5$ & 8 & ৪ & 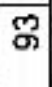 \\
\hline ul $-\mathrm{t} / \varepsilon>\mathrm{d}$ & $\infty$ & \% & $\mathscr{s}$ & g & צ & 요 & $\infty$ & \% & $\stackrel{\infty}{\infty}$ & बे & 5 & 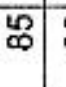 & क. & 8 & क: & $\infty$ & \$ & 2 & ळ & 8 \\
\hline$u !-l>d$ & $\tilde{\infty}$ & 8 & s. & ஃ & से & 8 & ○! & 学 & Ф & \% & $\stackrel{\infty}{\circ}$ & ळ| & 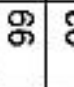 & హ) & के & 家 & 思 & $\infty_{\infty}^{\infty}$ & $\stackrel{\infty}{\sigma}$ & 용 \\
\hline u!-ZIレ $レ>d$ & สิ & क & \& & 욷 & 8 & 울 & (3) & ลे & \% & के & \% & ') & 욷 & ぬ & ळ: & 苛 & \$ & 8 & g) & g \\
\hline$u ! z>d$ & \% & 8) & \& & 8 & 号 & 8 & \& & 弯 & g & \% & \% & $\mathscr{L}$ & 웅ㅁㅇ & के & \% & 8 & \$ & के & g) & 음 \\
\hline u!- $\varepsilon>d$ & 우 & 함 & 운 & 웅 & : & @ & 으 & 이 & 하 & 우 & 운 & 인 & 은 & 8 & $\%$ & 8 & 38 & 웅 & 흐 & 음 \\
\hline$\left(\%\right.$ (\%) $\operatorname{sos}^{-}$(x) & $\stackrel{\square}{=}$ & $\stackrel{N}{\stackrel{N}{c}}$ & $\begin{array}{l}\vec{y} \\
0\end{array}$ & 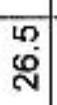 & $\begin{array}{l}\dot{0} \\
\sigma\end{array}$ & $\bar{J}$ & $\underset{\infty}{\forall}$ & $\stackrel{m}{=}$ & $\begin{array}{l}\vec{\nabla} \\
\stackrel{0}{\circ}\end{array}$ & $\begin{array}{l}m \\
\dot{m} \\
\dot{y}\end{array}$ & 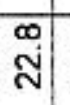 & 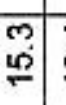 & 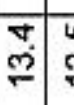 & & $\begin{array}{ll}40 \\
5\end{array}$ & 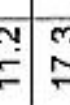 & 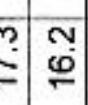 & 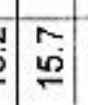 & के & $\begin{array}{l}\omega \infty \\
\stackrel{\omega}{\omega}\end{array}$ \\
\hline$\left(\%\right.$ \%) sOsn ${ }^{-}$pues & \begin{tabular}{l|} 
\\
8 \\
8
\end{tabular} & 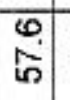 & î & 每 & $\begin{array}{l} \\
g \\
0\end{array}$ & $\begin{array}{l}m \\
\infty \\
\infty \\
c\end{array}$ & \begin{tabular}{l}
$\infty$ \\
\hdashline \\
0 \\
0
\end{tabular} & ì & 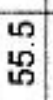 & $\begin{array}{l} \\
\vdots \\
\vdots \\
\end{array}$ & \begin{tabular}{l|l|} 
\\
$\infty$ \\
$\infty$ \\
0
\end{tabular} & $\begin{array}{l}m \\
0 \\
0\end{array}$ & 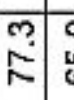 & 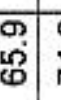 & & & 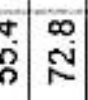 & $\mid$\begin{tabular}{c|c}
0 \\
$\infty$ \\
$\infty$ \\
$\infty$
\end{tabular} & $\begin{array}{l}0 \\
\mathbb{N} \\
\mathbb{N}\end{array}$ & 咅 \\
\hline 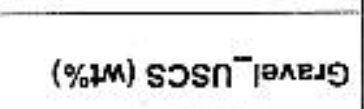 & స̃. & ָู & $\begin{array}{l}\text { a } \\
\stackrel{\sim}{*}\end{array}$ & \begin{tabular}{|c|}
\multirow{2}{*}{} \\
$\sigma$
\end{tabular} & \begin{tabular}{|l|}
$\infty$ \\
$\dot{d}$ \\
\end{tabular} & 운 & 市 & 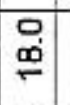 & 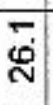 & $\overline{\mathrm{N}}$ & $\begin{array}{c}m \\
\infty \\
\infty\end{array}$ & $\begin{array}{l}\infty \\
\infty \\
\infty \\
-\end{array}$ & 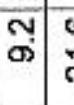 & $\stackrel{0}{\sim}$ & & ֶָ. & $\stackrel{0}{=}$ & 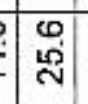 & $\begin{array}{r}\vec{m} \\
\vec{\sigma}\end{array}$ & 峞 \\
\hline qeT-dnojg sosn & $\begin{array}{l}\sum \\
\omega \\
\sum \\
\omega\end{array}$ & $\sum$ & $\sum$ & क & $\begin{array}{l}\sum \\
0 \\
\vdots \\
\vdots\end{array}$ & 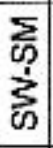 & $\begin{array}{l}\sum_{0} \\
\sum_{\omega} \\
\text { क }\end{array}$ & $\begin{array}{l}\sum_{W} \\
\sum_{\omega}\end{array}$ & $\sum_{\infty}$ & $\begin{array}{l}\text { 妾 } \\
\sum_{\omega} \\
\vdots\end{array}$ & $\sum_{\omega}$ & $\sum_{\infty}$ & $\begin{array}{l}5 \\
5 \\
\sum_{0}^{1}\end{array}$ & 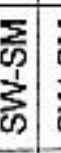 & 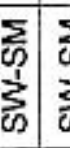 & & 5 & $\sum$ & $\begin{array}{l}\sum_{0} \\
\sum_{\omega}\end{array}$ & $\sum_{\infty}$ \\
\hline (шэ) чұdaم دамоา & 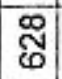 & ลิ & 인 & 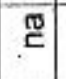 & 馬 & $\bar{\Phi}$ & 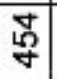 & \begin{tabular}{|l|}
0 \\
0 \\
0
\end{tabular} & $\bar{\sigma}$ & 이 & 要 & $\bar{d}$ & \begin{tabular}{l|l}
0 \\
0 \\
0
\end{tabular} & ్ֶ & है: & $\frac{2}{6}$ & $\frac{2}{50}$ & $\frac{0}{\square}$ & ్ํㅁ & 总 \\
\hline (แэ) पzdəa dəddn & \begin{tabular}{|l|} 
\\
0 \\
0
\end{tabular} & 0 & $\begin{array}{l}\infty \\
\vdots \\
\vdots\end{array}$ & 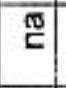 & 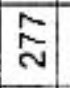 & : & 咨 & $\begin{array}{ll}0 \\
0\end{array}$ & i & 맏 & ⿷ & 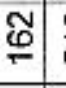 & $\frac{n}{n}$ & 8 & E) & 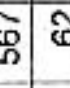 & ס) & क & 5 & 㞭 \\
\hline & ల & $\begin{array}{l}0 \\
\alpha \\
\alpha\end{array}$ & 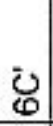 & פ & ن्ల & 己ِ & ల్ల & U. & 音 & o) & 묻 & & 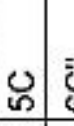 & ¿ & 04 & 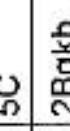 & \begin{tabular}{l|l}
$\frac{i}{2}$ \\
s.
\end{tabular} & 善 & . & y \\
\hline apoj ㄹ|ᅵues pla!t & 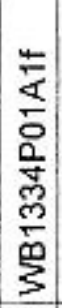 & 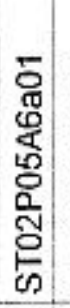 & $\begin{array}{l}\frac{0}{0} \\
\frac{\alpha}{0} \\
\frac{0}{2} \\
\frac{2}{2} \\
\frac{2}{3} \\
3\end{array}$ & $\begin{array}{l}\underset{\mathbb{E}}{\mathbb{E}} \\
\sum_{\mathbb{N}}\end{array}$ & $\begin{array}{l}\frac{5}{2} \\
\frac{1}{0} \\
\frac{1}{n} \\
\frac{1}{2} \\
\frac{2}{3}\end{array}$ & 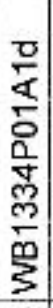 & 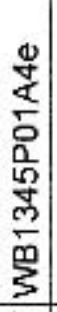 & $\begin{array}{l}\frac{0}{\Delta} \\
\frac{1}{0} \\
\frac{n}{N} \\
\frac{0}{m} \\
\frac{9}{3}\end{array}$ & 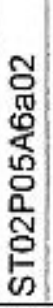 & 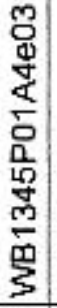 & $\begin{array}{l}\bar{g} \\
\bar{g} \\
\text { ğ } \\
\text { g. }\end{array}$ & 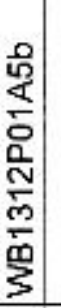 & 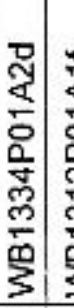 & 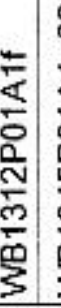 & 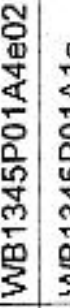 & 童 & 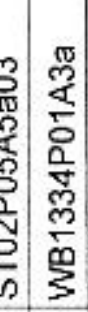 & 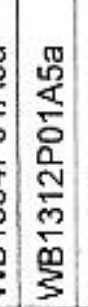 & $\begin{array}{l}\frac{d}{2} \\
\frac{1}{2} \\
\frac{0}{0} \\
\frac{N}{2} \\
\frac{m}{2} \\
\frac{m}{3}\end{array}$ & 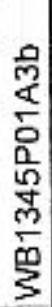 \\
\hline ㅁdues & & $\sim$ & m) & $\nabla$ & 60 & 6 & $N$ & $\infty$ & का & 웅 & $=$ & 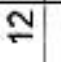 & m & $\bar{\nabla}$ & $\div:$ & E. & $=\stackrel{\infty}{\sim}$ & 9 & ন & $\bar{N}$ \\
\hline 1189 & & $\mathscr{q}$ & $\stackrel{8}{\square}$ & $\stackrel{8}{\%}$ & 号. & $\stackrel{8}{8}$ & 过 & 韋 & $\begin{array}{l}\text { 号 } \\
\text { ga }\end{array}$ & $\begin{array}{l}0 \\
g\end{array}$ & 号 & $\begin{array}{l}0 \\
\text { o }\end{array}$ & 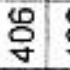 & 尊 & 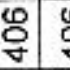 & $\begin{array}{l}8 \\
8 \\
y\end{array}$ & $8 \%$ & $\begin{array}{l}0 \\
y\end{array}$ & $\stackrel{8}{y}$ & $\stackrel{8}{\%}$ \\
\hline
\end{tabular}




\begin{tabular}{|c|c|c|c|c|c|c|c|c|c|c|c|c|c|c|c|c|c|c|c|c|}
\hline OOZ\#>d & $\stackrel{\mathbb{N}}{N}$ & $\varphi$ & $\mp$ & m & m & $N$ & 유 & का & का & 움 & $\omega$ & $F$ & $\infty$ & 㟧 & $\stackrel{\infty}{\leftarrow}$ & 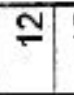 & 2 & $\stackrel{6}{2}$ & $N$ & $\approx$ \\
\hline $0 t \#>d$ & 8 & m & $\mathfrak{N}$ & 5 & ָั & $m$ & $\bar{N}$ & 年 & 守 & 寸 & $\mathscr{N}$ & g & $\stackrel{9}{\longrightarrow}$ & R & 8 & テ & 芯 & พ & $\mathbb{N}$ & F \\
\hline Ol\# $>d$ & ஐ & 5 & m & 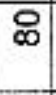 & 운 & 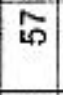 & $\bar{\nabla}$ & 8 & $\Phi$ & $\tilde{0}$ & 足 & $\hat{0}$ & $\stackrel{\infty}{N}$ & $\Phi$ & $\stackrel{0}{R}$ & ถั & $\mathscr{8}$ & 员 & $\stackrel{\infty}{q}$ & $\varnothing$ \\
\hline$t \#>d$ & 号 & $\mathscr{8}$ & 尔 & வ & षे & 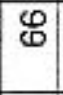 & $\overline{0}$ & 8 & $\Phi$ & $\bar{N}$ & 寸 & $\mathscr{2}$ & ข & 8 & ๘ & क & $N$ & R & $N$ & $\Sigma$ \\
\hline $\mathbf{u} \mid-8 / \varepsilon>d$ & 용 & $N$ & 9 & $\%$ & $E$ & $E$ & 9 & \begin{tabular}{|l|}
8 \\
0
\end{tabular} & \begin{tabular}{|l|l|}
10 \\
$\infty$
\end{tabular} & $E$ & 5 & 心 & ชิ & 녕 & 이 & ஜ & $\stackrel{10}{\infty}$ & M & 田 & ஓ \\
\hline$u \mid-t / \varepsilon>d$ & 8 & $\mathscr{\infty}$ & வ & $\stackrel{\infty}{\infty}$ & 8 & $\infty$ & $\mathscr{\varpi}$ & \% & 芯 & ळ & 曲 & б) & N & \% & $\mathscr{\sigma}$ & $\infty$ & 8 & $\mathscr{8}$ & 움 & 品 \\
\hline$u !-l>d$ & 음 & g & $\bar{\sigma}$ & 怘 & ภু & 요 & $\mathscr{H}$ & \% & 4 & 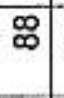 & $\widehat{6}$ & 8 & $\mathscr{\infty}$ & $g$ & $\mathscr{8}$ & 要 & ठั & Бे & 음 & $\bar{\sigma}$ \\
\hline$u_{l}-z / L b d$ & 8 & 9 & बे & \% & 4 & 9 & $\stackrel{\infty}{\infty}$ & \% & 's & ๙ิ & 9 & కొ & $\bar{\sigma}$ & g & 总 & $\infty$ & 号 & 号 & 응 & $\%$ \\
\hline$u !-z>d$ & 문 & $\mathscr{\sigma}$ & ڤั & \% & के & 8 & ৪ & g & 怘 & ๘ & Ф్ & 5 & 용 & 음 & 8 & 용 & 怘 & g & 응 & $\hat{\sigma}$ \\
\hline$u !-\varepsilon>d$ & 9 & 음 & 우 & 응 & 웅 & 웅 & 욤 & 8 & 용 & 으 인 & $\stackrel{8}{-}$ & 임 & 8 & 욤 & 웅 & 웅 & 움 & 8 & 움 & 응 \\
\hline 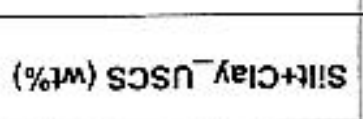 & $\begin{array}{l}\text { क) } \\
\stackrel{\omega}{N}\end{array}$ & $\stackrel{\text { L? }}{=}$ & के & $\begin{array}{l}\infty \\
\oplus \\
\oplus\end{array}$ & $\stackrel{\infty}{\sim}$ & $\stackrel{5}{=}$ & $\begin{array}{l}0 \\
0 \\
6\end{array}$ & $\begin{array}{c}\dot{m} \\
\stackrel{m}{\sigma}\end{array}$ & $\begin{array}{l}\text { O } \\
\text { ț }\end{array}$ & $\stackrel{\square}{\circ}$ & $\stackrel{\mathrm{N}}{\mathrm{N}}$ & $\begin{array}{l}\ddots \\
\stackrel{0}{*}\end{array}$ & $\begin{array}{l}\overrightarrow{2} \\
\text { \%े }\end{array}$ & $\overline{\grave{N}}$ & $\stackrel{\varphi}{\grave{N}}$ & 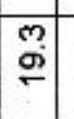 & \begin{tabular}{l|}
$\infty$ \\
$\infty$ \\
$\infty$
\end{tabular} & $\frac{ \pm}{\mathrm{N}}$ & 응 & $\frac{0}{\mathrm{~N}}$ \\
\hline$(\%)$ sosn so pues & is & ले & 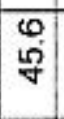 & $\begin{array}{l}N \\
\dot{N}\end{array}$ & $\begin{array}{l}\sigma \\
80 \\
0\end{array}$ & \begin{tabular}{l|}
$\infty$ \\
$\omega$ \\
$\omega$
\end{tabular} & $\begin{array}{l}\text { क } \\
\text { कi }\end{array}$ & $\begin{array}{l} \pm \\
N\end{array}$ & $\bar{g}$ & $\begin{array}{l}\infty \\
\text { 's } \\
0\end{array}$ & ल & $\begin{array}{l}9 \\
8 \\
0\end{array}$ & \begin{tabular}{|c|}
$m$ \\
0 \\
$q$ \\
\end{tabular} & 瓷 & $\begin{array}{l}0 \\
\Phi \\
0\end{array}$ & \begin{tabular}{l|} 
\\
$\dot{0}$ \\
\end{tabular} & $\begin{array}{l}\nabla \\
\dot{0}\end{array}$ & $\begin{array}{l}0 \\
\\
\end{array}$ & 品 & $\begin{array}{l}\Phi \\
00 \\
00\end{array}$ \\
\hline (\%¥M) sosn & $\stackrel{\sim}{\infty}$ & $\frac{m}{\sim}$ & $\begin{array}{l}0 \\
0 \\
\text { D }\end{array}$ & o & $\begin{array}{l}\text { m } \\
\text { ले }\end{array}$ & \begin{tabular}{|l|}
$\infty$ \\
$\mathbb{N}$
\end{tabular} & $\begin{array}{l}0 \\
\text { ஸे } \\
\text { N }\end{array}$ & $\begin{array}{l} \pm \\
\end{array}$ & \begin{tabular}{l|} 
\\
$\varphi$ \\
-
\end{tabular} & $\begin{array}{l}0 \\
\text { Ni }\end{array}$ & ल্ল & $\begin{array}{l}\overrightarrow{0} \\
\stackrel{2}{*}\end{array}$ & \begin{tabular}{l|} 
ले \\
g్ల.
\end{tabular} & \begin{tabular}{l|l|} 
\\
$\infty$ \\
$\infty$
\end{tabular} & $\begin{array}{l}\mathrm{v} \\
\mathrm{v} \\
\mathrm{v}\end{array}$ & \begin{tabular}{c|}
$\sim$ \\
$\stackrel{N}{N}$ \\
$\stackrel{N}{ }$
\end{tabular} & $\begin{array}{l}0 \\
\varphi \\
\varphi\end{array}$ & 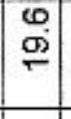 & $\begin{array}{l}\infty \\
\dot{v} \\
\sim\end{array}$ & ํㅗㅇ \\
\hline qEר-dnosg sosn & $\sum_{\text {W }}$ & $\begin{array}{l}\sum_{\infty} \\
\text { 1 } \\
\sum_{0}\end{array}$ & ఉ & ऊ & $\begin{array}{l}\sum_{0} \\
\sum_{0} \\
0\end{array}$ & $\begin{array}{l}\sum_{0} \\
\sum_{0}^{1} \\
\vdots\end{array}$ & 心 & $\begin{array}{l}\sum_{0} \\
w \\
\sum \\
\infty\end{array}$ & $\begin{array}{l}\sum \\
0 \\
\frac{1}{0} \\
0\end{array}$ & $\begin{array}{l}5 \\
0 \\
\substack{1 \\
0}\end{array}$ & $\begin{array}{l}\sum_{\infty} \\
\infty \\
\sum_{\infty}\end{array}$ & ๘ & ऊ & ऍ & $\sum_{0}^{5}$ & $\sum_{\infty}$ & $\sum_{\infty}$ & ळ & $\begin{array}{l}\sum_{\infty} \\
\frac{1}{\infty} \\
\infty\end{array}$ & $\bar{\aleph}$ \\
\hline (யכ) чұdəa גәмоา & $\stackrel{\mathbb{2}}{5}$ & 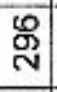 & $\stackrel{\circ}{=}$ & $\frac{10}{50}$ & $\overline{\mathrm{C}}$ & 学 & 9 & m & $E$ & $\begin{array}{l}\text { 总 } \\
\text {. }\end{array}$ & $\bar{\infty}$ & $\begin{array}{l}\mathscr{L} \\
\stackrel{4}{N}\end{array}$ & $\begin{array}{l}\text { लొ } \\
\text { ஸे }\end{array}$ & 믿 & 号 & E & $\begin{array}{l}\stackrel{\infty}{్} \\
\mathrm{~N}\end{array}$ & 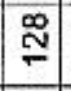 & 유 & ఫ \\
\hline (uo) uldog dəddn & $\mathbb{5}$ & $\begin{array}{l}0 \\
\end{array}$ & \% & $\stackrel{m}{\mathrm{~m}}$ & g & \begin{tabular}{|l|}
0 \\
$\stackrel{0}{2}$ \\
\end{tabular} & $\stackrel{\sigma}{=}$ & 总 & 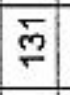 & 品 & 点 & $\stackrel{\mathscr{T}}{\square}$ & \begin{tabular}{|l|}
\multicolumn{1}{|c}{} \\
0
\end{tabular} & $\mathbb{\mathfrak { C }}$ & 惫 & 㐫 & $F$ & $m$ & ग़ & $\omega$ \\
\hline UOZ!」아 & $\stackrel{\mathbb{2}}{\mathrm{C}}$ & 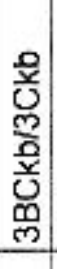 & $\begin{array}{l}\frac{0}{\mathrm{~m}} \\
\mathrm{~m} \\
\mathrm{v}\end{array}$ & 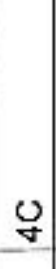 & 0 & 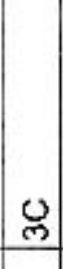 & 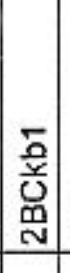 & 品 & $\begin{array}{c}0 \\
\mathrm{~N} \\
\mathbf{0} \\
\mathbf{y} \\
\mathrm{M} \\
\mathrm{N}\end{array}$ & 0 & 仓̊. & 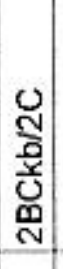 & $\begin{array}{l}\text { N } \\
\text { D্ } \\
\text { N }\end{array}$ & ฮ) & $\begin{array}{l}\text { 妾 } \\
\text { 号 }\end{array}$ & O & 0 & 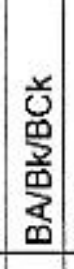 & 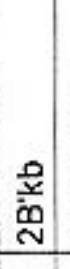 & 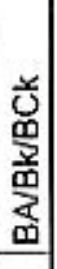 \\
\hline 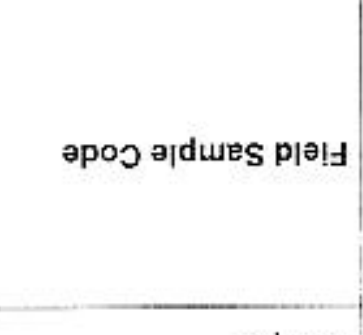 & 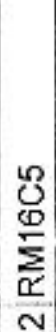 & 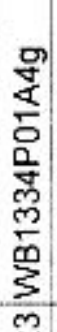 & 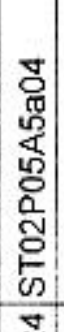 & 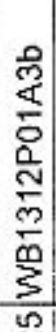 & 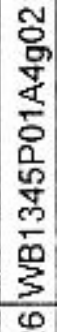 & 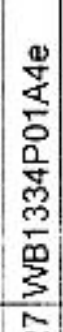 & \begin{tabular}{|l|} 
\\
0 \\
0 \\
0 \\
0 \\
0 \\
0 \\
0 \\
0 \\
0 \\
0 \\
0 \\
0 \\
0 \\
\end{tabular} & 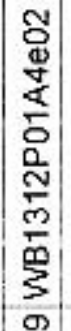 & 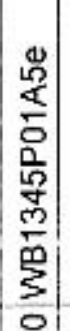 & 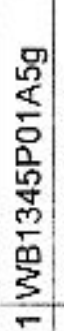 & 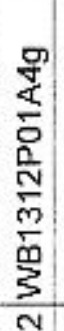 & 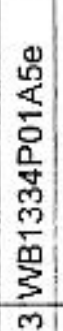 & 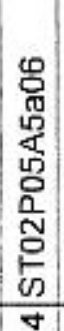 & $\frac{\sum_{0}}{\underline{D}}$ & 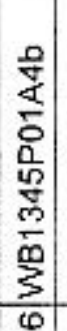 & 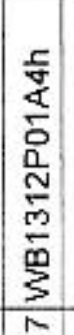 & 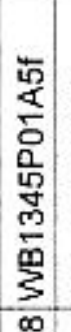 & 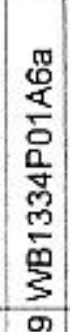 & 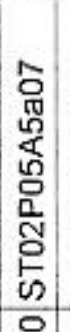 & 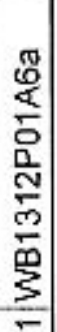 \\
\hline ә|dues & N & $\stackrel{\mathrm{N}}{\mathrm{N}}$ & ম & $\stackrel{\sim}{2}$ & $\stackrel{\leftrightarrow}{\sim}$ & $\widehat{N}$ & $\stackrel{\infty}{N}$ & i & স! & ले & लै & ले & ले & 邑 & 足 & $m$ & $\begin{array}{l}\infty \\
\infty\end{array}$ & ๙ & 움 & Ұ \\
\hline 40759 & $\stackrel{8}{\mathcal{O}}$ & $\stackrel{8}{8}$ & $\stackrel{9}{8}$ & $\mathscr{g}$ & $\stackrel{g}{\nabla}$ & $\stackrel{8}{8}$ & $\stackrel{8}{8}$ & $\stackrel{8}{8}$ & 8 & $\mathscr{q}$ & 9 & 8 & 号 & $\stackrel{9}{9}$ & 号 & $\begin{array}{l}0 \\
9 \\
\end{array}$ & $\stackrel{8}{8}$ & $\stackrel{9}{8}$ & $\begin{array}{l}8 \\
8 \\
\end{array}$ & 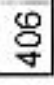 \\
\hline
\end{tabular}

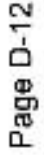




\begin{tabular}{|c|c|c|c|c|c|c|c|c|c|c|c|c|c|c|c|c|c|c|c|c|c|}
\hline $00 z \#>d$ & 에 & 우 & $\stackrel{m}{\div}$ & $\infty$ & \pm & ㄴ & का & 유 & $\stackrel{m}{-}$ & $\Phi$ & m/ & \begin{tabular}{|c|} 
N \\
\end{tabular} & $N$ & 6 & Ф & बा & Ф & 只 & ㄱ & 6 & $F$ \\
\hline $0 t \#>d$ & ल) & I) & 品 & $\Phi$ & 品 & छे & 8 & N & $\stackrel{0}{\mathbb{N}}$ & V & 塄; & ष & $\stackrel{\infty}{\div}$ & 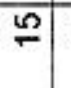 & 이 & 9 & I & m & $\bar{D}$ & $\stackrel{\infty}{\leftarrow}$ & $\bar{\varphi}$ \\
\hline $0 \mathrm{bH}>d$ & $\mathcal{Y}$ & $\frac{60}{8}$ & 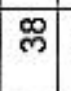 & g্v & 足 & $\bar{\infty}$ & \begin{tabular}{|l|l|}
0 \\
0
\end{tabular} & 守 & m & 8 & का & 4 & ले & 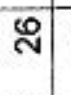 & P & $\pi$ & $\tilde{6}$ & $\Xi$ & $\bar{\infty}$ & m & ๗ \\
\hline$t \#>d$ & กี & $\bar{\infty}$ & 8 & I & क्ष & \begin{tabular}{|l|}
$\infty$ \\
$\infty$
\end{tabular} & $\Phi$ & 5 & $\mathscr{F}$ & $m$ & $m$ & $\mathbb{R}$ & ڤ & 忿 & 苾 & m్ & $\mathbb{N}$ & $\varphi$ & \% & 욿 & 요 \\
\hline$u !-8 / \varepsilon>d$ & $\widetilde{\sigma}$ & $N$ & 吕 & ष్ & 4 & ळ. & $\bar{\infty}$ & 2 & 8 & ఫ & \begin{tabular}{|l|l|l|}
$\infty$ \\
\end{tabular} & $\cong$ & N & กิ & 19 & $\bar{n}$ & $\bar{\sigma}$ & $\mathscr{0}$ & $\mathscr{~}$ & $\bar{r}$ & ষั \\
\hline $\mathbf{u l}_{-}-\nabla / \varepsilon>d$ & $\mathbb{N}$ & 8 & T & ष & 웅 & \% & $\bar{\sigma}$ & 5 & $\mathscr{\infty}$ & Б & के & क्ष & 8 & 8 & $\infty$ & g & g & m & : & $\bar{\sigma}$ & 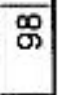 \\
\hline u!- $b>d$ & $\stackrel{\infty}{\infty}$ & 40 & 足 & 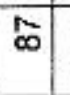 & 응 & 号 & \% & m & $\mathscr{\infty}$ & "̊ & 官 & g) & $\varphi$ & $\stackrel{\varphi}{\Gamma}$ & $\mathscr{\infty}$ & D & \% & চ & 잉 & 9 & g \\
\hline$u \mid-z / h b d$ & 9 & 5 & 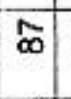 & สู & 웅 & g & \% & $\mathscr{O}$ & ริ & 今 & 果 & g & 品 & 10 & 5 & g & g & 8 & 옹 & $\mathscr{2}$ & g: \\
\hline u!-z>d & N & $\mathscr{g}$ & 8 & 잉 & 융 & : & \% & 5 & 号 & \% & क & 8 & g & $\bar{\sigma}$ & 5 & 음 & 8 & \% & 8 & ळ & 음 \\
\hline u! $\varepsilon>d$ & 음 & 욤 & 욤 & 음 & 응 & 은 & 8 & 8 & 8 & ㅇ & 8 & 음 & 응 & 웅 & 응 & 8 & 은 & 음 & 음 & 응 & 응 \\
\hline 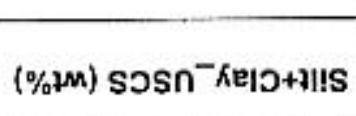 & $\begin{array}{l}\text { mo } \\
\text { m. }\end{array}$ & $\stackrel{n}{r}$ & $\begin{array}{l}40 \\
\text { Ṅ }\end{array}$ & $\begin{array}{l}\text { v } \\
\text { gn }\end{array}$ & $\underset{\infty}{\leftarrow}$ & $\begin{array}{l}0 \\
0 \\
0\end{array}$ & ஸٌ & $\begin{array}{c}\infty \\
\stackrel{\sigma}{\sigma} \\
\end{array}$ & $\begin{array}{c}\infty \\
\infty \\
\text { N }\end{array}$ & $\begin{array}{l}\mathrm{m} \\
\stackrel{\sigma}{\sigma}\end{array}$ & $\begin{array}{l}\infty \\
\infty \\
0\end{array}$ & $\begin{array}{l}0 \\
\text { สิ }\end{array}$ & $\begin{array}{l}\dot{J} \\
\dot{+}\end{array}$ & $\begin{array}{l} \\
\text { ó }\end{array}$ & 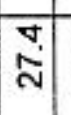 & $\begin{array}{l}m \\
j\end{array}$ & $\begin{array}{l}\stackrel{\sim}{\infty} \\
\infty\end{array}$ & $\begin{array}{c}\omega \\
\tilde{m}\end{array}$ & 岕 & $\begin{array}{l}\sigma \\
m \\
m\end{array}$ & $\begin{array}{l}q \\
\stackrel{+}{+}\end{array}$ \\
\hline (\%+M) sosn-pues & 官 & $\begin{array}{l}\text { d } \\
\text { 品 }\end{array}$ & \begin{tabular}{|c|}
$\varphi$ \\
$\omega$ \\
$\sigma$ \\
\end{tabular} & $\begin{array}{l} \\
g \\
g\end{array}$ & \begin{tabular}{|l|}
0 \\
$N$ \\
$N$
\end{tabular} & $\begin{array}{l}n \\
N\end{array}$ & $\begin{array}{l}N \\
0 \\
0\end{array}$ & 它 & \begin{tabular}{|c|} 
\\
$\rho^{\prime}$
\end{tabular} & $\frac{\varphi}{\dot{\omega}}$ & $\begin{array}{l}\infty \\
\\
\end{array}$ & $\begin{array}{l}\bar{n} \\
\stackrel{8}{\circ}\end{array}$ & $\begin{array}{l}\infty \\
\infty \\
\infty \\
\omega^{\circ}\end{array}$ & $\begin{array}{l}N \\
\dot{q}\end{array}$ & \begin{tabular}{l|}
$\omega 0$ \\
40 \\
9
\end{tabular} & $\begin{array}{l}0 \\
\stackrel{N}{v}\end{array}$ & \begin{tabular}{|l|}
0 \\
0 \\
0 \\
0
\end{tabular} & \begin{tabular}{c|} 
\\
\\
\end{tabular} & 产 & $\begin{array}{l} \\
0 \\
\dot{0} \\
0\end{array}$ & $\vec{i}$ \\
\hline (\%дM) sosn & $\overline{\mathrm{g}}$ & $\begin{array}{l} \\
\dot{\mathrm{N}}\end{array}$ & \begin{tabular}{|l|}
$\infty$ \\
\\
$m$
\end{tabular} & $\frac{N}{m}$ & $\dot{\infty}$ & \begin{tabular}{|l|} 
\\
\\
\end{tabular} & $\stackrel{N}{=}$ & 穴 & $\frac{5}{m}$ & $\stackrel{\dddot{\phi}}{\mathscr{\omega}}$ & $\begin{array}{l}m \\
\infty \\
\infty\end{array}$ & $\hat{\Sigma}$ & $\begin{array}{l}\infty \\
\dot{N} \\
\mathbb{N}\end{array}$ & 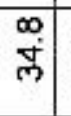 & Nָ & 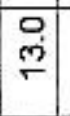 & \begin{tabular}{|l|} 
\\
$\stackrel{2}{\square}$
\end{tabular} & \begin{tabular}{l}
0 \\
\multirow{1}{*}{}
\end{tabular} & \begin{tabular}{|l|}
$\infty$ \\
\\
\end{tabular} & 蛋 & 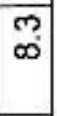 \\
\hline qe7-dnosg sosn & 5 & $\Sigma$ & 5 & एँ & $\sum_{\omega}$ & $\sum_{\infty}$ & $\underset{\omega}{\Sigma}$ & $\frac{\Sigma}{\omega}$ & $\bar{\Sigma}$ & $\frac{5}{\omega}$ & $\begin{array}{l}\sum \\
\bar{c} \\
\vdots \\
\omega\end{array}$ & क & $\begin{array}{l}\text { 市 } \\
\sum_{\varpi}\end{array}$ & $\sum_{\infty}$ & 立 & $\Sigma$ & $\Sigma$ & $\frac{\Sigma}{\omega}$ & $\sum$ & 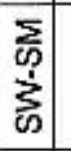 & 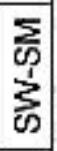 \\
\hline (யง) पदdea Jамоา & $\mp$ & 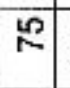 & \begin{tabular}{|l|}
$\mathscr{N}$ \\
\end{tabular} & 芯 & 맏 & 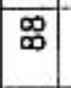 & $\mathbb{C}$ & 疋 & Б) & g & A & 可 & मे & ㅇ & $\stackrel{\mathscr{I}}{\rightleftharpoons}$ & $\mp$ & న & 要 & m & पे & 읃 \\
\hline (uv) чұdeg seddn & 0 & के & $\bar{\Phi}$ & ले & 묻 & \begin{tabular}{|l|}
$\infty$ \\
ட
\end{tabular} & $\stackrel{\pi}{5}$ & $F$ & $\underline{m}$ & D & 0 & व & 芯 & $\stackrel{\infty}{\sim}$ & 守 & 0 & $\stackrel{\infty}{\sim}$ & $\stackrel{\mathbb{O}}{\mathrm{I}}$ & $\stackrel{\Delta}{N}$ & 움 & $\bar{z}$ \\
\hline & 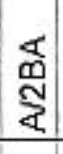 & $\frac{m}{m}$ & $\begin{array}{l}\text { m } \\
\text { 盖 } \\
\text { m }\end{array}$ & $\begin{array}{l}\mathbb{v} \\
\text { 惫 } \\
\text {. }\end{array}$ & 0 & U & $\stackrel{\mathbb{E}}{\mathrm{C}}$ & 聴 & 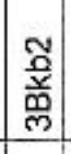 & 离 & 0 & జृ & 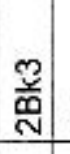 & $\begin{array}{l}\mathrm{E} \\
\text { 总 } \\
\text { N }\end{array}$ & $\begin{array}{l}\overline{\mathbf{0}} \\
\text { 㩊 } \\
\end{array}$ & $\begin{array}{l}4 \\
\text { 要 }\end{array}$ & 音 & $\stackrel{\mathbb{2}}{\mathrm{C}}$ & 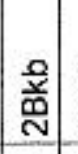 & $\begin{array}{l}\bar{Y} \\
\text { 兽 }\end{array}$ & 0 \\
\hline epo & 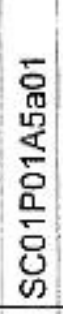 & 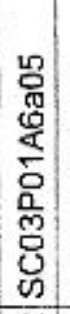 & 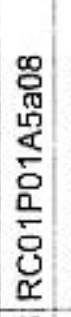 & 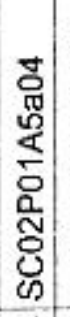 & 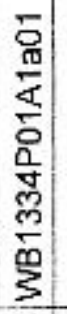 & $\begin{array}{l}\frac{5}{0} \\
\frac{\pi}{5} \\
5 \\
0 \\
0 \\
\frac{0}{0} \\
\frac{0}{0} \\
5 \\
5\end{array}$ & 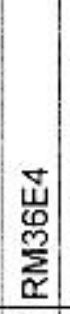 & 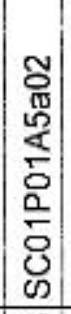 & 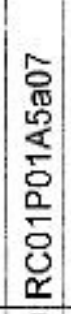 & 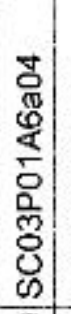 & 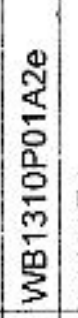 & 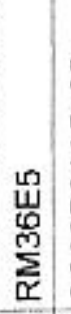 & 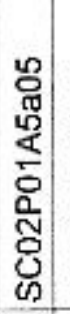 & 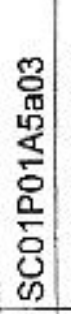 & 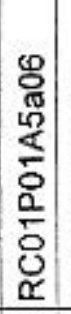 & 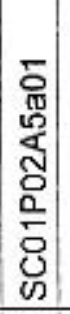 & $\begin{array}{l}0 \\
0 \\
0 \\
0 \\
5 \\
0 \\
0 \\
0 \\
0 \\
0 \\
0 \\
0\end{array}$ & 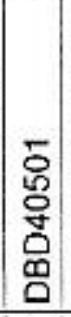 & 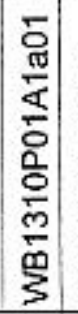 & 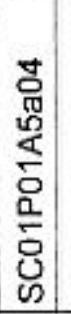 & 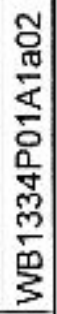 \\
\hline әрdues & - & $N$ & m & $\nabla$ & n & 0 & $N$ & $\infty$ & क & 은 & $\mp$ & 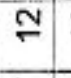 & $\stackrel{m}{=}$ & 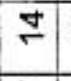 & 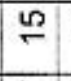 & $\stackrel{\varphi}{\oplus}$ & $\approx$ & $\stackrel{\infty}{\sigma}$ & क & 이 & $\bar{v}$ \\
\hline บวาย & 守 & 守 & 守 & S & 今 & 今 & 웅 & 今 & 8 & 今. & $\begin{array}{l} \\
\\
\end{array}$ & 守 & 守 & So & 今 & 守 & 总 & 守 & 守 & 亏ั) & to \\
\hline
\end{tabular}




\begin{tabular}{|c|c|c|c|c|c|c|c|c|c|c|c|c|c|c|c|c|c|c|c|}
\hline Ooz\# $>d$ & $\stackrel{\infty}{=}$ & \% & 9 & $\stackrel{\infty}{\leftarrow}$ & r & क & & 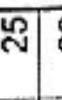 & & & $\pi$ & & & $1=$ & का & का? & $F \hat{m}$ & $\infty$ & $\infty$ \\
\hline 0 t $\#>d$ & $m$ & 우 & $\overline{\mathrm{m}}$ & 罚 & का & 9 & g & q & บ & స & है & $5 \mathscr{5}$ & 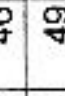 & 8 & $\bar{m}$ & $\bar{N}$ & 6 & $\operatorname{4}$ & $\stackrel{\infty}{\sim}$ \\
\hline Ol\# $>d$ & 守 & D & 임 & 号 & 离 & \$ & 6 & เి & 5 & m: & 8 & 8 & 8 & 8 & O & $9 i$ & 雨 & 8 & I \\
\hline$t \#>d$ & 8 & 苟 & 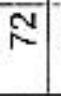 & 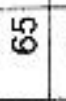 & क & वे & $E$ & N & $\bar{\varphi}$ & : & 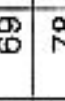 & 0.6 & 8 & 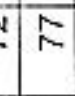 & की & $\widehat{\hat{\theta}}$ & 进 & 曲 & मे \\
\hline$u \mid-8 / \varepsilon>d$ & $\stackrel{\infty}{\sim}$ & $\mathscr{2}$ & 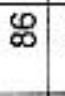 & న & ? & $\mathscr{8}$ & 市 & Ð & ⿷匚 & $\Phi$ & 岗|: & \begin{tabular}{l|l}
0 & $\infty$
\end{tabular} & $\bar{g}$ & 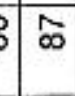 & N & $\bar{\infty} \mid \check{c}$ & бळ & 9 & क \\
\hline$u \mid-t / \varepsilon>d$ & б) & of & 8 & g) & హ) & क & \% & ৪) & g. & 9 & के & 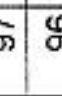 & $\begin{array}{l}9 \\
0\end{array}$ & 5 & 8. & : & $8 \%$ & 8 & $\stackrel{\infty}{\varnothing}-1$ \\
\hline u! $-t>d$ & की & g & 우 & g. & ু & ஃ & \$: & म. & Б) & Б) & 糹: & 8 & 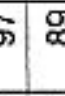 & $\%$ & รู & 우: & ๙ু & कू & A \\
\hline $\mathrm{u} \mid-z f \mathrm{l} b>\mathrm{d}$ & $\%$ & ஜ & ○. & (1) & $\$$ & g) & का & g & $\mathscr{8}$ & $\Phi$ & बढ. & 急 & $\begin{array}{c}8 \\
0\end{array}$ & 8 & 용 & ㅇㅇํ & $\%$ \% & 웅 & $\bar{\infty}$ \\
\hline 니-z>d & 5 & $\stackrel{8}{\circ}$ & 우 & 임 & 四 & 웅 & \% & 8 & $\bar{\Phi}$ & ळ & 思 & 艿 & $\begin{array}{ll}0 \\
0\end{array}$ & 8 & के & 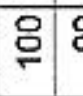 & $8 \%$ & $\%$ & 5 \\
\hline$u !-\varepsilon>d$ & $\stackrel{8}{\circ}$ & 8 & 음 & 움 & 8 & 임 & ㅇ․ & $\stackrel{\circ}{\circ}$ & 임 & $\$$ & 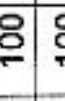 & 5 & 5 & 5 움 & 우 & 잉 & $8 \div$ & 5 & 웅 \\
\hline$(\%+M) \operatorname{sos}^{-}{ }^{-1}$ (E) & ন্d & $\bar{\sigma}$ & $\overline{=}$ & ָ̀ & مَّ & गें & \begin{tabular}{c|}
$\infty$ \\
$\stackrel{\infty}{\sigma}$
\end{tabular} & $\vec{m}$ & 官 & \begin{tabular}{l|l} 
\\
$\dot{0}$ \\
$\dot{0}$
\end{tabular} & $\bar{m}$ & : & $=$ & ก & 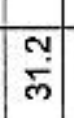 & $\stackrel{\infty}{\sim}$ & $\begin{array}{lll}0 \\
\dot{\nabla} \\
\dot{m}\end{array}$ & $\begin{array}{ll}0 \\
\stackrel{\infty}{*}\end{array}$ & $\hat{\tilde{N}}$ \\
\hline (\%1M) sosn'pues & กั่ & $\begin{array}{l}0 \\
\tilde{\mathscr{C}} \\
\end{array}$ & $\begin{array}{l}\text { पे } \\
\dot{0}\end{array}$ & ธ్ర & $\stackrel{\mathrm{N}}{\mathrm{N}}$ & 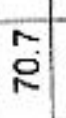 & $\begin{array}{l} \\
\varnothing \\
\end{array}$ & $\begin{array}{l} \\
\text { के } \\
\text { के }\end{array}$ & : & : & & $\frac{1}{0}$ & g & \begin{tabular}{l}
$\sqrt[N]{3}$ \\
\hdashline \\
\hdashline
\end{tabular} & \begin{tabular}{|l|} 
\\
g్ల
\end{tabular} & $\stackrel{m}{i}$ & \begin{tabular}{l|l}
$\dot{S}$ & $\stackrel{6}{5}$
\end{tabular} & $\begin{array}{c}0 \\
\bar{n}\end{array}$ & $\frac{d}{\dot{y}}$ \\
\hline (\%) & \begin{tabular}{|l|} 
\\
$\hat{\mathrm{N}}$
\end{tabular} & 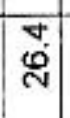 & $\begin{array}{l}\omega \\
\infty \\
-\infty\end{array}$ & त) & d্ & $\bar{\varphi}$ & $\begin{array}{l}\mathcal{N} \\
\varrho\end{array}$ & $\begin{array}{l}0 \\
\stackrel{0}{\circ}\end{array}$ & $\begin{array}{l}\text { O } \\
\text { ปे }\end{array}$ & ํ. & 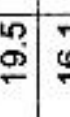 & 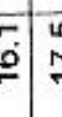 & $=$ & is & 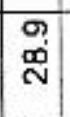 & $\underset{\sim}{\stackrel{5}{N}}$ & $\begin{array}{c}\dot{c o s} \\
= \\
= \\
\infty\end{array}$ & \begin{tabular}{l|l|}
0 \\
0 \\
$\dot{N}$
\end{tabular} & $\begin{array}{l}\text { क् } \\
\text { सें }\end{array}$ \\
\hline qE7-dnoגo sosn & क & $\begin{array}{l}\sum \\
\infty \\
\sum \\
\infty\end{array}$ & $\sum_{\infty}$ & $\sum_{\infty}$ & ळ) & $\sum_{\text {के }}$ & $\sum_{\infty}$ & $\sum_{\infty}$ & E & $\begin{array}{l}\sum_{0} \\
\sum_{\infty}^{1}\end{array}$ & 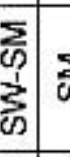 & $\Sigma$ & 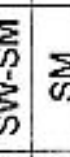 & $\frac{\Sigma}{w}$ & $\sum_{\infty}$ & 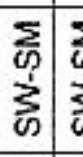 & $\sum_{\substack{\infty \\
\vdots \\
\infty}}^{\infty}$ & $\sum_{\infty}$ & इ \\
\hline (шэ) प1) & $\frac{P}{7}$ & 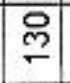 & क् & $E$ & 2 & \% & $\stackrel{2}{\circ}$ & N] & $\sigma$ & के & $\begin{array}{l}\infty \\
\vdots\end{array}$ & (פ) & हैं & 0 & 2 & సิ| & $\infty$ & ల్ల & ले \\
\hline (wo) ułded Jeddn & 웅 & ‡ & $E$ & $\overline{9}$ & ज़ & ल & क & $\mp$ & 0 & si & ? & 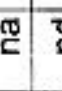 & क् & 0 & $\theta$ & $\mathbb{D}_{\infty}$ & fo & ल্ & $\pi$ \\
\hline uoz!noH & 善 & 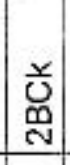 & 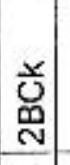 & 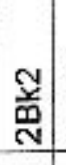 & 眔 & 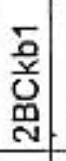 & 言 & 盖 & $<$ & 总 & $\begin{array}{l}\text { 总 } \\
\mathrm{a} \\
\end{array}$ & $\mathbb{\Xi}$ & 0 & 穼 4 & 总 & U & 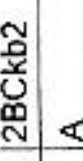 & 苇 & 眔 \\
\hline әро弓 әрdwes ple!ty & 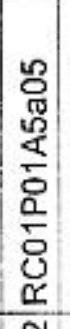 & 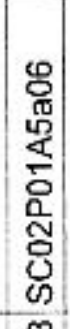 & 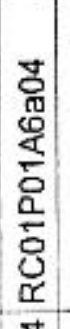 & 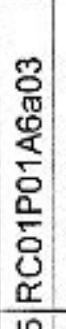 & 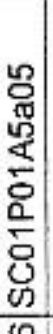 & $\begin{array}{l}\frac{a}{0} \\
\frac{\omega}{5} \\
\vdots \\
0 \\
\frac{0}{0} \\
\frac{m}{3} \\
\frac{9}{3}\end{array}$ & 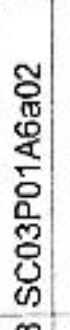 & 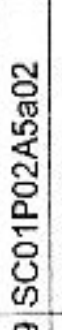 & 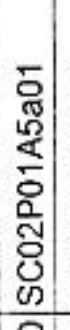 & 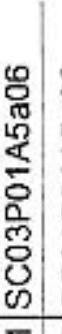 & 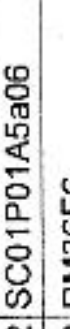 & 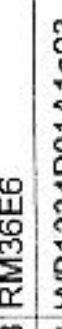 & 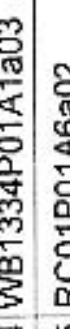 & 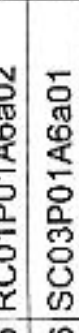 & $\begin{array}{c}0 \\
0 \\
0 \\
0 \\
0 \\
0 \\
0 \\
0 \\
0 \\
0 \\
0\end{array}$ & 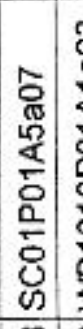 & 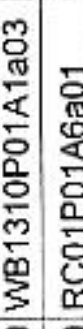 & 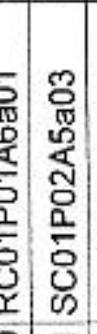 & 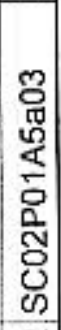 \\
\hline श्रdues & ก & 2 & A & М̆ & $\stackrel{N}{N}$ & 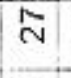 & $\stackrel{\infty}{\sim}$ & g & के & $\bar{m}$ & ल্লা: & m & ले & $\begin{array}{ll}0 \\
n\end{array}$ & m & $\stackrel{\infty}{m}$ & 兽 & $\overline{7}$ & \& \\
\hline yoगe日 & 高 & & $\hat{q}$ & $y$ & $\vec{v}$ & $\bar{g}$ & $y^{\prime}$ & $\vec{y}$ & 拿 & 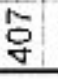 & 名! & $\underline{9}$ & & 弇 & 守 & 可! & $\begin{array}{l}3 \\
\bar{a}\end{array}$ & 5 & 吾 \\
\hline
\end{tabular}




\begin{tabular}{|c|c|c|c|c|c|c|c|c|c|c|c|c|c|c|c|c|c|c|c|c|c|}
\hline $00 Z \#>d$ & : & ㅁ & Ln & 只 & n) & $\div$ & $\stackrel{-1}{2}$ & $\infty$ & $\bar{\sigma}$ & $\infty$ & $\infty$ & $\stackrel{\mathscr{N}}{\sim}$ & $N$ & $\$ 2$ & ㄱ. & $\bar{m}$ & $N$ & 6 & 에 & 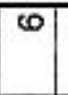 & का \\
\hline OtH $>d$ & 寸 & 虫 & g) & 贯 & $\infty$ & 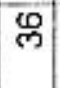 & i & ก & $\stackrel{N}{-1}$ & $\overline{\mathrm{N}}$ & 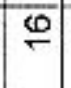 & 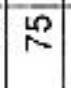 & 点 & $\bar{\nabla}$ & 落 & 寸 & i & क & 范 & $\stackrel{m}{-}$ & $\stackrel{\infty}{\sim}$ \\
\hline $\mathrm{OLH}>\mathrm{d}$ & กู & ఫ్ & : & 8 & N & 8 & $\mathscr{M}$ & लू & $\stackrel{\infty}{\leftarrow}$ & लि & 出 & 怘 & 守 & $g$ & छे & q & ले & D & $\mathscr{8}$ & $\approx$ & 塄 \\
\hline$t H>d$ & $\omega$ & 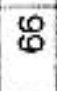 & กิ & 5 & 5 & 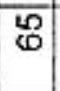 & พ & 8: & $\overparen{ఠ}$ & $\frac{48}{8}$ & 8 & 9 & $n$ & กิ & 6 & $m$ & ก & g & $\begin{array}{l}\infty \\
\infty\end{array}$ & 6 & $\frac{6}{4}$ \\
\hline$u !-8 / \varepsilon>d$ & ळ & $R$ & $\kappa$ & 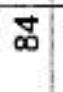 & N & $\mathscr{Q}$ & 8 & 2 & 电 & $\Phi$ & $\mathbb{N}$ & 今) & 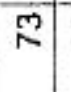 & 思 & $\stackrel{q}{\sim}$ & ষ & $\bar{N}$ & کू' & N & $\stackrel{\infty}{\sim}$ & $\mathscr{E}$ \\
\hline$u \vdash-\nabla / \varepsilon>d$ & สู & $\bar{\sigma}$ & สิ & 8 & $\bar{\sigma}$ & $\infty$ & ఐ & $\mathscr{\infty}$ & के & $\mathscr{D}$ & क् & 5 & 䁅 & $\mathscr{\infty}$ & g & Б & \begin{tabular}{|l|}
$\infty$ \\
$\infty$
\end{tabular} & $\mathscr{m}$ & $\mathscr{\infty}$ & 9 & $\mathscr{\infty}$ \\
\hline u!n $L>d$ & 5 & 9 & \% & 吉 & 8 & 8 & 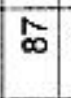 & 5 & \% & $\begin{array}{l}9 \\
\infty\end{array}$ & б) & 今 & 5 & $\Phi$ & న & 通 & 요 & $\stackrel{\infty}{\infty}$ & 8 & 48 & 品 \\
\hline u!ZI L $>d$ & 8 & $\mathscr{8}$ & $\mathscr{\infty}$ & 8 & $\mathscr{8}$ & में & \% & के & के & g & ๘ & \%ి & छ & $\bar{\sigma}$ & 边 & 5 & 要 & ร & Бे & 5 & 雨 \\
\hline u!-z $>d$ & $\mathscr{g}$ & M & 塄 & 覀 & m & 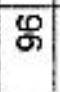 & 4 & 5 & \% & $\Phi$ & के & क & बे & \% & क & 栗 & 果 & 5 & 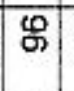 & 品 & \% \\
\hline u!- $\varepsilon>d$ & 욤 & 음 & 응 & 잉 & 응 & 인 & 8 & 8 & 8 & 8 & ○ & 응 & 용 & 요 & 음 & 으 & 응 & 은 & 은 & 은 & 은 \\
\hline 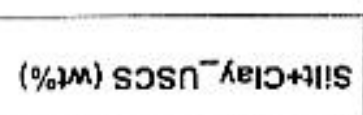 & $\begin{array}{l}\omega \\
\text { ले }\end{array}$ & స్ & क & m & 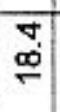 & $\begin{array}{l}\text { N̦ } \\
\text { ปे }\end{array}$ & $\begin{array}{l}m \\
\text { ñ }\end{array}$ & \begin{tabular}{l|}
$\infty$ \\
$\dot{\Psi}$ \\
\end{tabular} & 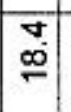 & $F$ & $\begin{array}{l}\infty \\
\infty \\
\infty\end{array}$ & $\begin{array}{l}0 \\
\text { g. }\end{array}$ & $\begin{array}{l}0 \\
\dot{J}\end{array}$ & $\stackrel{m}{\mathrm{~N}}$ & के & $\begin{array}{l} \\
\text { vi }\end{array}$ & \begin{tabular}{l|}
$\infty$ \\
$\dot{+}$ \\
\end{tabular} & $\hat{\mathbb{N}}$ & 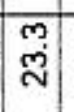 & $\begin{array}{l}\infty \\
\text { ㅁ․ }\end{array}$ & $\begin{array}{l}8 \\
5 \\
5\end{array}$ \\
\hline$(\%+M)$ sosn pues & $\begin{array}{l}\text { m } \\
\dot{q}\end{array}$ & $\begin{array}{l}0 \\
\text { N } \\
\text { n. }\end{array}$ & $\begin{array}{l}m \\
\stackrel{m}{6}\end{array}$ & $\begin{array}{c}0 \\
\infty \\
\infty \\
4\end{array}$ & $\stackrel{\circ}{9}$ & $\begin{array}{l}N \\
10 \\
0\end{array}$ & $\frac{n}{4}$ & $\begin{array}{l}\square \\
0 \\
0\end{array}$ & 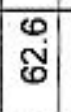 & 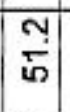 & is & \begin{tabular}{|c|} 
\\
$\dot{0}$
\end{tabular} & $\begin{array}{l} \\
\text { 吕 }\end{array}$ & $\begin{array}{l}0 \\
0 \\
5\end{array}$ & $\begin{array}{l}\text { 吕 } \\
\text { ஸे }\end{array}$ & 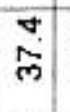 & $\begin{array}{l}0 \\
0 \\
6\end{array}$ & \begin{tabular}{|l|} 
\\
0 \\
$\sigma$ \\
\end{tabular} & $\begin{array}{l}0 \\
\text { กี่ }\end{array}$ & \begin{tabular}{l|}
$\infty$ \\
员 \\
\end{tabular} & $\frac{0}{15}$ \\
\hline (\%) sosก & 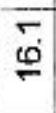 & $\stackrel{m}{\mathrm{n}}$ & $\stackrel{\infty}{\sim}$ & \begin{tabular}{|l|l|}
$\infty$ \\
$\infty$ \\
$\infty$ \\
\end{tabular} & $\begin{array}{l}\omega \\
\text { N̦ }\end{array}$ & $\begin{array}{l}\infty \\
\dot{\mathrm{d}}\end{array}$ & $\begin{array}{l}N \\
M \\
m\end{array}$ & $\begin{array}{c}\infty \\
\text { ஸें }\end{array}$ & $\begin{array}{l} \\
\\
\end{array}$ & $\frac{m}{m}$ & $\begin{array}{l} \\
\text { ஸे } \\
\text { | }\end{array}$ & $\begin{array}{l} \\
\sigma\end{array}$ & 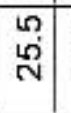 & $\frac{5}{m}$ & \begin{tabular}{l|}
0 \\
गें \\
|
\end{tabular} & $\underset{N}{\stackrel{N}{*}}$ & $\begin{array}{l}\infty \\
\mathrm{N}\end{array}$ & \begin{tabular}{|l|}
0 \\
8 \\
\end{tabular} & \begin{tabular}{c|}
$\infty$ \\
गे \\
ve
\end{tabular} & $\begin{array}{l}\infty \\
\infty \\
\infty\end{array}$ & m \\
\hline qe7-dnods sosn & $\sum_{W}$ & ङ & $\begin{array}{l}\sum_{0} \\
\vdots \\
\sum_{\omega}\end{array}$ & ๘ & $\Sigma_{\infty}$ & $\sum_{\infty}$ & $\sum_{\infty}$ & 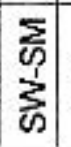 & $\sum_{\infty}$ & $\sum_{\omega}$ & $\sum_{\infty}$ & ए & $\begin{array}{l}\sum_{0} \\
\text { 足 } \\
\sum_{\infty}\end{array}$ & $\Sigma_{\omega}$ & $\bar{s}$ & $\sum$ & $\begin{array}{l}\sum \\
\text { क } \\
\text { क }\end{array}$ & क & $\sum_{\infty}$ & $\sum_{\infty}$ & $\sum_{\infty}$ \\
\hline (шэ) чұdad دамоา & $N$ & $\mathbb{T}$ & $\underset{\mathrm{g}}{\mathrm{g}}$ & $\mathbb{2}$ & $\mathbb{N}$ & $\stackrel{\sim}{\sim}$ & ల్ల & 은 & $\stackrel{\text { g }}{\leftarrow}$ & g & 怘 & 要 & ‡ & 胥 & ? & $\sigma$ & $\mp$ & @ & ㅁ. & 요 & $\stackrel{\mathbb{N}}{\sim}$ \\
\hline (ws) पłdeg Jadd & 0 & 蛋 & $\stackrel{9}{\circ}$ & 匹 & g & 1 & $\stackrel{\mathscr{N}}{\mathbf{N}}$ & Ф & 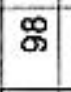 & 点 & $\mathbb{N}$ & 茎 & 夺 & $\stackrel{\mathbb{Z}}{\complement}$ & 四 & 0 & पे & 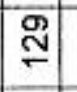 & 맏 & $\sigma$ & $\mp$ \\
\hline uozuoH & $<$ & $\stackrel{\mathbb{E}}{5}$ & $\begin{array}{l}\infty \\
\text { 想 } \\
\end{array}$ & O & $\frac{\mathrm{y}}{\mathbf{0}}$ & 妾 & 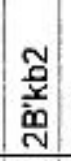 & 寅 & 迎 & 音 & 登 & $\stackrel{\mathbb{E}}{\Sigma}$ & 总 & $\mathbb{E}$ & 永 & $\begin{array}{l}\bar{\xi} \\
\text { 辛 } \\
\text { 帘 }\end{array}$ & 玄 & 竎 & 空 & $\begin{array}{l}\text { Ẽ } \\
\text { 离 } \\
\end{array}$ & $\frac{\omega}{0}$ \\
\hline opos әjdues pla! & 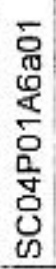 & 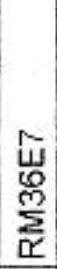 & \begin{tabular}{|c|}
0 \\
0 \\
0 \\
0 \\
0 \\
0 \\
0 \\
\\
0 \\
0 \\
0 \\
0
\end{tabular} & 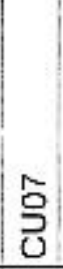 & 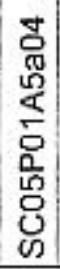 & 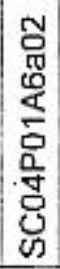 & 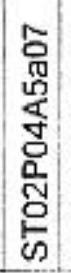 & 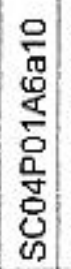 & 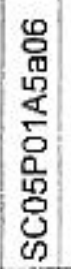 & $\begin{array}{l}8 \\
0 \\
0 \\
\mathbb{8} \\
5 \\
0 \\
8 \\
0 \\
0 \\
0 \\
0\end{array}$ & 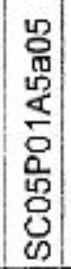 & $\mid \begin{array}{c} \\
0 \\
\frac{0}{2}\end{array}$ & 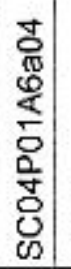 & $\begin{array}{l} \\
\stackrel{\mathrm{J}}{\mathrm{J}} \\
\mathrm{J}\end{array}$ & 㟔 & $\begin{array}{l}5 \\
0 \\
0 \\
0 \\
0 \\
0 \\
0 \\
0 \\
0 \\
0 \\
0\end{array}$ & $\begin{array}{l}0 \\
0 \\
0 \\
0 \\
0 \\
0 \\
0 \\
0 \\
0 \\
0 \\
0\end{array}$ & $\mid \begin{array}{l}0 \\
0 \\
0 \\
0 \\
0 \\
0 \\
0 \\
0 \\
0 \\
0 \\
0 \\
0\end{array}$ & 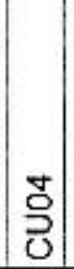 & $\begin{array}{l}0 \\
0 \\
0 \\
0 \\
8 \\
0 \\
0 \\
0 \\
0 \\
0 \\
0 \\
0\end{array}$ & 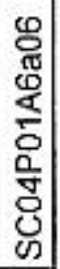 \\
\hline әdumes & $F$ & $N$ & $m$ & 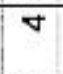 & 10 & 10 & $n$ & $\infty$ & क & 운 & $\mp$ & $\mathbb{N}$ & $m$ & 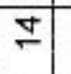 & $\stackrel{\sim}{\sim}$ & $\mathscr{\varphi}$ & $=$ & $\stackrel{\infty}{\sim}$ & क्) & ㅇ & $\bar{v}$ \\
\hline 4갑 & $\begin{array}{l}\infty \\
\stackrel{9}{q}\end{array}$ & $\begin{array}{l}\infty \\
\text { O }\end{array}$ & $\begin{array}{l}\infty \\
\text { o }\end{array}$ & 总 & g & 总 & $\begin{array}{l}\infty \\
\text { O }\end{array}$ & 罗 & 䙳 & $\begin{array}{l}\text { D } \\
\text { o }\end{array}$ & $\begin{array}{l}\text { Oo } \\
\text { g }\end{array}$ & $\begin{array}{l}9 \\
\text { g }\end{array}$ & $\begin{array}{l} \\
\\
\end{array}$ & $\begin{array}{l} \\
\end{array}$ & 总 & g & \begin{tabular}{l|}
$g$ \\
g
\end{tabular} & $\mid \begin{array}{l}\mid g \\
\text { g }\end{array}$ & $\begin{array}{l}\text { o } \\
\text { Oे }\end{array}$ & $\begin{array}{l}\text { g } \\
\text { Oे }\end{array}$ & 号 \\
\hline
\end{tabular}




\begin{tabular}{|c|c|c|c|c|c|c|}
\hline $00 z \#>d$ & क & का & का & $N$ & $\sqrt[N]{ }$ & का \\
\hline 0 t\# $>d$ & $\stackrel{\infty}{\sim}$ & $\mathcal{C}$ & : & 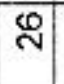 & 8 & $\mp$ \\
\hline Ol\# $>d$ & N & 9 & घ) & 9 & $m$ & $\mathbb{N}$ \\
\hline$t \#>d$ & 8 & $\bar{\sigma}$ & 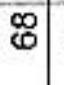 & 5 & $M$ & 5 \\
\hline$u[-8 / \varepsilon>d$ & $\infty$ & के & क & $E$ & ஜ্口 & $\pi$ \\
\hline$u[-t / \varepsilon>d$ & ळ) & ज. & 8) & एా & 8 & 8 \\
\hline$u]^{*} L>d$ & ๓ & ח & ๙ & 怘 & ষా & 8 \\
\hline ul-zI $レ>d$ & $\widehat{\sigma}$ & क & 品 & สู & $\Phi$ & 59 \\
\hline$u !-z>d$ & 䍐 & g & $\hat{n}$ & 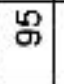 & 塄 & 5 \\
\hline U! $-\varepsilon>d$ & 8 & 인 & 임 & 吕 & 임 & 욤 \\
\hline 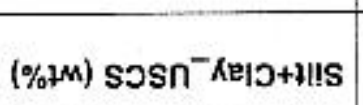 & $\begin{array}{l}\dot{v} \\
\stackrel{i}{*}\end{array}$ & $\begin{array}{l}\dot{v} \\
\dot{v}\end{array}$ & 몰 & $\begin{array}{l}\dot{0} \\
\stackrel{0}{0}\end{array}$ & $\begin{array}{l}\text { N } \\
\dot{N}\end{array}$ & $\stackrel{\text { D }}{\leftarrow}$ \\
\hline (\%?m) sosn-pues & $\begin{array}{l}\sigma \\
\mathscr{\emptyset} \\
\mathscr{\omega}\end{array}$ & $\begin{array}{c}\nabla \\
0 \\
0 \\
\end{array}$ & $\begin{array}{l}\text { क) } \\
\text { लिं }\end{array}$ & $\overline{\mathrm{C}}$ & $\begin{array}{l}\infty \\
\text { 心 }\end{array}$ & $\begin{array}{l}m \\
\text { gin }\end{array}$ \\
\hline (\%मM) sosn & $\approx$ & $N$ & 굥 & $\begin{array}{l}\text { Ln } \\
\text { స }\end{array}$ & $\frac{0}{\mathrm{~N}}$ & $\begin{array}{l}\text { 울 } \\
\text { N్ }\end{array}$ \\
\hline qeר-dnoגg sosn & $\begin{array}{l}\sum_{0,} \\
\sum_{\omega}\end{array}$ & क & ळ & $\begin{array}{l}\sum_{00} \\
\vdots \\
\vdots \\
0\end{array}$ & ह & ङ \\
\hline (uכ) uзdag дәмо 7 & 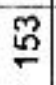 & $\stackrel{\text { I }}{\complement}$ & $\stackrel{\mathbb{O}}{\complement}$ & $\stackrel{9}{\circ}$ & $\mathbb{Z}$ & g \\
\hline (wo) undog seddn & $\stackrel{\mathbb{m}}{\mathrm{N}}$ & $\stackrel{\text { ( }}{\mathrm{C}}$ & $\stackrel{\mathbb{N}}{\mathrm{I}}$ & 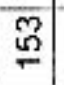 & $\stackrel{\mathbb{R}}{\check{2}}$ & 윤 \\
\hline 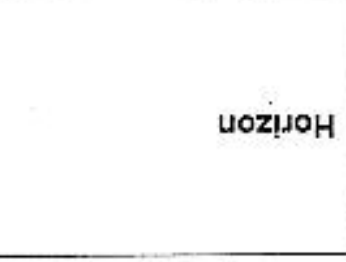 & 产 & $\stackrel{\mathscr{c}}{c}$ & $\mathbb{E}$ & \multicolumn{2}{|l|}{ 甶 } & 盖 \\
\hline 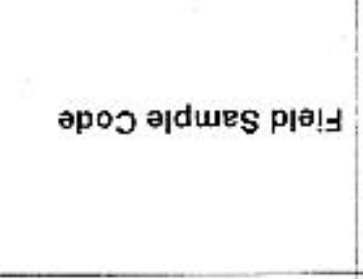 & 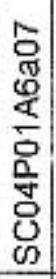 & 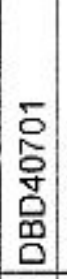 & 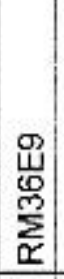 & 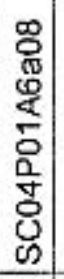 & $\frac{6}{5}$ & 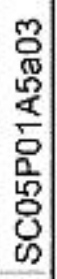 \\
\hline a|dues & N & N & $\grave{N}$ & ल) & 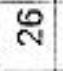 & $\hat{N}$ \\
\hline प갑 & 品 & $\begin{array}{l}\infty \\
⿱ 乛\end{array}$ & $\begin{array}{l}\infty \\
\dot{q} \\
\end{array}$ & $\begin{array}{l}0 \\
\dot{q} \\
q\end{array}$ & $\stackrel{\infty}{q}$ & $\begin{array}{l}0 \\
\text { Oे }\end{array}$ \\
\hline
\end{tabular}

$\stackrel{\oplus}{\vdots}$
首
0

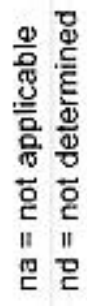




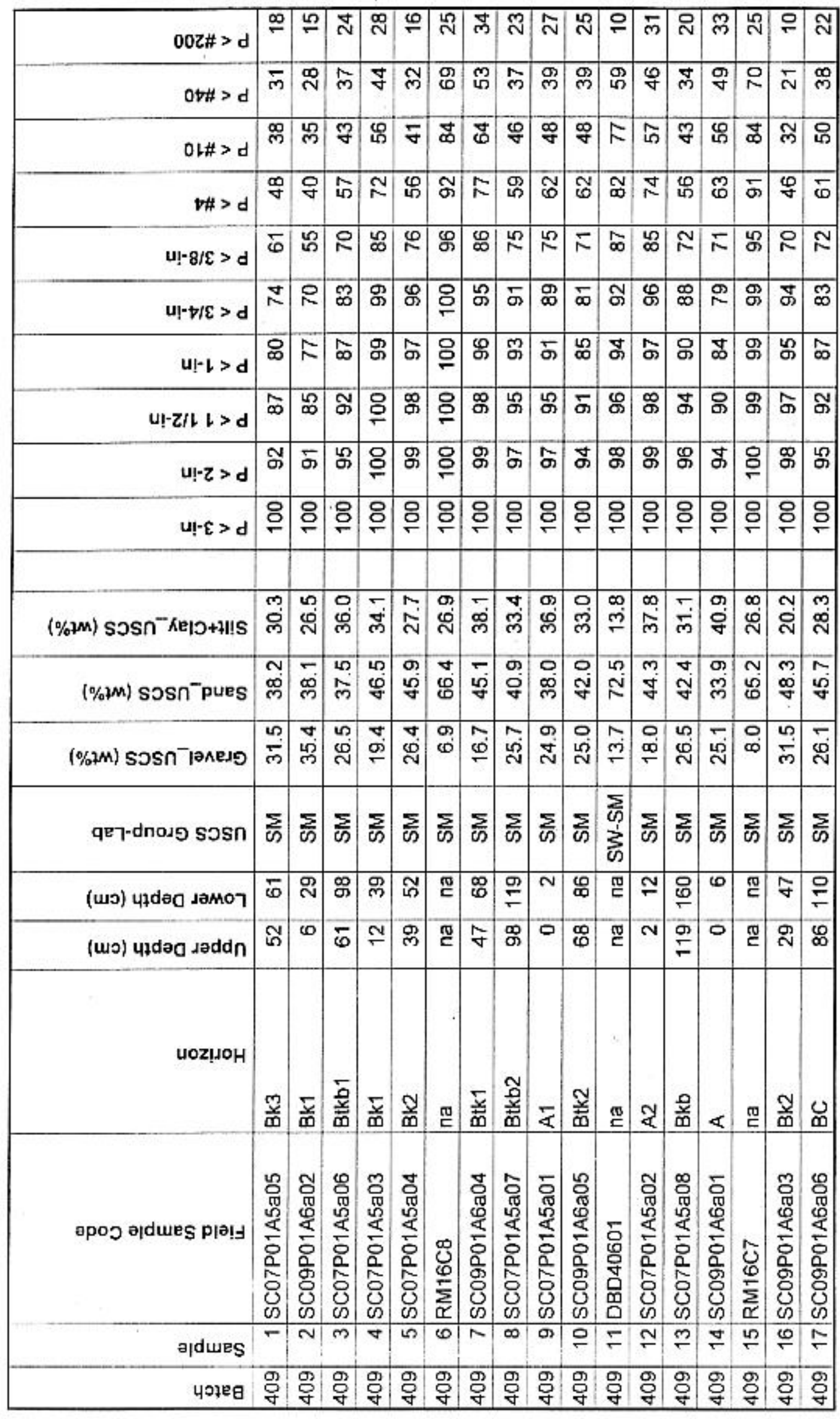




\begin{tabular}{|c|c|c|c|c|c|c|c|c|c|c|c|c|c|c|c|c|c|c|c|c|c|}
\hline $00 \mathrm{z} \#>d$ & $\stackrel{+}{T}$ & 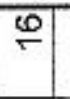 & का & \pm & $=$ & 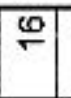 & $F$ & $F$ & 운 & 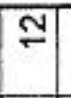 & 인 & กู & $=$ & $\approx$ & $\omega$ & 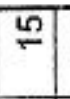 & $=$ & $\stackrel{\sim}{\sim}$ & $F$ & $\bar{N}$ & $\stackrel{m}{2}$ \\
\hline 0 t\# $>d$ & ळ్ల & $\mathbb{1}$ & 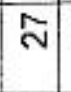 & 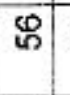 & $\bar{m}$ & 8 & ח & ำ & \% & 8 & लि & 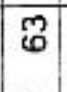 & is & 吕 & ले & 늉 & 政 & 政 & \begin{tabular}{|l|}
$g$ \\
\end{tabular} & $\stackrel{\infty}{\square}$ & $\mathscr{0}$ \\
\hline $0 \mathrm{~L} \#>d$ & $\varphi$ & ळ & 员 & 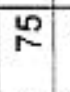 & $\frac{m}{8}$ & 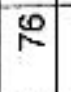 & 咅 & N & $\bullet$ & $N$ & g & $\frac{10}{N 2}$ & A & L & 5 & 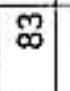 & $\tilde{6}$ & $\bar{T}$ & I & $F$ & N \\
\hline$t \#>d$ & $\mathscr{C}$ & ๙ & 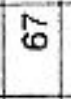 & m & $\begin{array}{l}\infty \\
\infty\end{array}$ & \begin{tabular}{|l|l|}
$\infty$ \\
\end{tabular} & ఏ & ఫ & R & 品 & 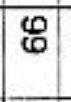 & ma & 市 & 罣 & 8 & 8 & 9 & 9 & $\mathscr{\infty}^{-}$ & $\infty$ & $\infty$ \\
\hline$u !-8 / \varepsilon>d$ & 9 & 号 & 品 & 占 & $m$ & 임 & $\bar{\sigma}$ & 8 & 贯 & $\mathscr{0}$ & 요 & 8 & छ & $\mathscr{m}$ & 品 & 5 & \begin{tabular}{|l|}
$\infty$ \\
$\infty$
\end{tabular} & ळ & สู & \% & חু \\
\hline $\mathbf{u}|-\downarrow| \varepsilon>d$ & $\mathscr{G}$ & 용 & \% & б & \begin{tabular}{l|}
$\infty$ \\
$\infty$
\end{tabular} & 题 & 心 & $\mathscr{G}$ & के & \% & 吉 & 男 & 잉 & $\stackrel{\infty}{\infty}$ & $\bar{\sigma}$ & 응 & 5 & 8) & 惫 & 悤 & ( \\
\hline$u !-i>d$ & जे & gọ & 吉 & m. & $\bar{\sigma}$ & 品 & 号 & 5 & 怘 & あँ & 뇽 & \& & 要 & \% & 8 & 은 & के & ชั & 密 & g & g \\
\hline $\mathrm{u} \mid-z / L \quad b>\mathrm{d}$ & 品 & 8 & \% & 甲 & 学 & 䑻 & 8 & 息 & g & $\mathscr{8}$ & 5 & g & 욤 & S & $\mathscr{\sigma}$ & 음 & 品 & 5 & \% & g & g \\
\hline$u !-z>d$ & क & 응 & 罷 & 5 & 5 & gi & g & g & g. & $\mathscr{\infty}$ & \% & क & 응 & 옹 & s. & 응 & g & б̆ & \% & 은 & 음 \\
\hline$u !-\varepsilon>d$ & 용 & 잉 & 웅 & 8 & 8 & 우 & 웅 & 웅 & 웅 & 음 & 8 & 8 & 8 & 응 & 웅 & 움 & 음 & 용 & 응 & 으 & 음 \\
\hline (\%جM) $\operatorname{sOSn}^{-}$- & 묻 & 믿 & 믇 & 豆 & प्र & 产 & g & एव & T & 马 & 马े & $\overline{\mathrm{g}}$ & E & D & 맏 & 豆 & 马 & 豆 & प् & $\bar{c}$ & 묻 \\
\hline (\%2m) sosn-pues & $\begin{array}{l}\infty \\
0 \\
4\end{array}$ & $\begin{array}{l}\infty \\
0 \\
0\end{array}$ & $\begin{array}{l}8 \\
6 \\
6\end{array}$ & $\bar{N}$ & in & $\bar{N}$ & $\begin{array}{l}\mathcal{M} \\
\mathscr{P}\end{array} \mid$ & $\begin{array}{l}\mathbb{N} \\
\mathbb{R}\end{array}$ & $\begin{array}{l}\mathrm{N} \\
\mathrm{N} \\
\mathrm{N}\end{array}$ & $\begin{array}{l}\mathrm{N} \\
\mathrm{N}\end{array}$ & \begin{tabular}{|l|} 
\\
\\
\end{tabular} & 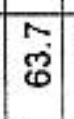 & \begin{tabular}{l|} 
\\
0 \\
$\infty$ \\
$N$
\end{tabular} & $\begin{array}{l}\infty \\
\mathbb{N} \\
N\end{array}$ & \begin{tabular}{l|}
$\infty$ \\
0 \\
\end{tabular} & \begin{tabular}{|l|} 
\\
0 \\
0
\end{tabular} & \begin{tabular}{l|} 
\\
$\dot{\theta}$
\end{tabular} & \begin{tabular}{l|} 
\\
\end{tabular} & $\bar{N}$ & $\begin{array}{l}10 \\
80 \\
0\end{array}$ & $\underset{N}{N}$ \\
\hline (\%7M) sosn & $\begin{array}{l}\infty \\
\dot{N}\end{array}$ & 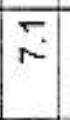 & क़ & 吕 & $\begin{array}{l}\mathrm{N} \\
\stackrel{\mathrm{N}}{ }\end{array}$ & $\stackrel{\infty}{\infty}$ & $\stackrel{\sim}{\stackrel{D}{\sim}}$ & $\stackrel{\sim}{\sim}$ & \begin{tabular}{|c|} 
\\
$\stackrel{\varphi}{L}$ \\
-
\end{tabular} & 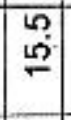 & \begin{tabular}{|l|}
$\infty$ \\
ปู่ \\
\end{tabular} & $\begin{array}{l}r \\
\stackrel{m}{2}\end{array}$ & $\stackrel{N}{O}$ & के & $\frac{0}{\mathrm{~N}}$ & \begin{tabular}{|c|} 
\\
$\infty$ \\
\end{tabular} & \begin{tabular}{|l|}
$\infty$ \\
$\infty$ \\
\end{tabular} & \begin{tabular}{|l|} 
\\
$\oplus$ \\
$\varphi$
\end{tabular} & $\stackrel{N}{=}$ & $\stackrel{\sim}{\circ}$ & $\stackrel{\nabla}{\circ}$ \\
\hline qE7-dno. פ sosก & 을 & 무 & $\mathrm{g}$ & $\mathrm{g}$ & 믇 & 믇 & 믇 & 믇 & 马 & 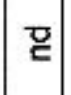 & 맏 & 묻 & 몯 & 믇 & g & 미 & 马 & 몯 & 읃 & 묻 & 믇 \\
\hline (що) чұdea دәмоา & Tू & $\stackrel{N}{N}$ & $\begin{array}{l} \\
\\
\end{array}$ & $\frac{\mathscr{N}}{\mathrm{N}}$ & $\frac{\sigma}{-}$ & 总 & 忿 & $\begin{array}{l} \\
0 \\
\text { 品 }\end{array}$ & N & 욤 & 운 & व & 今్ & के & \begin{tabular}{|l|}
0 \\
\\
\end{tabular} & \begin{tabular}{|l|}
8 \\
\\
\end{tabular} & $\stackrel{\mathscr{\infty}}{\stackrel{\infty}{\leftarrow}}$ & 恕 & 总 & $\begin{array}{l}m \\
\text { W }\end{array}$ & $\overline{\mathrm{N}}$ \\
\hline (wo) ułdeg dodd గ & $\stackrel{\mathbb{Z}}{C}$ & 旁 & ণ & N & $\stackrel{\circ}{\circ}$ & 里 & ஏे & $\bar{\kappa}$ & $\frac{\mathscr{N}}{\grave{N}}$ & $\bar{s}$ & $\stackrel{\sigma}{=}$ & $\mathbb{T}$ & $\stackrel{N}{N}$ & E & $\begin{array}{l}\infty \\
\text { 员 } \\
\text { 足 }\end{array}$ & ⿳亠丷厂 & 용 & $\stackrel{\mathscr{Q}}{\mathscr{C}}$ & స్ల్ & 点 & g \\
\hline UOZ!」OH & $\underset{\mathbb{C}}{\mathrm{E}}$ & $\begin{array}{l}\text { 点 } \\
\text { 思 }\end{array}$ & D & 号 & 高 & 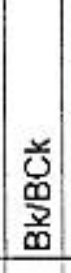 & 离 & 0 & U్ & 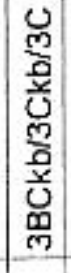 & 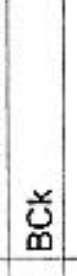 & (D) & U & 酋 & 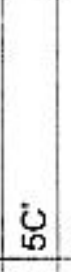 & 怘 & $\begin{array}{l}\mathbf{0} \\
\mathbf{m} \\
\text { ․ }\end{array}$ & 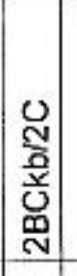 & 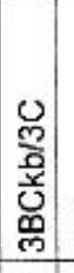 & 怘 & U \\
\hline apos 키wes pl!ㅓ & 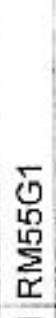 & 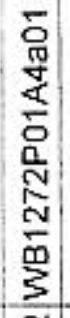 & 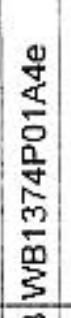 & 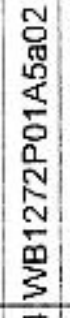 & 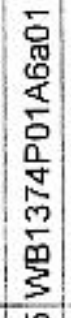 & 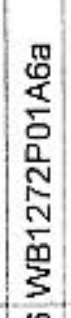 & 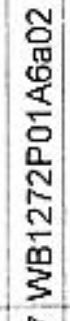 & $\mid \begin{array}{l}0 \\
0 \\
5 \\
5 \\
0 \\
\frac{0}{5} \\
0 \\
0 \\
0 \\
3\end{array}$ & 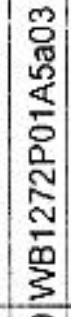 & 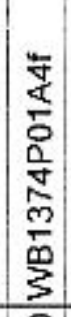 & 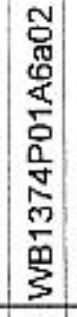 & 号 & 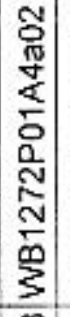 & 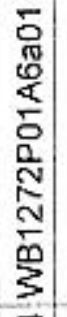 & 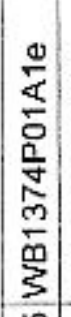 & $\begin{array}{l}0 \\
\frac{1}{\alpha} \\
0 \\
0 \\
\frac{N}{N} \\
\frac{0}{0} \\
\frac{3}{5}\end{array}$ & 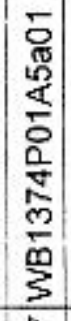 & 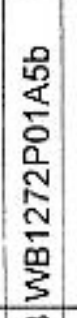 & 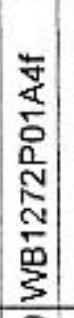 & 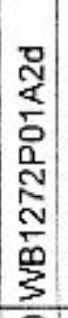 & 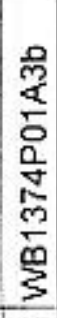 \\
\hline ㅋdures & $\sigma$ & $\mathrm{N}$ & m & 8 & 100 & $\omega$ & $\pi$ & $\infty$ & $\sigma$ & 은 & $=$ & - & $\stackrel{m}{-}$ & 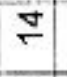 & 4 & 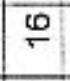 & 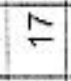 & $\stackrel{\infty}{-}$ & क & 이 & $\bar{N}$ \\
\hline yojeg & $\frac{2}{8}$ & $\frac{0}{4}$ & $\frac{0}{\frac{9}{4}}$ & $\frac{0}{\frac{1}{4}}$ & $\frac{0}{8}$ & $\frac{9}{4}$ & $\frac{9}{7}$ & $\frac{9}{4}$ & $\frac{0}{4}$ & $\frac{O}{8}$ & $\frac{\text { 인 }}{4}$ & $\frac{0}{\frac{1}{4}}$ & $\frac{0}{8}$ & $\frac{0}{4}$ & $\frac{9}{4}$ & $\frac{9}{8}$ & $\frac{O}{4}$ & 夏 & $\frac{O}{8}$ & $\frac{0}{4}$ & $\frac{8}{4}$ \\
\hline
\end{tabular}

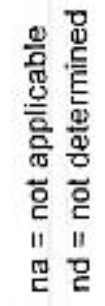




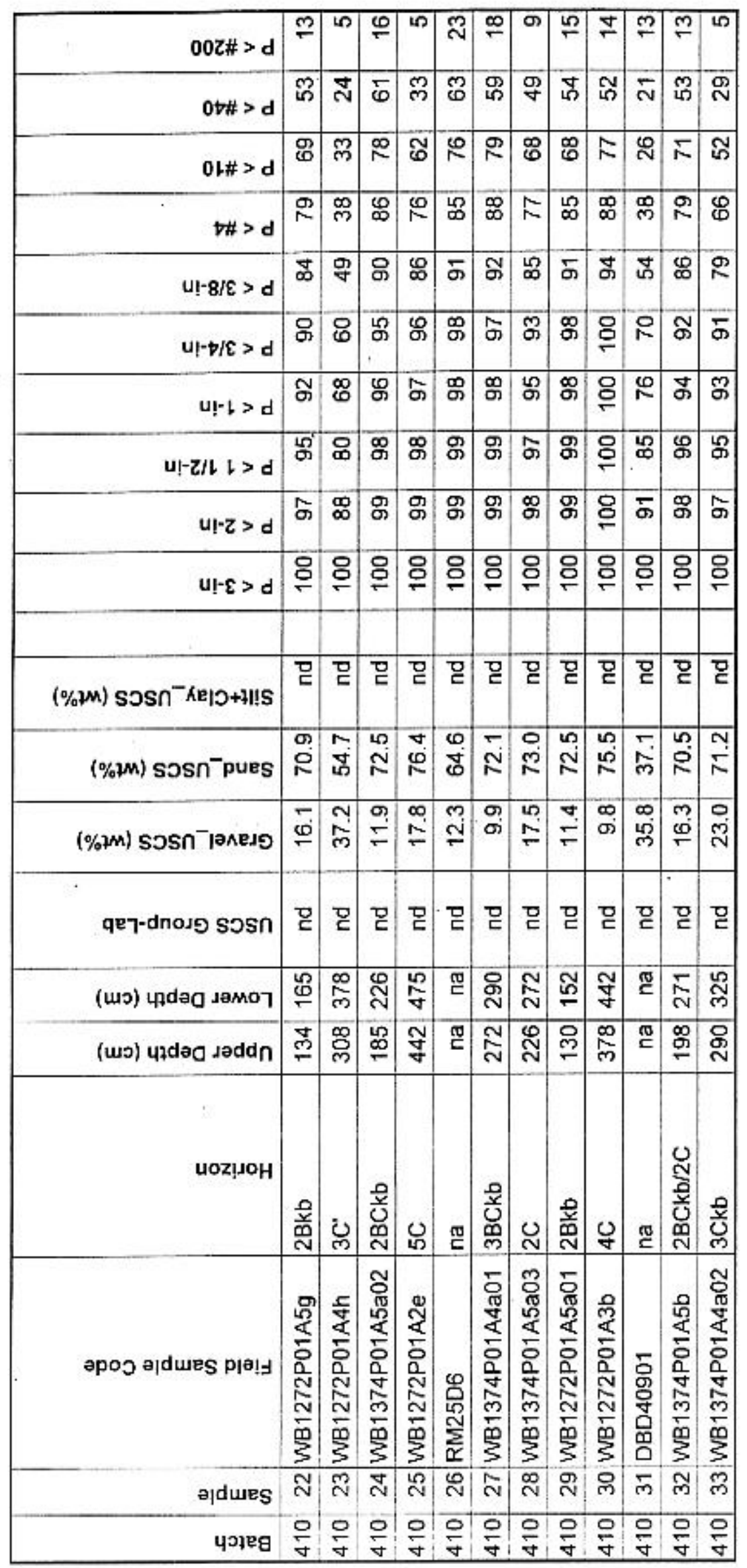


This Page Intentionally Left Blank 


\section{Distribution List}

\section{U. S. Department of Energy}

Jhon Carilli

Waste Management Division

U.S. Department of Energy

National Nuclear Security Administration

Nevada Site Office

P.O. Box 98518, M/S 505

Las Vegas, NV 89193-8518

B. M. Crowe

Apogen Technologies

U.S. Department of Energy

National Nuclear Security Administration

Nevada Site Office

P.O. Box 98518, M/S 505

Las Vegas, NV 89193-8518

U.S. Department of Energy

1

National Nuclear Security Administration

Nevada Site Office

Technical Library

P.O. Box 98518, M/S 505

Las Vegas, NV 89193-8518

U.S. Department of Energy

2 (electronic)

National Nuclear Security Administration

Nevada Site Office

Nuclear Testing Archive

Public Reading Facility

P.O. Box 98518, M/S 400

Las Vegas, NV 89193-8518

U.S. Department of Energy

1 (electronic)

Office of Scientific and Technical Information

Post Office Box 62

Oak Ridge, Tennessee 37831-0062 
Distribution List (continued)

\section{$\underline{\text { Bechtel Nevada }}$}

Max Dolenc 1

Bechtel Nevada

P.O. Box 98521, M/S NSF080

Las Vegas, NV 89193-8521

Environmental Management Library 1

Bechtel Nevada

P.O. Box 98521, M/S NLV094

Las Vegas, NV 89193-8521

Stuart Rawlinson

Bechtel Nevada

P.O. Box 98521, M/S NTS416

Las Vegas, NV 89193-8521

Dan Tobiason

1

Bechtel Nevada

P.O. Box 98521, M/S NTS416

Las Vegas, NV 89193-8521 